Safe Upper Levels for Vitamins and Minerals

May 2003

Expert Group on

Vitamins and Minerals 


\section{Contents}

Foreword 3

Executive Summary and Conclusions $\quad 5$

Introductory Chapters $\quad 9$

1. Background to the establishment of the EVM 10

2. About the EVM 13

3. Methods 15

4. The assessment of micronutrients $\quad 21$

5. General principles for assessing micronutrients 27

6. EVM risk assessments and how to interpret them 30

7. Public consultation 33

Risk Assessments 36

Part 1 - Water Soluble Vitamins

Biotin 36

Folic acid $\quad 42$

Niacin (nicotinic acid and nicotinamide) 52

Pantothenic acid $\quad 62$

Riboflavin $\quad 68$

Thiamin $\quad 74$

Vitamin $\mathrm{B}_{6}$

Vitamin $\mathrm{B}_{12}$

Vitamin C 100

Part 2 - Fat Soluble Vitamins

Vitamin A 110

B-Carotene $\quad 127$

Vitamin D 136

Vitamin E 145

Vitamin K 154

Part 3 - Trace elements

Boron $\quad 164$

$\begin{array}{ll}\text { Chromium } & 172\end{array}$

$\begin{array}{ll}\text { Cobalt } & 180\end{array}$

$\begin{array}{ll}\text { Copper } & 187\end{array}$

Germanium 197

lodine 203

Manganese 213

$\begin{array}{ll}\text { Molybdenum } & 219\end{array}$

$\begin{array}{lr}\text { Nickel } & 225\end{array}$

Selenium $\quad 232$

Tin $\quad 240$

$\begin{array}{ll}\text { Vanadium } & 246\end{array}$

Zinc $\quad 253$ 
Part 4 - Minerals

Calcium $\quad 264$

Fluoride $\quad 274$

Iron $\quad 275$

$\begin{array}{ll}\text { Magnesium } & 287\end{array}$

Phosphorus $\quad 293$

Potassium $\quad 299$

Silicon $\quad 306$

Sodium chloride $\quad 313$

Sulphur $\quad 320$

Annexes to the Report:

1. Membership of the Expert Group on Vitamins and Minerals 322 and details of the Secretariat

2. Assessment of information 324

3. Information submitted to the EVM 334

4. Current usage of vitamin and mineral supplements (VMS) in the UK 335

5. Members' interests 343

6. Consultation list respondents 346

7. Abbreviations 349

8. Glossary 351 


\section{Foreword}

The Expert Group on Vitamins and Minerals first met in 1999 to consider the safety in long-term use of vitamin and mineral supplements sold under food law with a view to recommending maximum advisable levels of intake. Since then it has endeavoured to work as openly as possible. Thus, its agendas and working papers have been freely available, and observers, nominated by Interest groups, took a full part in reviewing pertinent evidence, all with the objective of ensuring as full and balanced a review of evidence as was practicable. In keeping, the draft report was then made freely available for comment, so giving a second opportunity to ensure all relevant evidence was addressed, and addressed properly.

As a result of that consultation in the autumn of last year significant modifications were made to the draft. The determination of Safe Upper Levels or if that was not possible, Guidance Levels of intake had, of necessity, to take account of the possible effects on potentially susceptible individuals who could freely obtain vitamins and minerals and take them on a long-term basis. This implied that good quality large studies of appropriate duration were needed. The group could only work within the confines of existing evidence, and robust conclusions depended on the availability of adequate and relevant data, but in many cases the necessary information was incomplete.

The final version of the report was agreed unanimously by members, it will be for others to decide if our recommendations, which are, in essence, risk assessments, should be followed by the adoption of altered risk management strategies. In this context it should be emphasised that our recommendations do not address the medicinal uses of vitamins and minerals, which lie within the remit of the Medicines and Healthcare products Regulatory Agency.

I am extremely grateful to the members of the group for bringing us to logical and consistent conclusions, to the observers for their support in ensuring that all relevant data were properly considered, and to the secretariat for their unflagging contribution in bringing to completion a task that was more complex and took far longer to complete than expected.

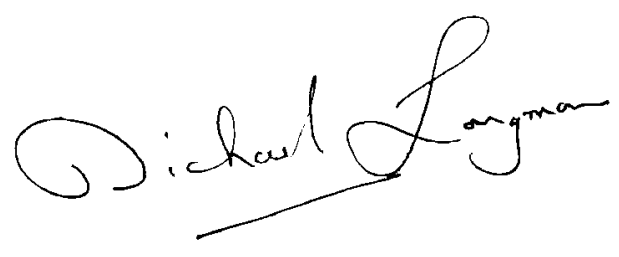

\section{PROFESSOR MICHAEL LANGMAN \\ CHAIRMAN}


Executive Summary

and Conclusions 


\section{Executive Summary and Conclusions}

The Expert Group on Vitamins and Minerals (EVM) is an independent expert advisory committee which was asked to advise on safe levels of intakes of vitamins and minerals in food supplements and fortified foods. The EVM was asked to consider only vitamins and minerals sold under food law. Review of nutritional or non-nutritional beneficial effects or non nutritional use in medicines was outside the terms of reference of the group. The use of vitamins and minerals in medicines is within the remit of the Medicines and Healthcare products Regulatory Agency (MHRA). The EVM considered a detailed review of the relevant evidence for each substance and then a risk assessment which was based on this evidence. The detailed reviews covered both nutritional and toxicological information. Thirty-four substances were assessed in detail. Safe Upper Levels (SULs) were recommended for eight vitamins and minerals and guidance was issued for twenty-two.

Recommendations for SULs made by the EVM depend on the availability of good data on both the nature and the frequency of adverse effects detected at different levels of intake. The database supporting the safety-in-use of vitamins and minerals is generally poor. There is a particular lack of reasonable-sized, well-designed comparative human studies of significant duration at different levels of intake, and with adequate assessment of possible adverse effects. Moreover, there has rarely been adequate consideration of potentially vulnerable population sub-groups such as children or older people. For some nutrients the EVM had to rely on animal data, with the use of empirical uncertainty factors in order to allow the extrapolation of the data to humans. For some vitamins and minerals the animal data were too limited for this purpose. As a consequence, the ability to give clear advice on SULs was often compromised and, in many cases, the EVM considered the evidence base inadequate to set a SUL. In such cases, and where possible, Guidance Levels have been given instead. These are based on very limited data; Guidance Levels must not be confused with, or used as, SULs.

The determination of SULs or Guidance Levels entails the determination of doses of vitamins and minerals that potentially susceptible individuals could take daily on a life-long basis, without medical supervision in reasonable safety. The setting of these levels provides a framework within which the consumer can make an informed decision about intake, having confidence that harm should not ensue. The levels so set will therefore tend to be conservative, and it is possible that for some vitamins and minerals larger amounts could be consumed for shorter periods without risk to health. However, there would be difficulties in deriving SULs for shorter term consumption because the available data are limited and relate to differing time periods. Although less susceptible individuals might be able to consume higher levels without risk to health, separate advice for susceptible individuals would be appropriate only if those individuals could recognise their own potential susceptibility.

The data were adequate to establish SULs for vitamin $B_{6}$ (page 81) B-carotene (page 127), vitamin E (page 145), boron (page 164), copper (page 187), selenium (page 232), zinc (page 253) and silicon (page 306). Guidance was given for biotin (page 36), folic acid (page 42), niacin (page 52), pantothenic acid (page 62), riboflavin (page 68), thiamin (page 74), vitamin $B_{12}$ (page 93), vitamin C (page 100), vitamin A (page 110), vitamin D (page 136), vitamin K (page 154), chromium (page 172), cobalt (page 180), iodine (page 203), manganese (page 213), molybdenum (page 219), nickel (page 225), tin (page 240), calcium (page 264), iron (page 275), magnesium (page 287), phosphorus (page 293) and potassium (page 299). 
The data were inadequate to establish either SULs or guidance for germanium (page 197), vanadium (page 246) and sodium chloride (page 313). Two minerals were not considered in detail: sulphur was excluded because intake is mainly as amino acids; fluoride was excluded as supplements are only available as licensed medicines, and fortification of food as a public health measure was not within the terms of reference of the EVM.

The EVM was not asked to consider the beneficial effects of the vitamins and minerals. Nutritional need was noted and SULs were not set at a level below dietary requirements. Some trace elements available in food supplements are unnecessary for human nutrition.

Food supplement products are used by consumers in a number of ways; these may include a desire to improve the perceived nutritional quality of their diet or self-medication (either as prophylaxis or treatment) for a range of symptoms or conditions. The remit of the EVM was to consider supplements sold under food law, thus, the non-nutritional efficacy of vitamins and minerals has not been considered since such effects would be classified as medicinal and would be within the remit of the Medicines and Healthcare products Regulatory Agency.

It has been argued that since vitamins and minerals are essential for human health, it is not appropriate to assess them in the same way in which other chemicals added to food are assessed. However, since there is much evidence that excessive intakes of some vitamins and minerals can cause harm, it is not appropriate to exclude essential nutrients from the safety assessment that is applied to other chemical substances which are added to foods.

The terms of reference of the EVM included the opportunity to advise on fortified foods. However, the EVM considered it preferable to frame its advice in terms of additional intake, which would cover both supplements and fortified foods. The SUL for a nutrient is expressed in two ways, depending upon the evidence base from which it was derived. In some cases the SULs are expressed as total intake, with an estimated safe margin for intake from both supplements and fortification within current high level dietary intake. In other cases, the SUL is expressed as a supplemental intake with an estimated safe total intake. This inconsistency arises from the nature of the data available on different vitamins and minerals, and the limited nature of the evidence base.

The data used for the dietary exposure assessments were generally taken from a National Diet and Nutrition Survey conducted in UK adults in 1986/7, which, despite its age, is the most comprehensive data set currently available? ${ }^{1}$. The market for both fortified foods and food supplements is changing and expanding. Where intake data are available these may now be somewhat outdated. It was not possible for the EVM to estimate how the market is likely to expand in the future, although this will be an important consideration for risk managers when providing advice based on the SULs that have been established. As the market and thus the exposure changes, risk managers giving advice based on these SULs will need to consider scenarios where individuals may be exposed to high levels of certain micronutrients depending on the combinations of products consumed. This changing pattern of micronutrient addition to different foods and of levels of consumption should thus be kept under review.

\footnotetext{
The most recent NDNS of adults aged 19-64 years was carried out in 2000/2001. The first report of the findings from this survey, covering the types and quantities of foods consumed, was published in December 2002. Reports on macronutrient and micronutrient intakes are due to be published in June 2003 with a further report on nutritional status and a summary report expected later in 2003.
} 
In the context of assessing intake, the EVM wishes to draw attention to the practice of using 'overages' within the food supplement industry. 'Overage' means the formulation of a product with more than the declared content of a particular nutrient to ensure that there is a sufficient level of the vitamin or mineral throughout the shelf-life of the product. This is particularly common with the fat soluble vitamins (for example vitamins A, D and E) as they are prone to oxidation. It should therefore be borne in mind that some supplements may have significantly higher levels of the vitamins and minerals at the start of their shelf-life than is indicated on the product label. Data on typical levels of overage are given in Annex 4. It is also essential that consistent, validated methods for the analysis of levels of vitamins and minerals in supplements and fortified foods are available, enabling the quoted levels to be independently verified. 
Introductory Chapters 
Introductory Chapters Chapter 1 - Background to the establishment of EVM

\section{Chapter 1 - Background to the establishment of EVM}

\section{UK regulation of products containing added vitamins and minerals}

In the UK, products containing added vitamins and minerals can be regarded in law as either foods or medicines ${ }^{2}$.

Those products for which claims are made for the treatment or prevention of disease, or which are administered to restore, correct or modify physiological functions, fall within the definition of a 'relevant medicinal product' and are subject to the requirements of the Medicines Act 1968, Directive 65/65/EEC. Such products require a marketing authorisation (licence) before they can be sold or supplied and are regulated in accordance with the Medicines for Human Use (Marketing Authorisations, etc.) Regulations 1994 [SI 1884/3144]. Products that are licensed as medicines are excluded from the definition of food in the Food Safety Act 1990. The control of medicines is the responsibility of the Medicines and Healthcare products Regulatory Agency (MHRA), which is an executive agency of the Department of Health.

In Great Britain, products that are not regulated as medicines may be regarded as foods and, as such, are subject to the general provisions of the Food Safety Act 1990, the Food Labelling Regulations, 1996 (as amended) and the Trade Descriptions Act 1968. Northern Ireland has similar but separate legislation. Food products containing added vitamins and minerals can be divided into two categories: fortified foods, such as margarine and breakfast cereals, and food supplements.

At present there are no rules on voluntary fortification of foods at EU or at UK level. Voluntary fortification of foods is permitted in the UK provided that the final foodstuff is safe and appropriately labelled. Similarly, until the EU Food Supplements Directive is implemented in national law in 2003, there is currently no definition of the term 'food supplement' in UK food law and there are no specific regulations that apply to food supplements. Fortified foods and food supplements fall within the scope of the Food Safety Act 1990, which makes it an offence to sell any food that is not safe for consumption, not of the nature, substance or quality demanded by the consumer or that is falsely or misleadingly described or labelled.

At present there are no specific limits on the levels of vitamins, minerals or other micronutrients which may be contained in supplements or fortified foods sold under food law, nor are there rules on the range of vitamins, minerals or other nutrients ${ }^{3}$ that they may contain. Food supplements can be sold without restriction, subject to the general provisions of the Food Safety Act, and, as for other foods, manufacturers are not required to demonstrate their efficacy before marketing, and food supplements are not subject to priorapproval unless genetically modified or otherwise 'novel'.

\footnotetext{
2 The regulation of products containing added vitamins and minerals is considered in working paper EVM/99/2/P which is available on the EVM website or from the Secretariat.

3 The exception to this is the amino acid tryptophan which, under the terms of the Tryptophan in Food Regulations 1990 may not be added to food.
} 


\section{Harmonisation of controls within the EU}

\section{Food supplements}

Until July 2002, when EU Directive 2002/46/EC on food supplements came into force, there were no harmonised controls on food supplements within the EU and each Member State applied its own rules. For example, many EU Member States, such as France, Spain and Italy, did not have specific legislation but usually treated products containing more than a certain multiple of the Recommended Daily Amount (RDA) (equivalent to the UK Reference Nutrient Intake (RNI)) as medicines. In other Members States, such as Denmark and Germany, products containing the RDA or a small multiple of it were acceptable as foods, but higher doses required licensing. EU Member States such as the Netherlands, with liberal safety-based regimes similar to that in the UK, were in the minority. In these Member States, some limits were applicable to particular vitamins and minerals, and these were generally based on risk assessment.

Directive 2002/46/EC on food supplements was adopted on 30th May 2002 and published in the Official Journal of the European Communities on 12th July 2002. Article 15 states that it must be implemented into Member States' law by 31st July 2003. The Directive establishes a framework for harmonised legislation covering the composition (at present limited to the vitamins and minerals that can be used) and labelling of food supplements. It also establishes a framework for setting maximum limits of vitamins and minerals in food supplements, although no such limits have yet been set.

In 1998, the Scientific Committee on Food (SCF), a committee of independent scientific experts which advises the European Commission, established a Task Force on Upper Levels to undertake a series of evaluations similar to those conducted by the EVM. Those opinions adopted by the SCF plenary group are published on the SCF website (http://europa.eu.int/ comm $/ \mathrm{food} / \mathrm{fs} / \mathrm{sc} / \mathrm{scf} /$ index_en.html). The European Commission will base its future proposal for EU rules on maximum limits of vitamins and minerals in food supplements on the advice of the SCF and its interpretation of Article 5 of the Food Supplements Directive.

\section{Fortified foods}

The European Commission published an informal draft proposal for a Directive relating to the addition of nutrients to foods in June 2000, which was discussed by Member States at a Commission working group in September 2000. The Commission followed this up in January 2003 by publishing a preliminary draft proposal for a Regulation on the addition of vitamins and minerals and of certain other substances to foods. 
Introductory Chapters Chapter 1 - Background to the establishment of EVM

\section{Establishment of the Expert Group on Vitamins and Minerals}

In view of the expanding market for food supplements, as well as the developments taking place within the EU with regard to harmonisation, the UK Government considered that it was timely to review the available data on the safe intake of vitamins and minerals from foods and food supplements. Thus in 1997, the Expert Group on Vitamins and Minerals was established.

\section{References}

The Medicines Act 1968

The Medicines Directive 65/65/EEC

Medicines for Human Use (Marketing Authorisations, Etc.) Regulations 1994 [SI 1884/3144]

The Trade Descriptions Act 1968.

The Food Safety Act 1990

The Food Labelling Regulations, 1996 (as amended).

Addition of Vitamins and Minerals to Food and Food Supplements. A Discussion paper prepared by DGIII of the European Commission. III/5934/97.

Directive 2002/46/EC of the European Parliament and of the Council of 10 June 2002 on the approximation of the laws of the Member States relating to food supplements, Official Journal of the European Communities (L183/51), 12 July 2002.

Preliminary draft proposal for a Regulation of the European Parliament and of the Council on the addition of vitamins and minerals and of certain other substances to foods.

SANCO/329/03, Brussels, 17 January 2003. 


\section{Chapter 2 - About the EVM}

\section{Terms of reference}

The terms of reference of the EVM were to:

- establish principles on which controls for ensuring the safety of vitamin and mineral supplements sold under food law can be based;

- review the levels of individual vitamins and minerals associated with adverse effects;

- recommend maximum levels of intakes of vitamins and minerals from supplements if appropriate;

- report to the Food Advisory Committee (FAC)4.

The EVM also agreed to advise on the levels of vitamins and minerals in fortified foods where appropriate.

\section{Membership}

Membership of the EVM is given at Annex 1. EVM Members were drawn from the Committee on Toxicity of Chemicals in Food, Consumer Products and the Environment (COT), the Committee on Safety of Medicines (CSM), the Committee on the Medical Aspects of Food and Nutrition Policy (COMA) and the Food Advisory Committee (FAC). The membership represented a range of expertise in toxicology, pharmacology, epidemiology, medicine and nutrition as well as a non-specialist Member to represent consumer interests. Two further EVM Members with nutritional and toxicological expertise were also nominated by the main interest groups.

The first meeting of the EVM took place in January 1999. Four observers, nominated by the main interest groups (food, health food, consumers and complementary medicines), were also appointed to act as a link between EVM and its main stakeholders. The observers were able to contribute to discussions of general issues, the individual reviews of the nutrients and subsequent draft sections of the report, but did not contribute to the risk assessment process until the final meetings of the group, when the consistency of the decisions reached at previous meetings was considered.

\section{Secretariat}

The Secretariat for the EVM was initially provided jointly by the Department of Health and the Ministry of Agriculture, Fisheries and Food. The Secretariat was transferred to the Food Standards Agency on the 1st April 2000.

Details of the Secretariat are provided in Annex 1. 
Introductory Chapters Chapter 2 - About the EVM

\section{Code of Practice and Declarations of Interest}

The Members and, where applicable, observers agreed to abide by the Code of Practice (document EVM/99/11) including the seven principles of public life as established by the Committee on the Standards in Public Life (i.e. the Nolan Committee). The Code of Practice covers matters such as the roles of the Chair, Members and Observers, and dealing with conflicts of interests.

Members were required to declare any interests prior to the discussion of the item concerned. Where a direct, personal interest was apparent, the Member concerned was not permitted to contribute to the risk assessment process. This was recorded in the minutes of the meeting. The observers were not required to declare interests since they were presumed to be representing interest groups. They were therefore unable to contribute to the risk assessment process.

\section{Openness}

The EVM agreed that its working methods should be as open as practicably possible. Accordingly, the agenda, minutes, draft scientific reviews of the individual nutrients and other working papers were made available in hard copy and were published on the EVM website (http://www.food.gov.uk/science/ouradvisors/vitandmin), subject to issues of copyright or confidentiality. Interested parties were invited to provide information in response to these papers, to be considered in updated versions at the end of the review process. However, preliminary risk assessments were not released because it was agreed that the risk assessments would need to be considered as a whole at the end of the EVM task in order to ensure consistency of approach in hazard characterisation and the use of uncertainty factors. Following this, the draft risk assessments and sections of the draft final report were released for public consultation.

\section{Involvement of interested parties}

The EVM has been keen to involve interested parties in its work. It has sought to achieve this through the activities of the observers and by issuing a call for information at the beginning of the task. As described above, the draft reviews concerning the hazard identification and characterisation were posted on the EVM website soon after completion for the consideration of interested parties, with a request for details of any relevant data not considered in the review. Since very limited data were available concerning the effects of high intakes of some micronutrients, the EVM particularly sought additional relevant information from a wide audience to ensure identification of as complete an evidence base as possible. Very little information or comment was received until the draft report was published for consultation. 


\section{Chapter 3 - Methods}

\section{Vitamins and minerals considered}

The EVM agreed to review the data on vitamins and minerals which were either essential to human health or which were available in dietary supplements. The exception to this policy was germanium, which is not known to be essential and has been withdrawn from the market in the UK due to toxicity. However, since germanium supplements can still be obtained by mail order, the EVM reviewed the data on germanium.

The list considered was:

$\begin{array}{ll}\text { Water Soluble Vitamins } & \text { Fat Soluble Vitamins } \\ \text { Biotin } & \text { Vitamin A } \\ \text { Folic acid } & \text { B-Carotene } \\ \text { Niacin } & \text { Vitamin D } \\ \text { Pantothenic acid } & \text { Vitamin E } \\ \text { Riboflavin } & \text { Vitamin K } \\ \text { Thiamin } & \\ \text { Vitamin } B_{6} & \\ \text { Vitamin } B_{12} & \\ \text { Vitamin C } & \\ & \\ \text { Trace Elements } & \\ \text { Boron } & \text { Minerals } \\ \text { Chromium } & \text { Calcium } \\ \text { Cobalt } & \text { Chloride } \\ \text { Copper } & \text { Fluoride } \\ \text { Germanium } & \text { Iron } \\ \text { lodine } & \text { Magnesium } \\ \text { Manganese } & \text { Phosphorus } \\ \text { Molybdenum } & \text { Potassium } \\ \text { Nickel } & \text { Sodium } \\ \text { Selenium } & \text { Sulphur } \\ \text { Silicon } & \\ \text { Tin } & \\ \text { Vanadium } & \\ \text { Zinc } & \end{array}$

During the course of the EVM's task, some alterations were made to this list. It became apparent that, as there were no relevant data available, chloride could not be considered alone; it was thus agreed that it would be considered as sodium chloride. It was also agreed that it was not possible to assess sulphur, since intake was largely in the form of amino acids rather than as elemental sulphur. Sulphur was excluded from the EVM's consideration and an explanatory statement prepared to set out the background to the decision (see page 320). It was agreed that fluoride was not within the remit of the EVM, because fluoride supplements are available only as licensed medicines and assessment of fluoride fortification involved a consideration of the risks and benefits which was not within the terms of reference of the EVM; therefore an explanatory statement was prepared (see page 274). 
Introductory Chapters Chapter 3 - Methods

The EVM considered a detailed review and risk assessment for each vitamin and mineral. The reviews and risk assessments were prepared either by the Secretariat or external contractors. The EVM discussed these, making changes and requesting further information as appropriate. Once all the reviews had been initially considered, they were updated with any newly published data (up to September $2001^{5}$ ) and any data submitted by interested parties as appropriate. The thirty-four risk assessments were then considered as a whole to ensure that a consistent approach had been taken in the evaluation of all the micronutrients. Copies of the reviews are on the EVM website (http://www.food.gov.uk/science/ouradvisors/vitandmin) and on a compact disk accompanying this report. This report contains the individual risk assessments, which describe the key data assessed by the EVM in reaching its provisional conclusions. When the various sections of the report were brought together and reviewed for clarity and consistency, any new data considered likely to have significant impact on conclusions were added.

\section{Types of evidence considered}

\section{Hazard assessment and characterisation}

The EVM has considered data from both human and animal studies. The types of information used are considered in more detail in Annex 2. Determining the weight of evidence as a whole requires assessment of the adequacy of each relevant study. The assessment could not be a simple addition of studies for or against a particular point but had to be based on consideration of the validity of the whole of the evidence.

\section{Hierarchy of evidence}

The EVM agreed that data from human studies would take precedence over data from animal investigations. The 'gold standard' for human studies is the double-blind placebo-controlled trial, particularly where there is reasonable evidence that those exposed are likely to be representative of the general population. Where available, such studies would have considerable weight; unfortunately very few of these are available for vitamins and minerals. It was considered appropriate to give greater weight to good quality animal data for both nutrition and toxicity where the human data were sparse or of poor quality.

In assessing the available evidence from all sources, validity of evidence depends not only on the appropriateness of the study type but also on the quality of its design, execution and analysis. The relationship between a micronutrient and an adverse event can be demonstrated by a number of different types of studies and designs. Sound conclusions will depend on sound evidence.

\footnotetext{
${ }^{5}$ The exceptions to this were vitamins A and E, where new data relevant to the risk assessment became available in January 2002 and July 2002 respectively.
} 


\section{Human studies}

The human database on the non-nutritional effects of vitamins and minerals is generally limited. This may be due partly to the difficulty of assessing the effects of micronutrients in isolation from the rest of the diet. Some epidemiological studies exist, for example where populations are exposed to high levels of minerals such as selenium or boron due to the local geology or other such circumstances. There are also some large-scale intervention trials, e.g. the Alpha Tocopherol Beta Carotene (ATBC) Prevention study in Finnish male smokers or the B-Carotene and Retinol Efficacy Trial (CARET), also in smokers, but these have tended to assess specific end points, such as the incidence of different cancer types or stroke. Other supplementation trials have been conducted, but these have usually been designed to assess the beneficial or metabolic effects of nutrients and there is often limited reporting of adverse effects. It is frequently unclear whether this was because they did not occur, because the evidence of adverse events was not specifically sought or because the events were not reported. Where reports of adverse effects are included, it is generally not clear whether they were obtained in a systematic and objective manner, or were self-reported.

The therapeutic use of high doses of some vitamins for the treatment or prevention of illness has been an important contributor to data on human exposure. Examples include $ß$-carotene, nicotinic acid, nicotinamide and pyridoxine $\left(B_{6}\right)$, but inadequate dose-response data make assessment of the likely risks associated with lower non-therapeutic intakes difficult to quantify. Case reports of accidental or deliberate poisonings are sporadic and uncontrolled, and thus can be difficult to interpret.

\section{Nutritional studies}

A range of nutritional studies in both human volunteers and laboratory animals has been considered. These studies have included investigations of the effects of deficiency or excess, nutrient balance, bioavailability or metabolism. The quality of these studies has varied.

\section{Animal toxicity and in vitro toxicity data}

The animal toxicity data considered by the EVM have included a range of repeat and single dose studies. Generally these have involved oral exposure. The animal database is also frequently of inadequate standard, many of the available studies being poorly conducted and/or reported, although these studies may have reflected the standards of the time. Few of the micronutrients have the extensive toxicity database comprising short and long term studies as well as reproductive, carcinogenicity and genotoxicity studies that would be required for the registration of other 'synthetic chemicals's such as drugs or food additives. Data from in vitro studies have also been considered.

\footnotetext{
${ }^{6}$ Although some vitamins and minerals may be extracted from natural sources, many will be chemically synthesised and in some cases differ from the chemical forms found in food.
} 
Introductory Chapters Chapter 3 - Methods

\section{Exposure assessment}

Nutritional surveys

Three different types of nutritional surveys were considered by the EVM. The National Food Surveys and Total Diet Studies provide nutrient intake data for the population, whereas the National Diet and Nutrition Surveys are detailed dietary surveys for individuals from specific population age groups. Further details on exposure assessment are provided in Annex 2.

The National Food Survey (NFS) ${ }^{7}$ reports annually on household food purchases. The NFS provides information on population trends in food consumption and nutrient intakes at the household level. The Total Diet Study (TDS) is a continuous study in which foods representing the average UK diet are analysed. The results are widely used to estimate exposure to nonnutrients, such as contaminants, as well as the intake of some nutrients. It is particularly useful for estimating intakes of some nutrients where food composition data are lacking. The National Diet and Nutrition Surveys (NDNS) are a rolling programme of detailed dietary surveys of individuals divided into specific population age groups: children aged $11 / 2-41 / 2$ years; young people aged 4-18 years; adults aged 19-64 years; adults aged 65 years and over. The mean and high level intakes for adults have been considered when estimating exposure/intake levels in the risk assessments. These detailed data are included in the EVM review of each individual nutrient. However, to simplify the maximum exposure estimates in the risk assessments, the intake data have been summarised to provide a single figure for mean and high level intakes of each nutrient from food sources only (excluding supplements), usually taken from the 1986/7 NDNS adult survey (see Table 1). Where NDNS data were not available, information on mean and high level consumption has been taken from the NFS. Where survey data were not available, exposure data from the published scientific literature have been used in the EVM assessments. The NDNS is the most comprehensive database available; however, in most cases, exposure information was taken from the 1986/87 survey and thus it may not accurately reflect current intakes in all cases since consumption patterns of food supplements and fortified foods have changed over recent years. Dietary intake includes intake from beverages such as tea but does not include exposure from drinking water, which has been considered separately where appropriate.

Data on the ranges of vitamin and mineral contents of food supplements available in the UK as well as their sales were supplied by the manufacturers and are given in Annex 4. Potential exposure to vitamins and minerals from food supplements was also estimated from the OTC Directory 2001-2002. These sources of information were used to provide a single estimate of maximum exposure from supplements, which is included for comparative purposes.

\section{Uncertainty}

In order to avoid an impression of precision that would be inappropriate in view of the uncertainties involved in deriving estimates of nutrient intake and safe upper levels, the EVM agreed that the values for intakes, Safe Upper Levels and Guidance Levels should be expressed to a maximum of two significant figures. 
Table 1: Mean and high level intakes of vitamins and minerals

\begin{tabular}{|c|c|c|c|}
\hline \multirow[t]{2}{*}{ Nutrient } & \multicolumn{2}{|c|}{$\begin{array}{l}\text { Daily exposure estimates from food } \\
\text { sources, excluding supplements, for } \\
\text { men and women (in } \mathrm{mg} \text { ) }\end{array}$} & Source of data \\
\hline & Mean & 97.5\% ile & \\
\hline \multicolumn{4}{|c|}{ Water-soluble vitamins } \\
\hline Biotin & 0.034 & 0.067 & Gregory et al., 1990 \\
\hline Folic acid & 0.26 & 0.49 & $" \quad "$ \\
\hline Niacin equivalents & 34 & 57 & $" \quad "$ \\
\hline Pantothenic acid & 5.4 & 9.7 & $" \quad "$ \\
\hline Riboflavin & 1.8 & 3.3 & $" \quad "$ \\
\hline Thiamin & 1.5 & 2.6 & $" \quad "$ \\
\hline Vitamin $B_{6}$ & 2.0 & 3.9 & $" \quad "$ \\
\hline Vitamin $B_{12}$ & 0.0062 & 0.020 & $" \quad "$ \\
\hline Vitamin C & 64 & 160 & $" \quad "$ \\
\hline \multicolumn{4}{|l|}{ Fat-soluble vitamins } \\
\hline Vitamin A (median) & $1.1(0.52)$ & 6.0 & $" \quad "$ \\
\hline Total carotene & 2.3 & 7.0 & $" \quad "$ \\
\hline Vitamin D & 0.003 & 0.009 & $" \quad "$ \\
\hline Vitamin E & 8.5 & 18 & $" \quad "$ \\
\hline Vitamin K & 0.068 & - & DEFRA, 2001 \\
\hline \multicolumn{4}{|l|}{ Trace elements } \\
\hline Boron & 1.5 & 2.6 & MAFF, 1997 \\
\hline Chromium & 0.1 & 0.17 & MAFF, 1999 \\
\hline Cobalt & 0.012 & 0.019 & MAFF, 1997 \\
\hline Copper & 1.4 & 3.0 & Gregory et al., 1990 \\
\hline Germanium & 0.004 & 0.007 & MAFF, 1997 \\
\hline lodine & 0.22 & 0.43 & Gregory et al., 1990 \\
\hline Manganese & 4.9 & 8.2 & MAFF, 1997 \\
\hline Molybdenum & 0.11 & 0.21 & $" \quad "$ \\
\hline Nickel & 0.13 & 0.21 & $" \quad "$ \\
\hline Selenium & 0.039 & 0.10 & MAFF, 1999 \\
\hline Tin & 1.8 & 6.3 & $" \quad "$ \\
\hline Vanadium & 0.013 & - & MAFF, 1980 \\
\hline Zinc & 9.8 & 17 & Gregory et al., 1990 \\
\hline \multicolumn{4}{|l|}{ Major minerals } \\
\hline Calcium & 830 & 1500 & $" \quad "$ \\
\hline Chlorine & 4400 & 7800 & $" \quad "$ \\
\hline Iron & 12 & 24 & $" \quad "$ \\
\hline Magnesium & 280 & 510 & $" \quad "$ \\
\hline Phosphorus & 1300 & 2100 & $" \quad "$ \\
\hline Potassium & 2800 & 4700 & $" \quad "$ \\
\hline Sodium & 2900 & 5100 & $" \quad "$ \\
\hline
\end{tabular}

Notes: Data are expressed as two significant figures. The median as well as the mean is provided for vitamin A because the mean is influenced by the individuals who have a very high vitamin A intake from liver and its products. Survey data were not available for silicon. Data from the published literature have been used.

Specific groups of consumers with potentially high intakes have been identified in the risk assessments of the individual vitamins and minerals as appropriate. Where food is consumed with similar frequency, children are inevitably exposed to higher levels of vitamins or minerals than adults because of their lower body weights. 
Introductory Chapters Chapter 3 - Methods

\section{References}

DEFRA (2001). Provisional estimate based on consumption data from National Food Survey 2000. National Food Survey 2000. The Stationery Office, London.

J Gregory, K Foster, H Tyler and M Wiseman. The dietary and nutritional survey of British adults. London: HMSO, 1990.

MAFF 1980 Total Diet Study.

MAFF (1997) 1994 Total Diet Study: metals and other elements. Food Surveillance Information Sheet No. 131.

MAFF (1999) 1997 Total Diet Study: Aluminium, arsenic, cadmium, chromium, copper, lead, mercury, nickel, selenium, tin, zinc. Food Surveillance Information Sheet No. 191.

OTC Directory 2001-2002. The Proprietary Association of Great Britain, Communications International Group, London. 


\section{Chapter 4 - The assessment of micronutrients}

Nutrients differ from other chemical substances used in foods in that they are essential for human health, so that adverse effects can result from intakes that are too low as well as too high. The balance between deficiency and toxicity is often expressed as below:

Figure 1. Theoretical dose-response relationships in humans

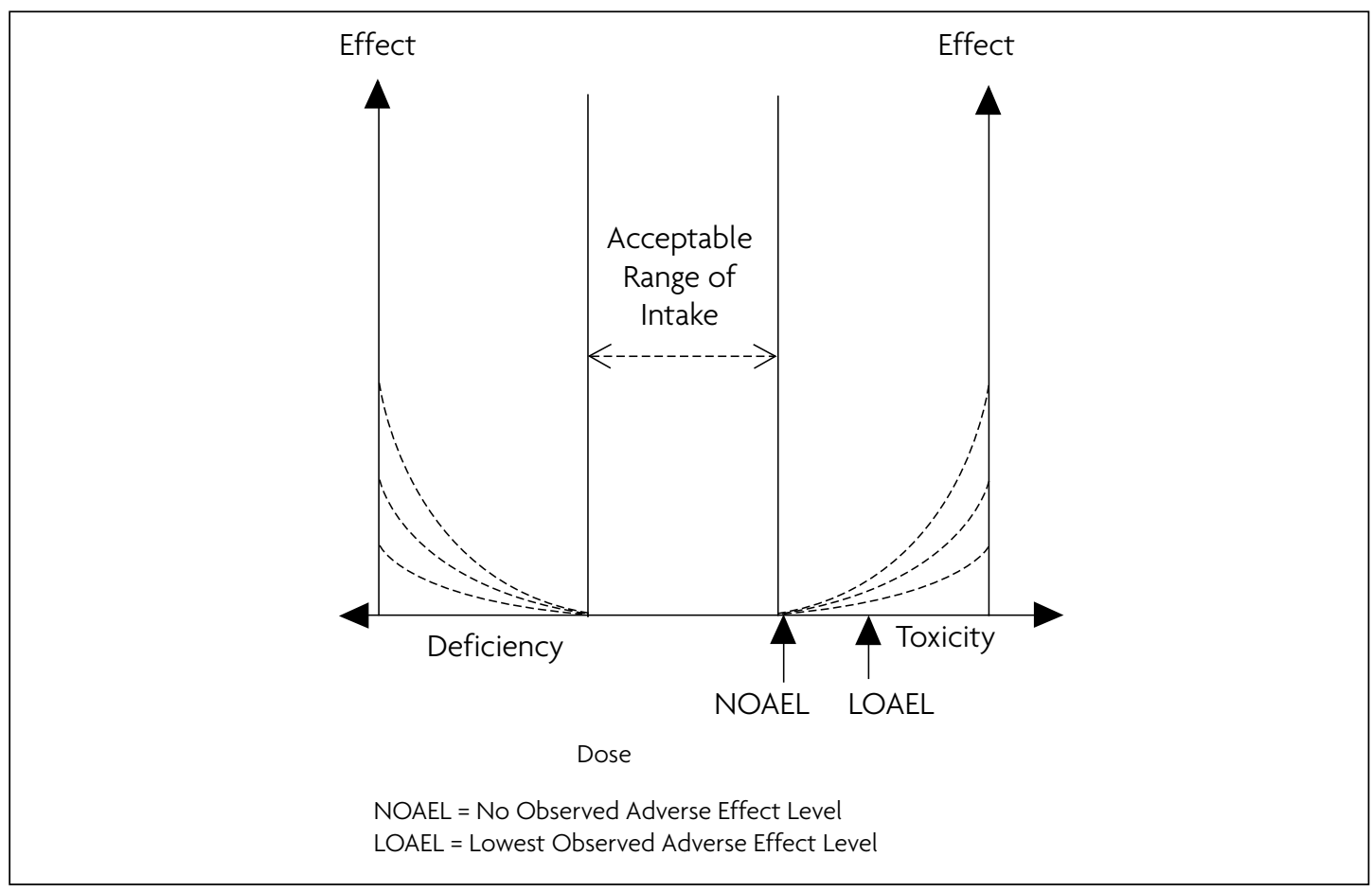

Within the acceptable range of intake, neither deficiency nor toxicity would occur in normally susceptible people. The margin between necessity (that is, the prevention of deficiency) and toxicity can range from a few fold for trace elements such as selenium, to orders of magnitude for some of the B group vitamins such as biotin or pantothenic acid. In practice, the magnitude of the adverse effects attributable to either deficiency or toxicity may differ, giving the curve, for example, a J shape.

Where the margins between necessity and toxicity are narrow, application of conventional methods of risk assessment, such as would be used in the establishment of an Acceptable Daily Intake (ADI), could result in recommended safe levels which would be below those that are essential. This would occur because of the use of uncertainty factors, which are applied when extrapolating data from laboratory animals to humans or from small human studies to the general population. 
Dietary Reference Values (DRVs) for energy and nutrients were most recently set for the UK in 1991 (COMA, 1991). They take account of the biological variation between individuals, which determines the different nutrient needs to meet specific criteria. Normal metabolic needs for healthy individuals such as growth are taken into account, although the DRVs make no allowances for the different energy and nutrient needs imposed by diseases.

For most nutrients, the DRVs comprise three levels of intake. The RNI is the amount of a nutrient which is adequate for nearly all (97.5\%) of a population group, the Estimated Average Requirement (EAR) is the average requirement for the population (i.e. adequate for $50 \%$ of the population) and the Lower Reference Nutrient Intake (LRNI) is the amount of a nutrient which is adequate for only $2.5 \%$ of a population group, those with the lowest needs. In establishing the DRVs it is assumed that requirements for a nutrient follow a normal distribution (see Figure 2, below) so that the RNI is notionally two standard deviations above the EAR and the LRNI is two standard deviations below it.

Figure 2. Nutritional requirements

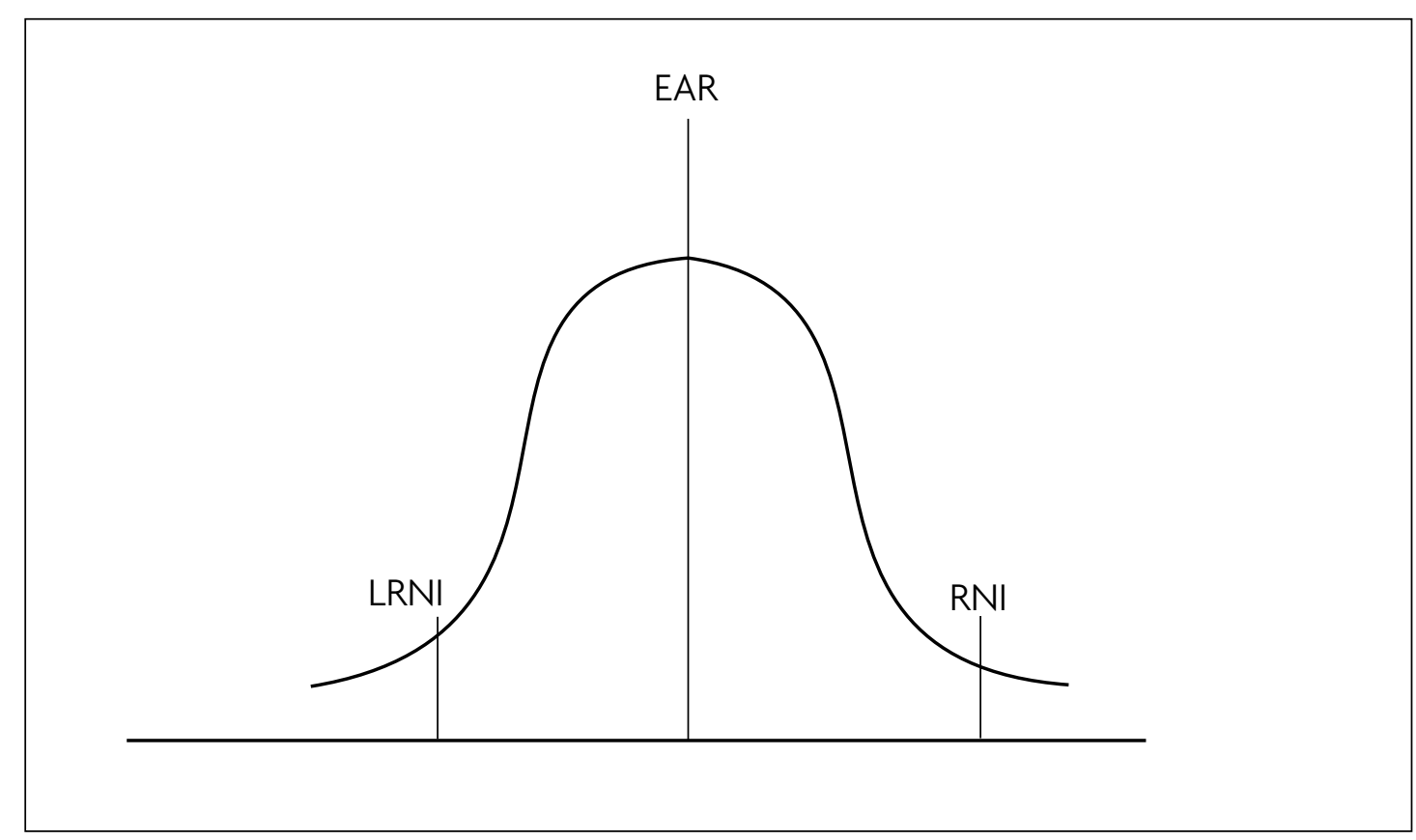

Where the acceptable range of intake is narrow, a level of intake necessary for an individual with a high requirement may be excessive or result in adverse effects for individuals with a lower requirement.

The Committee on Medical Aspects of Food and Nutrition Policy could not set an RNI for some nutrients as there were insufficient reliable data on human requirements to do so. In some instances COMA set a Safe Intake ${ }^{8}$.

8 A level or range of intake at which there is no risk of deficiency, and below a level where there is a risk of undesirable effects. 
A variety of approaches has been taken in the assessment of the safety of high intakes of nutrients ${ }^{9}$, ranging from schemes where the upper level is a set multiple of the population reference intake (for example, three-fold) or where a set fraction (such as 10\%) of an identified undesirable chronic dose is deemed acceptable. More detailed formulae have also been proposed; these have involved the use of fixed and/or variable safety factors whilst taking into account a population reference intake of the nutrient concerned (such as the US Recommended Dietary Allowance).

As a result of the variable quality of the available database, as well as the differing margins between necessity and toxicity, the EVM concluded that no single scheme was satisfactory and that it was more appropriate to consider each nutrient individually. Safe Upper Levels (SULs) were established where supported by adequate data and guidance was given on safe levels of intake where establishing SULs was not possible. The EVM SUL represents an intake that can be consumed daily over a lifetime without significant risk to health on the basis of available evidence. Where Guidance Levels have been established, these should not be used as SULs. Guidance Levels have been given as an approximate indication of levels that would not be expected to cause adverse effects, but have been derived from limited data and are less secure than SULs. Situations in which a SUL could not be set are discussed further in Chapter 6.

As described above, SULs or Guidance Levels are the doses of vitamins and minerals that susceptible individuals could take daily on a life-long basis, without medical supervision. The levels have been derived so that the consumer can have confidence that harm should not ensue from daily intake up to that level. The risk assessments, as outlined in this report, have usually focused on one or two adverse effects, which were identified as the critical effect(s) after consideration of all available safety data.

The SULs set by the EVM may be considerably lower than might be appropriate during medical treatment, where the balance between therapeutic benefit and safe use can be monitored. It also follows that exploration of potential benefits during clinical research would not necessarily be limited by the SUL or the guidance, which applies to unsupervised use by the general population.

\section{Uncertainty factors}

Safety or uncertainty factors are applied to allow for uncertainties in the use of data obtained from human or animal studies in order to establish the amount of a particular substance that can be consumed without harm. Typically, the intake level at which no adverse effects are observed in animal or human studies (i.e. the No Observed Adverse Effect Level or NOAEL see Figure 1) is divided by an appropriate uncertainty factor, with the result representing a level of intake that can be consumed by the general population daily over a lifetime without significant risk to health. Uncertainty factors are empirical values used in the absence of more detailed data. Safety or uncertainty factors have a long history of use by national and international regulatory bodies in order to establish safe levels of intake of compounds present in the human diet (IGHRC, 2003). The terms 'safety factor' and 'uncertainty factor' are largely interchangeable, but the latter is being used more in recent years.

\footnotetext{
9 Previous approaches to the assessment of nutrients are considered in working paper EVM/99/13 which is available on the EVM website or from the Secretariat.
} 
The numerical value of the uncertainty factor used varies in size depending on the nature of the database and the various uncertainties that have to be taken into account. The factors vary in size according to the type of data being considered, and could range from a value of 1 if adequate data were available for potentially vulnerable human subjects up to 100 or more if the safe intake had to be based on a study in animals, because adequate human data were not available. The most commonly used factors are to allow for inter-individual and inter-species variations. These factors allow for differences in sensitivity between individuals and between species that may result from differences in, for example, absorption, metabolism or biological effect of the substance under consideration. Uncertainty factors can also be applied to account for uncertainties due to:

- database deficiencies, such as the absence of a NOAEL requiring extrapolation from a Lowest Observed Adverse Effect Level (LOAEL);

- a poor database, such as the absence of adequate data on chronic or reproductive toxicity;

- a study involving few subjects;

- the severity of a particular adverse effect.

It is common practice in risk assessments to use separate factors for each area of uncertainty, with typical values being 10 for inter-individual variation, 10 for species differences and 3 for extrapolation of a LOAEL to a NOAEL. The separate factors are multiplied on the assumption that they are independent variables. The use of more refined values requires data specific to the chemical under consideration to support the use of a smaller or larger chemical-specific factor (Renwick, 1991, 1993).

The use of uncertainty factors has evolved since their introduction in the early 1960s ${ }^{10}$ (Lu, 1988). They were first used in the establishment of an Acceptable Daily Intake (ADI). A NOAEL from a study in animals was divided by a factor of 100, which was subsequently split into a factor of 10 for possible inter-species differences multiplied by a second factor of 10 for inter-individual (person to person) variation. That is:

$$
A D I=\quad \frac{\text { No Observed Adverse Effect Level }}{10 \times 10}
$$

Since then the process has been developed to allow the use of chemical-specific adjustment factors by sub-dividing the 10-fold factors into separate components representing toxicokinetic ${ }^{11}$ and toxicodynamic ${ }^{12}$ differences and, incorporating suitable chemical-specific experimental data where available (IPCS, 2001). There are no a priori reasons why species differences and human variability would be any different in relation to the toxicity of high doses of micronutrients, compared with any other form of chemical toxicity.

\footnotetext{
10 More detail on the history and use of uncertainty factors is contained in working papers EVM/99/14 and EVM/01/05, which are available on the EVM website or from the Secretariat.

11 Toxicokinetics is the uptake of potentially toxic substances by the body, the transformation they undergo, the distribution of the substance and its metabolites in the body tissues and the elimination of the substance and its metabolites from the body.

12 Toxicodynamics is the action of a potentially toxic substance in living systems, including reaction with or binding to cellular constituents and the biochemical and physiological consequences of these effects.
} 


\section{Use of uncertainty factors in assessment of micronutrients}

It has been suggested that very low uncertainty factors are required in the assessment of vitamins and minerals because large numbers of people consume vitamin and mineral supplements without apparent harm. Whilst an uncertainty factor would not be necessary if a NOAEL were available from comprehensive studies in sensitive human populations, the simple consumption of a vitamin without adequate health monitoring cannot be used as the basis for risk assessment. Relevant data from adequately conducted studies in humans given the vitamin or mineral and from epidemiological studies have been considered as part of the database. However, for most vitamins and minerals there is a lack of data to support the view that uncertainty factors are unnecessary, whereas there is clear evidence that excessive intakes of vitamins and minerals may be harmful.

The EVM agreed that it was appropriate to use uncertainty factors in assessing nutrients but the assessment should also take account of the lower advisory levels below which intake should not fall. The EVM also agreed that it was appropriate to use chemical-specific adjustment factors where possible, but recognised that extensive information on toxicokinetics and/or mode of toxicity was necessary for the calculation of such adjustment factors, and that there were few substances where the available database actually allowed this.

Where extrapolation from a LOAEL to a NOAEL was necessary, it was agreed that the size of the factor should vary depending on the available dose-response data and the severity of the adverse effect. For example, a severe or irreversible effect such as reproductive toxicity would result in a factor of 10, whereas a small change in biochemical parameters without associated clinical effect, such as changes in enzyme activities, would attract an uncertainty factor such as 3 or lower. The factor of 3 is commonly used when extrapolating from a LOAEL to a NOAEL for data derived from studies in experimental animals. This is because the dose levels used in such studies are commonly at 3-fold intervals. In deriving SULs or Guidance Levels from human data, the EVM similarly used the 3-fold factor unless the available data indicated that an alternative value was more appropriate.

The Interdepartmental Group on Health Risks from Chemicals (IGHRC, 2003) is currently undertaking a review of the use and application of uncertainty factors by different UK Governmental Departments and the relevant Scientific Advisory Committees. This review (in preparation) has shown that there is general consensus, both nationally and internationally, on the use of the usual 100-fold factor when using data from a study in animals to predict a safe level of exposure for the general population. 
Introductory Chapters Chapter 4 - The assessment of micronutrients

\section{References}

COMA (1991). Dietary Reference Values for Food Energy and Nutrients for the United Kingdom. Report of the Panel on Dietary Reference Values, Committee on Medical Aspects of Food and Nutrition Policy. HMSO, London.

IGHRC (2003). Interdepartmental Group on the Health Risks from Chemicals. Report on uncertainty factors: their use in UK government toxicological risk assessment (in preparation).

IPCS (2001). International Programme on Chemical Safety: Guidance document for the use of chemical-specific adjustment factors (CSAFs) for interspecies differences and human variability in dose-concentration response assessment. World Health Organisation, Geneva. http://www.ipcsharmonize.org/csaf-intro.html

Lu, F.C. (1988). Acceptable Daily Intake: inception, evolution, and application. Regulatory Toxicology and Pharmacology 8, 45-60.

Renwick, A.G. (1991). Safety factors and the establishment of Acceptable Daily Intakes. Food Additives and Contaminants 8, 135-150.

Renwick, A.G. (1993). Data-derived safety factors for the evaluation of food additives and environmental contaminants. Food Additives and Contaminants 10, 275-305. 


\section{Chapter 5 - General principles for assessing micronutrients}

As part of its terms of reference, the EVM considered the general principles that should underpin the establishment of Safe Upper Levels for vitamins and minerals. These general principles can be summarised as follows:

Nutrients should be assessed on an individual basis. The lack of complete and coherent bodies of evidence, particularly based on human studies, makes it appropriate that micronutrients are considered individually. However, it is important that where the quality and quantity of data are comparable they are handled consistently.

Where the database is inadequate to establish a SUL, guidance should be given. For the majority of vitamins and minerals, the available database is inadequate to establish Safe Upper Levels. For these nutrients, guidance has been given on the levels that would not be expected to be associated with adverse effects but acknowledging that such Guidance Levels may not be applicable to all life stages or for life-long intake.

Where no adverse effects have been identified, SULs are not necessary but some guidance should be given. For some nutrients, the available data indicate that known consumption levels are not associated with any adverse effects, that is, an effect level has not been established. However, the database for the nutrient may be sparse. It is clearly only possible to consider the available data and unwise to speculate about the possible effects at higher levels. Therefore guidance should be given for such nutrients in order to ensure that the highest levels at which no adverse effects have been identified are stated and the reliability of the database is described.

Uncertainty factors should be used when necessary in the assessment of SULs. There is no evidence that essential micronutrients should be regarded as inherently safe at high doses and much evidence, varying according to the substance concerned, that excessive intakes can cause harm. It is appropriate to use uncertainty factors in assessing nutrients, provided that the assessment takes into account the levels below which intake should not fall. An uncertainty factor to allow for human variability may be necessary if the safety data in humans is restricted to a small group of individuals, and where study data do not include all potentially vulnerable groups. Where evidence from studies in humans is limited, and/or there is evidence from animal studies that suggests possible risk, there is a need to apply an interspecies uncertainty factor to the animal data. The uncertainty factors used should be replaced by factors specific to the chemical concerned wherever possible. Where the database is inadequate, the numeric values of the uncertainty factors should be considered carefully to ensure that they are appropriate.

The recommended intakes of the nutrients should be taken into account. Recommended intakes do not need to be formally or numerically included in any calculations but should be considered in making the assessment, formulating guidance and in considering vulnerable groups, since some groups will be vulnerable to deficiency as well as to toxicity. 


\section{Introductory Chapters Chapter 5 - General principles for assessing micronutrients}

Intakes from all sources should be taken into account. In assessing the nutrients, intakes from all sources should be considered, except where the data demonstrate that adverse effects are specifically due to the effects of supplemental dosing. For example, a large dose of a particular nutrient might overwhelm physiological mechanisms resulting in effects that would not occur where exposure was through food and thus more gradual. In this case it should be clear that the Safe Upper Level applies to supplemental doses only.

The EVM also identified a number of more specific principles that apply to their assessment of the data. These are considered within the text as appropriate but have also been summarised below:

The most important studies in the risk assessment should be identified. These studies have been described in the assessments, with the relevant strengths and weaknesses of the studies being noted.

Gaps in the database should be acknowledged. Gaps in the database are inevitable and in many cases are large enough to hinder assessment.

The claimed benefits of individual nutrients at levels in excess of the recommended nutritional intake should be noted, but not taken into account. It is often claimed that some nutrients have benefits over and above their nutritional role. However, risk/benefit analysis is not within the remit of the EVM. Therefore, benefits have not been considered, but such claims have been noted for completeness. Where the reported benefit has been endorsed by an official body such as COMA, this has also been noted by the EVM. Where benefits are claimed by the authors of published studies or in marketing information, these have been neither evaluated nor endorsed by the EVM.

Good quality human data should be given more weight than data from laboratory animals. Although good quality animal data may provide valuable information on hazard identification, mechanisms and dose-response relationships, good quality data from humans are preferable because of the innate metabolic differences between laboratory animals and humans. Where human data are poor, animal data may be more valuable in establishing SULs. The use of data from studies in humans avoids the need for an inter-species uncertainty factor; if there is a large study of potentially vulnerable group(s) this would remove the need for an uncertainty factor for human variability.

Vulnerable groups should be identified. Some groups of the population may be particularly susceptible to the effects of a particular substance. Such groups might include children, older people or genetically diverse groups and should be specifically considered. The vulnerable groups are generally sub-groups of the healthy general population rather than individuals made vulnerable through disease, but, where necessary and where supporting evidence was available, consideration was given to groups made vulnerable through disease.

SULs should be expressed as total intakes where possible. Where possible, SULs or Guidance Levels should be expressed in terms of total micronutrient intakes (from all sources, including foods that are currently fortified). By inference, the difference between high level current dietary intakes of the nutrient and the SUL is an indication of the maximum potential range for 
additional intakes by means of additional supplementation or fortification. However, for the majority of nutrients considered by the EVM, the data were inadequate to establish total intakes. This generally occurred because many studies considered by the EVM had investigated the effects of supplemental intakes of the micronutrients without specifying the level already present in the diet. SULs or guidance for supplemental levels are given and a maximum total intake for the micronutrients can be estimated by adding the estimated dietary intakes to the supplemental SULs.

SUL (total intake) - (intake from dietary and other known exposures) = available margin for additional intake exposure from supplements or new sources of fortification.

SUL (supplemental intake) + (dietary and other known exposures) = estimated SUL (total)

The EVM noted that these calculations used the most recent available information on dietary intake. This was primarily from the 1986/87 dietary and nutritional survey of British Adults (see Chapter 3), which may not accurately reflect current intakes. Additional fortification of processed foods could result in increased dietary exposure to some vitamins and minerals, such that the total SUL could be exceeded. 


\section{Chapter 6 - EVM risk assessments and how to interpret them}

\section{Approaches to risk analysis ${ }^{13}$}

Risk analysis is conventionally considered as three separate processes: risk assessment, risk management and risk communication.

\section{Approach taken by EVM}

The EVM is concerned solely with risk assessment, which comprises:

- hazard identification;

- hazard characterisation (including dose-response assessment);

- exposure assessment; and

- risk characterisation.

The available database is reviewed and hazards (adverse effects) identified and characterised. The hazards are compared to known levels of exposure and the risk determined.

\section{Risk-benefit analysis}

The EVM has not conducted risk/benefit analyses of the nutrients since beneficial effects in excess of the nutritional value of the vitamins and minerals are not within its remit.

\section{How to interpret EVM risk assessments}

The available data have been summarised and a risk assessment conducted. The risk assessments have been set out in a standardised format. The studies to which EVM paid particular attention in the risk assessment have been identified. Following the section setting out the SUL or the guidance, a section of text has been included to explain the EVM view and provide any necessary qualification or aid to interpretation. Risk managers should bear in mind that where uncertainty factors are used they reflect a judgement on the uncertainty inherent in characterising the risk.

Although the risk assessments provide a brief summary of the available data and have been designed to stand alone, they are best read in conjunction with the detailed reviews. In particular it should be noted that the reviews contain the full reference list with only a selection of the references being included in the risk assessments. The individual reviews are available on the compact disk supplied with this report, on the EVM website or from the Secretariat. 


\section{Setting a Safe Upper Level (SUL)}

Where the database was sufficient to establish a SUL, the NOAEL or LOAEL identified has been given, together with any uncertainty factors that have been used and the reason for their use.

The SUL applies to chronic/lifetime intake by the general population. It is expressed in terms of $\mathrm{mg}$ per person per day (mg/person/day) and $\mathrm{mg}$ per $\mathrm{kg}$ body weight per day $(\mathrm{mg} / \mathrm{kg}$ bw/day), assuming a body weight of $60 \mathrm{~kg}$, in accordance with procedures defined by the World Health Organisation. SULs are given for both total and supplemental intake depending on the nature of the database. Estimates of upper levels of total intake are given where appropriate. The fraction of the SUL which is not accounted for by known dietary or other exposures can be taken to indicate a safe level of additional intake, bearing in mind changing eating patterns and the potential for some consumers to have intakes above the population intake value indicated. The SUL does not apply to high doses of vitamins or minerals used under medical supervision.

The SULs apply to healthy individuals in the general population and represent lifetime exposure. They can be applied to children by scaling for body weight or body surface area as appropriate, unless it is specifically indicated that children are particularly vulnerable to the effect concerned or have a greater requirement. However, it should be noted that inappropriate scaling may lead to a child's recommended intake not being met. Extrapolation of SULs based on studies in adults, to infants and children needs careful consideration, especially in relation to ensuring adequate nutritional intake of those vitamins and minerals for which the SUL in adults is close to the RNI. Other vulnerable groups have been identified in some cases and, where appropriate, have been accounted for in the SULs established or guidance given. In some cases, the data are inadequate to do this and other risk management strategies might be necessary.

\section{Situations in which a SUL cannot be set}

There are three situations where SULs for nutrients have not been established:

- For some nutrients, the available data indicate that known consumption levels are not associated with any adverse effects; that is, an effect level has not been established. For these nutrients, the text specifies the levels at which data are available. It should be noted that the effects of consuming the nutrient at levels in excess of this are unknown, may not be safe and that the database for such nutrients may be limited. Uncertainty factors have not been used in deriving guidance in such situations, unless the database is particularly limited.

- In contrast, where adverse effects have been identified but data are limited, suggested maximum intakes are given using appropriate uncertainty factors. However, these values are considerably less secure than the SULs and should be interpreted with caution. As with the SULs, Guidance Levels have been given as total or supplemental intakes. In a very few cases the database was so limited that it was not possible to provide guidance. 
Introductory Chapters Chapter 6 - EVM risk assessments and how to interpret them

- For some nutrients, such as sodium chloride, the adverse effect concerned does not have a defined threshold, but is a continual graded response such that risk increases with increasing intake i.e. there may be a small risk of an adverse effect at current levels of intake.

As with SULs, guidance is given for both total and supplemental exposure depending on the database. However, although guidance for total intakes has been estimated, margins for additional intake have not been since the database is too limited to allow this. 


\section{Chapter 7 - Public consultation on the EVM draft report}

A draft report on the Safe Upper Levels for Vitamins and Minerals was issued for a 12 week public consultation on 29th August 2002. Responses were invited from interested parties on:

- scientific evidence that may have been missed;

- the interpretation of the scientific evidence by the EVM; and

- any additional supporting scientific evidence that was considered appropriate.

One hundred and three responses were received in total, which covered a range of topics including the individual vitamins and minerals as well as more general points. Only four vitamins and minerals did not attract comment; these were: cobalt, potassium, thiamin and tin. The majority of respondents disagreed with the SULs and Guidance Levels set by the Group. Many of the respondents commented on the application of uncertainty factors. A list of respondents can be found in Annex 6. A consultation summary is available on the Food Standards Agency website: http://www.food.gov.uk/science/ouradvisors/vitandmin. Full copies of the consultation responses are available on request from the Food Standards Agency library.

The majority of respondents who sent in comments were interested members of the public (38\%), followed by academics (20\%), industry and complementary health practitioners (12\% each), the remainder coming from Local Government, NHS, consumer groups and the media.

The EVM met in early February 2003 to discuss the comments made during the public consultation and to review the draft report in light of comments received. All 103 submissions received from interested parties during the consultation were considered. However there were certain scientific issues for some vitamins and minerals that required more detailed assessment. Of the 103 submissions, thirty-seven respondents commented on the scientific content of the draft report as requested. Additional evidence, in the form of peer-reviewed papers published in scientific or medical journals, covered the following vitamins and minerals:

- folic acid: 7 additional papers.

- vitamin C: 1 additional paper.

- vitamin A: 1 additional paper.

- beta carotene: 1 additional paper.

- vitamin E: 2 additional papers.

- chromium: 5 additional papers.

- molybdenum: 1 additional paper.

- selenium: 1 additional paper.

- zinc: 1 additional paper.

- sodium chloride: 5 additional papers. 
Introductory Chapters Chapter 7 - Public consultation on the EVM draft report

The following risk assessments in this publication represent the final opinions of the EVM following its deliberations on all of the comments received. Some respondents commented on the general approach adopted by the EVM in the risk assessments. Many responders commented on issues outside the remit of the EVM. The majority of these related to the EU Food Supplements Directive. Other general issues such as the efficacy of high level nutrient intake and EVM structure were also raised. 


\section{Part 1}

Water Soluble

Vitamins 


\section{Risk Assessment}

\section{Biotin}

\section{General Information}

\section{Chemistry}

D-Biotin (biotin, coenzyme R, vitamin $\mathrm{H}$ ) is a water-soluble vitamin. It has a bicyclic ring structure. One ring contains a ureido group and the other contains a heterocyclic sulphur atom and a valeric acid side-group.

\section{Natural occurrence}

Biotin is derived from de novo synthesis by bacteria, primitive eukaryotic organisms including yeasts, moulds and algae, and some plant species.

\section{Occurrence in food, food supplements and medicines}

Biotin is widely distributed in natural foodstuffs but at very low levels compared to other water-soluble vitamins. Foods relatively rich in biotin include egg yolk, liver, kidney, muscle and organ meats, and some vegetables. Liver contains approximately $1 \mathrm{mg} / \mathrm{kg}$ biotin, whereas fruits and most other meats contain approximately $0.01 \mathrm{mg} / \mathrm{kg}$ biotin. Biotin, usually either in the form of crystalline D-biotin or brewer's yeast, is included in many dietary supplements, infant milk formulas and baby foods, as well as various dietetic products. The maximum dose in supplements sold in the UK is $2 \mathrm{mg}$. Several medicines containing biotin, which are available only from pharmacies, are licensed for the prevention and treatment of nutrient deficiency, supplementation of special diets and malabsorption. The maximum daily dose of biotin in licensed medicines is $0.50 \mathrm{mg}$.

\section{Recommended amounts}

Due to insufficient data, COMA was unable to set Dietary Reference Values for biotin, but considered that intakes between 0.010 and $0.20 \mathrm{mg} /$ day are both safe and adequate (COMA, 1991).

\section{Analysis of tissue levels and biotin status}

Measurement of biotin in plasma is not a reliable indicator of status. Changes in urinary excretion of biotin, or its metabolites bisnorbiotin, 3-hydroxyisovaleric acid and 3-methylcrotonylglycine, are good indicators of biotin status.

\section{Brief overview of non-nutritional beneficial effects}

Biotin has been claimed to be beneficial in the treatment of brittle nails, hyperinsulinaemia and impaired glucose tolerance and in sternocostoclavicular hyperostosis. Biotin supplements are also indicated in the management of inborn biotin-associated enzyme abnormalities such as deficiency of biotinidase, holocarboxylase synthetase and the individual carboxylase enzymes. 


\section{Function}

Biotin acts as an essential cofactor for the acetyl-CoA, propionyl-CoA, B-methylcrotonyl-CoA and pyruvate carboxylase enzymes, which are important in the synthesis of fatty acids, the catabolism of branched-chain amino acids and the gluconeogenic pathway. Biotin may also have a role in the regulation of gene expression arising from its interaction with nuclear histone proteins.

\section{Deficiency}

Biotin deficiency has been observed in individuals maintained on total parenteral nutrition, people who consume large amounts of uncooked egg white, sufferers from inherent or acquired biotin malabsorption, haemodialysis patients, and individuals receiving some forms of long-term anticonvulsant therapy. Pregnancy may be associated with marginal biotin deficiency. Signs of biotin deficiency include a fine scaly desquamating dermatitis and characteristic skin rash frequently observed around the eyes, nose and mouth, hair loss, conjunctivitis and ataxia. Biotin deficient infants show signs of hypotonia, lethargy, developmental delay and withdrawn behaviour, all of which are characteristic of a biotin deficiency-related neurological disorder. 'Egg white injury' may be associated with glossitis, anorexia, nausea, hallucinations, depression and somnolence. Inherited deficiencies in biotinidase and holocarboxylase synthetase result in multiple carboxylase deficiency. These deficiencies and those of specific carboxylase enzymes may produce the same or similar disorders and manifestations of biotin deficiency. Clinical manifestations of biotin deficiency are generally thought to result, directly or indirectly, from deficient activities of the carboxylase enzymes. Biotin deficiency has been shown to cause abnormal foetal development in animals.

\section{Interactions}

Some anticonvulsant drugs and alcohol may inhibit intestinal carrier-mediated transport of biotin. Steroid hormones and some anticonvulsant drugs may accelerate the catabolism of biotin in the tissues. Peroxisome proliferators have been shown to accelerate biotin catabolism in rats. However, the human relevance of this finding is questionable. Pantothenic acid and biotin may share common carriermediated uptake mechanisms in some tissues but at present there are no known clinical implications of this interaction.

\section{Absorption and bioavailability}

Biotin uptake from the small intestine occurs by a carrier-mediated process that operates with a high carrier affinity and also by slow passive diffusion. The carrier is driven by an electron-neutral sodium $\left(\mathrm{Na}^{+}\right)$gradient, has a high structural specificity and is regulated by the availability of biotin, with upregulation of the number of transporter molecules when biotin is deficient. The colon is also capable of absorbing biotin via a similar transport mechanism. Approximately $80 \%$ of biotin in plasma is in the free form and the remainder is either reversibly or covalently bound to plasma proteins. The existence of a specific biotin carrier protein in plasma is a subject of debate.

Factors determining the bioavailability of biotin present in the diet are uncertain. The bioavailability of biotin that is covalently bound to protein is reduced in individuals suffering from biotinidase deficiency. There are few data concerning the bioavailability of crystalline biotin supplements, but a recent study has suggested that doses as high as $22 \mathrm{mg}$ may be completely absorbed. The nutritional significance of biotin synthesis by bacteria present in the lower gut is a subject of controversy. 
Part 1 Water Soluble Vitamins

\section{Distribution and metabolism}

Uptake into tissues occurs by specific transport mechanisms dependent upon $\mathrm{Na}^{+}$gradients. Transplacental transport is thought to involve the active accumulation of biotin within the placenta followed by its passive release into the foetal compartment. Biotin is metabolically trapped within the tissues by its incorporation into carboxylase enzymes. In the normal turnover of cellular proteins, carboxylase enzymes are broken down to biocytin or oligopeptides containing lysyl-linked biotin. Biotin may be released for recycling by the hydrolytic action of biotinidase. Liberated biotin may be reclaimed in the kidney against a concentration gradient. Biotin not incorporated into carboxylase enzymes may be metabolised oxidatively at the sulphur present in the heterocyclic ring and/or at the valeric acid side chain.

\section{Excretion}

Biotin metabolites are not active as vitamins and are excreted in the urine. Very little biotin is thought to undergo biliary excretion and the substantial amounts of biotin that appear in the faeces are derived from colonic bacteria.

\section{Toxicity}

\section{Human data}

Anecdotal reports suggest that typical daily doses of $10 \mathrm{mg}$ are without adverse effects and toxicity has not been reported in individuals receiving as much as $200 \mathrm{mg}$ per day. Clinical data are limited but studies have reported no biotin-related adverse effects following the administration of $9 \mathrm{mg} /$ day for up to 4 years, $10 \mathrm{mg} /$ day for 15 days, $4 \mathrm{mg} /$ day for 3 weeks or $2.5 \mathrm{mg} /$ day for 6-15 months.

\section{Animal data}

The database on the toxicity of biotin in laboratory animals is limited. Acute toxicity in mice and rats after intravenous or oral dosage appears to be low. There is controversy as to whether high doses of biotin given sub-cutaneously cause adverse effects to the reproductive system in laboratory animals. Biotin-related disturbances in oestrus cycle, atrophic changes in ovaries, inhibition of foetal and placental growth and the increased resorption of foetuses were reported following administration of biotin $(50 \mathrm{mg} / \mathrm{kg}$ ) by injection to female Holzman rats, up to 3 weeks prior to mating. These effects were not observed in a similar study conducted in Ibm:RORO rats or ICR mice. These studies were not appropriate for risk assessment of oral doses.

\section{Carcinogenicity and genotoxicity}

No carcinogenicity data are available for biotin. It has been shown to be negative in the Ames test. However, further data on the mutagenicity testing of biotin are not available.

\section{Genetic variations}

No relevant genetic variations have been identified. 


\section{Mechanism of toxicity}

No relevant data are available.

\section{Dose-response characterisation}

No relevant data are available.

\section{Vulnerable groups}

No potential vulnerable groups have been identified.

\section{Studies of particular importance in the risk assessment:}

(For full review see http://www.food.gov.uk/science/ouradvisors/vitandmin/evmpapers or the enclosed CD)

\section{Human data}

\section{Maebashi et al., 1993}

Biotin ( $9 \mathrm{mg}$, in combination with $3 \mathrm{~g}$ of an anti-microbial drug, Miya-BM) was administered daily, by the oral route (in three divided doses), for up to 4 years, to 20 patients (sex and age unspecified) suffering from non-insulin dependent diabetes. The number of patients followed up after 24, 30, 36 and 48 months was 15, 15, 10 and 5, respectively. There was no control group included in the study. Fasting blood glucose levels decreased to normal within 2 months and remained normal thereafter with continuing treatment. Serum insulin levels were not significantly changed. The authors reported that there were no observed clinical aggravations or undesirable side effects. This study cannot be used for risk assessment because of the unusual design and the small number of subjects.

Velazquez et al., 1995

In a double blind placebo controlled study, protein deficient children ( $\mathrm{n}=22)$ were administered $10 \mathrm{mg}$ biotin/day for fifteen days. Plasma biotin concentrations and lymphocyte carboxylase enzymes were measured. The authors reported no adverse effects. This study cannot be used to define the safety of biotin in children with a normal protein intake. 
Part 1 Water Soluble Vitamins

\section{Exposure assessment}

Total exposure/intake:

Food

mean: $0.033 \mathrm{mg} /$ day $(1986 / 7 \mathrm{NDNS})$

97.5th percentile: $0.066 \mathrm{mg} /$ day

Supplements

up to $2 \mathrm{mg}$ /day (Annex 4)

Estimated maximum intake: $0.066+2=2.07 \mathrm{mg} /$ day

No potential high intake groups were identified.

\section{Risk assessment}

There are relatively few human data available on the oral toxicity of biotin. The data available are in the form of anecdotal case reports or from clinical trials or supplementation studies designed primarily to investigate beneficial effects of biotin. The latter rarely specifically report on the presence or absence of adverse effects.

The animal toxicity database for biotin is very limited, especially when given by the oral route.

\section{ESTABLISHMENT OF GUIDANCE LEVEL}

The data from studies in humans and animals are not adequate for the establishment of a Safe Upper Level.

The numerous clinical case reports in the literature describe the outcome of oral biotin administration to patients (infants, juveniles and adults) with biotin-responsive inborn errors of metabolism and other forms of biotin deficiency. Furthermore, in cases where foetal biotin-responsive disorders have been suspected, biotin has been administered prenatally, via the mother. Typically, doses of $10 \mathrm{mg} /$ day $(250 \mathrm{x}$ the average intake of the adult male in the UK) have been studied for therapeutic effects, without reported adverse side effects.

Supplemental doses of $9 \mathrm{mg} /$ day given to human volunteers for up to 4 years have not been associated with adverse effects. The study is limited in that it was performed in diabetics, there was no control group and only a few individuals remained in the study after 4 years. The authors concluded that there were no adverse effects related to the biotin treatment, although they reported a tendency for the treatment to lower blood sugar. Due to the low number of individuals studied, and the small proportion of volunteers followed up long term, an uncertainty factor of 10 for inter-individual variation has been applied in this case to allow for inter-individual variation. Thus, for guidance purposes only, a supplemental daily intake of $0.9 \mathrm{mg}$ biotin (equivalent to $0.015 \mathrm{mg} / \mathrm{kg}$ bw/day in a $60 \mathrm{~kg}$ adult) would not be expected to produce adverse effects, although this value may not be applicable to all life stages. Assuming a maximum intake of $0.066 \mathrm{mg} /$ day from food, an estimated total intake (from all sources) of $0.97 \mathrm{mg} /$ day biotin (equivalent to $0.016 \mathrm{mg} / \mathrm{kg}$ bw/day in a $60 \mathrm{~kg}$ adult) would not be expected to result in any adverse effects. 


\section{References}

COMA (1991). Dietary Reference Values for Food Energy and Nutrients for the United Kingdom. Report of the Panel on Dietary Reference Values, Committee on Medical Aspects of Food and Nutrition Policy. HMSO, London.

Maebashi, M., Makino, Y., Furukawa, Y., Ohinata, K., Kimura, S., Sato, T. (1993). Therapeutic evaluation of the effect of biotin in hyperglycemia in patients with non-insulin-dependent diabetes mellitus. Journal of Clinical Biochemistry and Nutrition 14, 211-218.

Velazquez, A., Teran, M., Baez, A., Gutierriez, J., Rodriguez, R. (1995). Biotin supplementation affects lymphocyte carboxylases and plasma biotin in severe protein-energy malnutrition. American Journal of Clinical Nutrition 61, 385-91. 
Part 1 Water Soluble Vitamins

\section{Risk Assessment $\quad$ Folic Acid}

\section{General information}

\section{Chemistry}

The term folate is used generically to describe the various derivatives of pteroylglutamic acid (PGA, folic acid), the common pharmaceutical and most stable form of the folate vitamins group, which is composed of three major subunits - pteridine, $p$-aminobenzoic acid, and glutamic acid.

Within this assessment, in accordance with the guidelines of the International Union of Pure and Applied Chemistry and International Union of Biochemistry and Molecular Biology (IUPAC-IUB) advisory panel, the term folic acid is used to indicate the parent compound, pteroylglutamic acid, and folate is used generically, to indicate one or a mixture of pteroylglutamates.

\section{Natural occurrence}

Folic acid (PGA) per se is not present in significant quantities in foods or in the human body. The derivatives of PGA which are predominantly present in the human body, and in plant- and animalderived foods, are reduced folates, mostly 5,6,7,8-tetrahydrofolates (THF), and also 7,8-dihydrofolate (DHF). Other modifications also occur.

\section{Occurrence in food, food supplements and medicines}

Folates are present in the majority of natural foods. Liver, yeast extract, green leafy vegetables, legumes and some fruits are especially rich sources. Dietary forms are broken down to monoglutamates during storage, processing and cooking. The synthetic pharmaceutical form used for food fortification and in supplements is folic acid (PGA), as this compound is more stable in comparison to other forms of the vitamin. Folic acid is widely available as a nutritional supplement, either alone or as a component of B complex or multivitamin preparations, in daily doses of up to $0.80 \mathrm{mg}$. Preparations providing a daily dose of $5 \mathrm{mg}$ are available on prescription only.

\section{Recommended amounts}

RNI for folate are $0.05,0.07,0.10$ and $0.15 \mathrm{mg} /$ day for infants and children $\leqslant 12$ months, $1-3$ years, $4-6$ years and $7-10$ years, respectively. The RNI for adults and children $\geqslant 11$ years old is 0.200 $\mathrm{mg} /$ day. Recommendations to allow for increased requirements during pregnancy and lactation are increments of 0.10 and $0.060 \mathrm{mg} /$ day, respectively, i.e. $0.30 \mathrm{mg} /$ day for pregnant women and 0.26 $\mathrm{mg} /$ day for breastfeeding women. The UK Department of Health (COMA) also recommends that 'women who could become pregnant' should take $0.40 \mathrm{mg} /$ day folic acid, in addition to normal dietary folate intake, and until the 12th week of pregnancy. An additional dose of $5 \mathrm{mg} /$ day is recommended for women at high risk of a neural tube defect (NTD)-affected pregnancy. 


\section{Analysis of tissue levels and folate status}

Folate status is usually measured by determination of serum and/or red cell folate levels, in which the predominant species is 5-methyl-THF. Serum folate is a short-term indicator of folate status; levels are normally within the range of 5-16 ng/mL (11-36 nmol/L folic acid activity). Red cell levels are more stable and reflect long term intake, levels $<140 \mathrm{ng} / \mathrm{mL}(317 \mathrm{nmol} / \mathrm{L})$ indicate reduced body stores. Thus, negative folate balance is indicated by a serum folate concentration $<3 \mathrm{ng} / \mathrm{mL}$, whilst folate deficiency is indicated by erythrocyte and liver folate levels $<120 \mathrm{ng} / \mathrm{mL}$ or $1.6 \mathrm{mg} / \mathrm{kg}$ (wet weight), respectively.

\section{Brief overview of non-nutritional beneficial effects}

Randomised controlled trials and observational studies indicate that peri-conceptional folic acid supplementation in women is associated with a significant reduction in the incidence of foetal NTDs. It has also been shown that folic acid supplementation is associated with a reduction of serum levels of homocysteine, but commensurate benefits for cardiovascular health are uncertain.

\section{Function}

Folate coenzymes within the cell are involved in one-carbon transfer reactions, including those involved in phases of amino acid metabolism, purine and pyrimidine synthesis, and the formation of the primary methylating agent, S-adenosylmethionine.

\section{Deficiency}

Folate deficiency results in reduced de novo DNA biosynthesis and thus, impairment of cell replication, with the most obvious effects relating to rapidly dividing cell-types, such as erythrocytes and other cells generated by the bone marrow, enterocytes and skin cells. The condition causes megaloblastic and macrocytic anaemia. Vitamin $\mathrm{B}_{12}$ deficiency also causes a macrocytic megaloblastic anaemia and should be excluded before folate treatment alone is given.

Nutritional folate deficiency may develop during pregnancy, infection, malignant disease, malabsorption syndromes (e.g. coeliac disease) or alcoholism, during some drug treatments and in the older people on restricted diets.

\section{Interactions}

Folic acid supplementation can interfere with a number of drugs (anti-folate drugs, drugs used to treat epilepsy, anti-inflammatory drugs). Folate, vitamin $\mathrm{B}_{6}$ and vitamin $\mathrm{B}_{12}$ metabolism are linked via the enzyme methionine synthase (which requires vitamin $B_{12}$ as a cofactor). Some authors have reported a negative effect of folate supplementation on zinc status. Some animal studies have suggested that iron deficiency may cause folate depletion. 
Part 1 Water Soluble Vitamins

\begin{abstract}
Absorption and bioavailability
The majority of dietary folate is absorbed within the proximal region of the small intestine by active, carrier-dependent mechanisms, and also by passive diffusion. Polyglutamate forms are first hydrolysed to monoglutamates by conjugase (hydrolase) enzymes within the enterocyte brush border. Ingested folic acid is enzymatically reduced and methylated within the intestinal lumen and enterocytes, although ingestion of high concentrations ( $>0.20-0.30 \mathrm{mg} / \mathrm{meal}$ ) result in the direct appearance of the compound, unmodified, in the plasma.
\end{abstract}

The absorption of natural folate from a typical North American diet has been suggested to be in the range of 50 to $75 \%$ of the amount ingested. Synthetic folic acid, as a food fortificant or supplement is more highly bioavailable than natural food folate.

\title{
Distribution and metabolism
}

Absorbed folate is carried via the portal blood to the liver, where a proportion (approximately 0.1 $\mathrm{mg} /$ day) is excreted into the bile and undergoes enterohepatic circulation and reabsorption. The liver is also the main storage site, containing approximately half of the total $(5-10 \mathrm{mg})$ body folate. The majority of plasma folate is present as 5-methyl-THF-monoglutamate. Within cells, folate is retained in the cytoplasm by polyglutamation. 5 Methyl-THF is not a good substrate for polyglutamation, and must be first converted, via a vitamin $\mathrm{B}_{12}$-dependent reaction, to THF. Alternatively, folic acid can be converted to polyglutamate (i.e. metabolically active) forms via a vitamin $B_{12}$-independent pathway.

\section{Excretion}

Folate is excreted in the urine, either as the metabolically active form or as breakdown products, and in the faeces.

\section{Toxicity}

\section{Human data}

\section{Therapeutic use}

Folic acid is generally considered safe for medicinal use, even at doses of about 10-20 mg/day. 'Indirect toxicity' may occur due to folic acid reversal of the haematological signs and symptoms of vitamin $B_{12}$ deficiency; this masking effect could allow the neuropathy associated with vitamin $B_{12}$ deficiency to develop untreated. Vitamin $B_{12}$ deficiency is most prevalent in older people and, in general, in those with impaired absorption. Adverse effects may, potentially, occur in specific groups, such as individuals being treated with drugs that interact with folic acid metabolism.

A small number of case reports have described hypersensitivity reactions to oral folic acid therapy (generally $\geqslant 1 \mathrm{mg} /$ day). One short-term, uncontrolled supplementation trial reported adverse symptoms (mental changes, sleep disturbances and gastrointestinal symptoms) in healthy volunteers given very high doses of folic acid ( $15 \mathrm{mg} /$ day) for 1 month, but other studies have not observed similar effects. 


\section{Supplementation trials}

A substantial number of supplementation studies have been carried out or are ongoing to assess the effectiveness of folic acid therapy in disease prevention (generally, either for the prevention of foetal NTD-pregnancies or cardiovascular disease, in high-risk groups). Many of these trials have shown beneficial effects associated with folic acid supplementation at levels up to $10 \mathrm{mg} /$ day for periods of several weeks or months. Very few adverse effects have been reported, although the majority of studies have not specifically addressed this issue.

Trials have shown that peri-conceptional supplementation with folic acid, or folic acid-containing multivitamin supplements, is associated with a significant reduction in the incidence of foetal NTDs. Consequently, COMA recommended that, for the prevention of foetal NTDs, women who could become pregnant should take a $0.40 \mathrm{mg}$ folic acid supplement daily, in addition to normal dietary folate intake. Women in high risk groups may be advised to take up to 10 times this level of supplementation. Such therapy is generally considered to be without adverse reproductive or developmental effects, although it has been noted that human trials carried out to date were not sufficiently powerful to identify rare or possibly slight adverse effects.

One large study of peri-conceptional multivitamin supplementation (including $0.8 \mathrm{mg} /$ day folic acid) in Hungary (1992) showed reduced incidence of NTDs, but with a significant increase in spontaneous abortions. Thus, it has been suggested that folic acid may prevent NTDs by causing spontaneous abortion of affected foetuses. However, this theory is not supported by recent studies using a genetically-predisposed NTD mouse model, in which the administration of folic acid to embryos in vitro normalised neural tube development without stimulating abortion.

In two studies of the effects of folic acid supplementation in early pregnancy, statistically significantly increased numbers of multiple births were reported ${ }^{14}$. The effect appeared to be limited to dizygotic twinning and the theory proposed by the authors of one of the studies was that this effect is due to increased survival of multiple pregnancies, rather than increased ovulation, as multiple pregnancies have greater micronutrient requirements.

\section{Animal data}

Data from toxicological studies of folic acid in experimental animals are limited. A number of reports have described nephrotoxicity associated with the parenteral administration of extremely high doses ( $\geqslant 75 \mathrm{mg} / \mathrm{kg}$ bw) of folic acid in rodents. Additionally, direct injection of high doses of folic acid or folates into the brain or spinal fluid has been shown to produce seizures in rats and mice. One study showed that high-dose dietary folic acid supplementation decreased the dose of pentylenetetrazol required to induce seizures in rats.

\footnotetext{
14 However, a recent study was published after 31 December 2002, the EVM's cut-off date for studies to be included in the risk assessment, which showed no association between folic acid supplementation and risk of multiple births (Li et al., Lancet 2003; 361: 380). The study was a population-based cohort study of 242,015 women, 127,018 of whom who took $0.4 \mathrm{mg}$ folic acid/day as a supplement before and/or during the first trimester of pregnancy. The relative risk for multiple births in the supplemented group was 0.91 ( $95 \% \mathrm{Cl}, 0.82-1.00)$.
} 
Part 1 Water Soluble Vitamins

Oral folic acid supplementation, alone has generally not shown reproductive or embryotoxic effects in animal models, although studies have shown that supplementation is associated with increased foetal folate levels. One group reported that the foetuses of rats fed diets containing approximately $2 \mathrm{mg} / \mathrm{kg}$ bw/day folic acid for 3 weeks during pregnancy showed reduced body weight and vertex-coccyx length compared with a control group of animals given a basal diet, although the validity of the reported statistical analysis is uncertain. Additionally, folic acid treatment has been reported to enhance the embryotoxic effects of certain drugs (pyrimethamine, valproic acid), and of zinc deficiency.

\section{Carcinogenicity and genotoxicity}

There are limited data to suggest that folic acid supplementation, in comparison to deficiency, may be associated with the promotion of tumours in animals that develop spontaneous tumours or are exposed to chemical carcinogens. However, this may be related to the role of folic acid in supporting cell replication. Data from in vitro and in vivo studies indicate that folic acid is not genotoxic.

\section{Vulnerable groups}

Groups vulnerable to adverse effects associated with folic acid supplementation include:

1] Individuals at risk of vitamin $B_{12}$ deficiency (most prevalent in the older people), in whom folic acid supplementation may mask the haematological signs and symptoms of this deficiency, allowing the associated myeloneuropathy to develop.

2] Patients treated with drugs which interfere with folate metabolism, and in whom folic acid supplementation may be associated with reduced effectiveness of the therapy or increased incidence of side effects.

\section{Genetic variations}

Population groups with a genetically determined increase in susceptibility to folic acid toxicity have not been identified. In contrast, some individuals have a genetic predisposition to deficiency. Subjects homozygous for a variant of the enzyme 5,10-methylene tetrahydrofolate reductase (MTHFR) show reduced activity of this enzyme, resulting in altered cellular distribution of one-carbon units, and associated with low plasma folate status and hyperhomocysteinaemia. Defects of other enzymes involved in homocysteine metabolism (e.g. cystathionine ß-synthase, methionine synthase) may also be associated with pathology related to low folate intake/status. In addition, congenital errors of various enzymes involved in folate metabolism have been described, associated with functional folate-deficiency.

\section{Mechanisms of toxicity}

Folic acid is generally considered safe and no likely mechanisms for toxicity have been hypothesised. The metabolism of folate and vitamin $B_{12}$ are linked by the enzyme methionine synthase, which is vitamin $B_{12}$ dependent and results in polyglutamate synthesis. High levels of folate can result in the production of polyglutamates by a vitamin $B_{12}$ independent mechanism, which may reverses the megaloblastic anaemia caused by vitamin $B_{12}$ deficiency. This complicates the diagnosis of vitamin $B_{12}$ deficiency and allows the neurological damage associated with it to continue. 


\section{Dose response characterisation}

Few systematic data exist regarding the level of folate intake required to mask vitamin $B_{12}$ deficiency (Koehler et al., 1997). The majority of available information relates to early case reports of folic acid therapy for the treatment of pernicious anaemia (mostly high doses, for example $\geqslant 5 \mathrm{mg} /$ day) (Heinle et al., 1947; Vilter et al., 1947; Bethell et al., 1948; Ross et al., 1948; Vilter et al., 1950; Will et al., 1959; Schwartz et al., 1950; Ellison and Curry, 1960; Marshall and Jandl, 1960; Baldwin et al., 1961; Hansen \& Weinfeld, 1962; Vilter et al., 1963). In general, data taken from these reports suggest that supplementation with $\leqslant 1 \mathrm{mg} /$ day folic acid does not mask vitamin $B_{12}$-associated anaemia in the majority of subjects. The effects of doses of between 1 and $5 \mathrm{mg} /$ day are unclear (cited by Chanarin, 1994; Bower \& Wald, 1995). Supplementation with $\geqslant 5 \mathrm{mg} /$ day folic acid is reported to reverse the haematological signs of vitamin $\mathrm{B}_{12}$-deficiency in at least $50 \%$ of subjects (discussed by Chanarin, 1994; Bower \& Wald, 1995; Savage \& Lindenbaum, 1995).

\section{Studies of particular importance in the risk assessment}

(For full review see http://www.food.gov.uk/science/ouradvisors/vitandmin/evmpapers or the enclosed CD).

\section{Human data}

Weissberg et al., 1950

This was a non-controlled study of the neurological effects of $20 \mathrm{mg} /$ day folic acid supplementation for 6-12 months, in 26 normal volunteers and 22 patients with non-pernicious anaemia. Prior to therapy, 6 of the normal subjects and 7 of the anaemic subjects showed some abnormal neurological signs (but not those of subacute combined spinal cord degeneration), which were not significantly altered during the therapy. Four subjects (1 normal, 3 anaemic) developed central nervous system changes during the folic acid treatment, but these changes were not considered to be related to the therapy.

Harvey et al., 1950

In this open, uncontrolled study, oral folic acid supplementation (20 mg/day for $3-12$ months) produced no indications of spinal cord or peripheral nerve damage in 40 healthy subjects without pernicious anaemia (13 subjects had mild hypochromic anaemia).

\section{MRC Vitamin Study Research Group, 1991}

This was a multicentre, randomised, double-blind, placebo-controlled trial carried out in the UK, in which a total of 1817 women with a previous NTD-affected pregnancy were assigned to 1 of 4 supplementation groups:
A] $4 \mathrm{mg} /$ day folic acid
B] $4 \mathrm{mg} /$ day folic acid + multivitamins (daily - $4000 \mathrm{IU}$ vitamin A, $400 \mathrm{IU}$ vitamin D, $1.5 \mathrm{mg}$ each vitamins $B_{1}$ and $B_{2}, 1.0 \mathrm{mg}$ vitamin $B_{6}, 40 \mathrm{mg}$ vitamin $C, 15 \mathrm{mg}$ nicotinamide)
C] placebo
D] multivitamins (as B], without folic acid) 
Part 1 Water Soluble Vitamins

The duration of therapy was from the date of randomisation until the 12th week of pregnancy. Statistical analysis showed a significantly reduced relative risk for NTDs associated with folic acid supplementation compared with no folic acid supplementation, i.e. groups $A$ and $B$ compared with groups $C$ and $D(R R=$ $0.28 ; 95 \% \mathrm{Cl}, 0.12-0.71)$. The authors also reported that possible adverse effects of folic acid to the foetus and the mother were examined. The authors concluded that there was no demonstrable harm from the folic acid supplementation, although the ability of the study to detect rare or slight adverse effects was limited. The incidences of general side effects (e.g. infertility, irregular menses, vomiting in pregnancy, upper respiratory illness) reported by women taking part in the trial were similar in all 4 groups.

\section{Animal data}

Chung et al., 1993

In a study of the synergistic effects of folic acid and the anti-malarial drug, pyrimethamine (an inhibitor of dihydrofolate reductase), groups of 10 pregnant female rats were supplemented with combinations of the anti-malarial drug, pyrimethamine, and/or folic or folinic acid, from days $7-17$ of gestation, by gavage. Folic acid treatment $(50 \mathrm{mg} / \mathrm{kg}$ bw/day) alone showed no significant maternal or embryotoxicity, as compared with vehicle-only treatment.

\section{Exposure assessment}

Total exposure/intake:

Food Mean: $0.26 \mathrm{mg} /$ day

97.5th percentile: $0.49 \mathrm{mg} /$ day $(1986 / 87 \mathrm{NDNS})$

Supplements $\quad$ up to $0.50 \mathrm{mg}$ in OTC supplements for males (OTC, 2001)

up to $0.80 \mathrm{mg}$ in OTC supplements for females (Annex 4)

Estimated maximum intake: $0.99 \mathrm{mg} /$ day for males

$1.29 \mathrm{mg} /$ day for females

No potential high intake groups have been identified.

\section{Implications of food fortification of wheat flour}

During the 1990s, the public health policy with regard to preventing NTDs aimed to ensure that women of childbearing age were aware of the importance of acquiring sufficient folic acid in the diet at the time of conception to minimise the risk of NTDs in their offspring. In the UK, as in the US, there have been calls for more active public policy, including food fortification to address this. The Committee on Medical Aspects of Food and Nutrition Policy (COMA) reviewed the links between folates, including folic acid, and disease (COMA, 2000), and:

- confirmed the link between low folate status and the risk of NTDs

- concluded that there is insufficient evidence on which to establish a conclusive link with cardiovascular disease 
- acknowledged that increasing the intake of folic acid might pose a risk to people with undiagnosed vitamin $B_{12}$ deficiency, particularly older people, and advised clinical vigilance to avoid any delays in diagnosis

- recommended that the current policy of encouraging women who could become pregnant to take $0.40 \mathrm{mg}$ folic acid as a supplement should continue

COMA further concluded that universal fortification of flour at $0.24 \mathrm{mg}$ per $100 \mathrm{~g}$ in food products as consumed would result in:

- a significant effect in preventing NTD-affected births and pregnancies without resulting in unacceptably high intakes in any group of the population. It is estimated that this would reduce the incidence of NTD-affected pregnancies by $41 \%$ and would have, for example, prevented 38 of the 93 NTD-affected births in England and Wales in 1998, 30 of the 74 in Scotland in 1997, and 6 of the 14 in Northern Ireland in 1998

- The average intake of folic acid of women aged 16-45 years would increase by $0.201 \mathrm{mg} /$ day, leading to a total folate intake of $0.405 \mathrm{mg} /$ day

- Approximately $7 \%$ of women in this age group would have total folate intakes in excess of $0.600 \mathrm{mg} /$ day

- Approximately $0.6 \%$ of people aged over 50 years would be exposed to levels of folic acid intake greater than $1 \mathrm{mg} /$ day

\section{Risk assessment}

Folic acid is generally considered as safe in therapeutic use. Adverse effects may, potentially, occur in specific groups, such as individuals being treated with drugs that interact with folic acid metabolism. Women at risk of a NTD-affected pregnancy appear to be able to take folate supplements at up to 4 $\mathrm{mg} /$ day, without adverse reproductive or developmental effects.

Folic acid may lead to reversal of the symptoms of vitamin $B_{12}$ deficiency, potentially allowing the neuropathy associated with vitamin $B_{12}$ deficiency to develop untreated. Vitamin $B_{12}$ deficiency is most prevalent in older people.

Few data are available from toxicological studies of folates in animals.

\section{ESTABLISHMENT OF GUIDANCE LEVEL}

There are insufficient data from animal or human studies to establish a Safe Upper Level for folic acid.

COMA recommended that, for the prevention of foetal NTDs, women who could become pregnant should take a $0.40 \mathrm{mg}$ folic acid supplement daily, in addition to normal dietary folate intake, until the 12th week of pregnancy. Women in high risk groups may be advised to take up to 10 times this level of supplementation.

We are aware of evidence that increased folate acid intake lowers serum homocysteine levels and may potentially help prevent adverse cardiovascular events. However, consideration of such effects is not within the remit of the EVM. We are also aware of recent studies which have suggested that increased folate intake may increase the incidence of multiple births. We note that this is an area of potential concern, but that there is currently no substantive evidence for such an effect. 
Part 1 Water Soluble Vitamins

The main concern regarding ingestion of excess folic acid is the consequential masking of vitamin $B_{12}$ deficiency. A general consistency of data indicates that supplementation with $\leqslant 1 \mathrm{mg} /$ day folic acid does not mask vitamin $\mathrm{B}_{12}$-associated anaemia in the majority of subjects, whereas supplementation with $\geqslant 5 \mathrm{mg} /$ day folic acid does. The effects of doses of between 1 and $5 \mathrm{mg} /$ day are unclear. No other significant adverse effects have been associated with ingestion of folic acid.

For guidance purposes only, in the general population a supplemental dose of $1 \mathrm{mg} /$ day (equivalent to $0.017 \mathrm{mg} / \mathrm{kg}$ bw/day in a $60 \mathrm{~kg}$ adult) would not be expected to cause adverse effects. Because of the consistency of the data, from a large number of studies in humans, no uncertainty factors have been applied. Assuming a maximum intake from food of approximately $0.49 \mathrm{mg} /$ day, a total dose of 1.5 $\mathrm{mg} /$ day (equivalent to $0.025 \mathrm{mg} / \mathrm{kg}$ bw/day in a $60 \mathrm{~kg}$ adult) would not be expected to have any adverse effects.

\section{References}

Baldwin, J.N., Dalessio, D.J. (1961). Folic acid therapy and spinal-cord degeneration in pernicious anemia. New England Journal of Medicine 264, 1339-1342

Bethell, F.H., Sturgis, C.C. (1948). The relation of therapy in pernicious anemia to changes in the nervous system: early and late results in a series of cases observed for a period of not less than 10 years and early results with folic acid. Blood 3, 57-67

Bower, C., Wald, N.J. (1995). Vitamin $B_{12}$ deficiency and the fortification of food with folic acid. European Journal of Clinical Nutrition 49, 787-793

Chanarin, I. (1994). Adverse effects of increased dietary folate. Relation to measures to reduce the incidence of neural tube defects. Clinical and Investigative Medicine 17, 244-252

Chung, M.K., Han, S.S., Roh, J.K. (1993). Synergistic embryotoxicity of combination pyrimethamine and folic acid in rats. Reproductive Toxicology 7, 463-468

COMA (2000). Department of Health. Report on Health and Social Subjects 50. Folic Acid and the Prevention of Disease. The Stationery Office. London

Ellison, A.B., Curry, A.B. (1960). Pernicious anemia masked by multivitamins containing folic acid. Journal of the American Medical Association 173, 240-243

Hansen, H.A., Weinfeld, A. (1962). Metabolic effects and diagnostic value of small doses of folic acid and $\mathrm{B}_{12}$ in megaloblastic anemias. Acta Medica Scandinavia 172, 427-443

Harvey, E.A., Howard, I., Murphy, W.P. (1950). Absence of a toxic effect of folic acid on the central nervous system of persons without pernicious anemia. New England Journal of Medicine 242, 446-447

Heinle, R.W., Welch, A.D. (1947). Folic acid in pernicious anemia: failure to prevent neurologic relapse. Journal of the American Medical Association 133, 739-741

Koehler, K.M., Pareo-Tubbeh, S.L., Romero, L.J., Baumgartner, R.N., Garry, P.J. (1997). Folate nutrition and older adults: challenges and opportunities. Journal of the American Dietetics Association 97, 167-173 
Marshall, R.A., Jandl, J.H. (1960). Responses to 'physiologic' doses of folic acid in the megaloblastic anemias. Archives of Internal Medicine 105, 352-360

MRC Vitamin Study Research Group (1991). Prevention of neural tube defects: results of the Medical Research Council Vitamin Study. Lancet 338, 131-137

Ross, M.D., Belding, M.D., Paegel, B.L. (1948). The development and progression of subacute combined degeneration of the spinal cord in patients with pernicious anemia treated with synthetic pteroylglutamic (folic) acid. Blood 3, 68-90

Savage, D.G., Lindenbaum, J. (1995). Folate-cobalamin interactions. In: Bailey, L.B. ed. Folate in Health and Disease. Marcel Dekker Inc, New York, pp. 237-285

Schwartz, S.O., Kaplan, S.R., Armstrong, B.E. (1950). The long-term evaluation of folic acid in the treatment of pernicious anemia. Journal of Laboratory and Clinical Medicine 35, 894-898

Vilter, R.W., Vilter C.F., Hawkins, R. (1947). Combined system disease and hematologic relapse occurring in persons with pernicious anaemia treated with synthetic folic acid (pteroyl-glutamic acid) for a period of two years. Journal of Laboratory and Clinical Medicine 32: 1426

Vilter, R.W., Horrigan, D., Mueller, J.F., Jarrold, T., Vilter, C.F., Hawkins, V., Seaman, A. (1950). Studies on the relationships of vitamin $B_{12}$, folic acid, thymine, uracil, and methyl group donors in persons with pernicious anemia and related megaloblastic anemias. Blood 5, 695

Vilter, R.W., Will, J.J., Wright, T., Rullman, D. (1963). Interrelationships of vitamin $B_{12}$, folic acid, and ascorbic acid in the megaloblastic anemias. American Journal Clinical Nutrition 12, 130-144.

Weissberg, J., McGavack, T.H., Vogel, M., Kenigsberg, S. (1950). The effect of folic acid on the central nervous system of normal subjects and subjects with anemia other than pernicious anemia. Blood 5, 148-154.

Will, J.J., Mueller, J.F., Brodine, C., Kiely, C.E., Firedman, B., Hawkins, B.R., Dutra, J., Vilter, R.W. (1959). Folic acid and vitamin $B_{12}$ in pernicious anemia: studies on patients treated with these substances over a tenyear period. Journal of Laboratory and Clinical. Medicine 53, 22-38. 


\section{Risk Assessment $\quad$ Niacin (Nicotinic Acid and Nicotinamide)}

\section{General information}

\section{Chemistry}

Niacin (vitamin $\mathrm{B}_{3}$ ) is the generic term for nicotinic acid (pyridine 3-carboxylic acid) and nicotinamide (nicotinic acid amide), and the coenzyme forms of the vitamin. Nicotinamide is the active form, which functions as a constituent of two coenzymes, namely, nicotinamide adenine dinucleotide (NAD) and nicotinamide adenine dinucleotide phosphate (NADP). These coenzymes in their reduced states (NADH/NADPH) are the principal forms of niacin that exist in animal tissues.

\section{Natural occurrence}

Free nicotinic acid and nicotinamide are present in nature in only small amounts. Nicotinic acid is mainly bound to macromolecules in plants, while nicotinamide is usually a component of NADP in the animal world. Nicotinic acid can be formed in humans from the metabolism of dietary tryptophan, and so niacin is not really a vitamin providing adequate tryptophan is available.

\section{Occurrence in food, food supplements and medicines}

Important sources of preformed niacin include beef, pork, wheat flour, maize (corn) flour, eggs and cows' milk. Human milk contains a higher concentration of niacin than cows' milk. In unprepared foods, niacin is present mainly in the form of the cellular pyridine nucleotides NAD and NADP. Enzymatic hydrolysis of the coenzymes can occur during the course of food preparation.

In the UK there is mandatory fortification of flour (except wholemeal and certain other specified types) with nicotinic acid at a level of not less than $1.6 \mathrm{mg} / 100 \mathrm{~g}$ flour for restoration purposes.

In the UK, niacin supplements are generally in the form of nicotinamide. Levels range from 0.25 to 150 $\mathrm{mg}$ per daily dose in multi-nutrient supplements and up to $250 \mathrm{mg}$ per daily dose in single nutrient products. Nicotinamide products with a maximum daily dose of $500 \mathrm{mg}$ are only available from pharmacists, and those preparations used to treat specific metabolic disorders are only available on prescription. Nicotinic acid is present in some General Sales List multivitamin preparations at levels up to $100 \mathrm{mg}$, and is available from the pharmacist to treat muscle cramp, and on prescription for use in hyperlipidaemias.

\section{Other sources of exposure}

No other sources of exposure have been identified. 


\section{Recommended amounts}

The UK RNI for niacin is $6.6 \mathrm{mg}$ niacin equivalent/1000 kcal, equivalent to 17 and $13 \mathrm{mg} /$ day in adult males and females respectively (COMA, 1991). An increment of $2.3 \mathrm{mg} /$ day niacin was recommended for lactating women. Under normal conditions, for an adult, the amount of tryptophan present in dietary protein provides adequate niacin without the need for any preformed vitamin.

\section{Analysis of tissue levels and niacin status}

There is no established laboratory method for the assessment of niacin status. In experimental animals, measurement of whole blood NADP can provide a sensitive indication of niacin depletion, while the determination of the urinary excretion of $\mathrm{N}$-methyl nicotinamide and its metabolite methyl pyridone carboxamide offers the only other method available.

\section{Brief overview of non-nutritional beneficial effects}

High dose treatment with nicotinic acid has been shown to reduce plasma cholesterol levels (by an average of 20 to $35 \%$ ). In the Coronary Drug Project, nicotinic acid was observed to reduce mortality due to a reduction in lethal coronary events. Supplementation studies have been shown to increase $\mathrm{NAD}^{+}$concentrations in lymphocytes. DNA strand breaks in lymphocytes exposed to oxygen radicals were shown to decrease proportionately to $\mathrm{NAD}^{+}$concentrations.

High dose nicotinamide treatment has also been claimed to offer protection against the development of insulin-dependent diabetes mellitus.

\section{Function}

Niacin is the functional factor of two important coenzymes, NAD and NADP, which activate over 200 dehydrogenases essential to electron transport and other cellular respiratory reactions. Despite their structural similarity, NAD and NADP have quite different metabolic roles. NAD functions as an electron carrier for intracellular respiration as well as a co-factor for enzymes involved in the oxidation of fats and carbohydrates, such as glyceraldehyde 3-phosphate, lactate, pyruvate and $\alpha$-ketoglutarate dehydrogenases. NADP functions as a hydrogen donor in reductive biosynthesis, such as in fatty acid and steroid synthesis and like NAD as a cofactor for enzymes, such as in the oxidation of glucose-6phosphate to ribose 5 -phosphate in the pentose phosphate pathway.

\section{Deficiency}

The most common symptoms of niacin deficiency are changes in the skin, mucosa of the mouth, stomach and intestinal tract and the nervous system. The changes in the skin are among the most characteristic in human beings. They are called 'pellagra', which means 'raw skin' and are most pronounced in the parts of skin exposed to sunlight. Other signs and symptoms include dizziness, vomiting, constipation or diarrhoea, and inflammation of the tongue and gastric mucosa. The neurological symptoms can include fatigue, sleeplessness, depression, memory loss and visual impairment. 
Part 1 Water Soluble Vitamins

\section{Interactions}

Interactions of niacin with drugs have been identified. Prolonged treatment of tuberculosis with isoniazid may lead to niacin deficiency due to its competition with pyridoxal phosphate, a co-enzyme necessary for the conversion of tryptophan to niacin.

Nicotinic acid-induced vasodilation, presenting as skin flushing, is inhibited by clonidine, and may exacerbate the vasodilatory effect of ganglion blocking agents. However, ganglion blocking antihypertensives are used extremely rarely now.

\section{Absorption and bioavailability}

In humans, niacin is rapidly absorbed from the stomach and intestine by a sodium carrier-mediated mechanism at low concentrations.

\section{Distribution and metabolism}

Niacin circulates in the plasma in the unbound form as both the acid and the amide. Each enters peripheral tissues by passive diffusion, followed by metabolic trapping by conversion to the pyridine dinucleotides, $\operatorname{NAD}(H)$ and $\operatorname{NADP}(H)$. Most is found as $N A D(H)$ and the oxidised form NAD.

The plasma half-life of nicotinic acid is relatively short, approximately one hour. Animal studies have shown that nicotinic acid rapidly disappears from the blood and is mainly concentrated in the liver, but also in adipose tissue and in the kidneys.

The main metabolites in humans are $\mathrm{N}$-methylnicotinamide, $\mathrm{N}$-methyl-2-pyridone-5-carboxamide and $N$-methyl-4-pyridone-5-carboxamide.

\section{Excretion}

The pattern of niacin products excreted after ingestion of the vitamin depends largely on the amount and form of niacin ingested and on the niacin status of the individual. However, the two major excretion products in humans are $\mathrm{N}$-methylnicotinamide and $\mathrm{N}$-methyl-2-pyridone-5-carboxamide, with minor amounts of the unchanged vitamin, nicotinamide- $N$-oxide and 6-hydroxynicotinamide also being excreted.

\section{Toxicity}

\section{Human data}

Reports of nicotinic acid toxicity in humans stem, in the main, from its use in the treatment of hypercholesterolaemia. Most adverse effects are dose related and generally subside with a reduction in dose or the cessation of treatment. Symptoms of acute toxicity include flushing, itching of the skin, nausea, vomiting and gastrointestinal disturbances. Additionally, jaundice, hyperglycemia, abdominal pain, elevated serum bilirubin, alkaline phosphatase and aminotransferase levels can be seen with ingestion of high levels of nicotinic acid (generally intakes of 3,000 mg/day or more) for long periods of 
time. In a small number of cases, anorexia, ophthalmological effects, skin hyperpigmentation and precipitation of incipient psychosis have been reported as side effects of nicotinic acid therapy. Sustained release preparations are reported to be more hepatotoxic than the crystalline form.

Evidence is sparse, but there have been case reports of liver dysfunction following long-term high dose (3000-9000 mg) nicotinamide therapy.

\section{Supplementation trials}

Hoffer (1969) reported a range of adverse effects, including headaches, heartburn, nausea, gastrointestinal disturbances and fatigue at doses of $3000 \mathrm{mg}$ supplemental nicotinamide/day for 3-36 months. However, few details were provided and comparison with controls was lacking. Other supplementation trials (Vague et al., 1987; Mendola et al., 1989; Chase et al., 1990; Pozzilli et al., 1995; Lampeter et al., 1998) have reported no adverse effects at intakes of nicotinamide up to $3000 \mathrm{mg} /$ day. However the studies in which the highest doses were given were primarily of beneficial effect in Type 1 diabetes mellitus patients and it is unclear how information on adverse effects was ascertained. Gastrointestinal effects and flushing have been reported at intakes of $50 \mathrm{mg}$ supplemental nicotinic acid/day and above.

Doses of up to $2000 \mathrm{mg} /$ day of nicotinic acid have reportedly been administered during pregnancy to niacin deficient women in developing countries, without evidence of foetal toxicity (Moghissi, 1981).

\section{Animal data}

Few data are available concerning the ingestion of nicotinic acid. Acute effects in dogs, associated with intake of $2000 \mathrm{mg} /$ day for less than 20 days, included weight loss, blood in the faeces and convulsions resulting in death with associated gastrointestinal and central nervous system changes. With administration of lower doses (up to $1000 \mathrm{mg} /$ day) for 8 weeks dogs gained weight and appeared in good health. Traces of albumin and sugar were found in urine.

Chronic administration of $1 \%$ nicotinamide in the diet to rats has been shown to inhibit growth.

\section{Reproductive toxicity}

In rats, ingestion of nicotinamide has been shown to cause growth retardation, which may be due, in part, to the reduced intake of food and water due to the palatability and in part to a deficiency in methionine, which is expended during the methylation of nicotinamide into its metabolites.

\section{Carcinogenicity and genotoxicity}

No carcinogenicity data are available for nicotinic acid.

In lifetime studies in the mouse, nicotinamide alone was not carcinogenic. However, in combination with streptozotocin, nicotinamide has been shown to cause islet cell tumours in rats. The resulting oncogenicity is likely to be the result of an imbalance between the degree of DNA damage and the level of inhibition of DNA repair.

No genotoxicity data are available for nicotinic acid or nicotinamide. 
Part 1 Water Soluble Vitamins

\section{Dose response characterisation}

Few data are available, but supplemental intakes of nicotinamide of up to $3000 \mathrm{mg} /$ day appear to be well tolerated. Flushing has been reported with bolus doses of nicotinic acid of $10 \mathrm{mg}$ or higher, but flushing is more consistently associated with supplemental intakes of nicotinic acid of $50 \mathrm{mg} /$ day and above.

\section{Mechanisms of toxicity}

No relevant data have been identified.

\section{Vulnerable groups}

Individuals with hepatic dysfunction or a history of liver disease, diabetes mellitus, active peptic ulcer disease, gout, cardiac arrhythmias, migraine headaches and alcoholism may be particularly susceptible to nicotinic acid effects.

\section{Genetic variations}

No data on genetic variations that increase vulnerability to niacin toxicity have been identified.

\section{Studies of particular importance in the risk assessment}

(For full review see http://www.food.gov.uk/science/ouradvisors/vitandmin/evmpapers or the enclosed CD)

\section{Human data - nicotinic acid}

Spies et al., 1938

In an uncontrolled study, 100 adult subjects were given single oral doses of 50 or $100 \mathrm{mg}$ nicotinic acid on an empty stomach. Flushing, burning and itching was reported in $5 \%$ of those receiving the $50 \mathrm{mg}$ dose and $50 \%$ of those given $100 \mathrm{mg}$. Flushing occurred in the majority of subjects receiving $200 \mathrm{mg}$ doses of nicotinic acid and was present 'to some extent' in all subjects receiving $500 \mathrm{mg}$ doses. It is unclear how the subjects were divided between dose groups. By studying cutaneous temperatures, it was determined that the increase was most marked over the ears and neck and less pronounced over the trunk, with the extremities being least affected.

\section{Sebrell and Butler, 1938}

In an uncontrolled study, groups of six subjects were given 10, 30 or 50 mg nicotinic acid daily for three months. Flushing was reported intermittently in 0, 2 and 4 individuals, respectively. The subjects received an identical diet. The authors noted that there was considerable inter-individual variation, which was not accounted for by body weight. 


\section{The Coronary Drug Project, 1975}

The study was conducted to investigate the possible use of nicotinic acid in coronary heart disease to reduce the incidence of a second myocardial infarction. The study demonstrated little immediate beneficial effect, but considerable toxicity. One third to one half of the 1119 patients taking $3000 \mathrm{mg}$ nicotinic acid/day for at least five years had increased serum levels of liver enzymes. An elevation in serum uric acid levels was noted in the nicotinic acid treated group (44\% of nicotinic acid treated individuals) and an increased incidence of acute gouty arthritis (6.4\% of nicotinic acid treated individuals), compared to controls ( $4.3 \%$ of individuals). Consistent with other reports, side effects included dermatological problems of flushing, itching and rash. Other significant complaints in nicotinic acid-treated individuals included gastrointestinal and urinary tract problems.

Knopp et al., 1985

A study in 71 patients compared the effects of regular nicotinic acid (37 individuals) and sustained release nicotinic acid (34 individuals). Treatment was for six months and subjects received an initial dose of $1500 \mathrm{mg} /$ day, increasing to $3000 \mathrm{mg} /$ day from the second month. However, due to a higher frequency of side effects among those receiving the sustained release formula, the daily intake was reduced to $2000 \mathrm{mg} /$ day for this group. Subjects treated with sustained release nicotinic acid experienced a significantly higher rate of gastrointestinal effects, compared with those on regular nicotinic acid. Dermatological problems were reported for both treatments.

Fraunfelder et al., 1995

In a retrospective survey, 102 patients taking nicotinic acid medication (3000-8000 mg/day) for hyperlipidaemia were more likely than those taking other lipid lowering agents to report blurred vision (26\%), dry eyes (20\%), eyelid oedema (10\%) and macular oedema $(2 \%)$. The ocular side effects were all reversible if treatment was discontinued and the authors reported the severity of effects to be dose dependent. For one patient, a reduction in dose from $3000 \mathrm{mg} /$ day to $1500 \mathrm{mg} /$ day was stated to have reversed the effects on her vision.

\section{Human data - nicotinamide}

Vague et al., 1987

In a study published as a letter, no adverse effects were reported in a double-blind trial in 16 Type 1 diabetics who received $3000 \mathrm{mg}$ nicotinamide per day or placebo for six months. The average age of the subjects was 22.1 years in the treatment group and 24.8 years in the placebo group. No adverse effects were noted but it is unclear how this was ascertained.

\section{Mendola et al., 1989}

In a single-blind trial, twenty newly diagnosed Type I diabetics, received $1000 \mathrm{mg}$ nicotamide/day or placebo for 45 days. The average age of the subjects was 18.3 years in the treatment group and 15.5 years in the placebo group. No adverse effects were observed when physiological, biochemical and haematological parameters were assessed. 
Part 1 Water Soluble Vitamins

\section{Chase et al., 1990}

In a double-blind study in 35 newly diagnosed Type I diabetics, aged between 6 and 18 years, individuals received $100 \mathrm{mg}$ nicotinamide per year of age per day, up to a maximum of $1500 \mathrm{mg} /$ day for 12 months. The authors stated that no significant adverse effects were encountered with the use of nicotinamide, although it is unclear how information on side effects was ascertained.

\section{Pozzilli et al., 1995}

In a double-blind trial following up the work of Mendola, a further 56 newly diagnosed Type I diabetics aged 5-35 years were given $25 \mathrm{mg} / \mathrm{kg}$ bw/day or placebo for 12 months. It was stated that biochemical tests including liver and kidney function were normal during follow up and that no adverse effects were noted in either treated or placebo patients.

Lampeter et al., 1998

The Deutsche Nicotinamide Intervention Study (DENIS) evaluated the clinical efficacy of high doses of nicotinamide in children at high risk of developing Type I diabetes. Fifty-five children were randomised into two groups and received either placebo or supplemental nicotinamide (1200 mg $/ \mathrm{m}^{2} /$ day) for a maximum duration of 3.8 years. Mean treatment time was 2.1 years. If it is assumed that the children in the study were of average height and weight, a total nicotinamide intake, including the contribution of the diet, of $1260 \mathrm{mg} /$ day or $42 \mathrm{mg} / \mathrm{kg}$ bw/day can be estimated in subjects receiving the nicotinamide supplement. The rates of diabetes onset were the same throughout the observation period in both groups. All biochemical and haematological parameters (alanine aminotransferase, aspartate aminotransferase, bilirubin, blood sedimentation rate, $\gamma$-glutamyl transferase, urea, uric acid, creatinine and lactate dehydrogenase) were within the normal range, and means did not differ between the groups throughout the study.

\section{Exposure assessment}

The exposure data below for intake from food are for niacin equivalents. These are defined as the niacin content of the food plus 1/60th the content of tryptophan, as nicotinic acid is formed in the body from the metabolism of tryptophan. It is not possible to distinguish the two forms of niacin in this survey data.

Food

Mean: 34 mg/day (from 1986/87 NDNS)

97.5th percentile: $57 \mathrm{mg} /$ day

Supplements

up to $250 \mathrm{mg} /$ day (as nicotinamide, or up to $150 \mathrm{mg} /$ day as nicotinic acid) (Annex 4; OTC, 2001)

Estimated maximum intake

of niacin equivalents: $\quad 57+250=307 \mathrm{mg} /$ day.

No potential high intake groups have been identified. 


\title{
Risk assessment
}

\author{
Nicotinic acid \\ Large doses of nicotinic acid are associated with a number of adverse effects in man. These have been \\ identified from the use of nicotinic acid in the treatment of hypercholesterolaemia. The effects \\ reported include flushing, skin itching, nausea, vomiting and gastrointestinal disturbance. The effects are \\ dose related and reversible on cessation of treatment.
}

At higher intakes of nicotinic acid over long periods of time, liver dysfunction has been reported. Symptoms such as elevated liver enzymes, elevated bilirubin levels and jaundice have been observed. Other adverse effects reported include hyperglycaemia and adverse ophthalmological effects such as blurred vision and cystoid macular oedema. No relevant animal data have been reported and the mechanism for nicotinic acid-induced toxicity is unclear.

\section{Nicotinamide}

Fewer data are available on the safety of nicotinamide. Studies in Type I diabetics have suggested that doses of up to $3000 \mathrm{mg} /$ day nicotinamide are not associated with adverse effects, although these investigations involved small numbers of subjects and it is unclear from the studies in which the highest doses were given how adverse effects would have been ascertained. Since the studies were undertaken in diabetics or in individuals at high risk of developing diabetes the applicability of the results to the general population is unclear. No relevant animal data have been identified.

\section{ESTABLISHMENT OF GUIDANCE LEVEL - NICOTINIC ACID}

There are insufficient data from human or animal studies to establish a Safe Upper Level for nicotinic acid.

Numerous reports exist, including both case reports and controlled clinical trials, in which doses of approximately $3000 \mathrm{mg} /$ day nicotinic acid have apparently caused hepatotoxic effects. For example, in a randomised double-blind study, one third or more of 1119 patients who received $3000 \mathrm{mg}$ nicotinic $\mathrm{acid} /$ day for up to 5 years were reported to have elevated levels of liver enzymes. Elevations in serum uric acid levels and an increased incidence of gout were also reported.

Flushing has been consistently reported at intakes of $50 \mathrm{mg} /$ day and above (Spies et al., 1938; Sebrell and Butler, 1938). If $50 \mathrm{mg} /$ day is taken as a LOAEL and an uncertainty factor of 3 is applied to extrapolate to a NOAEL, then a guidance level, for supplementation only, of 50/3 = 17 mg/day (equivalent to $0.28 \mathrm{mg} / \mathrm{kg}$ bw/day in a $60 \mathrm{~kg}$ adult) for nicotinic acid is derived. This guidance level is given for supplements only, as adverse effects appear to be related to acute, bolus intakes of nicotinic acid, rather than more sustained exposure as would occur with ingestion of nicotinic acid via food. Free nicotinic acid levels in food are low.

It should be noted that this guidance level is based on intakes of conventional formulations of nicotinic acid and, therefore, would not be applicable to sustained release preparations. Nicotinic acid contained in dietary supplements is not in the sustained release form, which is thought to be more hepatotoxic. 
Part 1 Water Soluble Vitamins

\section{ESTABLISHMENT OF GUIDANCE LEVEL- NICOTINAMIDE}

There are insufficient data from human or animal studies to establish a safe upper level for nicotinamide.

From the limited existing database the occurrence of nicotinamide toxicity appears to be quite low. Large doses of nicotinamide (up to $3000 \mathrm{mg} /$ day for periods of up to 3 years) appear to be well tolerated, as reported in numerous trials on the possible benefits of nicotinamide in patients with, or at risk of developing, diabetes. These trials, however, usually studied only one dose level, and the numbers of subjects involved in the trials was small. Two of the best conducted studies are those by Pozzilli et al. (1995) and Lampeter et al. (1998) and we have used these for guidance purposes. In these studies, doses of 25 and $42 \mathrm{mg} / \mathrm{kg}$ bw/day did not affect a range of biochemical parameters, including liver and kidney function tests in small groups of Type 1 diabetics (or those at high risk of developing the condition). Although no adverse effects were detected, the nature of the study population and the small numbers involved mean that these findings may not be applicable to the whole population. Although no adverse effects were identified, it is prudent in this case to apply a UF of 3 to account for inter-individual variability because of the nature of the study population. Thus, $25 / 3$ results in a guidance value, for supplementation only, of $8.3 \mathrm{mg} / \mathrm{kg}$ bw/day for nicotinamide. This is equivalent to $500 \mathrm{mg} /$ day supplemental nicotinamide in a $60 \mathrm{~kg}$ adult.

Assuming a maximum intake of $57 \mathrm{mg} /$ day from food, a total dose of $560 \mathrm{mg} /$ day (equivalent to 9.3 $\mathrm{mg} / \mathrm{kg}$ bw/day in a $60 \mathrm{~kg}$ adult) would not be expected to result in any adverse effects. There is a lack of data on the safety of nicotinamide in pregnancy, and no relevant animal data. This level does not therefore apply to pregnant women.

\section{References}

Chase, H.P., Butler-Simon, N., Garg, S., McDuffie, M., Hoops, S.L. and O’Brien, D. (1990) A trial of nicotinamide in newly diagnosed patients with Type 1 (insulin-dependent) diabetes mellitus. Diabetologia 33, 444-446.

COMA (1991). Dietary Reference Values for Food Energy and Nutrients for the United Kingdom. Report of the Panel on Dietary Reference Values, Committee on Medical Aspects of Food and Nutrition Policy. HMSO, London.

Coronary Drug Project Research Group. (1975) Clofibrate and niacin in coronary heart disease. Journal of the American Medical Association 231, 360-81.

Fraunfelder, F. W., Fraunfelder, F. T., Illingworth, D. R. (1995) Adverse ocular effects associated with niacin therapy. British Journal of Ophthalmology 79, 54-56.

Hoffer, A. (1969) Safety, side effects and relative nicotinic acid and nicotinamide. Schizophrenia 1, 78-87.

Knopp, R. H., Ginsberg, J., Albers, J. J. (1985) Contrasting effects of unmodified and time-release forms of niacin on lipoproteins in hyperlipidemic subjects: clues to mechanism of action of niacin. Metabolism 34, 642-650. 
Lampeter, E.F., Klinghammer, A., Scherbaum, W.A., Heinze, E., Haastert, B., Giani, G., Kolb, H. (1998) The Deutsche Nicotinamide Intervention Study: an attempt to prevent Type I diabetes. Diabetes 47, 980-984.

Mendola, G., Casamitjana, R. and Gomis, R. (1989) Effect of nicotinamide therapy upon B-cell function in newly diagnosed Type 1 (insulin-dependent) diabetic patients. Diabetologia 32, 160-162.

Moghissi, K.S. (1981) Risks and benefits of nutritional supplements during pregnancy. Obstetrics and Gynecology, 58, 68s-78s.

Pozzilli, P., Visalli, N., Signore, A., Baroni, M.G., Buzzetti, R., Cavallo, M.G., Boccuni, M.L., Fava, D., Gragnoli, C., Andreani, D., Lucentini, L., Matteoli, M.C., Crino, A., Cicconetti, C.A., Teodonio, C., Paci, F., Amoretti, R., Pisano, L., Pennafina, M.G., Santopadre, G., Marozzi, G., Multari, G., Suppa, M.A., Caqmpea, L., De Mattia, G.C., Cassone Faldetta, M., Marietti, G., Perrone, F., Greco, A.V., Ghirlanda, G. (1995) Double blind trial of nicotinamide in recent-onset IDDM (the IMDIAB III study). Diabetologia 38, 848-852.

Sebrell, W.H. and Butler, R.E. (1938) A reaction to the oral administration of nicotinic acid. Journal of the American Medical Association 111, 2286-2287.

Spies, T.D., Bean WB, Stone, R.E. (1938) The treatment of subclinical and classic pellagra. Use of nicotinic acid, nicotinic acid amide and sodium nicotinate, with special reference to the vasodilator action and the effect on mental symptoms. Journal of the American Medical Association 111, 584-592.

Vague, P., Vialettes, B., Lassmann-Vague, V., and Vallo, J. J. (1987) Nicotinamide may extend remission phase in insulin dependent diabetes. Lancet 1, 619-620. 


\section{Risk Assessment}

\section{Pantothenic acid}

\section{General information}

\section{Chemistry}

Pantothenic acid consists of a pantoic acid moiety amide-linked to a ß-alanine subunit. Pantetheine consists of pantothenic acid linked to a B-mercaptoethylamine group. In living systems, the compound is a component of coenzyme A (COA), which is composed of 4'-phosphopantetheine linked to adenosine 5'-monophosphate, modified by a 3'-hydroxyl phosphate. 4'-Phosphopantetheine is also found covalently linked to various proteins, particularly those involved in fatty acid metabolism.

\section{Natural occurrence}

The active vitamin is present in virtually all plant, animal and microbial cells.

\section{Occurrence in food, food supplements and medicines}

The majority of pantothenic acid within foods is present as CoA. Chicken, beef, potatoes, oat cereals, tomato products, liver, kidney, yeast, egg yolk, broccoli and whole grains are reported to be major sources of the vitamin, whilst very high levels are present in royal bee jelly $(511 \mathrm{mg} / \mathrm{kg})$ and in the ovaries of tuna and cod $(2300 \mathrm{mg} / \mathrm{kg})$. Cooking is reported to destroy $15-50 \%$ of pantothenic acid in meats and 37 to $78 \%$ of pantothenic acid is lost from vegetables during processing. Breakfast cereals may be fortified with 5-6 $\mathrm{mg}$ pantothenic acid/100 g.

Pantothenic acid derivatives sold as supplements or medicines are prepared synthetically as calcium pantothenate or panthenol, which are more stable than pure pantothenic acid. In the UK, dietary supplements generally contain up to $550 \mathrm{mg}$ pantothenic acid; licensed medicines may contain a maximum of $50 \mathrm{mg} /$ day pantothenic acid.

\section{Other sources of exposure}

The pantothenic acid derivative, panthenol, is added to some cosmetic products.

\section{Recommended amounts}

Dietary reference values for pantothenic acid have not been established in the UK. There is no detectable evidence of pantothenic acid deficiency within the general population, even during pregnancy and lactation. Therefore, human requirements for pantothenic acid are considered to be adequately provided by the diet. 


\section{Analysis of tissue levels and pantothenic acid status}

Blood, urine or tissue pantothenic acid levels may be measured by yeast or lactobacillus assay, or by radio-immunoassay. Assays of pantothenic acid in biological materials other than urine require that the compound be first hydrolysed from COA.

\section{Brief overview of non-nutritional beneficial effects}

It has been claimed that supplementation with pharmacological doses of pantothenic acid may alleviate the symptoms of rheumatoid arthritis and lupus erythematosus.

\section{Function}

Pantothenate, usually in the form of CoA-containing species (e.g. acetyl CoA, succinyl CoA), fulfils multiple roles in cellular metabolism and in the synthesis of many essential molecules.

\section{Deficiency}

Deficiency of pantothenic acid in humans is extremely rare. Pantothenic acid deficiency has been induced experimentally in human subjects by a diet virtually devoid of the vitamin, or by the administration of a metabolic antagonist (omega-methyl pantothenic acid). Signs and symptoms exhibited by subjects given the antagonist (not all of which were necessarily due to the deficiency) included irritability and restlessness, fatigue, apathy, malaise, sleep disturbance, gastrointestinal complaints (such as nausea, vomiting and abdominal cramps) neurological and other clinical effects such as numbness, paraesthesia, muscle cramps and staggering gait, hypoglycaemia and an increased sensitivity to insulin. Historically, pantothenic acid deficiency has been implicated in the 'burning feet' syndrome experienced by severely malnourished prisoners of war.

\section{Interactions}

Reports have indicated a sparing effect of some other vitamins, such as ascorbic acid and other Bvitamins, on pantothenic acid within the body. Biotin and pantothenic acid may share a common carrier-mediated uptake mechanism in the gastrointestinal tract and other tissues, although the physiological relevance of this is unknown. Some earlier reports have suggested that high dietary fat and low dietary protein may exacerbate pantothenic acid deficiency.

\section{Absorption and bioavailability}

Pantothenic acid is readily absorbed throughout the gastrointestinal tract. Ingested CoA is hydrolysed within the intestinal lumen, via the formation of dephospho-CoA, phosphopantetheine and pantetheine, to pantothenic acid. Uptake of these latter two compounds into intestinal tissues has been demonstrated, and subsequently the enzyme, pantetheinase, can hydrolyse pantetheine to pantothenic acid. Uptake into intestinal cells occurs both by a sodium-dependent active transport mechanism and by passive diffusion. Limited data are available regarding the bioavailability of dietary pantothenic acid. One study found that pantothenic acid in natural foods was approximately $50 \%$ bioavailable compared with calcium pantothenate given in a formula diet, as assessed by subsequent urinary excretion of the vitamin. 
Part 1 Water Soluble Vitamins

\section{Distribution and metabolism}

Absorbed pantothenic acid is transported to body tissues via the blood, primarily as bound forms within erythrocytes. Plasma levels do not correlate well with dietary intake. The majority of tissues import pantothenic acid via an active sodium co-transport mechanism. Analysis of rat tissues has shown high concentrations of pantothenic acid in the heart and kidneys. CoA is synthesised from pantothenic acid within cells, with the first, and apparently rate-limiting, step catalysed by pantothenate kinase.

\section{Excretion}

Catabolism of CoA leads to the formation of pantothenate, which is excreted in the urine. Excretion levels correlate well with dietary intake.

\section{Toxicity}

\section{Human data}

Case reports and some much earlier non-controlled studies describe a lack of acute or chronic toxic effects of pantothenic acid compounds (calcium or sodium pantothenate, panthenol) at very high doses (approximately 10,000 mg/day in some cases for a number of years), although such levels have been associated with diarrhoea and gastrointestinal disturbances. In more recent, controlled studies (generally carried out to assess the potential benefits of pantothenic acid supplementation in specific subgroups, for example, arthritic patients) no side effects have been reported for pantothenic acid supplementation at levels up to approximately $2000 \mathrm{mg} /$ day, for periods of several days to several weeks. However, the small numbers of participants and short duration of these studies limit the value of the data regarding any potential rare or long-term toxic effects.

One non-blind, non-randomised, non-placebo-controlled trial, designed to investigate the effectiveness of megavitamin therapy in improving the behaviour of 41 children with attention deficit disorder, showed significant increases in serum aspartate transaminase levels (indicative of liver damage) in 17 children after 12 weeks of multivitamin therapy (including doses of calcium pantothenate increasing during the study period to a maximum of $1200 \mathrm{mg} /$ day). This effect may have been associated with the nicotinamide component of the multivitamin supplement, although this could not be confirmed as the vitamins were not given separately.

\section{Animal data}

Data regarding the toxicity of pantothenic acid and its commonly-used pharmaceutical forms in experimental animals are limited because of the small numbers of animals used in the studies. In the early 1940's Unna \& Greslin reported acute and chronic toxicity tests with D-calcium pantothenate in mice, rats, dogs and monkeys (Unna and Greslin, 1940, 1941). Acute oral $\mathrm{LD}_{50}$ values were very high $\geqslant$ $10,000 \mathrm{mg} / \mathrm{kg}$ bw, mice and rats), with lethal doses producing death by respiratory failure. An oral dose of $1000 \mathrm{mg} / \mathrm{kg}$ bw produced no toxic signs in dogs or in one monkey. Oral dosing (500 or $2000 \mathrm{mg} / \mathrm{kg}$ bw/day to rats, $50 \mathrm{mg} / \mathrm{kg}$ bw/day to dogs, $200-250 \mathrm{mg} / \mathrm{kg}$ bw/day to monkeys) for 6 months produced no toxic signs or weight loss, or evidence of histopathological changes at autopsy. The offspring of rats 
supplemented with $500 \mathrm{mg} / \mathrm{kg}$ bw/day calcium pantothenate were fed diets supplemented with 500 $\mathrm{mg} / \mathrm{kg}$ bw/day calcium pantothenate from weaning; no evidence of toxicity or reduced weight gain, or histopathological changes were observed. The available data do not indicate reproductive or developmental toxicity of pantothenic acid or its commonly used pharmaceutical forms.

\section{Carcinogenicity and genotoxicity}

Calcium pantothenate, sodium pantothenate and panthenol were not mutagenic in bacterial tests. No in vivo genotoxicity or carcinogenicity data have been found.

\section{Mechanism of toxicity}

No data have been identified.

\section{Dose response characterisation}

No data have been identified.

\section{Vulnerable groups}

No vulnerable groups have been identified.

\section{Genetic variations}

No genetic variations in the metabolism or effects of pantothenic acid have been identified.

\section{Studies of particular importance in the risk assessment}

(For full review see http://www.food.gov.uk/science/ouradvisors/vitandmin/evmpapers or the enclosed CD).

\section{Human studies}

Goldman, 1950; Welsh, 1952, 1954

Supplementation of lupus erythematus patients with doses of pantothenic acid of $10,000 \mathrm{mg} /$ day or more led to gastrointestinal signs and symptoms.

The General Practitioner Research Group, 1980

This randomised, double-blind, placebo-controlled study was carried out to assess the effects of highdose pantothenate supplementation in alleviating symptoms of arthritis. A total of 47 (31 completed the trial) patients were treated with calcium pantothenate (total daily dose $-500 \mathrm{mg}$ on days $1-2,1000 \mathrm{mg}$ on days 3-5, $1500 \mathrm{mg}$ on days 6-9, $2000 \mathrm{mg}$ from day 10 onwards) and 47 (34 completed the trial) with placebo for a period of 8 weeks. The patients were suffering from a variety of arthritides, including 27 patients with rheumatoid arthritis. The authors stated that '... no side effects were recorded in 43 patients (91\%) on calcium pantothenate as compared to 37 (79\%) on placebo.' 
Part 1 Water Soluble Vitamins

\section{Animal studies}

\section{Unna \& Greslin, 1940, 1941}

These were reports of sub-chronic toxicity studies of D-calcium pantothenate in rats, dogs and monkeys (strains not specified). Young rats (10 males and 10 females per group) were fed doses of 50 or 200 $\mathrm{mg} /$ day (approximately 500 or $2000 \mathrm{mg} / \mathrm{kg}$ bw/day) D-calcium pantothenate, for 190 days. Growth and development were normal and did not differ significantly from those of a control group fed a standard diet. The authors reported that autopsies at the end of the feeding period did not reveal any gross or microscopic changes in the organs (not specified). Six adult dogs and 4 monkeys were fed $50 \mathrm{mg} / \mathrm{kg}$ bw/day and $1000 \mathrm{mg} /$ day (approximately $200-250 \mathrm{mg} / \mathrm{kg}$ bw/day) D-calcium pantothenate, respectively, for periods of 6 months. None of the animals showed any toxic signs or weight loss during the supplementation period, and again the authors reported that histopathological examination at the end of the supplementation period did not reveal any changes.

\section{Exposure assessment}

Total exposure/intake:

Food

Mean: $5.4 \mathrm{mg} /$ day (1990 NDNS)

97.5th percentile: $9.7 \mathrm{mg} /$ day

Supplements $\quad$ up to $550 \mathrm{mg} /$ day (Annex 4)

Estimated maximum intake $9.7+550=560 \mathrm{mg} /$ day

No potential high intake groups were identified.

\section{Risk assessment}

The available toxicological data on pantothenic acid are limited. However, case reports and some earlier, uncontrolled human studies suggested a lack of acute or chronic toxic effects of pantothenic acid compounds (calcium or sodium pantothenate, panthenol) at very high doses (approximately 10,000 $\mathrm{mg} /$ day, in some cases for a number of years). However, doses at such levels have been associated with diarrhoea and gastrointestinal disturbances. In more recent, controlled studies, no side effects have been reported with pantothenic acid supplementation at levels up to approximately $2000 \mathrm{mg} /$ day, for periods varying from several days to several weeks. These studies were generally designed to assess the potential benefits of pantothenic acid supplementation in specific subgroups, for example patients suffering joint disease.

Data regarding the toxicity of pantothenic acid and its commonly-used pharmaceutical forms in experimental animals are also limited. However, doses of 500 and $2000 \mathrm{mg} / \mathrm{kg}$ bw/day in rats and 200$250 \mathrm{mg} / \mathrm{kg}$ bw/day in dogs and monkeys, given in the diet for periods of six months, were not associated with adverse effects. 


\section{ESTABLISHMENT OF GUIDANCE LEVEL}

There are insufficient data from human or animal studies to establish a Safe Upper Level for pantothenic acid.

There are relatively few human data available on the oral toxicity of pantothenic acid from controlled trials. The limited available data have not identified target organ toxicity and the adverse effects that were noted were transient. The apparent low toxicity of pantothenic acid is supported by the available animal data. The General Practitioner Research Group study suggests that doses of $2000 \mathrm{mg}$ pantothenic acid/day are without effect, although this study investigated relatively few individuals, suffering from a variety of clinical conditions (rheumatoid arthritis, osteoarthritis, gout and spondylitis). Adverse effects were not a primary outcome measure, and though the authors specifically noted the absence of side effects in fewer placebo than treated individuals, the method by which these data were collected and the specific nature of the side effects noted are not discussed. An uncertainty factor of 10 is applied to allow for inter-human variability because of the small numbers of individuals involved, of which one third failed to complete the study, and the incomplete investigation of possible adverse effects. Based on these data, for guidance purposes only, a supplemental daily intake of $200 \mathrm{mg}$ (equivalent to $3.3 \mathrm{mg} / \mathrm{kg}$ bw/day for a $60 \mathrm{~kg}$ adult), in addition to that present in the diet, would not be expected to produce adverse effects in the general population. Assuming a maximum dietary intake of $10 \mathrm{mg} /$ day, this would equate to a total intake of $210 \mathrm{mg} /$ day, or $3.5 \mathrm{mg} / \mathrm{kg}$ bw/day for a $60 \mathrm{~kg}$ adult.

\section{References}

General Practitioner Research Group (1980) Calcium pantothenate in arthritic conditions. A report from the General Practitioner Research Group. Practitioner 224, 208-211.

Goldman, L. (1950) Intensive panthenol therapy of lupus erythematosus. Journal of Investigative Dermatology 15, 291-293.

Unna, K., Greslin, J.G. (1940) Toxicity of pantothenic acid. Proceedings of the Society for Experimental Biology and Medicine 45, 311-312.

Unna, K., Greslin, J.G. (1941). Studies on the toxicity and pharmacology of pantothenic acid. Journal of Pharmacology and Experimental Therapeutics 73, 85-90.

Welsh, A.L. (1952). Lupus erythematosus: treatment by combined use of massive amounts of calcium pantothenate or panthenol with synthetic vitamin E. Archives of Dermatology and Syphilology 65, 137-148.

Welsh, A.L. (1954). Lupus erythematosus: treatment by combined use of massive amounts of pantothenic acid and vitamin E. Archives of Dermatology 70, 181-198. 


\section{Risk Assessment}

\section{Riboflavin}

\section{General information}

\section{Chemistry}

Riboflavin is a water-soluble vitamin of the $B$ group (vitamin $B_{2}$ ). It is stable to mineral acids in the dark at $27^{\circ} \mathrm{C}$. Decomposition occurs in both acidic and alkaline solutions.

\section{Natural occurrence}

Riboflavin is present as an essential constituent of all living cells, and is therefore widely distributed in small amounts in foods.

\section{Occurrence in food, food supplements and medicines}

The major sources of riboflavin are milk, eggs, enriched cereals and grain, ice cream, liver, some lean meats, and green vegetables. Because riboflavin is degraded by light, loss will occur if foods are left out in sunlight, or any UV light. Riboflavin is stable when heated but will leach into cooking water. The pasteurisation process causes milk to lose about $20 \%$ of its riboflavin content. Alkalis, such as baking soda, also destroy riboflavin. Riboflavin is a permitted colouring agent in foods and pharmaceuticals. It is permitted at quantum satis level in most processed foods, but due to its instability when exposed to light, its use tends to be restricted to relatively few foods, such as salad dressings, confectionery and powdered drinks. It is included in multi-nutrient supplements available over the counter at levels up to $100 \mathrm{mg} /$ daily dose. It is also present in some multi-constituent products which can only be sold in pharmacies because other constituents cannot be sold without the supervision of a pharmacist. These are used for the prevention or treatment of nutrient deficiencies and contain up to $25 \mathrm{mg}$ riboflavin per daily dose.

\section{Other sources of exposure}

No other sources of exposure were identified.

\section{Recommended amounts}

The minimal requirement for riboflavin to prevent clinical signs of deficiency appears to be less than $0.35 \mathrm{mg} / 1000 \mathrm{kcal}$. COMA recommended a RNI of $1.1 \mathrm{mg} /$ day for women and $1.3 \mathrm{mg} /$ day for men (COMA, 1991). Turnover of riboflavin appears to be related to energy expenditure, and periods of increased physical activity are associated with a modest increase in requirement, but COMA saw no justification for reducing the RNIs for older people below those for younger adults. 


\section{Analysis of tissue levels and riboflavin status}

Riboflavin and its coenzyme derivatives, flavin mononucleotide (FMN) and flavin adenine dinucleotide (FAD), can be detected with high sensitivity using high pressure liquid chromatography. Riboflavin status can be assessed using the erythrocyte glutathione reductase activation test, in which an activity coefficient (EGRAC) $>1.30$ is indicative of biochemical riboflavin deficiency.

\section{Brief overview of non-nutritional beneficial effects}

Riboflavin has been suggested for the treatment of migraine, carpal tunnel syndrome, cataracts and a variety of skin conditions (acne, dermatitis, eczema, ulceration), and for muscle cramps. However, there is no firm evidence that it is effective in any condition that is not related to riboflavin deficiency.

\section{Function}

Clinically, riboflavin promotes normal growth and assists in the synthesis of steroids, red blood cells, and glycogen. FAD also play a role in oxidation-reduction reactions, interacting with a group of enzymes known as flavoproteins. Riboflavin helps to maintain the integrity of mucous membranes, skin, eyes and the nervous system. It supports the activity of antioxidants and is involved in the production of adrenaline by the adrenal glands. It is thought that riboflavin also aids the body in absorbing iron, since it is common for iron deficiency to accompany a deficiency in riboflavin.

\section{Deficiency}

Individuals who have inadequate food intake are at risk of deficiency, particularly children in developing countries. Other groups prone to riboflavin deficiency include older people with poor diet, chronic 'dieters', patients taking tranquillisers, persons who use fibre-based laxatives regularly, patients with hypothyroidism and women who exercise excessively. Riboflavin deficiency may arise in neonates during phototherapy for jaundice. It has also been associated with the development of cataracts and of rheumatoid arthritis. Riboflavin deficiency may occur as a result of inadequate nutrition or intestinal malabsorption. Riboflavin status can markedly influence the activity of hepatic microsomal drug metabolising enzymes.

Deficiency signs and symptoms include dry and cracked skin, sensitivity to bright light, itching, dizziness, insomnia, slow learning, weakness, sore throat, hyperaemia and oedema of the pharyngeal and oral mucous membranes, cheilosis, angular stomatitis, glossitis, seborrhoeic dermatitis, corneal vascularisation and anaemia associated with pure red cell hypoplasia of the bone marrow. The anaemia that develops in riboflavin deficiency is normochromic and normocytic and is associated with reticulocytopenia; leukocytes and platelets are generally normal. Administration of riboflavin to deficient patients causes reticulocytosis, and the concentration of haemoglobin returns to normal. 
Part 1 Water Soluble Vitamins

\section{Interactions}

The absorption of iron, zinc and calcium is impaired in riboflavin deficiency. Riboflavin impairs the antibiotic activity of streptomycin, erythromycin, tyrothricin, carbomycin and tetracyclines, but no inactivation occurs with chloramphenicol, penicillin or neomycin. Thyroid hormones, corticotrophin and aldosterone enhance the formation of FMN and FAD from riboflavin, while phenothiazines and possibly tricyclic antidepressants inhibit FAD formation. Ingestion of boric acid increases the excretion of riboflavin. Prior administration of probenecid decreases the renal clearance and gastrointestinal absorption of riboflavin.

A single report (Florsheim, 1994) has found riboflavin to increase the response of mice to ionising radiation. However, Pacernick et al. (1975) found that oral riboflavin had no effect on UV-induced skin tumours in hairless mice. Similarly, oral administration of riboflavin did not affect the sensitivity of homozygous or heterozygous Gunn rats to UV light.

\section{Absorption and bioavailability}

Riboflavin is readily absorbed from the small intestine, primarily by a specialised transport mechanism involving phosphorylation of the vitamin to FMN. Passive diffusion plays only a minor role at levels ingested in the diet. Riboflavin has been shown to undergo active secretion into, and saturable reabsorption from, the kidney tubules in rat, dog and human.

\section{Distribution and metabolism}

Riboflavin is distributed to all tissues. It is present in red blood cells, and appears to bind to a subfraction of immunoglobulins in plasma. Very little riboflavin is stored. Free riboflavin is transformed in the liver to form flavin coenzymes, (FAD and FMN), which are utilised as electron transfer factors in enzymatic reductions.

\section{Excretion}

When riboflavin is ingested in amounts approximately equivalent to the minimal daily requirement, only about $10-20 \%$ appears in the urine. As the intake is increased above minimal requirements, larger proportions are excreted unchanged.

Riboflavin is also found in faeces, sometimes in quantities exceeding that ingested. This probably represents the riboflavin synthesised by intestinal microorganisms, which is not absorbed.

\section{Toxicity}

\section{Human data}

No toxic or adverse reactions to riboflavin in humans have been identified. A harmless yellow discoloration of urine occurs at high doses. However, there has been at least one unconfirmed report of dermatitis following oral ingestion of a vitamin B complex that included riboflavin. Because riboflavin is a water-soluble vitamin, excess amounts are excreted. 


\section{Supplementation trials}

Few human data are available. However, no toxic symptoms have been reported at doses of up to 400 mg per day for at least 3 months, other than occasional minor side effects that were not clearly attributable to the compound.

\section{Animal data}

On the basis of the limited data available, riboflavin appears to be of very low toxicity when administered orally or by injection to animals. It has been reported that administration of an acute oral dose of $10,000 \mathrm{mg} / \mathrm{kg}$ to rats resulted in no toxic effects. There are a few studies in which riboflavin has been administered orally to experimental animals (rats, mice, rabbits, dogs) for periods of up to 22 months at doses of up to $25 \mathrm{mg} / \mathrm{kg}$ bw per day. No obvious toxicity was observed. However, only a limited number of endpoints was investigated and, overall, the information available on the toxicity of riboflavin in experimental animals is limited. Riboflavin has been reported to be of very low developmental toxicity in experimental animals.

\section{Carcinogenicity and genotoxicity}

Riboflavin has not been shown to be carcinogenic, although deficiency of riboflavin may predispose to the development of some tumours. In a limited study in rats, oral administration of $1.5 \mathrm{mg} / \mathrm{kg}$ per day of riboflavin for 22 months was not carcinogenic.

Riboflavin was not mutagenic in the Ames Salmonella test. One report found no evidence of mutagenesis in the umu or SOS chromotest. In another report, riboflavin had no effect on polyploidy in Chinese hamster lung cells. No other adequate reports on the genotoxicity of riboflavin in other test systems could be located.

\section{Mechanism of toxicity}

No relevant data have been identified.

\section{Dose response characterisation}

No relevant data have been identified.

\section{Vulnerable groups}

There is a theoretical possibility that neonates undergoing phototherapy for hyperbilirubinaemia may be at risk at this time from photoactivation of riboflavin.

\section{Genetic variations}

No relevant genetic variations have been identified. 
Part 1 Water Soluble Vitamins

\section{Studies of particular importance in the risk assessment}

(For full review see http://www.food.gov.uk/science/ouradvisors/vitandmin/evmpapers or the enclosed CD).

\section{Human data}

\section{Zempleni et al., 1996}

The pharmacokinetics and utilisation (flavocoenzyme synthesis) of orally and intravenously administered riboflavin were assessed in a study in healthy adults. After the determination of circadian rhythms of riboflavin concentrations in plasma and urine of four males and five females (control period), each subject received three different oral riboflavin doses $(20,40$, and $60 \mathrm{mg}$ ) and one intravenous bolus injection of riboflavin $(11.6 \mathrm{mg})$. Pharmacokinetic variables were calculated using a two-compartment open model. No adverse effects were reported at any of the dose levels studied.

\section{Schoenen et al., 1998}

In a prophylactic study of migraine in 28 patients with placebo control, high doses ( $400 \mathrm{mg}$ per day) of riboflavin for at least 3 months were well tolerated, only two minor, non-specific adverse events being reported. These could not be attributed unequivocally to treatment.

\section{Exposure assessment}

Total exposure/intake:

Food Mean: $1.8 \mathrm{mg} /$ day (from 1986/87 NDNS) 97.5th percentile: $3.3 \mathrm{mg} /$ day

Supplements up to $100 \mathrm{mg} /$ day (Annex 4)

Estimated maximum intake: $3.3+100=103 \mathrm{mg} /$ day

No potential high intake groups have been identified.

\section{Risk assessment}

In several human studies riboflavin was well tolerated with no reports of adverse events.

Data on the toxicity of riboflavin in experimental animals are sparse. Acute oral administration of riboflavin to rats produced no adverse effects. Riboflavin has also been administered orally to rats, mice, rabbits and dogs for long periods without obvious toxicity. However, in none of these studies was a full evaluation performed, and in most cases only a very limited number of endpoints was investigated. 


\section{ESTABLISHMENT OF GUIDANCE LEVEL}

There are insufficient data from human and animal studies to establish a Safe Upper Level for riboflavin, although the available data indicate that it is of low toxicity. No toxicological hazard has been identified for riboflavin from animal studies. The balance of evidence suggests that ingestion of riboflavin over prolonged periods of time is without harmful effects, even at many times the normal level of exposure. In a prophylactic study of migraine, doses of $400 \mathrm{mg}$ riboflavin per day for at least 3 months were well tolerated (Schoenen et al., 1998). Only two minor non-specific adverse effects, which could not be unequivocally attributed to the treatment, were reported in the 28 patients. Although there is no evidence of hazard at higher levels, an uncertainty factor of 10 is applied to allow for inter-human variability because of the small numbers of individuals involved, who may not be representative of the general population, and the incomplete investigation of adverse effects. This suggests that, for guidance purposes only, supplemental intakes of $40 \mathrm{mg}$ riboflavin/day (equivalent to $0.67 \mathrm{mg} / \mathrm{kg}$ bw for a $60 \mathrm{~kg}$ adult) would be unlikely to result in adverse effects. This is in addition to riboflavin provided by the diet. Assuming a maximum dietary intake of $3.3 \mathrm{mg} /$ day, a total daily intake of $43 \mathrm{mg}$ riboflavin (equivalent to $0.72 \mathrm{mg} / \mathrm{kg}$ bw/day in a $60 \mathrm{~kg}$ adult) would not be expected to result in any adverse effects. The available database is insufficient for us to comment on the safety of higher intakes, although these may be without adverse effects.

\section{References}

COMA. (1991) Committee on Medical Aspects of Food and Nutrition Policy: Dietary Reference Values for Food Energy and Nutrients for the UK.. HMSO, London.

Floersheim GL. (1994) Allopurinol, indomethacin and riboflavin enhance radiation lethality in mice. Radiation Research 139, 240-247.

Pacernick LJ, Soltani K, Lorincz AL (1975) The inefficacy of riboflavin against ultraviolet-induced carcinogenesis. Journal of Investigative Dermatology 65, 547-548.

Schoenen, J., Jacquy, J., Lenaerts, M. (1998) Effectiveness of high-dose riboflavin in migraine prophylaxis. A randomised controlled trial. Neurology 50, 466-470.

Zempleni, J., Galloway, J.R., McCormick, D.B. (1996) Pharmacokinetics of orally and intravenously administered riboflavin in healthy humans. American Journal of Clinical Nutrition 63, 54-66. 


\section{Risk Assessment}

\section{Thiamin (Vitamin $\mathbf{B}_{1}$ )}

\section{General Information}

\section{Chemistry}

Thiamin (vitamin $\mathrm{B}_{1}$ ) is a relatively heat- and acid-stable, water-soluble compound, containing a pyrimidine and a thiazole nucleus linked by a methylene bridge. Derivatives of thiamin include the mono-, pyro- and triphosphate forms and the synthetic hydrochloride and slightly less water-soluble mononitrate salt. Synthetic non water-soluble derivatives of thiamin are available but these are not used in food supplements.

\section{Occurrence in food, food supplements and medicines}

Foods providing rich sources of thiamin include unrefined grain products, meat products, vegetables, dairy products, legumes, fruits and eggs. In the UK there is mandatory fortification of white and brown flour with thiamin, to a level of not less than $0.24 \mathrm{mg} / 100 \mathrm{~g}$ flour, to replace losses during production; thus, cereal products are also a rich source of thiamin.

Mononitrate or hydrochloride derivatives of thiamin are present in multi-constituent medicinal products for the prevention (dose $1-5 \mathrm{mg}$ daily) or treatment (dose $10-35 \mathrm{mg}$ daily) of nutrient deficiencies. Supplements containing thiamin alone are also available (daily doses up to $300 \mathrm{mg}$ ).

\section{Recommended amounts}

Body stores of thiamin are limited and a regular intake is necessary. Thiamin requirement is related to energy consumption. The RNI for adults and children $\geqslant 1$ year is $0.4 \mathrm{mg} / 1000 \mathrm{kcal}$ and $0.3 \mathrm{mg} / 1000$ $\mathrm{kcal}$ in infants (COMA, 1991). Assuming food intakes of $2000 \mathrm{kcal} /$ day and $20 \%$ losses through cooking, this can be estimated to be 1.4 and $1 \mathrm{mg} /$ day for adult males and females respectively. In pregnancy and lactation, thiamin requirement increases to approximately $1.6-1.8 \mathrm{mg} /$ day.

\section{Analysis of tissue levels and thiamin status}

Thiamin status may be assessed by measurement of thiamin levels in blood or by urinary excretion, before and after loading. Erythrocyte transketolase (ETK) activity or its activation coefficient (ATK-AC) in haemolysed red blood cells is a functional measure of thiamin status.

\section{Brief overview of non-nutritional beneficial effects}

No reports of non-nutritional beneficial effects have been identified. Established therapeutic uses of thiamin supplements are largely related to the treatment or prophylaxis of deficiency. The effects of thiamin on spasmodic dysmenorrhoea, exercise performance, ventricular function, Alzheimer's disease, and leg cramps during pregnancy have been investigated, with inconclusive results. 


\section{Function}

Thiamin pyrophosphate (TPP) is a co-enzyme in several enzymatic reactions. TPP may also have a nonco-enzymic function during stimulation of neuronal cells and other excitable tissues, such as skeletal muscle.

\section{Deficiency}

The biological half-life of thiamin is approximately $10-20$ days and marginal deficiency can develop quite rapidly. Symptoms of sub-clinical deficiency include headache, tiredness, anorexia and muscle wasting. A regular daily thiamin intake of $\leqslant 0.2 \mathrm{mg} / 1000 \mathrm{kcal}$ results in clinical deficiency and the disease known as beriberi, which affects the cardiovascular and nervous systems. Thiamin deficiency can result in a disorder of the central nervous system known as Wernicke's encephalopathy, characterised by confusion, ataxia and coma. This condition is sometimes accompanied by a syndrome known as Korsakoff psychosis. Both conditions are typically found in alcoholics and co-exist in Wernicke-Korsakoff syndrome. In developed countries, most cases of thiamin deficiency are associated with chronic alcoholism where dietary intake of the vitamin may be low and absorption and utilisation impaired.

Thiamin deficiency may also be involved in foetal alcohol syndrome, characterised by growth retardation, psychomotor abnormalities and congenital malformations, in the offspring of alcoholic mothers.

\section{Interactions}

Alcohol can impair the uptake and utilisation of thiamin and these effects may contribute to the prevalence of thiamin deficiency in alcoholics. Alcohol also reduces cellular thiamin diphosphokinase activity. Thiamin is an acetylcholine antagonist, and thus may enhance the effect of neuromuscular blocking agents. 5-Fluorouracil appears to be antagonistic to thiamin, possibly through competition for phosphorylation, which is required by both entities for their activation.

\section{Absorption and bioavailability}

Thiamin in food appears to be highly available for absorption. Absorption of thiamin hydrochloride and other water-soluble forms of thiamin is dose-dependent. At physiological concentrations, intestinal uptake occurs mainly via a carrier-mediated transport mechanism. However, this process is saturable and at higher concentrations, uptake is predominately by slower passive diffusion.

\section{Distribution and metabolism}

In the blood and tissues, thiamin is present as the free form and mono-, di- (pyro) and triphosphorylated forms, which are interconvertible. Free and phosphorylated forms are transported within the erythrocytes, but plasma and cerebrospinal fluid contain only the free and monophosphorylated forms. Within the tissues, most thiamin present is converted to the pyrophosphate form. Liver contains the highest concentration of thiamin. Catabolic metabolism amounts to approximately $1 \mathrm{mg} /$ day, and most of this occurs in the liver. The mean thiamin content of human breast milk in the UK has been reported to be $0.16 \mathrm{mg} / \mathrm{L}$.

\section{Excretion}

Thiamin metabolites and thiamin in excess of requirements are excreted in the urine. The level of unchanged thiamin in the urine increases as intake increases. 
Part 1 Water Soluble Vitamins

\section{Toxicity}

\section{Human data}

The oral toxicity of thiamin and thiamin derivatives in humans is generally considered very low. Most reports of adverse effects with exposure to thiamin follow parenteral nutrition. High oral doses of thiamin hydrochloride ( $\geqslant 7000 \mathrm{mg}$ ) may cause headache, nausea, irritability, insomnia, rapid pulse and weakness. These symptoms are relieved following cessation of treatment or reduction of dose. There have been a very small number of reported adverse effects following lower doses from case reports. Three case reports concerned women, one who experienced muscle tremor, rapid pulse and nervous hyperirritability after taking daily doses of thiamin hydrochloride, reported to be $17 \mathrm{mg} / \mathrm{day}^{14}$. In another case, a patient suffered an anaphylactic reaction and subsequently died following a single oral dose of $100 \mathrm{mg}$ thiamin 2 months after repeatedly taking $100 \mathrm{mg}$ thiamin per day for a period of 15 days. One patient with thiamin-related contact dermatitis experienced an exacerbation of eczema following experimental provocation with an oral dose of $200 \mathrm{mg}$ thiamin. A fourth case-report involved a young man who contracted allergic encephalitis following an oral dose of thiamin (the amount and form are unclear).

No evidence has been identified on reproductive effects of thiamin or thiamin derivatives in humans.

\section{Supplementation trials}

In a supplementation study, one isolated individual, who had earlier received parenteral thiamin hydrochloride, experienced nausea and insomnia following a daily dose of $200 \mathrm{mg}$ thiamin hydrochloride per day for less than a week. Symptoms resolved when the dose was halved.

\section{Animal data}

The animal toxicity database is limited. Thiamin is of low acute toxicity but single oral doses of $3000-$ $5000 \mathrm{mg} / \mathrm{kg}$ bw thiamin/thiamin hydrochloride in rats and mice are lethal. Thiamin nitrate is even less acutely toxic, with no adverse effects being reported in mice following a single oral dose of 5000 $\mathrm{mg} / \mathrm{kg}$ bw. There is an absence of chronic and sub-chronic data for high-dose exposure to the watersoluble thiamin derivatives.

\section{Carcinogenicity and genotoxicity}

There has been no study on the carcinogenicity of thiamin. Thiamin hydrochloride has been shown to be non-mutagenic in a range of bacterial mutagenicity and in vitro chromosomal aberration tests.

\section{Genetic variations}

There are no known genetic variations resulting in increased susceptibility to thiamine toxicity.

\footnotetext{
15 It is noted that a dose of $17 \mathrm{mg}$ would have been inconsistent with the rate of urinary and likely faecal excretion quoted within the original article. It is suggested therefore, that this was a text error within the article that should have read ' $17 \mathrm{~g}$ ', equivalent to $17,000 \mathrm{mg}$.
} 


\section{Mechanisms of toxicity}

No mechanisms of toxicity have been identified.

\section{Dose-response characterisation}

No data have been identified.

\section{Vulnerable groups}

No vulnerable groups have been identified; however, the clinical trials indicate that there is a possibility that a very small number of people may be particularly sensitive (allergic) to thiamin.

\section{Studies of particular importance in the risk assessment}

(For full review see http://www.food.gov.uk/science/ouradvisors/vitandmin/evmpapers or the enclosed CD).

\section{Human data}

Mills, 1941

Thiamin-associated toxicity was reported in a 47 year old woman who had been taking 10,000 mg thiamin hydrochloride daily for $2 \frac{1}{2}$ weeks (presumably by the oral route, although this is unclear). Symptoms were reported to resemble those of over-dosage of thyroid extract: headache, increased irritability, insomnia, rapid pulse, weakness and trembling. Symptoms disappeared within 2 days following cessation of treatment but recurred $4 \frac{1}{2}$ weeks after the patient resumed a dose of $5 \mathrm{mg}$ per day ${ }^{16}$. Again, prompt relief soon followed cessation of intake. In the same report, Mills described symptoms similar to those of thyroid hyperactivity, with fine and coarse muscle tremor, rapid pulse and nervous hyperirritability in a young woman receiving an average of $17 \mathrm{mg}$ thiamin hydrochloride per day (again, this was presumed to be by mouth but this was not explicit) ${ }^{17}$. The woman was said to be excreting $12 \mathrm{mg} /$ day in her urine and passing stools smelling strongly of thiamin.

\section{Meador et al., 1993}

This was a study conducted in 17 Alzheimer's patients ( 9 males and 8 females), mean age 69 years, to assess possible beneficial effects of thiamin supplementation. Patients were treated with graduated doses of thiamin hydrochloride, up to $6000-8000 \mathrm{mg} /$ day, for 5-6 months. Subjects were reported to have tolerated the doses well without weakness or other side effects, with the exception of two subjects who developed nausea and indigestion at dose levels of 7000 and $7500 \mathrm{mg} /$ day. However, these individuals were subsequently returned to their own previously highest tolerated doses $(6500$ and $7000 \mathrm{mg} /$ day) without side effects. The study was limited in that there were small numbers involved and that $8 / 17$ subjects suffered significant mental impairment on objective mental tests, so that any effects may have been undereported.

\footnotetext{
16 The Mills report states $5 \mathrm{mg} /$ day. However, when citing the Mills data, Iber et al. (1982) state $5 \mathrm{~g} /$ day.

17 The dose reported here as $17 \mathrm{mg}$ is inconsistent with the quoted rate of urinary and likely faecal excretion and suggests that this is a text error within the Mills report that should read $17 \mathrm{~g}$. Such an error would be consistent with an earlier error within the same report, indicated by 1 ber et al. (see notes 15 and 16 ).
} 
Part 1 Water Soluble Vitamins

\section{Gokhale, 1996}

A randomised double-blind placebo-controlled study was carried out in 556 females (aged 12-21 years) from 14 schools and hostels in India, suffering from moderate to severe spasmodic dysmenorrhoea. The thiamin status of the participants is unclear. A daily oral dose of $100 \mathrm{mg}$ of thiamin hydrochloride was given for 90 days followed by placebo for 60 days or vice versa. No adverse effects were reported.

\section{Animal data}

Molitor, 1942

The lethal dose for thiamin in mice was reported to be $100 \mathrm{mg}$ (approximately $5000 \mathrm{mg} / \mathrm{kg}$ bw, assuming a bodyweight of $20 \mathrm{~g}$ ).

Leuschner, 1992

As a preliminary test to determine a maximum tolerated dose for an investigation into the antinociceptive properties of thiamin, no toxic effects were observed in female NMRI mice (weight, $21-28 \mathrm{~g}$ ) when administered oral doses of thiamin nitrate in $0.8 \%$ aqueous hydroxypropyl methylcellulose gel at doses up to $5000 \mathrm{mg} / \mathrm{kg}$ bw. The number of animals tested was not stated.

\section{Exposure assessment}

Total exposure/intake:

Food Mean: $1.50 \mathrm{mg} /$ day (from 1986/87 NDNS) 97.5th percentile: $2.6 \mathrm{mg} /$ day

Supplements up to $300 \mathrm{mg} /$ day (OTC, 2001)

Estimated maximum intake: $2.6+300=303 \mathrm{mg} /$ day

No potential high intake groups have been identified.

\section{Risk assessment}

Thiamin present in food is efficiently absorbed. However, water-soluble supplements, such as thiamin hydrochloride and thiamin mononitrate, are poorly absorbed due to saturation of transport mechanisms.

It is generally accepted that ingested thiamin has a very low toxicity in humans. Most data are either in the form of case reports of possible thiamin-associated adverse effects or from thiamin supplementation studies designed primarily to investigate potential beneficial effects. The latter do not always specifically report an absence of adverse effect.

The limited amount of human data indicates that adverse effects are generally CNS-related and occur only at very high doses. A small number of individuals may show an allergic response to lower doses, 
but reports of these lower dose-related events are rare. It is possible that this sub-population may be the same sub-group that is susceptible to adverse effects, e.g. anaphylaxis etc, following parenteral administration of thiamin.

The animal database is also very limited. A lethal dose of thiamin in rodents is preceded by CNS effects such as shock, muscle tremor, convulsions, respiratory disturbance and collapse, symptoms which are similar to acute thiamin toxicity in humans.

\section{ESTABLISHMENT OF GUIDANCE LEVEL}

There are insufficient data to establish a Safe Upper Level for thiamin. The oral toxicity of thiamin and thiamin derivatives in humans is generally considered to be very low. Most available documented data are either in the form of case reports of possible thiamin-associated adverse effects or from thiamin supplementation studies designed primarily to investigate potential beneficial effects. The latter generally involve the use of the synthetic non-water soluble derivatives (not included in this review and not currently found in dietary supplements) and do not always specifically report an absence of adverse effect. Reports of thiamin-associated toxicity in humans are rare and most relate to incidents following parenteral administration of the vitamin. High doses ( $\geqslant 5000 \mathrm{mg}$ ) of thiamin hydrochloride may cause headache, nausea, irritability, insomnia, rapid pulse and weakness; these symptoms are relieved following cessation of treatment or reduction of dose. There have been a very small number of reported adverse effects following lower doses. These comprise four case reports and one isolated individual taking part in a supplementation study.

No specific toxic effects of thiamin ingestion by humans have been identified. However, there is a paucity of large controlled human supplementation studies. Significant adverse effects have not been noted with the water-soluble forms of thiamin used in dietary supplements. These forms are poorly absorbed at high doses, which further restricts their toxicity.

One human supplementation study (Meador et al., 1993) reported that graduated doses of thiamin hydrochloride, up to 6000-8000 mg/day for 5-6 months, caused no adverse effects in a very small group of patients. These subjects were reported to have tolerated the doses well, without weakness or other side effects, with the exception of two subjects (out of seventeen) who developed nausea and indigestion at doses of $7000-7500 \mathrm{mg}$. The study may well have under-reported side effects since half of the subjects were suffering from significant mental impairment on objective measures. From the available database, it appears that higher doses of thiamin ( $\geqslant 7000 \mathrm{mg}$ ) may be associated with headache, nausea, irritability, insomnia, rapid pulse and weakness.

In a randomised double-blind placebo-controlled study by Gokhale et al. (1996), a daily oral dose of 100 $\mathrm{mg}$ thiamin hydrochloride (for sixty or ninety days) was given to 556 young females ( $12-21$ years). No adverse effects were reported. The thiamin status of the participants is unclear. Based on this study, a level of $100 \mathrm{mg} /$ day (equivalent to $1.7 \mathrm{mg} / \mathrm{kg}$ supplemental thiamin for a $60 \mathrm{~kg}$ adult) of supplemental thiamin would not be expected to result in adverse effects. No uncertainty factor has been applied since this guidance is based on human data with large numbers of subjects and no hazard has been identified from other studies. This level is for guidance only and is applicable to the water-soluble forms of thiamin only. It should be noted that the applicability of the study, which was conducted in young women, to the general population is uncertain and the possibility of rare hypersensitivity reactions cannot be excluded. 
Part 1 Water Soluble Vitamins

\section{References}

COMA (1991). Dietary Reference Values for Food Energy and Nutrients for the United Kingdom. Report of the Panel on Dietary Reference Values, Committee on Medical Aspects of Food and Nutrition Policy. HMSO, London.

Gokhale, LB (1996). Curative treatment of primary (spasmodic) dysmenorrhea. Indian Journal of Medical Research 103, 227-231.

Iber, F.L., Blass, J.P., Brin, M., Leevy, C.M. (1982). Thiamin in the elderly - relation to alcoholism and to neurological degenerative disease. American Journal of Clinical Nutrition 36, 1067-1082.

Leuschner, J. (1992). Antinociceptive properties of thiamin, pyridoxine and cyanocobalamin following repeated oral administration to mice. Arzneimittel-Forschung 42, 114-115.

Meador, K., Loring, D., Nichols, M., Zamrini, E., Rivner, M., Posas, H., Thompson, E., Moore, E. (1993). Preliminary finding of a high dose thiamin in dementia of Alzheimer's type. Journal of Geriatric Psychiatry and Neurology 6, 222-229.

Mills, C.A. (1941). Thiamin overdosage and toxicity. Journal of the American Medical Association 116, 2101. Molitor, H. (1942). Vitamins as pharmacological agents. Federation Proceedings 1, 309.

OTC (2001). OTC Directory 2001-2002, Proprietary Association of Great Britain. 


\section{Risk Assessment}

\section{Vitamin $B_{6}$ (Pyridoxine)}

\section{General Information}

\section{Chemistry}

Pyridoxine is a water-soluble vitamin. Pyridoxine is composed of three forms (vitamers), pyridoxine, pyridoxal and pyridoxamine, all of which are normally present in foods. Pyridoxine hydrochloride is photosensitive and will degrade slowly when exposed to light.

\section{Natural occurrence}

Pyridoxamine and pyridoxal are found in animal products, and pyridoxine in animals and plants.

\section{Occurrence in food, food supplements and medicines}

Pyridoxine is found in chicken (4.2 mg/kg), fish, liver, kidney, pork, eggs $(1.1 \mathrm{mg} / \mathrm{kg})$, milk, wheatgerm $(11.5 \mathrm{mg} / \mathrm{kg})$ and brewer's yeast $(25 \mathrm{mg} / \mathrm{kg})$. Other sources include brown rice $(5.5 \mathrm{mg} / \mathrm{kg})$, soybeans $(6.3 \mathrm{mg} / \mathrm{kg})$, oats, whole-wheat grains, peanuts and walnuts $(7.3 \mathrm{mg} / \mathrm{kg})$. Long-term storage, canning, roasting or stewing of meat and food processing techniques can destroy pyridoxine. Boiling reduces the pyridoxine content of food because of losses into the water. Pyridoxine is present in a number of food supplements generally at doses up to $10 \mathrm{mg} /$ day but some single dose food supplements may contain 50 to $150 \mathrm{mg}$. Single nutrient products (recommended maximum daily doses of $10-100 \mathrm{mg}$ ) are available without the supervision of a pharmacist.

As a licensed medicine, pyridoxine hydrochloride is also present in various multivitamin preparations for the prevention and treatment of vitamin deficiencies (maximum daily doses of $0.5-30 \mathrm{mg}$ ). Products containing pyridoxine (maximum daily dose of $10 \mathrm{mg}$ ) combined with other constituents are available from a pharmacist.

\section{Recommended amounts}

Recommended intakes of pyridoxine are based on protein intake. In the UK, the RNI is set at $15 \mu \mathrm{g} / \mathrm{g}$ protein for adults. This is equivalent to approximately 1.4 and $1.2 \mathrm{mg} /$ day in the UK for males and females respectively. In the US, the Recommended Daily Allowance is set at $1.3 \mathrm{mg} /$ day, and it is approximately $1.6 \mathrm{mg} /$ day in Australia. Pregnant and lactating women and older people, who have low vitamin $B_{6}$ levels, can usually increase their intake through a high-protein diet.

\section{Analysis of tissue levels and pyridoxine status}

Pyridoxal phosphate has been determined enzymatically using tyrosine apodecarboxylase or by fluorimetric methods. The preferred method is high-pressure liquid chromatography. Vitamin $\mathrm{B}_{6}$ status has also been assessed using erythrocyte aminotransferases and the tryptophan loading test; the latter is not a reliable indicator of vitamin $B_{6}$ status in persons receiving oestrogens or with increased secretion of glucocorticoids. 
Part 1 Water Soluble Vitamins

\section{Brief overview of non-nutritional beneficial effects}

Pyridoxine is an approved treatment for sideroblastic anaemias and pyridoxine-dependent errors of metabolism. Pyridoxine has also been claimed to alleviate the symptoms of a range of conditions including premenstrual syndrome, sickness during pregnancy, carpal tunnel syndrome, hyperhomocystinaemia (a risk factor for cardiovascular disease) and neuropathies.

\section{Function}

The cofactor forms of pyridoxine are pyridoxal-5'-phosphate and pyridoxamine-5'-phosphate. Pyridoxal phosphate is involved as a cofactor particularly in the metabolic transformation of amino acids, including decarboxylation, transamination and racemisation.

Vitamin $B_{6}$ is a cofactor in the conversion of tryptophan to 5-hydroxytryptamine and of methionine to cysteine. Pyridoxine can modify the action of steroid hormones in vivo by interacting with steroidreceptor complexes. Pyridoxine is essential for the manufacture of prostaglandins and for the formation of red blood cells.

Pyridoxine is involved in cellular replication and antibody production. An adequate supply of pyridoxine is necessary for the function of the nervous system. The vitamin is involved in the biosynthesis of several neurotransmitters, including serotonin, gamma amino-butyric acid (GABA), dopamine and noradrenaline and so has a role in the regulation of mental processes and mood. It is also involved in sodium-potassium balance, histamine metabolism, the conversion of tryptophan to niacin, absorption of vitamin $\mathrm{B}_{12}$ and the production of hydrochloric acid in the gastrointestinal tract.

\section{Deficiency}

Pyridoxine deficiency is unusual in humans. Children who had been given milk in which the pyridoxine had been destroyed by overheating, displayed various symptoms, including weakness, irritability, nervousness, susceptibility to noise, weight loss and insomnia. Adult volunteers on a pyridoxine deficient diet became depressed and irritable, with 'a loss of sense of responsibility'. They also experienced a greasy rash on the forehead and around the nose and cracking of the lips and tongue.

Pyridoxine dependency is a rare autosomal recessive disorder in which the enzyme glutamate decarboxylase, which is involved in the synthesis of GABA, has a defective binding site for pyridoxal phosphate. Much higher tissue levels of pyridoxal phosphate are necessary for the enzyme to have any significant activity. The condition results in seizures of prenatal or neonatal onset and treatment with large doses of pyridoxine is necessary to prevent severe mental retardation or death.

It has also been suggested that pyridoxine deficiency may be a factor in hyperhomocysteinaemia, which is associated with an increased risk of cardiovascular disease.

Pyridoxine deficiency has also been reported to be associated with immune dysfunction, kidney stones, cancer and carpal tunnel syndrome, although the evidence for these links is variable. 


\section{Interactions}

Pyridoxine requires riboflavin, zinc and magnesium to fulfil its physiological function in humans. Pyridoxine supplements reduce the therapeutic effect of levodopa, a naturally occurring amino acid used to treat Parkinson's disease. Pyridoxine also interacts with other drugs such as isoniazid, phenytoin, theophylline and phenobarbitone. It has been claimed that women taking oral contraceptives may have an increased requirement for pyridoxine.

\section{Absorption and bioavailability}

The phosphate forms of vitamin $B_{6}$ in food are dephosphorylated in the intestinal lumen, and pyridoxine, pyridoxal and pyridoxamine are taken up from the small intestine by an energydependent process. All three are converted to pyridoxal phosphate in the tissues.

A proportion of the vitamin $B_{6}$ present in plant-based foods is biologically unavailable because it is present as pyridoxine glycosides that are not hydrolysed by intestinal enzymes. These glycosides may be absorbed, but do not act as a coenzyme in the body and are excreted unchanged in the urine.

All three forms of vitamin $B_{6}$ (pyridoxine, pyridoxal and pyridoxamine) are readily absorbed in the small intestine. The extent of absorption is decreased following gastric resection or in patients with malabsorption syndrome. Excess pyridoxine is excreted in the urine, and an adequate daily intake is therefore essential.

\section{Distribution and metabolism}

Pyridoxine in food is converted to active forms in the liver, a process which requires zinc and riboflavin.

Vitamin $\mathrm{B}_{6}$ is stored in the liver, with about $50 \%$ also being present in muscle, bound to glycogen phosphorylase. Pyridoxine is also stored in the brain. The total body storage for adults is between 6 and $27 \mathrm{mg}$. Pyridoxine in the form of pyridoxal crosses the placenta, with foetal plasma concentrations being five times the level found in maternal plasma. The three forms of vitamin $B_{6}$ are present in body tissues, mainly as 5-phosphorylated derivatives of pyridoxal and pyridoxamine. The half-life of pyridoxine is 15-20 days, and it is not significantly bound to plasma proteins.

Pyridoxine, pyridoxal and pyridoxamine are all largely metabolised in the liver through phosphorylation by pyridoxal kinase. Pyridoxine phosphate is oxidised to the active coenzyme form, pyridoxal-5-phosphate, by an enzyme found mainly in liver. Pyridoxal-5-phosphate interconverts with pyridoxamine-5-phosphate through enzymatic transamination. The phosphorylated forms are hydrolysed by phosphatases.

Pyridoxal is oxidised in the liver to pyridoxic acid.

\section{Excretion}

Pyridoxic acid, the main excretory metabolite, is eliminated via the urine. 
Part 1 Water Soluble Vitamins

\section{Toxicity}

\section{Human data}

Long-term use of pyridoxine (generally in excess of $200 \mathrm{mg} /$ day) has been reported to result in paraesthesiae, somnolence and low serum folic acid levels. Large doses of pyridoxine (usually quoted as over $2000 \mathrm{mg} /$ day) can cause nerve damage. Symptoms include tingling in the hands and feet (paraesthesiae), a stumbling gait, perioral numbness, a characteristic 'stocking-glove' sensory loss and lack of muscle coordination. Duration of pyridoxine use is important as well as dosage, with lower doses of pyridoxine (500 mg/day or less) consumed for many months or years also being associated with neuropathy. In most, but not all reported cases the damage has been generally reversible. Night restlessness, vivid dreams, sun sensitivity and an acne-like rash may also occur with high doses (150 mg/day or more) of pyridoxine.

\section{Supplementation trials}

A number of pyridoxine supplementation trials in humans have investigated effects on conditions such as carpal tunnel syndrome, pre-menstrual syndrome, sickness during pregnancy and hyperhomocysteinaemia. The majority of these trials have not been placebo controlled and have either not considered or not reported adverse effects in detail. The available studies are considered in detail in the review of vitamin $B_{6}$ (http://www.food.gov.uk/science/ouradvisors/vitandmin/evmpapers) and the key studies for the risk assessment are discussed below.

\section{Animal data}

Symptoms of neuropathy have occurred at variable time intervals in different species following doses ranging from $50 \mathrm{mg} / \mathrm{kg}$ bw/day to $7000 \mathrm{mg} / \mathrm{kg}$ bw/day. Inter-species variability in response is apparent.

No effects on foetal development have been observed in animal studies. Doses of 250 and $500 \mathrm{mg} / \mathrm{kg}$ bw/day pyridoxine, administered intraperitoneally, produced histological changes in the testes of rats (reversible at the lower dose), and doses of $50 \mathrm{mg} / \mathrm{kg} /$ day administered intraperitoneally to female rats prevented prolactin release.

\section{Carcinogenicity and genotoxicity}

No published data on the carcinogenicity or genotoxicity of pyridoxine have been found.

\section{Mechanisms of toxicity}

Pyridoxal phosphate is thought to be responsible for the observed toxicity. Schwann cells in culture grow less well when provided with pyridoxal in the culture medium than when the vitamin $\mathrm{B}_{6}$ source is pyridoxine. The addition of pyridoxal to the culture medium decreased cell survival even in the presence of an adequate concentration of pyridoxine, suggesting a possible neurotoxic action of pyridoxal. It is not known whether pyridoxal is cytotoxic to other cell types in culture. 


\section{Dose-response characterisation}

The precise dose-response relationship between pyridoxine and peripheral neuropathy is uncertain in humans. Neuropathy has been associated with high doses of pyridoxine (generally over $2000 \mathrm{mg} /$ day), but the duration of exposure is also important, with neuropathy occurring after long periods of exposure to lower levels (500 mg/day or less) of supplemental pyridoxine.

\section{Vulnerable groups}

No groups particularly vulnerable to pyridoxine-induced toxicity have been identified.

\section{Genetic variations}

No genetic variations which increase vulnerability to toxicity of vitamin $B_{6}$ have been identified.

\section{Studies of particular importance in the risk assessment}

(For full review see http://www.food.gov.uk/science/ouradvisors/vitandmin/evmpapers or the enclosed CD).

\section{Human data}

Data are available from numerous human studies for consideration in the risk assessment of vitamin $B_{6}$. However, most of the available human data are unsatisfactory, with retrospective exploration of records, use of non-standardised end points and incomplete follow up. Other significant deficiencies were that the studies did not include adequate controls (similar patients who were not treated with vitamin B6), were often performed in small groups using observational methods, and were of a duration of treatment ( $<6$ months) too short to reveal neurological effects, even at very high doses. In many studies adverse effects were not examined. Examples from the available database are described in more detail below.

\section{Short term studies}

Del Tredici et al., 1985

This report studied the effect of vitamin $B_{6}$ on 24 patients with carpal tunnel syndrome treated for 2-4 months with 150 or $300 \mathrm{mg}$ vitamin $\mathrm{B}_{6}$ daily. Vitamin $\mathrm{B}_{6}$ was reported as having a beneficial effect on carpal tunnel syndrome, and no exacerbation of neurological symptoms or signs of peripheral neuropathy were reported. Distal motor latency was measured and patients completed a selfassessment questionnaire numerically ranking the severity of the symptoms. The short duration and small number of patients involved limit the usefulness of this study in setting a safe upper level.

\section{Brush, 1988}

In a retrospective survey of 336 women treated with pyridoxine alone or in combination with other agents, 5 subjects reported mild tingling and/or numbness at doses of $200 \mathrm{mg} /$ day, which were described in the paper as 'definite side effects'. Similar side effects were not observed in an earlier 
Part 1 Water Soluble Vitamins

group of women treated with up to $150 \mathrm{mg} /$ day. No control group of patients unsupplemented with vitamin $B_{6}$ was included. This study does not form an adequate basis for setting a safe upper level because of the lack of detail in the reporting, particularly with respect to duration of treatment.

Brush et al., 1988

The effects of supplemental vitamin $B_{6}$ in 630 women suffering from premenstrual syndrome were described from a retrospective survey of clinical records. The patients reported taking daily doses of vitamin $B_{6}$ ranging from 40 to $100 \mathrm{mg}$ early in the study and from 120 to $200 \mathrm{mg}$ later in the investigation period. The highest dose taken was $100 \mathrm{mg} /$ day or less in $77 \%$ of the patients. Patients were reported to have taken vitamin $B_{6}$ for periods from three months to five years, but only $20 \%$ of subjects had continued treatment for more than one year. The authors reported that indigestion and nausea were 'probably genuine side effects' and that peripheral sensory neuropathy was not observed. The limitations of this study are that symptoms were reported from a retrospective investigation of records and inadequate details were included.

\section{Bernstein and Lobitz, 1988}

In an open study, 16 patients received $150 \mathrm{mg}$ vitamin $\mathrm{B}_{6}$ daily for 6 months for the treatment of diabetic neuropathy. It was reported that one patient developed increased photosensitivity with increased tanning on minimal exposure to sunlight, but this subject elected to remain in the study. The patients underwent a monthly clinical evaluation by a neurologist, including a detailed electrophysiological study of motor and sensory nerves. No deterioration of peripheral nerve function was observed, though examinations were completed after only 4 months of supplementation in 10 subjects and after 5 months in 5 subjects. The limitations of this study are the short duration of treatment, the small number of patients studied and the incomplete follow-up of symptoms.

\section{Molimard et al., 1980}

In a double-blind study, 69 medical students received a tablet of placebo, 50 or $250 \mathrm{mg}$ vitamin $\mathrm{B}_{6}$ twice a day for 10 days (total dose being 100 or $500 \mathrm{mg} /$ day). The subjects were given a simple digit-coding task prior to treatment, immediately after treatment and 14 days later. The volunteers were also tested at the end of the treatment period on some numerical problems and on the content of the medical undergraduate course during the study period. Fifty eight of the volunteers completed the digit-coding tests, which showed a highly significant improvement with time (a learning effect) in all groups. There were no significant differences between groups in the uncorrected scores, but there was evidence of a dose-related decrease in the learning effect, which was highly statistically significant $(p<0.002)$ in the highest-dose group compared to the controls, but this was not statistically significant at the $5 \%$ level $(p<0.07)$ in the $100 \mathrm{mg} /$ day group. No other differences were observed between the three groups. In a second experiment, a group of 30 patients were randomised to receive placebo, $20 \mathrm{mg}$ or $1000 \mathrm{mg} /$ day pyridoxine for 15 days with subjects given a variety of tests before and after treatment. At the high dose, an adverse effect was reported for word recognition, but not for word or visual memorisation. Performance in the visual retention test was worse in the high dose pyridoxine group after treatment. The extremely short treatment period and the small number of participants limit the usefulness of this study.

Berger et al., 1992

Doses of 1000 or $3000 \mathrm{mg} /$ day pyridoxine were administered to three and two healthy volunteers respectively, until the development of symptoms of neuropathy in an experimental study. Symptoms occurred within 1.5 and 3.5 months in high-dose subjects. In subjects treated with $1000 \mathrm{mg} /$ day 
symptoms of neuropathy, indicated by numbness and pins and needles sensation in the toes, developed in two of the subjects after 4.5 and 7 months of treatment. Treatment of one subject was continued for over 14 months before quantitative sensory threshold test results became abnormal, an early indicator of neuropathy. An inverse relationship was observed between the daily dose calculated on a body weight basis, and the time taken for the development of toxicity. In other words as the dose decreased, the period of treatment before onset of adverse effects increased. Clinical symptoms continued to intensify for some weeks after pyridoxine was withdrawn. The very small number of participants involved in this study limits its usefulness in risk assessment.

\section{Bernstein and Dinesen, 1993}

This paper reported the effect of vitamin $B_{6}$ in the treatment of carpal tunnel syndrome. Sixteen patients received $200 \mathrm{mg}$ vitamin $\mathrm{B}_{6}$ per day for 3 months. No signs of peripheral neuropathy were found in the uninvolved ulnar nerves, and there was no evidence of new peripheral neuropathy during the 3 months of treatment. The limitations of this study are the short duration and the small number of patients involved.

\section{Schaumburg et al., 1983}

This paper describes case reports of seven patients who had ataxia and severe sensory nervous system dysfunction after vitamin $B_{6}$ consumption. Patients self-prescribed a maximum of $2-6 \mathrm{~g}$ vitamin $\mathrm{B}_{6}$ daily. Only two of the seven patients started taking the vitamin at such high doses, while others began with doses of 50 to $100 \mathrm{mg} /$ day. None experienced symptoms at doses below $2 \mathrm{~g}$ per day. Plasma pyridoxine was measured in one of the patients at the initial examination and found to be $30 \mathrm{ng} / \mathrm{mL}$ three hours after the subject's usual daily dose of $4 \mathrm{~g}$ vitamin $\mathrm{B}_{6}$. The reported duration of treatment ranged from two months to over three years. The small number of patients and the lack of reported details limit the usefulness of this study.

\section{Long term studies}

\section{Parry and Bredesen, 1985}

The paper describes 16 patients who developed neuropathy after taking high doses of pyridoxine (200$5000 \mathrm{mg} /$ day) for several months or more. The patients developed unstable gait, perioral numbness and a 'stocking-glove' sensory loss followed. Discontinuation of pyridoxine was followed by improvement in symptoms. The authors believed that the toxicity of pyridoxine was manifest through an effect on the dorsal root ganglion. Most patients took $2000 \mathrm{mg} /$ day or more pyridoxine (many of these had started at lower doses) and symptoms became apparent within a year of taking $2000 \mathrm{mg} /$ day or more. One patient had taken $200 \mathrm{mg}$ pyridoxine/day for 3 years and two others had taken $500 \mathrm{mg} /$ day for 1-2 years. Although the duration of this study is adequate, a limited number of patients were studied and few details are reported.

\section{Dalton and Dalton, 1987}

This paper represents a follow-up study of data presented in a letter to the Lancet in 1985, which described sensory neuropathy in 23 out of 58 women with premenstrual syndrome, who had serum vitamin $B_{6}$ concentrations above the normal range (Dalton, 1985). Serum vitamin $B_{6}$ levels were measured in all women taking vitamin $B_{6}$ supplements at a private clinic. Patients $(n=172)$ who had elevated serum vitamin $B_{6}$ levels (above the normal range in humans of 3.16 to $18 \mathrm{ng} / \mathrm{mL}$ ) were specifically asked to report symptoms such as tingling in the fingers, which could be interpreted as evidence of sensory 
Part 1 Water Soluble Vitamins

neuropathy. The patients were all taking $<50$ to $<500 \mathrm{mg} /$ day vitamin $\mathrm{B}_{6}$ for premenstrual syndrome. No control group of patients with similar premenstrual symptoms, but not taking vitamin $B_{6}$, were asked the same questions. Patients reported symptoms including paraesthesia, hyperaesthesia, bone pains, muscle weakness, numbness and fasciculation that were bilateral and most marked in the extremities. Neurological examinations were undertaken on those who reported symptoms, but neurophysiological tests were not undertaken. Findings on neurological examination included weakness, diminution but not loss of tendon reflexes, positive L'Hermitte's test and sensory loss in a stocking-glove distribution. Symptoms disappeared when vitamin $B_{6}$ was withdrawn and reappeared if treatment was resumed. Complete recovery occurred within 6 months of stopping vitamin $B_{6}$ treatment. Three women had subnormal serum levels of vitamin $B_{6}$ after stopping supplementation due to symptoms of sensory neuropathy. They were advised to take $50 \mathrm{mg}$ vitamin $\mathrm{B}_{6} /$ day, but within a month they again experienced symptoms of neuropathy and had serum vitamin $B_{6}$ levels of $>18 \mathrm{ng} / \mathrm{mL}$. The 103 women who presented with symptoms took a mean dose of $117 \pm 92 \mathrm{mg}$ vitamin $\mathrm{B}_{6}$ for a period of $2.9 \pm 1.9$ years, and in $70 \%$ of these subjects, serum levels were $>34 \mathrm{ng} / \mathrm{mL}$ (the upper limit of testing). In contrast, the group which did not report symptoms, took a mean dose of $116 \pm 66 \mathrm{mg}$ vitamin $\mathrm{B}_{6}$, for a period of $1.6 \pm 2.1$ years, and in $55 \%$ of these subjects, serum levels were $>34 \mathrm{ng} / \mathrm{mL}$. The difference in duration of treatment, was statistically significant $(P<0.01)$. Although the duration of treatment and the measurement of plasma vitamin levels in this study were useful, the study has limitations when being considered for risk assessment. The lack of a control group and potential bias introduced by the focussed questioning of the patients on their symptoms detract from the quality of these data. It should be noted that muscle weakness has not been observed at higher doses in other studies.

\section{Mitwalli et al., 1984}

Twenty two male patients with kidney stones secondary to hyperoxaluria were given doses (250 - 500 $\mathrm{mg} /$ day) of vitamin $B_{6}$ for 8 months to 6 years (average 2.3 years). Seven subjects underwent nerve conduction studies. No neurological complications were reported in the group. The usefulness of the findings of this study for risk assessment is limited by the low number of patients involved.

\section{Hawkins, 1986}

A lower than expected incidence of tardive dyskinesia was reported in a total of 58,139 schizophrenic patients who had been treated by 80 physicians, in some cases for many years, with neuroleptics (phenothiazines and/or haloperidol) and with high doses of a mixture of vitamins. The vitamin regimen consisted of three daily doses of vitamin C (1000 mg), vitamin $B_{6}(200 \mathrm{mg})$, vitamin E (200 lU) and either nicotinic acid or nicotinamide (1000 mg). This data set is of limited value for several reasons: there is a lack of detail, the results have not been published in a peer-reviewed journal, there was no check on compliance (which is a problem in such patients), the primary endpoint (tardive dyskinesia) is not relevant to vitamin $B_{6}$ neuropathy, the elicitation of minor vitamin $B_{6}$-related symptoms in schizophrenic patients would have been difficult, and any effect of vitamin $B_{6}$ might have been masked by the neuroleptic drug treatment and/or the very high doses of the other vitamins. In consequence, the usefulness of these observations for risk assessment is limited.

The study which reports neuropathy at the lowest dose (50 mg/day) is that of Dalton and Dalton (1987), but this investigation is flawed in a number of ways. For example, there was no control group, and since subjects were questioned about their symptoms, reports may be biased. However, this study has the advantage that plasma levels of the vitamin were measured. In women with elevated plasma levels, longer exposure was associated with clinical symptoms and in the absence of better quality data to confirm safety, it is not possible to dismiss this investigation. Brush (1988) and Parry and Bredesen (1985) 
reported neuropathy at doses of 200 or $500 \mathrm{mg} /$ day, the latter study involving exposure of several years' duration. However, small numbers of patients were involved and few data were provided. Other studies which investigated the effects of low doses of vitamin $B_{6}$ (Del Tredici et al., 1985; Bernstein and Lobitz, 1988; Bernstein and Dinesen, 1993) were somewhat better conducted but did not consider exposure of sufficient duration. For example, in Bernstein and Lobitz (1988) patients received $150 \mathrm{mg} /$ day for 6 months, in Del Tredici (1985) patients received 150 or 300 mg/day for 2-4 months and in Bernstein and Dinesen (1993) the subjects received $200 \mathrm{mg} /$ day for 3 months. These studies did not have placebo control groups and involved small numbers of patients, as well as being of durations that were insufficient for the development of symptoms, even had the doses been higher. Thus while the results of these studies are negative, they are not necessarily incompatible with those of Dalton and Dalton (1987).

\section{Animal data}

In many of the available animal studies vitamin $\mathrm{B}_{6}$ was administered by injection. Whilst these studies confirm that pyridoxine neurotoxicity is dose- and time-dependent in both rats and dogs, the data are not directly comparable to those generated by exposure via the oral route. In contrast to the majority of the human data, the animal studies described below were performed under controlled conditions making them useful for consideration in risk assessment. Small numbers of animals were involved, as is common practice for studies conducted in dogs.

Phillips et al., 1978

Pyridoxine hydrochloride was administered orally in gelatine capsules $(0,50$ or $200 \mathrm{mg} / \mathrm{kg}$ bw/day) to three groups of female beagles (four control and five per treatment group) over 100-112 days. Four of the five animals in the high dose group showed signs of ataxia and loss of balance after 45 days of treatment, while the fifth showed clinical signs after 75 days. Clinical signs of toxicity were not observed in the $50 \mathrm{mg} / \mathrm{kg} /$ day group, but bilateral loss of myelin in the dorsal nerve roots was observed histologically.

\section{Hoover et al., 1981}

Three adult beagle dogs (with a fourth dog serving as a control) received pyridoxine hydrochloride in gelatine capsules, $5 \mathrm{mg} / \mathrm{kg}$ bw/day for the first week, $100 \mathrm{mg} / \mathrm{kg}$ bw/day for the second week and $150 \mathrm{mg} / \mathrm{kg}$ bw/day from the $15^{\text {th }}$ to the $100^{\text {th }}$ day of the experiment. Neurological disease, characterised by proprioceptive defects, developed in each pyridoxine-treated dog and occurred in two dogs during the fourth week and in the third dog during the eighth week of administration. The dogs had spastic, dysmetric leg movements and lacked apparent sense of motion or position of the legs. This was characterised ultrastructurally by degeneration of axons, loss of axons, collapse of myelin sheaths, degeneration and loss of myelin and astrocytic scarring on histological examination.

\section{Krinke et al., 1980}

Six beagle dogs were administered $300 \mathrm{mg} / \mathrm{kg}$ bw/day pyridoxine hydrochloride in gelatine capsules, in addition to a balanced diet. The animals were examined in comparison with two control dogs. Within 9 days the treated animals developed a swaying gait, and eventually became unable to walk, but had no observable muscle weakness. Morphological examination showed widespread neuronal degeneration in the dorsal root ganglia and the Gasserian ganglia. Degeneration of sensory nerve fibres was apparent in peripheral nerves, dorsal columns of the spinal cord and the descending spinal tract of the trigeminal nerve. 
Part 1 Water Soluble Vitamins

\section{Exposure assessment}

Total exposure/intake:

Food

Mean: $2.0 \mathrm{mg} /$ day

97.5th percentile: $3.9 \mathrm{mg} /$ day (NDNS, 1986/87)

Supplements

up to $100 \mathrm{mg} /$ day (Annex 4)

Estimated maximum daily

exposure:

$3.9+100=104 \mathrm{mg}$

No potential high intake groups have been identified.

\section{Risk assessment}

The key adverse effect, for vitamin $B_{6}$ is neuropathy, which has been demonstrated in both humans and laboratory animals. The effect occurs after consumption of high doses and/or long duration. Generally the symptoms are reversible once the exposure is stopped but in some cases involving high doses, the effects are irreversible. Progressive sensory ataxia occurs, presenting initially as unstable gait and numb feet, then numbness in the hands, followed by profound impairment of position sense and vibration sense in the distal limbs. The senses of touch, temperature and pain are less affected.

Data from animal studies also demonstrate neurotoxicity, although some species differences are apparent. Doses as low as $50 \mathrm{mg} / \mathrm{kg}$ bw/day have been associated with a loss of myelin. Subtle effects such as changes in startle response have also been observed. The animal data also suggest that duration of exposure is important in the response to vitamin $B_{6}$.

\section{ESTABLISHMENT OF SAFE UPPER LEVEL}

As discussed above, the available human data are not adequate for risk assessment and the Safe Upper Level has been based on the most appropriate of the studies performed in animals under controlled conditions.

Phillips et al. (1978)

LOAEL: $50 \mathrm{mg} / \mathrm{kg}$ bw/day, based on the study by Phillips et al. (1978) in dogs

Uncertainty factors: $\quad 3$ for LOAEL to NOAEL extrapolation

10 for inter-species variation

10 for inter-individual variation 
Safe Upper Level (for daily consumption over a lifetime):
$50 / 300=0.17 \mathrm{mg} / \mathrm{kg}$ bw $/$ day supplemental pyridoxine (equivalent to $10 \mathrm{mg} /$ day for a $60 \mathrm{~kg}$ adult)

Excessive quantities of vitamin $B_{6}$ result in peripheral neuropathy in both animals and humans. The effect is dependent on both the dose and the duration of exposure.

In order to determine the levels at which vitamin $\mathrm{B}_{6}$ could be safely taken by the entire population over a lifetime, large well-controlled studies with careful symptomatic observation and attention to critical endpoints are required. Unfortunately much of the available data from studies in humans are unsatisfactory with retrospective exploration of records, use of unstandardised end points and incomplete follow up, often of small observational groups with short durations of treatment $(<6$ months) and without adequate control groups.

Despite the limitations in design of the human studies, the available data clearly indicate that pyridoxine causes neuropathy in humans and that the duration of exposure as well as dose is important. Therefore, in the absence of better quality data the reports of toxicity following long, low level exposure to vitamin $B_{6}$ cannot be entirely dismissed. Overall, the human data are inadequate to establish a Safe Upper Level, since the effect levels are unclear and the studies at low levels of intake are of limited quality.

Neuropathy is also observed in laboratory animals, and the data confirm the importance of dose and duration in determining the effects of vitamin $B_{6}$. In the absence of reliable human data at low levels of exposure, the Safe Upper Level is based on animal data, in which histological changes were apparent in the nerves of dogs treated with $50 \mathrm{mg} / \mathrm{kg}$ bw/day for 100-112 days (Phillips, 1978). Clinical signs of toxicity were not apparent in this group but were observed in the high dose group which received $200 \mathrm{mg} / \mathrm{kg}$ bw/day. Using uncertainty factors of 300 (consisting of 3 for LOAEL to NOAEL extrapolation of a histopathological change, 10 for inter-species and 10 for inter-individual variation) a Safe Upper Level of $0.17 \mathrm{mg} / \mathrm{kg}$ bw/day can be derived. This relates to supplemental pyridoxine because the basal pyridoxine content of the diet in the key study is unknown. This SUL is equivalent to $10 \mathrm{mg} /$ day in a $60 \mathrm{~kg}$ adult. These uncertainty factors are appropriate because the LOAEL in the dog related to a sub-chronic study and therefore may have underestimated the toxicity during chronic exposure. The need for an inter-species factor is supported by the fact that the LOAEL dose in dogs $(50 \mathrm{mg} / \mathrm{kg}$ bw/day) is equivalent to an intake of $3000 \mathrm{mg}$ per day in humans, which would produce severe toxicity in humans, suggesting that humans are probably more sensitive than dogs to these effects. Finally, the factor for human variability is necessary to allow for the greater size and diversity of the human population compared with the small number of dogs $(n=5)$ studied by Phillips et al. (1978).

In humans, a supplementary dose of $10 \mathrm{mg} /$ day represents a clear SUL, with no adverse effects being anticipated over a lifetime's exposure. Doses of $200 \mathrm{mg} /$ day vitamin $\mathrm{B}_{6}$ or more taken for long periods are associated with reports of neuropathy in some human subjects. The effect of taking vitamin $B_{6}$ at doses between 10 and $200 \mathrm{mg}$ is unclear. The risk posed by such exposure in the short term may be negligible, but the available data do not allow identification of a dose or duration of exposure above the SUL that would be of negligible risk.

It is unfortunate that no reliable and controlled studies have been conducted in the past 5 years to establish whether intakes between 10 and $200 \mathrm{mg} /$ day are safe in the long term. 
Part 1 Water Soluble Vitamins

\section{References}

Berger, A.R., Schaumburg, H.H., Schroeder, C., Apfel, S., Reynolds, R. (1992) Dose response, coasting, and differential fibre vulnerability in human toxic neuropathy: a prospective study of pyridoxine neurotoxicity. Neurology 42, 1367-1370.

Bernstein, A.L., Lobitz, C.S. (1988) A clinical and electrophysiologic study of the treatment of painful diabetic neuropathies with pyridoxine. In: Leklem JE, Reynolds, RD (eds) Clinical and Physiological Applications of Vitamin B-6. Alan R Liss, New York, pp 415-423.

Bernstein, A.L., Dinesen, J.S. (1993) Effect of pharmacologic doses of vitamin $B_{6}$ on carpal tunnel syndrome, electroencephalographic results, and pain. Journal of the American College of Nutrition 12, 73-76.

Brush, M.G. (1988) Vitamin B-6 treatment of premenstrual syndrome. Clinical and Physiological Applications of B-6. Alan R. Liss, New York, pp 363-379.

Brush, M.G., Bennett, T., Hansen, K. (1988) Pyridoxine in the treatment of premenstrual syndrome: a retrospective study in 630 patients. British Journal of Clinical Practice 41, 448-452.

Dalton, K., Dalton, M.JT. (1987) Characteristics of pyridoxine overdose neuropathy syndrome. Acta Neurologica Scandinavica 76, 8-11.

Del Tredici, A.M., Bernstein, A.L., Chinn, K. (1985) Carpal tunnel syndrome and vitamin B-6 therapy. In: Reynolds RD, Leklem JE (eds) Vitamin B-6: its Role in Health and Disease. Alan R Liss, New York, pp 459-462.

Hawkins, D.R. (1986) The prevention of tarditive dyskinesia with high dosage vitamins: a study of 58,000 patients. Journal of Orthomolecular Medicine 1(1), 24-29.

Hoover, D.M., Carlton, W.W., Henrikson, C.K. (1981) Ultrastructural lesions of pyridoxine toxicity in beagles dogs. Veterinary Pathology 18, 769-777.

Krinke, G., Schaumberg, H.H., Spencer, P.S., Suter, J., Thomann, P. Hess, R. (1980) Pyridoxine megavitaminosis produces degeneration of peripheral sensory neurons (sensory neuropathy) in the dog. Neurotoxicity 2, 13-24.

Mitwalli, A., Blair, G., Oreopoulos, D.G. (1984) Safety of intermediate doses of pyridoxine. Canadian Medical Association Journal 131, 14.

Molimard, R., Marillaud, A., Paille, A., Le Devehat, C., Lemoine, A. and Dougny, M. (1980). Impairment of memorization by high doses of pyridoxine in man. Biomedicine 32, 88-92.

Parry, G.J., Bredesen, D.E. (1985) Sensory neuropathy with low dose pyridoxine. Neurology 35, 1466-1468.

Phillips, W.E.J., Mills, J.H.I., Charbonneau, S.M., Tryphonas, L., Hatina, G.V., Zawidska, Z., Bryce, F.R., Munro, I.C. (1978) Sub-acute toxicity of pyridoxine hydrochloride in the beagle dog. Toxicology and Applied Pharmacology 44, 323-333.

Schaumburg, H., Kaplan, J. Windebank, A, Vick, N., Ramus, S., Pleasure, D., Brown, M.J. (1983) Sensory neuropathy from pyridoxine abuse. New England Journal of Medicine 309 (8), 445-448. 


\section{Risk Assessment}

\section{Vitamin $\mathbf{B}_{12}$}

\section{General Information}

\section{Chemistry}

Vitamin $\mathrm{B}_{12}$ (cobalamin, $\mathrm{Cbl}$ ) is a water-soluble vitamin and a member of a family of related molecules known as corrinoids which contain a corrin nucleus made up of a tetrapyrrolic ring structure. The centre of the tetrapyrrolic ring nucleus contains a cobalt ion that can be attached to methyl, deoxyadenosyl-, hydroxo- or cyano- groups.

\section{Natural occurrence}

Vitamin $B_{12}$ originates from bacteria, fungi and algae, and is present in virtually all animal tissues. Plants contain no vitamin $B_{12}$ beyond that derived from microbial contamination.

\section{Occurrence in food, food supplements and medicines}

Major dietary sources of vitamin $\mathrm{B}_{12}$, mainly in the forms of methyl, deoxyadenosyl- and hydroxocobalamin, include meat (e.g. $>0.1 \mathrm{mg} / \mathrm{kg}$ in lamb), particularly liver $(>0.1 \mathrm{mg} / \mathrm{kg}$ ) and fish (e.g. $0.03-0.1 \mathrm{mg} / \mathrm{kg}$ in salmon, $0.01-0.03 \mathrm{mg} / \mathrm{kg}$ in tuna). Hydroxocobalamin and, in particular, cyanocobalamin are synthetic forms used in vitamin supplements, pharmaceuticals and in the fortification of food. Methyl cobalamin has been used therapeutically outside the UK, for example, in Japan.

\section{Recommended amounts}

The RNI for vitamin $B_{12}$ in adults in the UK is $0.0015 \mathrm{mg} /$ day (COMA, 1991). There is no increment required during pregnancy but there is a recommended increment of $0.0005 \mathrm{mg} /$ day for breast feeding mothers.

\section{Analysis of tissue levels and vitamin $B_{12}$ status}

Measurement of vitamin $B_{12}$ in plasma is routinely used to determine deficiency, but may not be a reliable indication in all cases. In pregnancy, for example, tissue levels are normal but serum levels are low. Various other plasma markers have been identified (including methylmalonic acid, homocysteine, holotranscobalamin, anti-intrinsic factor antibodies) and methods devised (Schilling test, cobalamin absorbance test, serum gastrin deoxyuridine suppression test) to distinguish different causes of deficiency.

\section{Brief overview of non-nutritional beneficial effects}

Results of studies in humans have suggested that large doses of vitamin $B_{12}$ (particularly methyl cobalamin) may influence biological rhythms and thus may be beneficial in the treatment of sleep disorders. Vitamin $B_{12}$ has also been reported to increase light sensitivity by affecting melatonin secretion. Vitamin $B_{12}$, in combination with folic acid, has been suggested to be beneficial in certain disorders, such as idiopathic osteoarthritis and vitiligo. 
Part 1 Water Soluble Vitamins

\section{Function}

Vitamin $B_{12}$ serves as a cofactor to at least two enzymes, methionine synthase and methylmalonyl CoA mutase. Methionine synthase is pivotal in one-carbon metabolism, being crucial in the synthesis of the universal methyl donor S-adenosyl methionine and in the cellular import and metabolism of folate. Methylmalonyl CoA mutase converts L-methylmalonyl CoA to succinyl CoA and is important in even-chained fatty acid synthesis.

\section{Deficiency}

Dietary deficiency is rare in younger people living in the community but occurs more frequently in older people, particularly those living in institutional environments. Individuals adhering to vegan diets may also be at risk. Deficiency is mostly attributable to inherited or acquired defects resulting in malabsorption or the impairment of transport of the vitamin within the body. Deficiency impacts on the haematopoietic and nervous systems. Associated diseases include megaloblastic anaemia and neuropathies typically sub-acute combined degeneration of the spinal cord. Vitamin $B_{12}$ deficiency can lead to moderate hyperhomocysteinaemia, a possible risk factor for occlusive vascular disease.

Oral supplements are indicated prophylactically where there is a likelihood of deficiency in those whose gastrointestinal function is normal e.g. in individuals who are strict vegetarians. Inherited and acquired disorders relating to vitamin $B_{12}$ malabsorption are usually treated by repeated injection. However, oral administration of very high doses of vitamin $B_{12}$ has been shown to be effective in the treatment of pernicious anaemia.

\section{Interactions}

Steroid drugs, such as prednisone, have been reported to increase the absorption of vitamin $B_{12}$ in patients with pernicious anaemia. Excessive alcohol consumption and some drugs may decrease absorption of vitamin $\mathrm{B}_{12}$. Oral co-administration with ascorbic acid may result in destruction of vitamin $B_{12}$. Concurrent administration of chloramphenicol may lead to antagonism of the haematopoietic response to vitamin $B_{12}$.

\section{Absorption and bioavailability}

Vitamin $B_{12}$ requires intrinsic factor (IF), secreted mainly from the gastric parietal cells, to ensure adequate absorption at normal dietary intake levels. Thus the absorption of physiological doses of vitamin $B_{12}$ is limited to approximately $0.0015-0.002 \mathrm{mg} /$ dose or meal, due to saturation of the uptake system. Regardless of dose, approximately $1.2 \%$ of vitamin $B_{12}$ is absorbed by passive diffusion and consequently this process becomes quantitatively important at pharmacological levels of exposure. Protein binding in certain foods may reduce the bioavailability of the vitamin, particularly in individuals with impaired gastric acid and/or digestive enzyme secretion. The different forms of crystalline cobalamin appear to be absorbed or retained to different extents, depending on the dose. Differences are most apparent at low doses.

Ingested vitamin $\mathrm{B}_{12}$ is released from the food matrix by the action of digestive enzymes and gastric acid and becomes bound to salivary haptocorrin-binding proteins. As the $\mathrm{pH}$ rises further along the gut, and under the influence of pancreatic enzymes, vitamin $B_{12}$ is released from the salivary haptocorrin and becomes complexed with intrinsic factor (IF). The cobalamin-IF complex binds to a specific cell wall 
receptor of the ileal enterocyte and is internalised by endocytosis. Once inside the cell, the IF is degraded and the liberated vitamin is converted to the methyl or the deoxyadenosyl form, is bound to transcobalamin II (TC II) binding protein and then exported into the portal blood. In the general circulation, most cobalamin is bound to transcobalamin I (TC I) but the majority of cobalamin available for uptake into the tissues is that bound to TC II.

\section{Distribution and metabolism}

Vitamin $B_{12}$ is distributed into the liver, bone marrow and virtually all other tissues, including the placenta and breast milk of nursing mothers. The liver is the predominant storage site for vitamin $B_{12}$.

Uptake into cells occurs through receptor mediated endocytosis involving specific TC II cell wall receptors. Once inside the tissues/cells, the complex is degraded by the lysosomes, and the released cobalamin is metabolised either to methyl-cobalamin in the cytosol, where it binds to methionine synthase, or to deoxyadenosyl-cobalamin in the mitochondria, where it binds to methylmalonyl CoA mutase.

\section{Excretion}

Excretion occurs mainly via the faeces and urine, but also through the shedding of skin cells. Excretion is very slow, with significant enterohepatic cycling.

\section{Toxicity}

\section{Human data}

There are a few case reports of adverse effects associated with ingestion of vitamin $B_{12}$, either as a supplement, or following the consumption of yeast extract products, which also contain cyanocobalamin. Five cases of allergic reactions were reported, three of which were recurrences of symptoms in individuals who had been previously exposed to cobalamin by the parenteral route. One further case reported the occurrence of a skin eruption that resembled acne rosacea. Vitamin $B_{12}$ exposures were generally not specified.

No adverse effects were reported in an experiment designed to determine the uptake of single oral doses of cyanocobalamin (up to $100 \mathrm{mg}$ ). However, only three participants were administered the very high doses.

Oral studies have been conducted to investigate the effects of vitamin $B_{12}$ on pernicious anaemia. However, although no adverse effects are apparent, these studies are not relevant to the general population since absorption of vitamin $B_{12}$ is reduced in this condition. The effect of high oral-dose cyanocobalamin on plasma homocysteine levels in healthy females of child-bearing age and the benefits of cyanocobalamin in patients with seasonal affective disorder have been investigated. No adverse affects related to treatment were reported in any study including those in which individuals received up to $4.5 \mathrm{mg} /$ day cyanocobalamin for 14 days, $2.0 \mathrm{mg}$ /day cyanocobalamin for up to one year or $1.0 \mathrm{mg} /$ day cyanocobalamin for several years. Less information is available following the oral administration of the hydroxocobalamin form of vitamin $B_{12}$. However, no adverse effects were reported 
Part 1 Water Soluble Vitamins

in individuals administered $0.3 \mathrm{mg} /$ day for up to 12 months. No adverse effects were reported in a controlled study in which 125 individuals received $6.0 \mathrm{mg} /$ day methylcobalamin for up to 12 weeks.

Adverse reactions (not specified) were reported in one of 16 and in one of 23 oligozoospermia patients given 6 or $12 \mathrm{mg} /$ day methyl cobalamin, respectively, for 16 weeks, presumably via the oral route. However, this study was not controlled.

\section{Animal data}

The data-base on the oral toxicity of vitamin $B_{12}$ in laboratory animals is limited. Doses of 1.5 to 3.0 $\mathrm{mg} / \mathrm{kg}$ bw by intraperitoneal and subcutaneous administration were found to be acutely toxic in mice (CNS effects; convulsions, cardiac and respiratory failure and ultimately death). However, much higher doses ( $\geqslant 5 \mathrm{~g} / \mathrm{kg}$ bw) cyanocobalamin appeared to be tolerated by mice following oral administration. There is no evidence relating to vitamin $B_{12}$ and teratogenicity or adverse effects on fertility or postnatal development.

\section{Carcinogenicity and genotoxicity}

There is no evidence suggesting that vitamin $B_{12}$ is carcinogenic or genotoxic in vitro or in vivo. However, although data are not consistent, there is some limited evidence to suggest that high doses of vitamin $B_{12}$ may have tumour promoting activity.

\section{Mechanisms of toxicity}

No data have been identified.

\section{Dose-response characterisation}

No relevant data have been identified.

\section{Vulnerable groups}

No vulnerable groups have been identified.

\section{Genetic variations}

No genetic variations have been identified. 


\section{Studies of particular importance in the risk assessment}

(For full review see http://www.food.gov.uk/science/ouradvisors/vitandmin/evmpapers or the enclosed CD).

Waife et al., 1963

As part of a non-controlled supplementation trial in patients with pernicious anaemia $(n=27)$ no adverse reactions were reported in individuals receiving $0.3 \mathrm{mg}$ hydroxocobalamin/day for up to one year.

Berlin et al., 1968

No adverse effects attributable to vitamin $B_{12}$ (cyanocobalamin) were recorded in a long-term clinical trial of 64 patients with pernicious anaemia, and other types of vitamin $B_{12}$ deficiency given a daily oral dose of $0.5 \mathrm{mg}$ rising to $1.0 \mathrm{mg}$ of cyanocobalamin (without intrinsic factor) for 10 to 70 months (42 patients received treatment for over 4 years). However, as noted previously, systemic absorption in these patients is limited.

Juhlin and Olsson, 1997

One hundred patients with vitiligo were treated with oral folic acid $(5 \mathrm{mg})$ and vitamin $B_{12}$ (1.0 mg cyanocobalamin) twice daily, for up to 12 months. There were no reports of adverse effects. However, 27/100 and 48/100 participants had stopped taking the supplements after $1-2$ months and $3-6$ months, respectively but reasons for withdrawal were not stated.

Takahashi et al., 1999

As part of a double-blind study to assess the therapeutic effect of methyl cobalamin on sleep-wake disorder, patients were administered either 6.0 ( $n=21)$ or $0.03 \mathrm{mg} /$ day $(n=27)$ methylcobalamin for 8 weeks. The lower dose group was considered as a control group because ethical permission was not granted for the inclusion of a placebo control group. There was no report of any adverse effects. The route of administration was not clear from the information given. Data were not available for all patients at the end of 8 weeks (two of the $6.0 \mathrm{mg} /$ day dose group and three of the $0.03 \mathrm{mg} /$ day group).

\section{Exposure assessment}

Total exposure/intake ${ }^{18}$ :

Food Mean: $0.0062 \mathrm{mg} /$ day

$97.5^{\text {th }}$ percentile: $0.020 \mathrm{mg} /$ day $(1986 / 7$ NDNS)

Supplements $\quad$ up to $3.0 \mathrm{mg} /$ day (Annex 4)

Estimated maximum intake: $0.020+3.0=3.0 \mathrm{mg} /$ day

No potential high intake groups have been identified.

18 The survey data do not distinguish the different forms of vitamin $B_{12}$. Dietary vitamin $B_{12}$ is mainly in the methyl, deoxyadenosyl and hydroxocobalamin forms. Hydroxo- and particularly cyanocobalamin are the forms usually present in dietary supplements. 
Part 1 Water Soluble Vitamins

\section{Risk assessment}

Vitamin $B_{12}$ is a water-soluble vitamin. At physiological doses, as occurs in food, the amount absorbed is largely limited (approximately $0.002 \mathrm{mg} / \mathrm{meal}$ ) by the capacity of the intrinsic factor-wall receptor uptake system. At pharmacological levels of dosing, diffusion becomes more important as the route of absorption. Vitamin $B_{12}$ present in excess of the binding capacity of the liver, plasma and other tissues is excreted by glomerular filtration.

It is generally accepted that ingested vitamin $B_{12}$ (cobalamin) has a very low toxicity in humans. Most available documented data are either in the form of case reports of possible vitamin $\mathrm{B}_{12}$-associated adverse effects or from clinical trials or supplementation studies designed primarily to investigate potential beneficial effects. The latter generally involve the use of the cyanocobalamin or methylcobalamin forms of vitamin $B_{12}$ and do not always specifically report an absence of adverse effects.

The animal toxicity database for vitamin $B_{12}$ is very limited. Doses of 1.5 to $3.0 \mathrm{mg} / \mathrm{kg}$ bw by intraperitoneal and subcutaneous administration were found to be acutely toxic in mice (CNS effects; convulsions, cardiac and respiratory failure and ultimately death). However, much higher doses ( $\geqslant 5$ $\mathrm{g} / \mathrm{kg}$ bw) cyanocobalamin appeared to be tolerated by mice following oral administration.

\section{ESTABLISHMENT OF GUIDANCE LEVEL}

There are insufficient data from studies in humans and animals to set a Safe Upper Level for vitamin $B_{12}$.

Clinical studies have reported no adverse effects following administration of up to $6.0 \mathrm{mg} /$ day of methylcobalamin for several weeks and up to $1.0 \mathrm{mg} /$ day cyanocobalamin for several years. Clinical trials and supplementation studies involving up to 100 individuals to investigate the beneficial effects of oral cyanocobalamin have not reported any treatment-related adverse reactions following doses of 0.3 to $4.5 \mathrm{mg}$ for periods ranging from 14 days to several years. Cyanocobalamin is the type of vitamin $\mathrm{B}_{12}$ most frequently included in supplements in the UK. The study by Juhlin and Olsson (1997), supported by the absence of an identified hazard and widespread clinical experience with oral and parenteral treatment, suggests that supplemental intakes of $2.0 \mathrm{mg}$ cyanocobalamin/day should not produce any adverse effects and this intake can be used for guidance purposes. This is equivalent to $0.034 \mathrm{mg} / \mathrm{kg}$ $\mathrm{bw} /$ day in a $60 \mathrm{~kg}$ adult. No uncertainty factor is needed because human data from large numbers of individuals are available. However, it should be noted that this figure has been established in particular subgroups of the population, i.e. vitiligo suffers and those treated for pernicious anaemia, and may not be completely applicable to the general population. 


\section{References}

Berlin, H., Berlin, R., Brante, G. (1968). Oral treatment of pernicious anemia with high doses of vitamin $B_{12}$ without intrinsic factor. Acta Medica Scandinavica 184, 274-258.

COMA (1991). Dietary Reference Values for Food Energy and Nutrients for the United Kingdom. Report of the Panel on Dietary Reference Values, Committee on Medical Aspects of Food and Nutrition Policy. HMSO, London.

Juhlin, L., Olsson, M.J. (1997). Improvement of vitiligo after oral treatment with vitamin $B_{12}$ and folic acid and the importance of sun exposure. Acta Dermatol-venereologica 77, 460-462.

Takahashi, K., Okawa, M., Matsumoto, M., Mishima, K., Yamadera, H., Sasaki, M., Ishizuka, Y., Yamada, K., Higuchi, T., Okamoto, N., Furuta, H., Nakagawa ,H., Ohta, T., Kuroda, K., Sugita, Y., Inoue, Y., Uchimura, N., Nagayama, H., Miike, T., Kamei, K. (1999). Double-blind test on the efficacy of methylcobalamin on sleepwake rhythm disorders. Psychiatry and Clinical Neurosciences 53, 211-213.

Waife, S.O., Jansen, C.J., Crabtree, R.E., Grinnan, E.L., Fouts, P.J. (1963). Oral vitamin B 12 without intrinsic factor in the treatment of pernicious anemia. Annals of Internal Medicine 58, 810-817. 


\section{Risk Assessment}

\section{Vitamin C}

\section{General information}

\section{Chemistry}

Vitamin $C$ is a six-carbon compound structurally related to glucose, consisting of two inter-convertible compounds: L-ascorbic acid, which is a strong reducing agent, and its oxidised derivative, Ldehydroascorbic acid.

\section{Natural occurrence}

Most animals are able to synthesise vitamin C from glucose and galactose. However, primates and guinea pigs are unable to do so.

\section{Occurrence in foods, food supplements and medicines}

Food of plant origin, particularly citrus and soft fruits and leafy green vegetables, are major sources of vitamin C. Kidney and liver are good animal-derived sources of vitamin C. Vitamin C is readily lost during cooking, due to oxidation. Ascorbic acid is a permitted anti-oxidant in food with no specified limits on the level of fortification. Vitamin $C$ is present in numerous dietary supplements and in licensed medicines at doses of up to $3000 \mathrm{mg}$.

\section{Recommended amounts}

In 1991, COMA recommended a RNI for ascorbic acid of $40 \mathrm{mg} /$ day for adults, with an increase in pregnancy to $50 \mathrm{mg} /$ day, and during lactation to $70 \mathrm{mg} /$ day (COMA, 1991).

\section{Analysis of tissue levels and vitamin C status}

Plasma and urinary vitamin $C$ levels may be measured but reflect recent dietary intake rather than the level of vitamin $C$ in body stores. Leucocytes contain higher concentrations of vitamin $C$ than plasma, whole blood or serum but measurement of leucocyte vitamin $C$ is technically more difficult than estimation of plasma or urinary levels. A leucocyte vitamin C level below $0.01 \mathrm{mg}$ per $10^{8}$ cells is generally regarded as deficient.

\section{Brief overview of claimed non-nutritional beneficial effects}

It has been claimed that vitamin $C$ protects against the common cold. Beneficial effects on conditions such as cancer, vascular disease, cataracts, diabetes, asthma, arthritis, Parkinson's disease, autism and depression have also been suggested. 


\section{Function}

Vitamin $\mathrm{C}$ is a strong reducing agent and as an antioxidant is involved in prevention of the damaging effects of free radicals. Vitamin $C$ is involved in the synthesis of collagen, neurotransmitters and carnitine; it is an enzyme co-factor and also increases the gastrointestinal absorption of non-haem iron.

\section{Deficiency}

Vitamin C deficiency in humans leads to the clinical syndrome of scurvy. Early symptoms in adults include fatigue, weakness, aching joints and muscles. In later stages scurvy is characterised by anaemia, bleeding from the gums, petechial and sheet haemorrhages, and delayed wound healing.

\section{Interactions}

Absorption of metal ions may be altered by vitamin C.

\section{Absorption and bioavailability}

Gastrointestinal absorption of vitamin $C$ is efficient and occurs in the small intestine via a saturable active transport mechanism. Absorption efficiency of low oral doses of vitamin C (4-64 mg) may be as high as $98 \%$, but decreases with increasing doses of the vitamin.

\section{Distribution and metabolism}

Ascorbic acid is widely distributed in all tissues of the body, with higher levels found in the adrenal glands, pituitary and retina, and lower levels in kidney and muscle tissue.

Vitamin $C$ is oxidised to dehydroascorbic acid, which is hydrolysed to diketogulonic acid and then oxidised to oxalic and threonic acid. Some oxidation to carbon dioxide occurs at high doses.

\section{Excretion}

Unmetabolised vitamin $C$ and vitamin $C$ metabolites, such as oxalate, are largely excreted in the urine. Approximately $3 \%$ of a $60 \mathrm{mg}$ oral dose is excreted in the faeces. More of the vitamin is excreted unchanged at higher levels of vitamin $C$ intake.

\section{Toxicity}

\section{Human data}

Gastrointestinal effects are the most common adverse clinical events associated with acute, high doses of vitamin $C$ given over a short period of time. 
Part 1 Water Soluble Vitamins

Other suggested adverse effects include metabolic acidosis, changes in prothrombin activity and 'conditioned need' scurvy (where ingestion of an excessive amount of vitamin C during pregnancy may condition the offspring to require greater than the expected or recommended daily intakes of the vitamin).

Adverse effects related to the urinary route of excretion have been claimed, including renal stones, renal tubular disease and oxaluria. It has been suggested that vitamin $C$ consumption may increase oxalate excretion and cause the formation of urinary stones, and subjects with a predisposition to the formation of kidney stones may be more sensitive to increases in urinary oxalate associated with vitamin C. However, studies in humans have not revealed a substantial increase in urinary oxalate after high intakes of vitamin C, and the moderate increases reported may be an experimental artefact (see later).

Vitamin $C$ increases iron uptake from the gut and it is possible that this may be important in subjects with conditions such as haemochromatosis or in subjects heterozygous for this condition. However, when doses of vitamin C of $2000 \mathrm{mg} /$ day were given to healthy volunteers for 20 months or more, no effects on iron status were found.

\section{Supplementation trials}

Vitamin $C$ was administered (in combination with other vitamins and minerals) at doses of up to $1000 \mathrm{mg} /$ day for up to 5 years in two supplementation trials with good compliance and no reported adverse effects. Reduced vitamin $B_{12}$ levels in 3 (of 90) individuals consuming more than $1000 \mathrm{mg} /$ day over a minimum of 3 years were reported in an earlier trial, although the relevance of these observations has been questioned.

\section{Animal data}

Vitamin $C$ has low toxicity when large doses are given over a short period of time. High doses of vitamin $\mathrm{C}$ are associated with decreased growth rates in guinea pigs (50 mg/day), increased cholesterol levels in rats $(150 \mathrm{mg} / \mathrm{kg}$ bw/day) and interference with trace element metabolism in chicks. No effects on reproductive or developmental parameters have been reported. A conditioned increase in vitamin $C$ requirements has been reported in guinea pigs.

\section{Carcinogenicity and genotoxicity}

No data on carcinogenicity have been identified. Some positive in vitro mutagenicity tests have been reported, although results are generally mixed. However, the positive results tended to occur when vitamin $C$ was tested in the presence of copper. Short-term vitamin $C$ supplementation has been reported to cause an increase in modified DNA bases, and this has been attributed to pro-oxidant effects. In vitro, vitamin C caused an increase in DNA reactive metabolites formed from lipid hydroperoxides. There is no reliable evidence of mutagenicity in vivo.

\section{Vulnerable groups}

Individuals unable to regulate iron absorption due to haemochromatosis or thalassaemia may be vulnerable to any enhanced iron absorption caused by vitamin C. Although clear and substantial rises in urinary oxalate excretion have not been demonstrated during increased vitamin $C$ intake, it remains possible that individuals with risk factors such as uricosuria will be more sensitive. 


\section{Mechanism of toxicity}

Urinary stones may result from an increase in urinary oxalate excretion (see discussion below). Adverse effects attributed to vitamin $C$ may be due to increased sensitivity to oxidant stress, because vitamin $C$ can be pro-oxidant at very high concentrations.

\section{Dose-response characterisation}

The dose-response is unclear, as many studies have used only one dose level. However, where found, adverse effects are generally reported at levels in excess of $1000 \mathrm{mg}$ vitamin C/day.

\section{Genetic variations}

Polymorphisms in haptoglobin and transferrin have been reported to be associated with altered metabolism of vitamin C. Certain conditions may increase sensitivity to the adverse effects associated with this compound e.g. haemochromatosis, thalassaemia or a pre-disposition to urinary or renal stones.

\section{Studies of particular importance in the risk assessment}

(For full review see http://www.food.gov.uk/science/ouradvisors/vitandmin/evmpapers or the enclosed CD).

\section{Cameron and Campbell, 1974}

In a stepped study, healthy human volunteers were given vitamin $C$ with the dose increasing each week by $1000 \mathrm{mg}$. Abdominal distention, flatulence, diarrhoea and transient colic were described as 'fairly frequent' effects at supplemental dose levels of 3000-4000 mg daily. No other details were provided.

Cook et al., 1984

Seventeen adult volunteers were given $2000 \mathrm{mg}$ vitamin C/day with meals for 16 weeks in a study examining the effect of dietary iron absorption and assimilation on body iron status. Nine subjects continued in the study, taking these doses of vitamin C for 24 months. No subjective side effects were reported. Although vitamin $C$ enhances absorption of non-haem iron, no increase in serum ferritin levels was found, despite wide variation in initial iron status between the volunteers. The authors concluded that supplemental vitamin $C$ had a negligible effect on iron stores. Vitamin $C$ supplementation continued for a further 20 months in 4 iron-deficient and 4 iron-replete subjects. No effect of vitamin $C$ on body iron reserves (as measured by serum ferritin levels) was apparent and no intestinal adaptation to the enhancing effect of the vitamin had occurred. The authors considered several possible explanations for their findings. No adverse effects were reported in this study of large supplemental doses of vitamin $\mathrm{C}$ but the authors noted that the study did not exclude possible adverse effects in individuals who are heterozygous for the haemochromatosis gene (approximately $10 \%$ of the population). The study involved small numbers of participants with variable iron status and was not blinded, though compliance was checked. 
Part 1 Water Soluble Vitamins

Mai et al., 1990

Twenty patients with multiple sclerosis were randomised to receive either supplements providing 2000 $\mathrm{mg} /$ day vitamin C, $6 \mathrm{mg} /$ day sodium selenite and $480 \mathrm{mg} /$ day vitamin $\mathrm{E}$, or placebo for 5 weeks. The patients were interviewed about side effects after 2 weeks and 4 weeks of treatment. Out of the 10 patients receiving the active supplement, one reported slight facial erythema at week 2 , which subsequently subsided during continued treatment, one reported passing urine with a peculiar smell. Three of the 10 patients receiving the placebo reported an increased number of headaches.

\section{Urivetzky et al., 1992}

Fifteen patients with calcium oxalate renal stones (presumed by the authors to be at greatest risk from the effects of ascorbic acid), were given 100, 500, 1000, or $2000 \mathrm{mg}$ supplemental ascorbic acid on days 2 and 3 following operations to remove the stones. Intrarenal urine specimens were collected by nephrostomy catheter directly into EDTA and sodium thimerosol preservative to stabilise ascorbic acid and oxalate; oxalate was measured following the removal of ascorbic acid with sodium nitrite. The increase in urinary oxalate was statistically significant at doses of administered vitamin C of $500 \mathrm{mg}$ or more. The authors estimated that there was a $6-13 \mathrm{mg} /$ day increase in urinary oxalate excretion per $1000 \mathrm{mg} /$ day administered ascorbic acid, and concluded that vitamin C supplementation was associated with an increased risk of calcium oxalate renal stones.

\section{Wandzilak et al., 1994}

Fifteen healthy volunteers were given 1000, 5000 and 10,000 mg vitamin C each for 5 days, separated by 5 days with no supplementation. Twenty-four-hour urine samples were preserved by adding hydrochloric acid to reduce the $\mathrm{pH}$ to 2 and frozen. Ascorbate appeared to be converted nonenzymatically into oxalate during analysis, confounding the analytical measurement. This may explain the previously published results in other studies, where oxalate excretion was affected by ascorbate consumption. A moderate, dose-dependent increase in urinary oxalate was found in the volunteers. However, a clear trend was not apparent when the non-enzymatic ascorbate-to-oxalate conversion was taken into account. Subjects were advised to take the vitamin $C$ tablets at mealtimes to minimise potential adverse gastrointestinal effects. However, two out of 15 volunteers experienced diarrhoea when consuming 10,000 mg supplemental vitamin C/day and were unable to continue taking this level. The study was not blinded.

Levine et al., 1996; Levine et al., 1999

Steady state plasma ascorbic acid concentrations, urinary ascorbic acid and urinary oxalate concentrations (the latter determined by an enzymatic method reported to be free from interference by ascorbic acid) were measured in 7 young healthy non-smoking subjects. At vitamin C intakes of 1000 $\mathrm{mg} /$ day there were statistically significant increases in urinary oxalate excretion (though this was still within physiological limits) and uric acid excretion.

\section{Auer et al., 1998}

Ten healthy male volunteers (with no history of stone formation) ingested $4000 \mathrm{mg}$ vitamin $\mathrm{C}$ daily (in 4 doses per day) for 5 days. The urine collection procedure allowed the analysis of oxalate in the presence and absence of an EDTA preservative and for the analysis of ascorbic acid. In the preserved samples there was no significant increase in oxalate excretion at any stage of the protocol. Excretion of ascorbic acid increased when vitamin $C$ ingestion commenced but levels stabilised after 24 hours suggesting saturation of the metabolic pool. While transient and statistically significant changes 
occurred in some of the biochemical risk factors, it was concluded that large doses of vitamin $\mathrm{C}$ did not affect the principal risk factors associated with calcium oxalate kidney stone formation.

Gokce et al., 1999

In a randomised, double-blind, placebo-controlled study, flow-mediated dilation of the brachial artery was measured in 21 patients with coronary artery disease 2 hours after a single supplementary dose of $2000 \mathrm{mg}$ vitamin C and following a 30-day period during which $500 \mathrm{mg}$ vitamin C was taken daily. A significant increase in flow-mediated dilation of the brachial artery was observed, compared to controls, following the single dose of $2000 \mathrm{mg}$ vitamin C. This improvement was sustained after the 30-day supplementation period with $500 \mathrm{mg} /$ day vitamin $\mathrm{C}$. The report does not mention assessment of adverse effects.

Morton et al., 2001

A retrospective cohort study of 994 women, of whom 277 were regular users of vitamin C supplements, assessed the effects of vitamin $C$ supplementation on bone mineral density (BMD). Amongst the users of vitamin C supplements, supplemental intake ranged from $100 \mathrm{mg} /$ day to $5000 \mathrm{mg} /$ day; the mean was calculated to be $745 \mathrm{mg} /$ day. Average duration of vitamin C supplement use was 12.4 years. BMD levels were assessed at the hip, lumbar spine and ultradistal and midshaft radius of the arm. Vitamin $C$ supplement users were reported to have BMD levels $3 \%$ higher than non-users at the midshaft radius, femoral neck and hip, after adjusting for age, body mass index and total calcium intake. A significant increase in BMD of the femoral neck and a small increase in BMD of the total hip were still apparent in vitamin $\mathrm{C}$ supplement users even after adjusting for age, body mass index, exercise, alcohol consumption, smoking, physician-diagnosed osteoarthritis and use of calcium supplements, multivitamin supplements, thiazides, oestrogen, thyroid hormones and oral corticosteroids. No parameters other than BMD were assessed, thus no side effects were reported.

\section{Exposure assessment}

Total exposure/intake:

Food Mean: $64 \mathrm{mg} /$ day 97.5th percentile: $160 \mathrm{mg} /$ day (from 1986/87 NDNS)

Supplements up to $3000 \mathrm{mg} /$ day (Annex 4)

Estimated maximum

daily intake: $160+3000=3160 \mathrm{mg}$

Vegetarians are a potential high intake group. 
Part 1 Water Soluble Vitamins

\section{Risk assessment}

The available data suggest that vitamin $C$ is not associated with significant adverse effects and there are no obvious specific key toxic endpoints for vitamin $C$ dose given orally to healthy subjects. High oral doses of vitamin $C$ are associated with gastrointestinal effects, generally at doses of several grams, but have also been reported at doses of $1000 \mathrm{mg}(1 \mathrm{~g})$. There are few controlled studies specifically investigating this adverse effect. Controlled studies do not support anecdotal reports of other possible adverse effects, such as infertility.

Earlier suspicions of potential adverse effects, such as destruction of vitamin $B_{12}$, have not been confirmed following subsequent developments in analytical techniques. Data on increased oxalate excretion attributable to vitamin $C$ are conflicting. Some reported increases in urinary oxalate might be attributable to experimental artefact (Auer et al., 1998) though increased oxalate was apparent at doses of more than $500 \mathrm{mg} /$ day in subjects with calcium oxalate stones. In contrast, doses of $4000 \mathrm{mg} /$ day vitamin $C$ were not associated with increased urinary oxalate excretion in normal subjects.

Potential vulnerable groups include sufferers from disorders of iron metabolism or storage.

Vitamin $C$ has been reported to produce a variety of pro-oxidant effects. The significance of this for the general population is uncertain.

Vitamin $C$ has very low acute toxicity in animals and no effects on reproductive parameters have been reported. However high doses of vitamin $\mathrm{C}$ are associated with decreased growth rates in guinea pigs ( $50 \mathrm{mg} /$ day) and increased cholesterol levels in rats ( $150 \mathrm{mg} / \mathrm{kg} \mathrm{bw} /$ day).

\section{ESTABLISHMENT OF GUIDANCE LEVEL}

There are insufficient data to set a Safe Upper Level for vitamin C. The vitamin may be of low toxicity, though adverse effects, in particular on the gastrointestinal system, may occur in subjects consuming quantities of vitamin C greater than $1000 \mathrm{mg} /$ day. The precise dose at which such effects occur is variable. These effects may be a serious problem for individuals with disordered gastrointestinal function. A limited study by Cameron and Campbell (1974) suggests a LOAEL for gastrointestinal effects in humans of 3000-4000 mg/day. For guidance purposes, based on a LOAEL of $3000 \mathrm{mg} /$ day, and applying an UF of 3 for LOAEL to NOAEL extrapolation, a supplemental dose of $1000 \mathrm{mg} /$ day supplement would not be expected to have any significant adverse effects. The dose is equivalent to $17 \mathrm{mg} / \mathrm{kg}$ bw/day in a $60 \mathrm{~kg}$ adult. A guidance level for total vitamin C intake has not been estimated since adverse effects appear to follow supplemental, bolus doses rather than intake of vitamin $C$ in food. It should be noted that higher levels of vitamin $\mathrm{C}$ may be without adverse effects in many individuals.

A number of potentially vulnerable groups have been identified; these include individuals who are heterozygous for haemochromatosis, and thalassaemia or those with a pre-disposition to urinary or renal stones. Data on the possible adverse effects of vitamin $C$ on these individuals are also conflicting, but, appear to occur at intakes above $1 \mathrm{~g} /$ day. 


\section{References}

Auer, B.L., Auer, D. and Rodgers, A.L. (1998) The effect of ascorbic acid ingestion on the biochemical and physiochemical risk factors associated with calcium oxalate kidney stone formation. Clinical Chemistry and Laboratory Medicine 36, 143-148.

Cameron, E. and Campbell, A. (1974). The ortho-molecular treatment of cancer. II. Clinical trial of high dose ascorbic acid supplements in advanced human cancer. Chemical and Biological Interactions 9 , 285-315.

COMA (1991). Dietary Reference Values for Food Energy and Nutrients for the United Kingdom. Report of the Panel on Dietary Reference Values, Committee on Medical Aspects of Food and Nutrition Policy. HMSO, London.

Cook, J.D. Watson S.S., Simpson K.M., Lipschitz D.A. and Skikne B.S. (1984). The effect of high ascorbic acid supplementation on body iron stores. Blood 64, 721-726.

Gokce N., Keaney J.F. Jr, Frei B., Holbrook M., Olesiak M., Zachariah B.J., Leeuwenburgh C., Heinecke J.W., Vita J.A. (1999). Long-term ascorbic acid administration reverses endothelial vasomotor dysfunction in patients with coronary artery disease. Circulation 99, 3234-3240.

Levine, M. Conry-cantilena, C. Wang, Y. Welch R.W., Washko P.W., Dhariwal K.R., Park J.B., Lazarev A., Graumlich J.F., King J., Cantilena L.R. (1996). Vitamin C pharmacokinetics in healthy volunteers. Proceedings of the National Academy of Sciences 93, 3704-3709.

Levine, M., Rumsey, SC. Daruwala, R. Park, JB. Wang, Y. (1999) Criteria and recommendations for vitamin C intake. Journal of the American Medical Association 281, 1415-1423.

Mai J., Sorensen P.S., Hansen J.C. (1990) High dose antioxidant supplementation to MS patients. Effects on glutathione peroxidase, clinical safety, and absorption of selenium. Biological Trace Element Research 24, 109-117.

Morton D.J., Barrett-Connor E.L., Schneider D.L. Vitamin C supplement use and bone mineral density in postmenopausal women. Journal of Bone Mineral Research 16, 135-40.

Urivetsky, M., Kessaris, D., Smith, A.D. (1992). Ascorbic acid overdosing: a risk for calcium oxalate nephrolithiasis. Journal of Urology 147, 1215-1218.

Wandzilak, T.R. D'Andre S.D., Davis P.A., Williams H.E. (1994). Effect of high dose vitamin C on urinary oxalate levels. Journal of Urology 151, 834-837. 
Part 2

Fat Soluble Vitamins 


\section{Risk Assessment}

\section{Vitamin A (Retinol)}

\section{General information}

\section{Chemistry}

The term 'vitamin A' refers to a group of fat-soluble compounds known as 'the retinoids'. Generally, their structure consists of a ß-ionone ring, a conjugated isoprenoid side chain and a polar terminal group. The parent compound all-trans-retinol and its fatty acid ester derivatives are referred to as pre-formed vitamin A, as opposed to the vitamin A precursors, such as B-carotene. Following the recommendations of the Food Agricultural Organisation (FAO) and World Health Organisation (WHO), estimations of vitamin $A$ requirement and food content take into account the different activities and variable absorption of vitamin A and provitamin A, and are expressed in terms of 'retinol equivalent' (RE) where $1 \mu \mathrm{gRE}=1 \mu \mathrm{g}$ retinol $=1.78 \mu \mathrm{g}$ retinyl palmitate $=6 \mu \mathrm{g}$ B-carotene $=12 \mu \mathrm{g}$ other carotenoids with provitamin A activity $=3.33 \mathrm{IU}$ vitamin A activity from retinol.

\section{Natural occurrence}

The primary sources of vitamin A are the precursor compounds, the carotenoids, which are largely plant derived and retinyl esters found in foods of animal origin.

\section{Occurrence in food, food supplements and medicines}

Foods rich in pre-formed vitamin A (retinol, retinyl esters) include dairy products (90-300 $\mu \mathrm{g} \mathrm{RE} / \mathrm{kg}$ ), fortified margarine (approximately $330 \mu \mathrm{g} R / \mathrm{kg}$ ), liver (approximately $3500 \mu \mathrm{gEE} / \mathrm{kg}$ ) and fish oils. Other sources of exposure include single- or multi-vitamin supplements (at levels up to $2400 \mu \mathrm{g}$ RE per daily dose) and synthetic forms prescribed for therapeutic purposes, e.g. certain skin disorders.

\section{Other sources of exposure}

No other sources of exposure have been identified.

\section{Recommended amounts}

The UK RNI ranges set by COMA are $700 \mu \mathrm{g}$ RE/day for adult males and $600 \mu \mathrm{g}$ RE/day for adult females (COMA, 1991). The US recommended dietary allowance (RDA) is $1000 \mu \mathrm{g}$ RE/day for adult males and $800 \mu \mathrm{g} R /$ day for adult females. The $\mathrm{WHO}$ recommended dietary intake (RDI) is $600 \mu \mathrm{g} R /$ day for adult males and $500 \mu \mathrm{g}$ RE/day for adult females. 


\section{Analysis of tissue levels and vitamin A status}

The hepatic concentration of retinyl esters is the most objective measure of vitamin A status, but cannot be readily determined in living individuals. The plasma retinol concentration is under homeostatic control by the synthesis of retinol binding protein, and therefore is an insensitive indicator of status, except in cases of extreme depletion when other signs of deficiency are already evident. Hypervitaminosis A may be characterised by elevated levels of plasma retinyl esters, which normally contribute to $<5 \%$ of blood vitamin A. Other methods used to determine vitamin A status in humans include dietary assessment, clinical evaluation, retinal function tests and assessment of retinol-binding proteins.

\section{Brief overview of non-nutritional beneficial effects}

Vitamin A is used in the treatment of some skin disorders including acne, psoriasis and ichthyosis. Vitamin A has been suggested to be beneficial when used as a chemo-preventative or adjuvant agent in the treatment of some cancers and may improve lung function in chronic obstructive pulmonary disease. Vitamin A is used as a treatment for abnormal dark-adaptation and is included in eye drops used to treat blurred vision, cataracts, glaucoma, conjunctivitis and dry eyes. Vitamin A (along with zinc) has been suggested to be a useful adjunct in the treatment of anaemia with iron. Vitamin $A$ is also taken as an anti-oxidant dietary supplement.

\section{Function}

Vitamin A is essential to the processes of vision, reproduction, embryonic development, morphogenesis, growth and cellular differentiation. With the exception of the visual process, most processes are related to the control of gene expression, with vitamin A metabolites, such as retinoic acid, acting as nuclear receptor-ligands.

\section{Deficiency}

Vitamin A deficiency is a problem largely in developing countries. It can lead to night blindness and xerophthalmia, growth retardation, keratinisation of epithelia, impaired hearing, taste and smell, increased susceptibility to infection, increased child mortality and reduced male fertility. In pregnancy, vitamin A deficiency can result in malformations in offspring. Deficiency in the developed world is usually limited to those with absorption difficulties, increased susceptibility to opportunistic infections, chronic liver disease and alcoholics.

\section{Interactions}

Vitamin A may potentiate the development of intracranial hypertension when taken in combination with tetracycline and minocycline type antibiotics.

The catabolism of retinol and retinoic acid in the human liver may be mediated by cytochrome P450. Drugs such as ketoconazole, which inhibit cytochrome P450, can significantly increase the half-life of retinoic acid. 
Part 2 Fat Soluble Vitamins

Hypervitaminosis A may decrease vitamin C tissue storage. Vitamin A may antagonise the action of vitamin $\mathrm{K}$ in blood clotting function.

Hypervitaminosis A may have an anti-thyroid effect. Deficiency in zinc may adversely affect mobilisation of vitamin A from hepatic stores and absorption of vitamin A from the gut.

Vitamin A deficiency may result in impaired iron absorption and decrease its utilisation for erythropoiesis.

Alcohol may potentiate vitamin A-induced hepatotoxicity. Competitive inhibition of alcohol dehydrogenase may lead to decreased synthesis of retinoic acid, resulting in functional vitamin A deficiency, which has been postulated to be involved in foetal alcohol syndrome.

\section{Absorption and bioavailability}

Approximately $80 \%$ of dietary pre-formed vitamin A is absorbed but this may be reduced if diets are low in fat or individuals are suffering from fat malabsorption syndrome. The form of preparation influences the rate and extent of absorption of vitamin A from supplements. Thus, aqueous dispersions and emulsions achieve higher plasma levels, at a faster rate, with lower faecal losses, than oily solutions. The pharmacokinetic handling of retinol from foods, such as liver, differs to that obtained from supplements.

Dietary retinyl ester is released from food by proteolytic digestion and hydrolysed to retinol in the gut.

\section{Distribution and metabolism}

The retinol is taken up into enterocytes, undergoes re-esterification and is incorporated into chylomicra, which are released into the circulation via the lymph. Following the breakdown of chylomicra by serum lipases, the retinyl esters are released, taken up by hepatocytes and re-hydrolysed. The resulting retinol is transferred to the stellate (fat storing) cells and stored in the form of long-chain fatty esters. Approximately $90 \%$ of the body's vitamin A is stored in the liver this way. The availability of hepatic stores of vitamin A may be decreased if protein status is low.

Plasma retinol is usually maintained under tight homeostatic control and concentrations do not alter significantly unless hepatic stores are severely depleted. If hepatic storage capacity is exceeded, plasma levels of retinyl ester increase, but plasma levels of retinol itself do not.

Mobilised retinol is transported in plasma bound to retinol-binding protein and transthyretin. Uptake into extra-hepatic tissues occurs via a receptor-mediated process. Once inside the cell, retinol undergoes a complex series of metabolic oxidations, isomerisations and conjugations, most of which are reversible. Several enzymes are involved in these reactions, including cytochromes P450. Cellular binding proteins direct the reactions. Other intracellular binding proteins facilitate transport of specific vitamin A metabolites, such as retinoic acid, into the nucleus of the cell, where they interact with the retinoid nuclear receptors (RARs and RXRs) and participate in the control of gene expression for differentiation and growth.

\section{Excretion}

Oxidised products are excreted in the urine or conjugated with glucuronic acid and excreted in the urine or bile. 


\title{
Toxicity
}

\author{
Human data \\ Acute toxicity \\ Symptoms of acute vitamin A toxicity include abdominal pain, anorexia, vomiting, blurred vision, \\ irritability, headache, and in neonates and infants, bulging of fontanelles. Acute toxicity is associated \\ with doses well in excess of 100,000 $\mu \mathrm{g}$ RE and 10,000 $\mu \mathrm{g}$ RE, in adults and children, respectively. \\ Infants of $<6$ months have been shown to develop acute symptoms following a single dose of 7500 - \\ $15,000 \mu \mathrm{g}$ RE, whereas a dose of 30,000 $\mu \mathrm{g}$ RE appears to be well-tolerated in older infants ( 6 and 9 \\ months of age).
}

\section{Chronic and sub-chronic toxicity}

Symptoms of chronic toxicity include dry thickening of the skin, cracking of lips, conjunctivitis, erythematous eruption, alopecia, reduced bone mineral density, bone joint pain, chronic headache, intracranial hypertension and hepatotoxicity. The onset and severity of toxic manifestations are dependent on dose, duration and the manifestation in question. Damage to the eyes, bone and liver may be permanent, but most other symptoms are reversible. Chronic toxicity in adults is generally attributed to supplemental doses of $>7500-15,000 \mu \mathrm{g} R /$ day, over weeks, months or years. However, there have been cases of toxicity associated with lower doses of $\sim 1500-3,000 \mu \mathrm{g} R /$ day. Determination of a threshold dose for chronic toxicity may be confounded by pre-existing disease, alcohol abuse, drug therapy and limited knowledge of dietary intake. Extreme vitamin A intolerance may have a genetic basis, although the precise metabolic defect has not yet been elucidated.

Epidemiological data have shown that the risk of hip fracture in postmenopausal women may be doubled when dietary retinol intake is $>1500 \mu \mathrm{g} R /$ day, compared to an intake of $500 \mu \mathrm{g}$ RE/day. Other supporting epidemiological data have shown that hip fracture risk is higher in Northern European countries, particularly Scandinavia, where dietary intake of retinol is higher. Differences in fracture are such that Swedish men have a higher hip fracture rate than English or Swiss women.

\section{Developmental toxicity}

There are a number of case reports of vitamin A-associated birth defects. However, only one case involved a vitamin A exposure of $<7500 \mu \mathrm{g} R /$ day. Since 1990, seven epidemiological studies (five case-control and two prospective) have been reported. Four studies failed to establish an association between vitamin A exposure and birth defects, whereas three studies determined a teratogenic effect at varying levels.

\section{Supplementation trials}

In the (B-Carotene Retinol Efficacy study) CARET trial, a combination of $7500 \mu \mathrm{g} r e t i n o l / d a y ~ a n d ~ 30,000$ $\mu \mathrm{g} /$ day $B$-carotene resulted in an increase in lung cancer in male smokers and males who had been exposed to asbestos. However, this and the ABTC study ${ }^{19}$ indicated strongly that the $B$-carotene 
component was responsible for this effect. A chemopreventive study in workers exposed to blue asbestos found that 45/512 subjects taking $7500 \mu \mathrm{g} R /$ day elected to change treatment because of associated headaches. A modest increase in skin cancer was apparent in subjects given $7500 \mu \mathrm{g}$ retinol/day compared to isotretinoin or controls. In a chemopreventative trial, patients with lung cancer or head and neck cancer, were given $90,000 \mu \mathrm{g}$ RE/day as retinyl palmitate for one year followed by $45,000 \mu \mathrm{g}$ RE/day for a further year. Side effects including dryness, and itching of skin and bleeding and hair loss were reported by $45 \%$.

\section{Animal data}

\section{Acute and chronic toxicity}

Vitamin A toxicity in animals is dependent upon dose, formulation, duration, species, and age. Lethal doses in rat and mouse are $>2,500,000 \mu \mathrm{g} / \mathrm{kg}$ bw. Chronic toxicity in adult rats begins at about $3000 \mu \mathrm{g}$ RE/kg bw/day. Younger animals may be more susceptible. Hypervitaminosis A causes anorexia, weight loss, anaemia, cachexia and ultimately death in rats, hamsters, mice and dogs. Chronic manifestations include effects on skin (hair loss, localised erythema and thickened epithelium), effects on internal organs (fatty infiltration of the liver and fatty changes in the heart and kidney, testicular changes, degeneration of myocardial fibres), blood effects (decreased haemoglobin, transient increases in circulating lipids and serum cholesterol, elevated serum triglycerides), skeletal effects (limping gait, spontaneous fractures, hind leg paralysis, increased osteoclast activity, decreased osteoblast activity, bone elongation without thickening, reduced formation of dentine and atrophy of odontoblasts), subcutaneous and intramuscular haemorrhage, hypothrombinaemia, internal haemorrhage, and inflammation of nasal passage, gut, and conjunctiva. Hypervitaminosis A results in the development of gallstones in the hamster.

\section{Developmental toxicity}

Retinol is teratogenic in laboratory animals, with the following order of increasing susceptibility: rat and mouse, hamster, monkey, and rabbit. The lowest teratogenic doses reported in rat, monkey and rabbit are 35,000, 6000 and $2500 \mu \mathrm{RE} / \mathrm{kg}$ bw/day, respectively. Malformations are largely analogous to those associated with synthetic retinoid teratogenicity in humans, such as anencephaly, spinabifida, cleft lip, cleft palate, micronathia, microphthalmia, several types of malformation to the ear, teeth, salivary glands and aortic arch, ventricular septal defects, imperforate anus, omphalocele, renal agenesis, polycystic kidney, hydronephrosis, phocomelia, digit malformation, certain defects of the genitalia, pituitary, thyroid, thymus, skull, vertebrae, ribs, and muscles and situs inversus (Hathcock et al., 1990 and references therein). Permanent learning disabilities have been seen in F344 rats born to dams exposed $\geqslant$ $3000 \mu \mathrm{RE} / \mathrm{kg} /$ day.

\section{Carcinogenicity and genotoxicity}

There is no evidence to suggest that retinol or retinyl esters are carcinogenic in laboratory animals and at sub-toxic doses, vitamin A can be highly effective in preventing chemically induced tumours. Epidemiological studies have generally shown a negative association between vitamin A intake and cancer.

The weight of evidence suggests that neither retinol nor retinyl ester are genotoxic. 


\section{Mechanisms of toxicity}

Retinoic acid plays an important role in the control of expression of many genes, including Hox genes, which are vital for correct positional and sequential development of the embryo. The morphogenic action of retinoic acid is mediated through the actions of retinoic acid receptor (RAR and RXR) bound material on nuclear retinoic acid response elements. The most sensitive exposure period, in both humans and animals, coincides with development and differentiation of the facial neural crest and formation of the branchial apparatus (pharyngeal arches), structures from which most retinoidassociated birth defects are derived. The evidence related to vitamin $A$ in humans remains circumstantial, but there is a strong suggestion that retinoid teratogenicity may be the outcome of an exaggerated normal response, resulting from abnormal, inappropriate or prolonged transcriptional activation of critical genes in target embryonic cells.

Although there is some disagreement in the literature, much of the evidence so far indicates that the teratogenic effects of retinol and other naturally occurring retinoid compounds are due to their conversion to all-trans-retinoic acid. Interspecies differences make comparisons of teratogenic potency and extrapolation for human risk assessment difficult.

\section{Dose response characterisation}

Symptoms of chronic toxicity in humans, such as thickening and dryness of the skin, cracking of lips, conjunctivitis, erythmatous eruption, alopecia, reduced bone mineral density, bone joint pain, chronic headache, intracranial hypertension and hepatoxicity, are generally associated with supplemental doses of $7500 \mu \mathrm{gE} / \mathrm{kg}$ bw/day. However, decreases in bone density and increases in fracture risk have been reported with chronic exposure to lower doses of 1500 to $2000 \mu \mathrm{gE} / \mathrm{kg}$ bw/day.

In humans, teratogenic effects have been reported at 3000-9000 $\mu \mathrm{g} R E /$ day (equivalent to 50-150 $\mu \mathrm{g} \mathrm{RE} / \mathrm{kg}$ bw/day). The lowest teratogenic doses reported in rat, monkey and rabbit are 35,000, 6000 and $2500 \mu \mathrm{RE} / \mathrm{kg} /$ day, respectively.

\section{Vulnerable groups}

Retinol may represent a teratogenic risk, particularly within the first trimester of pregnancy. Other groups potentially vulnerable to vitamin A toxicity include the young; older people; those suffering from osteoporosis, chronic renal failure, diabetes mellitus or under-nutrition; haemodialysis patients and individuals with compromised liver function. An apparent vitamin A intolerance has been observed in some children (Carpenter et al., 1987). It has been suggested that there may be a genetic component to these cases. However, the basis of this has not been defined.

\section{Genetic variations}

No genetic variations that increase the likelihood of vitamin A toxicity have been identified. 


\title{
Studies of particular importance in the risk assessment
}

\author{
(For full review see http://www.food.gov.uk/science/ouradvisors/vitandmin/evmpapers or the \\ enclosed CD)
}

\section{Human studies - developmental toxicity}

Retinoids are recognised animal teratogens, and isotretinoin (13,cis-retinoic acid) is a known human teratogen; therefore, developmental toxicity is critical to the risk assessment of vitamin $A$.

\section{Martinez-Frias and Salvador, 1990}

This paper reported the results of a retrospective epidemiological study of prenatal exposure to high doses of vitamin A, using data from a Spanish hospital-based, case control registry (Spanish Collaborative study of Congenital Malformations, a surveillance programme which monitored $>10^{6}$ births). Newborns from 58 hospitals were examined for malformations during the first 3 days of life, and their mothers asked an open-ended question about drug exposures during pregnancy. The mothers were not directly asked about vitamin use. The investigators studied 12,625 cases and 12,525 controls, becoming 11,293 and 11,193, respectively, after eliminations for chromosomal malformations. They considered only women who had used $3000 \mu \mathrm{g}$ RE/day or more of retinyl palmitate, either alone or as a multivitamin. No information on dietary intake was provided. The number of exposures was very low (16 cases, 14 controls). There was no overall association between vitamin A exposure total supplementary vitamin A and birth defects (odds ratio (OR) $=1.1[95 \% \mathrm{Cl} 0.5,2.5] \mathrm{p}=0.4$ ). However, for retinyl palmitate exposure alone, (10, cases, 1 control), the odds ratio was 9.9 [95\% Cl 1.4, 430.1] $\mathrm{p}=0.006)$. The OR for exposure to vitamin $A$ in multivitamin complexes ( 6 cases, 13 controls) was not significantly different to 1 (OR= 0.5 [95\% Cl 0.2, 1.3] $p=0.08)$. The OR for exposures of $<12,000 \mu \mathrm{g} R /$ day was $0.5[95 \% \mathrm{Cl} 0.1,1.6] \mathrm{p}=0.15(5$ cases, 10 controls) and for exposures to $>12,000 \mu \mathrm{g}$ RE/day, OR=2.7 [95\% Cl, 0.8, 11.7] p=0.06 (11 cases, 4 controls). The authors concluded that their results suggested that a teratogenic effect might exist at high levels of exposure. However, their dose-response data are also consistent with no effect. Furthermore, the number of subjects exposed was very small and some exposures may have occurred too late on in pregnancy to be relevant to the birth defects found.

\section{Werler et al., 1990}

This paper reports a retrospective case control study from the Slone Epidemiology Unit Birth Defects Study, a multi-hospital surveillance programme. The aim of the study was to evaluate the relationship between vitamin A supplementation during pregnancy and malformations of structures derived, at least in part, from the cranial neural crest.

Birth defects were identified from hospital records. Women were interviewed within 6 months of delivery and asked whether they had taken any vitamins, iron or folic acid during the 6 months prior to or at any time during pregnancy. No data on vitamin A dose, number of supplements or diet were collected. Cases were 2,658 infants with primarily craniofacial and cardiac malformations. Controls were 2,609 infants with other malformations. Mothers of 6 controls used vitamin A supplements in each of the of the first 3 lunar months of pregnancy compared to mothers of 14, 14 and 10 cases in lunar months 1, 2 and 3, respectively. Relative risk estimates were $2.5[95 \% \mathrm{Cl} 1.0,6.2]$ for lunar month 1, $2.3[95 \% \mathrm{Cl} 0.9$, 5.8] for lunar month 2 and 1.6 [ $95 \% \mathrm{Cl} 0.6,4.5]$ for lunar month 3. No statistically significant association between vitamin A exposure and birth defects was made. However, the numbers of cases and controls exposed to vitamin A supplements were small. The authors also examined specific common defects 
and found no statistically significant positive association with vitamin A supplementation $(95 \% \mathrm{Cls}$ included 1). The authors also compared the risk to those taking multi-vitamins with those taking single vitamin A supplements. The risk was higher but did not achieve statistical significance.

\section{Rothman et al., 1995}

This paper reports a prospective study from an open prenatal screening programme at Boston University, in which 22,748 pregnant women were identified during prenatal screening. Nurse interviewers obtained information on diet, medication and illness during the first trimester and on family medical history. Information on pregnancy outcomes was obtained from obstetricians or from the mothers themselves. Three hundred and thirty-nine babies were born with birth defects and of these, 121 were identified as having defects originating in the cranial neural crest. The prevalence ratio of babies with defects born to women who had consumed $>4500 \mu \mathrm{g}$ RE/day pre-formed vitamin A from food and supplements compared to those born to mothers who consumed $\leqslant 1500 \mu \mathrm{g} R /$ day was 3.5 [95 \% Cl 1.7, 7.3]. For vitamin A from supplements alone, the prevalence ratio among babies born to women who consumed $\geqslant 3,000 \mu \mathrm{g} R /$ day compared to those born to mothers who consumed $\leqslant 1500$ $\mu \mathrm{g}$ RE/day was 4.8 [95\% Cl 2.2,10.5]. Using a smoothed regression curve, the authors suggested a threshold of near $3000 \mu \mathrm{g}$ R/day of supplemental vitamin A.

There has been some criticism of the design of this study and the analysis of the data. This study had no geographical or other population base and no denominator population, which may have introduced bias to the results. Furthermore, physicians identified malformations in only $76.5 \%$ of cases. In the remaining cases, the mother supplied information relating to incidence of malformation, so that there is uncertainty that all malformations were correctly classified.

\section{Khoury et al., 1996}

This is a communication (letter), in response to the publication of the Rothman study, from investigators reporting re-examination of data from a large population-based case-control study of major birth defects, conducted by the Centres for Disease Control. Using the same classification of defect as in the Rothman et al. study, the authors found no overall increased risk of birth defects and no increased risk of cranial neural crest defects among users of vitamin $A$ and/or multi-vitamin supplements (OR $=0.6$ [95\% $\mathrm{Cl} 0.28,1.29]$ and 0.69 [95\% $\mathrm{Cl} 0.24,1.91]$, respectively). However, no data were collected on vitamin A dosage although the authors noted that most multi-vitamins and supplements during the study period were expected to contain $<2400 \mu \mathrm{g}$ RE pre-formed vitamin A (which is less than the $3000 \mu \mathrm{g}$ $\mathrm{RE} /$ day suggested as a threshold by the Rothman study).

Shaw et al., 1996

This is a communication (letter) also in response to the publication of the Rothman study. The authors re-examined geographical population-based surveillance data from previously published studies of oral cleft and conotruncal defects of the heart (both structures associated with the cranial neural crest). The authors assumed that most preparations contained pre-formed vitamin A rather than $B$-carotene, that single supplements would have contained at least $3000 \mu \mathrm{g}$ RE and that combination exposures of single and multi-vitamin supplements would exceed $3000 \mu \mathrm{g}$ RE. There was no increased risk of either birth defect associated with the use of vitamin A supplements (OR $=0.55[95 \% \mathrm{Cl} 0.21,1.5]$ and $\mathrm{OR}=0.0[95 \%$ $\mathrm{Cl} 0.0,2.2]$, oral cleft and conotruncal heart defect studies, respectively). These data were limited in that interviews with the mothers were conducted an average of 3.5 years after delivery and lacked specific information regarding vitamin A dosage. 
Mills et al., 1997

This paper describes a geographically based case-control study (encompassing all pregnancies in California and Illinois between 1985-87) to examine whether moderate doses of vitamin A are associated with teratogenicity. The study group included women whose pregnancies resulted in offspring with neural tube defects ( $n=548$ ), offspring with major malformations other than neural tube defects $(n=387)$ and normal controls $(n=573)$. Study participants gave telephone interviews to trained interviewers between 1 and 5 months after the detection of the congenital anomaly, in order to estimate periconceptional vitamin A exposures from supplements, fortified cereals, meat and offal consumption. The proportion of women consuming 2400-7500 $\mu \mathrm{g}$ RE was no greater in either of the malformation groups than in the control group. Women exposed to $>2400 \mu \mathrm{gRE} /$ day and $>3000 \mu \mathrm{g} \mathrm{RE} /$ day had odds ratios for major malformations of 0.79 [95\% $\mathrm{Cl} 0.4,1.53$ ] and 0.73 [95\% $\mathrm{Cl} 0.27,1,96]$, respectively when compared to women consuming $<1500 \mu \mathrm{g}$ RE/day (the result for neural tube defects was similar). Consequently, no association was made between periconceptual exposure to vitamin $A$ at doses $>3000 \mu \mathrm{g}$ RE/day. However, unlike the study by Rothman et al., dietary recall was many months after the critical period of pregnancy.

\section{Mastroiacovo et al., 1999}

This paper describes a multi-centre prospective controlled study to evaluate whether foetuses exposed during organogenesis to high doses of vitamin A have a higher risk of major malformations than the general population. Thirteen European Teratology Information Services (all part of ENTIS - European Network Teratology Service) counselled women during pregnancy. Data were collected on 423 pregnancies exposed to a maternal daily dose of at least $3000 \mu \mathrm{g}$ supplemental RE during the first nine completed weeks of gestation. 394 women were followed up by telephone interview until the first few weeks after expected delivery date using standardised procedures. Information about malformations was obtained from mothers or doctors. No information on dietary intake was obtained. The occurrence of major structural malformations, excluding chromosomal and genetic diseases, was evaluated in 311 infants exposed to a median daily dose of 15,000 (range 3000-90,000) $\mu \mathrm{g}$ RE/day. Only three infants with major malformations were reported and no congenital malformations were reported among 120 infants exposed to $>15,000 \mu \mathrm{gE} /$ day. The birth prevalence ratios of major malformations compared to two internal control groups (i) exposed to high levels of vitamin A later on in pregnancy and (ii) nonteratogenic agent exposures were 0.28 [95\% $\mathrm{Cl} 0.06-1.23]$ and 0.50 [95\% $\mathrm{Cl} 0.14,1.76]$, respectively. Consequently, this study provided no evidence that there was an increased risk of major malformations associated with exposure to high doses of vitamin A during organogenesis. However, the sample size in this study was not large enough to enable the detection of differences in risk of less than 2.76 .

\section{Human studies - general toxicity}

Wald et al., 1985

In a randomised double-blind study, 376 people were studied and allocated to 1 of 7 dose regimens: 0 , $3000,4054,6757,8108,9460$ or $10,810 \mu \mathrm{g}$ RE/day, for 6 months. The study was designed to investigate the effect of supplementation on serum retinol levels. The only adverse effects noted related to skin and mucous membranes with $6 \%$ of the subjects receiving $4054 \mu \mathrm{g}$ RE or more reporting skin dryness and itching at their second clinic visit compared to $3 \%$ of subjects receiving 0 or $3000 \mu \mathrm{g} R /$ day. 
Hathcock et al., 1990

This paper is a review of vitamin A-related chronic and sub-chronic toxicity data in humans. Clinical case studies reviewed supported the view that exposure to doses $\geqslant 30,000 \mu \mathrm{g} R /$ day for short periods (days/few weeks) or exposure to $7500-15,000 \mu \mathrm{g}$ RE/day for several months or more can produce multiple adverse effects. Most evidence for toxicity at the lower end of this range was from reports of individuals who had concomitant hepatic damage from other contributing factors. Furthermore, information on dietary intake was not always available. The authors concluded that the data available were not sufficient to provide a specific minimum threshold for adverse effects but that $7500 \mu \mathrm{g} R /$ day was nutritionally excessive and carried some risk of toxicity. Similarly, data did not permit identification of a safe upper limit for the intake range. It was noted that an intake of $3000 \mu \mathrm{g} R \mathrm{E} /$ day was more than adequate for good nutrition but was low enough to avoid toxicity in most people. However the effects of vitamin A between $3000 \mu \mathrm{g} R /$ day and $7500 \mu \mathrm{g}$ RE/day could not be predicted.

\section{Human studies - bone toxicity 20}

Freudenheim et al., 1986

In a 4 year clinical trial, the effect of usual intakes of energy and 14 nutrients on single-photon absorptiometric measurement of mineral content in arm bones was assessed in 99 women aged 35-60 given either a calcium supplement or placebo. In the post-menopausal women of the treatment group, there was an inverse correlation between vitamin A intake and the rate of change in ulna bone mineral content. In a single patient receiving a high supplemental dose (average intake $4392 \mu \mathrm{g} R \mathrm{R} /$ day) bone loss was very rapid with no other reason apparent.

Sowers and Wallace, 1990

Vitamin A intake, serum retinol concentrations, radial bone mass and fracture history were evaluated in 246 postmenopausal women. More than $36 \%$ of the population used a vitamin A supplement, with $8 \%$ using a supplement containing $>2000 \mu \mathrm{g}$ RE/day. No relationship was observed between radial bone mass and fracture history and vitamin A intake or serum retinol. No statistically significant relationship was observed between serum retinol and bone mass after adjustment for factors associated with bone mass such as age, when the population was stratified by supplement use. When serum retinol values were divided into tertiles, no relationship was observed with bone mass when the comparison included adjustment for age, muscle area and use of thiazide anti-hypertensives. However, it has been noted (Binkley and Krueger, 2000) that this study had inadequate power to test an association between bone mass and vitamin A intakes $>2000 \mu \mathrm{g}$ RE/day (only $8 \%$ of the study population had intakes exceeding this level); $36 \%$ of the population were aged $<60$ years of age and were therefore likely to be heterogenous with regards to oestrogen depletion bone loss; and the site where bone mass was measured, i.e. central radius, is considered less responsive to change.

\footnotetext{
20 A cohort study of serum retinol levels and fracture risk in 266 men followed up over 30 years was recently published by K. Michaëlsson et al. (N. Engl. J. Med. 2003; 348: 287-294). This study was published after the cut off date of 31 December 2002 for data to be considered by the EVM and it has therefore not been used for risk assessment. This prospective cohort study showed a statistically significant association between serum retinol levels and risk of fracture. The relative risk for all fractures in men in the highest quintile of serum retinol compared to middle quintile was 1.64 (95\% Cl, $1.12-2.41)$.
} 


\section{Houtkooper et al., 1995}

The relationships between total energy intake, nutrient intake, body composition and exercise group status with rates of change in bone mineral density were measured in 66 pre-menopausal women taking calcium supplements. Nutrients were not significant variables in regression models predicting bone mineral density slopes at any femur site, but retinol intake was associated with decreased bone mineral density.

\section{Melhus et al., 1998}

This paper reported two studies, a randomly selected cross-sectional study, involving 175 females (28-74 years) and a nested case-control study, involving 247 women (40-60 years), who had first hip fracture 2-64 months after enrolment, and 873 age-matched controls (selected from a mammography study cohort). Retinol intake was estimated from dietary records and a food-frequency questionnaire. There was no reported use of vitamin A supplements.

In multivariate analysis, intake of pre-formed retinol was negatively associated with bone mineral density. For intake greater than 1,500 $\mu \mathrm{g} R E /$ day, compared with less than $500 \mu \mathrm{g} /$ day, bone mineral density was reduced by $10 \%$ at the femoral neck ( $p=0.05), 14 \%$ at the lumber spine $(p=0.001)$ and $6 \%$ for the total body $(p=0.009)$. The risk for hip fracture was apparently doubled (odds ratio 2.1 [ $95 \% \mathrm{Cl} 1.1-$ 4.0]). For every 1000 ( $\mathrm{g}$ increase in daily intake, risk of hip fracture increased by $68 \%$ [ $95 \% \mathrm{Cl} 18-140 \%$, $p=0.006]$. Smoking was found to be a confounding factor.

The authors suggested that their study was limited by the possibility of information bias resulting from questioning case-patients after hip fracture had occurred, as was done for some covariates such as physical activity. In addition, data on thyroid hormone therapy and family history of osteoporosis were not available. Furthermore, the authors could not rule out confounding influences of an unidentified dietary factor but suggested that the possibility of a high degree of random error in the assessment of retinol intake might lead to an underestimation of the true risk of hip-fracture associated with high levels.

The rationale for this study was based on the findings of the MEDOS study group (Johnell et al., 1992) that hip fracture rates varied across Europe, being 11 and 7 fold higher for women and men respectively in Northern Europe than in Southern Europe, particularly in Sweden and Norway. The difference in European rates was sufficiently marked that fracture rates were higher in Swedish men than in Swiss or English women. The authors further noted that the difference in incidence was higher between countries than between sexes suggesting that an important genetic or environmental factor was involved. Known risk factors were not thought to explain the finding. Melton (1995) also reported that hip fracture rates were higher in Scandinavia than in comparable populations in North America. When dietary patterns in Europe were compared in different European towns, retinol intakes were found to be 6 fold higher in Scandinavia, compared to Southern Europe (Cruz et al., 1991).

\section{Ballew et al., 2001}

The association between fasting serum levels of retinyl esters and bone mineral density (BMD) was studied in 5790 non-pregnant participants aged 20 years or older. Data were also collected on age, body mass index, smoking, alcohol consumption, use of dietary supplements, diabetes, physical activity and, in women, use of oral contraceptives or oestrogen replacement therapy, menopausal status and parity. These covariates were controlled for using multiple linear regression. The study showed no significant association between fasting serum retinyl esters and BMD, as assessed at the femoral neck, trochanter, intertrochanter and total hip. 
Feskanich et al., 2002

As part of the Nurses' Health Study, the relationship between high vitamin A intake from food and supplements and hip fracture was assessed. The study included 72,337 post-menopausal women aged 34-77 and the period 1980 to 1998 was considered in the analysis. During this period, 603 hip fractures occurred resulting from low or moderate trauma. After controlling for confounding factors, women in the highest quintile of vitamin A intake ( $\geqslant 3000 \mu \mathrm{g} R /$ day) had a significantly elevated relative risk (RR) for hip fractures (RR, 1.48; $95 \% \mathrm{Cl} 1.05$ to 2.07; P for trend 0.03) compared to women in the lowest quintile of intake ( $<1250 \mu \mathrm{g} R$ R/day). The increased risk was primarily attributable to retinol (RR, 1.89; $95 \% \mathrm{Cl}, 1.33-2.68)$. The association of retinol with hip fracture was reduced in women taking postmenopausal oestrogens. ß-Carotene did not contribute to fracture risk. Women currently taking a specific vitamin A supplement had a non-significant $40 \%$ increased risk of hip fracture (RR, 1.4; $95 \%$ $\mathrm{Cl}$ 0.99-1.99) compared to those not taking the supplement. Among women not taking supplements, retinol from food was significantly associated with fracture risk (RR, 1.69, 95\% Cl 1.05-2.74).

Overall the authors concluded that long-term intake of diet high in retinol may promote the development of osteoporotic hip fractures in women and that the amount of retinol in supplements and fortified foods may need to be reassessed. The authors noted that the study cohort was largely comprised of white women and that the findings were not necessarily applicable to other ethnic groups.

Promislow et al., 2002

The association between BMD and bone loss, and total and supplemental retinol intake was investigated in 570 women and 388 men, aged 55 to 92 at completion of the study. Dietary intake of vitamin $A$ was assessed by dietary questionnaire over a four year period. This ended four years before the start of a 4-year period in which annual measurements of BMD and bone loss were made. Retinol intake was associated with decreased BMD and increased bone loss at total intakes above $840 \mu \mathrm{g} /$ day, even after adjustment for age, body mass index, weight change, calcium intake, years menopausal (women), use of steroids, cigarettes, alcohol, thiazides, thyroid hormones and supplemental retinol. Supplemental retinol use was also associated with decreased BMD and increased bone loss.

\section{Animal data}

Developmental toxicity

Vitamin A has been shown to be teratogenic in animals. The data are summarised below:

Hendrickx et al., 1997a,b; Wiegand et al., 1998 and Miller et al., 1998

Hendrickx et al. (1997a,b), Wiegand et al. (1998) and Miller et al. (1998), reported a teratogenicity study in which dose-related increased rates of abortions and malformations were observed in Cynomolgus monkeys administered oral doses, during early pregnancy (gestation days 16-27), of 0, 2250, 6000, 12,000 and $24,000 \mu \mathrm{gE} / \mathrm{kg}$ bw/day retinyl palmitate. Incidences of malformation were $1 / 21$ and $5 / 11$ at doses of 6000 and $24,000 \mu \mathrm{RE} / \mathrm{kg}$ bw/day, respectively (intermediate dose not reported). The higher dose affected structures derived from the cranial neural crest. These malformations were similar to those observed with isotretinoin (13-cis-retinoic acid) in both monkeys and humans. The spectrum of defects differed slightly to that observed in isotretinoin syndrome in that there was a higher frequency of abnormality of craniofacial structures, thymus and heart defects were less severe and there were no brain malformations. Maternal toxicity was observed (nature not specified). 
Part 2 Fat Soluble Vitamins

\section{Bone toxicity}

\section{Leelaprute et al., 1973}

Gross bone lesions characterised by resorption of parts of the pelvis, fibulae, and scapulae with bone thinning were observed in growing female rats (initial average weight 147g) treated with 7500-22,500 $\mu \mathrm{g}$ $\mathrm{RE} /$ day retinol as palmitate or retinol (vitamin A alcohol) for 17 days. Soft tissue calcification also occurred. The animals were given the doses either orally or by intraperitoneal injection. Intraperitoneal administration was associated with greater retinol toxicity, though not with vitamin A palmitate.

Frankel et al., 1986

A single oral dose of $82,000 \mu \mathrm{RE} / \mathrm{kg}$ bw given to adult rats, had no effect on biologically active parathyroid hormone (bioactive PTH) concentrations. Secretions of bioactive PTH were not altered by incubation of rat thyroparathyroid complexes with retinol in vitro. In 3-week-old rats given 15,000 $\mu \mathrm{g}$ RE, 3 times a week for 6 weeks, osteoclast numbers were higher and osteoid lower than in the controls. Serum bioactive PTH was not detectable and serum 25-hydroxyvitamin D was significantly lower than in controls. At $7500 \mu \mathrm{g}$ RE, 3 times a week for 3 weeks, serum bioactive PTH was suppressed to undetectable levels but there was no effect on serum 25-hydroxyvitamin D. Serum calcium and 25-hydroxyvitamin D levels were lower in vitamin D intoxicated rats which were also given $7500 \mu \mathrm{g} R \mathrm{E}$, 3 times a week.

The authors considered that the skeletal changes caused by high levels of vitamin A were independent of the effects on PTH but could be caused by the changes in vitamin D metabolites. However, these pathological changes could be modified by secondary changes in calcium metabolism and in the metabolism of calcium-regulating hormones.

Hough et al., 1988

Young rats (initial weight 100g) were treated with 3000 or $7500 \mu \mathrm{g}$ RE/day retinyl palmitate for 21 days by stomach tube. Tibial histomorphometry revealed increased bone resorption (increased osteoclast size and number) and reduced bone formation. There was also a paucity of trabecular surfaces covered with osteoid. Spontaneous limb fractures and increased skeletal turnover (as measured by serum alkaline phosphatase and urinary hydroxyproline excretion) were also observed in the high dose group. Serum calcium and magnesium levels were unremarkable but serum phosphorus levels were significantly elevated in the control animals. Circulating levels of the potent bone resorbers, PTH, 1,25-dihydroxyvitamin $D$ and 25-hydroxyvitamin $D$ were comparable in the treated animals and the controls suggesting that vitamin A was having a direct effect on bone. 


\section{Exposure assessment}

Total exposure/intake:

Food

Median: $520 \mu \mathrm{g}$ RE/day ${ }^{21}$ (from 1986/87 NDNS)

97.5th percentile: $6050 \mu \mathrm{g}$ RE/day

Supplements

Up to $2400 \mu \mathrm{g}$ RE/day (Annex 4)

Estimated maximum intake: $6050+2400=8450 \mu \mathrm{RE} /$ day

High intake groups include people who consume liver and liver products regularly.

\section{Risk assessment}

Acute vitamin A toxicity in humans is rare, but is more likely to occur following ingestion of high dose supplements, rather than following high intakes of vitamin A from food. Vitamin A accumulates in the body and, therefore, individuals who have regular high daily intakes of vitamin A might suffer adverse effects from chronic hypervitaminosis A. Although most manifestations of chronic vitamin A toxicity are reversible on cessation of dose, permanent damage to liver, bone and vision, and chronic muscular and skeletal pain may occur in some cases.

Epidemiological studies have indicated that exposure to high levels of vitamin A during pregnancy might increase the risk of birth defects. The available data do not allow identification of a threshold dose, although one study has suggested that effects may occur at modest intakes. Vitamin A has also been shown to be teratogenic in animals.

Recent epidemiological data have indicated that post-menopausal women with long-term high intakes of vitamin A have an increased risk of hip-bone fracture. Other supporting epidemiological data have indicated that this effect may occur in men as well as women. These findings are supported by animal data, which have indicated that retinol has a direct effect on bone, possibly via an interaction with vitamin $\mathrm{D}$, and an effect on parathyroid hormone and therefore calcium metabolism.

\section{ESTABLISHMENT OF GUIDANCE LEVEL}

It is not possible to establish a Safe Upper Level for vitamin A. There are two threads of evidence regarding potential adverse effects of vitamin $A$, one on teratogenicity and one on the risk of bone fracture, which suggest different levels of intake at which adverse effects may occur. Both of these ranges appear to overlap with dietary intakes of vitamin $\mathrm{A}$.

${ }^{21}$ The median intake, rather than the mean, is provided because the mean appears artificially high. It is skewed by the individuals who have a very high vitamin A intake from liver and its products. 
Part 2 Fat Soluble Vitamins

A number of epidemiological studies have suggested that high doses of vitamin A may be teratogenic, i.e. that they could cause malformations in the unborn child. The precise threshold for this effect is uncertain. The study by Rothman et al. reports that supplemental doses of $>3000 \mu \mathrm{g} R /$ day, in addition to the diet, may be teratogenic; this is the lowest dose level associated with such an effect. It was also noted that a small excess of cranial defects was found in the offspring of women consuming $5000 \mu \mathrm{g}$ RE/day from food and supplements, compared to those taking $1660 \mu \mathrm{g}$ RE/day. Other studies indicate that the threshold may be higher. However, given the severity of the effect, it is prudent to take $3000 \mu \mathrm{g}$ RE/day as the threshold for teratogenicity. The Group note and endorse the current advice that women who are pregnant or who wish to become pregnant should not take dietary supplements containing vitamin A except on medical advice.

In studies of long-term dietary intake, vitamin A has been associated with decreased bone density and increased risk of hip fracture. This finding is supported by investigations in laboratory animals in which vitamin A has been reported to affect calcium metabolism as well as to have a direct effect on bone. Other supportive epidemiological data suggest that the effect may also occur in men since fracture risk is increased in both males and females in Scandinavian countries, where retinol intake is also higher than in Southern Europe.

The risk of hip fracture is a continuous graded response associated with exposure levels that include average dietary intakes. It is not possible to identify an intake that is without some degree of risk. However, the available data indicate that total intakes greater than $1500 \mu \mathrm{g} R /$ day may be inappropriate. This corresponds to $25 \mu \mathrm{RE} / \mathrm{kg}$ bw/day in a $60 \mathrm{~kg}$ adult.

Data on retinol intakes from food and supplements suggest that high level consumers of liver and liver products and/or supplements may exceed intakes at which adverse effects have been reported in the literature. It should also be noted that dietary supplements may contain $20-100 \%$ more vitamin A than is stated on the label, due to the practice of using 'overages' within the food supplements industry to ensure that the product contains no less than the stated content of the vitamin throughout its shelf life. This may be particularly important given that the effect on fracture risk appears to be a graded response, with the risk of fracture increasing with increased intake.

\section{References}

Binkley, N., Krueger, D. (2000). Hypervitaminosis A and bone. Nutrition Reviews 58, 138-144.

Carpenter, T.O., Pettifor, J.M., Russell, R.M., Pitha, J., Mobarhan, S., Ossip, M.S., Wainer, S., Anast, C.S. (1987). Severe hypervitaminosis A in siblings: evidence of variable tolerance to retinol intake. Journal of Pediatrics 111, 507-512.

COMA (1991). Dietary Reference Values for Food Energy and Nutrients for the United Kingdom. Report of the Panel on Dietary Reference Values, Committee on Medical Aspects of Food and Nutrition Policy. HMSO, London.

Cruz, J.A,, Cruz, A.A., Moreiras-Varela, O., van Staveren, W.A., Trichopoulou, A., Roszkowski, W. (1991) Intake of vitamins and minerals. European Journal of Clinical Nutrition 45 (supp 3),

$121-138$. 
Feskanich, D., Singh, V., Willett, W.C., Colditz, G,A. (2002). Vitamin A intake and hip fractures among postmenopausal women. Journal of the American Medical Association 287, 47-54.

Frankel, T.L., Seshadri, M.S., McDowall, D.B., Cornish, C.J. (1986). Hypervitaminosis A and calciumregulating hormones in the rat. Journal of Nutrition 116, 578-87.

Freudenheim, J.L., Johnson, N.E., Smith, E.L. (1986). Relationship between usual nutrient intake and bonemineral content of women 35-65 years of age: longitudinal and cross-sectional analysis. American Journal of Clinical Nutrition 44, 863-876.

Hathcock, J.N., Hattan, D.G., Jenkins, M.Y., McDonald, J.T., Sundaresan, P.R., Wilkening, V.L. (1990). Evaluation of vitamin A toxicity. American Journal of Clinical Nutrition 52, 183-202.

Hendrickx, A.G., Hummler, H. (1997a). Teratogenicity of hypervitaminosis A in the monkey. The Federation of American Societies for Experimental Biology Journal 11, A412.

Hendrickx, A.G., Hummler, H., Oneda, S. (1997b). Vitamin A teratogenicity and risk assessment in the Cynolmolgus monkey. Teratology 55, 68.

Hough, S., Avioli, L.V., Muir, H. Gelderblom, D., Jenkins, G., Kurasi, H., Slatopolsky, E., Bergfeld, M.A., Teitelbaum, S.L. (1988). Effects of hypervitaminosis A on the bone and mineral metabolism of the rat. Endocrinology 122, 2933-9.

Houtkooper, L.B., Ritenbaugh, C., Aickin, M., Lohman, T.G., Going, S.B., Weber, J.L., et al (1995). Nutrients, body composition and exercise are related to change in bone mineral density in pre-menopausal women. Journal of Nutrition 125, 1229-1237.

Johnell, O., Gullberg, B., Allender, E., Kanis, J. A. (1992) The apparent incidence of hip fractures in Europe: A study of national register sources. Osteoporosis International 2, 298-302.

Khoury, M.J., Moore, C.A., Mulinare, J. (1996). Vitamin A and birth defects. The Lancet 347, 322.

Leelaprute, V., Boonpucknavig, V., Bhamarapravati, N., Weerapradist, W. (1973). Hypervitaminosis A in rats. Varying responses due to different forms, doses, and routes of administration. Archives of Pathology 96, 5-9.

Martinez-Frias, M.L., Salvador, J. (1990). Epidemiological aspects of prenatal exposure to high doses of vitamin A in Spain. European Journal of Epidemiology 6, 116-123.

Mastroiacovo, P., Mazzone, T., Addis, A., Elephant, E., Carlier, P., Vial, T., Garbis, H., Robert, E., Bonati, M., Ornoy, A., Finardi, A., Schaffer, C., Caramelli, L., Rodriguez-Pinilla, E., Clementi, M. (1999). High vitamin A intake in early pregnancy and major malformations: a multicentre prospective controlled study. Teratology 59, 7-11.

Melhus, H., Michaelsson, K., Kindmark, A., Bergström, R., Holmberg, L., Mallmin, H., Wolk, A., Ljunghall, S. (1998). Excessive dietary intake of vitamin A is associated with reduced bone mineral density and increased risk for hip fracture. Annals of Internal Medicine 129, 770-778. 
Part 2 Fat Soluble Vitamins

Melton, L.J. (1995). Epidemiology of fractures. In : Osteoporosis: Etiology, Diagnosis, and management Second edition (eds Riggs and Melton) Lippincot-Raven, Philadelphia.

Miller, R.K., Hrndrickx, A.G., Mills, J.L., Hummler, H., Wiegand, U.-W. (1998). Periconceptual vitamin A use: how much is teratogenic? Reproductive Toxicology 12, 75-88.

Mills, J.L., Simpson, J.L., Cunningham, G.C., Conley, M.R., Rhoads, G.G.. (1997). Vitamin A and birth defects. American Journal of Obstetrics and Gynecology 177, 31-36.

Rothman, K.J., Moore, L.L., Singer, M.R., Nguyen, U.-S.D.T., Mannino, S, Milunsky, A. (1995). Teratogenicity of high vitamin A intake. New England Journal of Medicine 333, 1369-1373.

Shaw, G.M., Wasserman, C.R., Block, G., Lammer, E.J. (1996). High maternal vitamin A intake and risk of anomalies of structures with a cranial neural crest contribution. The Lancet 347, 899-900.

Sowers, M.R., Wallace, R.B. (1990). Retinol, supplemental vitamin A and bone status. Journal of Clinical Epidemiology 43, 693-9.

Wald, N.J., Cuckle, H.S., Barlow, R.D., Thompson, P., Nanchahal, K., Blow, R.J., Brown, I., Harling, C.C., McCulloch, W.J., Morgan, J., Reid, A.R. (1985). The effect of vitamin A supplementation on serum retinol and retinol binding protein levels. Cancer letters 29, 203-213.

Werler, M.M., Lammer, E.J., Rosenberg, L., Mitchell, A.A. (1990). Vitamin A supplementation in relation to selected birth defects. Teratology 42, 497-503.

Wiegand U.-W., Hartmann S., Hummler H. (1998). Safety of vitamin A: recent results. International Journal of Vitamin Nutrition Research 68, 411-416.

Wolbach, B. (1947). Vitamin A deficiency and excess in relation to skeletal growth. Journal of Bone Joint Surgery 29, 171-92. 


\section{Risk Assessment}

\section{B-Carotene}

\section{General information}

\section{Chemistry}

B-Carotene $\left(\mathrm{C}_{40} \mathrm{H}_{56}\right)$ is a member of the carotenoid family of isoprenoid compounds, which are characterised by their polyunsaturated nature and antioxidant properties. The compound can exist in different geometrical forms (as cis- or trans- isomers); the majority of naturally-occurring B-carotene, as well as virtually all of the compound prepared by chemical synthesis, is the all-trans isomer.

\section{Natural occurrence}

ß-Carotene is synthesised in plants and microorganisms, but not in higher organisms.

\section{Occurrence in food, food supplements and medicines}

The main food sources of $B$-carotene are yellow and green (leafy) vegetables and yellow fruits. Commercially-available $B$-carotene is either synthetic or derived from palm oil, algae or fungi, and is widely used as a yellow colouring agent (EC160a) in foods and drinks. B-carotene is widely used in vitamin and mineral supplements at levels ranging from $0.4 \mathrm{mg}$ to $20 \mathrm{mg}$ per day, and is given medicinally in doses of up to $6 \mathrm{mg} /$ day for dietary deficiency of vitamin A and up to $300 \mathrm{mg} / \mathrm{day}$ in the reduction of photosensitivity in individuals with erythropoietic protoporphyria (EPP).

\section{Recommended amounts}

B-Carotene per se is not an essential nutrient and there are currently no dietary reference values specifically for $B$-carotene in the UK or USA. However, as B-carotene is a precursor for vitamin A, dietary intakes are traditionally expressed as part of the RNI for vitamin $A$ as retinol equivalents (RE). For adults the RNI for vitamin A is 600 and $700 \mu \mathrm{g}$ RE/day $(0.6$ and $0.7 \mathrm{mg})$ for females and males respectively (COMA, 1991). $6 \mu \mathrm{g}$ ß-carotene $=1 \mu \mathrm{g}$ RE.

\section{Analysis of tissue levels and $B$-carotene status}

B-Carotene status is assessed by measurement of plasma concentration.

\section{Brief overview of non-nutritional beneficial effects}

Observational studies in humans have shown that high intake of $B$-carotene-containing foods in the diet, as well as higher serum $B$-carotene levels, are associated with reduced risk of chronic diseases, such as coronary heart disease and cancer. It has been postulated that this association may be due to the antioxidant properties of the molecule. However, it is unknown whether ß-carotene intake is acting as a marker for other components of fruits and vegetables or another lifestyle factor, rather than having an effect in its own right. 
Part 2 Fat Soluble Vitamins

\section{Function}

B-Carotene is a precursor of vitamin A, and (as recently suggested) of other retinoid-like compounds. Its importance in any individual depends upon the level of pre-formed vitamin A in the diet.

\section{Deficiency}

B-Carotene is not classed as an essential vitamin, but it is a provitamin of vitamin A. Therefore deficiency as a clinical condition per se, is not clearly established.

\section{Interactions}

Interactions between ß-carotene and other carotenoids (such as lycopene, lutein and canthaxanthin) occur during absorption and/or metabolism.

\section{Absorption and bioavailability}

Dietary fat and bile salts facilitate absorption in the upper small intestine, which occurs via incorporation into multilamellar lipid micelles. It has been estimated that, in humans, 10 to $90 \%$ of the total $ß$-carotene consumed in the diet is absorbed, and that absorption decreases as intake increases. Availability from food products is lower than that of a water-dispersed formulation, due to the need for disruption (by pepsin and proteolytic enzymes and by cooking), of the matrix of fibre, polysaccharide and protein. Bioavailability is reduced in very low fat diets.

\section{Distribution and metabolism}

A proportion of absorbed $B$-carotene is converted to retinol within intestinal mucosal cells. Unaltered $ß$-carotene is transported via the lymph to the plasma where it is associated with lipoproteins. Tissue uptake and distribution are not well characterised. In the case of regular high intake, long-term accumulation occurs preferentially in adipose tissues.

Serum levels of $\beta$-carotene have been reported to be low in smokers, in individuals with a high alcohol intake, and in those with HIV infection. Low B-carotene status may be associated with conditions of impaired lipid absorption such as jaundice, liver cirrhosis and cystic fibrosis.

B-Carotene is mainly converted to retinol (vitamin A) in the cytosol of intestinal mucosal cells. Experiments in rats have shown that the process is regulated by the levels of $B$-carotene and of preformed vitamin A. In vitro studies have shown that other B-carotene derivatives may also occur, but their biological activity, and whether they are synthesised in vivo, is unknown.

Carotenoid absorption and metabolism vary considerably between animal species. No single species provides a good model for studying all aspects of the biokinetics and metabolism of $B$-carotene in humans. The rat is particularly unsuitable, due to the high efficiency of conversion to vitamin A, such that significant levels of unaltered $B$-carotene are absorbed only when very high doses are given, for prolonged periods of time. The pre-ruminant calf, the ferret and the Mongolian gerbil are suggested to be more useful models, although it is apparent that there are many differences between carotenoid absorption, distribution and metabolism in these animals and humans. 


\section{Excretion}

Absorbed $ß$-carotene is secreted into the bile and excreted in the faeces. It is also excreted in the sweat.

\section{Toxicity}

\section{Human data}

Until recently, ß-carotene was considered to show no toxicity in humans. Hypercarotenaemia (high plasma $B$-carotene) has not been associated with adverse effects other than reversible yellowing of the skin (hypercarotenodermia). Long-term, oral ß-carotene therapy, in doses up to $300 \mathrm{mg} /$ day has shown no toxic effects in individuals with erythropoietic protoporphyria (EPP). Vitamin A toxicity does not occur, as the metabolic conversion is regulated by vitamin A status. There have been no reports of reproductive toxicity or teratogenicity associated with high $B$-carotene intake, either before or during pregnancy. Two recent, large-scale supplementation trials testing the hypothesis that $B$-carotene supplementation in smokers would reduce the incidence of cancer, have shown an association of highdose $B$-carotene supplementation ( $20-30 \mathrm{mg} /$ day) with increased incidence of lung cancer in smokers and asbestos-exposed individuals. No statistically significant differences in other cancer types were observed in these studies. These are described in more detail below.

\section{Supplementation studies}

Two large-scale studies (Alpha-Tocopherol Beta-Carotene Prevention Study (ATBC) and ß-Carotene and Retinol Efficacy Trial (CARET)) have shown an association of $B$-carotene supplementation $(20 \mathrm{mg} /$ day, alone or in combination with $\alpha$-tocopherol; $30 \mathrm{mg} /$ day in combination with retinyl palmitate respectively) with increased incidence of lung cancer in smokers and individuals with previous high-level exposure to asbestos. However, another large-scale study involving B-carotene supplementation in a well-nourished population, the US Physicians' Health Study, showed no beneficial or adverse effects of ß-carotene supplementation (50 mg every other day), in a trial population which comprised $11 \%$ current smokers. Additionally, the Heart Protection Study, a large scale study of supplementation with $20 \mathrm{mg}$ ß-carotene, $60 \mathrm{mg}$ vitamin $\mathrm{E}$ and $250 \mathrm{mg}$ vitamin $\mathrm{C}$ daily for up to 5 years, showed no difference in cancer incidence compared to placebo controls. The designs of these studies are given below: 
Part 2 Fat Soluble Vitamins

Table. Study design, treatment (type, dose and duration) and predefined end-points in four major intervention trials involving B-carotene (adapted from International Agency for Research on Cancer, 1998)

\begin{tabular}{|c|c|c|c|c|}
\hline & ATBC & CARET $^{2}$ & $\mathrm{PHS}^{3}$ & $\begin{array}{l}\text { Heart Protection } \\
\text { Study }{ }^{4}\end{array}$ \\
\hline Study design & $\begin{array}{l}2 \times 2 \text { factorial: } \\
3 \text { treatment and } \\
1 \text { placebo groups }\end{array}$ & $\begin{array}{l}\text { Randomised, double- } \\
\text { blind, placebo- } \\
\text { controlled: } 1 \text { treatment } \\
\text { and } 1 \text { placebo groups }\end{array}$ & $\begin{array}{l}2 \times 2 \text { factorial: } \\
3 \text { treatment and } \\
1 \text { placebo groups }\end{array}$ & $\begin{array}{l}\text { Randomised, double- } \\
\text { blind, placebo- } \\
\text { controlled trial: } \\
1 \text { treatment group and } \\
1 \text { placebo group }\end{array}$ \\
\hline $\begin{array}{l}\text { Number of subjects } \\
\text { recruited }\end{array}$ & 29,133 & 18,314 & 22,071 & 20,536 \\
\hline Treatment and dose & $\begin{array}{l}\text { Daily: } \\
\text { a-tocopherol } 50 \mathrm{mg} \text { or } \\
\text { ß-carotene } 20 \mathrm{mg} \text { or } \\
\text { a-tocopherol + } \\
\text { ß-carotene }\end{array}$ & $\begin{array}{l}\text { Daily: } \\
\text { Retinol } 25000 \mathrm{IU}+ \\
\text { ß-carotene } 30 \mathrm{mg}\end{array}$ & $\begin{array}{l}\text { On alternate days: } \\
\text { Aspirin } 325 \mathrm{mg} \text { or } \\
\text { ß-carotene } 50 \mathrm{mg} \text { or } \\
\text { aspirin + } ß \text {-carotene }\end{array}$ & $\begin{array}{l}\text { Daily: ß-carotene } \\
20 \mathrm{mg} \text {, vitamin } \mathrm{E} \\
60 \mathrm{mg} \text { and vitamin C } \\
250 \mathrm{mg}\end{array}$ \\
\hline Duration & 6 years (median) & 4 years (mean) & $\begin{array}{l}11-12 \text { years (for } \\
\text { ß-carotene) }\end{array}$ & Up to 5 years \\
\hline Predefined end-points & $\begin{array}{l}\text { Lung cancer and other } \\
\text { major cancers, } \\
\text { incidence }\end{array}$ & $\begin{array}{l}\text { Lung and other } \\
\text { cancers, incidence }\end{array}$ & $\begin{array}{l}\text { Cardiovascular disease } \\
\text { and lung cancer, } \\
\text { incidence }\end{array}$ & $\begin{array}{l}\text { Major coronary } \\
\text { events, subsidiary } \\
\text { assessments of cancer } \\
\text { incidence and other } \\
\text { morbidity }\end{array}$ \\
\hline Results & $\begin{array}{l}\text { Increased lung cancer } \\
\text { in ß-carotene treated } \\
\text { individuals }\end{array}$ & $\begin{array}{l}\text { Increased lung cancer } \\
\text { in } ß \text {-carotene treated } \\
\text { individuals }\end{array}$ & $\begin{array}{l}\text { No treatment related } \\
\text { effect on cancer }\end{array}$ & $\begin{array}{l}\text { No significant effects } \\
\text { on incidence of } \\
\text { vascular disease, } \\
\text { cancer or other major } \\
\text { outcome }\end{array}$ \\
\hline
\end{tabular}

1 ATBC, Alpha-Tocopherol Beta-Carotene Prevention Study (The Alpha Tocopherol Beta Carotene Prevention study group, 1994)

2 CARET, B-carotene and Retinol Efficacy Trial (Omenn et al., 1996b)

3 US Physicians' Health Study (Hennekens et al., 1996)

$4 \mathrm{MRC} / \mathrm{BHF}$ Heart Protection Study of antioxidant vitamin supplementation (Heart Protection Study Collaborative Group, 2002)

Smaller-scale studies, involving varying levels of ß-carotene supplementation, up to $300 \mathrm{mg} /$ day, have shown no evidence of toxicity.

\section{Animal data}

No adverse effects of high-dose ß-carotene supplementation have been observed in standard toxicological studies in experimental animals. These include tests for acute toxicity (doses up to 5000 $\mathrm{mg} / \mathrm{kg}$ bw/day in rats), chronic toxicity/carcinogenicity (doses up to $1000 \mathrm{mg} / \mathrm{kg}$ bw/day for life in rats and mice; up to $250 \mathrm{mg} / \mathrm{kg}$ bw/day for 2 years in beagle dogs) and teratogenicity and reproductive toxicity (doses up to $1000 \mathrm{mg} / \mathrm{kg}$ bw/day for 3 generations, or during days 7 to 16 of gestation, in rats; up to $400 \mathrm{mg} / \mathrm{kg}$ bw/day during days 7 to 19 of gestation in rabbits). 


\section{Carcinogenicity and genotoxicity}

B-Carotene shows no genotoxicity in vitro or at high doses in vivo and was not carcinogenic in experimental rodent studies. However, B-carotene supplementation $(2.4 \mathrm{mg} / \mathrm{kg}$ bw/day, with or without exposure of the animals to cigarette smoke) was associated with the development of squamous cell metaplasia in the lungs of ferrets (Wang et al., 1999).

\section{Vulnerable groups}

Groups vulnerable to $ß$-carotene toxicity include current smokers, individuals with previous high-level exposure to asbestos, those with high alcohol intakes, and/or a history of myocardial infarction.

\section{Genetic variations}

No genetic variations that increase sensitivity to $ß$-carotene toxicity have been identified.

\section{Mechanism of toxicity}

A number of hypotheses have been suggested to account for the association between

B-carotene supplementation and lung tumourigenesis in smokers, including an imbalance of other carotenoids or antioxidant species, a pro-oxidant activity of ß-carotene at high oxygen tensions (in the lungs), induction of P450 enzymes and the production of damaging B-carotene oxidation products by components of cigarette smoke. However, the mechanism for the observed association remains to be established.

\section{Dose-response characterisation}

$20 \mathrm{mg} /$ day $ß$-carotene was associated with increased incidence of lung cancer in human smokers and individuals with previous high-level exposure to asbestos. Similar studies with lower doses have not been carried out.

\section{Studies of particular importance in the risk assessment}

(For full review see http://www.food.gov.uk/science/ouradvisors/vitandmin/evmpapers or the enclosed CD)

\section{Toxicity in humans}

The Alpha-Tocopherol, Beta-Carotene (ATBC) Trial (The Alpha-Tocopherol and Beta-Carotene Cancer Prevention Study Group, 1994; Albanes et al., 1996, Rapola et al., 1997)

This was a randomised, double-blind, placebo-controlled, $2 \times 2$ factorial trial to assess the potential efficacy of daily supplementation with $\alpha$-tocopherol and/or $\beta$-carotene in the prevention of lung cancer. Finnish, male smokers $(n=29,133)$ were given a daily supplement of $50 \mathrm{mg} \alpha$-tocopherol, $20 \mathrm{mg}$ ß-carotene, $50 \mathrm{mg} \alpha$-tocopherol $+20 \mathrm{mg}$ ß-carotene or placebo for a period of 5-8 years. Statistical 
analysis for a median follow-up period of 6.1 years showed no statistically significant change in the primary assessed endpoint, lung cancer incidence, in the $\alpha$-tocopherol-only treatment group, but a significant $18 \%$ increase was noted in men who received $B$-carotene supplementation compared with those who did not. There was no evidence of an interaction between $\alpha$-tocopherol and $\beta$-carotene in their effects on lung cancer. No significant changes were seen in the incidences of cancers other than lung cancer, but there was a significant increase in the risk of fatal coronary heart disease in individuals with a previous myocardial infarction in the $ß$-carotene-treated groups.

\section{The Beta-Carotene and Retinol Efficacy Trial (CARET) (Omenn et al., 1996a,b).}

This was a multicentre, randomised, double-blind, placebo-controlled primary prevention trial of ßcarotene and retinol supplementation in individuals considered to be at high risk of developing lung cancer (14,254 male and female heavy smokers and 4060 males with previous high-level occupational asbestos exposure). The aim of the investigation was to assess the effects of daily supplementation with a combination of $30 \mathrm{mg}$ B-carotene $+25000 \mathrm{IU}$ retinol (as retinyl palmitate), compared with a placebo, upon the primary measured endpoint - the incidence of lung cancer. The trial was terminated approximately 2 years prematurely (after a mean intervention time of 4 years) when a trend towards increased incidence of cancer in participants receiving supplementation with ß-carotene became evident. At this time, statistical analysis showed a significant $28 \%$ increase in the relative risk of lung cancer in the supplementation group compared with the placebo group. No statistically significant differences in other cancer types were observed.

The US Physicians' Health Study (US-PHS) (Hennekens et al., 1996).

This was a randomised, double-blind, placebo-controlled, $2 \times 2$ factorial trial involving 22,071 male physicians (of whom $11 \%$ were current smokers) to assess the effects of aspirin and/or $\beta$-carotene supplementation on the incidence of cardiovascular disease and cancer. Supplementation, on alternate days, of either $325 \mathrm{mg}$ aspirin, $50 \mathrm{mg}$ ß-carotene, $325 \mathrm{mg}$ aspirin $+50 \mathrm{mg}$ ß-carotene, or placebo, was given for 6 years, at which time aspirin supplementation was discontinued whilst the randomised $\beta$-carotene component of the trial continued for another 6 years. After an average 12 years of follow-up, statistical analysis showed no significant effect of $B$-carotene supplementation on overall cancer incidence, the incidences of specific cancer types (including lung cancer), myocardial infarction, stroke, death due to cardiovascular pathology, all important cardiovascular events, or death from all causes. It has been suggested that, as current smokers comprised only $11 \%$ of the total study population the PHS trial had limited capacity to detect adverse effects in this subgroup (in contrast with ATBC where $100 \%$ of the study population were smokers, and CARET, which included only current or recently-quit heavy smokers and individuals with previous high-level asbestos exposure).

\section{Heart Protection Study Collaborative Group, 2002}

20,536 adults with coronary artery disease, other occlusive arterial disease or diabetes were allocated to receive either $20 \mathrm{mg}$ ß-carotene/day, together with $600 \mathrm{mg} /$ day vitamin E and $250 \mathrm{mg} /$ day vitamin C, or placebo for up to 5 years in this randomised, placebo controlled trial. Small but statistically significant increases in total plasma cholesterol, LDL cholesterol and triglycerides were observed in patients on the active treatment compared to controls. However, no significant differences in the incidences of all-caused mortality, or in deaths due to vascular or non-vascular events, were observed. Nor were there any significant differences in the incidence of non-fatal myocardial infarctions or coronary death, non-fatal or fatal strokes, coronary or non-coronary revascularisation, or in the incidence of cancer or of hospitalisation for non-cancer causes. 


\section{Toxicity in animals}

The vast majority of standard toxicological studies in experimental animals have shown no adverse effects of high dose ß-carotene supplementation. One study (Wang et al., 1999) has described an association of $ß$-carotene supplementation with the development of squamous metaplasia in the lungs of ferrets:-

\section{Wang et al., 1999}

This study used a ferret model to assess the effects of cigarette smoke and B-carotene supplementation, alone and in combination, on lung histopathology/biochemistry. Four groups of 6 males were treated with dietary ß-carotene supplementation ( $2.4 \mathrm{mg} / \mathrm{kg}$ bw/day), cigarette smoke exposure, both, or neither, for a period of 6 months. Histopathological analysis revealed that all ß-carotene treated animals showed an increase in cell proliferation and squamous metaplasia in lung tissue, and this was further enhanced in the animals that were also exposed to cigarette smoke. Animals exposed to cigarette smoke alone, did not show these changes. The histopathological endpoint, squamous metaplasia, may not, however, be directly related to carcinogenesis (Lotan, 1999). Furthermore, these findings are not consistent with the results of human studies, in which B-carotene supplementation showed no effect on sputum atypia or metaplasia in smokers or asbestos workers (McLarty et al., 1995; van Poppel et al., 1997).

\section{Exposure assessment}

Total exposure/intake

Food ${ }^{22} \quad$ Mean: $2.3 \mathrm{mg} /$ day in adults

97.5th percentile: $7.0 \mathrm{mg} /$ day (NDNS, 1986/7)

Supplements $\quad$ up to $20 \mathrm{mg} /$ day (Annex 4)

Estimated maximum daily intake: $7.0+20=27 \mathrm{mg}$

No potential high intake groups have been identified.

\section{Risk assessment}

B-carotene is of low toxicity in both animals and man, and prior to the publication of a number of intervention studies was thought to be without adverse effect, other than a yellowing of the skin, which occurred after sustained high intake. However, supplementation of smokers and subjects previously exposed to asbestos has been associated with an increased risk of lung cancer. The mechanism for this effect is unknown but it seems likely that $ß$-carotene has a tumour promoting effect of some type. 
Part 2 Fat Soluble Vitamins

\section{ESTABLISHMENT OF SAFE UPPER LEVEL}

Key study:

Effects observed at:

Uncertainty Factor:

Safe Upper Level for daily consumption over a lifetime:
The Alpha Tocopherol and Beta Carotene Cancer Prevention Study Group (1994).

$20 \mathrm{mg} /$ person/day supplemental ß-carotene.

3 (for LOAEL to NOAEL extrapolation).

$20 / 3=7 \mathrm{mg} /$ day supplemental $ß$-carotene (equivalent to

$0.11 \mathrm{mg} / \mathrm{kg}$ bw/day in a $60 \mathrm{~kg}$ adult)

Epidemiological studies have shown an association between supplementation with ß-carotene and an increase in lung cancers in smokers and in individuals who have been heavily exposed to asbestos. The Safe Upper Level applies only to the general population, i.e. non-smokers and those not exposed to asbestos.

There is no evidence that $ß$-carotene supplementation has any effect on non-smokers. However, until the mechanism for the promotion of lung tumours is established it remains uncertain whether other coexposures could have the same effect as observed in smokers. As a matter of prudence, therefore, the EVM has set a Safe Upper Level for supplementation, based on the ATBC study. This study was chosen for use in deriving a Safe Upper Level, as of the four large-scale studies investigating the effects of $ß$ carotene supplementation on smokers and/or those exposed to high levels of asbestos, this study has shown effects at the lowest level of supplemental intake. The LOAEL from this study was $20 \mathrm{mg} / \mathrm{day}$. Applying an uncertainty factor of 3 , to extrapolate from a LOAEL to a NOAEL, results in a Safe Upper Level for supplementation of $7 \mathrm{mg} /$ day. This is equivalent to $0.11 \mathrm{mg} / \mathrm{kg}$ bw day for a $60 \mathrm{~kg}$ adult. This Safe Upper Level applies to supplements only, as there is no evidence to suggest that current levels of ß-carotene intake from food results in adverse effects.

The dose at which supplemental ß-carotene promotes carcinogenesis in smokers and workers exposed to asbestos is unknown. However, there is no evidence that dietary $ß$-carotene is associated. The EVM recommends that, as a matter of prudence, smokers or those exposed to asbestos should not take $ß$ carotene supplements.

\section{References}

Albanes, D., Heinonen, O.P., Taylor, P.R., Virtamo, J., Edwards, B.K., Rautalahti, M., Hartman, A.M., Palmgren, J., Freedman, L.S., Haapokoski, J., Barrett, M.J., Pietinen, P., Malila, N., Tala, E., Liippo, K., Salomaa, E-R., Tangrea, J.A., Teppo, L., Askin, F.B., Taskinen, E., Erozan, Y., Greenwald, P., Huttunen, J.K. (1996). $\alpha$ Tocopherol and $\beta$-carotene supplements and lung cancer incidence in the alpha-Tocopherol, betaCarotene cancer prevention study: effects of base-line characteristics and study compliance. Journal of the National Cancer Institute 88, 1560-1570.

The Alpha-Tocopherol, Beta Carotene Cancer Prevention Study Group. (1994). The effect of vitamin E and beta carotene on the incidence of lung cancer and other cancers in male smokers. New England Journal of Medicine 330, 1029-1035. 
COMA (1991). Dietary Reference Values for Food Energy and Nutrients for the United Kingdom. Report of the Panel on Dietary Reference Values, Committee on Medical Aspects of Food and Nutrition Policy. HMSO, London.

Heart Protection Study Collaborative Group (2002). MRC/BHF heart protection study of antioxidant vitamin supplementation in 20536 high-risk individuals: a randomised placebo-controlled trial. Lancet 350, 23-33.

Hennekens, C.H., Buring, J.E., Manson, J-A, E., Stampfer, M., Rosner, B., Cook, N.R., Belanger, C., LaMotte, F., Gaziano, J.M., Ridker, P.M., Willett, W., Peto, R. (1996). Lack of effect of long-term supplementation with beta carotene on the incidence of malignant neoplasms and cardiovascular disease. New England Journal of Medicine 334, 1145-1149.

Lotan, R. (1999). Lung cancer promotion by ß-carotene and tobacco smoke: relationship to suppression of retinoic acid receptor- $\beta$ and increased activator protein-1. Journal of the National Cancer Institute 91, 7-8.

McLarty, J.W., Holiday, D.B., Girard, W.M., Yanagihara, R.H., Kummet, T.D., Greenberg, S.D. (1995). $B$-carotene, vitamin A, and lung cancer chemoprevention: results of an intermediate endpoint study. American Journal of Clinical Nutrition 62 (Suppl.6), 1431S-1438S.

Omenn, G.S., Goodman, G.E., Thornquist, M.D., Balmes, J., Cullen, M.R., Glass, A., Keogh, J.P., Meyskens, F.L., Valanis, B., Williams, J.H., Barnhart, S., Cherniack, M.G., Brodkin, C.A., Hammer, S. (1996a). Risk factors for lung cancer and for intervention effects in CARET, the beta-carotene and retinol efficacy trial. Journal of the National Cancer Institute 88, 1550-1558.

Omenn, G.S., Goodman, G.E., Thornquist, M.D., Balmes, J., Cullen, M.R., Glass, A., Keogh, J.P., Meyskens, F.L., Valanis, B., Williams, J.H., Barnhart, S., Hammer, S. (1996b). Effects of a combination of beta-carotene and vitamin A on lung cancer and cardiovascular disease. New England Journal Medicine 334, 1150-1155.

van Poppel, G., van Aspert, A., Heynen, T., Vooys, G.P., Ockhuizen, T. (1997). The effect of beta-carotene on sputum cytology in smokers: a preliminary study. European Journal Cancer Prevention 6, 294-299.

Rapola, J.M., Virtamo, J., Ripatti, S. Huttunen, J.K., Albanes, D., Taylor, P.R., Heinonen, O.P. (1997). Randomized trial of alpha-tocopherol and beta-carotene supplements on incidence of major coronary events in men with previous myocardial infarction. Lancet 349, 1715-1720.

Wang, X.D., Liu, C., Bronson, R.T., Smith, D.E., Krinsky, N.I., Russell, M. (1999). Retinoid signaling and activator protein-1 expression in ferrets given beta-carotene supplements and exposed to tobacco smoke. Journal of the National Cancer Institute 91, 60-66. 


\section{Risk Assessment}

\section{Vitamin D}

\section{General information}

\section{Chemistry}

Vitamin D refers to a group of fat-soluble seco-steroid compounds. Two nutritionally significant compounds are vitamin $D_{2}$ (ergocalciferol) and vitamin $D_{3}$ (cholecalciferol). Vitamin $D_{3}$ is metabolised to the active steroid hormone 1,25-dihydroxyvitamin $D_{3}$ by successive hydroxylations in the liver and kidney. Vitamin $D_{2}$ is metabolised to 1,25-dihydroxyvitamin $D_{2}$ by the same enzyme systems. One milligram of vitamin $D$ is equivalent to 40,000 international units.

\section{Natural occurrence}

Vitamin $D_{3}$ (cholecalciferol) is produced photochemically from 7-dehydrocholesterol in the skin by exposure to sunlight or ultraviolet light. Vitamin $D_{2}$ is formed similarly from ergosterol in plants, fungi and lower life forms.

\section{Occurrence in food, food supplements and medicines}

Vitamin $D$ is found in only a few foodstuffs, with fatty fish and fish oils, liver, milk and eggs being the main natural sources. In most industrialised countries, including the UK, processed milk, some powdered milks, margarine, breakfast cereals, bread and chocolate bars are fortified with vitamin D. Human milk contains low levels of vitamin D, but infant formula is fortified with $0.001-0.0025 \mathrm{mg} / 100 \mathrm{kcal}$.

Vitamin $D$ is present in a range of food supplements (including fish oil products) and licensed medicines. Both vitamin $D_{2}$ and vitamin $D_{3}$ are used in food supplements, at levels up to $0.0125 \mathrm{mg}$ per daily dose, and for food fortification.

\section{Other sources of exposure}

No data have been identified.

\section{Recommended amounts}

The establishment of recommended intakes of vitamin $D$ is difficult because it is produced endogenously as a result of exposure to sunlight, which cannot readily be quantified. COMA has not set a RNI value for those individuals leading a normal lifestyle. For individuals confined indoors, pregnant and lactating women, an intake of $0.01 \mathrm{mg} /$ day was recommended by COMA. RNls of 0.0085 and $0.007 \mathrm{mg} /$ day were recommended for infants aged 0-6 months and 6 months to 3 years respectively (COMA, 1998). 


\section{Analysis of tissue levels and vitamin D status}

The conventional marker of vitamin D status is plasma 25-hydroxyvitamin D. This marker is employed because it reflects the main storage form of precursor substrate for vitamin $D, 1,25$-dihydroxyvitamin $D$, the formation of which is under homeostatic control.

\section{Brief overview of non-nutritional beneficial effects}

Vitamin D analogues have been reported to induce cell differentiation and reduce proliferation, and based on in vitro and epidemiological studies it has been suggested that vitamin D could protect against both prostate and colon cancer. This hypothesis was not commented on by COMA in its review of diet and cancer. It has been proposed that the anti-proliferative effect of vitamin D may be of benefit in the treatment of psoriasis (and treatment with the vitamin $\mathrm{D}$ analogue calcipotriol is of established benefit). There are also some claims that vitamin D may inhibit or stop the development of a number of autoimmune disorders, such as rheumatoid arthritis, and that it or its analogues may suppress transplant rejection.

\section{Function}

Vitamin $D$ is metabolised to the steroid hormone 1,25-dihydroxyvitamin $D$, a process which is promoted by parathyroid hormone (PTH). 1,25-Dihydroxyvitamin D regulates calcium and phosphate metabolism via three target tissues: kidney, small intestine and bone. In the kidney, 1,25-dihydroxyvitamin D regulates calcium transport in the proximal tubule; in the small intestine, it regulates calcium and phosphate uptake from the gut. 1,25-dihydroxyvitamin D is also involved in the maintenance of plasma calcium levels via bone resorption and formation. 1,25-dihydroxyvitamin $\mathrm{D}$ regulates the synthesis of PTH by a negative feedback mechanism.

Vitamin $D_{2}$ and $D_{3}$ are generally assumed to have equal levels of efficacy in humans, although recent data suggest that vitamin $D_{3}$ may be more efficient at increasing serum 25 -hydroxyvitamin $D$ levels (Trang et al., 1998).

\section{Deficiency}

Prolonged vitamin D deficiency in infants and children results in rickets. In adults, vitamin D deficiency results in osteomalacia, the clinical symptoms of which include skeletal pain and muscle weakness and pathological fractures. Less severe vitamin D deficiency (usually referred to as vitamin D insufficiency) is associated with secondary hyperparathyroidism and increased bone loss, leading to high risk of fractures.

Groups such as black, Asian, institutionalised older people, and those who habitually cover the skin may form less vitamin $D$ endogenously as a result of exposure to sunlight, and are vulnerable to vitamin $D$ deficiency. Individuals with a decreased capacity for intestinal absorption of vitamin D, for example following partial gastrectomy, are also at increased risk of vitamin D deficiency, as are patients with liver, renal and cardiopulmonary diseases. 
Part 2 Fat Soluble Vitamins

\section{Interactions}

The cytotoxic agent actinomycin and imidazole antifungal agents interfere with vitamin $D$ activity by inhibiting the conversion of 25-hydroxyvitamin D to 1,25-dihydroxyvitamin D by the kidney enzyme, 25-hydroxyvitamin D-1-hydroxylase. Lead has also been reported to inhibit vitamin D synthesis. Some anticonvulsant drugs may interfere with hepatic metabolism of vitamin $D$ and so raise requirements.

\section{Absorption and bioavailability}

Vitamin $D$ is absorbed from the small intestine as bile salt-dependent micelles and circulated in the body via the lymph. Absorption of polar derivatives, such as 25 -hydroxyvitamin $D$, is more efficient and less dependent on bile salts. These polar derivatives are generally not present in any significant amount in food or food supplements, although small amounts of 25-hydroxyvitamin D are found in meat and breast milk.

\section{Distribution and metabolism}

There is substantial storage of vitamin $D$ in adipose tissue. Vitamin $D$ in plasma is bound to vitamin D-binding protein and transported to the liver where it undergoes 25 -hydroxylation and is released into the circulation bound to $\alpha-2$ globulin. Additional 1-hydroxylation then occurs in the kidney, producing the active form of the vitamin. This reaction is regulated by parathyroid hormone (PTH), which is secreted in response to low plasma calcium levels.

\section{Excretion}

Vitamin $D$ is principally excreted in the bile. It is also metabolised to water-soluble metabolites, such as calcitroic acid, and excreted in the urine.

\section{Toxicity}

\section{Human data}

Excessive vitamin D intake may lead to hypercalcaemia and hypercalciuria. Vitamin D promotes the absorption of calcium and the resorption of bone resulting in the deposition of calcium in soft tissues, diffuse demineralisation of bones and irreversible renal and cardiovascular toxicity. Patients with sarcoidosis are abnormally sensitive to vitamin $\mathrm{D}$, due to uncontrolled conversion of the vitamin to its active form in the granulomatous tissue. Although the condition is uncommon, it would be a potential hazard if affected individuals were to take supplementary vitamin D.

One study has suggested that moderate levels $(0.025-0.050 \mathrm{mg} /$ day) of vitamin D may enhance renal stone formation in susceptible individuals.

Data on the comparative toxicity of vitamin $D_{2}$ and $D_{3}$ in humans are lacking. 


\section{Supplementation trials}

A number of supplementation trials have reported no adverse effects at intakes of $0.010-0.045 \mathrm{mg}$ vitamin D/day. The effects at higher doses have been more variable. For example, in a study in which 63 older individuals (females over 60 years, males over 65 years) were given $0.05 \mathrm{mg} /$ day supplementary vitamin D (form unspecified) for 6 months, 2 subjects developed hypercalcaemia (Johnson et al., 1980). However, in a 5 month study in which 61 younger adults (mean age, 41 years) were given $0.10 \mathrm{mg} /$ day supplementary vitamin $D_{3}$, serum calcium levels were not significantly increased (Vieth et al., 2001). The difference may reflect differing prior states with hyperparathyroidism or hypovitaminosis D possibly occurring in the elderly.

\section{Animal data}

In animals, excess vitamin D causes hypercalcaemia, resulting in deposition of calcium in soft tissues and bone demineralisation, anorexia, weight loss, anaemia and weakness.

In studies in rhesus monkeys, vitamin $D_{3}$ was shown to be significantly more toxic than vitamin $D_{2}$. The authors noted that although less toxic, vitamin $D_{2}$ was still functional in this species.

Excess vitamin $D_{2}$ during gestation in rabbits led to decreased foetal viability, an increased number of abortions, and supravalvular lesions in the offspring. High doses of vitamin D appear to affect maternal calcium, phosphate and cholesterol homeostasis and neonatal calcium homeostasis. In rodents, administration of high levels of vitamin $\mathrm{D}_{2}$ during gestation resulted in retarded foetal and placental growth, loss of ossification of foetal bones and foetal skeletal degeneration, resulting particularly in facial malformations.

\section{Carcinogenicity and genotoxicity}

There are no data relating to the carcinogenicity of vitamin D. No in vivo or in vitro genotoxicity data have been identified.

\section{Mechanism of toxicity}

Vitamin $D$ is involved in calcium metabolism and can increase calcium uptake from the gut, reabsorption of calcium from the kidney and resorption of calcium from bone. Such responses are not well characterised.

\section{Genetic variations}

Children born with the rare autosomal recessive condition, vitamin D dependency rickets Type II, are vulnerable to rickets and alopecia. The condition is characterised by high circulating levels of 1,25dihydroxyvitamin $D_{3}$ and functionless vitamin $D$ receptors. Such individuals would be less susceptible to high intakes of vitamin $D$.

The gene coding for the vitamin $\mathrm{D}$ receptor has been reported to be polymorphic, and sensitivity to vitamin $\mathrm{D}$ has been shown to vary depending on genotype. 
Part 2 Fat Soluble Vitamins

\section{Dose response characterisation}

Due to the unknown contribution of vitamin $D$ formed through exposure to sunlight, the dose response relationship of vitamin $D$ and hypercalcaemia or hypercalciuria is difficult to determine in humans. Human supplementation studies using less than $0.02 \mathrm{mg} /$ day were not associated with adverse effects, whereas adverse effects have been reported at higher levels of intake in some studies. The majority of studies have reported significant changes at supplementation levels of approximately $0.10 \mathrm{mg} /$ day or greater.

A problem in characterisation arises from the difficulty in ascertaining whether a particular serum calcium level on vitamin D supplementation does or does not indicate normality. In deficient individuals serum calcium levels would be expected to rise less than in those initially replete. Such a situation could prevail in older people and in Asian populations.

\section{Vulnerable groups}

Infants are at risk of developing hypervitaminosis $D$; hypercalcaemia has been reported at vitamin $D$ intakes of $0.050 \mathrm{mg} /$ day and above (see later). Adults with disease states such as sarcoidosis, Mycobacterium infections and idiopathic hypercalciuria and hypercalcaemia more vulnerable to hypercalcaemia resulting from moderate vitamin $D$ intakes ( $>0.025 \mathrm{mg} /$ day).

\section{Studies of particular importance in the risk assessment}

(For full review see http://www.food.gov.uk/science/ouradvisors/vitandmin/evmpapers or the enclosed CD).

\section{Human data}

Johnson et al., 1980

A randomised, double-blind, placebo-controlled clinical trial of dietary supplementation with $0.05 \mathrm{mg}$ vitamin $D$ per day (form unspecified) for six months was carried out in females over 60 and males over 65 years of age $(n=63)$. Serum calcium levels were significantly raised in the vitamin $D$-treated group and two of the subjects developed hypercalcaemia (serum calcium $>2.75 \mathrm{mmol} / \mathrm{L}$ ).

Narang et al., 1984

A clinical trial of vitamin D supplementation (form unspecified) was carried out in healthy subjects aged 21-60 years and patients with pulmonary tuberculosis. Groups were given 0.01, 0.02, 0.03, 0.06 and $0.095 \mathrm{mg}$ vitamin $D$ per day for 3 months. In the healthy subjects $(n=30)$, serum calcium levels were significantly raised in the 0.06 and $0.095 \mathrm{mg} /$ day groups, although levels only exceeded the normal range $(>2.75 \mathrm{mmol} / \mathrm{L}$ ) in the $0.095 \mathrm{mg} /$ day group. Serum calcium levels were non-significantly raised in the $0.01,0.02$ and $0.03 \mathrm{mg} /$ day groups.

\section{Honkanen et al., 1990}

This randomised clinical trial of dietary supplementation with $0.045 \mathrm{mg}$ vitamin $\mathrm{D}_{3}$ and $1558 \mathrm{mg}$ calcium per day was performed in 52 independently-living and institutionalised elderly subjects treated for 11 weeks (27 controls, 25 treated). Serum calcium, 25-hydroxyvitamin D and creatinine levels were measured before and after the trial period. Serum calcium and creatinine levels did not significantly 
differ in control or treated groups after the trial period, and were not outside the normal ranges in any individuals.

\section{Chapuy et al., 1992}

The effect of supplementation for 18 months with $0.020 \mathrm{mg} /$ day vitamin $D_{3}$, together with $1200 \mathrm{mg} /$ day calcium, on hip fracture incidence was investigated in this randomised, placebo-controlled study in healthy women (mean age 84 years). Of the 1634 subjects who received the active supplements, 877 completed the study. However, the rate of dropout from the study was similar in both treated and placebo groups and there were no significant differences in the reasons reported for withdrawal (death, non-compliance, inability to walk during the study, loss to follow-up, intercurrent illness, gastrointestinal effects). Serum calcium levels were measured every 6 months and one subject in the treated group withdrew from the study due to mild hypercalcaemia (serum calcium $2.8 \mathrm{mmol} / \mathrm{L}$ ), which was reported to be due to primary hyperparathyroidism. No other subjects had hypercalcaemia at any point during to the study and it was reported that no subjects developed renal calculi.

Jacobus et al., 1992

A retrospective study was carried out on eight US patients with hypervitaminosis $D$, thought to be caused by consumption of milk excessively fortified with vitamin $D_{3}$. Serum vitamin $D_{3}$ concentrations varied considerably in these patients. A proposed explanation for this was that apparently the fortification of the milk was sporadic and only at times in excess. All patients had elevated serum 25hydroxyvitamin $D$ levels, with $6 / 8$ having elevated serum vitamin $D_{3}$ concentrations. Seven of the eight patients had hypercalcaemia. All patients drank locally produced milk (118-710 mL/day) which contained vitamin $D$ levels ranging from undetectable to $6.1 \mathrm{mg} / \mathrm{L}$. The recommended level for milk fortification in the US is $0.01055 \mathrm{mg} / \mathrm{L}$.

\section{Dawson-Hughes et al., 1997}

A randomised double-blind placebo-controlled clinical trial investigated dietary supplementation with $0.0175 \mathrm{mg}$ vitamin $D_{3}$ and $500 \mathrm{mg}$ calcium per day for 3 years in 389 subjects of 65 years or older. Although primarily a study of effects on bone mineral density, serum calcium and creatinine were monitored. There was a small but significant increase in serum calcium levels over the lifetime of the study in the treated group, but levels were within the normal range. One treated subject withdrew from the trial due to hypercalciuria.

Vieth et al., 2001

The safety of supplementation with $0.10 \mathrm{mg} /$ day vitamin $D_{3}$ for 2 to 5 months was investigated in healthy men and women with a mean age of 41 years. The number of treated individuals with hypercalciuria was not significantly different to the number of untreated controls with hypercalciuria, although the study was of low statistical power to detect such differences. There was no evidence of hypercalcaemia; mean serum calcium concentrations were within the normal range $(2.2-2.6 \mathrm{mmol} / \mathrm{L})$ in all subjects.

\section{Animal data}

Haschek et al., 1977

Pigs received standard diets that had optimal calcium and phosphate and provided intakes of vitamin $D_{3}$ of $0.00132 \mathrm{mg} / \mathrm{kg}$ bw/day for one week following weaning. They were injected with ${ }^{45} \mathrm{Ca}$ and, 3 days 
Part 2 Fat Soluble Vitamins

later, divided into two groups, one of which received standard diet (control) and one of which received diet providing $0.825 \mathrm{mg} / \mathrm{kg}$ bw/day vitamin $\mathrm{D}_{3}$. Two pigs from each group were sacrificed 1, 2, 3, 4, 7 and 14 days after division into the two treatment groups. Pigs fed the high level of vitamin D lost weight; anorexia, weakness, rough hair coat and laboured breathing were also observed. In the treated group, hypercalcaemia began at 12 hours after starting the high vitamin D diet, and progressed rapidly after two days. Interpretation of the radioisotope studies indicated that bone was the primary source of the increased plasma calcium, since food intake and, therefore calcium absorption from the gut, was depressed. Calcium was released at a rapid rate from pre-labelled bone undergoing breakdown.

\section{Chineme et al., 1977}

Pigs were fed diets providing vitamin $D_{3}$ intakes of approximately $0.00132,0.0066,0.033$ or $0.165 \mathrm{mg} / \mathrm{kg}$ bw/day. The resulting hypercalcaemia, seen in the upper two dose groups, was thought to result primarily from intestinal absorption of calcium, not from bone loss and tissue degeneration, as hypercalcaemia per se was not always associated with soft tissue calcification in pigs.

\section{Exposure assessment}

Total exposure/intake:

Food

Mean: $0.003 \mathrm{mg} /$ day

$97.5^{\text {th }}$ percentile: $0.009 \mathrm{mg} /$ day (from 1986/87 NDNS)

Supplements $\quad$ Up to $0.0125 \mathrm{mg}$ (Annex 4; OTC, 2001)

Estimated maximum intake $0.009+0.0125=0.022 \mathrm{mg} /$ day

No potential high intake groups have been identified.

\section{Risk assessment}

Excess vitamin D may lead to hypercalcaemia and hypercalciuria. Hypercalcaemia results in the deposition of calcium in soft tissues, diffuse demineralisation of bones and irreversible renal and cardiovascular toxicity. Moderate levels of vitamin D intake may enhance renal stone formation in predisposed individuals. It has been suggested that excess vitamin D may be linked to heart disease, but there is limited evidence for this.

Data are available from a range of human supplementation studies, but the levels of vitamin $D$ intake at which hypercalcaemia or hypercalciuria occurs vary between studies. Likely reasons for this include differences in populations studied; for example, several of the studies are in older people, a group vulnerable to vitamin D deficiency, while other studies are in younger adults, who are not likely to be vitamin $D$ deficient. Individuals and groups are also likely to differ in their exposure to vitamin $D$ sources other than supplementation, such as consumption of vitamin D-fortified foods and through exposure to the sun.

Excess vitamin $D$ during gestation in rats and rabbits led to a number of adverse reproductive effects. 


\section{ESTABLISHMENT OF GUIDANCE LEVEL}

A safe upper level suitable for long-term intake by the whole population cannot be established based on the available data from studies in humans or animals. The human data are adequate to provide guidance. High intakes of vitamin D are associated with elevated levels of calcium, which in turn lead to soft tissue calcification, demineralisation of bones, and renal and cardiovascular toxicity. The effect is apparent in both human case reports and in animal studies.

Assessing the risk of toxicity in man is complicated. On the one hand, significant numbers of the UK population, notably older people and Asian populations not exposed to sunlight, may ordinarily be at risk of deficiency. By contrast, apart from the rare individuals with granulomatous diseases, who are abnormally sensitive to the effects of vitamin $D$, there are others, notably older people, who have a high prevalence of primary hyperparathyroidism. Others, with tertiary hyperparathyroidism due to prolonged vitamin $D$ deficiency, may show falsely low serum calcium levels in response to vitamin $D$. There is therefore a particular need for large studies covering all sections of the population. These are generally lacking.

The highest level of vitamin $D$ supplementation at which no effect on calcium was observed was 0.10 $\mathrm{mg} /$ day, reported by Vieth et al. (2001). This was from a 5 month supplementation study of vitamin $D_{3}$ in 63 adults aged $23-56$ years. In contrast, the lowest level of vitamin $D$ at which effects have been observed was $0.05 \mathrm{mg} /$ day (Johnson et al., 1980) from a 6 month study of supplementation with vitamin $D$ (of unspecified form) in females above the age of 60 and males above the age of 65 . Two out of the 63 subjects developed hypercalcaemia (serum calcium $>2.75 \mathrm{mmol} / \mathrm{L}$ ). The reason for the difference is not known but may be due to difference in other sources of vitamin $D$ exposure or in the study populations.

Taking the evidence as a whole, long-term exposures of up to $0.025 \mathrm{mg} /$ day vitamin $D$ appear to be well-tolerated and may be necessary to prevent deficiency in some groups. Higher levels (for example, $0.045 \mathrm{mg} /$ day; Honkanen et al., 1990) may be tolerated without adverse effects over the short-term under medical supervision and may be necessary to correct a deficiency. The use of an uncertainty factor is not appropriate because the value is derived from an overview of a number of human studies which measured sensitive biochemical markers of calcium homeostasis.

For guidance purposes only, a level of $0.025 \mathrm{mg} /$ day supplementary vitamin $D$ would not be expected to cause adverse effects in the general population. This is equivalent to $0.0004 \mathrm{mg} / \mathrm{kg}$ bw/day for a 60 $\mathrm{kg}$ adult. Due to the difficulties in assessing total vitamin D exposure, an estimate for total intake has not been provided. Such an intake, or more, might well be required under medical supervision in managing overt or occult deficiency states. It should be noted that scaling on a body weight basis to children and infants may not be appropriate for vitamin $D$ as it may lead to the recommended intake for an infant not being met. 
Part 2 Fat Soluble Vitamins

\section{References}

Chapuy, M.C., Arlot, M.E., Duboeuf, F., Brun, J., Crouzet, B., Arnaud, S., Delmas, P.D., Meunier, P.J. (1992) Vitamin $\mathrm{D}_{3}$ and calcium to prevent hip fractures in elderly women. New England Journal of Medicine 327, 1637-1642.

Chineme, C.N., Krook, L., Pond, W.G. (1976) Bone pathology in hypervitaminosis D: an experimental study in young pigs. Cornell Veterinary Medicine 66, 387-412.

COMA (1998). Nutrition and Bone Health: with particular reference to calcium and vitamin D. Report of the Subgroup on Bone Health, Working Group on the Nutritional Status of the Population, Committee on Medical Aspects of Food and Nutrition Policy. The Stationery Office, London.

Dawson-Hughes, B., Harris, S.S., Krall, E.A., Dallal, G.E. (1997) Effect of calcium and vitamin D supplementation on bone density in men and women 65 years of age or older. New England Journal of Medicine 337, 670-676.

Haschek, W.M., Krook, L., Kallfelz, F.A., Pond, W.G. (1977) Vitamin D toxicity. Initial site and mode of action. Cornell Veterinary Medicine 68, 324-364.

Honkanen, R., Alhava, E., Parviainen, M., Talasniemi, A., Mönkkönen, R. (1990) The necessity and safety of calcium and vitamin D in the elderly. Journal of the American Geriatrics Society 38, 862-866.

Jacobus, C.H., Holick, M.F., Shao, Q., Chen, T.C., Holm, I.A., Kolodny, J.M., Fuleihan, G.E., Seely, E.W. (1992) Hypervitaminosis D associated with drinking milk. New England Journal of Medicine 326 (18), 1173-1177.

Johnson, K.R., Jobber, J., Stonawski, B.J. (1980) Prophylactic vitamin D in the elderly. Age and Ageing 9, 121-127.

Narang, N.K., Gupta, R.C., Jain, M.K. (1984) Role of vitamin D in pulmonary tuberculosis. Journal of the Association of Physicians of India 32, 185-188.

Trang, H.M., Cole, D.E.C., Rubin, L.A., Pierratos, A., Siu, S., Vieth, R. (1998) Evidence that vitamin $D_{3}$ increases serum 25 -hydroxyvitamin $D$ more efficiently than does vitamin $D_{2}$. American Journal of Clinical Nutrition 68, 854-858.

Vieth, R., Chan, P-C. R., MacFarlane, G.D. (2001) Efficacy and safety of vitamin $D_{3}$ intake exceeding the lowest observed adverse effect level. American Journal of Clinical Nutrition

73, 288-94. 


\title{
Risk Assessment $\quad$ Vitamin E
}

\section{General information}

\author{
Chemistry \\ The term vitamin $\mathrm{E}$ is used as a generic designation for a group of eight lipid-soluble compounds \\ synthesised by plants. These compounds fall into two classes, tocopherols and tocotrienols, which \\ exhibit the biological antioxidant activity of vitamin E. Vitamins in both classes are designated by the \\ Greek letters $\alpha, B, \gamma$ and $\delta$. The most biologically active antioxidant is $d$ - $\alpha$-tocopherol. Vitamin $E$ \\ activity is expressed as $d$ - $\alpha$-tocopherol equivalents. Where activity is given as International Units (IU), \\ $1 \mathrm{IU}$ of $d$ - $\alpha$-tocopherol (RRR- $\alpha$-tocopherol) is equivalent to $0.67 \mathrm{mg}$. If the vitamin $\mathrm{E}$ is present in the \\ form of $d$ l- $\alpha$-tocopherol (all-rac- $\alpha$-tocopherol), then $0.91 \mathrm{mg}$ is equivalent to $1 \mathrm{IU}$.
}

\section{Natural occurrence}

Vitamin $\mathrm{E}$ is synthesised only by plants and is, therefore, found primarily in plant products, the richest source being plant oils. All higher plants (that is plants other than algae) appear to contain $\alpha$-tocopherol in leaves and other green parts, while $\gamma$-tocopherol is generally present in lower concentrations. Animal tissues tend to have low concentrations of vitamin $\mathrm{E}$, with the highest levels occurring in fatty tissues though this varies according to the intake of vitamin $E$.

\section{Occurrence in food, food supplements and medicines}

Plant oils are the main dietary sources of vitamin E $(560-1600 \mathrm{mg} / \mathrm{kg}$ in soybean oil, $530-1620 \mathrm{mg} / \mathrm{kg}$ in corn oil and $50-150 \mathrm{mg} / \mathrm{kg}$ in olive oil), with meat $(0.5-1.6 \mathrm{mg} / \mathrm{kg})$, poultry $(1.6-4.0 \mathrm{mg} / \mathrm{kg})$ and dairy products $(0.4-10.0 \mathrm{mg} / \mathrm{kg}$ ) providing only moderate amounts. The amount of vitamin $\mathrm{E}$ in foods at the point of consumption is difficult to assess as it depends upon the effects of processing, storage and preparation. Vitamin $E$ is present in a variety of dietary supplements at doses of up to $268 \mathrm{mg} /$ day in multi-constituent products and $670 \mathrm{mg} /$ day in single constituent products. Vitamin $\mathrm{E}$ is also present in licensed medicinal products; the highest doses authorised are 20-100 mg.

\section{Recommended amounts}

Foods containing large amounts of polyunsaturated fatty acids (PUFAs) will generally contain large amounts of vitamin $\mathrm{E}$. In addition, the requirement for vitamin $\mathrm{E}$ increases with the amount of dietary PUFAs consumed. Thus, owing to the widely differing requirements based on the PUFA intake, it is generally considered more realistic to give ranges of acceptable intake rather than a fixed level. Using this approach, in a NDNS survey (Gregory et al, 1990) of 1629 adults only $0.7 \%$ had serum tocopherol:cholesterol ratios lower than $2.25 \mu \mathrm{mol} / \mathrm{mmol}$ (below this erythrocytes have a tendency to haemolyse). The 2.5 and 97.5 centile intakes were $3.5 \mathrm{mg}$ and $19.5 \mathrm{mg}$ in men and $2.5 \mathrm{mg}$ and $15.2 \mathrm{mg}$ in women. This gives a median intake of $9.3 \mathrm{mg} \alpha$-tocopherol equivalent/day for men and $6.7 \mathrm{mg} /$ day for women. COMA considered these ranges and concluded that daily intakes of $4 \mathrm{mg}$ and $3 \mathrm{mg}$ of $\alpha$ tocopherol equivalents could be adequate for men and women respectively (COMA, 1991). Potential deficiency, however, could be excluded if the lower intakes are maintained over prolonged periods. Intakes of $3.8-6.2 \mathrm{mg} /$ day appear to be satisfactory for pregnant and lactating women. 
Part 2 Fat Soluble Vitamins

\section{Analysis of tissue levels and vitamin E status.}

Measurement of serum plasma concentration of $\alpha$-tocopherol provides the simplest and most direct evidence of vitamin E status. Values of $5-20 \mu \mathrm{g} / \mathrm{mL}$ for adults and children of twelve years or older and values of $3-15 \mu \mathrm{g} / \mathrm{mL}$ for children under twelve years indicate acceptable levels of intake. Another widely used indicator of vitamin $\mathrm{E}$ status is the extent of haemolysis of red blood cells in the presence of hydrogen peroxide. A high degree of haemolysis accompanies vitamin E deficiency (i.e. greater than $20 \%$ ); however, this is not specific to vitamin E deficiency.

\section{Brief overview of non-nutritional beneficial effects.}

It has been claimed that vitamin $\mathrm{E}$ can prevent free radical damage. In a cancer prevention study, the incidence of prostate cancer was $32 \%$ lower and mortality was $41 \%$ lower in men taking $\alpha$-tocopherol, either with or without $B$-carotene, than in those not taking the vitamin supplement. Vitamin $E$ is thought to have a role in the prevention of atherosclerosis, through inhibition of oxidation of low-density lipoprotein. Vitamin E has been reported to relieve the symptoms of fibrocystic breast disease. It has also been claimed that menopausal symptoms may be relieved by vitamin $\mathrm{E}$.

Vitamin $\mathrm{E}$ has been used to treat scleroderma and it has been reported that it may prevent retrolental fibroplasia and intracranial haemorrhage in premature infants. In animal studies, vitamin $E$ has been found to reduce UV-induced acute and chronic skin damage.

\section{Function}

It is unclear whether vitamin E functions solely as a lipid antioxidant, or whether it might also be required for the function of some other critical, but unknown metabolic factor. However, current information suggests that the effects of vitamin $E$ are consistent with an antioxidant role. In this regard, vitamin $E$ is thought to have basic functional importance in the maintenance of membrane integrity in virtually all cells of the body. Non-antioxidant functions have also been proposed for $\alpha$-but not ß-tocopherol including modification of gene transcription and expression.

\section{Deficiency}

The clinical manifestations of vitamin E deficiency vary considerably between species. In general, the targets are the neuromuscular, vascular and reproductive systems. The various signs of vitamin $\mathrm{E}$ deficiency are believed to be manifestations of membrane dysfunction, resulting from oxidative degradation of polyunsaturated membrane phospholipids and/or the disruption of other critical cellular processes.

\section{Interactions}

Vitamin E may exacerbate the effects of vitamin $\mathrm{K}$ deficiency, thus affecting blood coagulation. This has been reported in animal studies and in case reports of humans taking large doses the basis for the interaction is unclear. Vitamin $\mathrm{E}$ may also interfere with vitamin $\mathrm{A}$ absorption. 


\section{Absorption and bioavailability}

$\alpha$-Tocopherol is absorbed unchanged from the small intestine by non-saturable, passive diffusion. Tocotrienol esters are first hydrolysed by pancreatic esterase. Absorption appears to occur mostly in the upper and middle thirds of the small intestine. The absorption efficiency of tocopherol and its esters is generally considered to be variable. It has been reported that in human studies, absorption of $\alpha$-tocopherol and its acetate ester was in the region of $21-86 \%$ over 24 hours.

\section{Distribution and metabolism}

Vitamin E does not appear to have a specific carrier protein in the plasma, but it is rapidly transferred from chylomicrons to plasma lipoproteins, to which it binds non-specifically. The vitamin is taken up by the liver and released in low density lipoprotein (LDL). Most absorbed tocopherols are transported unchanged to the tissues. Kinetic studies indicate that the body has two pools of the vitamin: a 'labile' pool which turns over rapidly and a 'fixed' pool which turns over slowly. The labile pool predominates in tissues such as plasma and liver, as the tocopherol contents of those tissues are depleted rapidly under conditions of vitamin $\mathrm{E}$ deprivation. In contrast, the adipose vitamin $\mathrm{E}$ resides predominately in the bulk lipid phase, which appears to be a fixed pool of the vitamin.

\section{Excretion}

At normal intake levels, vitamin $\mathrm{E}$ is conjugated with glucuronic acid and this conjugate is excreted (via bile) in the faeces. Up to $30-70 \%$ of vitamin $E$ is excreted via this route with less than $1 \%$ being excreted in the urine. Some vitamin E may be eliminated via the skin.

\section{Toxicity}

\section{Human data}

Vitamin $E$ has low toxicity. Humans and animals appear to be able to tolerate levels of the vitamin two orders of magnitude above nutritional requirements, e.g. $1000-2000 \mathrm{lu} / \mathrm{kg}$ diet without untoward effects. At very high doses, however, vitamin $\mathrm{E}$ can produce signs indicative of antagonism with the function of the other fat-soluble vitamins (vitamins A, D, K). Isolated reports of adverse effects in humans consuming up to $1000 \mathrm{IU}$ of vitamin E per day include headache, fatigue, nausea, double vision, muscle weakness, mild creatinuria and gastrointestinal distress.

In the Alpha-Tocopherol, Beta-Carotene (ATBC) study, vitamin E supplementation was associated with an increased risk of mortality from haemorrhagic stroke, whilst in the Cambridge Heart Antioxidant Study (CHAOS) a small excess of cardiovascular deaths was observed in the supplemented group. These are described below:

\section{Supplementation studies}

Many human studies, both small and large scale, have considered the effect of vitamin E on various biochemical and physiological parameters. These have included large-scale intervention studies, designed to assess the effects of vitamin $\mathrm{E}$ on conditions such as cancer and heart disease, which have not generally considered more minor side effects. In smaller scale trials, few adverse effects have been reported. However, creatinuria, breast pain, diarrhoea and fatigue and dizziness have been noted in some investigations. 
Part 2 Fat Soluble Vitamins

\section{Animal data}

Animals with hypervitaminosis $\mathrm{E}$ have been found to show impaired bone mineralisation, reduced hepatic storage of vitamin A and coagulopathy. In each case, these signs could be corrected with increased dietary supplements of the appropriate vitamin (i.e. vitamins $D, A$ and $K$, respectively). The antagonism appeared to occur during absorption.

\section{Carcinogenicity and genotoxicity}

No carcinogenicity studies have been identified in laboratory animals, however, some limited chronic studies exist which do not suggest that vitamin $\mathrm{E}$ is carcinogenic. No in vivo or in vitro genotoxicity studies have been identified. Co-incubation with vitamin $\mathrm{E}$ has been reported to reduce the mutagenic effect of chemicals such as malonaldehyde and ß-propiolactone in some Ames test studies.

\section{Mechanism of toxicity}

Vitamin $\mathrm{E}$ has been reported to interact with vitamin $\mathrm{K}$. The mechanism of this is uncertain, but it has been suggested that the metabolite tocopherylquinone, which is structurally similar to vitamin $\mathrm{K}$ may inhibit vitamin $\mathrm{K}$ metabolism and thus coagulation.

\section{Dose-response characterisation}

No relevant data have been identified.

\section{Vulnerable groups}

No vulnerable groups have been identified.

\section{Genetic variations}

Familial isolated vitamin E deficiency is a rare autosomal recessive neurodegenerative disorder with symptoms similar to those of Friedrich's ataxia. Supplementation with vitamin E can prevent the onset of the disease if given before irreversible damage occurs.

\section{Studies of particular importance in the risk assessment}

(For full review see http://www.food.gov.uk/science/ouradvisors/vitandmin/evmpapers or the enclosed $C D$ ).

\section{Gillilan et al., 1977}

Forty-eight patients with stable angina completed a double-blind crossover study of two 6 month periods of treatment with $1600 \mathrm{lU} /$ day $d$ - $\alpha$-tocopherol succinate (equivalent to $1072 \mathrm{mg} d$ - $\alpha$ tocopherol) or placebo. No statistically significant differences were apparent in a number of cardiac parameters including systolic time interval assessment of left ventricular function and the multistage, 
maximal exercise test. The subjects were questioned regarding possible side effects and underwent periodic urinalysis, blood count and blood chemistry analysis, measures of prothrombin time, chest Xray and ECG. No deleterious side effects were observed. There was a slightly increased incidence of gastrointestinal disturbance during the placebo phase; further details are not provided. It was stated that there was 'no exacerbation of hypertension, congestive heart failure or skeletal muscle complaints could be attributed to vitamin E therapy'. No further details are provided. No differences were found in the various clinical chemistry indices.

\section{Cambridge Heart Antioxidant Study (CHAOS): Stephens et al., 1996}

2002 patients with atherosclerosis were entered into a double-blind placebo-controlled study and followed up for a median of 510 days (range 3-981). The treatment group received a dose of $800 \mathrm{IU}$ vitamin E (537 mg as $d$ - $\alpha$-tocopherol)/day for the first 546 patients and $400 \mathrm{IU} /$ day (268 $\mathrm{mg}$ as $d$ - $\alpha$ tocopherol)/day for the remainder. Treatment significantly reduced the risk of cardiovascular death and non-fatal myocardial infarction (MI) (a composite endpoint). However, this decrease was due to the decrease in Ml since, when separated out, there was a slight non-significant excess of cardiovascular deaths in the treatment group ( $R R, 1.18,95 \% \mathrm{Cl}, 0.62-2.27, \mathrm{p}=0.61)$. The authors speculated that the finding might be due to chance or might reflect a difference in biological events leading to death and those leading to non-fatal Ml and noted that the results of larger studies would be necessary to clarify this. The study was not designed to look at any dose response effects and there was no randomisation of the subjects within the different treatment groups.

\section{Meydani et al., 1998}

Eighty-eight healthy older subjects (aged $>65$ years) were entered into a double-blind placebocontrolled study, receiving placebo, 60, 200 or $800 \mathrm{IU} \mathrm{dl}$ - $\alpha$-tocopherol/day $(34,134$ or $537 \mathrm{mg} d$ - $\alpha$ tocopherol equivalents) for four months. The groups contained 17, 19, 18 and 19 subjects respectively. The study was designed to assess the effect of supplementation on general health and measured an extensive range of parameters. It was stated that no side effects were reported. Supplementation had no effect on plasma concentration of other anti-oxidant vitamins and minerals, glutathione peroxidase, superoxide dismutase or total cysteine. There was no significant effect of vitamin E on serum non-specific immunoglobulin concentrations or anti-DNA and anti-thyroglobulin antibodies. The cytotoxic ability of neutrophils against Candida albicans was not compromised. Vitamin E had no effect on body weight, plasma total proteins, albumin, glucose, plasma lipids or the lipoprotein profile, total bilirubin, serum liver enzymes, blood count, platelet number, bleeding time, haemoglobin, haematocrit, urinary or serum creatine levels. The authors concluded that supplementation had no detrimental effect on health.

\section{Ascherio et al., 1999}

In the Health Professional Follow-up Study, 43,738 men were recruited and followed for up to 8 years. Vitamin $E$ intake was assessed by food frequency questionnaire which included questions on supplement use. None of the subjects had cardiovascular disease or diabetes. A total of 328 strokes occurred: 210 ischaemic, 70 haemorrhagic and 48 unclassified. After adjustment, the relative risks for ischaemic and total stroke were not affected by vitamin $E$ intake. The association of vitamin $E$ with haemorrhagic stroke was also non-significant but the confidence intervals were wide. The authors concluded that vitamin E supplements did not affect stroke risk but that modest effects could not be ruled out. 
Part 2 Fat Soluble Vitamins

\section{GISSI-Prevenzione Investigators, 1999}

11,324 patients who had survived recent myocardial infarction were randomly assigned supplements of n-3 PUFA (1 g daily), $300 \mathrm{mg}$ vitamin E (as synthetic $\alpha$-tocopherol), both or neither for 3.5 years. The primary combined efficacy endpoint was death, non-fatal myocardial infarction and stroke. Smokers and ex-smokers were evenly distributed through the groups. Vitamin $E$ treatment had no effect on the combined or separate endpoints.

\section{ATBC study: The Alpha-Tocopherol, Beta-Carotene Cancer Prevention Study Group, 1994; Leppälä et al., 2000}

In a randomised, double-blind placebo-controlled study, a total of 29,133 Finnish male smokers were randomly assigned to one of four treatment groups: $50 \mathrm{mg} \mathrm{dl}$ - $\alpha$-tocopherol/day (equivalent to $55 \mathrm{IU}$ ), $20 \mathrm{mg} /$ day $\beta$-carotene, both $\alpha$-tocopherol and $\beta$-carotene, or placebo. Follow-up continued for five to eight years. Lung cancer incidence was not affected by $\alpha$-tocopherol treatment, but the incidence of prostate cancer was reduced. $\alpha$-Tocopherol had no apparent effect on total mortality but was associated with an increase in mortality from haemorrhagic stroke (7.8 deaths per 10,000 person years in the $\alpha$-tocopherol group versus 5.2 deaths per 10,000 person years in the no $\alpha$-tocopherol groups). In contrast, deaths from ischaemic stroke and ischaemic heart disease were reduced in the $\alpha$-tocopherol group. Subsequent analysis of the risk factors for stroke indicated that vitamin $\mathrm{E}$ increased the risk of sub-arachnoid haemorrhage (RR, $2.45,95 \% \mathrm{Cl} 1.08-5.55)$ and decreased the risk of cerebral infarction (RR, 0.7: $95 \% \mathrm{Cl} 0.55-0.89$ ) in hypertensive men but had no effect on normotensive men.

HOPE study: Yusuf et al., 2000

A total of 9,541 subjects (6996 men and 2545 women) aged 55 or over at high risk for cardiovascular events were enrolled in a trial with a $2 \times 2$ factorial design. The participants received either $400 \mathrm{IU}$ vitamin $E$ (from natural sources - no further details provided) or placebo and either ramipril or matching placebo for a mean of 4.5 years. The primary endpoint was a combination of myocardial infarction and stroke and death from cardiovascular causes. The secondary outcomes included unstable angina, congestive heart failure, revascularisation or amputation, death from any cause, complications of diabetes and cancer. There were no significant differences in the numbers of deaths from cardiovascular causes (RR 1.05; $95 \% \mathrm{Cl}$ 0.9-1.22) between those receiving vitamin $\mathrm{E}$ or placebo or in any of the secondary outcomes. There were no differences in adverse effects between the vitamin $E$ and placebo group or in the numbers of patients who stopped taking the study medication. There was also no difference in the incidence of haemorrhagic stroke between the groups.

\section{Primary Prevention Project (PPP), 2001}

In a controlled open $2 \times 2$ factorial trial, 4495 people were randomised to receive low dose aspirin (100 mg/day) or no aspirin, and vitamin E (300 mg/day as synthetic $\alpha$-tocopherol) or no vitamin $E$ to investigate the prevention of cardiovascular events in people with one or more major cardiovascular risk factors (hypertension, hypercholesterolaemia, diabetes, obesity or family history). The mean follow up period was 3.6 years. The main combined endpoint was the cumulative rate of cardiovascular death, non-fatal myocardial infarction and non-fatal stroke. Predefined analyses included cardiovascular deaths, total deaths, and total cardiovascular events. Smokers and ex-smokers were evenly distributed through the groups. Vitamin $\mathrm{E}$ had no effect on any pre-specified endpoint. 


\section{Heart Protection Study Collaborative Group, 2002}

In a randomised placebo-controlled trial of antioxidant vitamin supplementation, 20,536 high risk adults (those with coronary heart disease, other occlusive vascular disease or diabetes) were given a daily supplement of $600 \mathrm{mg} \mathrm{dl}$ - $\alpha$-tocopherol (equivalent to $660 \mathrm{lU}$ ), $250 \mathrm{mg}$ vitamin $\mathrm{C}$ and $20 \mathrm{mg}$ B-carotene or placebo for five years (Heart Protection Study Collaborative Group, 2002). No difference in all cause mortality was revealed. Compliance was $83 \%$ on average in each treatment group, adverse experiences were sought at each follow up visit (every 4 months for the first year and every 6 months thereafter) and no significant side effects were reported. It is unclear whether minor side effects were reported or investigated. There was no significant difference in the number having a haemorrhagic stroke. Current and ex-smokers were evenly distributed between groups.

\section{Exposure assessment}

Total exposure/intake (as $d$ - $\alpha$-tocopherol equivalents):

Food

Mean: $8.5 \mathrm{mg} /$ day

97.5 percentile intake: $18 \mathrm{mg} /$ day (1986/7 NDNS)

Supplements

up to $670 \mathrm{mg} /$ day (Annex 4)

Estimated maximum

$18+670=690 \mathrm{mg} /$ day

daily intake:

No potential high intake groups have been identified.

\section{Risk assessment}

Very high doses of vitamin E have been reported to cause a few sporadic adverse effects. These include headache, fatigue, gastrointestinal distress, double vision, muscle weakness and mild creatinuria. High levels of vitamin $E$ may also antagonise the effects of the other fat-soluble vitamins. Vitamin $E$ also has an anti-platelet and anti-coagulant effect. A number of human supplementation studies on vitamin $E$ are available. Unexpected findings were apparent in two large trials, one concerned with heart disease (CHAOS), and one which considered a range of health endpoints in male smokers (ATBC). An increased risk of mortality from haemorrhagic stroke was found in the treatment group of the ATBC study; this was considered to be biologically plausible given the effect of vitamin $\mathrm{E}$ on platelets and the authors noted that this finding should be subject to careful review. The level of vitamin E supplementation involved (55 IU/day, equivalent to $37 \mathrm{mg} d$ - $\alpha$-tocopherol equivalents/day) was relatively low, being about 4 times the average daily dietary intake. It is possible, however, that an interaction might have occurred between vitamin $\mathrm{E}$ and smoking which was not apparent in the rest of the population studies. Further analysis of the data suggests that the effect was only apparent in hypertensive subjects. The CHAOS study reported a non-significant excess in deaths from cardiovascular disease in the treatment group; but the authors considered that this might be a chance effect. In the MRC/BHF, HOPE and PPP studies where subjects at high risk of cardiovascular events were given higher doses of vitamin $E$, similar effects were not reported. In the observational study of male health professionals (Ascherio et al., 1999) 
Part 2 Fat Soluble Vitamins

the relative risk of total and ischaemic stroke was not affected by vitamin $E$ intake, and there was no significant association between haemorrhagic stroke and vitamin $E$ intake. These large intervention studies do not report more minor side effects.

Other smaller human supplementation studies are available, but not all of these investigate side effects in detail.

A significant effect of vitamin $E$ is potential interference with vitamin $\mathrm{K}$. This has been demonstrated in animal studies and in isolated case reports of subjects taking large doses of vitamin $E$. However, the Meydani study did not find an increase in prothrombin time in subjects taking $800 \mathrm{IU} /$ day vitamin $\mathrm{E}$. Work with in-patients taking the anti-coagulant warfarin suggested similar results. However, there are few data on these haematological effects at higher doses, so it is not possible to establish a threshold.

As noted above, vitamin $\mathrm{E}$ has been reported to have adverse effects on clotting in animal studies. There are few other animal data on vitamin $\mathrm{E}$.

\section{ESTABLISHMENT OF SAFE UPPER LEVEL}

Key studies: Gillilan et al. (1977); Meydani et al. (1996); Stephens et al. (1996)

NOAEL: 800-1600 IU/day (540 - 970 mg $d$ - $\alpha$-tocopherol equivalents/day)

Uncertainty Factor: 1

Safe Upper Level consumption over a lifetime:

$800 \mathrm{IU}$ (540 mg $d$ - $\alpha$-tocopherol equivalents/day) supplemental for daily vitamin $\mathrm{E}$ (equivalent to $9.0 \mathrm{mg} / \mathrm{kg}$ bw/day in a $60 \mathrm{~kg}$ adult)

In the trials by Gillilan et al. (1977) and Meydani et al. (1996) the biochemical and physiological effects of vitamin $\mathrm{E}$ were investigated in some detail and the findings indicate that supplemental doses of 800 to $1600 \mathrm{IU} /$ day are without apparent adverse effect. The results were derived from small groups that may not be representative, thus an additional uncertainty factor could be applied to account for interindividual variation. However, the results of the larger CHAOS trial (Stephens et al., 1996) support the view that $800 \mathrm{lU} /$ day supplemental vitamin $\mathrm{E}$ would not result in any adverse effects and, taking the three studies together, no further uncertainty factors are necessary. A Safe Upper Level of 800 IU/day (540 mg $d$ - $\alpha$-tocopherol equivalents/day) supplemental vitamin $E$ is recommended. This is equivalent to $9.0 \mathrm{mg} / \mathrm{kg}$ bw/day for a $60 \mathrm{~kg}$ adult. Assuming an intake of $18 \mathrm{mg} /$ day from food, a total intake of $560 \mathrm{mg} d$ - $\alpha$-tocopherol equivalents/day would not be expected to result in any adverse effect. This is equivalent to $12.4 \mathrm{mg} / \mathrm{kg}$ bw/day.

A study in male smokers has suggested that $55 \mathrm{IU} /$ day vitamin $\mathrm{E}$ (equivalent to $37 \mathrm{mg} d$ - $\alpha$-tocopherol equivalents/day) may increase the risk of mortality from haemorrhagic stroke in hypertensive subjects who smoked. Although biologically plausible, the significance of this finding is uncertain. It has not been repeated in other studies in subjects at high risk of cardiovascular events treated with higher doses of vitamin $\mathrm{E}$ (up to $600 \mathrm{mg} /$ day); however, if it is an effect related to smoking there may have been too few smokers in these studies for any effect to be apparent. In addition, a large observational study of male health professionals did not report this association. 


\section{References}

The Alpha-Tocopherol, Beta-Carotene Cancer Prevention Study Group (1994). The effect of vitamin E and beta-carotene on the incidence of lung cancer and other cancers in male smokers. New England Journal of Medicine 330, 1029-1035.

Ascherio, A., Rimm, E.B., Hernan, M.A., Giovanucci, E., Kawachi, I., Stampfer, M.J., Willett, W.C., (1999) Relation of consumption of vitamin $\mathrm{E}$, vitamin $\mathrm{C}$ and carotenoids to risk for stroke among men in the United States. Annals of Internal Medicine 130, 963-970.

COMA (1991). Dietary Reference Values for Food Energy and Nutrients for the United Kingdom. Report of the Panel on Dietary Reference Values, Committee on Medical Aspects of Food and Nutrition Policy. HMSO, London.

Gillilan, R. E., Mondell, B., Warbasse, J.R., (1977) Quantitative evaluation of vitamin E in the treatment of angina pectoris. American Heart Journal 93, 444-449.

GISSI-Prevenzione Investigators (1999). Dietary supplementation with n-3 polyunsaturated fatty acids and vitamin E after myocardial infarction: results of the GISSI-prevenzione trial. Lancet 354, 447-455.

Gregory, J., Foster, K., Tyler, H., Wiseman, M (1990). The Dietary and Nutritional Survey of British Adults. London: HMSO.

Heart Protection Study Collaborative Group (2002). MRC/BHF Heart protection study of antioxidant vitamin supplementation in 20, 536 high-risk individuals: a randomised placebo-controlled trial. Lancet 360, 23-32.

Leppälä, J.M., Virtamo, J., Fogelholm, R., Albanes, D., Taylor, P.R., Heinonen, O.P (2000) Vitamin E and beta-carotene supplementation in high risk for stroke. Archives of Neurology 57, 1503-1509.

Meydani, S. N., Meydani, M., Blumberg, J.B., Leka, L.S., Pedrosa, M., Diamond, R., Schaefer, E.J., (1998) Assessment of the safety of supplementation with different amounts of vitamin $E$ in healthy older adults. American Journal of Clinical Nutrition 68, 311-318.

Primary Prevention Project (2001). Low-dose aspirin and vitamin E in people at cardiovascular risk: a randomised trial in general practice. Lancet 357,89-95.

Stampfer, M.J., Willett, W., Castelli, W.P., Taylor, J.O., Fine, J., Hennekens, C.H., (1983) Effect of vitamin E on lipids. American Journal of Clinical Pathology 79, 714-716.

Stephens, N.G., Parsons, A., Schofield, P.M., Kelly, F., Cheeseman, K., Mitchison, M.J. (1996) Randomised controlled trial of vitamin $\mathrm{E}$ in patients with coronary disease: Cambridge Heart Oxidation Study (CHAOS). Lancet 347, 781-786.

Virtamo, J., Rapola, J.M., Ripatti, S., Heinonen, O.P., Taylor, P.R., Albanes, D., Huttunen, J.K. (1998) Effect of vitamin $\mathrm{E}$ and beta carotene on the incidence of primary nonfatal myocardial infarction and fatal coronary heart disease. Archives of Internal Medicine 158, 668-675.

Yusuf, S., Dagenais, G., Pogue, J.. Bosch, J., Sleight, P. (2000). Vitamin E supplementation and cardiovascular events in high-risk patients. The Heart Outcomes Prevention Evaluation Study Investigators. New England Journal of Medicine 342, 154-160. 


\section{Risk Assessment}

\section{Vitamin K}

\section{General Information}

\section{Chemistry}

Vitamin $\mathrm{K}$ is not a single compound but a group of homologous fat-soluble compounds derived from 2-methyl-1,4-naphthoquinone. Phylloquinone (2-methyl-3-phytyl-1,4-naphthoquinone) is designated as vitamin $\mathrm{K}_{1}$. The menaquinones, collectively known as vitamin $\mathrm{K}_{2}$, are a large series of compounds containing an unsaturated side chain with differing numbers of isoprenyl units at the 3 position in the methyl-1,4-naphthoquinone nucleus. Depending on the number of isoprenyl units the individual compounds are designated as menaquinone- $n-(\mathrm{MK}-n)$. Several synthetic water-soluble compounds containing the 2-methyl-1,4-naphthoquinone structure also exist. These include menadione (vitamin $\mathrm{K}_{3}$ ) and menadiol (vitamin $\mathrm{K}_{4}$ ) (see table).

Table: Summary of the nomenclature of the various forms of vitamin $\mathrm{K}$

\begin{tabular}{|l|l|l|l|}
\hline Vitamin $\mathrm{K}_{1}$ & Name & $\begin{array}{l}\text { Synonyms/chemical name } \\
\text { 2-methyl-3-phytyl-1,4-naphthoquinone } \\
\text { phytomenadione } \\
\text { phytoandione } \\
\text { phytylmenadione }\end{array}$ & Plants \\
\hline Vitamin $\mathrm{K}_{2}$ & Menaquinone & $\begin{array}{l}\text { Menatetranone } \\
\text { Menaquinone K4 } \\
\text { Vitamin MK-4 }\end{array}$ & Gram +ve bacteria \\
\hline Vitamin $\mathrm{K}_{3}$ & Menadione & 2-methyl-1,4-naphthoquinone & Synthetic \\
\hline Vitamin $\mathrm{K}_{4}$ & Menadiol & $\begin{array}{l}\text { Menaquinol } \\
\text { 2-methyl-1,4-naphthoquinol } \\
\text { 2-methyl-1,4-naphthohydroquinone } \\
\text { reduced menadione }\end{array}$ & Synthetic \\
\hline Natural occurrence & & & \\
\hline
\end{tabular}

Vitamin $\mathrm{K}_{1}$ is synthesised by plants. The vitamin $\mathrm{K}_{2}$ series is synthesised by various $\mathrm{Gram}$-positive bacteria present in the jejunum and ileum.

\section{Occurrence in food, food supplements and medicines}

Dietary vitamin $\mathrm{K}$ is largely obtained from green leafy vegetables and vegetable oils, with lesser amounts present in dairy products, meat and eggs. Vitamin $\mathrm{K}$ is also present as $\mathrm{K}_{1}$ and $\mathrm{K}_{2}$ in multi-vitamin food supplements and as $\mathrm{K}_{7}$ and $\mathrm{K}_{4}$ in licensed medicines. Vitamins $\mathrm{K}_{1}$ and $\mathrm{K}_{4}$ are used in the treatment or prophylaxis of haemorrhage as a result of low blood level of prothrombin or Factor VII, malabsorption or use of coumarin anticoagulants.

UK food supplements may contain up to $0.045 \mathrm{mg}$ vitamin $\mathrm{K}$ (either as $\mathrm{K}_{1}$ or $\mathrm{K}_{2}$ ) for general consumption and $0.20 \mathrm{mg}$ in supplements intended for women from pre-conception to nursing. 


\section{Other sources of exposure}

Vitamin $\mathrm{K}$ (as the $\mathrm{K}_{2}$ series) is also provided by the bacterial flora in the intestine, and this contributes significantly towards the daily requirement of the vitamin.

\section{Recommended amounts}

Although vitamin $\mathrm{K}$ is known to be essential, recommendations on adequate nutritional intakes have not been precisely established, because of the unquantified contribution made by the intestinal bacteria. COMA considered that an intake of $0.001 \mathrm{mg} / \mathrm{kg}$ bw/day was probably adequate with regard to the coagulation function of vitamin $\mathrm{K}$ and noted that synthetic preparations of $\mathrm{K}_{3}$ were best avoided for nutritional purposes, due to their link with haemolysis and liver damage in the newborn (COMA, 1991).

The Infant Formula and Follow-on Formula regulations (1995) recommend a minimum vitamin $\mathrm{K}$ intake of $0.004 \mathrm{mg} / 100 \mathrm{kcal}$.

\section{Analysis of tissue levels and vitamin $\mathrm{K}$ status}

Functional tests of blood clotting were initially used to assess vitamin $\mathrm{K}$ status. However, a radioimmunoassay which measures the ratio of prothrombin to partially carboxylated prothrombin in plasma (the latter is formed during vitamin $\mathrm{K}$ deficiency) is now available. Measurement of the carboxylation of serum osteocalcin, and plasma vitamin $\mathrm{K}_{1}$ are also used to determine vitamin $\mathrm{K}$ status. However, the measurement of plasma vitamin $\mathrm{K}_{1}$ is of limited value because it reflects recent dietary intake.

\section{Brief overview of non-nutritional effects}

Vitamin $\mathrm{K}$ (in particular, the water-soluble form) has been reported to potentiate the analgesic effect of opiates and salicylates, and induce radiosensitisation.

\section{Function}

Vitamin $\mathrm{K}$ catalyses the carboxylation of a number of protein factors involved in blood clotting including prothrombin, forming the calcium binding sites on glutamyl side chains in the protein. Once carboxylated, the glutamates are referred to as gamma-carboxyglutamic acid (GLA).

GLA-containing proteins are also found in the bone. For example, bone GLA protein (or osteocalcin) is thought to be involved in the limitation of bone growth, and matrix GLA protein may be involved in mobilisation and deposition of bone calcium.

Kidney GLA proteins may be involved in reabsorption of calcium by the kidney tubules and solubilisation of calcium salts in urine.

GLA-containing proteins have also been found in the placenta, pancreas, spleen and lungs, but the majority of these have not yet been characterised.

Vitamin K-dependent proteins are also thought to have roles in cell signalling and brain lipid metabolism. 
Part 2 Fat Soluble Vitamins

\section{Deficiency}

Vitamin $\mathrm{K}$ is widely available from the diet and is also provided by gut bacteria. Thus, deficiency is generally secondary to conditions such as malabsorption or impaired gut synthesis. Hospitalised patients can be at risk of vitamin $\mathrm{K}$ deficiency following the use of antibiotics or gastrointestinal surgery. Newborn babies have low levels of vitamin $\mathrm{K}$, which may result in haemorrhagic disease of the newborn.

\section{Interactions}

It has been shown experimentally that excessive vitamin $\mathrm{E}$ exposure results in increased prothrombin times which are normalised by vitamin $K_{2}$. The effects of vitamin $K_{1}$ are also antagonised by excessive oral intakes of vitamin A.

Vitamin $\mathrm{K}_{1}$ interacts with coumarin anticoagulants. The effect of 2-methyl-1,4-napthoquinone (vitamin $\mathrm{K}_{3}$ ) is antagonised by actinomycin $\mathrm{D}$. The carcinogenic activity of benzo(a)pyrene has been shown to be increased by vitamin $K_{1}$, as a result of induction of cytochrome P450 1A1 (CYPIAT). In contrast, vitamin $K_{3}$ decreased CYPIA1 activity.

\section{Absorption and bioavailability}

Vitamin $\mathrm{K}$ is readily absorbed, but there are conflicting reports on whether this is higher from supplements or from a food matrix. The bioavailability of vitamin $\mathrm{K}_{1}$ is the same from raw and cooked broccoli and is unaffected by the fat content of the meal.

Absorption of vitamin $\mathrm{K}_{1}$ takes place in the proximal small intestine by a saturable, energy dependent process. Absorption of bacterial menaquinones $\left(\mathrm{K}_{2}\right)$ occurs in the terminal ileum by passive diffusion. Absorption of vitamin $\mathrm{K}$ is enhanced by bile salts and pancreatic juice. Overall, $40-70 \%$ of vitamin $\mathrm{K}$ may be absorbed in the jejunum, but absorption is lower elsewhere in the digestive system.

Vitamin $\mathrm{K}_{3}$ is believed to be absorbed by passive diffusion in the distal intestine and colon.

\section{Distribution and metabolism}

Vitamin $\mathrm{K}$ is transported via the lymph in chylomicrons and concentrated in the liver prior to wide distribution. The body pool of vitamin $\mathrm{K}$ is low. The metabolism of vitamins $\mathrm{K}_{1}$ and $\mathrm{K}_{2}$ involves the formation of an epoxide and a quinone, which can then be reduced by $\mathrm{NAD}(\mathrm{P}) \mathrm{H}$ reductases. The reduced form is involved in carboxylation reactions. The carboxylic acid derivatives of vitamin $\mathrm{K}_{1}$ are conjugated with glucuronic acid. In rats, vitamin $\mathrm{K}_{1}$ can be converted to $\mathrm{K}_{2}$ in the tissues.

Vitamin $\mathrm{K}_{3}$ is thought to be metabolised by conjugation with sulphate, phosphate and glucuronide.

\section{Excretion}

Approximately $30-40 \%$ of vitamin $\mathrm{K}$ is excreted, via the bile, in the faeces as partially degraded, conjugated water-soluble metabolites. Smaller quantities (approximately 15\%) are excreted in the urine as water-soluble metabolites. 


\section{Toxicity}

\section{Human data}

There are relatively few reports of human toxicity following ingestion.

High doses of water-soluble vitamin $\mathrm{K}_{3}$ (menadione) may result in oxidative damage, red cell fragility and the formation of methaemoglobin. High doses of vitamin $\mathrm{K}_{3}$ given to premature infants to treat intracranial and pulmonary haemorrhage have been reported to have caused hyperbilirubinaemia and overloading of the immature liver, resulting in kernicterus and brain toxicity. Fewer cases have been reported recently as a safer level has been established.

Local hypersensitivity reactions to dermal injections of vitamin $\mathrm{K}$ (mostly of the $\mathrm{K}_{1}$ form) have been reported. They are thought to be delayed-type hypersensitivity reactions.

\section{Animal data}

Acute doses of vitamin $\mathrm{K}_{1}$ (phylloquinone) up to $25,000 \mathrm{mg} / \mathrm{kg}$ bw caused no fatalities in rats, mice or chicks.

In contrast, oral vitamin $\mathrm{K}_{3}$ (menadione) administration to rats, chicks and mice resulted in anaemia and death. Parenteral administration of high doses of $\mathrm{K}_{3}$ to dogs caused methaemoglobinaemia, urobilinuria and urobilinogenuria, and fatal liver damage.

No data on reproductive toxicity have been identified.

\section{Carcinogenicity and genotoxicity}

No data on carcinogenicity have been identified.

Vitamin $\mathrm{K}_{1}$ was negative in the Ames bacterial mutagenicity test, but there are conflicting reports of its ability to cause sister chromatid exchange in human and animal leukocytes.

\section{Mechanisms of toxicity}

Menadione causes oxidative damage and lipid peroxidation.

\section{Dose response characterisation}

No data have been identified. 
Part 2 Fat Soluble Vitamins

\section{Vulnerable groups}

Individuals with glucose-6-phosphate dehydrogenase deficiency are more susceptible to the development of methaemoglobinaemia, and this would include oxidative damage caused by menadione (vitamin $\mathrm{K}_{3}$ ). Infants are vulnerable to vitamin $\mathrm{K}_{3}$ toxicity. An initial suggestion that intramuscular administration of vitamin $\mathrm{K}$ was associated with childhood cancer has not been supported in further studies.

\section{Genetic variations}

No data have been identified.

\section{Studies of particular importance in the risk assessment}

(For full review see http://www.food.gov.uk/science/ouradvisors/vitandmin/evmpapers or the enclosed CD).

\section{Human data}

Israels et al., 1987

Sister chromatid exchange (SCE) was measured in human leukocytes taken from adult and placental blood. In the presence of $1 \mu \mathrm{M}$ vitamin $\mathrm{K}_{1}$, the mean number of SCEs per metaphase increased significantly from $3.32 \pm 0.219$ in placental blood to $5.76 \pm 0.219$ and from $5.13 \pm 0.273$ to $7.81 \pm 0.326$ in adult blood. Co-incubation with vitamin $\mathrm{K}_{1}$ did not affect the number of SCEs caused by benzpyrene or mitomycin C.

Cornelissen et al., 1991

The numbers of SCEs were measured in the peripheral blood lymphocytes of six newborn babies 24 hrs after intra-muscular injection with $1 \mathrm{mg}$ vitamin $\mathrm{K}_{1}$ (as the drug Konakion) and in six control neonates. The mean number of SCEs per metaphase was $8.88+1.22$ in the vitamin $K_{1}$ group compared to $9.05 \pm 1.14$ in the controls. The mean number of chromosome aberrations per 100 mitoses was $3.00 \pm 2.61$ in the vitamin $\mathrm{K}$ group compared to $2.50 \pm 1.87$ in the controls. Plasma vitamin $\mathrm{K}_{1}$ concentration ranged from 0.0255 to $2.55 \mu \mathrm{M}$. The authors concluded that there was no evidence that vitamin $\mathrm{K}_{1}$ treatment caused genotoxicity. However, it is possible that taking the sample 24 hours after treatment may have been too early to detect SCEs.

\section{Craciun et al., 1998}

Eight elite female athletes were given $10 \mathrm{mg} /$ day vitamin $\mathrm{K}_{1}$ for one month and markers of bone health were assessed before and after treatment. Four of the eight athletes had been amenorrhoeic for more than 1 year whilst the others had been taking oral contraceptives. The athletes' dietary vitamin $\mathrm{K}$ intake was stated to be in excess of the $0.001 \mathrm{mg} / \mathrm{kg}$ bw/day RDA value. At baseline, the amenorrhoeic group was biochemically vitamin $\mathrm{K}$ deficient as assessed by the calcium binding activity of circulating osteocalcin. In all subjects, supplementation was associated with an increase in the calcium binding capacity of osteocalcin, indicating correction of a deficiency. In the amenorrhoeic group 
supplementation was associated with a $15-20 \%$ increase in bone formation markers and a $20-25 \%$ decrease in bone resorption markers. No adverse effects were noted at $10 \mathrm{mg} /$ day.

Booth et al., 1999

In a trial in a metabolic unit using a $3 \times 15$ day crossover design, both younger and older male and female subjects were fed a mixed diet containing $0.10 \mathrm{mg}$ vitamin $\mathrm{K}_{1}$ per day. During two of the 15-day residency periods, the diet was supplemented with either broccoli $\left(0.377 \mathrm{mg}\right.$ total $\mathrm{K}_{1}$ per day) or $\mathrm{K}_{1}$ fortified oil (0.417 $\mathrm{mg}$ total $\mathrm{K}_{1}$ per day). No adverse effects were noted at 0.377-0.417 $\mathrm{mg}$ total $\mathrm{K}_{1}$ per day.

\section{Animal data}

\section{Molitor and Robinson, 1940}

Toxicity studies were conducted in mice, chicks and rats. Vitamin $K_{1}$ in single oral doses up to 25,000 $\mathrm{mg} / \mathrm{kg}$ bw, produced no fatalities in mice, whereas vitamin $\mathrm{K}_{3}$ was highly toxic. Intraperitoneal studies showed that doses of $200 \mathrm{mg} / \mathrm{kg}$ bw of vitamin $\mathrm{K}_{3}$ caused $100 \%$ mortality in mice but intraperitoneal doses of vitamin $\mathrm{K}_{1}$ as high as $25,000 \mathrm{mg} / \mathrm{kg}$ bw failed to cause death. In sub-chronic studies in rats, daily feeding over a 30 day period of $500 \mathrm{mg} / \mathrm{kg}$ bw of vitamin $\mathrm{K}_{3}$ was fatal and smaller doses resulted in pronounced anaemia. Daily doses as high as $2000 \mathrm{mg} / \mathrm{kg}$ bw vitamin $\mathrm{K}_{1}$ produced no ill effects.

Israels et al., 1987

Five foetal sheep were given a $1 \mathrm{mg}$ dose of vitamin $\mathrm{K}_{1}$ into the femoral vein via a catheter. The mean number of SCEs per metaphase increased from $3.94 \pm 0.15$ at 15 minutes pre-injection to $5.38 \pm 0.23$ at 24 hours post-injection. Before treatment the level of vitamin $\mathrm{K}_{1}$ was not detectable, but increased to $0.3 \mu \mathrm{M}$ after treatment. The increase in SCE was low but was stated to be statistically significant. No positive controls were used and it is difficult to assess the biological importance of the result.

The in vitro dose response curve for SCE induction was investigated in both foetal and adult sheep leukocytes incubated with vitamin $\mathrm{K}_{1}$. The doses tested ranged from $0.1 \mathrm{nM}$ to $1 \mu \mathrm{M}$. At $0.1 \mathrm{nM}$ the number of SCEs in the adult cells (from the dam) were not significantly different from the solvent control. However, the number of SCEs in the foetal cells was significantly increased compared to the controls. The increase in SCEs in the adult cells became significant at $10 \mathrm{nM}$. The actual numbers of SCEs at different dose levels were not given but the figure indicates that SCEs in adults increased from approximately 6 to 9 per metaphase and in the foetus from 4.3 to 7.5 per metaphase.

\section{Edenharder et al., 1999}

Vitamins $\mathrm{K}_{1}, \mathrm{~K}_{3}, \mathrm{~K}_{4}$ and 1,4 naphthoquinone caused a concentration-related reduction in the mutagenicity of six heterocyclic amines in the Ames Salmonella assay. The mechanism for the anti-mutagenic effect was related to reduced activities of 7-ethoxyresorufin-O-deethylase (EROD) and 7-methoxyresorufin-Odemethylase (MROD), markers for cytochrome P450 1A1 and 1A2 activity. In further enzyme kinetic experiments, vitamins $\mathrm{K}_{3}$ and $\mathrm{K}_{4}$ behaved as competitive inhibitors of 2-amino-3-methyl-imidazo[4,5- $f$ ] quinoline induced mutagenesis. The mechanism of the anti-mutagenic effect of vitamin $\mathrm{K}_{1}$ was not investigated. 
Part 2 Fat Soluble Vitamins

\title{
Exposure assessment
}

\author{
Total Exposure/intake:
}

Food

Mean: 0.068 mg/day (based on National Food Survey 2000, DEFRA, 2001). Data for 97.5 th percentile not available.

Supplements

up to $0.20 \mathrm{mg} /$ day (Annex 4; OTC, 2001)

Estimated maximum intake: $0.068+0.20=0.27 \mathrm{mg} /$ day

No potential high intake groups have been identified.

\section{Risk assessment}

The data are sparse but the different forms of vitamin $\mathrm{K}$ are associated with different adverse effects. There are relatively few reports of human toxicity for vitamin $\mathrm{K}_{1}$ (phylloquinone) and it is stated to be well tolerated in animal studies. Although there are some reports of genotoxicity in vitro and in vivo, no effect was found in cells taken from babies given prophylactic intramuscular vitamin $\mathrm{K}_{1}$. The significance of the positive genotoxicity findings is uncertain.

High doses of vitamin $\mathrm{K}_{3}$ (menadione) may result in oxidative damage, red cell fragility and the formation of methaemoglobin. Hyperbilirubinaemia, resulting in kernicterus and toxicity to the neonatal brain occurred in premature infants given high doses of vitamin $\mathrm{K}_{3}$. Local hypersensitivity reactions to injections have been reported. In animal studies, vitamin $\mathrm{K}_{3}$ administration has resulted in anaemia, haemoglobinaemia, urobilinuria and urobilinogenuria. High doses have also been reported to cause liver damage.

Vitamin $\mathrm{K}_{3}$ has demonstrated some mutagenic activity in the Ames test, possibly as a result of the structure of the side chain.

Few data are available relating to vitamins $\mathrm{K}_{2}$ and $\mathrm{K}_{4}$.

\section{ESTABLISHMENT OF GUIDANCE LEVEL}

There are insufficient data from studies in humans or animals to establish a Safe Upper Level for vitamin $\mathrm{K}$. There are clear differences in the toxicity of different forms of vitamin $\mathrm{K}$.

Vitamin $\mathrm{K}_{1}$ (phylloquinone) the form occurring naturally in food is not associated with adverse effects at high doses in animal studies. Acute doses up to $25,000 \mathrm{mg} / \mathrm{kg}$ bw did not cause fatalities in rats, mice or chicks. Human supplementation studies in small numbers of subjects suggest that, although having biological activity, doses of up to $10 \mathrm{mg} /$ day for 1 month are not associated with adverse effects. Applying an uncertainty factor of 10 for inter-individual variation because of the very limited human database, indicates that for guidance purposes, a daily supplementary intake of $1 \mathrm{mg} /$ day would be 
unlikely to result in adverse effects. This is equivalent to $0.017 \mathrm{mg} / \mathrm{kg}$ bw in a $60 \mathrm{~kg}$ adult. There are insufficient data available to provide guidance on total intakes.

The water soluble forms of vitamin $\mathrm{K}$, particularly $\mathrm{K}_{3}$ (menadione) are more active and can be toxic at high doses in both humans and animals. Use of menadione in food supplements is therefore undesirable.

\section{References}

Booth, S. L., O'Brien-Morse, M.E., Dallal, G.E., Davidson, K.W. and Gundberg, C.M. (1999). Response of vitamin $\mathrm{K}$ status to different intakes and sources of phylloquinone-rich foods: comparison of younger and older adults. American Journal of Clinical Nutrition 70, 368-377.

COMA (1991). Dietary Reference Values for Food Energy and Nutrients for the United Kingdom. Report of the Panel on Dietary Reference Values, Committee on Medical Aspects of Food and Nutrition Policy. HMSO, London.

Cornelissen, M., Smeets, D., Merkx, G. De Abreu. R, Kollee, L and Monnens, M. (1991). Analysis of chromosome aberrations and sister chromatid exchange in peripheral blood lymphocytes of newborns after vitamin K prophylaxis at birth. Pediatric Research 30, 550-553.

Craciun, A. M., Wolff, J, Knapen M.H.J., Brouns, F. and Vermeer, C. (1998). Improved bone metabolism in female elite athletes after vitamin K supplementation. International Journal of Sports Medicine 19 , 479-484.

Edenharder, R, Worf-Wandelburg A, Decker, M. and Platt, KL. (1999). Antimutagenic effects and possible mechanisms of action of vitamins and regulated compounds against genotoxic heterocyclic amines from cooked food. Mutation Research 444, 235-248.

Israels, L. G., Friesen, E., Jansen, A.H. and Israels, E.D. (1987) Vitamin $\mathrm{K}_{1}$ increases sister chromatid exchange in vitro in human leukocytes and in vivo in fetal sheep cells: A possible role for 'vitamin K deficiency' in the fetus. Pediatric Research 22, 405-408.

Molitor, H. and Robinson, H.J. (1940) Oral and parenteral toxicity of vitamin $\mathrm{K}_{\mathrm{r}}$, phthicol and 2-methyl-1,4naphthoquinone. Proceedings of the Society for Experimental Biology and Medicine 43, 125-128. 
Part 3

Trace elements 


\section{General information}

\section{Chemistry}

Boron is a non-metallic element, which is found abundantly in nature, though only in compounds and in combination with sodium and oxygen. Examples of compounds containing boron are borax $\left(\mathrm{Na}_{2} \mathrm{~B}_{4} \mathrm{O}_{7}, 10 \mathrm{H}_{2} \mathrm{O}\right)$ and boric acid $\left(\mathrm{H}_{3} \mathrm{BO}_{3}\right)$. Within this risk assessment the word boron is used as a generic term for boron compounds. For comparative purposes doses of boron compounds are expressed as boron equivalents.

\section{Natural occurrence}

Boron is found (in the form of borates) in the oceans, rocks, coal, shale and some soils. Boron accumulates in plants.

\section{Occurrence in foods, food supplements and medicines}

Boron is present in foods, particularly nuts $(14 \mathrm{mg} / \mathrm{kg})$, fresh fruits $(3 \mathrm{mg} / \mathrm{kg})$ and green vegetables $(2 \mathrm{mg} / \mathrm{kg})$. Boron is present in a number of multi-vitamin and mineral food supplements at levels up to $10 \mathrm{mg}$, but not in licensed medicines in the UK.

\section{Other sources of exposure}

Intakes of boron from drinking water are estimated to be 0.2-0.6 mg/day. Exposure from cosmetics and consumer products (where boron is used as a preservative and $\mathrm{pH}$ adjuster) has been estimated to be up to $0.47 \mathrm{mg} /$ day.

\section{Recommended amounts}

COMA was unable to make specific recommendations for boron intake. The World Health Organisation (WHO) acceptable range of intake is 1-13 mg boron/day for adults.

\section{Analysis of tissue levels and boron status}

The preferred methods for analysis of boron in bone and plasma are inductively coupled plasma atomic emission spectroscopy and more commonly inductively coupled plasma mass spectrometry. Blood and urine measurements of boron are suitable indicators of excessive boron intake.

\section{Brief overview of non-nutritional beneficial effects}

It has been claimed that boron alleviates some symptoms of osteoarthritis and rheumatoid arthritis. 


\section{Function}

Boron is an essential element in plants and is presumed to be essential in animals, since boron deprivation in both experimental animals and humans causes changes in biological function which are reversible by restoration of boron. The function of boron is unknown, but it may be involved in metabolism and utilisation of various elements (including calcium, copper, magnesium), glucose, triglycerides, reactive oxygen and oestrogen.

\section{Deficiency}

Boron deficiency has not been observed definitively in human populations. However, it has been suggested that boron deficiency could be a contributing factor to Kashin-Beck disease (KBD), a musculoskeletal deficiency, which may cause severe joint deformity. Kashin-Beck disease affects children in China and the former Soviet Union, and has also been linked to selenium deficiency. A crosssectional survey in China found significantly lower boron levels in the hair of children with KBD than in that of local children without KBD. Other authors have reported that short periods of restricted boron intake may affect brain function and cognitive performance in healthy humans.

In animals, boron deficiency is associated with symptoms which include reduced growth rate and decreased blood steroid hormone levels.

\section{Interactions}

Studies in animals and in humans have demonstrated that boron interacts with the metabolism of calcium, reducing its rate of excretion and increasing serum levels, but the mechanism is uncertain.

\section{Absorption and bioavailability}

Boron as borate is readily, and almost completely, absorbed from the human gut. The mechanism has not been defined.

\section{Distribution and metabolism}

Boron, as borate, is distributed evenly, via passive diffusion, throughout body fluids. The highest levels are found in bone, which possibly represents a second kinetic compartment, as elimination kinetics for bone differ from those of soft tissue and body fluids. It has been shown that boron compounds can cross the human placenta.

Boron compounds are not metabolised in biological systems.

\section{Excretion}

Boron is predominantly excreted in the urine as borate. 


\section{Toxicity}

\section{Human data}

Data from accidental poisonings indicate that the acute, lethal dose of boric acid is $3000-6000 \mathrm{mg}$ for infants and 15,000-20,000 mg for adults. Clinical effects include irritability, seizures and gastrointestinal disturbances. There have also been reports of inflammation, congestion, oedema, exfoliative dermatitis, exfoliation of the mucosa, and findings of cloudy swelling and granular degeneration of renal tubular cells. Clinical symptoms of boron toxicity have been reported within the dose range 100 to $55,500 \mathrm{mg}$ depending on age/body weight. Inter-individual variability appears to be high.

Long-term exposure to boron in animal studies has been reported to affect male fertility. However, borax miners had a non-significant excess of births compared to the general population and a nonsignificant excess of female births compared to males in a study of 542 occupational workers in a borax mine (Whorton et al., 1994). No effects on fertility were apparent in a Turkish population exposed to boron in drinking water at levels up to $29 \mathrm{mg} / \mathrm{L}$.

\section{Supplementation studies}

No adverse effects were identified in female volunteers supplemented with $3 \mathrm{mg}$ boron/day for 1 year (Meacham et al., 1994). However, $10 \mathrm{mg}$ boron/day for 4 weeks increased plasma oestradiol and testosterone levels in adult male volunteers (Naghii and Samman, 1997). Plasma oestradiol was also increased in peri-menopausal women supplemented with $2.5 \mathrm{mg}$ boron/day for 60 days (Nielsen and Penland, 1999).

\section{Animal data}

Boron compounds have moderate acute toxicity, with lethal doses in the range 400-900 $\mathrm{mg}$ boron equivalents $/ \mathrm{kg}$ body weight. Symptoms and signs include depression, ataxia, convulsions and weight loss. Damage to the kidneys and nerve cells were observed on histopathological examination.

Long-term exposure to boron has been associated with reduced body weights and decreased pancreas, spleen and kidney weights.

Boron has been shown to cause specific adverse effects in the male reproductive tract in all species, including shrunken scrota, inhibited spermiation and degeneration of seminiferous tubules with loss or absence of germ cells. However, mating behaviour in the animals was unaffected. Developmental toxicity has been reported following administration of boron to pregnant rats and rabbits.

\section{Carcinogenicity and genotoxicity}

There is no evidence of carcinogenicity from oral exposure to boron compounds in rats and mice. Boron compounds are not mutagenic in bacterial or in vitro mammalian cell mutation assays. 


\section{Mechanism of toxicity}

Boric acid may affect DNA synthesis in germ cells and energy metabolism in Sertoli cells, possibly affecting the production of early germ cells. The mechanism by which boron inhibits spermiation is uncertain.

\section{Dose-response characterisation}

In humans, the lethal dose of boric acid is $3000-6000 \mathrm{mg}$ for infants and $15000-20000 \mathrm{mg}$ for adults. Clinical symptoms are apparent within the dose range 100 to $55500 \mathrm{mg}$ depending on age/body weight.

\section{Vulnerable groups}

Pregnant women are a potential vulnerable group, as indicated by results of animal reproductive studies.

\section{Genetic variations}

No genetic variations have been identified.

\section{Studies of particular importance in the risk assessment}

(For full review see http://www.food.gov.uk/science/ouradvisors/vitandmin/evmpapers or the enclosed CD).

\section{Weir and Fisher, 1972}

Ten Sprague Dawley rats of each sex per group were fed diets containing borax or boric acid for 90 days, at levels providing 2.6, 8.8, 26.3, 87.5 or $263 \mathrm{mg}$ boron $/ \mathrm{kg}$ bw/day. At the highest dose $100 \%$ mortality occurred. At $87.5 \mathrm{mg}$ boron $\mathrm{kg} / \mathrm{bw} /$ day, liver, kidney, spleen, testes, ovaries and body weights were decreased. Histological changes were observed in the adrenals at $87.5 \mathrm{mg}$ boron $/ \mathrm{kg}$ bw $/$ day. Testicular atrophy occurred at 26.3 and $87.5 \mathrm{mg} / \mathrm{kg}$ bw/day. Detailed results were provided for the $87.5 \mathrm{mg} / \mathrm{kg}$ bw/day dose group only.

Thirty five Sprague Dawley rats/sex/group were fed diets containing borax or boric acid for 2 years at levels providing 5.9, 17.5 and $58.5 \mathrm{mg}$ boron/kg bw/day. Growth rate was decreased, absolute and relative testes weights were decreased and relative brain and thyroid weights were increased at the top dose. Histological changes in the testes were observed in the top dose group. No other effects were apparent. Limited results were reported.

Five beagle dogs/group were fed diets containing borax or boric acid for 90 days, at levels providing $0.44,4.4$ or $44 \mathrm{mg}$ boron/ $\mathrm{kg}$ bw/day. In the group given the highest dose, one death occurred, histological changes in the adrenals and thyroid, and severe testicular atrophy were observed, and whereas relative weights of thyroid and testes were reduced, those of liver and brain were increased. 
Four beagle dogs of each sex per group were fed diets containing borax or boric acid for 2 years, at levels providing 1.45, 2.93 and $8.75 \mathrm{mg}$ boron $/ \mathrm{kg}$ bw/day. No treatment related effects were found. Limited results were reported in the paper. In a second study, additional groups were given $29.25 \mathrm{mg}$ boron $/ \mathrm{kg}$ bw/day for 38 weeks. Histological changes in the testes were apparent, but (based on one animal) were reversible when treatment was stopped.

Eight male and sixteen female Sprague Dawley rats/group were fed diets containing borax or boric acid for 14 weeks prior to mating and for 3 subsequent generations. The doses were provided 5.9, 17.5 and $58.5 \mathrm{mg}$ boron $/ \mathrm{kg}$ bw/day. No adverse effects were reported in the low and mid dose groups. The high dose animals were, however, sterile when mated with control animals. Decreased ovulation and lack of viable sperm were apparent. Limited results were provided in the paper.

\section{Heindel et al., 1992}

Feed containing boric acid at levels providing 13.6, 28.5, $57.7 \mathrm{mg}$ boron $/ \mathrm{kg}$ bw/day was given to pregnant Sprague Dawley rats throughout gestation (an additional group received $94.2 \mathrm{mg}$ boron $/ \mathrm{kg} \mathrm{bw} /$ day on days 6-25 only). Decreased body weight gain was found in the dams in the 57.7 and $94.2 \mathrm{mg}$ boron $/ \mathrm{kg}$ bw/day dose groups and increased liver and kidney weights at $28.5 \mathrm{mg}$ boron $/ \mathrm{kg}$ bw/day and above. Average foetal body weight/litter was reduced in the offspring of the dams in all dose groups. Pre-natal mortality was increased in the $94.2 \mathrm{mg}$ boron $/ \mathrm{kg}$ bw/day group only. The incidence of malformed foetuses was increased at $28.5 \mathrm{mg}$ boron/ $\mathrm{kg}$ bw/day and above. The malformations and anatomical variations included enlarged lateral ventricles of the brain and shortening of rib XIII. The authors noted that developmental toxicity was occurring at lower doses than maternal toxicity.

Feed containing boric acid was given to groups of 10 pregnant Swiss mice throughout gestation. The average doses provided by the feed were $43.3,79.0$ or $175 \mathrm{mg}$ boron/ $\mathrm{kg}$ bw. Minor renal lesions were found in the dams at all doses, and decreased body weights and increased relative kidney weights at the highest dose. In the mice given the highest dose, the number of resorptions and malformed foetuses was increased, and foetal body weights were reduced in offspring. The malformations and anatomical variations included an increased incidence of short rib XIII and a decreased incidence of rudimentary or full ribs at lumbar 1 , and were present at all dose levels. The authors argued that since the renal lesions were minor and occurred in 2/10 mice in the low dose group compared with $8 / 10$ and 10/10 in the higher dose groups, that this dose level approached a NOAEL for maternal toxicity. Thus, as with the rat, developmental toxicity was occurring at lower doses than maternal toxicity.

\section{Price et al., 1996}

This study is a follow up to the Heindel et al. rat study and was designed to establish a NOAEL. Groups of 60 pregnant female Sprague Dawley rats were given feed containing boric acid throughout gestation. The average doses provided in the feed were $0,3.3,6.4,9.6,13.1$ or $25.2 \mathrm{mg}$ boron $/ \mathrm{kg}$ bw/day. The only maternal effect observed was increased relative kidney weight at the $25.2 \mathrm{mg}$ boron $/ \mathrm{kg} \mathrm{bw} / \mathrm{day}$ level. There was no effect on pre-natal mortality. Foetal body weights were reduced in the offspring of the 13.1 and $25.2 \mathrm{mg}$ boron $/ \mathrm{kg}$ bw/day groups but had recovered by birth. The incidence of short rib XIII was increased in foetal rats at 13.1 and $25.2 \mathrm{mg}$ boron $/ \mathrm{kg}$ bw/day, but at $25.2 \mathrm{mg}$ boron $/ \mathrm{kg}$ bw $/$ day only in pups of 21 days of age. The incidence of wavy rib was also increased in foetal rats at 13.1 and $25.2 \mathrm{mg}$ boron $/ \mathrm{kg}$ bw/day but was reversed in pups of 21 days of age. A slight decrease in the incidence of extra lumbar ribs was observed at $25.2 \mathrm{mg}$ boron $/ \mathrm{kg}$ bw/day in the day 20 foetuses. The authors concluded that the NOAEL was $9.6 \mathrm{mg}$ boron/kg bw/day for foetal effects and $13.1 \mathrm{mg}$ boron $/ \mathrm{kg}$ bw/day for 
maternal toxicity. Developmental toxicity was noted to be occurring at lower doses than maternal toxicity. This is a well-reported study and is consistent with findings of Heindel et al.

\section{Exposure assessment}

Total exposure/intake:

Food

Mean: $1.5 \mathrm{mg} /$ day (1994 TDS).

97.5th percentile: $2.6 \mathrm{mg} /$ day

Water

$0.2-0.6 \mathrm{mg} /$ day (IPCS 1998)

Supplements

up to $10 \mathrm{mg} /$ day (Annex 4)

Cosmetics and

consumer products

up to $0.47 \mathrm{mg} / \mathrm{day}$

Maximum estimated intake: $2.6+0.6+10+0.47=14 \mathrm{mg} /$ day

Vegetarians have been identified as a potential high intake group.

\section{Risk assessment}

In animals, the main toxic effect associated with boron involves the reproductive system. Boron caused specific adverse effects in the male reproductive tract in rats, mice and dogs, including shrunken scrota, inhibited spermiation and degeneration of seminiferous tubules with loss or absence of germ cells. Boron also caused a reduction in ovulation in female rats and renal lesions in female mice. Boron also causes developmental toxicity, with foetal malformations occurring in mice and rats at levels lower than those associated with maternal toxicity.

The human data are scarce, although a study in two Turkish populations (Sayli et al., 1998a) demonstrated that higher levels of boron in well water appeared not to affect reproduction. Similarly, Whorton et al. (1994) examined the standardised birth ratio (SBR) in US borate workers. No effects on fertility were apparent (the SBR was increased compared to the general population), a small but insignificant excess of female births was found in both studies. Neither study investigated more sensitive markers of reproductive toxicity or the effects of boron on neonatal body weight, this being the limiting toxicity in the animal studies.

The human epidemiology data are negative but could be compatible with the available animal data since they involved lower exposure levels than used in the animal studies. However, limited conclusions can be drawn from these studies due to the relative insensitivity of the end-points measured. There was no effect on fertility, but more subtle effects on sperm quality (which could be significant in sub-fertile men) or on other aspects of reproductive health could not be ruled out. The supplementation trial by Naghii and Samman, found that $10 \mathrm{mg}$ boron/day for 4 weeks significantly increased plasma oestradiol 
levels in male volunteers. The clinical significance of the increase is uncertain. The study is of interest, but limited due to the small numbers involved and the lack of follow up. Other studies have not demonstrated this effect but lower doses of boron were used. Boron has also been reported to enhance the effects of oestrogen in post-menopausal women taking oestrogen therapy. Further work on the effects of boron on the endocrine system is therefore desirable.

\section{ESTABLISHMENT OF SAFE UPPER LEVEL}

Key Study:

NOAEL:

Uncertainty

Factor:

Safe Upper Level for daily consumption over a lifetime:
Price et al. (1996).

$9.6 \mathrm{mg}$ boron/kg bw/day.

10 for inter-species variation $\times 6$ for inter-individual variation in humans $=60$

$9.6 / 60=0.16 \mathrm{mg}$ boron $/ \mathrm{kg} \mathrm{bw} /$ day (equivalent to $9.6 \mathrm{mg}$ boron/day for a $60 \mathrm{~kg}$ adult).

The key adverse effect associated with boron is reproductive toxicity. However, the human data are limited regarding reproductive toxicity and so the SUL is based on animal studies. Reproductive toxicity is apparent in laboratory animals, with characteristic effects being produced in the testes. A clear doseresponse relationship has been demonstrated. The NOAELs for reproductive effects are $4.4 \mathrm{mg}$ boron $/ \mathrm{kg}$ bw/day in dogs and $9.6 \mathrm{mg}$ boron $/ \mathrm{kg}$ bw/day in rodents. Similar effects are observed in different animal species.

A Safe Upper Level can be derived from animal data with the appropriate use of uncertainty factors. Although the Weir and Fisher study resulted in the lower NOAEL of $4.4 \mathrm{mg} / \mathrm{kg} b w /$ day, the number of animals in this study was low and the differences between the dose levels tested were large, so that the lowest dose at which adverse effects were observed was ten times this level. Considering the totality of the data, the highest intake without significant adverse effects (the NOAEL) is $9.6 \mathrm{mg} / \mathrm{kg}$ bw/day, based on the study of Price et al. (1996) and so this has been used to establish an SUL. The usual uncertainty factor of 10 for differences between animals and humans was used to allow for the known differences in renal clearance of boron between animals and humans, and to allow for the possibility that human tissues may be inherently more sensitive than rat tissues. The factor of 10 for inter-individual variability in humans was revised to a chemical-specific adjustment factor of 6 as proposed by Dourson et al. (1998). This comprises a factor of 1.8 to allow for glomerular filtration in some women being 2 standard deviations below the average, and a factor of 3.1 to allow for possible, but undefined, inter-subject variability in tissue sensitivity. Pregnant women are a potential vulnerable group given the effects of boron on foetal weights. However, the critical animal studies cover this endpoint and there appears to be no need for an additional uncertainty factor. This results in a safe upper level for total intake of $9.6 \mathrm{mg}$ boron/day for a $60 \mathrm{~kg}$ adult. This is higher than the present maximum UK intake from food, water and consumer products (estimated to be up to $3.7 \mathrm{mg}$ boron/day), suggesting a margin of $6 \mathrm{mg} /$ day for supplemental intake. 


\section{References}

Dourson, M., Maier, A., Meek, B., Renwick, A., Ohanian, E., Poirier, K. (1998) Re-evaluation of toxicokinetics for data-derived uncertainty factors. Biological Trace Element Research 66, 453-463.

Heindel, J.J, Price, C.J., Field, E.A., Marr, M.C., Myers, C.B., Morrissey, R.E., Schwetz, B.A. (1992)

Developmental toxicity of boric acid in mice and rats. Fundamental And Applied Toxicology 18, 266-277.

IPCS (1998) Environmental Health Criteria 204: Boron. World Health Organization, Geneva, Switzerland.

Meacham, S.L., Taper, L.J., Volpe, S.L. (1994) Effects of boron supplementation on bone mineral density and dietary, blood, and urinary calcium, phosphorus, magnesium, and boron in female athletes.

Environmental Health Perspectives 102, 79-82.

Naghii, M.R., Samman, S. (1997) The effect of boron supplementation on its urinary excretion and selected cardiovascular risk factors in healthy male subjects. Biological Trace Element Research 56, 273-286.

Nielsen F.H., Penland J.G. (1999). Boron supplementation of peri-menopausal women affects boron metabolism and indices associated with macromineral metabolism, hormonal status and immune function. Journal of Trace Elements in Experimental Medicine 12, 251-261.

Price, C.J., Strong, P.L., Marr, M,C,, Myers. C,B,, Murray, F.J. (1996a) Developmental toxicity NOAEL and postnatal recovery in rats fed boric acid during gestation. Fundamental and Applied Toxicology 32, 179-193.

Sayli, B.S., Tuccar, E., Elhan, A.H. (1998). An assessment of fertility in boron-exposed Turkish subpopulations. Reproductive Toxicology 12, 297-304.

Weir, R.J., Fisher, F.S. (1972) Toxicologic studies on borax and boric acid. Toxicology and Applied Pharmacology 23, 351-364.

Whorton, M.D., Haas, J.L., Trent, L., Wong, O. (1994) Reproductive effects of sodium borates on male employees: birth rate assessment. Occupational and Environmental Medicine 51, 761-767. 


\section{General information}

\section{Chemistry}

Chromium is a metallic element that can exist in a variety of oxidation states; oxidation states other than $0,+2,+3$ and +6 are uncommon. Biologically, trivalent (III) and hexavalent (VI) chromium are most important. Within this risk assessment the word chromium refers to ionic chromium except where specific chromium compounds are mentioned.

\section{Natural occurrence}

Trivalent chromium is ubiquitous in nature, occurring in air, water, soil and biological materials. Hexavalent chromium compounds are man-made and do not occur naturally in the environment.

\section{Occurrence in foods, food supplements and medicines}

Chromium in foods or supplements is in the trivalent form. Processed meats $(0.23 \mathrm{mg} / \mathrm{kg})$, whole grain products (bread and miscellaneous cereals $0.13-0.14 \mathrm{mg} / \mathrm{kg}$ ), pulses and spices are the better sources of chromium, but chromium levels are low in staple foods.

Chromium is not contained in any licensed medicines, but is present (together with other nutrients) in a number of supplements that may only be sold under the supervision of a pharmacist, for use in malabsorptive states, conditions leading to hypoproteinaemia and perioperative nutritional support, at levels up to $0.2 \mathrm{mg}$. It is also present in a number of multivitamin and mineral food supplements at levels up to $0.6 \mathrm{mg}$.

\section{Recommended amounts}

COMA has set no RNIs but suggested that an adequate level of intake for trivalent chromium lies above $0.025 \mathrm{mg} /$ day for adults and between 0.0001 and $0.001 \mathrm{mg} / \mathrm{kg} /$ day for children and adolescents (COMA, 1991). COMA also noted that no adverse effects were observed at intakes of $1000-2000$ $\mathrm{mg} /$ day trivalent chromium. The US National Research Council (NRC) specify an Estimated Safe and Adequate Daily Dietary Intake (ESADDI) of $0.05-0.2 \mathrm{mg} /$ day for adults and $0.01-0.04 \mathrm{mg} /$ day for infants ( $0-0.5$ years).

\section{Analysis of tissue levels and chromium status}

Plasma or serum levels reflect trivalent chromium intake, but chromium tissue stores do not rapidly equilibrate with blood chromium, so fasting plasma or serum concentrations may not be an indication of chromium status. Elevated serum chromium may be a good indicator of excessive exposure to chromium. 


\title{
Brief overview of non-nutritional beneficial effects
}

Beneficial effects of trivalent chromium on adult-onset type II diabetes mellitus and glucose tolerance have been claimed. Increased lean body mass and decreased total body fat have been attributed to trivalent chromium and chromium compounds have been used to enhance weight loss.

\section{Function}

Trivalent chromium has been shown to potentiate insulin action and thereby influences carbohydrate, lipid and protein metabolism.

\section{Deficiency}

In humans, deficiency has only been observed in patients on long-term parenteral nutrition. The symptoms observed were impaired glucose tolerance and glucose utilisation, weight loss, neuropathy, elevated plasma fatty acids, depressed respiratory quotient and abnormalities in nitrogen metabolism.

\section{Interactions}

Chromium interacts with iron by affecting its binding to transferrin, and has been shown to impair iron metabolism and storage.

\begin{abstract}
Absorption and bioavailability
Intestinal absorption of trivalent chromium is low $(0.5-2.0 \%)$. The mechanism of absorption has not been clearly defined, but it appears to involve processes other than passive diffusion. Chromium picolinate is absorbed more effectively than other forms of trivalent chromium.
\end{abstract}

\section{Distribution and metabolism}

Absorbed trivalent chromium does not enter blood cells, but binds to plasma proteins such as transferrin and is transported to the liver. In contrast, hexavalent chromium does penetrate red blood cells, where it is reduced by glutathione to trivalent chromium, which binds to haemoglobin. Excess hexavalent chromium is taken up into the kidneys, spleen, liver, lungs and bone.

\section{Excretion}

Ingested trivalent chromium remains largely unabsorbed and is excreted via the faeces. Absorbed chromium is mainly excreted via urine, with only small amounts being eliminated in perspiration and bile. 


\section{Toxicity}

\section{Human toxicity}

Data on the acute effects of oral exposure to chromium are available from two case reports on accidental or intentional ingestion of chromium at levels of $7.5 \mathrm{mg} / \mathrm{kg}$ bw (as hexavalent dichromate) and $4.1 \mathrm{mg} / \mathrm{kg}$ bw (as hexavalent chromic acid) respectively. Death was preceded by gastrointestinal haemorrhage and severe kidney and liver damage. Haematological effects have also been reported in individuals consuming sublethal doses. Ingestion of basic chromium sulphate (trivalent) (48 g in $400 \mathrm{~mL}$, equivalent to $800 \mathrm{mg} / \mathrm{kg}$ ) caused death by cardiogenic and renal shock, pancreatitis, haemorrhage and gut mucosal necrosis.

Chronic exposure to hexavalent chromium is reported to induce renal failure, anaemia, haemolysis and liver failure. Anaemia, haemolysis and liver and renal dysfunction have also been reported in case studies describing chronic exposure to trivalent chromium, but where follow up was reported, these were reversible, and parameters had returned to normal in one year.

\section{Supplementation studies}

Trivalent chromium (usually in the form of picolinate) has been investigated in a number of human supplementation studies. The studies have investigated the effects of chromium on a variety of end points ranging from serum chromium to DNA damage. Doses of up to $1 \mathrm{mg} /$ day chromium have been used, for periods up to 64 weeks. No adverse effects were observed, although in many of the studies it is unclear whether such effects were investigated.

\section{Animal data}

The available data are limited, but oral exposure to trivalent chromium and hexavalent chromium compounds has resulted in adverse gastrointestinal, hepatic, renal, immunological, neurological, developmental and reproductive effects.

Trivalent chromium compounds are less toxic than hexavalent chromium compounds, with chronic intakes of up to $750 \mathrm{mg} / \mathrm{kg}$ bw/day not being associated with adverse effects. Doses of $14 \mathrm{mg} / \mathrm{kg}$ bw/day hexavalent chromium for 3 weeks resulted in decreased body weights.

Both forms of chromium have been reported to reduce fertility, foetal weight, and crown length and increase post-implantation losses in mice.

\section{Carcinogenicity and genotoxicity}

There is no clear evidence of carcinogenicity where chromium has been tested in rats via the oral route. No data are available relating to the mutagenicity in vivo of oral exposure to trivalent chromium.

In general, in vitro mutagenicity tests have yielded positive results for hexavalent chromium, and negative results for trivalent. However, two studies in mammalian cells have shown that chromium picolinate (a form of trivalent chromium) may cause DNA damage. The authors of one study proposed that the picolonate moiety was responsible for the activity. However, the authors of another study 
proposed a mechanism involving reduction to chromium II and production of hydroxyl radicals. Chromium nicotinate and chromium III chloride were also investigated in one of these studies and did not damage DNA. The significance of these observations is unclear and no in vivo genotoxicity data are available.

\section{Mechanism of toxicity}

Chromium (VI) induces oxidative stress in vivo.

\section{Dose-response characterisation}

No data have been identified.

\section{Vulnerable groups}

No vulnerable groups have been identified.

\section{Genetic variations}

No genetic variations have been identified.

\section{Studies of particular importance in the risk assessment}

(For full review see http://www.food.gov.uk/science/ouradvisors/vitandmin/evmpapers or the enclosed CD).

\section{Human data}

\section{Wasser and Feldman, 1997}

This was a clinical report of renal failure in a patient taking supplements of $0.6 \mathrm{mg}$ chromium picolinate (trivalent) daily for six weeks to aid weight loss. It is reported that tests had shown normal renal function 2 years previously. Findings from a renal biopsy supported a diagnosis of nephrotoxicity, which the authors attributed to ingestion of chromium.

Cerulli et al., 1998

This was a clinical report of observation of a single patient after ingestion of $1.2-2.4 \mathrm{mg} /$ day chromium picolinate for $4-5$ months. She presented with weight loss, anaemia, haemolysis, liver dysfunction and renal failure. In addition to her intake of over-the-counter chromium supplements, the patient was receiving medication for schizophrenia and depression, but this medication was not believed to be related to the symptoms presented. 
Jeejeebhoy, 1999

This was a review paper describing the results of 19 randomised controlled human supplementation trials, in which individuals received between 0.175 and $1 \mathrm{mg}$ trivalent chromium/day for durations of between 6 and 64 weeks. No evidence of toxic effects was identified. Studies considered in the review include:

\section{Anderson et al., 1985}

In a double-blind crossover study, 76 adult subjects were given $0.2 \mathrm{mg} /$ day chromic chloride (trivalent) or placebo for three months. Mean basal serum chromium increased significantly from $0.13 \pm 0.02$ (SE) $\mathrm{ng} / \mathrm{mL}$ to $0.38 \pm 0.02$ (SE) $\mathrm{ng} / \mathrm{mL}$. This was primarily a study of efficacy and did not report any adverse effects.

Roeback et al., 1991

In a randomised, double blind, placebo-controlled trial, 63 male patients, prescribed beta-blockers for the treatment of hypertension, were given $0.6 \mathrm{mg} /$ day trivalent chromium or placebo for 8 weeks. A low number of side effects were reported, including mild gastrointestinal symptoms and decreased appetite; however, these were of similar low frequency in both treated and control groups.

\section{Abraham et al., 1992}

In a randomised clinical trial, 76 patients with atherosclerotic disease were given $0.15 \mathrm{mg} /$ day chromium chloride or placebo for a period of 7 to 16 months. Mean serum chromium levels were significantly increased from $2.69 \mathrm{nmol} / \mathrm{L}$ to $12.12 \mathrm{nmol} / \mathrm{L}$. Clinical chemistry indicated no adverse effects on liver or renal function and there were no haematological abnormalities. The authors reported that both placebo and chromium supplement were well tolerated and that patients reported no adverse effects.

Campbell et al., 1999

In a randomised, double-blind study, 23 men aged 50-75 years were given $0.924 \mathrm{mg} /$ day chromium (as picolinate) or placebo for a period of 12 weeks, during which time the subjects participated in a resistance training programme. Five subjects did not complete the study due to reasons unrelated to chromium or placebo treatment (injury, aggravation of injuries, family commitments). The authors did not report any treatment- or placebo-related effects, but this was primarily a study of efficacy.

\section{Animal data}

Ivankovic \& Preussman, 1975

This was a chronic toxicity/carcinogenicity study, in which trivalent chromium (as chromic oxide) was incorporated into diet at 0, 1, 2, or $5 \%$ (equivalent to 150,300 or $750 \mathrm{mg}$ chromium $/ \mathrm{kg}$ bw/day). The compound was baked into bread and fed to rats 5 out of 7 days per week for 840 days (a total of 600 feeds). At the weekends a control diet and a vegetable supplement were given. No adverse effects were observed at any dose level. In a preliminary 90-day study using the same dosing regime, a decrease in organ weights was apparent at the top dose. No statistical analysis was given and the findings were not followed up. Although samples were taken for histological analysis in the main study, the results are not reported. On the basis of this study, it can be concluded that chromic oxide is not carcinogenic, or overtly toxic. 
Anderson et al., 1997

Trivalent chromium was given to rats in the diet. A lack of toxicity was demonstrated at levels up to $100 \mathrm{mg}$ chromium per $\mathrm{kg}$ of diet as chromium chloride or picolinate for 24 weeks. Assuming a dietary intake of $15 \mathrm{~g}$ and a $100 \mathrm{~g}$ rat, the intake in the $100 \mathrm{~g}$ chromium per $\mathrm{kg}$ diet would be $1.5 \mathrm{mg} /$ day or $15 \mathrm{mg} / \mathrm{kg}$ bw/day. The animals receiving chromium picolinate had significantly higher renal and hepatic chromium, indicating higher absorption of this compound. There were no histological changes in the liver and kidneys but other organs were not examined histologically.

\section{Reproductive toxicity}

\section{Elbetieha \& Al-Hamood, 1997}

Reproductive toxicity was assessed after exposure of sexually mature male and female mice to trivalent (and hexavalent) chromium in drinking water for 12 weeks. Females received approximately 500 or $1250 \mathrm{mg} / \mathrm{kg}$ bw/day and the males, approximately 250 or $1250 \mathrm{mg} / \mathrm{kg}$ bw/day trivalent chromium (as chromium chloride). Reduced fertility (assessed by number of pregnant females, viable foetuses and resorptions) was apparent in male mice at the highest dose. In females, exposure to trivalent chromium resulted in a decreased number of implantation sites and viable foetuses at both doses. A parallel study was used to assess organ weights at doses of 500 and $1250 \mathrm{mg} / \mathrm{kg}$ bw/day trivalent chromium. In males, bodyweights were significantly reduced and testes weights increased at both doses. Seminal vesicle weights were decreased at the top dose. Female mice were tested only at $120 \mathrm{mg} / \mathrm{kg}$ bw/day, which resulted in increased ovarian weight and decreased uterine weight.

\section{Exposure assessment}

Total exposure/intake:
Food
Mean: $0.10 \mathrm{mg} /$ day
97.5th percentile: $0.17 \mathrm{mg} /$ day (1997 TDS)
Water
up to $0.002 \mathrm{mg} /$ day (estimated intake from 2 litres of water containing $<0.001 \mathrm{mg} / \mathrm{L}$ )
Supplements
up to $0.6 \mathrm{mg} /$ day (Annex 4)
Maximum estimated intake: $0.17+0.002+0.6=0.77 \mathrm{mg} /$ day
No potential high intake groups have been identified. 


\section{Risk assessment}

The data on oral chromium toxicity are limited. However, it is apparent that the toxicity of chromium varies depending on the valency state, with hexavalent $(\mathrm{VI})$ chromium, being generally more toxic than trivalent (III) chromium. This risk assessment concentrates on the evaluation of trivalent chromium as this is the form found in food and dietary supplements.

Ingested trivalent chromium has a low level of toxicity, due partly to its poor absorption. Chromic acid at chronic doses of up to $750 \mathrm{mg}$ chromium $/ \mathrm{kg}$ bw/day given in food to adult animals for periods of up to 24 weeks was not associated with adverse effects. Absorption was not demonstrated in this study. Chromium picolinate and chromium chloride were not associated with adverse effects at doses of $15 \mathrm{mg}$ chromium/kg bw/day. Increased levels of tissue chromium indicated that absorption had occurred. Higher doses of chromium (approximately $100 \mathrm{mg} / \mathrm{kg}$ bw/day) are associated with reproductive and developmental effects, although these may be secondary to parental toxicity.

In general, hexavalent chromium has given positive results in in vitro mutagenicity tests, whereas trivalent chromium compounds have been negative. However, chromium picolinate, a synthetic chromium compound with a higher solubility and lipophilicity than other trivalent chromium compounds, has caused DNA damage in mammalian cells. The significance of these observations is unclear and no in vivo genotoxicity data are available.

Limited data from human supplementation studies have indicated that doses up to $1 \mathrm{mg} /$ day of trivalent chromium compounds in general were not associated with adverse effects, although it is unclear what adverse effects were evaluated. The human studies were conducted in a variety of small groups and investigated a range of different endpoints, so limited conclusions may be drawn from these. Two case reports associating chromium picolinate with renal failure exist but the significance of these is uncertain.

\section{ESTABLISHMENT OF GUIDANCE LEVEL}

Overall, there are insufficient data from human or animal studies to derive a Safe Upper Level for chromium, although the oral toxicity of poorly absorbed trivalent chromium appears to be low.

There are few available data on chromium (III) toxicity in humans by the oral route. Acute chromium toxicity is associated with vomiting, diarrhoea, haemorrhage and blood loss into the gastrointestinal tract resulting in cardiovascular shock. The available animal data are also limited but adverse gastrointestinal, hepatic, renal, immunological, neurological, developmental and reproductive effects have been reported.

The study by Anderson et al. (1997) indicated that $15 \mathrm{mg} / \mathrm{kg}$ bw/day chromium (as chromium chloride) was not associated with adverse effects in the rat. Based on this study, and allowing uncertainty factors of 10 for inter-species variation and 10 for inter-individual variation, a total daily intake of about $0.15 \mathrm{mg} / \mathrm{kg}$ bw/day (or $10 \mathrm{mg} /$ person) would be expected to be without adverse health effects. This value can be used for guidance purposes and applies to trivalent chromium only. Chromium picolinate is also excluded from this guidance. Unlike other forms of trivalent chromium, chromium picolinate was shown to cause DNA damage in mammalian cells in vitro. Additionally, two case reports have associated renal failure with the use of chromium picolinate supplements. The significance of such results is unclear but may be due to the higher solubility and lipophilicity of chromium picolinate than other trivalent chromium compounds, which enable it to be readily absorbed and able to pass through 
membranes and enter cells. Further research is therefore needed to investigate the safety of this compound.

\section{References}

Abraham, A.S., Brooks, B.A. and Eylath, U. (1992) The effects of chromium supplementation on serum glucose and lipids in patients with and without non-insulin-dependent diabetes. Metabolism 41, 768-771.

Anderson, R.A., Bryden, N.A., Polansky, M.M. (1985) Serum chromium of human subjects: effects of chromium supplementation and glucose. American Journal of Clinical Nutrition 41, 571-577.

Anderson, R.A., Bryden, N.A., Polansky, M.M. (1997). Lack of toxicity of chromium chloride and chromium picolinate in rats. Journal of the American College of Nutrition 16, 273-279.

Campbell, W.W., Joseph, L.J., Davey, S.L., Cyr-Campbell, D., Anderson, R.A., Evans, J.W. (1999). Effects of resistance training and chromium picolinate on body composition and skeletal muscle in older men. Journal of Applied Physiology 86, 29-39.

Cerulli, J., Grabe, D.W., Gauthier, I., Malone, M., McGoldrick, M.D. (1998) Chromium picolinate toxicity. Annals of Pharmacotherapy 32, 428-431.

COMA (1991). Dietary Reference Values for Food Energy and Nutrients for the United Kingdom. Report of the Panel on Dietary Reference Values, Committee on Medical Aspects of Food and Nutrition Policy. HMSO, London.

Elbetieha, A. and Al-Hamood, M. (1997) Long-term exposure of male and female mice to trivalent and hexavalent chromium compounds: effect on fertility. Toxicology 116, 39-47.

Ivankovic, S. and Preussman, R. (1975) Absence of toxic and carcinogenic effects after administration of high doses of chromium oxide pigment in sub-acute and long term feeding studies in rats. Food and Cosmetics Toxicology 13, 347-351.

Jeejeebhoy, K.N. (1999) The role of chromium in nutrition and therapeutics and as a potential toxin. Nutrition Reviews 57, 329-335.

Junaid, M., Murthy, R.C., Saxena, D.K. (1995) Chromium fetotoxicity in mice during late pregnancy. Veterinary and Human Toxicology 37, 320-322.

Roeback, J.R. Jr, Hla, K.M., Chambless, L.E., Fletcher, R.H. (1991) Effects of chromium supplementation on serum high-density lipoprotein cholesterol levels in men taking beta-blockers. Annals of Internal Medicine 115, 917-924.

Wasser, W.G. and Feldman, N.S. (1997) Chronic renal failure after ingestion of over-the-counter chromium picolinate. Annals of Internal Medicine 126, 410. 


\section{Risk Assessment}

\section{Cobalt}

\section{General information}

\section{Chemistry}

Cobalt is a transition metal that exists in oxidation states +2 and +3 . Within this risk assessment the word cobalt refers to ionic cobalt except where specific cobalt compounds are mentioned.

\section{Natural occurrence}

Cobalt is widely distributed in the environment, accounting for $0.001 \%$ of the earth's crust. It forms bivalent and trivalent compounds, those of biological interest being bivalent.

\section{Occurrence in food, food supplements and medicines}

High concentrations of cobalt are found in fish $(0.01 \mathrm{mg} / \mathrm{kg})$, nuts $(0.09 \mathrm{mg} / \mathrm{kg})$, green leafy vegetables $(0.009 \mathrm{mg} / \mathrm{kg})$ and fresh cereals $(0.01 \mathrm{mg} / \mathrm{kg})$, and most of the cobalt ingested is inorganic. The mean population intake of cobalt is $0.012 \mathrm{mg} /$ day. There is no evidence of cobalt being used in food supplements. However, cobalt (as the sulphate) is included in some multi-constituent licensed medicines, at a maximum daily dose of $0.25 \mathrm{mg}$.

\section{Other sources of exposure}

Atmospheric concentrations of cobalt are usually around $1 \mathrm{ng} / \mathrm{m}^{3}$, but levels up to $10 \mathrm{ng} / \mathrm{m}^{3}$ have been reported in heavily industrialised cities. Occupational exposure to cobalt occurs mainly by inhalation. Uncontaminated samples of fresh water contain concentrations of cobalt ranging from 0.001 to $0.010 \mathrm{mg} / \mathrm{L}$.

\section{Recommended amounts}

Recommended intakes of cobalt have not been set as the only form of cobalt required by the body is vitamin $B_{12}$, of which cobalt is an integral part. In the UK, COMA has set a RNI value for vitamin $B_{12}$ of $1.5 \mu \mathrm{g} /$ day (equivalent to $0.006 \mu \mathrm{g}$ cobalt) for adults, including pregnant women (COMA, 1991). This amount is considered adequate not only to reduce the risk of megaloblastic anaemia in normal individuals, but also to provide sufficient stores to withstand a period without intake. During lactation, an increment of $0.5 \mu \mathrm{g}$ vitamin $\mathrm{B}_{12} /$ day is recommended. For infants, the RNI for vitamin $\mathrm{B}_{12}$ is $0.3 \mu \mathrm{g} /$ day.

\section{Analysis of tissue levels and cobalt status}

Cobalt can be measured in whole blood, serum and various tissues. There are no recognised markers or indicators of cobalt status. 


\section{Brief overview of non-nutritional beneficial effects}

No suggested non-nutritional beneficial effects have been identified.

\section{Function}

Cobalt is an essential trace element being an integral part of vitamin $B_{12}$, which is essential for folate and fatty acid metabolism.

\section{Deficiency}

Although cobalt is an essential trace element, cobalt deficiency has not been reported in humans. A wasting disease in cattle, of which a key feature is anaemia, has been demonstrated to be due to cobalt deficiency in pastures.

\section{Interactions}

No compounds that interact with cobalt have been identified.

\section{Absorption and bioavailability}

The extent of gastrointestinal absorption of cobalt depends upon the dose, with very low doses being almost completely absorbed, whereas larger doses are less well absorbed. Nutritional factors also influence absorption, for example, absorption is reduced by amino acids, and increased in iron deficiency.

\section{Distribution and metabolism}

In human autopsy studies, the liver (the organ where vitamin $B_{12}$ is stored) contains the highest concentration of cobalt (approximately $20 \%$ of the total body content). In the human body there is no evidence of accumulation of cobalt with age.

\section{Excretion}

Cobalt is mainly excreted in the urine but also in the faeces. Independent of the route of exposure, most cobalt is eliminated rapidly, with a small proportion being eliminated slowly and having a half-life of the order of years. 
Part 3 Trace Elements

\section{Toxicity}

\section{Human data}

Cardiomyopathy was reported in heavy beer drinkers in the 1960s as a result of the use of cobalt chloride as a foam stabiliser, present in beer at concentrations of $1-1.5 \mathrm{ppm}$. Ethanol and cobalt have an additive effect, reducing blood flow to the heart and thus causing anoxia and damage to the heart muscle. Animal studies indicate that protein (particularly tryptophan, DL-methionine and L-cysteine) deficiency is a risk factor for cobalt toxicity.

Few other data on human toxicity are available where exposure is by ingestion. The majority of the available papers refer to reports from case studies. For example, Carson et al. (1986) reported cases of acute effects following ingestion of excess amounts of cobalt salts (30 mg/day) for the treatment of anaemias, including gastrointestinal upset, skin rashes and hot flushes. Features of chronic toxicity include effects on the heart, thyroid and possibly the kidney (Carson et al., 1986). Patients receiving 0.17 - $3.9 \mathrm{mg}$ cobalt $/ \mathrm{kg}$ bw/day (equivalent to $10-234 \mathrm{mg}$ in a $60 \mathrm{~kg}$ adult) for 6 days to 8 months, usually to treat anaemia, showed a $20-90 \%$ depression in iodine uptake, resulting in goitre and classic signs of hypothyroidism.

\section{Animal data}

Animal studies have shown that cobalt accumulates in the myocardium and causes myocytolysis.

Detrimental effects on sperm production and fertility have been observed in rats.

\section{Carcinogenicity and genotoxicity}

No carcinogenicity data are available following exposure by the oral route. Mixed results were obtained with cobalt compounds in a range of in vitro and in vivo genotoxicity tests.

\section{Mechanisms of toxicity}

No relevant data have been identified.

\section{Dose response characterisation}

There are insufficient data to establish a dose response relationship.

\section{Vulnerable groups}

No vulnerable groups have been identified.

\section{Genetic variation}

No genetic variations have been identified 


\section{Studies of particular importance in the risk assessment}

(For full review see http://www.food.gov.uk/science/ouradvisors/vitandmin/evmpapers or the enclosed CD)

\section{Human data}

Seghizzi et al., 1994

A review of the literature from the 1960s described endemics of cardiomyopathy with mortality rates of up to $50 \%$ in heavy consumers of cobalt-fortified beer (containing cobalt concentrations of $1-1.5$ ppm). The intake of cobalt in such consumers was estimated to be $6-8 \mathrm{mg}$ daily. Dietary protein deficiency may be an important factor in cobalt induced cardiomyopathy, and zinc and magnesium deficiency may also play a part.

\section{Animal data}

Pedigo et al., 1988

Cobalt chloride was administered to groups of 10 mice in drinking water providing 23,42 or $72 \mathrm{mg}$ cobalt $/ \mathrm{kg}$ bw/day for up to 13 weeks. The highest dose level resulted in a dose- and time-dependent decrease in testicular weight and decreased epididymal sperm concentration, along with decreases in both motility and percent motile forms of sperm. Fertility was decreased at week 13 . There was a dosedependent decrease in testicular weights (as a ratio to body weight), whilst serum testosterone levels were increased 5 to 7 fold at all dose levels.

Paternain et al., 1988

Cobalt chloride (25, 50 or $100 \mathrm{mg}$ cobalt $/ \mathrm{kg}$ bw/day) was administered to mice on days 6 to 15 of gestation by oral gavage. An increase in stunted foetus/litter rates was observed at 50 and $100 \mathrm{mg}$ cobalt $/ \mathrm{kg}$ bw/day, although this was not statistically significant. The number of corpora lutea, implants, resorptions, live and dead foetuses and sex distribution were unaffected by treatment. Maternal toxicity (decreased weight gain and food consumption) was seen at all dose levels. An erythropoietic effect was apparent in the top dose group.

Anderson et al., 1992

Cobalt chloride administered to groups of 10 mice in drinking water providing $72 \mathrm{mg}$ cobalt $/ \mathrm{kg} \mathrm{bw} / \mathrm{day}$ for a thirteen week period, resulted in seminiferous tubule degeneration. Groups of mice were evaluated at 7, 9, 11 and 13 weeks treatment. Initial changes involved vacuolation of Sertoli cells and formation of abnormal spermatid nuclei, followed by the presence of multi-nucleated cells and the sloughing off of cells. Continued degeneration resulted in shrinkage of tubules and the accumulation of some calcified necrotic debris. Groups of mice were allowed to recover for 20 weeks after the treatment period. Repopulation of the tubules was observed in a few animals only. 


\section{Cardiovascular effects}

In addition to the above papers, consideration was given to the studies of Morvai et al. and Sandusky et al. which provided evidence of cardiovascular effects of cobalt from animal models, substantiating the observations that have been made in humans.

\section{Sandusky et al., 1981}

Groups of dogs were maintained on normal diet or protein and thiamine deficient diet and received $500 \mathrm{mg}$ of cobalt chloride twice weekly by intravenous infusion. Dyspnoea and exercise intolerance was observed in all the dosed animals and tachycardia in those maintained on protein and thiamine deficient diet.

Morvai et al., 1993

Administration of cobalt chloride ( $50 \mathrm{mg}$ cobalt/ $\mathrm{kg}$ bw/day) by oral gavage to rats for 3 weeks, caused incipient multifocal myocytolysis and a significant decrease in blood pressure and the blood flow to the heart. Alcohol accentuated the effect of cobalt on blood flow.

\section{Exposure assessment}

Total exposure/intake:

Food

Mean: $0.012 \mathrm{mg} /$ day (1994 TDS)

97.5th percentile: $0.019 \mathrm{mg} /$ day

Water

$0.02 \mathrm{mg} /$ day (Estimated from IARC 1991 - range in water $0.001-0.01 \mathrm{mg} / \mathrm{L}$ and consumption of $2 \mathrm{~L} /$ day)

Supplements

$\mathrm{O}$ (present only as $\mathrm{B}_{12}$ ) (Annex 4)

Estimated maximum

intake:

$$
0.019+0.02=0.039 \mathrm{mg} / \text { day }
$$

No potential high intake groups have been identified.

\section{Risk assessment}

Cardiomyopathy was reported in heavy beer drinkers in the 1960s as a result of the use of cobalt chloride as a foam stabiliser. Cobalt without alcohol caused damage to the heart muscle, whilst both alcohol and cobalt reduced blood flow (cobalt significantly so), the combination having an additive effect. It has been suggested that the anoxia caused by the combination of alcohol and cobalt exacerbates the cardiotoxic effects of cobalt. Protein deficiency also appears to be a risk factor. Since cobalt chloride is no longer used as an additive in beer, no further cases have been reported. Although this condition was related to oral cobalt, a combination of exposures is necessary. 
Few other human data are available. Case reports suggest that acute intakes of $>30 \mathrm{mg} /$ day cobalt may cause gastrointestinal upset, skin rashes and hot flushes. Chronic cobalt intakes of $0.17-0.39 \mathrm{mg} / \mathrm{kg}$ (10.2 - $23.4 \mathrm{mg}$ total in a $60 \mathrm{~kg}$ adult) may depress iodine uptake.

The cardiotoxic effects of cobalt can also be reproduced in animals. Other adverse effects related to cobalt include degeneration of the seminiferous tubules and subsequently decreased fertility. There are no data available to establish whether this effect also occurs in humans. It is therefore prudent to assume that it could.

\section{ESTABLISHMENT OF GUIDANCE LEVEL}

There are insufficient data to set a Safe Upper Level for cobalt. There is no evidence of cobalt being used in food supplements, however, cobalt (as the sulphate) may be included in some multi-constituent licensed medicines, at a maximum daily dose of $0.25 \mathrm{mg}$.

In case reports cobalt is described to cause decreased iodine uptake in man (. $10 \mathrm{mg}$ ) or acute effects such as gastrointestinal upset and skin rashes in man (reported with intakes of $30 \mathrm{mg} /$ day).

Cobalt is known to have adverse effects on the heart in both animals and man. In man, cardiomyopathy occurred in heavy consumers of beer containing cobalt chloride as an additive providing a cobalt intake of $6.8 \mathrm{mg} /$ day. The combined effect of alcohol and cobalt and possibly protein deficiency was probably necessary to cause this condition. Cobalt chloride is no longer used for this purpose.

In laboratory animals, cobalt is associated with adverse effects on spermatogenesis and, ultimately, fertility. Doses of $23 \mathrm{mg}$ cobalt $/ \mathrm{kg}$ bw/day caused minor testicular effects, the severity of the effect then increasing in a dose related manner. This is the lowest dose at which toxic effects have been observed in animals. It is not known whether the effects on spermatogenesis and fertility also occur in humans exposed to cobalt but it would be prudent to assume that they do. If the conventional uncertainty factors were to be applied (10 for LOAEL to NOAEL extrapolation $\times 10$ for inter-species variation $\times 10$ for inter-individual variation $=1000$ ) for guidance purposes only, an intake of $0.023 \mathrm{mg} / \mathrm{kg}$ total cobalt would not be expected to result in any adverse effects. This is equivalent to $1.4 \mathrm{mg} /$ day in a $60 \mathrm{~kg}$ adult. This level is lower than the doses causing decreased iodine uptake or acute effects in humans, and below the level associated with cardiomyopathy in beer drinkers (see above). It is also substantially higher than the present daily intake of cobalt in the UK (estimated to be approximately $0.030 \mathrm{mg}$ from food and water). 


\section{References}

Anderson, M.B., Pedigo, N.G., Katz, R.P., George, W.J. (1992). Histopathology of testes from mice chronically treated with cobalt. Reproductive toxicology 6, 41-50.

Carson, B.L., Ellis, H.V., McCann, J.L. (1986). Toxicology and Biological Monitoring of Metals in Humans. Lewis Publishers Inc. Michigan, USA.

COMA (1991). Dietary Reference Values for Food Energy and Nutrients for the United Kingdom. Report of the Panel on Dietary Reference Values, Committee on Medical Aspects of Food and Nutrition Policy. HMSO, London.

Morvai, V., Szakmáry, É., Tátrai, E., Ungváry, G., Folly, G. (1993). The effects of simultaneous alcohol and cobalt chloride administration on the cardiovascular system of rats. Acta Physiologica Hungarica 81, 253-261.

OTC (2001). OTC Directory 2001-2002. Proprietary Association of Great Britain.

Paternain, J.L, Domingo, J.L., Corbella, J. (1988). Developmental toxicity of cobalt in the rat. Journal of Toxicology and Environmental Health 24, 193-200.

Pedigo, N.G., George, W.J., Anderson, M.B. (1988). Effects of acute and chronic exposure to cobalt on male reproduction in mice. Reproductive Toxicology 2, 45-53.

Sandusky, G.E., Henk, W.G. Roberts, E.D. (1981). Histochemistry and ultrastructure of the heart in experimental cobalt cardiomyopathy in the dog. Toxicology and Applied Pharmacology 61, 89-98.

Seghizzi, P., D’Adda, F., Borleri, D., Barbic, F., Mosconi, G. (1994). Cobalt cardiomyopathy. A critical review of the literature. The Science of the Total Environment 150, 105-109. 


\section{Risk Assessment}

\section{Copper}

\section{General Information}

\section{Chemistry}

Copper has two valency states, cuprous (copper I) and cupric (copper II). It occurs in nature mainly in the form of its oxide, $\mathrm{Cu}_{2} \mathrm{O}$ and sometimes as the chloride, $\mathrm{CuCl}_{2}$ which, in the presence of humidity and oxygen, is oxidised to the basic copper (II) chloride, $\mathrm{Cu}(\mathrm{OH}) \mathrm{Cl}$. The most important copper compounds in the aquatic environment are cupric chloride, cuprous nitrate and cupric sulphate. Within this risk assessment the word copper refers to ionic copper, except when specific copper compounds are mentioned.

\section{Natural occurrence.}

Copper is found in a wide variety of mineral salts and organic compounds as well as in the metallic form.

\section{Occurrence in food, food supplements and medicines}

Food is the major source of copper intake, with particularly high concentrations being found in nuts ( $8 \mathrm{mg} / \mathrm{kg})$, shellfish and offal $(40 \mathrm{mg} / \mathrm{kg})$.

Copper is present in mineral and multivitamin plus mineral dietary supplements at doses up to $2 \mathrm{mg} /$ day. Licensed medicines may be obtained under the supervision of a pharmacist as Pharmacy (P) medicines, with a maximum recommended dose of $4 \mathrm{mg}$, or from various retail outlets as General Sales List (GSL) products, with a maximum recommended daily dose of $1 \mathrm{mg}$.

\section{Other sources of exposure}

Other sources of copper exposure include emissions from mines, smelters and foundries. Environmental copper can also arise from the burning of coal for power generation and from municipal waste incinerators. However, the contribution of airborne copper to total daily intake is negligible. Copper is also used as an anti-fouling agent for boats. Buffered copper sulphate can be used as a pesticide in organic farming.

High concentrations of copper can be dissolved from copper plumbing, depending on the hardness, $\mathrm{pH}$ and quality of the water. Dissolved copper levels are higher in acidic waters, especially where pipe runs are long and water can stagnate. Thus, drinking water can make an important contribution to copper intake in some circumstances (the UK standard is $3 \mathrm{mg} / \mathrm{L}$, based on aesthetic considerations). However, levels in UK water are generally less than $1 \mathrm{mg} / \mathrm{L}$. 
Part 3 Trace Elements

\section{Recommended amounts}

COMA has set a RNI of $1.2 \mathrm{mg} /$ day for copper (COMA, 1991). The US safe designation for adults is $1.5-3.0 \mathrm{mg} /$ day.

\section{Analysis of tissue levels and copper status}

Present analytical methods lack sensitivity, particularly for marginal copper deficiency. The use of erythrocyte copper-zinc superoxide dismutase activity as a measure of copper status is under investigation. Tissue copper measurements considered to be of use are serum copper, caeruloplasmin, urinary copper and hair copper all of which are depressed in frankly copper-deficient subjects. Plasma copper levels may be raised in pregnancy, infection and inflammation.

\section{Brief overview of non-nutritional beneficial effects}

Copper supplements are generally only used to correct copper deficiency. Benefits have been claimed for copper-containing supplements as anti-oxidants for general use, and specifically for arthritis and osteoporosis. There is speculation that copper might have a role in preventing age-related decline in tissue function, and possibly in ensuring healthy foetal brain development.

\section{Function}

Copper is an essential micronutrient normally subject to effective homeostatic control. It is involved in the function of several enzymes, including cytochrome c oxidase, amino acid oxidase, superoxide dismutase and monoamine oxidase. Copper is thought to be required for infant growth, host defence mechanisms, bone strength, red and white cell maturation, iron transport, cholesterol and glucose metabolism, myocardial contractility and brain development.

\section{Deficiency}

Copper deficiency may result from an inherited defect, such as Menke's syndrome, or may be an acquired condition. Common clinical features include anaemia, neutropenia and bone abnormalities. Less frequent signs and symptoms include hypopigmentation of the hair, hypotonia, impaired growth, increased susceptibility to infection, abnormalities in metabolism of glucose and cholesterol and cardiovascular changes.

\section{Interactions}

Various dietary components affect the absorption of copper. For example, absorption is higher from a diet high in animal protein compared with plant protein. Milk proteins have varying effects on copper status, with whey protein having a negative effect on copper absorption. High dietary ascorbic acid has been suggested to reduce intestinal absorption of copper, but it also increases hepatic uptake and biliary excretion of copper. Studies in rats have shown that dietary fructose exacerbates the effects of copper deficiency, by elevating faecal and urinary excretion of copper, but this has not been demonstrated in humans. Other animal studies suggest that when copper status is low, copper absorption is affected by high concentrations of iron, and to a greater extent when high concentration ascorbic acid is also present. 
Zinc and copper may interact, with high concentrations of one element inhibiting the absorption of the other.

\begin{abstract}
Absorption and bioavailability
Copper is mainly absorbed in the duodenum bound to specific proteins as $\mathrm{Cu}(\mathrm{II})$. Both passive diffusion and carrier mediated transfer occur. A small amount of copper is absorbed from the stomach. Absorption of copper is decreased when copper ingestion is high. Cellular mechanisms such as induction of metallothionein, which sequesters copper, have also been suggested to be part of this homeostatic process. Infants are apparently unable to absorb copper to the same extent as adults, and are often found to be in negative copper balance.
\end{abstract}

\title{
Distribution and metabolism
}

Copper distribution occurs in two phases, via specific carrier proteins, first to the liver and then to other body tissues. On leaving intestinal cells, copper is initially bound predominantly to albumin and transcuprein and transported mainly to the liver hepatocytes, where it is incorporated into caeruloplasmin (the major form for transport to other tissues), excreted into the bile or incorporated into superoxide dismutase (SOD). Absorbed copper is incorporated into 3 main fractions in the liver cytosol; a high molecular weight pool, a 30,000 kDa pool (which appears to be SOD), and a 10,000 kDa pool (mainly composed of metallothionein). Copper binds to a range of unidentified components of both high and low molecular weight, but none of these has yet been related to the copper complexes identified in the liver.

\section{Excretion}

Homeostasis of copper is maintained largely by the rate of excretion. The major route of copper excretion is via the bile, which is directly correlated with absorbed dose.

\section{Toxicity}

\section{Human data}

Acute copper toxicity is rare, but can occur as a result of food or beverage contamination. However, the emetic properties and unpleasant taste of copper salts prevent their frequent accidental or deliberate ingestion. Signs of acute copper toxicity include salivation, epigastric pain, nausea, vomiting and diarrhoea. Individual susceptibility varies, but vomiting has been associated with consumption of beverages contaminated with copper ranging from 25 to $840 \mathrm{mg} / \mathrm{L}$. Intakes of 25 - $75 \mathrm{mg}$ copper have been quoted as emetic doses but lower intakes have resulted in the same symptoms when taken on an empty stomach. Ingestion of $\geqslant 100 \mathrm{~g}$ copper sulphate may produce intravascular haemolysis, acute hepatic failure, acute tubular renal failure, shock, coma or death.

Few data are available on chronic copper toxicity in humans. 
Indian childhood cirrhosis (ICC) is a fatal disorder associated with accumulation of massive levels of copper in the liver. Although ICC has been attributed to boiling and storing milk in copper and brass vessels, thus elevating copper content, there also appears to be an element of genetic predisposition in many cases of ICC. Isolated cases of idiopathic copper toxicosis (ICT), identical in nature to ICC, have also been reported in non-Indian communities in the US and Europe.

Wilson's disease is an autosomal recessive inherited disorder of copper metabolism, which is normally manifest in late childhood. There is a failure of normal copper excretion into the bile and of incorporation into caeruloplasmin. As a result, copper accumulates and causes toxicity, primarily in the liver and brain. Clinical manifestations may include liver disease, and neurological and psychiatric disturbances.

Increased copper levels in adults with untreated Wilson's disease, or in children who have recovered from ICC, have been associated with a possible increased incidence of hepatoma. High copper levels have also been cited as a possible risk factor for heart disease.

In the normal population, there is no evidence that elevated copper intake is associated with cancer incidence.

\section{Supplementation studies}

Few adverse effects have been reported in human supplementation studies where copper has been given in food or as a tablet. For example, in a study in which $10 \mathrm{mg} /$ day was given for 12 weeks no changes in serum enzyme activities was apparent. However in other studies enzyme activities increased in subjects with lower than median copper status. In studies of copper given in solution, increased incidences of vomiting, diarrhoea and other gastrointestinal symptoms have been reported.

\section{Animal data}

Toxicity is highly species dependent. For example, while pigs and rats are relatively tolerant, sheep develop copper toxicosis at low dietary intakes of copper.

Repeat dose studies in rats and mice have demonstrated lesions in the forestomach (attributable to irritation). Liver and kidney toxicity, anaemia, and changes in systolic blood pressure and enzyme activity have also been reported in rats.

Hepatic failure has been demonstrated in rabbits receiving $10 \mathrm{mg} / \mathrm{kg}$ copper sulphate by gavage for 33-440 days.

Limited effects on reproduction in rats have also have been reported. These include changes to the reproductive organs and growth retardation in the offspring. At higher doses, developmental abnormalities have been observed.

\section{Carcinogenicity and genotoxicity}

The Long-Evans Cinnamon (LEC) rat is a rodent model of Wilson's disease, characterised by caeruloplasmin deficiency, hepatic copper accumulation, and hepatocellular injury ultimately resulting in hepatocellular carcinoma. 
Copper compounds were negative in the majority of bacterial mutation assays reported.

Chromosomal aberrations have been produced in isolated rat hepatocytes incubated with copper sulphate.

\section{Mechanisms of toxicity}

The liver mitochondrion appears to be an important target in copper hepatotoxicity, and oxidative damage may be involved in its pathogenesis.

\section{Dose response characterisation}

In humans, mild gastrointestinal effects have been reported following consumption of water containing $3 \mathrm{mg}$ copper/L, probably due to the irritant effect of copper in solution. Metabolic and supplementation studies suggest that dietary doses of 7-10 $\mathrm{mg}$ copper/day do not result in adverse effects.

In laboratory animals, forestomach lesions were seen in rats given feed containing $2000 \mathrm{mg} / \mathrm{kg}$ or greater copper sulphate for 13 weeks (equivalent to $32 \mathrm{mg}$ copper $/ \mathrm{kg}$ bw/day in male rats and $46 \mathrm{mg}$ copper $/ \mathrm{kg}$ bw/day in female rats). In mice the lesion occurred in animals given $4000 \mathrm{mg} / \mathrm{kg}$ bw/day (equivalent to $184 \mathrm{mg}$ copper/ $\mathrm{kg}$ bw/day in male mice and $262 \mathrm{mg}$ copper $/ \mathrm{kg}$ bw/day in female mice). In the same study, liver toxicity in rats was observed at doses of $65-67 \mathrm{mg} / \mathrm{kg}$ bw/day copper (and in one male receiving $32 \mathrm{mg} / \mathrm{kg}$ bw/day) and kidney toxicity and anaemia was observed in rats at doses of 32-34 mg copper/kg bw/day. Other effects such as changes in blood pressure and enzyme activities have been reported at lower doses. Effects on the reproductive organs of rats have been observed at doses $\geqslant 45 \mathrm{mg}$ copper $/ \mathrm{kg}$ bw/day. Adverse effects on foetal development in rats and mice occur at doses of 65-80 mg copper/ $\mathrm{kg}$ bw/day, with foetal abnormalities in mice being observed at $159 \mathrm{mg}$ copper/kg bw/day.

\section{Vulnerable groups}

Haemodialysis patients and subjects with chronic liver disease are potentially sensitive to copper excess. See also 'Genetic variations' below.

Children may be at increased risk of copper toxicity due to a combination of efficient uptake and immature biliary excretion. However, copper is accumulated in the third trimester of pregnancy without apparent adverse effect, suggesting that neonates may be resistant to high levels of hepatic copper.

\section{Genetic variations}

Subjects with Wilson's Disease and, possibly, ICC are sensitive to excess copper intake. 


\section{Studies of particular importance in the risk assessment}

(For full review see http://www.food.gov.uk/science/ouradvisors/vitandmin/evmpapers or the enclosed CD).

Pratt et al., 1985

As part of a double-blind study of back pain management, 14 adult volunteers received a supplement of $10 \mathrm{mg}$ copper/day as copper gluconate for 12 weeks or a placebo. There was no increase in the levels of copper, zinc or magnesium in serum, urine or hair. Haematocrit, triglyceride, SGOT, GGT, LDH and cholesterol levels were not significantly affected by treatment. The authors concluded that the results supported the view that excess copper was excreted and homeostasis maintained in non-Wilson's disease subjects. Adverse effects of nausea, heart burn and diarrhoea occurred in copper and placebo takers, but the numbers stated are too few for reliable comparison.

\section{Turnland et al., 1989}

Eleven young male volunteers were confined to a metabolic ward for 90 days and given a sequence of adequate ( $1.68 \mathrm{mg} /$ day), low ( $0.785 \mathrm{mg} /$ day) and high $(7.53 \mathrm{mg} /$ day) copper diets for 24,42 and 24 days respectively. Absorption of a stable copper isotope ${ }^{65} \mathrm{Cu}\left(\right.$ as $\left.{ }^{65} \mathrm{CuO}\right)$ from the high copper diet, was lower (12\%) than that absorbed (36.3\%) from the adequate copper diet, and from the low copper diet (55.6\% for the first 35 days of treatment), and declined linearly with time. Thus in the last 6 days of the study, copper balance was negative in the high dose treatment group suggesting endogenous excretion of copper retained earlier in the study. The authors concluded that where copper intake was high, reduced fractional absorption was insufficient to prevent the absorption of some excess copper, but that the excess was then eliminated by increased endogenous losses. No adverse effects were noted, but because the experiment was designed to investigate copper balance, markers of copper toxicity were not considered.

\section{Olivares et al., 1998}

Healthy infants living in Santiago were randomly assigned to two groups receiving drinking water containing $<0.1 \mathrm{mg} / \mathrm{L}(\mathrm{n}=48)$ or $2 \mathrm{mg} / \mathrm{L}(\mathrm{n}=80)$ copper (as copper sulphate) from 3-12 months of age. The mothers of the infants also consumed the same water. Within the groups, the infants were divided into 2 subgroups: those who were formula-fed and those who were breast-fed. Formula-fed infants in the low and high copper intake groups received 0.8-1.2 and 2.3-2.5 mg copper/day, respectively. Breastfed infants in the low and high copper intake groups received 0.1-1.6 and 0.1-2.0 mg copper/day, respectively. Values for copper intake included copper intake from food, but not from breast milk. At 6 , 9 and 12 months serum concentrations of copper, caeruloplasmin, transaminases and $\gamma$-glutamyl transferases were measured, as an indication of copper status. No adverse effects were observed, but small differences were apparent in biochemical estimates of copper status. There were no significant differences in copper status between the 4 groups of infants during the study. However, there were differences between the breast-fed and formula-fed infants in serum copper and erythrocyte metallothionein levels and in the serum caeruloplasmin activity of individuals with high and low copper intake levels at points during the study. A greater incidence of diarrhoea in formula-fed infants would seem likely to reflect their enhanced risk of enteric infection. Overall, the authors concluded that consumption of drinking water containing copper at the $\mathrm{WHO}$ guideline level of $2 \mathrm{mg} / \mathrm{L}$ did not result in adverse effects. 


\section{Pizarro et al., 1999}

Sixty healthy adult female volunteers living in urban Santiago were randomised into four groups $(n=15)$ who received drinking water containing $0,1,3$ or $5 \mathrm{mg}$ copper/L for 2 weeks followed by a further week of standard tap water. The copper was added in the form of copper sulphate. A double-blind latin square design was used so that each volunteer experienced all of the copper concentrations and acted as their own control. Untreated tap water contained $0.02 \mathrm{mg}$ copper/L. Average dietary intake of copper by the study population was estimated to be $1.7 \mathrm{mg} /$ day. Water consumption and gastrointestinal symptoms were recorded daily. Average water consumption was $1.64 \mathrm{~L} /$ day regardless of copper concentration. Twenty-one subjects reported gastrointestinal disturbances during the study (nine subjects presented with diarrhoea (some with abdominal pain and vomiting) and twelve subjects presented with abdominal pain, nausea or vomiting. One third of the 60 subjects had mild diarrhoea during the study but this was not associated with copper concentration. A copper concentration of $3 \mathrm{mg} / \mathrm{L}$ or above was associated with an increased incidence of nausea, vomiting or abdominal pain $15,2,17$ and 15\% in the 0, 1, 3 and $5 \mathrm{mg} / \mathrm{L}$ groups respectively). Final serum copper, caeruloplasmin and liver enzyme levels were measured in all the volunteers but did not differ from baseline. The numbers involved were small and the incidence of diarrhoea in the whole group appears quite high; the reasons for the latter were discussed. The authors suggested that the subjects could have been more sensitive to symptoms since they were specifically asked to record them.

\section{Animal studies}

Hébert et al., 1993

Rats and mice were given up to 10,000 mg copper $/ \mathrm{kg}$ (estimated intake up to $35 \mathrm{mg}$ copper $/ \mathrm{kg}$ bw $/$ day in male rats, $30 \mathrm{mg}$ copper $/ \mathrm{kg}$ bw/day in female rats, $131 \mathrm{mg}$ copper $/ \mathrm{kg}$ bw/day in male mice and $171 \mathrm{mg}$ copper $/ \mathrm{kg}$ bw/day in female mice) copper sulphate pentahydrate in drinking water for 2 weeks. Concentrations of 300-1000 mg copper/ $\mathrm{kg}$ sulphate (up to $28 \mathrm{mg}$ copper $/ \mathrm{kg}$ bw/day in rats and up to $35 \mathrm{mg}$ copper/ $\mathrm{kg}$ bw/day in mice) did not have any overt adverse effects on F344 rats or B6C3F1 mice, whereas concentrations of 3000-10,000 mg copper sulphate $/ \mathrm{kg}$ (equivalent to $30-44 \mathrm{mg}$ copper $/ \mathrm{kg}$ bw/day in rats and 56-171 mg copper/ kg bw/day in mice) were rapidly lethal. Copper exposure was lower in the top dose rats due to markedly reduced drinking water consumption. Body weights were depressed at $>3000 \mathrm{mg} / \mathrm{kg}$ copper sulphate (30 and $44 \mathrm{mg}$ copper/ $\mathrm{kg}$ bw/day in female and male rats and 56 and $61 \mathrm{mg}$ copper/ kg bw/day in male and female mice) in both species. Clinical signs observed at these dose levels included ruffled fur, emaciation, abnormal posturing, hypoactivity, dyspnea and tremors. The only histological lesions observed in rats (males only) were increased numbers of protein droplets in the epithelium of the proximal convoluted tubules. In mice in the highest dose group, most tissues showed cellular depletion, due to decreased water consumption. No effects were produced at $1000 \mathrm{mg}$ copper sulphate/ $\mathrm{kg}$ (equivalent to $26 \mathrm{mg}$ copper/ $\mathrm{kg}$ bw in female rats and 24 and $35 \mathrm{mg}$ copper $/ \mathrm{kg}$ bw in male and female mice) in female rats and mice, but protein droplets were reported in male rats at all doses.

Rats and mice were given up to 16,000 mg copper sulphate pentahydrate $/ \mathrm{kg}$ in feed for 2 weeks. Concentrations of 4000-16000 mg copper sulphate $/ \mathrm{kg}$ (equivalent to $91 \mathrm{mg}$ copper $/ \mathrm{kg} \mathrm{bw} /$ day and above in male rats, $159 \mathrm{mg}$ copper/ $\mathrm{kg}$ bw/day and above in female rats, $193 \mathrm{mg}$ copper $/ \mathrm{kg} \mathrm{bw} /$ day and above in male mice and $212 \mathrm{mg}$ copper/kg bw/day and above in female mice) caused significant reductions in body weight gain in both species. Body weights were reduced in rats given $8000 \mathrm{mg} / \mathrm{kg}$ or more copper sulphate (equivalent to $192 \mathrm{mg}$ copper/ $\mathrm{kg}$ bw/day in females and $194 \mathrm{mg}$ copper $/ \mathrm{kg}$ bw/day in males) and in mice in the top dose group (704 mg copper/ $\mathrm{kg}$ bw/day for male mice and $767 \mathrm{mg}$ 
copper $/ \mathrm{kg}$ bw/day for female mice). Hyperplasia and hyperkeratosis on the limiting ridge of the forestomach were present in both species (this was more severe in mice) and was attributed to irritation. A dose-related increase in liver damage (inflammation and changes in clinical chemistry) was apparent in the rats, as was damage to the renal tubules. Depletion of iron stores occurred in rats but not in mice, and histologic changes indicated microcytic anaemia also in rats. The NOAEL for the 2 week feed studies was $1000 \mathrm{mg}$ copper sulphate $/ \mathrm{kg}$ in rats (equivalent to $23 \mathrm{mg}$ copper $/ \mathrm{kg}$ bw/day) in males and females respectively based on liver, kidney and forestomach effects. In mice, effects on the liver and kidney were not observed but the NOAEL based on the forestomach was $2000 \mathrm{mg} / \mathrm{kg}$ copper sulphate (equivalent to 91 and $102 \mathrm{mg}$ copper/kg bw) in males and females respectively.

A 13 week feeding study in $\mathrm{F} 344$ rats and $\mathrm{B} 6 \mathrm{C} 3 \mathrm{Fl}$ mice was also carried out. The rats and mice received diet containing $500-8000 \mathrm{mg} / \mathrm{kg}$ and 1000-16,000 mg/kg copper sulphate pentahydrate respectively. Body weights were significantly reduced in male rats at 4000 and $8000 \mathrm{mg} / \mathrm{kg}$ copper sulphate (equivalent to $65 \mathrm{mg}$ copper $/ \mathrm{kg}$ bw/day and $138 \mathrm{mg}$ copper $/ \mathrm{kg}$ bw/day) and in females at $8000 \mathrm{mg} / \mathrm{kg}$ (equivalent to $132 \mathrm{mg}$ copper $/ \mathrm{kg}$ bw/day). A dose related decrease in body weights was apparent in mice at doses of $4000 \mathrm{mg} / \mathrm{kg}$ copper sulphate or more (equivalent to $184 \mathrm{mg}$ copper $/ \mathrm{kg}$ bw/day and above in males and $262 \mathrm{mg}$ copper/ $\mathrm{kg}$ bw/day and above in females). Hyperplasia and hyperkeratosis on the limiting ridge of the forestomach were present in both species, though rats were more severely affected at the same dose levels. This was attributed to the irritant effects of the copper sulphate. Damage to the renal tubules, and a dose-related increase in liver damage (inflammation and changes in clinical chemistry) was apparent in the rats. Depletion of iron stores occurred in rats but not in mice, and changes in haematologic parameters at 13 weeks indicated microcytic anaemia in rats confirming the findings of the two week feed study. The NOAEL for the 13 week studies was $1000 \mathrm{mg} / \mathrm{kg}$ copper sulphate in rats (equivalent to 16 and $17 \mathrm{mg} / \mathrm{kg}$ bw/day copper in males and females respectively) based on liver, kidney and forestomach effects. In mice, effects on the liver and kidney were not observed but the NOAEL based on reduced body weights was $1000 \mathrm{mg} / \mathrm{kg}$ (43 mg copper $/ \mathrm{kg}$ bw/day) in male mice and $2000 \mathrm{mg} / \mathrm{kg}$ (124 mg copper $/ \mathrm{kg}$ bw/day) in females. Forestomach effects were reported in the mice at $4000 \mathrm{mg} / \mathrm{kg}$ or more copper sulphate.

\section{Exposure assessment}

Total exposure/intake:

Food

$1.4 \mathrm{mg} /$ day (mean from 1986/87 NDNS)

$3.0 \mathrm{mg} /$ day $\left(97.5^{\text {th }}\right.$ percentile from $1986 / 87$ NDNS)

Supplements

up to $2 \mathrm{mg} /$ day (Annex 4; OTC, 2001)

Drinking Water

up to $6 \mathrm{mg} /$ day (assuming $2 \mathrm{~L} /$ day consumption and the maximum permitted water copper concentration of $3 \mathrm{mg} / \mathrm{L}$.)

Estimated maximum

daily intake:

$3.0+2+6=11 \mathrm{mg}$

No potential high intake groups were identified. 


\section{Risk assessment}

Acute copper toxicity in humans is rare due to the emetic properties and unpleasant taste of the compounds. There are relatively few data on lower level or chronic oral copper exposure in man. Copper is kept under tight homeostatic control to prevent the accumulation of excess amounts. Where dietary copper is high, absorption is reduced and, in particular, biliary excretion increased. Other mechanisms, which sequester excess copper within the cell, may also occur. Copper toxicity occurs when such defences are overwhelmed. Thus, in man, liver toxicity has only been seen in genetically determined conditions such as Wilson's disease and in Indian Childhood Cirrhosis where hepatic copper accumulation occurs. There is no evidence for copper carcinogenicity in the general population, although an elevated incidence of hepatoma has been suggested in untreated Wilson's disease patients or subjects recovering from ICC.

In the general human population, the key adverse effects usually associated with excess copper intake are gastrointestinal, resulting from consumption of copper in water or beverages.

There are some animal data for copper, although few from adequate chronic studies. Forestomach lesions, liver and kidney toxicity and anaemia were reported in a sub-chronic toxicity study. Reproductive effects have been reported in laboratory animals, but these findings are not consistent.

\section{ESTABLISHMENT OF SAFE UPPER LEVEL}

Key studies:

Hebert et al., (1993)

Supporting studies:

Turnland et al. (1989), Pratt et al. (1985)

NOAEL:

$16 \mathrm{mg} / \mathrm{kg}$ bw/day from a 13-week study in rats.

Uncertainty

10 for inter-species variation $\mathrm{x} 10$ for intra-individual

Factors: variation (total 100)

Safe Upper Level $16 / 100=0.16 \mathrm{mg} / \mathrm{kg}$ bw $/$ day (equivalent to $10 \mathrm{mg} /$ day in a for total daily $60 \mathrm{~kg}$ adult).

consumption over

a lifetime:

Copper is kept under tight homeostatic control to prevent the accumulation of excess amounts. Copper toxicity occurs when such defences are overwhelmed.

Balance studies and supplementation studies in small numbers of human volunteers suggest that doses of $7.5 \mathrm{mg}$ to $10 \mathrm{mg}$ copper/day in food or supplements are not associated with adverse effects. In controlled studies on the effects of copper levels in drinking water. Pizarro et al. (1999) reported that water containing $3 \mathrm{mg}$ copper $/ \mathrm{L}$ was associated with gastrointestinal disturbance in adults, whereas water containing $1 \mathrm{mg} / \mathrm{L}$ was not. Olivares and colleagues reported that water containing $2 \mathrm{mg}$ copper/L (resulting in intakes of up to $2.5 \mathrm{mg} /$ day in formula fed infants and $2 \mathrm{mg} /$ day + an undefined contribution from breast milk in breast-fed infants) were not associated with adverse effects. No differences in liver function tests related to copper intakes were apparent. It is noted that there was a 
higher rate of diarrhoea in formula-fed infants. However, since breast-feeding is known to protect against diarrhoea this is not unexpected. The gastrointestinal disturbance reported by Pizarro et al. appears to be the result of a direct irritant effect from copper in water and is not so apparent when copper is present in a food matrix.

Whilst liver toxicity occurs in humans who accumulate large quantities of copper in the liver, this is as a result of genetically determined conditions such as Wilson's disease and Indian Childhood Cirrhosis and is not relevant to the general population. Copper balance studies indicate that decreased absorption and increased excretion occurs when excess copper is consumed so that homeostasis is maintained.

A NOAEL of $16 \mathrm{mg} / \mathrm{kg}$ bw/day was identified in a well-reported sub-chronic toxicity study in rats. Higher doses resulted in damage to the forestomach, kidney and liver. If uncertainty factors of 10 for inter-species variation and 10 for intra-individual variation (total 100) are applied to this, a Safe Upper Level of $0.16 \mathrm{mg} / \mathrm{kg}$ bw day for total intake of copper is derived. This is equivalent to $10 \mathrm{mg} /$ day in a $60 \mathrm{~kg}$ adult. This is consistent with the data from small scale human studies which suggest that up to $10 \mathrm{mg} /$ day supplemental copper may be without adverse effect. The worst-case maximum estimated daily exposure from food and water is $9 \mathrm{mg} /$ day copper suggesting that there is a margin of $1 \mathrm{mg} /$ day for supplementation or other additional intake.

The exposure estimate suggests that individuals in the UK could theoretically consume in excess of $6 \mathrm{mg} /$ day copper from water alone if it was assumed that $2 \mathrm{~L} /$ day water was consumed containing copper at the statutory limit of $3 \mathrm{mg} / \mathrm{L}$. This was also the dose associated with gastrointestinal effects in the Pizarro study. However, in practice copper levels in UK drinking water are much lower, so this level of exposure is unlikely to occur. There is no evidence that copper intakes in water in the UK present any risk to health.

\section{References}

COMA (1991) Committee on Medical Aspects of Food and Nutrition Policy: Dietary Reference Values for Food Energy and Nutrients for the UK. HMSO, London.

Hebert, C.D., Elwell, M.R., Travlos, G.S., Fitz, C.J., Bucher, J.R. (1993) Subchronic toxicity of cupric sulfate administered in drinking water and feed to rats and mice. Fundamental and Applied Toxicology 21, 461-475.

Olivares, M., Pizarro, F., Speisky, H., Lonnerdal, B., Uauy, R. (1998) Copper in infant nutrition: safety of World Health Organization provisional guideline value for copper content of drinking water. Journal of Pediatric Gastroenterology and Nutrition, 26, 251-257.

OTC (2001). OTC Directory 2001-2002, Proprietary Association of Great Britain

Pizarro, F., Olivares, M., Uauy, R., Contreras, P., Rebelo, A., Gidi, V. (1999) Acute gastrointestinal effects of graded levels of copper in drinking water. Environmental Health Perspectives 107, 117-121.

Pratt, W.B, Omdahl, J.L, Sorenson, J.R.J. (1985). Lack of effects of copper gluconate supplementation. American Journal of Clinical Nutrition 42, 681-682.

Turnlund, J.R., Keys, W.R., Anderson, H.L., Acord, L.L. (1989) Copper absorption, excretion, and retention in young men at three levels of by use of the stable isotope ${ }^{65} \mathrm{Cu}$. American Journal of Clinical Nutrition 49,870 . 


\section{Risk Assessment}

\section{Germanium}

\section{General information}

\section{Chemistry}

Germanium is a non-metallic element, which can exist in valence states of 2 and 4 . Within this risk assessment, the word germanium refers to ionic germanium, except when specific compounds are mentioned.

\section{Natural occurrence}

Germanium is found in a range of minerals and ores. Both organic and inorganic germanium compounds occur.

\section{Occurrence in food, food supplements and medicines}

Germanium is present in foods including beans, tomato juice, oysters, tuna and garlic. Germanium is not currently present in any licensed medicines. Germanium food supplements were voluntarily withdrawn by industry in the UK due to toxicity but can be obtained by mail order over the internet and are available in other parts of the world (for example, supplements containing $100 \mathrm{mg} \mathrm{Ge-132).}$

\section{Other sources of exposure}

No data were identified.

\section{Recommended amounts}

Germanium is not considered to be an essential trace element.

\section{Analysis of tissue levels and germanium status}

No data on the analysis of tissue levels and germanium status have been identified.

\section{Brief overview of non-nutritional beneficial effects.}

Germanium has been claimed to have beneficial effects on a number of conditions including cancer, AIDS, liver disease, hypertension, arthritis, food allergies and malaria. The results of trials using spirogermanium (an organic compound) as a cancer treatment are inconclusive. 
Part 3 Trace Elements

\section{Function}

There is no known biological function for germanium. It has been suggested that germanium may be involved in carbohydrate metabolism.

\section{Deficiency}

No deficiency disease has been described. It has been suggested that germanium deficiency could be a contributory factor in Kashin-Beck disease (an osteo-arthritic condition affecting children in China and the former Soviet Union) but this is based on a reported association from a single study.

\section{Interactions}

It has been suggested that germanium can interact with silicon in bone metabolism. It can interfere with the action of loop diuretic drugs and inhibit the activity of a number of enzymes including lactate and alcohol dehydrogenase. Hexabarbital-induced sleeping times are increased in mice treated with germanium compounds suggesting that inhibition of cytochrome P450 activity may also occur. Organic germanium compounds have been reported to inhibit the detoxication enzyme glutathione-S-transferase.

\section{Absorption and bioavailability}

Germanium compounds are readily absorbed following oral exposure.

\section{Distribution and metabolism}

Germanium is distributed throughout the body tissues, particularly the kidney and thyroid. Organic germanium is thought not to accumulate to the same extent as inorganic germanium compounds but few data on germanium metabolism are available.

\section{Excretion}

Germanium is excreted largely in the urine. Some biliary and faecal excretion also occurs.

\section{Toxicity}

Inorganic forms of germanium compounds are more toxic than organic germanium compounds.

\section{Human data}

Germanium toxicity in humans has generally occurred following consumption of inorganic germanium as a food supplement. Initial symptoms include anorexia, weight loss, fatigue and muscle weakness. This is followed by renal dysfunction and failure, which can be fatal. Peripheral neuropathy has also been reported. Where patients have recovered, renal function has not returned to normal. Transient neurotoxic side effects have been reported from the use of spirogermanium in clinical trials.

No data on trials in healthy human volunteers have been identified. 


\section{Animal data}

Germanium has low acute oral toxicity. Symptoms of acute toxicity from large doses include sedation, vasodilation, ptosis, cyanosis and tremors with death resulting from respiratory paralysis. Symptoms of chronic and sub-chronic effects of inorganic germanium include weight loss, changes in organ weights, progressive neuropathy (characterised by de- and re-myelination, nerve oedema and changes to Schwann cells) and renal damage. Organic compounds are less toxic but produce weight loss and decreased red blood cell counts. Few data are available on reproductive toxicity.

\section{Carcinogenicity and genotoxicity}

Sodium germanate was not carcinogenic in rats. Mutagenicity data are limited, but negative results were obtained with the germanium compounds tested.

\section{Vulnerable groups}

No vulnerable groups have been identified.

\section{Genetic variations}

No genetic variations resulting in increased susceptibility to germanium toxicity have been identified.

\section{Mechanism of toxicity}

The precise mechanism is uncertain but specific pathological effects on the mitochondria of the kidney and nervous system have been observed.

\section{Dose-response characterisation}

The toxicity of inorganic germanium compounds is clearly progressive. It is difficult to define a threshold since the available data consist of human case reports and animal studies where few dose levels have been used. Fatal renal failure in humans has occurred following cumulative doses of $>20 \mathrm{~g}$ germanium. In animals no toxicity was reported for inorganic germanium when the animals were dosed with $5 \mathrm{mg}$ germanium/kg bw/day for 4 weeks only. However, adverse effects were apparent at the same dose level over longer periods of exposure. Organic germanium compounds are less toxic but adverse effects have been reported. 


\section{Studies of particular importance in the risk assessment}

(For full review see http://www.food.gov.uk/science/ouradvisors/vitandmin/evmpapers or the enclosed CD).

\section{Human data}

Schauss, 1991

18 case reports of germanium-induced renal toxicity were reviewed, two of which were fatal. The patients took supplements of germanium dioxide or Ge-132, an organic form. The cumulative doses consumed ranged from 16-328 g over a 4 to 36 month period. However it is considered possible that the Ge-132 may have been contaminated with inorganic germanium compounds.

\section{Animal data}

Kanisawa and Schroeder, 1967

A group of 135 Swiss CD mice were given drinking water containing $5 \mathrm{mg} / \mathrm{L}$ sodium germanate from weaning throughout their lifespan. The control group consisted of 198 mice. The authors estimated that germanium intake was $0.35 \mathrm{mg} / \mathrm{kg}$ bw/day with an additional $0.019 \mathrm{mg} / \mathrm{kg}$ germanium being supplied by the diet. The body weights of the treated males were lower throughout the study, becoming significantly so in the second year. The body weights of the treated females were lower than the controls in the second year of treatment, but this was only significant at 15 months. Few historical control data were provided, not all of the animals were autopsied, and only tissues considered to be abnormal were microscopically evaluated. Sodium germanate was not found to be carcinogenic.

Sanai et al., 1991

Groups of female Wistar rats were fed $75 \mathrm{mg} / \mathrm{kg}$ bw germanium dioxide or $120 \mathrm{mg} / \mathrm{kg}$ carboxyethylgermanium sesquoxide $\left(\mathrm{GeO}_{2}, \mathrm{Ge}-132\right)$ for 24 weeks in the diet. The doses contained equivalent quantities of germanium. Treatment was then withdrawn for a further 16 week recovery period. By week 24, weight loss, anaemia, liver dysfunction, and increased blood urea nitrogen, sodium phosphate and creatinine were apparent in the $\mathrm{GeO}_{2}$ group only. Vacuolar degeneration and granular deposition were observed in the degenerated renal tubules of this group. The scores for tubular degeneration were $95 \pm 9 \%, 3 \pm 1 \%$ and $1 \pm 1 \%$, in the $\mathrm{GeO}_{2}$, Ge-132 and control groups respectively. After discontinuation of the treatment, fibrosis became prominent in the $\mathrm{GeO}_{2}$ treated group even at week 40, 16 weeks after treatment. No effects on renal histology were found in the control or Ge-132 groups. The only adverse effects observed in the Ge-132 group were elevated liver weights at week 16 and decreased liver weights compared to the controls following the recovery period.

Anger et al., 1992

Groups of 30 male and female Wistar rats were given an oral dose of $1000 \mathrm{mg} / \mathrm{kg}$ bw/day Ge-132 five days a week for 6 months (the route was unclear but appears to be stomach tube/gavage). Three deaths occurred in the treated group as a result of accidental damage to the animals made when administering the dose. A small decrease in body weight was observed in the treated animals. A significant decrease in erythropoiesis and leukocyte ratios was also found. The main toxic effect was a slight renal dysfunction, characterised by increased serum creatinine levels (significant in males only). Urinary germanium 
excretion was constant and related to achieved dose. It is stated that no preferential accumulation of germanium occurred, but it is apparent that germanium levels in the kidney and liver were 3 times higher than those in tissues such as the heart or the lung. The study is limited, in that it is not well-reported and few details are provided on many aspects of the investigation. However it suggests that higher and/or more sustained doses of Ge-132 are associated with renal dysfunction in male rats.

Yim et al., 1999

Sprague Dawley rats were treated with doses of 5, 10, 20, 50, 100 or $150 \mathrm{mg} / \mathrm{kg}$ germanium dioxide dissolved in water and administered by orogastric tube for up to 24 weeks. The aim of the experiment was to determine the earliest onset of germanium-induced myopathy and the minimum dose of $\mathrm{GeO}_{2}$ that would cause the effect. The earliest pathological changes in the muscle fibres observed by electron microscopy were abnormalities of mitochondrial shape and size and increased numbers of mitochondria. A number of rats died during the study. The minimal dose of $\mathrm{GeO}_{2}$ causing myopathy was $10 \mathrm{mg} / \mathrm{kg}$ bw/day for 4 or more weeks.

\section{Exposure assessment}

Total exposure/intake

Food mean: $0.004 \mathrm{mg} /$ day

97.5th percentile: $0.007 \mathrm{mg} /$ day (1994 TDS)

Estimated maximum intake: $0.007 \mathrm{mg} /$ day

No potential high intake groups were identified.

\section{Risk assessment}

Germanium is not considered to be an essential element although it has been marketed as such and was available in the UK as a dietary supplement prior to its voluntary withdrawal.

Germanium causes specific toxic effects on the kidney, the muscle and the nervous system in both animals and man. A number of human case reports are available, many of these involving fatal renal failure. However, the incidence of renal toxicity as a fraction of all those taking germanium supplements is uncertain. The effects of germanium on the kidney are not completely reversible following the cessation of treatment. Comparable data are available from animal studies.

Germanium toxicity has been largely associated with consumption of inorganic germanium dioxide and it has been suggested that organic germanium compounds do not cause adverse effects. For example Sanai et al. (1991) showed that an organic germanium compound carboxyethylgermanium sesquoxide (Ge-132) did not cause tubular degeneration in rats compared to a comparable dose of germanium dioxide over the same 16 week period. However, Anger et al. (1992) demonstrated moderate tubular damage when a higher dose of Ge-132 was given over a longer time period. Sources claiming the lack of toxicity of 
organic germanium are generally secondary and are not available in the peer-reviewed scientific literature. Although organic germanium compounds are less toxic than their inorganic equivalents, adverse effects are still apparent. The mechanisms of germanium-induced target organ toxicity are uncertain.

Naturally-occurring germanium present in food does not appear to be associated with any adverse effects.

\section{EVM OPINION}

Germanium is not considered to be an essential element. Germanium is a cumulative toxin causing serious, and potentially fatal, adverse effects on the kidneys. Naturally-occurring germanium present in food does not appear to be associated with any adverse effects, but there are insufficient data to define a NOAEL for chronic exposure in either animals or humans at levels in excess of this. Whilst organic forms of germanium appear to be less toxic than inorganic ones, at present there are also insufficient data to establish a NOAEL or to rule out cumulative toxicity of a similar type to that of inorganic germanium, albeit occurring at higher doses.

Dietary supplements containing germanium were voluntarily withdrawn in the UK but may be obtained by mail order over the Internet.

Given the cumulative nature of germanium toxicity there are insufficient data to set a Safe Upper Level for any amount of germanium in excess of that provided by the diet.

\section{References}

Anger, F., Anger, J.P, Guillou, L. and Papillon (1992). Subchronic oral toxicity (six months) of carboxyethylgermanium sesquoxide $\left[\left(\mathrm{HOOCCH}_{2} \mathrm{CH}_{2} \mathrm{Ge}\right)_{2} \mathrm{O}_{3}\right] n$ in rats. Applied Organometallic Chemistry 6, 267-272.

Kanisawa, M. and Schroeder, H. (1967). Life term studies on the effects of arsenic, germanium, tin and vanadium on spontaneous tumours in mice. Cancer Research. 27, 1192-1195.

Sanai, T., Okuda, S., Onoyama, K., Oochi, N., Takaichi, S., Mizuhira, V. and Fujishima, M. (1991). Chronic tubulointerstitial changes induced by germanium dioxide in comparison with carboxyethylgermanium sesquoxide. Kidney International 40, 882-890.

Schauss, A. G. (1991) Nephrotoxicity and neurotoxicity in humans from organogermanium compounds and germanium dioxide. Biological Trace Element Research 29, 267-280.

Yim, S.Y, Lee, I.Y, and Kim, T.S (1999). Enzyme histochemical study of germanium dioxide-induced mitochondrial myopathy in rats. Yonsei Medical Journal 40, 69-75. 


\section{Risk Assessment}

\section{lodine}

\section{General information}

\section{Chemistry}

lodine is a non-metallic group VII element (a halogen). At room temperature it is a blue-black solid which sublimes into a gaseous form. It reacts readily, common compounds being iodides (e.g. potassium iodide) and iodates (e.g. potassium iodate). lodine can exist in oxidation states $-1,1,5$ and $7,-1$ (iodide) being the most common. In this risk assessment, the word iodine refers to both the elemental and ionic forms of iodine.

\section{Natural occurrence}

lodine is present in seawater, igneous rocks and some soils.

\section{Occurrence in foods, food supplements and medicines}

High levels of iodine are present in marine fish (up to $2.5 \mathrm{mg} / \mathrm{kg}$ ), shellfish (up to $1.6 \mathrm{mg} / \mathrm{kg}$ ) and sea salt (up to $1.4 \mathrm{mg} / \mathrm{kg}$ ). Levels in cereals and grains vary depending on the iodine content of the soil. The food colour erythrosine is also rich in iodine. In the UK, iodine is also present in cows' milk (average level $0.15 \mathrm{mg} / \mathrm{kg}$ ), probably as a result of the use of supplemented cattle feeds and iodophors as teat sterilants. The content of iodine in raw food is reduced by cooking. lodine, as iodide, is present in multivitamin and mineral supplements (providing up to $0.49 \mathrm{mg}$ iodine/day) and is a component of kelp products. It is also present in licensed medicines, topical antiseptics and radiographic contrast agents.

lodine intakes in children are higher than those in adults, because of a greater consumption of milk, and are likely to be higher in the winter than the summer because winter milk contains more iodine.

\section{Other sources of exposure}

lodine intake from water is estimated to be generally less than $0.03 \mathrm{mg} /$ day.

\section{Recommended amounts}

COMA established a Lower Reference Nutrient Intake (LRNI) and a RNI for iodine of 0.07 and 0.14 $\mathrm{mg} /$ day, respectively (COMA, 1991).

\section{Analysis of tissue levels and iodine status}

Urinary iodine excretion, or blood levels of thyroxine (T4) or thyroid stimulating hormone (TSH) have been used to estimate iodine status. 
Part 3 Trace Elements

\section{Brief overview of non-nutritional beneficial effects}

lodine is claimed to assist with weight loss, rheumatism, ulcers, hair loss and the maintenance of healthy arteries, nervous tissue and nails.

\section{Function}

lodine forms part of the thyroid hormones, thyroxine (T4) and triiodothyronine (T3). Receptors binding T3 and T4 have been found in the cell nucleus and in mitochondria. These hormones are involved in the maintenance of metabolic rate, cellular metabolism and integrity of connective tissue. Thyroid hormones are necessary for the development of the nervous system in the foetus and infant.

\section{Deficiency}

A variety of mechanisms exist to compensate for low levels of iodine intake. These include enlargement of the thyroid gland (goitre). Only when these mechanisms fail do the clinical signs of hypothyroidism (also known as myxoedema) develop. Symptoms and signs of hypothyroidism include lethargy, weakness, weight gain, poor concentration, oedema, myalgia, dry skin, delayed tendon reflexes and slow heart rate. In pregnancy, iodine deficiency is associated with an increased risk of miscarriage, stillbirth and congenital abnormality. Cretinism is the result of iodine deficiency in the developing foetus, and is characterised by mental retardation, deaf mutism, and spastic diplegia. A less common form of cretinism is the myxoedematous type, which is characterised by hypothyroidism and dwarfism.

\section{Interactions}

lodine interacts with selenium and possibly with vanadium.

Natural goitrogens (which impair thyroid hormone synthesis) may be present in soybeans, peanuts and walnuts, or may be formed (for example, thiocyanate) from foods such as corn, maize, cassava, potato, cauliflower and broccoli. Pollutants derived from coal (such as 2 and 5 methyl resorcinol) have also been reported to act as goitrogens.

\section{Absorption and bioavailability}

The few data available suggest that the bioavailability of iodine in food is high.

Inorganic iodine (generally in the form of iodide) is readily absorbed, largely from the small intestine. It can also be absorbed through the skin, absorption being increased when the skin is damaged.

\section{Distribution and metabolism}

Once absorbed, iodine is distributed rapidly throughout the extracellular fluid, secreted into saliva, and enters the gastrointestinal system, from where it is reabsorbed. lodine can cross the placenta and is secreted into human breast milk. 
lodine is taken up and stored in the thyroid gland mainly as iodinated tyrosines and thyronines, within a large protein (thyroglobulin), for the synthesis of thyroid hormones (T4 and T3). Excess iodide is excreted in the urine. T4 and T3 are released into the blood (by passive diffusion) after proteolysis from thyroglobulin, and circulate in protein-bound and free forms. The free forms of the hormones enter tissues, in particular the liver (for T4) and skeletal muscle (for T3). The thyroid gland is the only significant store of iodine.

\section{Excretion}

lodine is largely excreted in the urine, mainly in the form of iodine. Very small amounts of iodine may be excreted in sweat, faeces and exhaled air.

\section{Toxicity}

\section{Human data}

Several biological mechanisms protect against iodine toxicity; these include reduced iodine uptake and preferential production of the more heavily iodinated thyroid hormones. Not all exposed subjects will react to excess iodine.

Clinical features of acute iodine toxicity that have been produced following accidental or deliberate ingestion, or medical procedures such as wound irrigation, include gastrointestinal disturbance (vomiting and diarrhoea), metabolic acidosis, seizure, stupor, delirium and collapse. Sensitivity reactions, such as iodide mumps, iododerma and iodide fever may also occur following treatment with iodine-containing drugs, or the use of radiographic contrast media.

Chronic and sub-chronic toxicity have also been identified. Excess iodine or iodide intake may disrupt thyroid function, resulting in the induction of hypothyroidism with or without goitre, hyperthyroidism (thyrotoxicosis) and changes in the incidence and types of thyroid malignancies. Responses of this type frequently occur where there is general high iodine intake or where intervention has taken place to correct iodine deficiency.

The pattern of effects changes over time as iodine exposure in the population changes. For example, in the UK, the possibility of iodine induced hyperthyroidism associated with prior iodine deficiency appears to be less common due to recent increases in the iodine intake of the population as a whole.

\section{Supplementation studies}

Measures of serum thyroid hormone levels, such as T3, T4 and TSH are used as indicators of iodine disturbances in human studies. Increased TSH and decreased T3 and T4 levels have been noted at supplemental doses of 0.5-1.5 mg/day and above for 2 weeks. Significant reductions in further iodine uptake have been noted with supplemental doses of $2 \mathrm{mg}$ iodine/day. Dietary iodine intakes were uncertain. 
Part 3 Trace Elements

\section{Animal data}

Symptoms of acute iodide or iodate toxicity include diarrhoea, with alternating periods of hyperactivity, weakness, prostration, convulsions and death.

Symptoms of sub-chronic toxicity include reduced weight gain and haemolysis, in addition to specific effects on the thyroid.

\section{Carcinogenicity and genotoxicity}

No data have been identified on the carcinogenicity of iodine. Both iodine deficiency and excess can promote tumour formation in animals pre-exposed to known carcinogens. Metaplasia of the thyroid was reported in rats given potassium iodide in drinking water for two years. This was thought to occur via a non-genotoxic proliferation dependent mechanism. Human epidemiological studies have shown variations in the incidence of thyroid cancer, depending on the levels of iodine available in water supplies in these areas. The type of cancer appears to differ depending on whether iodine levels are deficient or excess. Changes to the pattern of thyroid tumours have been noted after prophylaxis. The mutagenicity data for iodine are generally negative.

\section{Mechanism of toxicity}

The adverse effects of high levels of iodine are largely due to the derangement of thyroid hormone metabolism, the thyroid-pituitary axis and the compensatory mechanisms that exist to protect such metabolism against low or high levels of iodine intake. Previous exposures to iodine and the complex effects of pre-existing thyroid conditions also influence the effects of subsequent exposure.

\section{Dose-response characterisation}

The threshold level of iodine necessary to induce thyrotoxicosis is uncertain and appears to vary depending on previous iodine exposure. The available data are inadequate to establish a dose-response relationship.

\section{Vulnerable groups}

Pregnant and lactating women, and neonates are considered to be vulnerable groups as iodine freely crosses the placenta and is expressed in breast milk, and goitre and hypothyroidism have been reported to occur in the offspring of mothers exposed to pharmacological doses of iodine and iodide.

\section{Genetic variations}

No genetic variation in the uptake and toxicity of excess iodine has been identified. 


\title{
Studies of particular importance in the risk assessment
}

\author{
(For full review see http://www.food.gov.uk/science/ouradvisors/vitandmin/evmpapers or the \\ enclosed CD).
}

Saxena et al., 1962

This study investigated the minimal effective dose necessary to suppress radioactive iodine uptake. Increasing doses of stable iodine (as sodium iodide) were given to children with normal thyroid function. Doses of $0.1,0.3,0.6$ or $1 \mathrm{mg}$ iodide/day were given to children aged 1-3, 4-6 or 9-11 years of age. The 24 hour uptake of ${ }^{131}$ I was measured every two weeks until uptake declined to $5 \%$ or until there was no change between successive uptakes. lodide was then discontinued. No toxic effects were observed. Thyroid hormone concentrations were not assessed. The length of the study in the particular subgroups is uncertain, but appears to have been no longer than 3 months. The degree of suppression of radioactive iodine uptake was related to dose, with maximum suppression of ${ }^{131} \mathrm{l}$ being achieved with a dose of 1.5-2 mg iodide per square meter of body surface. This was equivalent to a dose of 1-2 mg iodide in children and 3-4 mg in adults. ${ }^{131}$ uptake rapidly returned to normal once the iodide was discontinued.

\section{Freund et al., 1966}

The health status and thyroid function of representative subjects of a prison population were assessed before and during use of iodinated water for 9 months. The group initially comprised 133 euthyroid prisoners, but was gradually reduced to 70 , due to discharge from the institution. Water containing $1 \mathrm{mg}$ iodine $/ \mathrm{L}$ resulted in a marked decrease in the uptake of test doses of radioactive iodine, to a level of $7 \%$. However, protein bound iodine levels did not change significantly until the iodine concentration of the water was increased to $5 \mathrm{mg} / \mathrm{L}$ for 2 months (following 7 months exposure at the lower level), radioactive iodine uptake then fell to $2 \%$. Serum thyroxine concentration did not change regardless of the iodine concentration of the water. No information is provided on individual iodine intake, but water consumption can be assumed to have been approximately $2 \mathrm{~L} /$ day. Prisoners continued to receive iodine from the diet, including iodised salt. No effects on thyroid function were reported in non-prison personnel who swam in water iodinated at a level of $5 \mathrm{mg} / \mathrm{L}$. No evidence of iodine allergy was apparent. Two (of fifteen) male inmates had impaired iodine organification, after consuming water (for at least 3 months) containing $1 \mathrm{mg}$ iodine $/ \mathrm{L}$, as measured by the change in thyroidal ${ }^{131} \mathrm{I}$ concentration following administration of perchlorate. The clinical significance of this effect is unclear, since individual T4 concentrations remained unchanged throughout the study i.e. no patients demonstrated iodineinduced hypothyroidism.

\section{Stockton and Thomas, 1978}

No significant change in serum thyroxine levels was seen following the iodination of a prison water supply at a concentration of 0.5 to $0.75 \mathrm{mg} / \mathrm{L}$ (estimated intake $1-1.5 \mathrm{mg} /$ day) for up to 15 . During the same period, 177 women in the prison gave birth to 181 full term infants none of whom had enlarged thyroids. Ninety-nine mothers (of 101 infants) had been in prison for $\geqslant 122$ days, whilst eighty mothers had been imprisoned for $<118$ days (10-118). This study has been published as an abstract only. 
Sternthal et al., 1980

Single doses of 10-100 mg iodide (as sodium iodide), were given to adult volunteers. In a subsequent experiment, 12 daily doses of $10-100 \mathrm{mg}$ sodium iodide were given. All doses greater than $10 \mathrm{mg}$ suppressed 24 -hour uptake of ${ }^{123} \mathrm{I}$ to between $0.7 \%$ and $1.5 \%$. Continued daily administration of $15 \mathrm{mg}$ iodide or more resulted in 24-hour uptake values of less than $2 \%$ (compared with baseline values of 17 to $23 \%$ ). Pooling the 24 hour uptake values of the subjects taking $30-100 \mathrm{mg}$ iodide resulted in a significant increase in serum TSH and significant decreases in T3 and T4 levels. These values returned to normal following the withdrawal of treatment. No adverse effects were reported.

\section{Braverman, 1987}

Doses of approximately $150 \mathrm{mg}$ iodine given to normal adults for 1-3 weeks induced a small but significant decrease in serum T3 and T4 levels, with a small, significant compensatory increase in serum TSH, and TSH response to thyrotrophin-releasing hormone (TRH). However, the values for these parameters were within the normal range. Similar changes in thyroid function were reported in male volunteers given 200 $\mathrm{mg} /$ day of the iodine-rich colour erythrosine for 14 days. The increase in urinary iodine excretion was approximately $1.5 \mathrm{mg}$ daily, which is equivalent to a 2-7 fold increase in dietary iodine intake. No effect was found when the volunteers were given $60 \mathrm{mg}$ erythrosine/day. The effects caused by $200 \mathrm{mg}$ erythrosine/day were reproduced when $1.5 \mathrm{mg} /$ iodine was administered. The author considered that these subtle changes in thyroid function represented physiological adaptation to iodine excess.

Serum T3 and T4 concentrations and free T4 index were unchanged in normal subjects who used iodine-containing mouthwash daily for 6 months. (A small but significant increase in TSH levels was measured; this was within the normal range and considered a normal physiological adaptive response. The amount of iodine absorbed was equivalent to $2-4 \mathrm{mg} /$ day (based on increased urinary excretion).

Paul et al., 1988

Subjects with a normal functioning thyroid ( 9 men and 23 women) between the ages of 26 and 56 were given doses of 0.25 , 0.5 or $1.5 \mathrm{mg}$ supplemental iodine/day for 14 days. There were small but significant decreases in serum T3 and T4 concentrations following the administration of $1.5 \mathrm{mg} /$ day as well as a small compensatory increase in serum TSH levels and in TSH response to TRH. No effects were observed in the subjects given the lower levels of iodine.

\section{Gardner et al., 1988}

Thirty healthy men aged 22-40 were randomly assigned to receive $0.5,1.5$ or $4.5 \mathrm{mg}$ iodide/day for 2 weeks. Several thyroid parameters being measured on day 1 and day 15. Mean serum T4 levels and free T4 index values were decreased at the 1.5 and $4.5 \mathrm{mg} /$ day dose. No changes were observed in serum T3 concentrations at any dose. Doses of $0.5 \mathrm{mg} /$ day resulted in a significant increase in the serum TSH response to TRH and the two larger doses resulted in increases in both basal and TRH-stimulated serum TSH concentrations.

\section{Chow et al., 1991}

A randomised controlled trial was performed to investigate the effects of low levels of iodine supplementation in healthy women and in women with sub-clinical Hashimoto's thyroiditis (one of the two major types of autoimmune lymphocytic thyroiditis, characterised by an enlarged thyroid). From a group of 225 women screened for thyroid microsomal antibody, 20 antibody positive women and 30 antibody negative controls were recruited into a trial comparing iodide and placebo. Additional groups 
of older subjects from iodine sufficient and iodine deficient areas were also enrolled. The subjects received a supplement of $0.5 \mathrm{mg}$ iodide/day or placebo. Dietary iodine intake was estimated to be approximately $0.25 \mathrm{mg} /$ day. Free thyroxine (T4) and thyrotrophin levels were measured after 14 and 28 days treatment. No changes in thyroid function were seen in the placebo group. All the iodide supplemented groups showed a small decrease in free thyroxine and increase in thyrotrophin levels. In two subjects thyrotrophin levels rose beyond the reference range and in a further three subjects, initially elevated thyrotrophin levels increased further. The effects seen were comparable in the normal and antibody positive subjects. The authors noted that the changes would not be of clinical significance in the general population but that a small shift in the mean value for these indices in a population tended to result in greater effects at the extremes of the distribution. The authors further concluded that dietary intakes in the UK should be monitored since total intakes of $0.75 \mathrm{mg} /$ day could adversely affect thyroid function.

\section{Garber et al., 1993}

Healthy hyperlipidaemic subjects (104) were placed on a low fat diet for 12 weeks. Between weeks 4 and 12 , they were randomised into a control group $(n=53)$ or were given an iodine enriched egg per day (iodine enriched eggs are produced by hens fed a diet containing kelp and contain an average of $0.71 \mathrm{mg}$ iodine/egg). No information was provided on the iodine content of the rest of the diet. Some subjects from each group continued in the study for an additional 4-8 weeks; there were 19 test subjects and 21 controls by week 16. Plasma and urinary iodine levels were significantly higher in the test subjects compared to the controls. No significant adverse clinical effects were reported (with the exception of one report of an allergic-type response shortly after beginning egg consumption). There were no differences in clinical chemistry values between the two groups. Thyroid function tests (T3, T4, T3 uptake, TSH, thyroid binding globulin and free T4 index) were normal in 17 (of 19) subjects and 20 (of 21) controls. The remaining subjects ( 2 test and 1 control) had elevated TSH levels. A report was provided for just 1 of these subjects, and revealed high iodine excretion, suggesting exposure to other sources of iodine. Unfortunately, the subject was not followed up and it was unknown whether clinically significant hypothyroidism developed.

Three of the studies cited above (Freund et al., 1966; Saxena et al., 1962; Sternthal et al., 1980) reported on blockage of radioactive iodine uptake following repeated doses of stable iodine. The purpose of these studies was to identify the minimum daily dose of iodine necessary to prevent thyroidal uptake of radioactive iodine in the event of nuclear fallout. The mechanism by which stable iodine blocks thyroidal uptake of radioactive iodine is not well established but may involve isotope dilution, saturation of iodide transport mechanism, interference with intrathyroidal organification of iodide (acute Wolff-Chaikoff effect), or inhibition of hormone release (Zanzonico and Becker, 2000). Thyroid radioactive iodine uptake is inversely proportional to iodine intake (Merck, 2001). In normal subjects the Wolff-Chaikoff effect is transient and overcome by prolonged exposure. Furthermore, when assessed in these studies, serum thyroid hormone concentrations were unaffected. It is therefore unclear whether this minimum inhibition dose can be considered an adverse effect. 


\section{Exposure assessment}

Total exposure/intake:

Food

Mean: $0.22 \mathrm{mg} /$ day $(1986 / 87 \mathrm{NDNS})$

97.5th percentile: $0.43 \mathrm{mg} /$ day

Drinking water

$<0.03 \mathrm{mg} /$ day (estimated intake from 2 litres of water containing $<0.015$ $\mathrm{g} / \mathrm{L})$

Supplements $\quad$ up to $0.49 \mathrm{mg} /$ day (Annex 4)

Estimated maximum intake: $0.43+0.03+0.49=0.95 \mathrm{mg} /$ day

Children are a potential high intake group, because iodine intakes in children are higher than those in adults due to higher milk consumption.

\section{Risk assessment}

The observed effects at lower levels of iodine exposure (as opposed to overt poisoning) seem to result from the derangement of thyroid hormone metabolism and the complex feedback mechanisms that govern it. Changes in thyroid parameters that represent normal compensating mechanisms are difficult to distinguish from those which indicate the onset of adverse or toxic effects in human studies as iodine exposure increases. Small changes in the levels of thyroid hormones may be of little significance, whereas larger changes or phenomena such as blocking of further iodine uptake or increased thyroid weight may be of more significance, suggesting that iodine intakes are at a level where permanent change or more significant symptoms could occur. The response to increased iodine intake also seems to vary depending on previous iodine exposure. Disruption of normal thyroid metabolism by increased iodine intake may manifest as either hyperparathyroidism or hypoparathyroidism (mimicking the hypothyroidism caused by iodine deficiency).

Subjects exposed to pharmacological doses of iodine, frequently as the result of medical treatment, may exhibit effects such as iodine fever or mumps or iododerma. The mechanisms for such reactions are uncertain, as is the nature of any susceptible groups. The relevance of such sensitivity reactions to the risk assessment process is therefore unclear.

Although there are human data available, much are unsuitable for risk assessment purposes since a large number of case reports or epidemiological studies have inadequate, if any, estimates of iodine exposure. Similarly, there are few, if any, good quality animal studies where clear adverse effects can be defined and thus animal studies have not been considered further. 


\section{ESTABLISHMENT OF GUIDANCE LEVEL}

There are insufficient data from human or animal studies to establish a Safe Upper Level for iodine.

A few studies (Paul et al., 1988; Chow et al., 1991; Gardner et al., 1988) have reported that supplemental doses of 0.5 to $1.5 \mathrm{mg}$ iodine/day produced small changes in the levels of thyroid hormones. Other studies (Saxena et al., 1962; Freund et al., 1966) indicate that supplemental doses of $2 \mathrm{mg} /$ day, in addition to the iodine present in the diet, result in the blockage of further iodine uptake. Although $0.5 \mathrm{mg}$ supplemental iodine/day has been reported to alter thyroid parameters in some studies, the changes are likely to represent normal feedback processes rather than adverse effects.

Taken together the data suggest that, for guidance purposes only, a supplemental intake of $0.5 \mathrm{mg} /$ day (equivalent to $0.003 \mathrm{mg} / \mathrm{kg}$ bw in a $60 \mathrm{~kg}$ adult), in addition to the iodine present in the diet would not be expected to have any significant adverse effects in adults. No uncertainty factors have been applied as these data come from a number of well-reported, controlled human studies. Assuming an intake of iodine from the diet of $0.43 \mathrm{mg} /$ day, this equates to a total intake, which would be expected to be without adverse effects, of $0.94 \mathrm{mg} /$ day (equivalent to $0.015 \mathrm{mg} / \mathrm{kg}$ bw/day in a $60 \mathrm{~kg}$ adult). It is possible that some consumers of foods with high levels of iodine, particularly children, may occasionally exceed this guidance level from normal dietary sources, but compensatory mechanisms exist and allay concerns for this potentially vulnerable group.

\section{References}

Braverman, L. E. (1987) lodine excess and thyroid function. Dietary iodine and other aetiological factors in hyperthyroidism. Conference report, Southampton 1987. MRC Environmental Epidemiology Unit, Scientific Report No. 9.

Chow, C.C., Phillips, D.I.W., Lazarus, J.H., Parkes, A.B. (1991) Effect of low dose iodide supplementation on thyroid function in potentially susceptible subjects: are dietary iodide levels in Britain acceptable? Clinical Endocrinology 34, 423-416.

COMA (1991). Dietary Reference Values for Food Energy and Nutrients for the United Kingdom. Report of the Panel on Dietary Reference Values, Committee on Medical Aspects of Food and Nutrition Policy. HMSO, London.

Freund, G., Thomas, W.C. Jr., Bird, E.D., Kinman, R.N., Black, A.P. (1966) Effect of iodinated water supplies on thyroid function. Journal of Clinical Endocrinology 26, 619-624.

Garber, D.W., Henkin, Y., Osterlund, L.C., Woolley, T.W., Segrest, J.P. (1993) Thyroid function and other clinical chemistry parameters in subjects eating iodine enriched eggs. Food and Chemical Toxicology, 31 247-251.

Gardner, D.F., Centor, R.M., Utiger, R.D. (1988) Effects of low dose oral iodide supplementation on thyroid function in normal men. Clinical Endocrinology 28, 283-288.

Merck (2001) The Merck Manual. Chapter 8: Thyroid Disorders. www.merck.com/pubs/mmanual/section2/chapter8/8a.htm 
Paul, T., Meyers, B., Witorsch, R.J., Pino, S., Chipkin, S., Ingbar, S.H., Braverman, L.E. (1988) The effect of small increases in dietary iodine on thyroid function in euthyroid subjects. Metabolism 37, 121-124.

Saxena, K. M., Chapman, E.M., Pryles, C.V. (1962) Minimal dosage of iodide required to suppress uptake of iodine-131 by normal thyroid. Science 138, 430-431.

Sternthal, E., Lipworth, L., Stanley, B., Abreau, B.S., Fang, S.H., Braverman, L.E. (1980) Suppression of thyroid radioiodine uptake by various doses of stable iodine. New England Journal of Medicine 303, 1083-1088.

Stockton, L. K. and Thomas, W. C. (1978) Absence of neonatal goitre during maternal use of iodinated water. Clinical Research 26, 536A.

Zanzonico, P.B., Becker, D.V. (2000) Effects of time of administration and dietary iodine levels on potassium iodide (KI) blockage of thyroid irradiation by ${ }^{131}$ from radioactive fallout. Health Physics 78 , 600-667. 


\section{Risk Assessment}

\section{Manganese}

\section{General information}

\section{Chemistry}

Manganese is an abundant metallic element that can exist in a variety of oxidation states. $\mathrm{Mn}^{2+}$ and $\mathrm{Mn}^{3+}$ are the most biologically important. Within this risk assessment, the word manganese refers to ionic manganese, except when specific manganese compounds are mentioned.

\section{Natural occurrence}

Manganese is present both naturally and as a result of contamination in soils, sediments and water.

\section{Occurrence in food, food supplements and medicines}

Manganese is present in foods, particularly green vegetables $(2 \mathrm{mg} / \mathrm{kg})$, nuts $(14.9 \mathrm{mg} / \mathrm{kg}$ ), bread $(8 \mathrm{mg} / \mathrm{kg})$ and other cereals $(6.81 \mathrm{mg} / \mathrm{kg})$. Tea is a rich source of manganese, containing $2.71 \mathrm{mg} / \mathrm{kg}$ and is the largest contributor to manganese intake. It is present in several licensed medicines, in combination with other substances, in use for prevention and treatment of nutrient deficiencies and other related conditions. Manganese is also present in a number of multi-vitamin and/or mineral food supplements at levels up to $10 \mathrm{mg}$.

\section{Other sources of exposure}

The level of manganese in drinking water ranges from 0.001 to $0.1 \mathrm{mg} / \mathrm{L}$, but is mostly around $0.01 \mathrm{mg} / \mathrm{L}$. Exposure to manganese may also occur through inhalation of airborne particles by miners, smelters and workers in the manufacture of dry batteries.

\section{Recommended amounts}

COMA and the $\mathrm{WHO}$ were unable to set a specific recommendation for manganese intake. The EU Scientific Committee for Food (SCF) considered a 'safe and adequate intake' to be 1-10 mg/person/day. The US National Research Council (NRC) specified Estimated Safe and Adequate Daily Dietary Intakes (ESADDIs) of 0.3-1, 1-3 and 2-5 mg/day for infants, children and adults respectively.

\section{Analysis of tissue levels and manganese status}

A method for assessing manganese status has not been defined, because of an inconsistent relationship between tissue measures and external exposures. Measurement of manganese-specific superoxide dismutase (MnSOD) activity and the ratio between manganese-specific superoxide dismutase and zinccopper superoxide dismutase activity has been proposed as a method for assessment, but may be confounded by elevated cytokine levels and disease states which increase MnSOD expression, independent of manganese status. 
Part 3 Trace Elements

\section{Brief overview of non-nutritional beneficial effects}

There have been claims of a variety of beneficial effects on diabetes, fatigue, memory, dizziness, rheumatoid arthritis, schizophrenia, some cases of epilepsy and the Parkinsonian side effects of phenothiazines.

COMA considered there might be a plausible basis for manganese affecting bone health, but that there is currently insufficient evidence to support dietary recommendations in this respect (COMA, 1998).

\section{Function}

Manganese is a component of a number of enzymes and activates a range of others. Glycosyl transferases are specifically activated by manganese.

\section{Deficiency}

In humans, manganese deficiency has only been observed under experimental conditions where decreased levels of cholesterol and clotting proteins were measured. Black hair was found to redden, fingernail growth slowed and scaly dermatitis was observed. In animals, manganese deficiency is associated with a variety of symptoms including skeletal malformations, and impaired growth and reproductive function.

\section{Interactions}

Manganese and iron compete for absorption sites. Fibre, phytate, calcium, phosphorus and magnesium may also interfere with manganese absorption. It has been suggested that ethanol may enhance manganese toxicity.

\section{Absorption and bioavailability}

Absorption takes place in the small intestine via a carrier-mediated mechanism; passive diffusion may also occur. Absorption is generally low but appears to be higher in infants and young animals.

Bioavailability of manganese from different food types is variable, but appears to be generally low, due to poor solubility.

\section{Distribution and metabolism}

In the portal blood manganese may bind to albumin and $\alpha_{2}$ macroglobulin. A small proportion of manganese is oxidised to $\mathrm{Mn}^{3+}$, and enters the systemic circulation, possibly by binding to transferrin. Manganese accumulates in mitochondria-rich tissues such as liver and pancreas. Manganese also accumulates in the brain, particularly in the globus pallidus, striatum and substantia nigra.

\section{Excretion}

Manganese is excreted largely in the faeces, mostly as a result of biliary excretion, although some direct secretion also occurs. A small amount of manganese is excreted in the urine. 


\section{Toxicity}

\section{Human data}

Occupational exposure, for example in manganese miners and smelters, to high levels of inhaled manganese has been associated with manganism, a neurotoxic condition similar to Parkinson's disease. Drinking water contaminated with manganese has also been associated with neurological and behavioural effects. There is an association between manganese accumulation and liver disease but this may be due to impaired biliary excretion caused by the liver disease rather than manganese toxicity. Effects on the immune system have been reported.

\section{Supplementation studies}

Supplementation trials of human volunteers with 15 or $9 \mathrm{mg}$ manganese/day for 124 days and 'many months' respectively have not reported any adverse effects, but it is unclear whether information on such effects was specifically sought.

\section{Animal data}

Manganese has low acute toxicity but neurotoxic effects have been observed in animals chronically fed high concentrations of manganese salts in the diet. High doses of manganese have also resulted in anaemia as a result of iron sequestration. Fertility is reduced by high doses of manganese but other reproductive parameters are unaffected.

\section{Carcinogenicity and genotoxicity}

Manganese has been tested in bioassays in rats and mice. No clear evidence of carcinogenicity has been found in either species. Some positive in vitro mutagenicity tests have been reported, although results are generally mixed. Studies in mice have indicated that manganese is not mutagenic in vivo.

\section{Mechanism of toxicity}

Several hypotheses have been proposed to explain the neurotoxicity of manganese, including irreversible oxidation of dopamine, via the reduction of $\mathrm{Mn}^{3+}$ to $\mathrm{Mn}^{2+}$ or interference with calcium. Other hypotheses include inhibition of mitochondrial respiration, decreased glutathione peroxidase and oxidant damage.

\section{Dose-response characterisation}

Manganese is a known neurotoxin at high occupational levels of inhalation exposure. However, it has also been suggested that exposure from lower levels in drinking water may result in more subtle neurological effects in human populations. Neurological effects have been reported at estimated intakes of 3.6-4.6 mg manganese from water, though comparable intakes have been negative in other studies. Other more limited data suggest that adverse effects may occur at even lower intake levels in children. In laboratory animals, adverse effects have been reported following long term exposure to manganese at doses greater than 50-200 mg/kg bw/day. Detailed neurological examinations were performed in only one study in mice which detected effects at $\geqslant 130 \mathrm{mg} / \mathrm{kg}$ bw/day. 


\section{Vulnerable groups}

Anaemic individuals may be vulnerable to the toxic effects of manganese due to the increased absorption that occurs in states of iron deficiency. Groups with impaired biliary clearance, such as patients with liver disease or older people, may also be susceptible to manganese accumulation and toxicity. It has also been reported that ethanol and long-term use of anti-psychotic drugs increases the susceptibility of humans to manganese toxicity.

\section{Genetic variations}

No genetic variations increasing susceptibility to manganese have been identified.

\section{Studies of particular importance in the risk assessment}

(For full review see http://www.food.gov.uk/science/ouradvisors/vitandmin/evmpapers or the enclosed CD).

\section{Kondakis et al., 1989}

This was a retrospective study of three cohorts exposed to water containing $0.0036-0.015,0.08-0.25$ or $1.8-2.3 \mathrm{mg} / \mathrm{L}$ manganese. The subjects were all aged over 50 years and had been exposed to manganese for $>10$ years. No estimates of the actual amount of water consumed or the manganese content of the diet were provided, although the 3 cohorts were considered to be comparable. Assuming $2 \mathrm{~L} /$ day water consumption, intakes for the three groups can be estimated to be 0.0072-0.03, 0.16-0.5 and 3.6-4.6 mg manganese plus dietary manganese. There was no difference in blood manganese levels in subjects from the 3 areas, but hair manganese was higher in subjects in the area with the highest level of manganese. It has been noted (US DHHS) that hair manganese is a useful measure of exposed versus unexposed populations but that it is of limited use in assessing individual exposure. The authors suggested that this could be because blood levels are highly variable and depend on how recent intake is, and thus could mask the influence of manganese stored in body tissues. A history was taken and a physical examination conducted by a neurologist unaware of the manganese exposure status. Neurological signs and symptoms were scored and were elevated in subjects from the highest manganese area. The symptoms scored ranged from depression, fatigue and hallucinations to tremor and impaired reflexes. Some of the symptoms were relatively subjective (e.g. fatigue) and it is uncertain whether one or two unusual 'outlier' individuals with a number of symptoms and/or signs may have produced the observed elevation in the high intake group. The authors noted that an effect was apparent at exposure levels which were negative in occupational studies. The authors attributed this finding to the age of the subjects, making them more susceptible to neuronal loss with ageing. The study was limited by inconsistent evidence of significant excess exposure and the subjective ascertainment of clinical effects.

\section{Vieregge et al., 1995}

This was a retrospective study of two cohorts exposed to water containing $<0.05 \mathrm{mg} / \mathrm{L}$ or $>0.3 \mathrm{mg} / \mathrm{L}$ (range 0.3-2.16 mg/L) manganese. The subjects in the two groups were aged 41-84 (mean $=57.5$ ) years and 41-86 (mean 56.9) years. Long duration of exposure (10-40 years) to manganese had taken place. No estimates of the actual amount of water consumed or of the manganese content of the diet were 
provided by the authors, but the cohorts were considered to be comparable. Assuming $2 \mathrm{~L} /$ day water consumption, manganese intakes of 0.1 and $4.3 \mathrm{mg}$ from water can be estimated. A neurologist blinded to the group status of the participants conducted the examination. No significant differences in blood manganese levels or neurological scores were found between the two groups. The authors noted the conflict with the findings of Kondakis et al., which they attributed to the greater age of the participants in that study. This study was also limited by the lack of exposure data.

\section{Exposure assessment}

Total exposure/intake:

Food

Mean: $4.9 \mathrm{mg} /$ day

97.5th percentile: $8.2 \mathrm{mg} /$ day (TDS, 1994)

Supplements $\quad 10 \mathrm{mg}$ manganese/day (Annex 4)

Water

$0.1 \mathrm{mg} /$ day (assuming UK level of $0.05 \mathrm{mg} / \mathrm{L}$ and consumption of $2 \mathrm{~L} /$ day).

Estimated maximum

intake:

$8.2 m g+10 m g+0.1 m g=18 m g /$ day

Tea drinkers are a potential high intake group, though the bioavailability of manganese in tea is unclear and may be low due to the presence of tannins.

\section{Risk assessment}

Cumulative exposure to manganese causes neurotoxicity in both humans and animals. Occupational exposure to manganese by inhalation has been associated with manganism, a condition similar to Parkinson's disease. This condition occurs as a result of inhalation exposure of high levels of manganese and is not relevant to the assessment of lower levels of manganese in food.

It has generally been considered that manganese uptake from oral exposure is subject to homeostatic control. However, there is also a suggestion of less severe neurotoxic effects at lower levels of exposure from consuming drinking water containing higher levels of manganese. The reported symptoms include muscle pain, fatigue, tremor, memory problems and impaired reflexes. Although other human data are available as noted above and in the review, it is not possible to draw conclusions from these due to problems with co-exposure, study design or lack of data.

Animal data are also available and indicate similar neurotoxic effects to those reported in humans. However, the neurotoxic effects are inevitably of a less subtle nature than the symptoms assessed in human studies and so these have not been considered further. Animal studies have also reported adverse effects on haematology and reproductive parameters. 


\section{ESTABLISHMENT OF GUIDANCE LEVEL}

The data are insufficient to establish a Safe Upper Level for manganese.

Manganese is known to cause neurotoxicity at high levels of exposure. In humans, this occurs as a result of inhalation exposure. However, there is also a suggestion of less severe neurotoxic effects occurring at lower levels of exposure from the consumption of contaminated well or drinking water. Two large epidemiological studies show apparently conflicting results where subjects were exposed to water containing manganese at levels of 1.8-2.3 mg/L (Kondakis et al., positive) and 0.3-2.1 mg/L (Vieregge et al., negative). However, these studies may not be incompatible given the higher range of manganese levels measured in the Kondakis study and the greater age of the participants (increasing age theoretically conferring potentially greater vulnerability). A major limitation of both studies is the failure to provide water consumption or dietary manganese intake data. If it is assumed that water consumption is $2 \mathrm{~L} /$ day, the resulting intakes from water would have been approximately $3.6-4.6$ and $0.6-4.4 \mathrm{mg} /$ day. The contribution from food is more difficult to estimate. Little information on diet is provided in the Vieregge study. It should also be noted that the effect level in the Kondakis study is apparently lower than occupational exposure levels not associated with adverse effects. The authors considered that this may be due to the increased sensitivity of the ageing brain to manganese.

Other epidemiological studies suggest adverse effects at lower levels of manganese exposure but these are too limited in design to draw any conclusions. For guidance purposes, it is reasonable to assume that in the general population, a supplemental intake of up to $4 \mathrm{mg}$ manganese/day in addition to the diet would be unlikely to produce adverse effects (equivalent to $0.07 \mathrm{mg} / \mathrm{kg}$ bw for a $60 \mathrm{~kg}$ adult) based on the NOAEL from the Vieregge study. No uncertainty factor is required as the NOAEL is based on a large epidemiological study. Using the NOAEL from the Kondakis study, it can be assumed that up to $0.5 \mathrm{mg} /$ day manganese (equivalent to $0.008 \mathrm{mg} / \mathrm{kg}$ bw for a $60 \mathrm{~kg}$ adult) in addition to the diet would not result in adverse effects in older people. Assuming a dietary intake of $8.2 \mathrm{mg}$, acceptable total manganese intakes can be estimated to be $12.2 \mathrm{mg} /$ day in the general population (equivalent to $0.2 \mathrm{mg} / \mathrm{kg}$ bw in a $60 \mathrm{~kg}$ adult) and $8.7 \mathrm{mg} /$ day (equivalent to $0.15 \mathrm{mg} / \mathrm{kg}$ bw in a $60 \mathrm{~kg}$ adult) for older people.

Some population groups may be exposed to high levels of manganese as a result of tea consumption. The significance of this level of exposure is uncertain.

\section{References}

COMA (1998). Nutrition and Bone Health: with particular reference to calcium and vitamin D. Report of the Subgroup on Bone Health, Working Group on the Nutritional Status of the Population, Committee on Medical Aspects of Food and Nutrition Policy. The Stationery Office, London

Kondakis, X. G., Makris, N., Leotsinidis, M., Prinou, M., Papapetropoulos, T. (1989) Possible health effects of high manganese concentration in drinking water. Archives of Environmental Health 44, 175-178

US Department of Health and Human Services, Agency for Toxic Substances and Disease Registry (1997). Draft toxicological profile for manganese update

Vieregge, P., Heinzow, B. Korf, G., Teichert, H.F., Schleifenbaum, P., Mosinger, H.U. (1995) Long term exposure to manganese in rural well water has no neurological effects. Canadian Journal of Neurological Sciences 22, 286-289 


\section{Risk Assessment Molybdenum}

\section{General information}

\section{Chemistry}

The transition element molybdenum exists in five oxidation states (II - IV), the predominant states are Mo (IV) and Mo (VI). Within this risk assessment, the word molybdenum refers to ionic molybdenum except where the specific compound is stated.

\section{Natural occurrence}

Molybdenum does not exist naturally in the metallic state, but occurs in association with other elements. The predominant form of molybdenum occurring in soil and natural waters is the molybdate anion, $\mathrm{MoO}_{4}^{-2}$.

\section{Occurrence in food, food supplements and medicines}

Generally, foodstuffs from above ground plant material, such as legumes, leafy vegetables and cauliflower, contain relatively high concentrations of molybdenum compared with food from tubers or animals. The 1994 TDS found the highest levels of molybdenum in nuts (0.96 mg/ $\mathrm{kg}$ fresh weight), canned vegetables $(0.31 \mathrm{mg} / \mathrm{kg}$ fresh weight) and cereals $(0.23 \mathrm{mg} / \mathrm{kg}$ fresh weight). Few data are available on the variability of molybdenum content of individual plant species, but it is affected by the soil molybdenum concentration and $\mathrm{pH}$, with molybdenum uptake increasing with soil pH. Foodstuffs from plants grown in alkaline or neutral soils with high molybdenum concentrations can be expected to have the highest molybdenum levels.

Molybdenum is available in food supplements at levels up to $0.33 \mathrm{mg}$ and licensed medicines. The latter are used (maximum daily dose $0.25 \mathrm{mg}$ ) to treat patients with malabsorption states and conditions leading to hypoproteinaemia and in perioperative nutritional support.

\section{Other sources of exposure}

Most natural waters contain low levels of molybdenum. The WHO recommends a maximum level of molybdenum in drinking water of $0.07 \mathrm{mg} / \mathrm{L}$ and notes that concentrations of molybdenum in drinking water are typically less than $0.01 \mathrm{mg} / \mathrm{L}$. However, in areas near mining sites, molybdenum concentrations up to $0.2 \mathrm{mg} / \mathrm{L}$ have been reported (WHO, 1993).

\section{Recommended amounts}

COMA has not set a RNI value for molybdenum. WHO (1993) estimated a daily requirement for molybdenum of between 0.1 and $0.3 \mathrm{mg} /$ day for adults.

\section{Analysis of tissue levels and molybdenum status}

Molybdenum can be measured in whole blood, serum and various tissues. 
Part 3 Trace Elements

\section{Brief overview of non-nutritional beneficial effects}

Molybdenum has been reported to be beneficial to various groups of individuals, including those with sulphite sensitivity, asthmatics with elevated urinary ratios of sulphites to sulphates, and those intolerant to intravenous sulphur contianing amino acids.

Molybdenum has been claimed to reduce the incidence and severity of dental caries.

\section{Function}

The basis of the importance of molybdenum is in its role in metalloenzymes. All of the molybdoenzymes are oxidoreductases, which exploit the variable valency states of molybdenum. The molybdenum in molybdoenzymes is inserted as part of a prosthetic group, known as the 'molybdenum cofactor'. In humans, xanthine oxidase and sulphite oxidase are important molybdoenzymes.

\section{Deficiency}

Molybdenum deficiency has not been identified in free-living human or animal species. It has, however, been identified in a single subject receiving total parenteral nutrition and can be achieved in animal studies. In goats, a molybdenum deficient diet was associated with reduced fertility and increased mortality in both the mothers and the offspring.

In a rare inherited metabolic disorder, molybdenum deficiency is associated with genetic deficiency of the molybdenum pterin cofactors. Neurological disorders, abnormal urinary metabolites, dislocated ocular lenses and failure to thrive are observed. The disorder is fatal by the age of 2-3 years.

\section{Interactions}

It is recognised that molybdenum interacts with copper and sulphates in living organisms, but the mechanism of this interaction has not been elucidated. The presence of dietary copper and sulphate affects the amount of molybdenum absorbed and retained by the body.

\section{Absorption}

Absorption of molybdenum varies over a wide range (25-93\%). Reports suggest that soluble molybdenum compounds are readily absorbed, whilst insoluble compounds are not.

\section{Distribution}

Molybdenum occurs in low concentrations in all the fluids and tissues of the body. The greatest amounts are found in the kidney, liver, small intestine and adrenals. It is found largely as molybdoenzymes. In plasma, molybdenum is bound specifically to $\alpha_{2}$-macroglobulin.

\section{Excretion}

Molybdenum is primarily excreted in the urine and experimental data from humans suggest that up to $80 \%$ of the absorbed amount is excreted via urine. Normally only small amounts are excreted in the faeces with exceptions in certain gastrointestinal disorders. 


\section{Toxicity}

\section{Human data}

Few data are available on human toxicity following ingestion. Food or water must contain more than $100 \mathrm{mg} / \mathrm{kg}$ to produce signs of toxicity, which include diarrhoea, anaemia and high levels of uric acid in the blood. Elevated uric acid levels, which are associated with the onset of gout, are hypothesised to be caused by stimulation of xanthine oxidase by high molybdenum intake. Occupational exposure, by inhalation, to molybdenum containing dusts has been associated with pneumoconiosis.

\section{Animal data}

Although non-ruminant animals will develop symptoms of toxicity when fed high molybdenum diets, ruminants are much more sensitive. Thus the toxicity seen in these species cannot be related to the effects that would be expected in man. The toxicity is primarily expressed as a copper deficiency and the ambient sulphate level has a marked effect on the interaction between copper and molybdenum. Molybdenum toxicity in animals is commonly referred to as molybdenosis or teart. In appearance it is similar to the disease of copper deficiency. Signs of molybdenum toxicity in animals include anaemia, anorexia, profound diarrhoea, joint abnormalities, osteoporosis, hair discoloration, reduced sexual activity and death.

\section{Carcinogenicity and genotoxicity}

There is no evidence of carcinogenicity of molybdenum. No data are available on the genotoxicity of molybdenum compounds in vitro, although there is limited evidence of genotoxicity from in vivo tests, the reliability of which is questionable.

\section{Mechanism of toxicity}

No data have been identified.

\section{Dose response characterisation}

No data have been identified.

\section{Vulnerable groups}

No vulnerable groups have been identified.

\section{Genetic variations}

No genetic variations have been identified. 


\section{Studies of particular importance in the risk assessment}

(For full review see http://www.food.gov.uk/science/ouradvisors/vitandmin/evmpapers or the enclosed CD).

\section{Human data}

Kovalsky et al., 1961

In a retrospective study of environmental exposure to increased molybdenum (form unclear), medical reports revealed a prevalence of gout in $31 \%$ and $18 \%$ of the exposed and control populations, respectively. Intake was calculated to be $10-15 \mathrm{mg}$ molybdenum per day in the exposed population compared to $1-2 \mathrm{mg} /$ day in the control area. It is possible that the $1-2 \mathrm{mg}$ intake produced a low level of response. Symptoms included arthralgia in the hand, feet and knee joints, accompanied by elevated blood and urine molybdenum levels.

\section{Animal data}

Fairhall et al., 1945

Molybdenum (as the trivalent form molybdenite, and as the hexavalent forms molybdenum trioxide, calcium molybdate and ammonium molybdate) was administered in the diet to rats at levels of 10 $500 \mathrm{mg}$ molybdenum/animal/day, for periods up to 232 days. No signs of toxicity were observed in the groups ingesting molybdenite. In the groups receiving hexavalent molybdenum, signs of toxicity were observed at all dose levels and included loss of appetite, weight loss, a tendency to become quiet and listless and premature death. One hundred percent mortality was recorded in the groups receiving $>100 \mathrm{mg}$ hexavalent molybdenum/animal/day. Limited experimental details were provided.

\section{Arrington \& Davies, 1953}

Hexavalent molybdenum (sodium molybdate) was administered in the diet to groups of 2-5 weanling or mature rabbits at levels of $140,500,1000,2000$ and $4000 \mathrm{mg} / \mathrm{kg}$ diet $(4,15,30,60$ or $120 \mathrm{mg} / \mathrm{kg}$ bw/day - estimated) for up to 12 weeks (average survival being 3 - 54 days in the higher dose groups). In the highest dose group 100\% mortality was observed and 80\% mortality in the group receiving $60 \mathrm{mg} / \mathrm{kg}$ bw/day. Anaemia, anorexia, loss of weight, alopecia, dermatosis and effects on the skeletal system were noted in animals receiving $30 \mathrm{mg} / \mathrm{kg}$ bw/day and above. Limited experimental details were provided. Haemoglobin levels appear to decrease by $>50 \%$ within a few weeks of treatment. It is uncertain whether this is a specific effect or related to factors such as haemodilution. Data for the $500 \mathrm{ppm}$ (4 and $15 \mathrm{mg} / \mathrm{kg}$ bw) group are not provided so it is uncertain whether the decrease would have also have been apparent at this level.

\section{Schroeder \& Mitchener, 1971}

Sodium molybdate (hexavalent molybdenum) was administered in drinking water $(0.0025 \mathrm{mg} / \mathrm{kg}$ $\mathrm{bw} /$ day) and diet $(0.07 \mathrm{mg} / \mathrm{kg} \mathrm{bw} /$ day) in lifetime studies over three generations of mice. In the first and second generations, $15 / 238$ and $7 / 242$ premature deaths, respectively, were observed, compared to 0/209 and 6/248 in the control populations. In the third generation increased numbers of premature deaths, maternal deaths, failure to breed, runting and production of single sex or dead litters were observed. 


\section{Exposure assessment}

Total exposure/intake:

Food

Mean: $0.11 \mathrm{mg} /$ day (1994 TDS)

97.5th percentile: $0.21 \mathrm{mg} /$ day

Drinking Water

$0.02 \mathrm{mg} /$ day (estimated from $0.01 \mathrm{mg} / \mathrm{L}-\mathrm{WHO}, 1993$ )

Supplements

up to $0.33 \mathrm{mg}$ molybdenum (Annex 4)

Estimated maximum intake: $0.21+0.02+0.33=0.56 \mathrm{mg} /$ day

No potential high intake groups were identified.

\section{Risk assessment}

Few data are available concerning oral molybdenum toxicity in humans, but some data exist that suggest an increased incidence in gout-like symptoms (joint pains and increased serum uric acid) in populations with a high molybdenum intake ( $1-15 \mathrm{mg} /$ person/day). The form of the molybdenum is uncertain. Few details of this study are available.

Some data are available from limited animal studies, which suggest that intakes of trivalent molybdenum of up to $500 \mathrm{mg} / \mathrm{rat} /$ day may be without adverse effects, although these are not sufficiently reliable to draw any conclusions. It should be noted from the animal studies that signs of toxicity were observed in studies in which hexavalent molybdenum was administered.

\section{ESTABLISHMENT OF A GUIDANCE LEVEL}

There are insufficient data from human or animal studies to establish a Safe Upper Level for molybdenum.

There are few reliable data on the oral toxicity of molybdenum. A limited human study (Kovalsky et al., 1961) suggests that intakes of $>1 \mathrm{mg} /$ day could be associated with an increase in gout-like symptoms joint pains and increased serum uric acid levels. The form of molybdenum to which the population was exposed is unclear, but as exposure is via water and locally grown/produced food, it is most likely to be the more toxic hexavalent molybdate ion. However, this epidemiological evidence is not robust, as there is no means of unbiased assessment, no control group and the exposure to molybdenum cannot accurately be determined. Lack of evidence makes it impossible to determine whether higher intakes would be problematic.

The available animal data are also limited. Signs of general/systemic toxicity have been reported at doses of $30-75 \mathrm{mg} / \mathrm{kg}$ bw/day hexavalent molybdenum (e.g. molybdate), whereas molybdenite, a trivalent form of molybdenum, appears to be less toxic. A multi-generation study in which mice were fed $0.0025 \mathrm{mg} / \mathrm{kg}$ bw $/$ day and $0.075 \mathrm{mg} / \mathrm{kg}$ sodium molybdate in drinking water and food respectively 
Part 3 Trace Elements

also produced adverse effects such that the colony was not viable beyond the $F_{2}$ generation. The level at which this effect occurred was lower than intakes causing adverse effects in other studies. The limited details available on the study make it impossible to establish whether the effect was real, or whether an effect unrelated to treatment occurred as a result of inbreeding.

The molybdenum intake from the UK diet (estimated maximum intake $0.23 \mathrm{mg} /$ day) is not expected to present any risk to health, but there are insufficient data on the safety of molybdenum intakes in excess of those naturally occurring in the diet to be able to provide further guidance.

\section{References}

Arrington, L.R. and Davies, G.K. (1953) Molybdenum toxicity in the rabbit. Journal of Nutrition 51, 295-304.

Fairhall, L.T., Dunn, R.C., Sharpless, N.E., Pritchard, E.A. (1945) The toxicity of molybdenum. US Public Health Service. Public Health Bulletin No. 293, 1-36.

Kovalsky, V.V., Yarovaya, G.A., Shmavonyan, D.M. (1961) The change in purine metabolism of humans and animals under the conditions of molybdenum biogeochemical provinces. Zhurnal Obshchei Biologii 22, 179-191 (Full translation of original Russian paper obtained).

Schroeder, H.A and Mitchener, M. (1971) Toxic effects of trace elements on the reproduction of mice and rats. Archives of Environmental Health 23, 102-106.

WHO (1993) Guidelines for drinking water quality. Second edition. World Health Organisation, Geneva. 


\section{Risk Assessment}

\section{Nickel}

\section{General information}

\section{Chemistry}

Nickel is an abundant metallic element which can exist in valency states of $0,+1,+2$ and +3 and form a variety of compounds. Within this risk assessment, the word nickel refers to ionic nickel, unless specific compounds are stated.

\section{Natural occurrence}

Nickel and nickel compounds are found in soil, water, animals and plants.

\section{Occurrence in food, food supplements and medicines}

Nickel is present in a variety of foods particularly pulses, oats $(0.18 \mathrm{mg} / \mathrm{kg}$ in miscellaneous cereals $)$ and nuts $(1.77 \mathrm{mg} / \mathrm{kg})$. Lower levels of nickel are present in drinking water. Nickel is present in a few multimineral food supplements at levels of approximately $0.005 \mathrm{mg} /$ daily dose. The average intake of nickel in the UK is $0.13 \mathrm{mg} /$ day from food, and $0.02-0.04 \mathrm{mg} /$ day from drinking water.

\section{Other sources of exposure}

Nickel-plated objects, such as jewellery, coins and nickel-containing implants, are sources of internal and dermal exposure.

\section{Recommended amounts}

COMA was unable to set recommended amounts for nickel intake.

\section{Analysis of tissue levels and nickel status}

Markers for nickel status have not been established. Nickel may be analysed in serum.

\section{Brief overview of non-nutritional beneficial effects}

No non-nutritional beneficial effects of nickel have been clearly identified.

\section{Function}

Nickel is present in a number of enzymes in plants and microorganisms. In humans, nickel influences iron absorption and metabolism, and may be an essential component of the haemopoietic process. 
Part 3 Trace Elements

\section{Deficiency}

Nickel deficiency has not been observed in humans. In animals deficiency is associated with disturbances such as reduced growth, impaired reproductive function and reduced haematopoiesis.

\section{Interactions}

It has been suggested that high levels of nickel may impair absorption or utilisation of iron when iron status is low. It has been hypothesised (on the basis of their chemistry and biology and limited in vitro studies) that magnesium and nickel may interact, but no toxic effects have been observed to result from such interaction in vivo.

\section{Absorption and bioavailability}

Absorption of nickel takes place in the small intestine via a carrier-mediated mechanism but passive diffusion may also occur. In a fasting state, the fraction of nickel salts absorbed after ingestion as a solution is between $20-25 \%$. However absorption of nickel from solution can be reduced to less than $1 \%$ when ingested with food, ascorbic acid, milk, tea, coffee and orange juice.

\section{Distribution and metabolism}

Nickel binds to albumin, histidine and $\alpha 2$ macroglobulin, and is distributed widely throughout the tissues. The highest nickel levels are found in the bone, lung, kidney, liver and endocrine glands. Nickel is also found in breast milk, saliva, nails and hair. Transplacental transfer of nickel has been demonstrated in rodents.

\section{Excretion}

Absorbed nickel is excreted in the urine predominantly in the form of low molecular weight complexes. Non-absorbed nickel is eliminated via the faeces together with a fraction of absorbed nickel that is excreted in the bile. Nickel is also eliminated in sweat.

\section{Toxicity}

\section{Human data}

Acute nickel exposure is associated with a variety of clinical symptoms and signs which include gastrointestinal disturbances (nausea, vomiting, abdominal discomfort and diarrhoea), visual disturbance (temporary left homonymous hemianopia), headache, giddiness, wheezing and cough.

Chronic inhalation of nickel and its compounds is associated with an increased risk of lung cancer.

Trials investigating nickel sensitisation are reviewed below. No additional data have been identified. 


\section{Animal data}

Nickel has moderate to low acute toxicity. In laboratory animals, long-term exposure to nickel is associated with adverse effects on haematological parameters, decreased body weights and changes in organ weights. These effects are generally produced at doses greater than 5 to $10 \mathrm{mg} / \mathrm{kg} \mathrm{bw} / \mathrm{day}$.

In reproductive toxicity studies, nickel is associated with an increase in perinatal mortality. Abnormal embryonic development has been reported following high doses of nickel chloride given by intraperitoneal injection, which were also associated with maternal toxicity.

\section{Carcinogenicity and genotoxicity}

In animal studies, orally administered nickel was not found to be carcinogenic. Nickel compounds cause chromosome aberrations in cultured cells in vitro but are generally negative or weakly positive in in vitro bacterial mutation assays. Chromosome aberrations have been observed in vivo in both humans and laboratory animals following exposure to nickel by inhalation and nickel is carcinogenic by this route.

\section{Vulnerable groups}

Approximately $7-10 \%$ of the population (predominately women) are affected by nickel allergic dermatitis. There is evidence suggesting that nickel ingestion may contribute to the exacerbation of eczema in sensitised individuals.

Iron-deficient individuals may be vulnerable to increased intestinal absorption of nickel, and thus to nickel sensitisation and adverse effects on haematopoiesis.

\section{Genetic variations}

No genetic variations that influence adverse reactions to nickel have been identified.

\section{Mechanism of toxicity}

Gastrointestinal effects are thought to be due to irritation rather than to specific toxicity. The chemical structure of the nickel atom is conducive to formation of antigens by binding to peptides or proteins, hence its strong sensitising potential. Nickel is thought to bind to histone proteins, generating potentially DNA damaging oxygen radicals through a redox cycling process. The interaction is dependent on the delivery of ionic nickel to target sites. Other mechanisms explaining the carcinogenicity of nickel following inhalation, such as inhibition of DNA repair, interference with cell signalling, calcium metabolism and transcription factors, have been suggested. The carcinogenic effects following inhalation are not relevant to low level oral exposure to nickel.

\section{Dose-response characterisation}

The lowest reported oral dose associated with acute effects in humans was $0.05 \mathrm{mg} / \mathrm{kg}$ bw $(1.2 \mathrm{mg}$ in a $60 \mathrm{~kg}$ adult). 
The dose response associated with sensitisation is difficult to define since sensitised subjects differ widely in their sensitivity, so that even a very small exposure could theoretically trigger a response in some individuals. Doses as low as $0.6 \mathrm{mg}$ have resulted in exacerbation of eczema in sensitised individuals.

Adverse effects are produced in laboratory animals, at doses above 5 to $10 \mathrm{mg} / \mathrm{kg}$ bw/day, although some studies have reported effects at lower doses.

\section{Studies of particular importance in the risk assessment}

(For full review see http://www.food.gov.uk/science/ouradvisors/vitandmin/evmpapers or the enclosed CD).

\section{Human studies}

Christensen and Moller, 1975

This was a double-blind crossover study on the provocation of eczema. 12 patients were given a placebo or nickel sulphate challenge. Oral nickel sulphate (5.6 mg nickel) provoked a reaction in $9 / 12$ sensitised subjects. The test was repeated with the reversed medication on a later occasion. The placebo did not provoke a response.

Kaaber et al., 1978

Seventeen out of 28 patients with chronic nickel dermatitis experienced aggravation of symptoms when given a $2.5 \mathrm{mg}$ oral dose of nickel, but no effect with placebo. Dermatitis improved in 9 of the 17 patients when they were put on a low nickel diet for 6 weeks and worsened when the normal diet was resumed. The nickel content of the diet was not measured but nickel excretion was measured in 14 of the patients and was found to decrease, indicating that nickel in the normal diet can contribute to dermatitis.

\section{Gawkrodger et al., 1986}

In a double blind crossover study, an oral dose of $5.6 \mathrm{mg}$ (but not $0.4 \mathrm{mg}$ or $2.5 \mathrm{mg}$ ) nickel produced a reaction in nickel-sensitised subjects more frequently than the placebo.

Nielsen et al., 1990

In a single-blind cross-over study, 12 nickel sensitive subjects were given a supplementary high nickel diet for 4 days. The additional dose of nickel was $0.49 \mathrm{mg}$ by analysis, this dose was lower than doses used in other oral challenge studies where reactions were not provoked. Hand eczema was exacerbated in 10/12 patients.

\section{Menne and Maibach, 1991}

Following a review of 12 published nickel challenge studies (1975-1988) using a variety of designs, the authors concluded that a dose-response existed for exposure to oral nickel and the exacerbation of dermatitis. Thus, a minority of nickel sensitive subjects would react to doses of $<1.25 \mathrm{mg}$ but the majority will react to oral doses of $5.5 \mathrm{mg}$ nickel. 


\section{Nielsen et al., 1999}

Eight non-allergic volunteers were given nickel in drinking water, followed by scrambled egg meals at different time intervals. When the nickel was ingested 30-60 minutes before the meal, peak serum nickel levels were reached 1 hour after water intake and were thirteen fold higher than when the nickel was ingested with the meal. Where the nickel and meal were taken together, a delayed peak in serum levels occurred 3 hours after ingestion. Nine out of 20 nickel-sensitised patients with vesicular hand eczema of the pompholyx type, developed flare-up of symptoms 12 hours after ingesting as little as $0.012 \mathrm{mg} / \mathrm{kg}$ body weight nickel in solution $(0.003 \mathrm{mg} / \mathrm{mL})$, on a empty stomach, with abstinence from food maintained for a further 4 hours. The dose given can be estimated to be equivalent to $0.720 \mathrm{mg}$ in a $60 \mathrm{~kg}$ adult. Twenty age-matched non nickel-sensitive controls, with similar hand eczema symptoms, exhibited no exacerbation following oral nickel challenge. The dermatologists assessing the symptoms were not informed of the status of the patients.

\section{Animal studies}

\section{Ambrose et al., 1976}

Groups of 25 rats of each sex were fed nickel, as nickel sulphate hexahydrate, in the diet for 2 years. The doses were estimated to be $0,5,50$ or $125 \mathrm{mg} / \mathrm{kg}$ bw/day. Decreased body weights, significantly increased heart and decreased liver weights were observed in both the mid and top dose groups. Analyses of blood, urine and tissue histology revealed no significant effects, but few data were reported. Poor survival of animals from the control and all dose groups was a major limitation of this study.

Groups of 3 beagle dogs of each sex were fed diets containing nickel, as nickel sulphate hexahydrate, for 2 years. The doses were estimated to be 0,3, 29 or $70 \mathrm{mg} / \mathrm{kg}$ bw/day. In the highest dose group, decreased body weights and increased kidney and liver weights were observed. No significant haematological effects were observed, and histological changes were seen only in the high dose group.

In a 3 generation rat study, groups of 20 rats of each sex were fed diets containing nickel, as nickel sulphate hexahydrate, for 11 weeks prior to breeding of three generations $\left(F_{0}, F_{1}, F_{2}\right)$, throughout pregnancy and lactation. The doses were estimated to be 5,25 or $50 \mathrm{mg} / \mathrm{kg}$ bw/day nickel. No effects on fertility, gestation, viability or lactation were observed. An increased number of stillborn rats was apparent in all treatment groups of the $\mathrm{F} 7$ generation but not in the $\mathrm{F} 2$ and $\mathrm{F} 3$ generations. The number of siblings per litter and siblings weaned decreased as the dose of nickel increased. Body weights were markedly reduced in the offspring of the dams exposed to $50 \mathrm{mg} / \mathrm{kg}$ nickel. No statistical analysis was reported.

Smith et al., 1993

Groups of 34 female rats were given nickel chloride in drinking water for 11 weeks prior to breeding and throughout 2 gestation (G1 and G2) and lactation (L1 and (L2) periods. The average doses provided by the water were estimated to be $1.33,6.8$ or $31.8 \mathrm{mg} / \mathrm{kg}$ bw/day nickel. No overt toxicity was reported. There was a dose-related increase in pups born dead or dying shortly after birth. This was significant at top dose at $\mathrm{Gl}$ and all doses at $\mathrm{G} 2$. Body weight gain was reduced in dams in the mid and top dose group in G1. Reproductive performance was unaffected by treatment. Although it is of unusual design, this was a well-conducted and reported study. 


\section{Exposure assessment}

Total exposure/intake:

Food Mean: $0.13 \mathrm{mg} /$ day (1997 TDS)

97.5th percentile: $0.21 \mathrm{mg} /$ day

Water

$0.04 \mathrm{mg} /$ day (maximum) from water (NAS/NRC)

Supplements

up to $0.005 \mathrm{mg} /$ day (Annex 4; OTC, 2001).

Estimated maximum intake: $0.21+0.04+0.005=0.26 \mathrm{mg} /$ day

No potential high intake groups have been identified.

\section{Risk assessment}

The carcinogenicity of nickel compounds which occurs through inhalation mainly as a result of occupational exposure does not appear to be relevant to oral exposure from low levels in food, although data are lacking in this area.

In animals, nickel caused a decrease in bodyweight in dogs and an increase in kidney weight at a dose of $70 \mathrm{mg} / \mathrm{kg}$ bw/day. In reproductive toxicity studies in rats, nickel was shown not to affect reproduction at doses up to $50 \mathrm{mg} / \mathrm{kg}$ bw/day. However, there was an increase in the number of pups stillborn or dying shortly after birth with the numbers stillborn increasing as dose increased from 5 to $50 \mathrm{mg} / \mathrm{kg}$ bw/day. At $50 \mathrm{mg} / \mathrm{kg}$ bw/day, there was a significant decrease in body weight of the pups.

The key toxic endpoint for nickel in humans is the aggravation of nickel sensitisation, for which a threshold is unclear but which is possible at the levels of nickel found in food. It is also possible that oral intakes of nickel as low as $0.49 \mathrm{mg}$ (Nielsen, 1990) could trigger symptoms, particularly in the fasting state. Although certain sensitised subjects will be aware that exposure to nickel may be responsible for their dermatological symptoms, other subjects may only be aware that they are unable to tolerate certain jewellery. They also may not be aware that their dermal symptoms could be aggravated by food.

\section{ESTABLISHMENT OF GUIDANCE LEVEL}

Nickel is a potent skin sensitiser, with 7-10\% of the UK population reportedly affected. Dietary nickel can cause flare-ups of dermatitis and while there is some discussion about the levels at which this effect occurs, it appears that levels as low as 0.49 to $0.72 \mathrm{mg}$ may be able to trigger a reaction, particularly if taken on an empty stomach. 
In animals, nickel has fairly non-specific toxic effects, but appears to be associated with increased perinatal mortality in multi-generation studies. Using the LOAEL of $1.3 \mathrm{mg} / \mathrm{kg}$ bw/day derived from the study by Smith et al. (1993) and applying the conventional uncertainty factors of 3 for LOAEL to NOAEL extrapolation, 10 for inter-species variation and 10 for intra-individual variation, indicates that total nickel intake of $0.0043 \mathrm{mg} / \mathrm{kg}$ bw/day would not be expected to have effects in non-sensitised individuals (equivalent to $0.26 \mathrm{mg} /$ day in a $60 \mathrm{~kg}$ adult).

Prevalence of nickel sensitivity is high and many individuals may not be aware that they are sensitised. Furthermore, the absorption of nickel is greater when taken on an empty stomach and in the absence of food, as may occur with supplements. It is therefore not possible to set a safe upper level or guidance for supplemental intake of nickel. UK dietary intake of nickel in food is not expected to result in harmful effects.

\section{References}

Ambrose, A.M., Larson, P.S., Borzelleca, J.F., Hennigar Jr., G.R. (1976) Long term assessment of nickel in rats and dogs. Journal of Food Science and Technology 13, 181-187.

Christensen, O.B. and Moller, H. (1975) External and internal exposure to the antigen in the hand eczema of nickel allergy. Contact Dermatitis 1, 136-141.

Gawkrodger, D.J., Cook, S.W., Fell, G.S., Hunter, J.A.A. (1986) Nickel Dermatitis: the reaction to the oral nickel challenge. British Journal of Dermatology 115, 33-38.

Kaaber, K., Veien, N.K., Tjell, J.C. (1978) Low nickel diet in the treatment of patients with chronic nickel dermatitis. British Journal of Dermatology 98, 197-201.

Menne, T. and Maibach, H.I. (1991) 'Systemic contact-type dermatitis', in Dermatotoxicology 4th Edition, eds Marzulli, F.N. and Maibach, H.I., Hemisphere Publishing Corporation, London, pp. 453-467.

Nielsen, G.D., Jepsen, L.V., Jorgensen, P.J., Grandjean, P.J., Brandrup, F. (1990) Nickel-sensitive patients with vesicular hand eczema: oral challenge with diet naturally high in nickel. British Journal of Dermatology $122,299-308$.

Nielsen, G.D., Soderberg, U., Jorgensen, P.J., Templeton, D.M., Rasmussen, S.N., Anderson, K.E., Grandjean, P.J. (1999) Absorption and retention of nickel from drinking water in relation to food intake and nickel sensitivity. Toxicology and Applied Pharmacology 154, 67-75.

Smith, M.K., George, E.L., Stober, J.A., Feng, H.A., Kimmel, G.L. (1993) Perinatal toxicity associated with nickel chloride. Environmental Research 61, 200-211. 


\section{Risk Assessment}

\section{Selenium}

\section{General information}

\section{Chemistry}

Selenium is a metallic group $\mathrm{VI}$ element that is abundant and which can exist in 4 oxidation states $(-2,+1$, +2 and +6$)$. Within this risk assessment, the word selenium refers to ionic selenium, except when specific selenium compounds are mentioned.

\section{Natural occurrence}

Selenium is found in soils and rocks and consequently may then accumulate in plants.

\section{Occurrence in foods, food supplements and medicines}

Selenium is present in foods particularly fish $(0.32 \mathrm{mg} / \mathrm{kg})$, offal $(0.42 \mathrm{mg} / \mathrm{kg})$, brazil nuts $(0.25 \mathrm{mg} / \mathrm{kg})$, eggs $(0.16 \mathrm{mg} / \mathrm{kg})$ and cereals $(0.02 \mathrm{mg} / \mathrm{kg})$. In foods, selenium is generally present as the amino acid derivatives selenomethionine and selenocysteine. Selenium is present in several licensed medicines both alone and in combination with other substances, and is present in a number of food supplements at doses up to $0.3 \mathrm{mg} /$ daily dose.

\section{Recommended amounts}

COMA set RNIs of 0.075 and $0.060 \mathrm{mg}$ selenium/day for males and females respectively, and $0.075 \mathrm{mg}$ selenium/day for lactating women (COMA, 1991). The lower limit of the WHO safe range of the population mean intake to meet requirements is $0.040 \mathrm{mg}$ selenium/day.

\section{Analysis of tissue levels and selenium status}

Selenium can be measured directly in plasma, serum, red cells, nails and hair. It can also be determined indirectly by measuring tissue glutathione peroxidase activity, although a plateau in activity may occur with higher levels of selenium intake not producing a corresponding increase in activity.

\section{Brief overview of non-nutritional beneficial effects}

Selenium has been claimed to reduce the incidence of a range of cancers, although the COMA report on Nutritional aspects of the Development of Cancer (1998) considered there to be insufficient evidence for such a link. Beneficial effects of selenium intake on AIDS symptoms, male fertility, skin disorders, anxiety and asthma have also been claimed. 


\section{Function}

The biologically active form of selenium is selenocysteine. Selenocysteine is incorporated into selenoproteins, of which over thirty have been identified to date. The selenoproteins include the glutathione peroxidases, which protect against oxidative damage, the iodothyronine deiodinases (involved in the production of the hormone triiodothyronine from thyroxine), selenoprotein $\mathrm{P}$ (which is involved in antioxidant and transport functions) and the thioredoxin reductases (maintenance of the intracellular redox state).

\section{Deficiency}

In humans, selenium deficiency is associated with Keshan disease, an endemic cardiomyopathy which particularly affects children and women of child-bearing age, and possibly also Kashin-Beck disease, a musculoskeletal disorder.

\section{Interactions}

Since selenium is an essential component of the enzyme tetraiodothyronine 5'-deiodinase 1 (which is involved in iodine metabolism) there is a complex interaction between selenium and iodine. Severe selenium deficiency may increase the hypothyroid stress caused by iodine deficiency. However, supplementation with selenium alone may aggravate iodine deficiency by accelerating iodine loss. This interaction is likely to be significant only in cases of severe deficiency.

Selenium interacts with other metals and ascorbic acid. Selenium status affects the metabolism and thus the toxicities of some xenobiotics.

\section{Absorption and bioavailability}

Selenium compounds are readily absorbed from the small intestine. The extent of absorption depends on the nature of the compound, with soluble selenate and selenomethionine being most readily absorbed.

\section{Distribution and metabolism}

Selenium is widely distributed throughout the body and can be detected in breast milk. Selenium has also been reported to cross the placenta in animals. Selenium levels are slightly higher in the liver and kidneys than in other tissues. Selenium can be incorporated into selenoproteins, bind to selenium binding proteins or be incorporated into volatile methylated metabolites, which are subsequently excreted.

\section{Excretion}

Selenium is largely excreted in the urine, with some volatile metabolites being excreted in the breath. Some faecal excretion occurs, particularly after chronic administration. 
Part 3 Trace Elements

\section{Toxicity}

The toxicity of selenium depends on the nature of the selenium compound, particularly its solubility. Thus, insoluble selenium sulphide is much less toxic than selenite, selenate and selenomethionine. Selenium toxicity is cumulative.

\section{Human data}

Acute selenium toxicity in humans is characterised by hypersalivation, emesis and a garlic aroma on the breath due to the excretion of volatile selenium metabolites. These effects may be accompanied by gastrointestinal effects (severe vomiting and diarrhoea), hair loss, neurological disturbance (restlessness, spasms, tachycardia) and fatigue.

Chronic selenium poisoning, or selenosis, is associated with changes to the hair and nails, skin lesions and clinical neurological effects such as peripheral hypoaesthesia, acroparasthaesiae, pain and hyperreflexia; numbness, convulsions and paralysis may then develop. Studies undertaken in subjects living in seleniferous areas of the USA and China indicate that selenosis is associated with intakes greater than $0.91 \mathrm{mg} /$ day $(0.015 \mathrm{mg} / \mathrm{kg}$ bw for a $60 \mathrm{~kg}$ adult).

\section{Supplementation trials}

Supplementation of human volunteers with $0.2 \mathrm{mg} /$ day for 10 years did not result in selenosis (Clark et al., 1992). Other data from supplementation trials indicate that doses of up to $0.388 \mathrm{mg}$ selenium/day for shorter periods are without apparent ill effect, although a formal clinical examination for symptoms and/or signs of selenosis was not always made.

\section{Animal data}

Selenium has moderate to high acute oral toxicity. Acute toxicity results in effects on the nervous system, liver and lungs. Chronic exposure results in reduced growth rates and weight gain. High levels of selenium exposure have adverse effects on some reproductive parameters, such as the oestrous cycle in females and sperm concentration and quality in males. Multi-generation studies suggest that selenium reduces post-natal survival and weight of the offspring. Specific teratogenic effects have been observed in birds, fish, sheep and pigs and in hamsters at maternally toxic doses. Selenium is not teratogenic in the macaque monkey. Some studies have indicated that pre-weanling animals are more sensitive to selenium toxicity.

\section{Carcinogenicity and genotoxicity}

Selenium sulphide is carcinogenic in rats and mice. Other selenium compounds are not carcinogenic. The results of in vitro mutagenicity tests are inconsistent. Selenium compounds are largely negative in the available in vivo mutagenicity tests. An increase in chromosomal aberrations in hamster bone marrow has been reported, but this occurred at lethal doses of sodium selenite only. 


\section{Mechanism of toxicity}

No specific mechanisms have been identified. However, redox cycling of auto-oxidisable metabolites, glutathione depletion, inhibition of protein synthesis, depletion of S-adenosyl-methionine and the replacement of sulphur by selenium in critical sulphydryl groups have been suggested.

\section{Dose-response characterisation}

The epidemiological studies of Yang and colleagues demonstrated a dose response relationship for selenium toxicity. In adult humans the estimated intake associated with the onset of selenosis (clinical selenium poisoning, which includes the occurrence of nail, hair and skin lesions) is $0.910 \mathrm{mg} /$ day $(0.015 \mathrm{mg} / \mathrm{kg} \mathrm{bw} /$ day). Acute selenium poisoning is harder to assess but appears to occur at doses of $0.5 \mathrm{mg} / \mathrm{kg}$ bw and above.

In animals, doses of soluble selenium compounds of approximately $0.5 \mathrm{mg} / \mathrm{kg}$ bw $/$ day are not associated with any adverse effects.

\section{Vulnerable groups}

In subjects suffering from iodine as well as selenium deficiency, supplementation with selenium alone may aggravate the iodine deficiency. However, this is likely to be significant only in cases of severe deficiency and would not be applicable to the UK.

\section{Genetic variations}

No relevant genetic variations have been identified.

\section{Studies of particular importance in the risk assessment}

(For full review see http://www.food.gov.uk/science/ouradvisors/vitandmin/evmpapers or the enclosed CD)

Studies in seleniferous areas

Yang et al., 1983

The intake of selenium was investigated in areas of China where selenium intakes were classified as high (with or without endemic selenosis), adequate or deficient. The average daily intake of selenium was $0.75 \mathrm{mg} /$ day (range 0.24-1.51) in areas where selenium intake was high but where there was no selenosis. In areas of chronic selenosis, selenium intake was 3.2-6.99 mg/day. Intake was estimated from information on dietary habits and by measuring the selenium content of typical cereal and vegetable staples. 
Yang et al., $1989 a$

Individual selenium intake was calculated for the inhabitants of areas with low, moderate or high selenium levels (selenosis cases occurred in high selenium areas only). Selenium intake was then correlated with selenium levels in a range of tissues from the same individuals. At physiological levels of selenium intake, whole blood selenium levels were the best reflection of intake, whereas at higher intake levels, hair, finger and toenails were more sensitive markers. Selenium intake was calculated from a three day dietary survey, collecting and weighing samples of raw and cooked food.

Yang et al., $1989 \mathrm{~b}$

Individual selenium intake was calculated for the inhabitants of areas with low, moderate or high selenium levels (1989a study described above). Selenium intake was then correlated with a range of biochemical parameters. Morphological changes in the fingernails were used as the main diagnostic criterion of selenosis and this occurred largely in adults. There was no correlation between blood selenium levels and clinical signs attributed to day to day variations in selenium intake causing variations in blood selenium levels. However, long term selenosis was apparent in 5 patients with blood selenium concentrations in excess of 1.054 to $1.854 \mathrm{mg} / \mathrm{L}$. This concentration was estimated to represent a selenium intake of $0.91 \mathrm{mg} /$ day and was considered by the authors to be the level indicating marginal selenium toxicity. Prothrombin time increased significantly from 12.2 seconds to 13.4-13.5 at selenium intake levels greater than $0.85 \mathrm{mg} /$ day; it is unclear whether this is outside the normal range for this population. White cell count also increased with selenium intake. Whole blood reduced glutathione levels were lower in the high selenium intake areas compared to the low ones. No evidence of liver toxicity was apparent but the authors suggested that the population may have adapted to high selenium intakes. No specific neurological symptoms were found. There was no evidence of birth defects in humans (although it was noted that malformed chickens were hatched from locally produced eggs). The authors suggested that the maximum safe level of selenium intake was $0.4 \mathrm{mg} /$ day (using a safety factor of 2).

Longnecker et al., 1991

Over a 2 year period, 142 subjects were recruited from ranches in the USA where a high selenium intake was suspected. Subjects underwent physical examinations, completed health questionnaires, and provided tissue samples. Duplicate-plate collections were used to estimate selenium intake. Specific questions were asked about neurological symptoms, and changes to hair and nails. A range of clinical chemistry tests were also conducted. Approximately half of the subjects had selenium intakes $>0.2 \mathrm{mg} /$ day (average $0.239 \mathrm{mg} /$ day, range 0.068 to $0.724 \mathrm{mg} /$ day). No physical characteristics of selenium toxicity, or any other significant effects were apparent. Increasing selenium intake was associated with increasing serum alanine aminotransferase (ALT) levels but the differences, even at the extremes of intake, were considered to be clinically insignificant. The increase in prothrombin time found by Yang was not apparent in this study.

Yang and Zhou, 1994

In a follow-up study of the 5 selenosis patients described in Yang et al., (1989b) a decrease in average blood selenium levels from 1.346 to $0.968 \mathrm{mg} / \mathrm{L}$ was reported which was attributed to dietary changes. The decrease in blood selenium levels was associated with a loss of clinical signs of selenosis. The corresponding safe selenium intake was equivalent to approximately $0.8 \mathrm{mg} /$ day), which was suggested by the authors to represent the mean NOAEL. The lower limit of the $95 \%$ confidence interval for the correlation equivalent to an intake of $0.6 \mathrm{mg} /$ day, was taken to approximate the maximum individual 
safe selenium intake. Based on these data, Yang and Zhou confirmed the previous recommendation that $0.4 \mathrm{mg} /$ day as the maximum safe daily dietary intake for selenium.

The reporting of the work of Yang and colleagues is confusing since Yang et al. (1989b) reported that there was no correlation between blood selenium and selenium intake due to daily variation in food supply. However, a regression equation was then used to estimate selenium intakes in subjects with selenosis from their blood levels. This appears to be based on work in Yang et al. (1989a) which used plots of average blood selenium levels and average selenium intakes to derive a regression equation. Generally, blood selenium is considered to be a reliable indicator of selenium intake where the composition of the diet is not very varied.

Studies in non-seleniferous areas

Clark et al., 1996

In a double blind, randomised placebo controlled trial, 1312 patients (with a history of basal cell or squamous cell carcinoma) were given a $0.2 \mathrm{mg}$ selenium supplement, or placebo, for up to 10.3 years (mean 4.5 years) in an attempt to establish whether selenium had a protective effect on skin cancer. Patients were evaluated for signs of selenosis (pathological nail changes, brittle hair and garlic breath) every 6 months. The dietary selenium intake of the participants was unknown but mean baseline serum selenium levels were comparable between the groups. No dermatological signs of selenium toxicity were found. A total of 14 and 21 patients from the control and selenium supplementation groups, respectively, withdrew from the trial as result of adverse effects (mainly gastrointestinal symptoms). Within each group the patients reporting adverse effects did not have significantly different plasma selenium concentrations from those not reporting such effects.

\section{Exposure assessment}

Total exposure/intake:

Food Mean: $0.039 \mathrm{mg} /$ day 97.5 percentile: $0.1 \mathrm{mg} /$ day (1994 TDS)

Supplements up to $0.3 \mathrm{mg} /$ day (Annex 4)

Estimated maximum intake: $0.1+0.3=0.4 \mathrm{mg} /$ day

No potential high intake groups have been identified 


\section{Risk assessment}

Selenium has a variety of toxic endpoints in both animals and humans. In man, the first signs of chronic toxicity appear to be pathological changes to the hair and nails, followed by adverse effects on the nervous system. Changes in biochemical parameters have also been reported. The available studies indicate the development of selenosis is associated with selenium intakes in excess of $0.85 \mathrm{mg} / \mathrm{day}$ (0.014 mg/kg bw for a $60 \mathrm{~kg}$ adult).

Supplementation studies in humans indicate that up to $0.3 \mathrm{mg} /$ day additional selenium is not associated with overt adverse effects over a short period of time, although specific symptoms have not always been investigated. However, the study by Clark, which specifically considered symptoms of selenosis, indicated that $0.2 \mathrm{mg} /$ day additional selenium for up to 10 years did not result in symptoms of selenosis.

In addition to reduced growth rates, similar symptoms to those in humans are found in animals treated with selenium.

Selenium sulphide is carcinogenic but other selenium compounds are not. Selenium compounds are not mutagenic in vivo. Adverse effects have been reported on the reproductive system of various animals, though not primates as determined by Tarantal et al. (1991). Reproductive toxicity is not an issue that has been examined in detail in the available human epidemiological studies.

\section{ESTABLISHMENT OF SAFE UPPER LEVEL}

Key Studies:

Yang et al. (1989 a and b)

LOAEL:

$0.91 \mathrm{mg}$ selenium/day

Uncertainty Factor:

2 (LOAEL to NOAEL extrapolation)

Safe Upper Level:

$0.91 / 2=0.45 \mathrm{mg}$ total selenium $/$ day

for daily consumption

over a lifetime

The most sensitive indicators of selenium toxicity are changes in the nails and hair. In a study by Yang et al. (1989a and b) conducted in an area of China where dietary selenium exposure is high, selenium intakes were correlated with blood levels to determine the intakes at which marginal selenium toxicity occur. This was at a total intake of $0.91 \mathrm{mg} /$ day selenium.

There are some discrepancies in the NOAELs described by Yang and colleagues in that the range of intakes in high selenium areas that was not associated with selenosis were given as $0.24-1.51 \mathrm{mg} / \mathrm{day}$ whereas a LOAEL of $0.91 \mathrm{mg}$ selenium/day was determined in a later study. This may have resulted from the way in which the dietary intakes were calculated but it has also been argued that subjects may have become sensitised to selenium as a result of earlier outbreaks of selenosis. A follow up study (Yang and Zhou, 1994) indicated that the symptoms of selenosis could be reversed if selenium intake was reduced. 
The intake of $0.91 \mathrm{mg}$ selenium/day produced slight effects and was close to a NOAEL. Because of this an uncertainty factor of 2 was applied for LOAEL to NOAEL extrapolation. Because this is based on a population study, an uncertainty factor for inter-individual variation is not required. A Safe Upper Level for total selenium intake of $0.45 \mathrm{mg} /$ day can therefore be derived (equivalent to $0.005 \mathrm{mg} / \mathrm{kg} \mathrm{bw} /$ day in a $60 \mathrm{~kg}$ adult). This Safe Upper Level is consistent with the findings of other groups who have reported that intakes of $0.2 \mathrm{mg} /$ day supplemental selenium and $0.24 \mathrm{mg} /$ day total selenium are not associated with any adverse effects (Clark et al., 1996; Longnecker et al., 1991). Assuming a maximum intake of $0.1 \mathrm{mg} /$ day from food, a margin of $0.35 \mathrm{mg}$ /day selenium is available for supplementation or other additional intake. No vulnerable groups have been identified.

\section{References}

Clark, L.C., Combs, G.F. Jr, Turnbull, B.W., Slate, E.H., Chalker, D.K., Chow, J., Davis, L.S., Glover, R.A., Graham, G.F., Gross, E.G., Krongrad, A., Lesher, J.L. Jr, Park, H.K., Sanders, B.B. Jr, Smith, C.L., Taylor, J.R. (1996) Effects of selenium supplementation for cancer prevention in patients with carcinoma of the skin. Journal of the American Medical Association 276, 1957-1963.

COMA (1991). Dietary Reference Values for Food Energy and Nutrients for the United Kingdom. Report of the Panel on Dietary Reference Values, Committee on Medical Aspects of Food and Nutrition Policy. HMSO, London.

Longnecker, M.P., Taylor, P.R., Levander, O. A., Howe, S.M., Veillon, C., McAdam, P.A., Patterson, K.Y., Holden, J.M., Stampfer, M.J., Morris, J.S., Willett, W.C. (1991) Selenium in diet, blood and toenails in relation to human health in a seleniferous area. American Journal of Clinical. Nutrition 53, 1288-94.

Tarantal, A.F., Willhite, C.C., Lasley, B.L., Murphy, C.J., Miller, C.J., Cukierski, M.J., Book, S.A., Hendrickx, A.G. (1991) Developmental toxicity of L- selenomethionine in Macaca fascicularis. Fundamental and Applied Toxicology 16, 147-160.

Yang, G. and Zhou, R. (1994) Further observations on the human maximum safe dietary selenium intake in a seleniferous area in China. Journal of Trace Elements and Electrolytes in Health and Disease 8, 159-165.

Yang, G., Wang, S., Zhou, R., Sun, S. (1983) Endemic selenium intoxication of humans in China. American Journal of Clinical Nutrition 37, 872-881.

Yang, G., Zhou, R., Yin S., Gu, L., Yan, B., Liu, Y., Liu, Y., Li, X. (1989a) Studies of safe maximal daily selenium intake in a seleniferous area in China. I. Selenium intake and tissue selenium levels of the inhabitants. Journal of Trace Elements and Electrolytes in Health and Disease 3, 77-87.

Yang, G., Yin S., Zhou, R., Gu, L., Yan, B., Liu, Y., Liu, Y. (1989b) Studies of safe maximal daily selenium intake in a seleniferous area in China. II. Relation between selenium intake and the manifestation of clinical signs and certain biochemical alterations in blood and urine. Journal of Trace Elements and Electrolytes in Health and Disease 3, 123-130. 


\section{Risk Assessment}

\section{Tin}

\section{General information}

\section{Chemistry}

Tin is a metallic element. It is rarely found as the metallic element in nature and is usually found combined with other substances, most commonly as the dioxide $\left(\mathrm{SnO}_{2}\right)$. It has oxidation states of II and IV and the corresponding oxides of these states are amphoteric. Organotin compounds can be synthesised and are highly toxic, but these are not found in food, beverages or food supplements. Within this risk assessment, the word tin refers to ionic tin, except where specific tin compounds are mentioned.

\section{Natural occurrence}

The occurrence of tin in various organisms from different localities is highly dependent on the existence of local mineral sources of tin. Although not commonly present in fresh water, it occurs at a concentration of about $0.003 \mathrm{mg} / \mathrm{kg}$ in seawater.

\section{Occurrence in food, food supplements and medicines.}

The presence of tin in fresh food of both vegetable and animal origin is highly dependent on the concentration of tin in the soil of the area in which the food is produced. Canning may result in dissolution of the tin lining of the can, particularly if the products are acidic. The regulatory limit for total tin in canned products is $200 \mathrm{mg} / \mathrm{kg}$. The major dietary sources of tin are fruit products and canned vegetables. Stannous chloride, $\mathrm{SnCl}_{2}$, is a permitted food additive (E512). Tin is present in some multivitamin and mineral food supplements at levels of up to $0.01 \mathrm{mg}$ in the daily dose recommended by the manufacturer.

No medicinal products containing tin have been licensed.

\section{Other sources of exposure}

Trace quantities of tin are detectable in the air, the concentrations in any area depending on the extent of industrial activity. Water and airborne tin provide only minimal exposure for humans.

\section{Recommended amounts}

There are no recommended daily intakes for tin compounds since it has not been proven to be an essential element. 


\section{Analysis of tissue levels and tin status}

Tin levels can be measured by spark source mass spectrometry, radioactivation analysis or $\mathrm{x}$-ray fluorescence analysis. Tin concentrations in fluids, tissues, or organs of the human body have been measured. There is no measurement used to indicate tin status.

\section{Brief overview of non-nutritional beneficial effects}

Tin is present in some dietary supplements and has been claimed to delay hair loss, male pattern baldness and hearing loss.

\section{Function}

There is no proven biological function for tin. It has been suggested that, because of its coordination chemistry, it may contribute to macromolecular structure and function at the active site of metalloenzymes.

\section{Deficiency}

Naturally occurring deficiency of tin in free-living humans or animal species has not been demonstrated. Experiments on induced deficiency of experimental animals in isolators have been criticised and other evidence claimed to support the hypothesis that tin is an essential element is not convincing.

\section{Interactions}

In humans, ingestion of tin has been shown to lower net retention of zinc. It can also alter the excretion of selenium in faeces and urine, but without affecting the overall balance. In rats the absorption of copper has been reported to be decreased by ingestion of tin.

\section{Absorption and bioavailability}

The gastrointestinal absorption of tin is low. The solubility and bioavailability of inorganic tin compounds varies and is dependent on the oxidation state.

\section{Distribution and metabolism}

The tissues and organs that accumulate the highest concentrations of tin are bone, lymph nodes, liver, lung, ovary, testis and kidney. No metabolism of tin has been demonstrated.

\section{Excretion}

The majority of ingested inorganic tin is excreted in the faeces (about $95-99 \%$ of ingested dose in animal studies), with the remainder in the urine. 
Part 3 Trace Elements

\section{Toxicity}

\section{Human data}

Reports of acute poisoning have been associated with high concentrations of tin in drinks or solid foods. Subjects presented with gastrointestinal effects (abdominal cramps, nausea, vomiting, diarrhoea), and headache and chills within 1-2 hours of consuming the implicated foodstuff, and recovered within 1-2 days. However, there is little consistency in the reports in terms of the nature of the foodstuff, or the concentration and chemical form of the tin, all of which may influence human toxicity.

\section{Animal data.}

In studies of the acute oral administration of tin to animals, cats have shown a vomiting response, similar to that of humans.

Most studies of the subchronic toxicity of tin have been carried out in rats, a species which lacks an emetic reflex. Reduction in body weight gain, reduced appetite and reduced feed conversion efficiency have been reported. These, together with abdominal distension and at autopsy, distended caeca, reddening of the mucosal surface of the stomach and histopathologic changes in the gastrointestinal tract (from the stomach to the ileum), are indicative of gastrointestinal effects in the rat. Haematological changes included reduced haematocrit and haemoglobin, which are indicative of anaemia, although no biologically significant changes to leucocytes occurred. These effects may result from disturbance to the pathways of haem biosynthesis and degradation, as tin reduces the activity of the enzyme 5 aminolaevulinate dehydratase and increases haem oxidase.

Pathological changes to the pancreas, including atrophy with single-cell necrosis and destruction of complete acini, have been suggested as being characteristic of tin toxicity. Pathological changes to the liver, spleen, kidneys, adrenals and testicles have also been reported. Various authors have investigated the effects of tin, given orally or parenterally, on calcium balance and bone metabolism in experimental animals. Some changes were detected at low doses of tin. The results of reproductive and developmental toxicity studies were negative.

The available data on chronic administration of tin are limited and it is difficult to draw conclusions due to adverse effects being observed in all groups (including controls). However, the available data indicate that doses of up to $100 \mathrm{mg} / \mathrm{kg}$ bw/day as stannous chloride are well-tolerated and do not affect survival.

\section{Carcinogenicity and genotoxicity}

Various tin compounds were not carcinogenic in long-term studies in animals. The conformation of DNA is disrupted by in vitro exposure to stannous chloride, but it has been suggested that this is a result of the generation of reactive oxygen species under the conditions used. Mutagenicity studies on tin have not been identified.

\section{Mechanism of toxicity}

No relevant data have been identified. 


\section{Dose-response characterisation}

There are no data on which to determine a threshold of intake at which adverse gastrointestinal effects will not occur in humans.

\section{Vulnerable groups}

No vulnerable groups have been identified.

\section{Genetic variation}

No genetic variations in the susceptibility to tin have been identified.

\section{Studies of particular importance in the risk assessment}

(For full review see http://www.food.gov.uk/science/ouradvisors/vitandmin/evmpapers or the enclosed CD).

\section{De Groot et al., 1973}

Groups of 10 male and 10 female weanling Wistar-derived rats were administered diets containing either stannous oxide or stannous chloride for 90 days, at concentrations of 0, 300, 1000, 3000 and $10,000 \mathrm{mg} / \mathrm{kg}$ of diet. There were no toxic effects of stannous oxide at any dose. Animals receiving the $10,000 \mathrm{mg} / \mathrm{kg}$ stannous chloride diet showed effects within the first 7 days, including abdominal distension and loss of appetite. This particular group was terminated in the ninth week because of a high rate of mortality. Upon autopsy, various gross pathological conditions were found, including distension of the intestines, small oedematous pancreases and greyish-brown livers. Histopathological evaluations revealed moderate testicular degeneration, severe pancreatic atrophy, a spongy state of the white matter of the brain, acute bronchopneumonia, enteritis and distinct liver changes (atypical homogenous liver cell cytoplasm and mild proliferation of bile duct epithelium). In view of the marked reduction in appetite in the $10,000 \mathrm{mg} / \mathrm{kg}$ group, it is difficult to assess the extent to which tin was responsible for the observed pathological changes. Animals fed the $3000 \mathrm{mg} / \mathrm{kg}$ diet showed some abdominal distension and loss of appetite during the first 2 weeks of the 90-day study. After the second week, appetite and growth returned to normal. Significantly lower haemoglobin levels were detected in both sexes between the fourth and ninth week. However, by the end of the study only males on the $3000 \mathrm{mg} / \mathrm{kg}$ stannous chloride diet had lower $(\mathrm{p}<0.05)$ haemoglobin and haematocrit values.

Autopsy and histological evaluations of the $3000 \mathrm{mg} / \mathrm{kg}$ group showed only minor treatment-related changes in some of the animals of both sexes (e.g., atypical homogenous cytoplasm of the hepatocytes and bile duct epithelial proliferation). There were no treatment-related effects in rats fed the 300 or $1000 \mathrm{mg} / \mathrm{kg}$ stannous chloride diets. Leucocyte total and differential counts of blood taken at autopsy were unaffected, as were the serum activities of glutamate-oxaloacetate and glutamate-pyruvate transaminases. At termination, there was a dose-related decrease in the activity of serum alkaline phosphatase in both male and female rats. However, this achieved statistical significance only at the $3000 \mathrm{mg} / \mathrm{kg}$ dietary concentration of stannous chloride. The authors indicated that unpublished pair feeding experiments suggest that the growth depression observed in some of the tin feeding 
experiments cannot be explained by a lack of palatability of the diet. In addition, on the basis of other studies involving iron supplementation and deficiency, the authors suggested that the toxicity of cationic tin compounds is inversely dependent on the iron content of the diet. The authors suggested that the tin compounds that cause adverse effects in vivo had no-effect levels corresponding to $1000 \mathrm{mg} / \mathrm{kg}$ of stannous chloride in the diet, which is equivalent to $450-650 \mathrm{mg} / \mathrm{kg}$ of tin, providing an intake in the range of $22-33 \mathrm{mg} \mathrm{tin} / \mathrm{kg}$ bw per day.

\section{Dreef-van der Meulen et al., 1974}

Male and female Wistar-derived rats were fed dietary concentrations of stannous chloride dihydrate increasing by weekly increments from $1000 \mathrm{mg} / \mathrm{kg}$ in the first week to $8000 \mathrm{mg} / \mathrm{kg}$ in weeks 8 to 13 . Body weight in the male animals was significantly reduced at the end of this period. Haemoglobin concentration and haematocrits were reduced compared to controls in males and females. Reduced serum alkaline phosphatase activity was recorded in female rats. Autopsy revealed pancreatic atrophy as well as changes to the gastrointestinal tract. Histological studies confirmed these observations, with severity of pancreatic changes ranging from necrosis of individual acinar cells to complete destruction of the pancreas. In addition, changes in the kidney and thyroid were observed. The authors considered the pancreatic atrophy to be the most specific manifestation of the toxicity of tin. Effects on the pancreas were observed in this study, which utilised an increasing concentration of tin in the diet, from $1000 \mathrm{mg} / \mathrm{kg}$ up to a final dose of $8000 \mathrm{mg} / \mathrm{kg}$ (equivalent to $310 \mathrm{mg} / \mathrm{kg}$ bw/day).

\section{Fritsch et al., 1978}

In a study of 6 months duration, groups of 10 male Sprague-Dawley CD rats were administered diets containing 0 (control), 4000, or $8000 \mathrm{mg}$ tin $/ \mathrm{kg}$ of diet as stannous chloride. Groups of five rats were treated in the same manner to provide control and treated animals for histological studies. Body weights were recorded twice weekly and in the 8th, 16th and 24th weeks the consumption of diet was measured. It is noted that all the treated animals exhibited a fatty degeneration, with lymphocytic infiltration and atrophy of the exocrine tissue of the pancreas. The kidneys and adrenal medulla were oedematous and there was karyolysis in the adrenal cortex. In the gastrointestinal tract there were generalised signs of irritation, with oedema and excessive mucus in the fundal and pyloric regions of the stomach. There was congestion in the jejunum and oedema with lymphocytic infiltration in the caecum. Effects on the pancreas were observed at $4000 \mathrm{mg} / \mathrm{kg}$ of tin in the diet (equivalent to $240 \mathrm{mg} / \mathrm{kg}$ bw/day).

\section{Exposure assessment}

Total exposure/intake:

Food

Mean: $1.8 \mathrm{mg} /$ day in adults.

97.5th percentile: $6.3 \mathrm{mg} /$ day (TDS, 1994)

Supplements $\quad 0.01 \mathrm{mg} /$ day (Annex 4; OTC, 2001)

Estimated maximum intake: $6.3+0.01=6.31 \mathrm{mg} /$ day

No potential high intake groups were identified. 


\section{Risk assessment}

Gastrointestinal effects are the main manifestation of toxicity associated with ingestion of foods or drinks contaminated with tin. These are caused by the irritant action of soluble inorganic tin compounds. Recovery from the effects is rapid.

Some subchronic feeding studies have resulted in haematological changes in rats. Other reports of other chronic carcinogenicity studies and one multigeneration reproduction study have either not recorded any such effects, or have noted that the observed changes were transient. Pancreatic atrophy has also been observed in subchronic studies in rats.

\section{ESTABLISHMENT OF GUIDANCE LEVEL}

There is no convincing evidence to suggest that tin is an essential element and it should be noted that tin in food is generally considered to be a contaminant.

There are insufficient data from human or animal studies to establish a safe upper level for tin. Nausea and vomiting have been reported following ingestion of food contaminated with tin. However, the balance of evidence suggests that the concentration of tin in contaminated foodstuff is critical to the development of acute gastrointestinal effects, and that tin concentrations above $200 \mathrm{mg} / \mathrm{kg}$ foodstuff are more likely to be associated with this. The regulatory limit of $200 \mathrm{mg} / \mathrm{kg}$ for the concentration of tin in foodstuffs therefore provides protection against the occurrence of episodes of acute human poisoning by tin. No groups vulnerable to this form of toxicity are known to exist.

For guidance purposes it should be noted that subchronic toxicity studies in rats indicate that pancreatic atrophy occurs at doses of about $240 \mathrm{mg}$ tin/ $\mathrm{kg}$ bw/day (Fritsch et al., 1978). In addition, changes to liver cells and anaemia have been observed in a study in which a NOAEL of 22-33 mg tin $/ \mathrm{kg}$ bw/day can be derived (de Groot et al., 1973). Applying uncertainty factors of 10 for inter-species variation and 10 for inter-individual variability to this NOAEL, gives a daily intake of about $0.2-0.3 \mathrm{mg} \mathrm{tin} / \mathrm{kg} \mathrm{bw} /$ day. The lower end of this range $0.22 \mathrm{mg} / \mathrm{kg} \mathrm{bw} /$ day, has been used for guidance purposes only and would be expected not to produce adverse effects in humans. This is equivalent to $13 \mathrm{mg}$ tin/day in a $60 \mathrm{~kg}$ adult

Current maximum dietary intakes are estimated at $6.3 \mathrm{mg} /$ day.

\section{References}

De Groot, A.P., Feron, V.J., Til, H.P. (1973) Subacute toxicity of inorganic tin as influenced by dietary levels of iron and copper. Food and Cosmetics Toxicology 11, 955-962.

Dreef-van der Meulen H. C., Feron V. J. and Til H. P. (1974) Pancreatic atrophy and other pathological changes in rats following the feeding of stannous chloride. Pathologia Europaea 9, 185-192.

Fritsch P., de Saint Blanquat G. and Derache R. (1978) Etude nutritionnelle et toxicologique, chez le rat, d'un contaminant alimentaire: l'étain [Nutritional and toxicological study of rats fed a diet containing tin]. Toxicology 8, 165-175.

OTC (2001) OTC Directory 2001-2002, Proprietary Association of Great Britain 


\section{Risk Assessment}

\section{Vanadium}

\section{General information}

\section{Chemistry}

Vanadium occurs as two natural isotopes, ${ }^{50} \mathrm{~V}$ and ${ }^{51} \mathrm{~V}$, the latter being the naturally occurring radioisotope. It forms oxidation states of $-1,0,+2,+3,+4$ and +5 . Within this risk assessment, the word vanadium refers to ionic vanadium, except when specific compounds are mentioned.

\section{Natural occurrence}

Metallic vanadium does not occur in the free form in nature but does occur in over 65 naturally occurring minerals. The predominant forms in biological materials are vanadate $\left(+5 \mathrm{~V}, \mathrm{VO}_{3}{ }^{-}\right)$and vanadyl $\left(+4 \mathrm{~V}, \mathrm{VO}^{+2}\right)$, respectively.

\section{Occurrence in food, food supplements and medicines}

Beverages, fats, oils, fresh fruits and vegetables contain the lowest levels of vanadium, whereas whole grains, seafood, meats and dairy products contain more $(0.005-0.03 \mathrm{mg} / \mathrm{kg})$. A few foods, including spinach, parsley, mushrooms and oysters, contain relatively high amounts of vanadium $(>0.10 \mathrm{mg} / \mathrm{kg})$. Vanadium is present in a number of multi-vitamin/mineral dietary supplements at levels of approximately $0.025 \mathrm{mg}$ per day. There are no licensed medicines containing vanadium.

\section{Other sources of exposure}

Exposure to vanadium by inhalation may occur occupationally. In the production of vanadium pentoxide, dust concentrations of the pentoxide can range from 0.1 to $30 \mathrm{mg} / \mathrm{m}^{3}$, and concentrations of $0.5-5 \mathrm{mg} / \mathrm{m}^{3}$ are not uncommon in the production of vanadium metal and vanadium catalysts.

\section{Recommended amounts}

Vanadium has not yet been proven to be an essential trace element for mammals.

\section{Analysis of tissue levels and vanadium status}

Vanadium can be measured in whole blood, serum and various tissues. Markers of vanadium status have not been established. 


\section{Overview of non-nutritional beneficial effects}

It has been claimed that vanadium may be a possible treatment for diabetes mellitus (types I and II). Information on the internet suggests that the element is used by body builders, although the rationale for this and any possible effects are uncertain.

\section{Function}

No specific function has been identified for vanadium in higher animals. In vitro and animal studies suggest that vanadium may function as an oxidation-reduction catalyst, and may regulate the sodium, potassiumadenosine triphosphatase enzyme, however, this has not been proven.

\section{Deficiency}

In humans, the reported signs of deficiency are questionable, although it has been suggested that low intakes may be associated with cardiovascular disease.

\section{Interactions}

Although no specific data have been identified, it is possible that vanadium may interfere with the storage and metabolism of iron, because absorbed vanadium is bound to transferrin.

\section{Absorption and bioavailability}

Intestinal absorption of vanadium is low, less than $5 \%$. The mechanism of absorption has not been defined.

\section{Distribution}

Absorbed vanadium is mainly transported in the plasma, associated with transferrin. Concentrations reported in human blood vary widely, with levels in whole blood and serum in the range of $0.01-0.4 \mathrm{mg} / \mathrm{L}$. The concentrations in all tissues are low, but are higher in the liver, kidney and lung. Vanadium is also present in breast milk and saliva and passes through the blood brain barrier. Small amounts have been identified in the placenta. Based on animal studies, bones and teeth retain the highest concentrations of vanadium.

\section{Excretion}

Ingested vanadium is predominantly eliminated unabsorbed via the faeces. Absorbed vanadium is mainly excreted via the urine. 
Part 3 Trace Elements

\section{Toxicity}

\section{Human data}

The toxicity of vanadium compounds increases as valency increases, $\mathrm{V}^{5+}$ being the most toxic. In humans, exposure by inhalation causes diverse toxic effects on the respiratory, digestive, and central nervous systems, the kidney and skin. There are very few reported cases of vanadium toxicity in humans, when it is taken by mouth.

\section{Supplementation trials}

Supplementation of human volunteers with vanadyl compounds at oral doses of 50-125 mg/day caused cramps, loosened stools and 'green tongue' in all patients, and fatigue and lethargy in a minority.

\section{Animal data}

Orally administered vanadium has low overt toxicity, but is reported to have adverse effects on reproduction and development in both males and females. There is some evidence of increased preand post-implantation foetal loss and significant accumulation of vanadium in the foetus. Skeletal anomalies and reduced ossification in the offspring, as well as an increased incidence of cleft palate have been reported.

\section{Carcinogenicity and genotoxicity}

Lifetime studies in animals indicate that vanadium is not carcinogenic. Positive results have been obtained in some in vitro mutagenicity tests.

\section{Mechanism of toxicity}

No data have been identified

\section{Dose-response characterisation}

No data have been identified

\section{Vulnerable groups}

No vulnerable groups have been identified in human studies. However, animal data suggest that high doses of vanadium may have adverse effects on reproduction in both males and females and on development of offspring.

\section{Genetic variations}

No genetic variation of effects with vanadium have been identified. 


\section{Studies of particular importance in the risk assessment}

(For full review see http://www.food.gov.uk/science/ouradvisors/vitandmin/evmpapers or the enclosed CD).

\section{Human data}

\section{Dimond et al., 1963}

Oral supplements of ammonium vanadium tartrate (increasing doses in the range of 25, 50, 75 and 125 $\mathrm{mg} /$ day) were administered to at least 6 volunteers for a minimum of six weeks. The total daily dose was limited by cramps and diarrhoea in all six patients. Haematological studies and urinalysis revealed no abnormalities. Non-significant and temporary decreases in cholesterol levels were observed in two (of six) patients. Doses of $50 \mathrm{mg}$ or more resulted in 'green tongue'. Two patients complained of fatigue and lethargy. Few subjects were involved and the dose was increased during the study. The authors suggested that the tolerated dose varied from 50 to $125 \mathrm{mg}$, although it was also noted that 'green tongue' developed at this dose range. No effects were observed at $25 \mathrm{mg}$ ammonium vanadium tartrate per day in 4 subjects, which is equivalent to approximately $5 \mathrm{mg}$ vanadium $/$ day $(0.08 \mathrm{mg}$ vanadium $/ \mathrm{kg}$ bw/day).

Somerville et al., 1992

$125 \mathrm{mg} /$ day of diammonium oxy-tartrato vanadate was administered for approximately five months, to patients with coronary heart disease. Half the subjects reported abdominal pains, anorexia and weight loss.

\section{Cohen et al., 1995.}

Vanadyl sulphate, administered at $100 \mathrm{mg} /$ day for three weeks to six patients with non-insulin dependant diabetes mellitus (NIDDM) in a single-blind placebo controlled trial, resulted in mild gastrointestinal effects: nausea, mild diarrhoea and abdominal cramps. This is equivalent to approximately $0.52 \mathrm{mg} / \mathrm{kg}$ bw/day as vanadium.

\section{Goldfine et al., 1995}

$125 \mathrm{mg}$ sodium metavanadate/day was administered to ten diabetic patients ( 5 insulin dependant and 5 with NIDDM) for two weeks. Mild gastrointestinal intolerance and diarrhoea were reported in both groups, including vomiting in one patient, which limited the total daily dose to $75 \mathrm{mg}$ in this patient. No biochemical evidence of toxicity was detected by screening, which included electrolytes, blood urea nitrogen, creatinine, liver function studies, thyroid functions, urine analysis and a complete blood count. The daily dose of $125 \mathrm{mg} /$ day was equivalent to approximately $0.83 \mathrm{mg} / \mathrm{kg}$ bw/day as vanadium)

\section{Boden et al., 1996}

Vanadyl sulphate was administered at $100 \mathrm{mg} /$ day (equivalent to approximately $0.52 \mathrm{mg} / \mathrm{kg}$ bw/day as vanadium) to eight NIDDM patients for a total of four weeks. Two of the eight patients reported no adverse effects. Adverse gastrointestinal effects were reported in the other six patients, including nausea, diarrhoea, abdominal cramps and flatulence. In one patient, diarrhoea and abdominal cramps lasted for eleven days. In the others all symptoms disappeared during the first week of treatment. 
Halberstam et al., 1996

Transient gastrointestinal effects were reported in seven diabetic patients given $100 \mathrm{mg} /$ day vanadyl sulphate (equivalent to approximately $0.52 \mathrm{mg} / \mathrm{kg}$ bw/day as vanadium) for three weeks. These included nausea, mild diarrhoea and abdominal cramps, which subsided after one week of treatment. Additionally, some patients noted discolouration of the stool; however, stool examinations were negative for occult blood.

\section{Animal data}

\section{Domingo et al., 1986}

Sodium metavanadate was administered to adult male rats by oral gavage $(0,5,10$ and $20 \mathrm{mg} / \mathrm{kg}$ bw/day, equivalent to approximately $0,2,4$ and $8 \mathrm{mg}$ vanadium/ $\mathrm{kg}$ bw/day) for sixty days, before mating with females that received the same vanadate doses for the 14 days prior to mating. Treatment of the females was continued during the periods of gestation and lactation. No significant effects on fertility, reproduction and parturition were observed; the number of dead foetuses and resorptions was increased in the highest dose group, but this was not significant. The development of the offspring (body weight, body length and tail length) was significantly decreased from birth and during the lactation period, at all dose levels, which were equivalent to approximately 2 to $8 \mathrm{mg} / \mathrm{kg} \mathrm{bw} /$ day as vanadium.

\section{Paternain et al., 1990}

Vanadyl $\left(\mathrm{V}^{+4}\right)$ sulphate pentahydrate $(0,37.5,75$ and $150 \mathrm{mg} / \mathrm{kg}$ bw/day) was administered by oral gavage to pregnant mice on days 6 to 15 of gestation. Maternal toxicity, embryotoxicity and foetotoxicity (including teratogenicity) were reported at 75 and $150 \mathrm{mg} / \mathrm{kg}$ bw/day, which is equivalent to approximately $7.5 \mathrm{mg} / \mathrm{kg}$ bw/day as vanadium.

\section{Llobet et al., 1993}

Sodium metavanadate was given in drinking water to male mice for 64 days before mating with untreated females. The concentrations of sodium metavanadate used were $0,0.1,0.2,0.3$ and $0.4 \mathrm{mg} / \mathrm{mL}$, giving doses of 0,20,40,60 and $80 \mathrm{mg} / \mathrm{kg}$ bw/day. A significant decrease in the pregnancy rate was seen at 60 and $80 \mathrm{mg} / \mathrm{kg}$ bw/day. Decreased body and epididymis weights were observed in the $80 \mathrm{mg} / \mathrm{kg}$ bw/day group, but testicular weights were not altered at any of the doses administered. The sperm count was significantly diminished at 60 and $80 \mathrm{mg} / \mathrm{kg}$ bw/day, but sperm motility was unaffected and histopathological examination revealed that the testes were normal. The development of subsequent offspring was not assessed. No effects were observed with sodium metavanadate at $40 \mathrm{mg} / \mathrm{kg}$ bw/day, which is equivalent to approximately $16 \mathrm{mg} / \mathrm{kg}$ bw/day as vanadium. 


\section{Exposure assessment}

Total exposure/intake:

Food

Mean: $0.013 \mathrm{mg} /$ day (1980 UK TDS)

97.5 percentile: No data available

Supplements

up to $0.025 \mathrm{mg} /$ day (Annex 4)

Drinking Water

$0.01 \mathrm{mg} /$ day (estimated from $0.005 \mathrm{mg} / \mathrm{L}, \mathrm{WHO}$ 1988)

Estimated maximum

daily intake*

$0.013+0.025+0.01=0.05 \mathrm{mg} /$ day

No potential high intake groups were identified.

* Note that no data on high level intakes are available, so this is an underestimate.

\section{Risk assessment}

Few data are available. Vanadium compounds have been reported to cause gastrointestinal effects in supplementation studies in human volunteers.

In rodents, orally administered vanadium has low overt toxicity, but is reported to have adverse effects at high doses on reproduction and development in both males and females. It is unclear whether these effects could also occur in humans.

\section{EVM OPINION}

It has not been proven that vanadium is an essential element in humans, although vanadium-dependent enzymes occur in lower organisms. There are insufficient data from human or animal studies to establish a safe upper level for vanadium.

Supplementation of human volunteers with vanadium compounds at doses of 50-125 mg/day has been reported to cause cramps, loosened stools and 'green tongue' in all patients and fatigue and lethargy in a minority of participants. Few human data are available and interpretation is limited by the small number of participants and the relatively short duration of studies.

Data from animal studies suggest that vanadium has adverse effects on both male and female reproduction and on the development on the subsequent offspring. There are insufficient data to establish whether or not this effect could occur in humans.

The available studies are inadequate to support the safe use of vanadium in supplements. It should be noted that vanadium supplements aimed at body builders are available (particularly via the internet) at doses between 7.5 and $10 \mathrm{mg} /$ day. Supplements containing these levels of vanadium may not be safe. 


\section{References}

Boden, G. Chen, X, Riuz, J, van Rossum J.D., Turco, S. (1996) Effects of vanadyl suphate on carbohydrate and lipid metabolism in patients with non-insulin dependent diabetes mellitus. Metabolism 45, 1130-1135.

Cohen, N., Halberstam, M., Shlimovich, P., Chang, C.J. Shamoon H, and Rosetti L. (1995) Oral vanadyl sulphate improves hepatic and peripheral insulin sensitivity in patients with non-insulin dependent diabetes mellitus. Journal of Clinical Investigation 95, 2501-2509.

Dimond, E.G., Caravaca, J. and Benchimol, A. (1963) Vanadium: Excretion, toxicity, lipid effect in man. American Journal of Clinical Nutrition 12, 49-53.

Domingo, J.L., Paternain, J.L, Llobet, J.M. and Corbella, J. (1986) Effects of vanadium on reproduction, gestation, parturition and lactation in rats upon oral administration. Life Sciences 39, 819-824.

Goldfine, A.B., Simondson D.C., Folli, F., Patti, ME, Kahn, F. (1995) Metabolic effects of sodium metavanadate in humans with insulin dependent and non-insulin dependent diabetes mellitus in vivo and in vitro studies. Journal of Clinical Endocrinology and Metabolism 80, 3311-3320.

Halberstam, M., Cohen, N. Shlimovich, P. Rossetti, L., Shamoon, H.(1996) Oral vanadyl sulphate improves insulin sensitivity in NIDDM but not in obese nondiabetic subjects. Diabetes 45, 659-666.

Llobet, J.M., Colomina, M.T., Sirvent, J.J. Domingo, J.L. and Corbella, J. (1993) Reproductive toxicity evaluation of vanadium in male mice. Toxicology 80, 199-206.

Paternain, J.L., Domingo, J.L., Gomez, M., Ortega, A. and Corbella. J. (1990) Developmental toxicity of vanadium in mice after oral administration. Journal of Applied Toxicology 10, 181-186.

Somerville, J. and Davies, B (1992) Effect of vanadium on serum cholesterol. American Heart Journal 64, 54-56.

WHO (1988). International Programme on Chemical Safety, Environmental Health Criteria 61. World Health Organisation, Geneva. 


\section{Risk Assessment}

\section{Zinc}

\section{General information}

\section{Chemistry}

Zinc is an abundant group IIB post-transition metallic element. It occurs in nature as the sulphide (ZnS), in blende and sphalerite, as the silicate (ZnSiO4), in calamine, willemite and zinc spar, and as the oxide $(\mathrm{ZnO})$ in zincite. Within this risk assessment document the word zinc refers to ionic zinc except where specific zinc compounds are mentioned.

\section{Natural occurrence}

Zinc is present in the earth's crust and in seawater. Zinc is found in all plant and animal tissues, particularly inside the nuclei.

\section{Occurrence in food, food supplements and medicines}

Meat and cereal products are rich in zinc. Zinc salts are also widely available in food supplements. In the UK, zinc supplements provide intakes up to $50 \mathrm{mg} /$ day.

Zinc is present in a number of licensed medicines both alone and with other ingredients, and is used for the treatment and prevention of deficiency. The maximum daily dose of products available from a pharmacist is $150 \mathrm{mg}$, and of products available on the General Sales List is $5 \mathrm{mg}$.

\section{Other sources of exposure}

Intake of zinc from drinking water is normally very low, but may be increased by dissolution of zinc from pipes and contaminated wells. The UK current regulatory limit in water (based on taste and appearance) is $5 \mathrm{mg} / \mathrm{L}$. Many cases of food poisoning incidents have resulted from the storage of food or drink in galvanised containers.

\section{Recommended amounts}

The UK RNI ranges set by COMA are 5.5-9.5 mg/day for males and 4.0-7.0 mg/day for females (COMA, 1991). The US Recommended Daily Allowance (RDA) is $15 \mathrm{mg} /$ day for males and $12 \mathrm{mg} /$ day for females.

\section{Analysis of tissue levels and zinc status}

Reliable, validated measures of zinc status are not available. Plasma, blood, hair and urinary zinc can be measured, but these may be affected by conditions unrelated to zinc deficiency. 
Part 3 Trace Elements

\section{Brief overview of non-nutritional beneficial effects.}

Zinc sulphate is used to treat Wilson's disease. Studies have also reported its use to enhance wound healing and slow macular degeneration. Zinc has also been claimed to ameliorate a variety of conditions including the common cold.

\section{Function}

Zinc is an essential constituent of more than two hundred metalloenzymes. Zinc plays a key role in the synthesis and stabilisation of genetic material and is necessary for cell division and the synthesis and degradation of carbohydrates, lipids and proteins.

\section{Deficiency}

Zinc deficiency results in effects including poor prenatal development, growth retardation, mental retardation, impaired nerve conduction and nerve damage, reproductive failure, dermatitis, hair loss, diarrhoea, loss of appetite, loss of taste and smell, anaemia, susceptibility to infections, delayed wound healing and macular degeneration.

\section{Interactions}

Zinc and copper are mutually antagonistic, each interfering with the gastrointestinal uptake of the other thus potentially leading to imbalance. Similarly, zinc and iron compete for absorption. Excess zinc may also decrease magnesium and calcium uptake, and conversely, high levels of calcium in the diet can decrease zinc absorption. Zinc salts reduce the bioavailability of fluoroquinoline antibiotics.

\section{Absorption and bioavailability}

Absorption of zinc salts from food is approximately $20-40 \%$, being higher from fish and meat but lower from cereals, where phytate content impairs absorption. The absorption of zinc salts depends on their solubility. Zinc is absorbed both by passive diffusion and an unknown membrane carrier process, which requires energy.

\section{Distribution and metabolism}

The mechanism for transfer across the gut wall is by both passive diffusion and an unknown membrane associated carrier-mediated process, which requires energy and occurs throughout the small intestine. Following internalisation in the intestinal cell, zinc associates with metallothionein. Once in plasma, zinc binds to a number of proteins including albumin, transferrin, $\alpha$-2-macroglobulin and ceruloplasmin (ferroxidase). Little is known about uptake, but zinc is found in all cells, particularly inside the nuclei, of all tissue types. Some tissues are particularly rich in zinc. These include the choroid of the eye, adrenal gland, skin, certain areas of the brain, pancreas and the prostate gland. 


\section{Excretion}

Since zinc is not stored, the balance between absorption and excretion (homeostasis) is essential to the maintenance of a broad spectrum of zinc-dependent functions. Zinc excretion is largely via the faeces, which contain unabsorbed zinc as well as biliary and pancreatic secretions.

\section{Toxicity}

\section{Human data}

The symptoms of acute zinc salt toxicity include abdominal pain, nausea and vomiting. Other reported effects include lethargy, anaemia and dizziness. Prolonged use of high doses of zinc can result in secondary deficiency of copper. Symptoms of this include hypocupraemia, impaired iron mobilisation, anaemia, leukopenia, neutropenia, decreased superoxide dismutase (SOD) (particularly erythrocyte SOD (ESOD)), decreased ceruloplasmin, decreased cytochrome c oxidase, increased plasma cholesterol, increased LDL:HDL cholesterol, decreased glucose clearance, decreased methionine and leucine enkephalins, abnormal cardiac function and impairment of pancreatic enzymes, amylase and lipase. It has also been suggested that excess zinc is atherogenic.

\section{Supplementation trials}

A variety of supplementation studies are available. Many of these investigate the effects of zinc supplementation on various medical conditions or physiological systems. However, some work has also been undertaken suggesting that high levels of zinc can adversely affect copper and iron status.

\section{Animal data}

Very high doses of zinc can cause minor neural degeneration, acinar cell necrosis and metaplasia in the pancreas, decreased haematocrit and decreased white blood cell count. Very high doses have also been shown to cause reproductive toxicity in rats. Lower doses have resulted in reduced ceruloplasmin activity and decreased haemoglobin levels.

\section{Carcinogenicity and genotoxicity}

Zinc has been found to give positive results in some in vitro and in vivo genotoxicity tests. No data have been identified on the carcinogenicity of zinc.

\section{Vulnerable groups}

A small study suggests that zinc supplementation increases the levels of glycosylated haemoglobin in diabetics.

\section{Genetic variations}

Sufferers from haemochromatosis may absorb larger amounts of zinc indicating possible increased risk of zinc-induced copper deficiency. 
Part 3 Trace Elements

\section{Mechanisms of toxicity}

No data have been identified on the mechanisms of toxicity.

\section{Dose response characterisation}

In humans, the acute toxic effects of zinc occur at doses of approximately $200 \mathrm{mg}$ or more. ESOD activity, thought to be the most sensitive measure of copper status, was shown to decrease following supplementation with $50 \mathrm{mg} /$ day or more for 12 days. However, other studies have reported no effect at doses of $50 \mathrm{mg} /$ day. Supplementation with $50 \mathrm{mg} /$ day for up to 10 weeks has further resulted in reductions in haematocrit and serum ferritin. Doses greater than $100 \mathrm{mg} /$ day have resulted in altered ratios of HDL:LDL cholesterol. Copper deficiency and sideroblastic anaemia, associated with chronic zinc ingestion, were reported in one individual who had taken non-prescribed zinc supplements of $26.6-40 \mathrm{mg} /$ day for at least 2 years.

In animal studies, acute effects occur at doses greater than $480 \mathrm{mg} / \mathrm{kg}$ bw/day. Reproductive toxicity in rats has been observed at doses above $200 \mathrm{mg} / \mathrm{kg}$ bw/day.

\section{Studies of particular importance in the risk assessment}

(For full review see http://www.food.gov.uk/science/ouradvisors/vitandmin/evmpapers or the enclosed CD).

\section{Secondary copper deficiency}

General

Sandstead, 1995

This paper discusses the inhibitory effect of zinc on copper absorption and the risk of secondary copper deficiency increasing with high zinc:copper molar ratios. The signs of secondary copper deficiency include decreased ESOD, increased LDL cholesterol, decreased HDL cholesterol, decreased glucose clearance, decreased methionine and leucine enkephalins and abnormal cardiac function. ESOD activity is thought to be particularly sensitive to decreased copper status. The author suggested a preliminary reference dose of $9 \mathrm{mg} /$ day for $60 \mathrm{~kg}$ adults for over the counter (OTC) zinc, assuming high bioavailability and uncertain copper intakes.

The Age-Related Eye Disease Study Research Group, 2002

The effects of supplementation for 5 years with $80 \mathrm{mg} /$ day zinc (as zinc oxide) plus either antioxidants (500 mg/day vitamin C, $268 \mathrm{mg} /$ day vitamin E, $15 \mathrm{mg} /$ day ß-carotene) or $2 \mathrm{mg} /$ day copper (as copper oxide) on serum levels of zinc, copper, lipids and haematocrit were reported in this paper. No significant differences in serum haematocrit, copper or lipids were observed at the end of the 5 -year period compared to placebo controls. However, only $20 \%$ of participants were followed up for the full 5 years and no group received zinc alone. It is therefore difficult to draw conclusions from this study. 


\section{$H D L, L D L$ and cholesterol}

Hooper et al., 1980

Twelve healthy male volunteers (23-25 years) were given $160 \mathrm{mg} /$ day zinc (as zinc sulphate, taken with meals) for 5 weeks. Eight control subjects received placebo treatment. After 5 weeks treatment, a 25\% reduction $(p<0.001)$ in HDL-cholesterol levels was measured. No effect on total cholesterol, LDLcholesterol, or triglyceride was found. No decline in these parameters was apparent in the controls. HDL levels continued to decline for a further two weeks after cessation of treatment. Eleven weeks after the cessation of treatment, the HDL values had returned to near baseline. Plasma copper was not decreased throughout. The amounts of zinc and copper provided by food and beverages were not stated. This was a small scale, presumably single-blinded, study.

\section{Pachotikarn et al., 1985}

Twenty three male volunteers were given $50 \mathrm{mg}$ zinc/day (as gluconate) for 6 weeks. Subjects were asked to avoid foods high in copper, phytate and fibre during the study. A statistically non-significant increase (approximately 16\%) in HDL cholesterol and a decrease in total cholesterol with a significant decrease in diastolic blood pressure were measured. Dietary zinc and copper intakes were in the range 11.9-12.4 and 0.93-1.01 mg/day during the study. The zinc:copper intake ratio was estimated to be 60:1. Haematocrit, haemoglobin and plasma copper levels were not significantly altered from baseline. No control group was included in the study, so it is not possible to assess the potential effect of dietary changes on the parameters measured.

\section{Black et al., 1988}

Groups of 9-13 male volunteers (19-29 years) were given supplements of 0 (placebo), 50 or 75 mg zinc/day (as gluconate, consumed after breakfast with water) for 12 weeks. HDL cholesterol was decreased by approximately $14 \%$ at weeks 6 and 12 of treatment in the higher dose group and at week 12 in the lower dose group. However, at weeks 8 and 10, levels of HDL cholesterol in both treated groups were higher than the controls. It was noted that week 10 was a holiday in which the volunteers left the campus. Serum zinc levels increased in the high but not the low dose group. Serum copper levels were not altered. Mean dietary copper levels were 2.1, 1.8 and $1.7 \mathrm{mg} /$ day for the placebo, $50 \mathrm{mg}$ and $75 \mathrm{mg}$ zinc supplement groups, respectively. Mean dietary zinc intakes were 12.6, 14.1 and $9.8 \mathrm{mg} / \mathrm{day}$. This was a double-blind study. Details of diet were collected to establish copper and zinc intake from food. The number of participants was small and the protocol demanding since a number of dietary restrictions were requested (high phytate and high copper foods). This was monitored by the use of bi-weekly 3-day dietary records. However, limited compliance with the dietary restrictions may explain some of the variations. In the high dose group, total and saturated fat and protein intakes were lower than in the other groups.

\section{Samman and Roberts, 1988}

As part of a double blind crossover study, 20 female (mean age 27 years) and 21 male volunteers (mean age 28 years) were given $150 \mathrm{mg}$ ( 3 x 50mg) zinc/day (as sulphate) for 6 weeks. Plasma zinc increased significantly on treatment. There were no changes in total plasma cholesterol levels in either sex. However, in females, LDL cholesterol was decreased by $9 \%$ while ESOD and caeruloplasmin activities were reduced. Plasma copper and haematocrit remained unchanged. Gastrointestinal and other effects were reported by some of the subjects leading to 6 females dropping out of the study (see also Samman and Roberts, 1987 below). Differences between the sexes were attributed to females receiving a 
higher dose on a mg/kg basis (mean bodyweights were 73.9 and 61.3 for males and females respectively). Copper intake was not stated.

ESOD and other enzyme activities

Taper et al., 1980

Groups of 5 or 6 adult women of child bearing age, were fed dietary regimes containing $2 \mathrm{mg} /$ day copper and 8, 16, or $24 \mathrm{mg} /$ days zinc for 18 days. Plasma levels of zinc and copper were increased and decreased, respectively. However, zinc had no effect on copper retention at any dose and the negative copper balance observed in each treatment group was unrelated to zinc dose. There was no functional assessment of copper status in this study.

\section{Fischer et al., 1984}

Groups of 13 healthy males, were given a placebo or a supplement of $50 \mathrm{mg}$ zinc/day (as gluconate taken as two doses) for 6 weeks. The volunteers were divided so that baseline caeruloplasmin and ESOD activity were the same within the two groups. In the treatment group plasma zinc levels were elevated. ESOD activity decreased following 4 weeks of treatment being reduced by a maximum of $20 \%$ after 6 weeks. Plasma copper and caeruloplasmin activity were unaffected by zinc treatment. The amounts of zinc and copper provided by food and beverages were not stated. The time taken for ESOD to decline was thought to be related to erythrocyte turnover. This was a reasonable double blind study, though the numbers are small and there is no information on dietary copper or zinc intake.

Festa, 1985

Nine healthy male volunteers were given a basal egg-white diet that provided recommended levels of all essential nutrients, with the exceptions of zinc and protein where the diet provided $1.8 \mathrm{mg}$ zinc and $16.4 \mathrm{~g}$ nitrogen daily. Copper intake was $2.63 \mathrm{mg} /$ day. Zinc carbonate was added to give a total zinc intake of $20.7 \mathrm{mg} /$ day (week 1), $18.5 \mathrm{mg} /$ day (weeks 3, 5, 6, 8, 9), $1.8 \mathrm{mg} / \mathrm{d}$ (week 2) 1.8 or $8.0 \mathrm{mg} /$ day (week 4) and 4, 6 or $8 \mathrm{mg} /$ day (week 7). It was reported that a total zinc intake of $18.5 \mathrm{mg} /$ day for 2 weeks following on from a week at a lower intake resulted in reduced apparent retention and increased excretion of copper. Mean plasma copper concentrations remained within the normal range throughout the study, but mean faecal copper excretion was elevated over copper intake in week 6 . However, the biological significance of this result is uncertain. Negative copper balance was not repeated in week 9 and there was no measurement of any functional index of copper status.

Yadrick et al., 1989

Nine female volunteers (25-40 years) were given supplements of $50 \mathrm{mg} /$ day zinc (as twice daily gluconate capsules) for 10 weeks. ESOD activity, haematocrit and serum ferritin were significantly reduced (by 50\%, 5\% and 30\% respectively) compared with pre-treatment levels. Effects on ferritin and haematocrit but not ESOD were ameliorated when equal ( $\mathrm{mg}$ for $\mathrm{mg}$ ) iron supplementation was given to a second group of nine women (at the start of study, the volunteers were divided so that baseline ferritin and ESOD levels were the same). The amount of zinc and copper provided by food and beverages during the study was not stated. This was a reasonable double blind study, though the numbers were small and there is no information on dietary copper or zinc intake. 


\section{Abdallah and Samman, 1993}

Six healthy female volunteers (age range 18-36 years), were given zinc capsules (50 mg/day as zinc sulphate, taken with breakfast) for 12 days. A significant, 20\%, decrease in ESOD activity was measured. Dietary zinc was estimated as 9-12 mg/day. Copper intake was not stated. Small increases in erythrocyte and plasma zinc were observed but were not significant. Stomach cramps and a 'burning sensation' after swallowing were reported by some volunteers. The study involved small numbers and there was no control group. The results are reasonably consistent with those of Yadrick (see above).

Cunningham et al., 1994

Following supplementation with $50 \mathrm{mg}$ /day zinc (as gluconate) for 28 days, $45 \%$ and $20 \%$ increases in $\mathrm{HbA}_{\mathrm{lc}}$ were reported in insulin dependent diabetes mellitus (IDDM) patients ( $\mathrm{n}=14,18-37$ years) and nonIDDM individuals ( $n=15,23-38$ years), respectively, suggesting glycosylation becomes altered in a milieu of zinc excess. In this study, plasma and erythrocyte copper did not differ significantly from baseline levels. Dietary zinc was estimated to be 11.2 and $8.7 \mathrm{mg} /$ day in the IDDM and non-IDDM individuals respectively, reflecting the sex distribution in the groups. Copper intake was not stated. Small numbers were involved in this study and there were no placebo control subjects.

Davis et al., 2000

A study was conducted on a metabolic ward, 25 healthy post-menopausal women were fed diets containing low ( $1 \mathrm{mg} /$ day) or high (10 mg/day) copper for 180 days. For one 90 day period, the women were given a supplement of $50 \mathrm{mg}$ zinc/day and for the other 90 day period, a placebo. The basal diet contained $3 \mathrm{mg}$ zinc/day. Zinc supplementation significantly increased extracellular superoxide dismutase activity but did not affect erythrocyte superoxide dismutase. This effect was more apparent when the subjects received the low copper diet. The study is unusual in that it was conducted on a metabolic ward, where a high degree of control over the zinc and copper intakes of the subjects was possible.

\section{Other adverse effects}

Samman and Roberts, 1987

This paper describes details of the side effects that occurred in the study of Samman and Roberts (1988), described above. Symptoms were reported by $84 \%$ of the female and $18 \%$ of the male volunteers. These included headaches, abdominal cramps, nausea, loss of appetite and vomiting. The symptoms were particularly apparent when small meals or no food was taken with the supplements (contrary to instructions). It was considered that, although a sex difference could not be ruled out, the higher number of symptoms in the female volunteers could be due to their lower body weight. Six subjects dropped out of the study, 5 due to the abdominal cramps and one due to persistent headaches. All were women receiving a dose of $0.86 \mathrm{mg} / \mathrm{kg}$. 
Hale et al., 1989

Health questionnaires, electrocardiograms, and laboratory and medication use data from a health screening program for older people were compared for 69 participants taking zinc supplements $(20$ to $150 \mathrm{mg} /$ day for an average duration of 8 years) with 1,832 subjects who were not taking supplements. No association was found between zinc supplementation and increased risk of developing cardiovascular disease. The zinc treated group had lower mean creatinine and total protein levels. After controlling for age and sex the group had lower serum uric acid and higher mean cell haemoglobin levels. The red blood cell count was decreased in women taking zinc. No significant differences were found in triglyceride or cholesterol levels but HDL cholesterol was not measured. Limited details and no actual values for the parameters were provided on this study. No data on dietary intake of zinc or copper were provided.

\title{
Exposure assessment
}

\author{
Food \\ Mean: $9.8 \mathrm{mg} /$ day \\ 97.5 percentile:17 mg/day (from 1986/87 NDNS) \\ Water \\ up to $10 \mathrm{mg} /$ day (assuming $2 \mathrm{~L} /$ day consumption at maximum \\ UK concentration of $5 \mathrm{mg} / \mathrm{L}$ ) \\ Supplements \\ up to $50 \mathrm{mg} /$ day (Annex 4) \\ Estimated maximum intake: $17+10+50=77 \mathrm{mg} /$ day
}

No potential high intake groups were identified.

\section{Risk assessment}

Consumption of zinc supplements by human volunteers has been reported to cause gastrointestinal effects, including cramping and nausea. This was particularly apparent when the supplements were taken with little or no food.

Excess levels of dietary zinc interfere with the gastrointestinal absorption of copper, potentially leading to secondary copper deficiency. Signs of this include decreased ESOD (erythrocyte superoxide dismutase) activity, increased LDL cholesterol and decreased HDL cholesterol, decreased glucose clearance and abnormal cardiac function. One of the most sensitive markers of this appears to be ESOD activity. Supplemental doses of $50 \mathrm{mg}$ cause significant decreases in the activity of this enzyme.

Iron and zinc in the diet each affect the gastrointestinal absorption of the other. Few data are available, but high levels of iron are known to interfere with zinc uptake, and more limited data suggest that the reverse interaction also occurs. This has not been considered in detail. 
Two vulnerable groups have been identified; diabetics, since the study by Cunningham et al. (1994) indicates that $\mathrm{HbA}_{\mathrm{lc}}$ is increased in conditions of zinc excess, and sufferers of haemochromatosis who have enhanced gastrointestinal absorption of iron, cobalt, lead and possibly zinc, potentially predisposing them to accumulation of zinc. However, there are insufficient data to establish the level of zinc intake at which adverse effects could occur in these groups.

Data are also available from animal studies that indicate high zinc levels can have a negative effect on copper balance. These have not been considered in detail in the risk assessment, as considerable data from human studies are available.

\section{ESTABLISHMENT OF SAFE UPPER LEVEL}

Key studies: Yadrick et al. (1989), Fischer et al. (1984), Black et al. (1988), Cunningham et al. (1994), Davis et al. (2000).

\section{LOAEL:}

Uncertainty factor:

Safe Upper Level: for daily consumption over a lifetime.

$$
50 \mathrm{mg} / \text { day }
$$

\section{2 (LOAEL to NOAEL extrapolation)}

$50 / 2=25 \mathrm{mg}$ zinc/day for supplemental zinc

Zinc affects iron and copper uptake at supplemental doses of $50 \mathrm{mg} /$ day and above. However, this is not apparent in all studies. The key endpoint is the reduction of copper absorption by zinc. Where the contribution of dietary zinc was also assessed, the lowest level at which effects were apparent (reduced activity of the copper-dependent enzyme ESOD being the most sensitive) was approximately 58-62 $\mathrm{mg} /$ day (50 mg supplements plus approximately $8-12 \mathrm{mg} /$ day from food). There is no evidence of adverse effects from dietary zinc intake. In addition, since the contribution of zinc from the diet in many of the studies is uncertain, and may have been altered under study conditions, it is appropriate to set a safe upper level for supplemental zinc but not total intake. Taking the LOAEL as $50 \mathrm{mg} /$ day and by applying an uncertainty factor of 2 to account for LOAEL to NOAEL extrapolation, a Safe Upper Level of $50 / 2=25 \mathrm{mg} /$ day for supplemental zinc is derived (equivalent to $0.42 \mathrm{mg} / \mathrm{kg}$ bw/day in a $60 \mathrm{~kg}$ adult). An uncertainty factor of 2 has been used rather than 3 since the effect concerned is a small and inconsistent change in a biochemical parameter. No uncertainty factor is needed for inter-individual variability because this safe upper level is supported by a large number of human studies. Assuming a maximum intake of $17 \mathrm{mg} /$ day from food, a total intake of $42 \mathrm{mg} /$ day (equivalent to $0.7 \mathrm{mg} / \mathrm{kg}$ bw/day in a $60 \mathrm{~kg}$ adult) would not be expected to result in any adverse effects. 
Part 3 Trace Elements

\section{References}

Abdallah, S.M., Samman, S. (1993). The effect of increasing dietary zinc on the activity of superoxide dismutase and zinc concentration in erythrocytes of healthy female subjects. European Journal of Clinical Nutrition 47, 327-332.

Black, M.R., Medeiros, D.M., Brunett, E., Welke, R. (1988). Zinc supplements and serum lipids in young adult white males. American Journal of Clinical Nutrition 47, 970-975.

COMA (1991). Dietary Reference Values for Food Energy and Nutrients for the United Kingdom. Report of the Panel on Dietary Reference Values, Committee on Medical Aspects of Food and Nutrition Policy. HMSO, London.

Cunningham, J.J., Fu, A., Mearkle, P.L., Brown, R.G. (1994). Hyperzincuria in individuals with insulindependant diabetes mellitus: Concurrent zinc status and the effect of high-dose zinc supplementation. Metabolism 43, 1558-1562.

Davis, C.D., Milne, D.B., Nielsen, F.H. (2000). Changes in Dietary Copper Affect Zinc-Status Indicators of Post-Menopausal Women, Notable Extracellular Superoxide Dismutase and Amyloid Precursor Proteins. American Journal of Clinical Nutrition 71, 781-788.

Festa, M.D., Anderson, H.L., Dowdy, R.P., Ellersiek, M.R. (1985). Effect of zinc intake on copper excretion and retention in men. American Journal of Clinical Nutrition 41, 285-292.

Fischer, P.W., Giroux, A., L’Abbe, M.R. (1984). Effect of zinc supplementation on copper status in adult man. American Journal of Clinical Nutrition 40, 743-746.

Hale, W.E., May, F.E., Thomas, R.G., Moore, M.T., Stewart, R.B. (1988). Effect of zinc supplementation on the development of cardiovascular disease in the elderly. Journal of Nutrition for the Elderly 8, 49-57.

Hooper, P.L. , Visconti, L., Garry, P.J., Johnson, G.E. (1980). Zinc lowers high-density lipoprotein-cholesterol levels. Journal of the American Medical Association 244, 1960-1961.

Pachotikarn, C., Madeiros, D.M., Windham, F. (1985). Effect of oral zinc supplementation upon plasma lipids, blood pressure, and other variables in young adult white males. Nutrition Reports International 32, 373-382.

Samman, S., Roberts, D.C. (1988). The effect of zinc supplements on lipoproteins and copper status. Atherosclerosis 70, 247-252.

Samman, S., Roberts, D.C. (1987). The effect of zinc supplements on plasma zinc and copper levels and the reported symptoms in healthy volunteers. Medical Journal of Australia 146, 246-9.

Sandstead, H.H. (1995). Requirements and toxicity of essential trace elements, illustrated by zinc and copper. American Journal of Clinical Nutrition 61 (Suppl), 621S-624S.

Taper, L.J., Hinners, M.L., Ritchey, S.J. (1980). Effects of zinc intake on copper balance in adult females. American Journal of Clinical Nutrition 33, 1077-1082.

Yadrick, M.K., Kenney, M.A., Winterfeldt, E.A. (1989). Iron, copper and zinc status: response to supplementation with zinc or zinc and iron in adult females. American Journal of Clinical Nutrition 49, $145-150$. 
Minerals 


\section{General information}

\section{Chemistry}

Calcium is an alkaline earth metal belonging to Group II of the periodic table. It is a divalent cation with an atomic weight of 40 . Calcium shows a single oxidation state of +2 .

\section{Natural occurrence}

Calcium does not exist freely in nature, but occurs abundantly as limestone $\left(\mathrm{CaCO}_{3}\right)$, gypsum $\left(\mathrm{CaSO}_{4} \cdot 2 \mathrm{H}_{2} \mathrm{O}\right)$, and fluorite $\left(\mathrm{CaF}_{2}\right)$. Many calcium compounds (e.g. fluorspar and calcium carbonate) are almost insoluble in water, but there are exceptions (e.g. calcium chloride and calcium nitrate). Within this risk assessment, the word calcium refers to ionic calcium, except where specific calcium compounds are described.

\section{Occurrence in food, food supplements and medicines}

Foods particularly rich in calcium are milk (1200 mg/kg), cheese $(730-12000 \mathrm{mg} / \mathrm{kg}$ ) and other dairy products (except butter), green leafy vegetables (except spinach), soybean products, bread and other baked goods made from calcium fortified flour (variable levels), almonds ( $2400 \mathrm{mg} / \mathrm{kg}$ ), brazil nuts $(1700 \mathrm{mg} / \mathrm{kg}$ ) and hazelnuts $(1400 \mathrm{mg} / \mathrm{kg})$. In the West, more than $70 \%$ of calcium intake is from milk and dairy products.

Fortified foods such as bread and baked products and some breakfast cereals now contribute significantly more calcium to the diet than would be the case without fortification. UK law requires that all flour, with the exception of wholemeal flour, be supplemented with 2350-3900 mg calcium carbonate (equivalent to $940-1560 \mathrm{mg}$ calcium) per kg. Wholemeal flour contains naturally $380 \mathrm{mg}$ calcium per $\mathrm{kg}$.

Calcium in dietary supplements is available either alone or in combination with other minerals beneficial to bone health (such as vitamin $\mathrm{D}$, magnesium and zinc) or in multivitamin preparations with added minerals. Concentrations typically range from $133 \mathrm{mg} /$ tablet, taken once daily, to $800 \mathrm{mg} /$ tablet, taken 3 times per day. Calcium in dietary supplements is available in many different forms, including calcium carbonate and various calcium chelates. Calcium carbonate contains the highest concentration of elemental calcium ( $400 \mathrm{mg} / \mathrm{g}$ ), but is of low solubility and therefore less bioavailable than calcium chelates. Antacid preparations contain calcium carbonate (with a maximum daily dose of $4000 \mathrm{mg}$ calcium).

\section{Other sources of exposure}

In different geographical areas, the concentration of calcium in drinking water varies from less than $100 \mathrm{mg}$ calcium per litre in soft water areas, to up to $300 \mathrm{mg}$ per litre in hard water areas. The calcium content of mineral water also varies widely $(<10 \mathrm{mg} / \mathrm{L}$ to $>300 \mathrm{mg} / \mathrm{L})$. 


\section{Recommended amounts}

Dietary Reference Values are based on calcium requirements for bone formation, minimised bone resorption and retention of calcium. Recommendations vary for different stages of growth and reproduction (COMA, 1998).

A Lower Reference Nutrient Intake (LRNI) of $240 \mathrm{mg} /$ day has been calculated for infants. However, absorption from infant formula is less than from breast milk (40\% as opposed to $66 \%$ ) and so a RNI of $525 \mathrm{mg} /$ day is recommended.

The calcium intake required for healthy bone formation and skeletal growth increases from the age of 1 to 10 years; RNIs of 350, 450 and $550 \mathrm{mg} /$ day are recommended for children of ages 1-3, 4-6 and 7-10 years respectively. For adolescents (11-18 years) RNIs are $800 \mathrm{mg} /$ day for females and $1000 \mathrm{mg} /$ day for males, due to an increased requirement for calcium at a time of increased skeletal growth.

RNIs for adults are based on calcium loss and retention and are $700 \mathrm{mg} /$ day. No additional intake is considered necessary for pregnancy, although an increase of $550 \mathrm{mg}$ /day over the RNI is recommended during lactation.

\section{Analysis of tissue levels and calcium status}

Calcium in the body may be measured in the blood or in bone. However, blood calcium concentrations are tightly regulated and are not reflective of calcium status. Blood calcium levels are only seen outside the normal range in conditions such as severe malnutrition or hyperparathyroidism.

There are several techniques for measuring the amount of calcium in individual bones at different ages, but results from the different methods do not correlate very well. A recently developed technique, using neutron activation analysis, enables total body calcium to be measured in living persons. Bone mineral content (BMC), the concentration of mineral at a specific skeletal site, and bone mineral density (BMD), the bone mineral content per unit area of skeletal site, are used as indicators of calcium insufficiency and as predictors of increased risk of fracture, when compared to a reference range, adjusted for age and gender. Change in BMC and BMD are useful indicators of calcium status in adults; change in $\mathrm{BMC}$ is a useful indicator of calcium retention in children.

\section{Brief overview of non-nutritional beneficial effects}

In some, but not all studies, calcium supplementation at doses of approximately $1250-2000 \mathrm{mg} /$ day, has been shown to reduce colonic and rectal epithelial cell proliferation in subjects at risk from developing colon cancer.

Epidemiological studies and clinical trials have suggested that dietary calcium may have a beneficial effect on primary hypertension (in non-pregnant women). Some studies have also indicated an association between calcium intake and decreased blood pressure in children.

Traditionally, calcium restriction has been advised in the prevention of calcium stones. However, recent evidence suggests that dietary calcium restriction may actually increase the risk. 
Evidence from a limited number of studies has suggested that calcium supplementation may inhibit the development of breast cancer, particularly in the presence of vitamin $D$.

\section{Function}

In the vertebrate skeleton, calcium provides rigidity in the form of calcium phosphate $\left(\mathrm{Ca}_{10}(\mathrm{OH})_{2}\left(\mathrm{PO}_{4}\right)_{6}\right.$, also known as hydroxyapatite), embedded in collagen fibrils. Calcium is also a key component in the maintenance of cell structure. Membrane rigidity, viscosity and permeability are partly dependent on local calcium concentrations.

Calcium fulfils important physiological roles as a cofactor for many enzymes (e.g. lipase), as an important component of the blood clotting mechanism and through an active role as an intracellular signal. Changes in intracellular calcium concentration, in response to a physiological stimulus, such as a hormone or neurotransmitter, can act as an intracellular signal. This signalling controls events such as cell aggregation, muscle contraction and cell movement, secretion, transformation and cell division, as well as muscle protein degradation.

\section{Deficiency}

A negative calcium balance occurs when net calcium absorption fails to compensate for urinary calcium losses. Calcium absorption is impaired in individuals with conditions of fat malabsorption (e.g. in syndromes such as pancreatic insufficiency, bile duct obstruction and coeliac disease) because of the lack of vitamin $D$ as a transporter. Acute hypocalcaemia is frequently seen following parathyroid surgery and may occur during thyroid surgery through inadvertent interference with the parathyroid glands.

The possible effects of calcium deficiency are numerous and wide-ranging. Signs of calcium deficiency are manifest in the bones and teeth of all young animal species, including humans. Effects include stunted growth, poor quality bones and teeth, and bone malformation.

\section{Interactions}

Interactions between calcium and other dietary constituents may affect the efficiency of calcium absorption. Phytic acid (found in unleavened bread, raw beans, seeds, nuts and grains) can reduce calcium absorption by forming an insoluble salt, calcium phytate.

Calcium is thought to have an inhibitory effect on iron absorption from the diet, even at low levels. High calcium diets have also been shown to reduce net zinc absorption and balance.

The phosphate ion can form insoluble complexes with calcium, and therefore potentially decrease calcium absorption. It has been suggested that the dietary phosphate:calcium ratio has to be very high (exceeding 3:1) to interfere significantly with calcium availability. However, some studies have shown that phosphorus intake had little or no effect on overall calcium balance, and that variations in phosphorus intake were not associated with differences in calcium absorption. Complexation of phosphorus with aluminium inhibits phosphorus absorption, which increases excretion of calcium. Thus, long-term use of antacids can cause calcium loss. 


\section{Absorption and bioavailability}

About $25-50 \%$ of dietary calcium is absorbed and delivered to the exchangeable calcium pool. Most of the calcium in food is in the form of complexes with other dietary constituents, which must be broken down and the calcium released in a soluble and ionised form before it can be absorbed.

Calcium crosses the intestinal mucosa by both active and passive transport mechanisms. The active transport mechanism is a saturable, transcellular process which involves the calcium-binding protein, calbindin. Calbindin is regulated by the active form of vitamin $D\left(1,25\right.$-dihydroxy-vitamin $\left.D_{3}\right)$. The passive transport mechanism is a nonsaturable, paracellular process, which is of low efficacy and is unaffected by calcium status or parathyroid hormone. Both processes occur throughout the small intestine, although the efficiency of calcium absorption is much greater from the duodenojejunal segments of the intestine, than from the ileal segments.

During periods of additional calcium requirement, the absorptive capacity of the gut is increased, and renal excretion is regulated.

\section{Distribution and metabolism}

Total body calcium is about $30 \mathrm{~mol}(1200 \mathrm{~g})$. Of this, $1 \%$ is located in the serum, lymph and other fluids, the remaining $99 \%$ being found in the bone and teeth. The concentration of ionised calcium in serum is closely regulated within $10 \%$ of approximately $100 \mathrm{mg} / \mathrm{L}$.

Calcium circulates in the plasma in three forms: bound to plasma proteins (45\%); in complexes with citrate, phosphate or bicarbonate (about 10\%); and as free calcium ions (about 45\%). Free ionised calcium is the most physiologically important form.

\section{Excretion}

Calcium excretion tends to equal intestinal calcium absorption in adults of a good nutritional state. Absorbed calcium is excreted in the urine, and to a lesser extent, in the sweat. Urinary calcium excretion varies widely among individuals, ranging from 100-200 mg/day. Faecal excretion of calcium consists mainly of unabsorbed dietary calcium, the remainder coming from shed epithelial cells and digestive juices. 


\section{Toxicity}

\section{Human data}

Excessive calcium intake may lead to hypercalcaemia (serum calcium levels in excess of $10.5 \mathrm{mg} / \mathrm{dl}$ ). Features of hypercalcaemia are progressive lethargy, confusion and ultimately coma (at serum calcium concentrations above $14 \mathrm{mg} / \mathrm{dL}$ ). These effects are reversible and are directly related to the degree of hypercalcaemia. Headache, elevated cerebrospinal fluid protein and, rarely, convulsions, may also occur in patients with hypercalcaemia. However, hypercalcaemia more commonly results from an excessive ingestion of both calcium and alkali, such as antacid tablets, calcium supplements and milk (providing vitamin D which promotes calcium absorption), a condition which is known as milk-alkali syndrome (MAS).

Clinical signs of MAS are hypercalcaemia, alkalosis and renal insufficiency. MAS can be acute, intermediate (Cope's syndrome) or chronic (Burnett's syndrome) depending on the duration of exposure and symptoms. Hypercalcaemia, hyperphosphatemia and renal insufficiency are reversible after acute or intermediate exposure. However, chronic MAS is associated with irreversible or only partially reversible renal insufficiency and metastatic calcinosis (deposition of calcium in soft tissue). Historically, the majority of patients developing MAS have been middle-aged males ingesting milk and absorbable alkali, but this has decreased with the use of modern medication for peptic ulcer disease. More recently, case reports have described the syndrome occurring in predominantly female patients taking calciumcontaining drugs for conditions such as autoimmune disease, organ transplantation, chronic renal failure and osteoporosis. The syndrome has been reported to occur at calcium carbonate intakes of $4000 \mathrm{mg} /$ day and above.

Although MAS has usually been observed after ingestion of large quantities of antacid tablets or other calcium-containing medication with vitamin D, two cases of MAS have been described resulting purely from ingestion of food. Both cases arose from the practice of consuming betelnut paste containing calcium carbonate from oyster shells.

Hypercalcaemia commonly occurs as a result of hyperparathyroidism and malignancy. Breast cancer, lung cancer, and multiple myeloma are the neoplasias most commonly associated with hypercalcaemia, due either to osteolytic secondary deposits or to ectopic parathyroid-like hormone production, typically in lung cancer.

\section{Supplementation studies}

A range of supplementation studies have been reported with supplementary calcium doses of up to $2000 \mathrm{mg} /$ day. These have generally failed to show adverse effects, but data on adverse effects are limited.

A study of supplementation of the diet of pregnant women with $2000 \mathrm{mg}$ calcium/day (as calcium carbonate) showed no significant increase in the incidence of urolithiasis. Women with a history of, or high risk for, developing renal disease were excluded from the study (Levine et al., 1997). 
In a randomised, double-blinded controlled clinical trial of pregnant adolescents, a reduced incidence of pre-term delivery and low birth weight was observed in the supplemented group receiving $2000 \mathrm{mg}$ additional calcium/day (Bucher et al., 1996). No adverse effects were reported.

Numerous clinical trials have been reported in which patients with a history of adenomatous colonic polyps have received calcium supplements. These trials mainly consist of double-blind placebocontrolled studies, with patients receiving between 250 and $2000 \mathrm{mg}$ calcium (usually in the form of calcium carbonate), over periods ranging from 4 weeks to 4 years. Few adverse effects have been reported in individuals participating in such trials, although this may be partly because the effects were not deliberately sought. A low incidence of gastrointestinal side effects, such as severe abdominal pain or diarrhoea, has been observed in studies where side effects have been actively ascertained.

\section{Animal data}

Limited animal data concerning calcium toxicity are available. In rodents and dogs, dietary administration of excess calcium leads to kidney calcification, enlarged kidneys and reduced glomerular filtration rate.

An investigation of the effect of providing pregnant mice with $8.2 \%$ calcium (as carbonate and lactate) in the diet (equivalent to an intake of approximately $12,200 \mathrm{mg} / \mathrm{kg}$ bw/day) compared to controls given diets containing $1.2 \%$ calcium (approximately $1800 \mathrm{mg} / \mathrm{kg}$ bw/day) on foetal development resulted in significantly decreased foetal weights, and retarded skeletal and dental calcification. No gross abnormalities were detected.

In rats maintained on a high calcium diet throughout pregnancy and lactation (providing an estimated calcium intake of $3790 \mathrm{mg} / \mathrm{kg}$ bw/day), offspring were born significantly hypocalcaemic and had lower growth rates and focal alopecia. These effects were reversible when the pups were weaned onto a normal diet. Hypercalcaemic lactating dams also produced milk with a higher calcium concentration than controls, which may have contributed to the response seen in the offspring.

\section{Carcinogenicity and genotoxicity}

No relevant data on carcinogenicity or genotoxicity have been identified.

\section{Mechanisms of toxicity}

Acute hypercalcaemia can impair renal function by causing vasoconstriction with consequent decreases in both the renal blood flow and glomerular filtration rate. Hypercalcaemia increases absorption of bicarbonate in the proximal tubule, thus predisposing the patient to metabolic alkalosis. Chronic hypercalcaemia, hyperphosphataemia and metabolic alkalosis promote irreversible renal calcification.

\section{Dose response characterisation}

No relevant data are available. 


\section{Vulnerable groups}

Patients with renal failure are particularly susceptible to developing hypercalcaemia when taking calcium supplements. Individuals without renal failure taking diuretics may also be at increased risk.

Patients with absorptive or renal hypercalcuria, primary hyperparathyroidism and sarcoidosis may have a higher risk of renal stone formation following calcium supplementation.

\section{Genetic variations}

No data on genetic variations that increase the toxicity of calcium have been identified.

\section{Studies of particular importance in the risk assessment}

(For full review see http://www.food.gov.uk/science/ouradvisors/vitandmin/evmpapers or the enclosed CD).

\section{Human data}

\section{Kleibeuker et al., 1993}

Calcium carbonate tablets were well tolerated by participants in this intervention study. No mention of side effects was made when individuals were specifically asked about them. Individuals received 1500 $\mathrm{mg}$ calcium/day for twelve weeks.

Wu et al., 1996

This paper reports two cases of milk-alkali syndrome. Both cases showed classic MAS symptoms, i.e. hypercalcaemia and renal insufficiency. The authors estimated that the patients had ingested $9000 \mathrm{mg}$ and $6000 \mathrm{mg}$ of calcium carbonate (3600 and $2400 \mathrm{mg}$ calcium) per day, respectively. These are the first reports of MAS not caused iatrogenically.

Levine et al., 1997

Supplementation of 2,295 pregnant women with 2,000 mg calcium/day (as calcium carbonate) resulted in no significant increase in the incidence of urolithiasis. This study excluded women with a history of, or a high risk of, developing renal disease.

\section{Hofstad et al., 1998}

116 patients received 1,600 mg calcium/day (as calcium carbonate) or placebo for 18 months. Five patients in the treatment group experienced constipation compared to one in the controls. Diarrhoea was reported in 5 calcium and 7 placebo subjects and bloating (8 patients) was equally distributed between groups.

Baron et al., 1999

No toxicity was associated with calcium supplementation during a randomised double blind, placebo controlled trial on patients with a history of colorectal adenomas. The 930 received $3000 \mathrm{mg}$ of calcium carbonate/day (equivalent to 1,200 mg calcium per day) and were examined after 1 and 4 years of treatment. 
Bonithon-Kopp et al., 2000

665 adult patients with a history of polyps were randomised into 3 groups receiving $2000 \mathrm{mg}$ calcium/day (as calcium gluconolactate and carbonate) fibre or placebo for 3 years. 552 patients completed the follow up examination after 3 years, although 94 of these had stopped treatment early. The total number of patients reporting side effects were 26/176, 19/198 and 12/178 in the calcium, fibre and control groups, respectively. Major side effects (severe abdominal pain or diarrhoea) were also reported to be more frequent $(6 / 176)$ in the calcium group compared to the control $(3 / 178)$ or fibre $(3 / 198)$ groups, which might have been a chance finding.

\section{Animal data}

\section{Fairney and Weir, 1970}

The effect of maternal hypercalcaemia during pregnancy and lactation on the development of the offspring was investigated. Rats were maintained on high calcium diets, containing $3 \%$ calcium in diet and $4000 \mathrm{mg} / 100 \mathrm{~mL}$ drinking water (equating to an estimated total intake of $3790 \mathrm{mg} /$ day), throughout pregnancy and lactation. In comparison to controls (receiving $0.8 \%$ calcium in diet and $1.1 \mathrm{mg}$ calcium $/ 100 \mathrm{~mL}$ drinking water), the offspring of treated rats were born with significant hypocalcaemia and had lower growth rates and focal alopecia. These effects were reversible when the pups were weaned onto a normal diet.

Shackelford et al., 1993

The effect of moderately increased dietary calcium on foetal development was investigated in pregnant rats. The doses were selected to resemble the increases recommended by the 1984 US National Institutes of Health Consensus Development Conference Panel on Osteoporosis. All animals were fed nutritionally adequate diets and received 0.5 (control), $0.75,1.00$ or $1.25 \%$ dietary calcium as calcium carbonate. Treatment was for 6 weeks prior to mating, during mating and for the first 20 days of gestation. Foetal bodyweights and lengths remained similar between treatment and control groups. There were no significant increases in external, visceral or skeletal variations of the foetuses, when compared to the control animals.

\section{Exposure assessment}

Total exposure/intake:

Food Mean: $830 \mathrm{mg} /$ day (from 1990 NDNS)

97.5th percentile: $1500 \mathrm{mg} /$ day

Water up to $600 \mathrm{mg} /$ day (assuming $2 \mathrm{~L} /$ day consumption at $300 \mathrm{mg} / \mathrm{L}$ )

Supplements $\quad$ up to $2400(3 \times 800) \mathrm{mg} /$ day $($ OTC, 2001)

Estimated maximum intake: $1500+600+2400=4500 \mathrm{mg} /$ day

No potential high intake groups were identified. 


\section{Risk assessment}

In humans, the main adverse effect associated with high levels of calcium intake is milk-alkali syndrome, resulting in hypercalcaemia, alkalosis and renal impairment. Symptoms can include abdominal pain, hypertension, headaches and tissue calcification. The condition has been reported in a small number of subjects taking calcium-containing medication. Previously, MAS was more common in males taking absorbable alkali and milk, but is now more common in females taking calcium-containing medication.

Data on the effects of high doses of calcium in animals are sparse. However tissue calcification and adverse reproductive effects have been reported.

\section{ESTABLISHMENT OF GUIDANCE LEVEL}

There are insufficient data from studies in animals or humans to establish a Safe Upper Level for calcium. High intakes of calcium in vulnerable human subjects can result in milk-alkali syndrome, a condition characterised by hypercalcaemia, alkalosis and renal impairment. This is associated with symptoms such as hypertension, neurological problems, abdominal pain and tissue calcification. Milkalkali syndrome has not been investigated in controlled studies, but numerous case reports are available. The condition tends to occur as a result of consumption of large amounts of calcium via calciumcontaining medication with or without vitamin D, with intakes of calcium carbonate as low as $4,000 \mathrm{mg} /$ day (1,600 mg calcium) having been associated with this condition. However, since milk-alkali syndrome generally occurs in subjects taking medication or with underlying medical conditions, it is not considered relevant to the general population.

Numerous human trials have been reported in which calcium supplements have been given to subjects with colonic polyps or who are at risk of such polyps. Some investigations have reported gastrointestinal effects in small numbers of the many patients studied taking doses of 1600 or $2000 \mathrm{mg} /$ day supplemental calcium, but many of the studies are limited by inadequate reporting of side effects. No uncertainty factor is needed because the assessment is based on studies of large numbers of people. Taken together the data suggest that, for guidance purposes, doses up to 1,500 mg/day supplemental calcium would not be expected to result in any adverse effect, but that higher doses could result in adverse gastrointestinal symptoms in a few people. An estimate for total calcium intakes has not been made as the effect is related to calcium in supplemental doses. 


\section{References}

Baron, J. A., Beach, M., Mandel, J.S., Van Stolk, R.U., Haile, R.W., Sandler, R.S., Rothstein, R., Summers, R.W., Snover, D.C., Beck, G.J., Bond, J.H., Greenberg, E.R. (1999) Calcium supplements for the prevention of colorectal adenomas. New England Journal of Medicine 340, 101-107.

Bonithon-Kopp, C., Kronborg, O., Giacosa, A., Räthe, U., Faivre, J. (2000) Calcium and fibre supplementation in prevention of colorectal adenoma recurrence: a randomised intervention trial. Lancet 356, 1300-06.

Bucher, H.C., Guyatt, G.H., Cook, R.J., Hatala, R., Cook, D.J., Lang, J.D. and Hunt, D. (1996). Effect of calcium supplementation on pregnancy-induced hypertension and pre-eclampsia. Journal of the American Medical Association 275, 1113-1117.

COMA (1998). Nutrition and Bone Health: with particular reference to calcium and vitamin D. Report of the Subgroup on Bone Health, Working Group on the Nutritional Status of the Population, Committee on Medical Aspects of Food and Nutrition Policy. The Stationery Office, London.

Fairney, A. and Weir, A.A. (1970) The effect of abnormal maternal plasma calcium levels on the offspring of rats. Journal of Endocrinology 48, 337-345.

Hofstad, B., Vatn, M.H., Anderson, S.N., Andersen, S.N., Owen, R.W., Larsen, S., Osnes, M. (1998) The relationship between faecal bile acid profile with or without supplementation with calcium and antioxidants on recurrence and growth of colorectal polyps. European Journal of Cancer Prevention 7 , 287-294.

Kleibeuker, J.H., Welberg, J.W.M., Mulder, N.H., van der Meer, R., Cats, A., Limburg, A.J., Kreumer, W.M.T., Hardonk, M.J., de Vries, E.G.E. (1993) Epithelial cell proliferation in the sigmoid colon of patients with adenomatous polyps increases during oral calcium supplementation. British Journal of Cancer 67, 500-503.

Levine, R.J., Hauth, J.C., Curet, L.B. Sibai B.M., Catalano P.M., Morris C.D., DerSimonian R., Erterlitz J.R., Raymond E.G., Bild D.E., Clements J.D. and Cutler J.A. (1997) Trial of calcium to prevent eclampsia. New England Journal of Medicine 337, 69-76.

OTC (2001). OTC Directory 2001-2002, Proprietary Association of Great Britain.

Shackelford, M.E., Collins, T.F.X., Welsh, J.J., Black T.N., Ames M.J., Chi R.K. O’Donell M.W. (1993) Foetal development in rats fed ain-76a diets supplemented with excess calcium. Food and Chemical Toxicology 31, 953-961.

Wu K. D., Chuang, R.-B., Wu, F.-L., Jan, I-S, Tsai, K-S. (1996) The milk-alkali syndrome caused by betelnuts in oyster shell paste. Clinical Toxicology 34, 741-745. 


\section{EVM Statement}

\section{Fluoride}

At the start of its task, the EVM drew up a list of vitamins and minerals to be considered. These were vitamins and minerals which were either generally considered to be essential and/or which were available as food supplements. Fluoride is thought to be essential, though this has been difficult to demonstrate experimentally. Although fluoride supplements are only available as licensed medicines, fluoride was included in the EVM programme as it has been used to fortify particular foodstuffs on a local basis in order to improve dental health.

Two of the major sources of exposure (drinking water and dental products, the latter being considered either licensed medicines or cosmetics) are neither foods nor food supplements.

The EVM has concluded that it is inappropriate to comment on fluoride with regard to food fortification since this is carried out as a public health measure. Determining maximum levels of supplementation has to take place within the context of local exposure and involves a consideration of risks and benefits which is not within the terms of reference of the EVM.

The review commissioned by the FSA for the EVM will remain available for information purposes on the EVM website or from the Secretariat. This should not be taken to mean that the EVM has endorsed or approved this review. 


\section{Risk Assessment}

\section{Iron}

\section{General information}

\section{Chemistry}

Iron, a transition metal, is ubiquitous in biological systems. In solid form the element exists free, or in iron-containing compounds. In aqueous solution, it exists in one of two oxidation states, $\mathrm{Fe}^{2+}$, the ferrous form, and $\mathrm{Fe}^{3+}$, the ferric form. Iron has a particularly high redox potential in solution. Within this risk assessment, the word iron refers to ionic iron, except where specific iron compounds are mentioned.

\section{Natural occurrence}

Iron is found in certain minerals, and in nearly all soils and in mineral waters

\section{Occurrence in food, food supplements and medicines}

Dietary sources rich in iron include liver, meat, beans, nuts, dried fruits, poultry, fish, whole grains or enriched cereals, soybean flour and most dark green leafy vegetables. Iron in foods occurs in two main forms: haem and non-haem. The major sources of haem iron in the diet are haemoglobin and myoglobin from meat, poultry and fish. Non-haem iron consists mainly of iron salts, derived from plant and dairy products. Most of the non-haem iron present as foods is in the ferric form.

Fortification of food with iron is common in developing countries, where deficiency of the element is widespread. In the UK there is mandatory fortification of white and brown flour at a level not less than $16.5 \mathrm{mg}$ iron $/ \mathrm{kg}$ flour. Many breakfast cereals are fortified on a voluntary basis; levels vary but are typically within the range of 70 to $120 \mathrm{mg} / \mathrm{kg}$.

Inorganic dietary iron supplements are generally available as ferrous salts (chloride, fumarate, gluconate, glycerophosphate, succinate, sulphate), which are more readily absorbed than ferric salts. Ferrous sulphate and succinate are the most commonly available.

\section{Other sources of exposure}

Water is a potential source of iron. The limit in UK water supplies is $0.2 \mathrm{mg} / \mathrm{L}$.

\section{Recommended amounts}

Estimated average daily iron requirements in the UK are 8.7 and $6.7 \mathrm{mg}$ for males aged 11-18 and 19+ years, respectively (COMA, 1991). For women in the 11-50 years age group the estimated average daily iron requirement is $11.4 \mathrm{mg}$, whilst that for postmenopausal (50+ years) women is $6.7 \mathrm{mg}$. Estimated average daily requirements for children are $1.3 \mathrm{mg}$ (0-3 months), $3.3 \mathrm{mg}$ (4-6 months), $6.0 \mathrm{mg}$ (7-12 months), $5.3 \mathrm{mg}$ (1-3 years), $4.7 \mathrm{mg}$ ( $4-6$ years) and $6.7 \mathrm{mg}$ (7-10 years). It has been estimated that the total 
amounts of iron required for a full gestation is $680 \mathrm{mg}$. Existing body iron stores should provide this requirement, assuming adequate iron stores at conception, cessation of menstruation and increased intestinal absorption throughout gestation.

\section{Analysis of tissue levels and iron status}

A number of haematological and biochemical tests may be used to characterise iron nutritional status. Serum ferritin, serum iron concentration, total iron binding capacity (TIBC) and transferrin saturation (Fe/TIBC) indicate the level of iron supply to the tissues. Transferrin receptors are expressed on cell surfaces in proportion to the cells requirement for iron. As functional iron depletion occurs, more transferrin receptors appear on the cell surfaces. The concentration of cleaved extracellular domains, or soluble serum transferrin receptors, rises in parallel. Elevated serum ferritin and transferrin saturation are useful as indicators of iron overload. In healthy subjects, an accurate determination of iron status can be made using combined measures of serum ferritin, transferrin saturation and erythrocyte protoporphyrin.

\section{Brief overview of non-nutritional beneficial effects}

No relevant data have been identified.

\section{Function}

The majority of functional iron within the body is present in haem proteins, such as haemoglobin, myoglobin and cytochromes, which are involved in oxygen transport or mitochondrial electron transfer. Many other enzymes also contain or require iron for their biological function.

Total body iron averages approximately $3800 \mathrm{mg}$ in men and $2300 \mathrm{mg}$ in women. Approximately one third of body iron in men and one eighth in women is in the form of storage iron. Iron is stored mainly in the liver within the iron storage proteins, ferritin and haemosiderin. Small amounts of ferritin are also present in the plasma, although there are a number of differences from tissue ferritin and plasma has a low iron content, even in iron-overloaded individuals.

Many of the key biological functions of iron in living systems rely on the high redox potential, enabling rapid conversion between the $\mathrm{Fe}^{2+}$ and $\mathrm{Fe}^{3+}$ forms. The redox potential is, however, also potentially harmful in terms of the capacity for oxidative damage to cellular components such as fatty acids, proteins and nucleic acids. However, iron within the body (whether it is being stored, transported or as a component of various catalytic pathways) is normally bound to carrier proteins and/or molecules with antioxidant properties, which minimise the capacity of the free ion to cause oxidative stress.

\section{Deficiency}

Iron deficiency generally develops slowly, and may not be clinically apparent until iron stores are exhausted and the supply of iron to the tissues is compromised, resulting in iron-deficiency anaemia. Groups that are vulnerable to iron deficiency include: infants over 6 months, toddlers, adolescents and pregnant women (due to high requirements); older people and people consuming foods high in iron absorption inhibitors (due to poor absorption); menstruating women or individuals with pathological blood loss (due to high blood losses). 


\section{Interactions}

Interactions may occur between iron and other metals close to iron in the periodic table, such as copper, manganese, zinc and chromium. Studies in rats have shown that iron supplementation impairs the absorption of zinc, and this has raised concerns that iron supplements may have adverse effects on zinc nutrition in humans. However, the magnitude of such an effect is less apparent in human studies than in rats. In general, calcium inhibits the absorption of iron. This may be relevant in consumption of iron supplements with milk or calcium-rich foods.

\section{Absorption and bioavailability}

Modulation of absorption of iron from the gastrointestinal tract is the primary mechanism for regulation of body iron levels. The amount of iron absorbed from the diet can vary widely and depends on body iron stores and physiological requirement (generally, the rate of erythrocyte production).

Absorption of haem and non-haem iron involves different mechanisms. In general haem iron uptake, which is via a specific haem receptor, occurs approximately 2- to 3-fold more extensively than that of non-haem iron and is largely independent of other dietary components. The mechanism by which nonhaem iron enters intestinal mucosal cells is not clearly established, although there appear to be separate mechanisms for the uptake of ferrous and ferric iron. Uptake of non-haem iron depends initially on a low pH to effect solubilisation. Iron chelators, such as ascorbic acid, increase absorption by maintaining iron in solution. In the absence of chelators, ferric iron is generally less well absorbed than ferrous iron, due to its low solubility at higher $\mathrm{pH}$. Dietary supplements are mostly inorganic salts. Iron supplements are also available in the form of the iron protein complex, ferritin, but poor absorption is reported.

Iron absorption from a diverse diet has been estimated to be approximately $15 \%$. Women and children generally have lower iron stores than men, and thus absorb a greater percentage of the amount ingested. This is particularly pronounced during pregnancy with absorption of dietary iron increasing throughout gestation. Conversely, absorption is lower in postmenopausal women, in whom iron stores are generally high.

\section{Distribution and metabolism}

Iron is transported by the plasma transport protein, transferrin. In healthy adults approximately onethird of the total iron binding capacity is saturated. In conditions of iron overload or atransferrinaemia, non-protein-associated iron may also be detected in the plasma. Turnover of the total plasma iron pool (approximately $3 \mathrm{mg}$ ) is more than 10 -fold every day. Approximately $80 \%$ of iron leaving the plasma is delivered to erythroid bone marrow. Iron in circulating erythrocytes is returned to plasma transferrin by means of reticuloendothelial cell phagocytosis.

Iron uptake by cells (other than during absorption from the intestinal lumen) occurs via binding of transferrin to the transferrin receptor, which is subsequently internalised within an endocytic vesicle. Recent studies have identified a number of novel proteins which are also likely to be involved in iron transport into and within cells, although the function of these proteins in iron transport has yet to be determined. 


\section{Excretion}

Little of the absorbed iron is excreted. Very small losses occur in the faeces, by desquamation of gastrointestinal cells, in haemoglobin and bile, and via the urine. Substantial iron loss can occur through loss of blood. Average, total daily iron losses for healthy adults are $1.0 \mathrm{mg}$ for men and $1.3 \mathrm{mg}$ for premenopausal women (assuming an average blood loss of $30-40 \mathrm{~mL}$ per menstrual cycle). Daily iron losses for children have not been measured directly but are estimated as 0.2 and $0.5 \mathrm{mg}$ for infants and children aged $6-11$ years, respectively.

\section{Toxicity}

\section{Human data}

Acute and sub-chronic toxicity

Most cases of acute iron poisoning occur in children, due to accidental ingestion of iron supplements intended for adults. The acute toxic dose of iron in infants is considered to be approximately $20 \mathrm{mg} / \mathrm{kg}$ bw, associated with gastrointestinal irritation, whilst systemic effects do not generally occur at doses < $60 \mathrm{mg} / \mathrm{kg}$ bw. The lethal dose in children is approximately $200-300 \mathrm{mg} / \mathrm{kg}$ bw. Iron poisoning in adults is rare. Individual case reports suggest that a dose of approximately $100 \mathrm{~g}$ (approximately 1400 $\mathrm{mg} / \mathrm{kg} \mathrm{bw}$ ) iron is lethal, although survival may occur with treatment.

High doses of iron supplements are frequently associated with gastrointestinal effects, especially constipation, but also with nausea, diarrhoea and vomiting. The severity and occurrence of the effect depends upon the formulation of the supplement and the amount of iron released in the stomach.

Severe gastrointestinal damage has been described following iron overdose. Reports of mucosal injury resulting from iron tablet ingestion at therapeutic levels are rare. Very few systematic studies of the potential of therapeutic doses of iron to cause or promote gastric or oesophageal ulceration have been conducted. The limited data available suggest that tissue damage due to iron tablets can be demonstrated histopathologically. It is unclear whether therapeutic doses of iron are likely to initiate gastrointestinal damage.

\section{Chronic toxicity and carcinogenicity}

Chronic iron overload may result from parenteral administration (as therapeutic iron or blood transfusions). Increased oral iron intake does not generally result in significant iron overload unless the iron is in a highly bioavailable form or there is an accompanying genetic defect, or increased demand (e.g. anaemia), causing increased iron absorption. 'Generalised iron overload' has been arbitrarily defined as an excess total body iron of more than $5 \mathrm{~g}$ in adults, and 'severe iron overload' as an excess of $10 \mathrm{~g}$ or more, associated with iron-induced tissue damage, including cirrhosis of the liver and impaired heart and endocrine function. The term haemochromatosis, previously used to describe massive iron overload associated with iron-induced tissue damage, is now usually restricted to the primary, geneticallydetermined disturbance of iron metabolism, hereditary haemochromatosis ( $\mathrm{HHC})$, an autosomal recessive disorder, associated with homozygosity for a specific mutation (Cys282Tyr) of the HLA-linked, HFE gene. The overall prevalence of HHC in Caucasian populations is approximately $1 / 250$ with an estimated carrier frequency of approximately 0.1. The disorder is characterised by unregulated dietary 
iron absorption, excess to bodily requirement, leading to accumulation of excess iron in the parenchymal cells of the major organs of the body, primarily the liver, pancreas and heart. This results in irreversible tissue damage, with clinical disease (cirrhosis and hepatocellular carcinoma, diabetes and heart failure) being manifested in middle age. Heterozygotes for HHC may show mildly increased signs of iron storage, but significant iron loading does not occur in the absence of other disorders related to excess iron-loading.

Secondary iron overload is defined as a quantitative increase in total body iron that is not the result of a genetically determined increase in iron absorption. The iron originates from either parenteral administration and/or increased absorption. Parenteral iron is initially cleared by the macrophages of the reticuloendothelial system, whilst iron absorbed from the gut may be taken up directly by hepatocytes. Pathology is most severe when there is both parenteral administration and increased absorption, as often occurs, for example, in the chronic inherited anaemia syndromes, ß-thalassaemia and sideroblastic anaemia. In such cases iron chelation therapy is required to prevent the development of severe pathology and early death.

Many epidemiological studies have found associations between markers of high body iron status and increased risk of either cardiovascular disease or cancer in the general population. Interpretation of such studies is difficult because the biochemical indices used to assess body iron status may have been altered by the existence of chronic disease. The results of epidemiological studies suggesting that individuals heterozygous for $\mathrm{HHC}$ show increased risk of chronic disease, particularly cardiovascular disease, are also controversial.

Iron supplementation is common during pregnancy, generally during the 2nd and 3rd trimesters (at levels up to approximately $200 \mathrm{mg} /$ day), with no reports of adverse effects other than gastrointestinal irritation. The relatively small number of reports that are available suggest that acute iron overdose during pregnancy is not associated with substantially increased foetal iron levels.

\section{Supplementation studies}

A large number of studies involving oral iron supplementation have been reported. Analysis of all reports was not possible, but was limited to those identified by database search as randomised, controlled trials. Many of these studies were carried out with the aim to improve iron status in population groups who were known or likely to be at risk of iron deficiency, such as pre-school children, juveniles, adolescent girls and pregnant and non-pregnant women, often in developing countries where such deficiency is common. Apart from gastrointestinal side effects, such studies have generally not addressed issues of potential adverse effects associated with oral iron supplementation.

Supplementary doses of 100-200 mg iron/day and above have been associated with nausea, vomiting and epigastric pain (Ganzoni et al., 1974; Hallberg et al., 1966b; Reddaiah et al. 1989). Other studies have reported a range of gastrointestinal effects, including diarrhoea, nausea, vomiting, constipation and epigastric pain, following supplementary doses of between 50 and $220 \mathrm{mg} /$ day (Hallberg et al., 1966a; Lokken and Birkland, 1970; Blot et al., 1981; Brock et al., 1985; Coplin et al., 1991; Liguori, 1993; Frykman et al., 1994). However, such effects were variable and appeared to vary depending on the formulation of the iron supplement given, with fewer adverse effects reported by subjects given supplementary iron as chelated iron or haem iron than by subjects given ferrous sulphate.

Several studies have suggested that excess iron intake could result in decreased serum zinc levels. Decreased maternal serum zinc concentrations were reported following iron supplementation at doses 
of more than $60 \mathrm{mg} /$ day during pregnancy (Breskin et al., 1983; Hambidge et al., 1983, 1987) and Dawson et al. (1989) reported that daily supplementation of pregnant women with $18 \mathrm{mg}$ iron, in combination with a multivitamin supplement (on average from 13 weeks of gestation to term), resulted in a significant decrease in serum zinc level in the third trimester, compared with women who were given an equivalent multivitamin supplement without iron. Conversely, Sheldon et al. (1985) reported that supplementation with $480 \mathrm{mg} /$ day ferrous fumarate (160 mg/day elemental iron), from the first or second trimester of pregnancy to term, had no effect on maternal serum zinc concentrations. As plasma zinc levels are not considered to be a good index of body zinc status the significance of these findings is unclear.

One study found an association between supplementation with ferrous sulphate (equivalent to $60 \mathrm{mg}$ elemental iron/day) during pregnancy and reduced birth weight in women who were carriers for the sickle cell anaemia genotype (Menendez et al., 1995).

\section{Animal data}

\section{Acute toxicity}

Oral lethal doses of 675 and $1230 \mathrm{mg} / \mathrm{kg}$ bw were reported for ferrous sulphate and phospholipidmicroencapsulated ferrous sulphate, respectively, in Swiss strain mice. An acute oral $\mathrm{LD}_{50}$ value of $2800 \mathrm{mg} / \mathrm{kg}$ bw (560 mg/kg bw iron) was reported for ferrous bisglycinate chelate (Ferrochel) in Sprague-Dawley rats.

\section{Sub-acute, sub-chronic and chronic toxicity}

Loading with high doses of carbonyl iron, a form of iron with higher bioavailability than other forms, has been used most to assess the effects of dietary iron overload in experimental animals. Chronic, highdose supplementation in rats and mice results in rapid hepatic iron deposition, with a pattern similar to that seen in human HHC, and associated with cellular changes but not, in the majority of studies, with the development of fibrosis or cirrhosis. The main targets, in addition to the liver, were the heart, pancreas and the spleen.

A number of genetic mouse models have also been developed to mimic dietary iron overload in humans. The haemochromatosis (HFE -/-) gene-knockout mouse exhibits a pattern of iron overload similar to that seen in human HHC, and develops even greater levels of iron overload in association with high-dose dietary iron supplementation. These mice may, thus, provide a good model for future studies of iron overload in humans with HHC.

\section{Reproductive and developmental toxicity}

A multigeneration study in rats showed no adverse effects of $20 \mathrm{mg} / \mathrm{kg}$ bw/week maternal iron supplementation (by intramuscular injection, but not during pregnancy) on the numbers of offspring produced or their growth weights, with no significant evidence of excess iron transfer across the placenta. A study of maternal iron poisoning in an ovine model also showed that extremely elevated maternal serum iron concentrations were not accompanied by corresponding increases in foetal serum iron levels.

One study showed that iron gluconate was teratogenic after intraperitoneal administration to pregnant mice on the 8th and 9th days of gestation, the most pronounced defect being exencephaly. 


\section{Carcinogenicity and genotoxicity}

A number of studies have shown that supplementary iron added to the diet enhances the development of neoplasia in animals that produce spontaneous tumours, are inoculated with tumour cells, or are exposed to chemical carcinogens. However, high-level (1200 - $1500 \mathrm{mg} / \mathrm{kg}$ bw/day) dietary carbonyliron supplementation had no effect on the initiation or promotion of hepatocarcinoma in the SoltFarber model of hepatocarcinogenesis in rats. There appear to have been relatively few studies carried out to assess the effects of dietary iron overload, in the absence of chemical carcinogens, on tumourigenesis in experimental animals. Supplementation of the diet with $80 \mathrm{mg} / \mathrm{kg} \mathrm{bw} /$ day iron in mice fed a high-fat diet was associated with increased mitotic and labelling indices in colonic crypts, but low-level dietary iron supplementation in rats (approximately $5.1 \mathrm{mg} / \mathrm{kg}$ bw/day iron, for 5 days) was associated with a significantly increased frequency of colonic crypt cell mitoses. Supplementation of BALB/C) mice with levels of carbonyl-iron up to $4,500 \mathrm{mg} / \mathrm{kg}$ bw/day, for periods of up to 12 months, was not associated with the development of hepatic fibrosis or hepatocellular carcinoma, although there was evidence of nuclear changes in hepatocytes in the animals receiving the highest dose supplement for 12 months.

The majority of ferric and ferrous iron salts that have been assessed, and also carbonyl iron, have produced negative results in gene mutation assays, but were positive for viral-enhanced cell transformation in Syrian hamster embryo cells, in vitro, or in a mouse lymphoma assay.

\section{Mechanisms of toxicity}

In the presence of available cellular reductants, iron may act as a catalyst in the initiation of free radicalmediated reactions. The resultant oxyradicals or lipid hydroperoxides have the potential to damage a variety of cellular structures, including lipids in organelle membranes, nucleic acids, proteins, and carbohydrates, which could result in the disruption of numerous cellular functions. However, the relationship of such effects to the progressive fibrosis associated with chronic iron overload in humans is currently unclear.

\section{Dose response characterisation}

Although there are many studies on iron in humans, there is no relevant information on the dose response because the studies are correcting deficiency and frequently only test one dose level. With regards to gastrointestinal effects in iron-replete subjects, the form of the iron affects the findings and therefore it is hard to compare doses.

The animal database is too limited to draw any conclusions regarding dose response.

\section{Vulnerable groups}

Individuals with pre-existing gastrointestinal tract disease or chronic hepatitis, have been shown to be vulnerable to the toxic effects of iron. 


\section{Genetic variations}

Several inherited diseases, for example, hereditary haemochromatosis (HHC), are associated with increased iron absorption. Hereditary anaemias, such as thalassaemia or sideroblastic anaemia, frequently require treatment by repeated blood transfusions, which may result in iron overload and toxicity.

\section{Studies of particular importance to the risk assessment}

(For full review see http://www.food.gov.uk/science/ouradvisors/vitandmin/evmpapers or the enclosed CD)

Blot et al., 1981

In this study 132 pregnant women were given $105 \mathrm{mg}$ iron (form not stated) plus $500 \mathrm{mg}$ ascorbic acid, with or without $350 \mathrm{mg}$ folic acid for 90 days. $14 \%$ of subjects reported severe gastrointestinal effects (diarrhoea or constipation) and 16\% minor effects. There was no difference in incidence between the groups, but there was no placebo group.

Brock et al., 1985

The tolerability of supplemental iron delivered from a wax-matrix tablet of ferrous sulphate was compared to that from a conventional ferrous sulphate tablet in a single blind, parallel group study. Both tablets delivered $50 \mathrm{mg}$ of iron. The incidence of adverse gastrointestinal effects was significantly greater amongst subjects taking the conventional tablets, than amongst those taking the wax-matrix preparation. Of those taking the wax-matrix formulation only $19 \%$ experienced a severe or moderate adverse effect (306 reports), compared to 50\% (1021 reports) of those taking the conventional tablets.

Coplin et al., 1991

The tolerability of supplemental iron (50 mg iron) in the chelated form of bis-glycino iron was compared with that of ferrous sulphate in a randomised double-blind cross-over trial. Of the 38 participants, $37 \%$ experienced moderate to severe adverse gastrointestinal effects whilst taking ferrous sulphate, compared to $21 \%$ who experienced similar side effects whilst taking the chelate formulation.

\section{Frykman et al., 1994}

In a controlled double-blind cross-over trial, haem and non-haem iron were administered to 100 healthy volunteers for periods of 1 month each. Groups of participants were given one of two different iron preparations: Group 1: two tablets of $1.2 \mathrm{mg}$ haem iron from porcine blood, plus $8 \mathrm{mg}$ non-haem iron as iron fumarate. Group 2: one tablet of $60 \mathrm{mg}$ iron as iron fumarate. The study was divided into three consecutive periods of one month each and all participants received a placebo for one of the last two periods. Participants assessed side effects by keeping individual symptom diaries, a multiple-choice questionnaire was used for daily evaluation with listing of effects known to be related to iron therapy. The number of reports of obstipation and the total side effects, were significantly higher for the nonhaem iron treatment, than for the haem iron or placebo. Constipation was reported in $35 \%$ and all gastrointestinal side effects were reported by $25 \%$ of participants during the non-haem iron phase of the trial. The effects reported for the haem iron treatment were indistinguishable from the placebo. 
Liguori, 1993

In a prospective, controlled, double-blind, two-placebo controlled, multicentre trial the tolerability of ironproteinsuccinylate (ITF 282) was compared with a ferrous sulphate controlled release tablet. 1095 patients were randomised to receive either two ITF 282 tablets per day (60 mg iron per tablet) or one controlled release ferrous sulphate tablet per day, containing $105 \mathrm{mg}$ iron, both treatments lasted 60 days. The general tolerability was reported to be favourable with both treatments, but significantly more favourable with ITF 282. With ITF 282, 63 patients (11.5\%) complained of 69 adverse reactions (25 heartburn, 19 constipation, 25 abdominal pain), compared with 141 adverse events ( 33 heartburn, 31 epigastric pain, 23 constipation, 32 abdominal pain, 8 skin rash, 14 nausea) reported by 127 (26.5\%) of those taking ferrous sulphate.

\section{Lokken and Birkelan, 1979}

In a randomised, double-blind crossover study, 19 young women received a dose of $120 \mathrm{mg} /$ day $(2 \times 60)$ iron as ferrous fumarate or placebo for 2 periods of 8 weeks. Seven participants experienced gastrointestinal discomfort, 2 while taking placebo.

\section{Exposure assessment}

Total exposure/intake:

Food

Mean: 12 mg /day

97.5th percentile: $24 \mathrm{mg} /$ day (from 1986/87 NDNS)

Water

$0.4 \mathrm{mg} /$ day (assuming $2 \mathrm{~L} /$ day at UK limit of $0.2 \mathrm{mg} / \mathrm{L}$ )

Supplements

$20 \mathrm{mg} /$ day (up to $60 \mathrm{mg} /$ day for particular conditions, e.g. pregnancy) (Annex 4)

Estimated maximum intake: $24+0.4+20 \mathrm{mg}=44 \mathrm{mg} /$ day

\section{Risk assessment}

In humans acute iron poisoning is associated with severe gastrointestinal damage which may include haemorrhagic gastroenteritis. Blood and other fluid loss may lead to shock and coma. In some cases, apparent recovery may take place, possibly due to a latency period during which the iron is distributed throughout the body. Systemic iron toxicity is characterised by multi-system damage, principally in the liver, metabolic acidosis, coagulopathies and cardiovascular collapse. Acute poisoning is relatively unusual in adults, the lethal dose being approximately $100 \mathrm{~g}$, but is more common in children.

Iron overload as a result of dietary intake is unusual in the normal population and only a handful of case reports exist describing this phenomenon. This may be due to the reduction in iron absorption that occurs as exposure increases. 
Individuals with conditions such as hereditary haemochromatosis $(\mathrm{HHC})$ are particularly vulnerable to iron overload, which occurs as a result of enhanced uptake. In subjects heterozygous for the condition, a small increase in iron storage may occur. It has been suggested that heterozygous subjects (up to $1 \%$ of the population) may have an increased risk of cardiovascular disease but this remains controversial. Similarly, the suggestion that high iron status may be associated with other chronic conditions remains unresolved.

Studies in rodents suggest a pattern of iron overload comparable with that seen in haemochromatosis, with cellular changes but not with fibrosis occurring. Reproductive studies in rodents have shown no significant evidence of iron transfer across the placenta. This is supported by a study in an ovine model where maternal iron poisoning did not result in increases in foetal serum iron levels. However one study reports that iron gluconate is teratogenic, causing exencephaly in mice following administration on the 8th and 9th days of gestation.

\section{ESTABLISHMENT OF GUIDANCE LEVEL}

Overall, there are insufficient appropriate data to establish a Safe Upper Level for iron. Many supplementation studies have been conducted, generally in iron-deficient groups and none of them are applicable to the population as a whole. For iron-replete individuals in non-developing countries, the most common side effects reported are gastrointestinal in nature, usually constipation but nausea, vomiting and epigastric pain have also been reported. These effects are reported to follow supplement doses of between 50 and $220 \mathrm{mg}$ iron/day, the frequency increasing at higher dose levels. The severity and occurrence of effects depends on the formulation of the supplement (Coplin et al., 1991; Liguori, 1993). A number of studies have examined the effects of different iron formulations.

For guidance purposes, a supplemental intake of approximately $17 \mathrm{mg} /$ day (equivalent to $0.28 \mathrm{mg} / \mathrm{kg}$ bw/day for a $60 \mathrm{~kg}$ adult) would not be expected to produce adverse effects in the majority of people. This is derived by dividing the lower end of the range found to have an effect by an uncertainty factor of 3 to allow for extrapolation from a LOAEL to a NOAEL. This is based on data referring to ferrous iron (Fe II), which is the form of iron used in supplements currently available in this country. No additional uncertainty factor is needed for inter-individual variation because the assessment is based on studies on large numbers of people. A safe upper level for total iron has not been estimated, as gastrointestinal effects are associated with iron in supplements rather than in foods.

The guidance value of $17 \mathrm{mg} /$ day calculated above, does not apply to the small proportion of the population who have increased susceptibility to iron overload, via a mechanism of unregulated (increased) absorption from the diet, associated with the homozygous haemochromatosis genotype (estimated prevalence, approximately $0.4 \%$ in Caucasian populations). It is not possible to give quantitative information on the difference in susceptibility between this group and normal subjects.

It should be recognised that many of the available studies do not look at side effects in detail and information on the long-term implications of iron supplementation on iron status and storage is lacking. 


\section{References}

Blot, I., Papriernik, E., Kaltwasser, J.P., Werner, E., Tchernia, G. (1981) Influence of routine administration of folic acid and iron during pregnancy. Gynecology and Obstetric Investigations 12, 294-304.

Breskin, M.W., Worthington-Roberts, B.S., Knopp, R.H., Brown Z., Plovie, B., Mottet, N.K., Mills, J.L. (1983). First trimester serum zinc concentrations in human pregnancy. American Journal of Clinical Nutrition 38, 943-953.

Brock, C., Curry, H., Hanna, C., Knipfer, M. and Taylor, L. (1985) Adverse effects of iron supplementation: a comparative trial of a wax matrix iron preparation and conventional ferrous sulphate tablets. Clinical Therapeutics 7, 568-573.

COMA (1991). Dietary Reference Values for Food Energy and Nutrients for the United Kingdom. Report of the Panel on Dietary Reference Values, Committee on Medical Aspects of Food and Nutrition Policy. HMSO, London.

Coplin, M., Schuette, S., Leichtmann, G., Lashner, B. (1991) Tolerability of iron: A comparison of bisglycino iron II and ferrous sulphate. Clinical Therapeutics 13, 606-612.

Dawson, E.A., Albers, J.A., McGanity, W.J. (1989). Serum changes due to iron supplementation in teen-age pregnancy. American Journal of Clinical Nutrition 50, 848-852

Frykman, E. Bystrom, M. Jansson, U. Edberg, A. Hansen T. (1994) Side effects of iron supplements in blood donors; superior tolerance of heme iron. Journal of Laboratory and Clinical Medicine 123, 561-564.

Ganzoni, A.M., Tondung, G., Rhymer, K. (1974). Orale Eisenmedikation. Vertraglichkeit von Eisensulfat und Eisensulfat + Bernsteinsaure. Einfluss auf die Hamoglobinkonzentration Gesunder. Deutsche Medizinische Wochenschrift 99, 1175-1178.

Hallberg, L., Hogdahl, A.-M., Nilsson, L., Rybo, G. (1966a). Menstrual blood loss - a population study. Acta Obstetricia et Gynaecologica Scandinavica 45, 320-351.

Hallberg, L., Ryttinger, L., Sollvell, L. (1966b). Side effects of oral iron therapy. Acta. Medica Scandinavica 459 (suppl.), 3-10.

Hambidge, K.M., Krebs, N.F., Jacobs, M.A., Favier A., Guyette L., Ikle D.N., (1983). Zinc nutritional status during pregnancy: a longitudinal study. American Journal of Clinical Nutrition 37, 429-442

Hambidge, K.M., Krebs, N.F., Sibley, R.N., English, J. (1987). Acute effects of iron therapy on zinc status during pregnancy. Obstetrics and Gynecology 70, 593-596.

Liguori, L. (1993) Iron protein succinylate in the treatment of iron deficiency: controlled, double-blind, multicenter clinical trialon over 1,000 patients. International Journal of Clinical Pharmacology, Therapy and Toxicology 31, 105-123.

Lokken, P. and Birkeland, J.M. (1979) Dental discolourations and side effects with iron and placebo tablets. Scandinavian Journal Of Dentistry Research 87, 275-278. 
Part 4 Minerals

Menendez, C., Todd, J., Alonso, P.L., Francis N., Lulat S., Ceesay S., Ascaso C., Smith T., M'Boge B., Greenwood B.M. (1995). The response to iron supplementation of pregnant women with the haemoglobin genotype AA or AS. Transactions of the Royal Society of Tropical Medicine and Hygiene 89, 289-292.

Reddaiah, V.P., Raj, P., Ramachandran K., Nath L.M., Sood S.K., Madan N., Rusia U.

(1989). Supplementary iron dose in pregnancy anemia prophylaxis. Indian Journal of Pediatrics 56, 109-114.

Sheldon, W.L., Aspillaga, M.O., Smith, P.A., Lind, T. (1985). The effects of oral supplementation on zinc and magnesium levels during pregnancy. British Journal of Obstetrics and Gynaecology 92, 892-898. 


\section{Risk Assessment}

\section{Magnesium}

\section{General information}

\section{Chemistry}

Magnesium is a metallic element of group 2 of the periodic table and has an atomic weight of 24.3.

\section{Natural occurrence}

Magnesium is the eighth most abundant element in the earth's crust. It does not occur as a pure metal in nature, but it is found in large deposits as magnesite, dolomite and other minerals. In this risk assessment the word 'magnesium' refers to ionic magnesium, except where specific magnesium salts are described.

\section{Occurrence in food, food supplements and medicines}

Magnesium is ubiquitous in foods, but the content varies substantially. Leafy vegetables, as well as grains and nuts, generally have a higher magnesium content $(60-2700 \mathrm{mg} / \mathrm{kg})$ than meats and dairy products (less than $280 \mathrm{mg} / \mathrm{kg}$ ). A number of magnesium salts are used as food additives. Magnesium salts are also used in food supplements at levels providing up to $750 \mathrm{mg} /$ day, and licensed medicines to treat malabsorption, as a perioperative nutritional support or in special diets, and are included in antacid and laxative formulations.

\section{Other sources of exposure}

The magnesium content of water is variable. The concentration depends on the region of its source and its manner of storage. 'Hard' water has a higher concentration of magnesium salts.

\section{Recommended amounts}

COMA has calculated a RNI of $300 \mathrm{mg} /$ day for adult males and $270 \mathrm{mg} /$ day for adult females (COMA, 1991). The RNI for infants and children ranges from 55 to $280 \mathrm{mg} /$ day. An RNI for magnesium during pregnancy was not calculated by COMA.

\section{Analysis of tissue levels and magnesium status}

Tissue levels of magnesium are determined by atomic spectroscopy.

\section{Brief overview of non-nutritional beneficial effects}

Dietary magnesium has been claimed to benefit bone health, but further studies are necessary to confirm this suggestion. 
There have been clinical trials and case reports of the therapeutic effects of magnesium in potentially life-threatening conditions, such as the cardiac arrhythmia 'torsade de pointes', digitalis toxicity, bronchospasm, alcohol withdrawal syndrome, ischaemic heart disease and myocardial infarction. It has also been suggested that magnesium possibly plays a role in the regulation of blood pressure.

Magnesium salts are effective cathartic agents and are also used in some antacid preparations.

\section{Function}

Magnesium is required as a cofactor for many enzyme systems. It is required for protein synthesis and for both anaerobic and aerobic energy generation and for glycolysis, either indirectly as a part of magnesium-ATP complex, or directly as an enzyme activator. Magnesium plays a multifunctional role in cell metabolism, (particularly at the level of key phosphorylations), and has a critical role in cell division. It has been suggested that magnesium is necessary for the maintenance of an adequate supply of nucleotides for the synthesis of RNA and DNA. Magnesium regulates the movement of potassium in myocardial cells and is also known to act as a calcium channel blocker.

Magnesium is an important element in the metabolism and/or action of vitamin D, and is essential for the synthesis and secretion of parathyroid hormone.

\section{Deficiency}

Magnesium deficiency has been linked to cardiovascular, skeletal, gastrointestinal and central nervous system disorders and to the use of loop diuretics.

Magnesium is essential for the normal function of the parathyroid gland and for vitamin D metabolism. Magnesium depletion markedly disturbs calcium homeostasis, and hypocalcaemia is a common manifestation of moderate to severe magnesium deficiency.

\section{Interactions}

Calcium homeostasis is controlled in part by a magnesium-requiring mechanism, which releases parathyroid hormone. Several magnesium-activated enzymes are inhibited by calcium.

\section{Absorption and bioavailability}

The net absorption of magnesium from the diet is typically approximately 50 percent. High levels of dietary fibre from fruits, vegetables, and grains decrease magnesium absorption. Dietary protein is also known to influence intestinal magnesium absorption.

Magnesium is absorbed along the entire intestinal tract, but the sites of maximal absorption appear to be the distal jejunum and ileum. It has been suggested that absorption occurs by both an unsaturable passive and saturable active transport system. Thus, in both adults and children, the fractional absorption of magnesium is inversely proportional to the amount ingested. 


\section{Distribution}

Magnesium is abundant in the body with the largest amounts found in bone. It is also found in a variety of other tissues including muscle, liver, heart and kidneys. In plasma, half of magnesium present is in the ionised form. About $20 \%$ is bound to proteins, the remaining $80 \%$ is unbound. Most intracellular magnesium is found bound to the endoplasmic reticulum.

\section{Excretion}

Magnesium is excreted primarily in the urine. The extent of urinary excretion, and thereby the homeostasis of magnesium, is influenced by a wide variety of hormones, including calcitonin, thyroxine, glucocorticoids, glucagons and angiotensin. Under normal conditions, the kidney tubule reabsorbs $95 \%$ of the filtered load of magnesium and about $5 \%$ is excreted in urine.

\section{Toxicity}

\section{Human data}

No adverse effects have been associated with the ingestion of magnesium as a naturally occurring substance in foods. However, adverse effects have been seen with excessive magnesium intake as a consequence of the use of various magnesium salts for pharmacological/medicinal purposes.

The primary manifestation of excessive ingestion of magnesium from non-food sources is osmotic diarrhoea, which is reversible. Magnesium has a well-known cathartic effect and is used in medicines for this purpose.

\section{Animal data}

There are only a limited number of studies on the toxicity of magnesium in animals. Studies on the short-term toxicity of magnesium administered by the intravenous route demonstrated that the $\mathrm{LD}_{50}$ value in rats is $174 \mathrm{mg} / \mathrm{kg}$ bw in females and $206 \mathrm{mg} / \mathrm{kg}$ bw in males, and in dogs is in excess of $1200 \mathrm{mg} / \mathrm{kg}$ bw, but such data are of no value for this risk assessment. High doses (1000 mg/kg bw/day three times daily) of magnesium sulphate administered subcutaneously in reproductive studies in rats resulted in lower food consumption and decreased body weight gains in the dams and produced delayed differentiation in their pups.

\section{Carcinogenicity and genotoxicity}

Mice given magnesium chloride in the diet for 96 weeks ( $2 \%$ in diet, equivalent to approximately $3000 \mathrm{mg} / \mathrm{kg}$ bw/day) exhibited no evidence of compound-related carcinogenicity.

In vitro genotoxicity tests were negative for magnesium chloride and magnesium sulphate. 


\section{Dose response}

No relevant data have been identified.

\section{Mechanism of toxicity}

No relevant data have been identified.

\section{Vulnerable groups}

No vulnerable groups have been identified.

\section{Genetic variations}

No genetic variations have been identified.

\section{Studies of particular importance in the risk assessment}

(For full review see http://www.food.gov.uk/science/ouradvisors/vitandmin/evmpapers or the enclosed CD)

\section{Nagy et al., 1988}

No adverse effects were reported in this study in which 20 patients with duodenal ulcers received up to $1200 \mathrm{mg}$ of magnesium per day in the form of an aluminium-magnesium-hydroxycarbonate antacid over a 6-week trial period in a randomised, prospective cross-over clinical trial.

\section{Marken et al., 1989}

Diarrhoea and other gastrointestinal effects were reported in a number of individuals participating in this randomised double-blind placebo-controlled cross-over study. The dose of magnesium in this study was $470 \mathrm{mg} /$ day (given as $800 \mathrm{mg} /$ day magnesium oxide) for 60 days.

\section{Zemel et al., 1990}

This study investigated the effect of magnesium supplementation on blood pressure, erythrocyte cation metabolism and serum lipid levels in 13 patients with mild hypertension. The study employed a 3-week placebo run-in period and no adverse effects were reported following a dose of $486 \mathrm{mg}$ magnesium twice daily for three months (given as magnesium aspartate).

\section{Paolisso et al., 1992}

In this double-blind cross-over study, doses of $393 \mathrm{mg} /$ day magnesium (as magnesium pidolate) were well tolerated by 12 older subjects (mean age 77.8 years) over a 4-week period. 
Bashir et al., 1993

This was a randomised double-blind placebo-controlled cross-over trial, in which 21 patients with stable congestive heart failure, secondary to coronary heart disease, received $384 \mathrm{mg}$ magnesium/day (as magnesium chloride) for 6 weeks. Gastrointestinal effects, including diarrhoea, were reported in 6 out of the 21 subjects receiving magnesium.

\section{Stendig-Lindberg et al., 1993}

No diarrhoea or gastrointestinal effects were reported in a long-term study in which a group of 31 postmenopausal women received daily supplements of magnesium hydroxide (providing up to $750 \mathrm{mg} /$ day magnesium) for 6 months followed by $226 \mathrm{mg} /$ day of magnesium for 18 months.

\section{Altura et al., 1994}

No adverse effects were reported in 18 healthy 18-38 year old males who were given diets enriched with magnesium oxide for 6 days, providing a daily dose of $452 \mathrm{mg}$ magnesium.

\section{Exposure assessment}

Food:

Mean: $280 \mathrm{mg} /$ day (NDNS 1986/7)

97.5th percentile: $510 \mathrm{mg} /$ day

Drinking water:

$100 \mathrm{mg} /$ day (assuming an intake of $2 \mathrm{~L} /$ day, estimated from $50 \mathrm{mg} / \mathrm{L}-\mathrm{EC}$ directives)

Supplements:

$750 \mathrm{mg} /$ day (Annex 4)

Estimated maximum intake: $510+100+750=1400 \mathrm{mg} /$ day

No potential high intake groups have been identified.

\section{Risk assessment}

The common effect of excessive ingestion of magnesium is osmotic diarrhoea. However, this effect was only observed in a limited number of studies of variable quality.

There are only limited data on the oral and general toxicity of magnesium in animals. The available data suggest a lack of carcinogenicity at doses of up to $3000 \mathrm{mg} / \mathrm{kg}$ bw/day. Mutagenicity tests on magnesium salts have also been negative. 


\section{ESTABLISHMENT OF GUIDANCE LEVEL}

There are insufficient data to establish a Safe Upper Level for magnesium. Although a few studies reported mild and reversible diarrhoea in a small percentage of patients and healthy volunteers at levels of 384 to $470 \mathrm{mg} /$ day, these symptoms were not observed in the majority of studies using similar or higher doses. For guidance purposes only, $400 \mathrm{mg} /$ day supplemental magnesium would not be expected to result in any significant adverse effects. This is equivalent to $6.7 \mathrm{mg} / \mathrm{kg}$ bw/day in a $60 \mathrm{~kg}$ adult. An uncertainty factor for human variability is not needed because the value is derived from a number of studies in humans, some of which reported no adverse effects at considerably higher doses. Guidance has not been given for total magnesium since the reported adverse effects are not associated with magnesium in food. The potential implications of this dose range for vulnerable groups such as infants and older people needs to be addressed by further studies.

\section{References}

Altura, B.T., Wilimzig, C., Trnovec, T., Nyulassy S., Altura B.M. (1994) Comparative effects of magnesium enriched diets and different orally administered magnesium oxide preparations on ionized $\mathrm{Mg}, \mathrm{Mg}$ metabolism and electrolytes in serum of human volunteers. Journal of the American College of Nutrition 13, 447-54.

Bashir, Y., Sneddon, J.F., Staunton, A., Haywood G.A., Simpson I.A., McKenna W.J., Camm A.J.(1993) Effects of long-term oral magnesium chloride replacement in congestive heart failure secondary to coronary artery disease. American Journal of Cardiology 72, 1156-1162.

COMA (1991). Dietary Reference Values for Food Energy and Nutrients for the United Kingdom. Report of the Panel on Dietary Reference Values, Committee on Medical Aspects of Food and Nutrition Policy. HMSO, London.

Marken, P.A., Weart, C.W., Carson, D.S., Gums J.G., Lopes-Virella M.F. (1989) Effects of magnesium oxide on the lipid profile of healthy volunteers. Atherosclerosis 77, 37-42.

Nagy, L., Tarnok, F., Past, T., Mozsik G., Deak G., Tapsonyi Z., Fendler K., Javor T. (1988). Human tolerability and pharmacodynamic study of Tisacid tablet in duodenal ulcer patients, a prospective, randomized, self-controlled clinicopharmacological study. Acta Medica Hungarica 45 (2) 231-247.

OTC (2001). OTC Directory 2001-2002, Proprietary Association of Great Britain.

Paolisso, G., Sgambato, S., Gambardella, A., Pizza G., Tesauro P., Varricchio M., D’Onofrio F. (1992). Daily magnesium supplements improve glucose handling in elderly subjects. American Journal of Nutrition 55, 1161-1167.

Stendig-Lindberg, G., Tepper, R., Leichter, I. (1993) Trabecular bone density in a two year controlled trial of personal magnesium in osteoporosis. Magnesium Research 6, 155-163.

Zemel, P.C., Zemel, M.B., Urberg, M., Douglas F.L., Geiser R., Sowers J.R. (1990) Metabolic and hemodynamic effects of magnesium supplementation in patients with essential hypertension. American Journal of Nutrition 51, 665-669. 


\section{Risk Assessment}

\section{Phosphorus}

\section{General information}

\section{Chemistry}

Phosphorus is a group 5 element of the periodic table and has an atomic weight of 30.97. Within this risk assessment, the phosphorus refers to ionic phosphorus except where specific phosphorus compounds are mentioned.

\section{Natural occurrence}

Phosphorus is most commonly found in nature in its pentavalent form in combination with oxygen, as phosphate $\left(\mathrm{PO}_{4}^{3-}\right)$.

\section{Occurrence in food, food supplements and medicines}

Phosphorus is widely found in many food groups, largely as phosphate(s). Dietary sources that are rich in phosphorus include red meats (1600 mg/kg), dairy products (>900 mg/kg), fish (4000 mg/kg), poultry $(2100 \mathrm{mg} / \mathrm{kg}$ ) and bread and other cereal products $(>900 \mathrm{mg} / \mathrm{kg})$. A number of phosphate salts are used in foods and soft drinks as additives (JECFA, 1994). Phosphorus is also used in food supplements (at levels up to a daily dose of $1100 \mathrm{mg} /$ day) and licensed medicines, in the form of inorganic phosphate salts and sodium acid phosphate, respectively.

\section{Other sources of exposure}

Phosphorus is present in fertilisers, detergents, sewage effluent and surface waters. The Drinking Water in the Water Supply (Water Quality) Regulations 1989 specify a limit of 2.2 mg phosphorus per litre drinking water.

\section{Recommended amounts}

COMA (1991) noted that phosphorus requirements are conventionally set as equal to calcium, in mass terms i.e. $1 \mathrm{mg}$ phosphorus : $1 \mathrm{mg}$ calcium. However, these elements are present in the body in equimolar amounts and COMA took the view that the ratio in the diet should be set at $1 \mathrm{mmol}$ phosphorus: $1 \mathrm{mmol}$ calcium and considered that the Recommended Nutrient Intake (RNI) for phosphorus should be set equal to the calcium RNI in molar terms $(1 \mathrm{mmol}$ calcium $=40 \mathrm{mg}, 1 \mathrm{mmol}$ phosphorus $=30.9 \mathrm{mg}$ ).

COMA therefore calculated a RNI of $550 \mathrm{mg} /$ day of phosphorus for males and females aged 19-50 years. COMA derived an increment of $440 \mathrm{mg} /$ day for women during lactation, giving a total of $990 \mathrm{mg} /$ day. The RNI for infants and children ranges from 400 to $775 \mathrm{mg} /$ day. 


\section{Analysis of tissue levels and phosphorus status}

Phosphorus is abundant in the body with largest amounts found in bone. It is also found in all soft tissues including muscle, liver, heart and kidneys. Serum total phosphorus levels are measured by colorimetric methods.

\section{Overview of non-nutritional beneficial effects}

Treatment with oral or intravenous phosphate causes a decrease in serum calcium in hypercalcaemic adults. Thus, it has been reported to be useful in reducing hypercalcaemia and hypercalciuria. It is also used for the treatment of conditions of the bone, such as fractures, Paget's disease and multiple myeloma.

\section{Function}

Phosphorus is a constituent of all major classes of biochemical compounds. Structurally, phosphorus occurs as phospholipids, which are a major constituent of most biological membranes, and as nucleotides and nucleic acids. Phosphorus plays an important role in carbohydrate, fat and protein metabolism and is essential for optimum bone health. The energy that is required for most metabolic processes is derived from the phosphate bonds of adenosine triphosphate and other high energy phosphate compounds.

Clinical studies employing chronic phosphorus supplementation were the first to show that high phosphorus intakes influence the parathyroid-vitamin $D$ axis, which maintains calcium balance in the body. The phosphorus loading in humans operates through mechanisms of nutritional or secondary hyperparathyroidism similar to those observed in animals fed excess phosphorus.

\section{Deficiency}

Factors associated with phosphorus deficiency (hypophosphatemia) include liver disease, sepsis, alcoholism, diabetic ketoacidosis and the use of aluminium-containing antacids. The symptoms of a potentially fatal syndrome include anorexia, anaemia, muscle weakness, bone pain, rickets and ataxia. Hypophosphatemia in infants is known to occur in situations of poorly managed parenteral nutrition with inappropriate administration of fluid and electrolyte therapy or with rapid refeeding after prolonged dietary restriction. Preliminary studies have indicated that phosphate deficiency at birth is associated with the development of rickets in later life.

\section{Interactions}

Phosphorus absorption is reduced by ingestion of aluminium-containing antacids and by pharmacological doses of calcium salts. High phosphate diets cause a reduction in the urinary excretion of calcium.

\section{Absorption}

Net absorption of phosphorus from a mixed diet has been reported to range from 55 to $70 \%$ in adults and from 65 to $90 \%$ in infants and children. The intestinal absorption of phosphorus is greatest in the jejunum and decreases along the length of the small intestine. 


\section{Distribution and metabolism}

Approximately $80 \%$ of the body phosphorus is present in the skeleton and the remainder is distributed in soft tissues and extracellular fluid. About $70 \%$ of the phosphorus in blood is as a constituent of phospholipids; the remainder is present as inorganic phosphate, about $85 \%$ free and $15 \%$ protein-bound. Parathyroid hormone (PTH) is the major regulator of the balance of phosphorus and calcium. Thus, a low calcium and high phosphate diet induces the secretion of PTH, which decreases the serum concentration of phosphate by increasing its urinary excretion.

\section{Excretion}

Phosphorus is primarily excreted in the urine. The regulation of phosphorus excretion is apparent from early infancy. In infants, as in adults, the major site for the regulation of the amount of phosphorus retained by the body is the kidney.

\section{Toxicity}

\section{Human data}

The majority of the published data relating to the toxicity of phosphorus in humans focus on accidental or intentional ingestion of the more toxic forms of phosphorus that are not found in food or food supplements (e.g. elemental yellow phosphorus).

No human data on chronic toxicity of dietary forms of phosphorus were identified in the literature.

\section{Supplementation trials}

The predominant adverse reaction to orally administered phosphorus (as various phosphate salts, including sodium, potassium, ammonium and glycerol) in human supplementation studies is osmotic diarrhoea, which has been reported at intakes of $750 \mathrm{mg} /$ day and above. Other mild gastrointestinal effects, including nausea and vomiting have been noted in some studies.

\section{Animal data}

There are a limited number of studies on the oral toxicity of inorganic phosphate salts in experimental animals. Kidney lesions have been reported in rats following the administration of acute doses of phosphates (approximately $5000 \mathrm{mg} / \mathrm{kg}$ bw/day, equivalent to about $1200 \mathrm{mg} / \mathrm{kg}$ bw/day phosphorus). Pathological effects in the parathyroids, kidneys and bone have also been reported in subchronic studies at high doses (approximately $4000 \mathrm{mg} / \mathrm{kg}$ bw/day, equivalent to about $1000 \mathrm{mg} / \mathrm{kg}$ bw/day phosphorus).

No adverse effects on growth and reproduction were reported in an abstract report of long-term studies with phosphoric acid. 


\section{Carcinogenicity and genotoxicity}

No data on carcinogenicity or genotoxicity of dietary forms of inorganic phosphorus and phosphate salts were identified.

\section{Mechanism of toxicity}

No relevant data have been identified.

\section{Dose-response characterisation}

Data from human trials indicate a dose-related increase in osmotic diarrhoea from intakes of 750 $\mathrm{mg} /$ day and above.

\section{Vulnerable groups}

One study suggested that increased phosphorus intake may lead to increased bone resorption in postmenopausal women with osteoporosis (Goldsmith et al., 1976). However, a more recent study in postmenopausal women found no biochemical evidence of increased bone remodelling as a result of supplementation with phosphorus (Brixen et al., 1992).

Black and Asian people and older people may be susceptible to bone resorption as a result of high phosphorus intakes, as they are more susceptible to hypovitaminosis $D$, which decreases the absorption of calcium, and phosphorus has been shown to influence the parathyroid-vitamin D axis, causing an increase in serum calcium levels via bone resorption.

\section{Genetic variations}

No genetic variations conferring vulnerability to phosphate toxicity have been identified.

\section{Studies of particular importance in the risk assessment}

(For full review see http://www.food.gov.uk/science/ouradvisors/vitandmin/evmpapers or the enclosed CD)

\section{Human data}

Goldsmith et al., 1968

This was a study of the role of phosphate as an adjunct in the therapy of multiple myeloma. Phosphate supplements (given as mixtures of sodium and potassium salts and providing a daily dose of approximately 1000 to $2000 \mathrm{mg}$ phosphorus) were given to 14 patients orally for up to 15 months, or intravenously (duration not stated). Reductions in bone pain and urinary calcium were reported by the authors. None of the patients developed any evidence of extra-skeletal calcification during follow-up periods of up to 15 months. One patient developed an increase in pedal and pretibial oedema, which subsided when the phosphate salts were discontinued. Another patient complained of dyspepsia 
following the ingestion of phosphate supplements, although the same subject reported similar discomforts with other medications including placebo.

\section{Brixen et al., 1992}

This was a study of the effect of a short course of oral phosphate treatment on serum parathyroid hormone levels and markers of bone turnover. A group of postmenopausal women with reduced bone mineral density and a history of at least one fracture were randomised in a double-blind study and given oral phosphate (providing 750, 1500 or 2250 mg/day phosphorus) or placebo for 7 days; the subjects were followed for 4 months thereafter. The phosphate was administered as effervescent tablets containing a mixture of ammonium phosphate, potassium phosphate and glycerol phosphate. The urinary phosphate/creatinine ratio increased in a dose-related fashion whereas no significant changes were seen in serum phosphate or serum calcium. Serum parathyroid hormone rose significantly in the groups receiving 1500 and $2250 \mathrm{mg}$ /day phosphorus. No effect on serum parathyroid hormone levels could be demonstrated with the lowest dose of phosphorus. Gastrointestinal side effects were noted in a dose-related fashion in 2 of 19 patients receiving $750 \mathrm{mg} /$ day, in 3 of 19 patients receiving $1500 \mathrm{mg} /$ day and in 7 of 20 patients receiving $2250 \mathrm{mg} /$ day.

\section{Whybro et al., 1998}

This was a report of two studies carried out to test the effect of supplementation with phosphate on calcium homeostasis and bone turnover. Study 1 was a 1-week, randomised, controlled cross-over trial involving 10 healthy men supplemented with $1000 \mathrm{mg}$ phosphorus (as sodium acid phosphate). The control diet for these men contained $800 \mathrm{mg} /$ day of calcium and $800 \mathrm{mg} /$ day of phosphorus. Study 2 (involving 12 healthy men) was an escalating dose study of 0, 1000, 1500 and $2000 \mathrm{mg} /$ day phosphorus, given as phosphate for 1 week. The control diet contained $1000 \mathrm{mg} /$ day of calcium and $1000 \mathrm{mg} /$ day of phosphorus as phosphates. Both studies showed an increase in the urinary excretion of phosphate and a decrease in urinary calcium. Serum levels of parathyroid hormone were only elevated in the first study. There were no changes in serum phosphate, osteocalcin or urinary N-telopeptide. Diarrhoea was reported in one subject in study 2 when receiving phosphorus at $2000 \mathrm{mg} /$ day.

\section{Exposure assessment}

Food:

Mean: $1260 \mathrm{mg} /$ day (NDNS 1986/7)

97.5th percentile: $2110 \mathrm{mg} /$ day

Drinking water: $4.4 \mathrm{mg} /$ day (assuming $2 \mathrm{~L} /$ day at maximum level of $2.2 \mathrm{mg} / \mathrm{L}$ )

Supplements: up to $1100 \mathrm{mg} /$ day (Annex 4)

Estimated maximum $2110+4.4+1100=3200 \mathrm{mg}$

daily intake:

No potential high intake groups have been identified. 


\section{Risk assessment}

In humans, changes in serum parathyroid hormone levels have been reported in supplementation studies in postmenopausal women with reduced bone mineral density and a history of fracture and in healthy men. Osmotic diarrhoea and other mild gastrointestinal symptoms have also been reported in supplementation studies. However, these symptoms were only reported in a limited number of studies.

There are limited data on the oral and general toxicity of inorganic phosphate salts in animals. Pathological effects in the parathyroid gland, kidneys and bones have been observed in mature male rats fed a diet containing an excessively high level of sodium orthophosphate for 7 months. No adverse effects on growth and reproduction were reported in long-term studies with phosphoric acid.

\section{ESTABLISHMENT OF GUIDANCE LEVEL}

There are insufficient data from human and animal studies to establish a Safe Upper Level for inorganic phosphates. A few studies have reported diarrhoea and mild gastrointestinal symptoms at doses of 750 to $2250 \mathrm{mg}$ supplemental phosphorus/day. The osmotic diarrhoea reported in these supplementation studies was mild and reversible in nature. In addition, physiological changes in calcium and parathyroid hormone levels have been associated with intakes of $1500 \mathrm{mg} /$ day and above of supplemental phosphorus. Because persons with hypovitaminosis $D$ are vulnerable to hyperparathyroidism, an uncertainty factor of 3 has been applied to the NOAEL of $750 \mathrm{mg} /$ day to allow for inter-individual variation. Based on these limited studies, and for guidance purposes only, a supplemental intake of $250 \mathrm{mg} /$ day would be expected not to produce adverse effects, including mild gastrointestinal upset. This is equivalent to $4.2 \mathrm{mg} / \mathrm{kg}$ bw in a $60 \mathrm{~kg}$ adult. Assuming a maximum intake of $2100 \mathrm{mg} /$ day from food and water, an estimated total intake of $2400 \mathrm{mg} /$ day (equivalent to $40 \mathrm{mg} / \mathrm{kg} \mathrm{bw} /$ day in a $60 \mathrm{~kg}$ adult) would not be expected to result in any adverse effects.

\section{References}

Brixen, K., Nielsen, H.K., Charles, P., Mosekilde, L. (1992) Effect of a short course of oral phosphate treatment on serum parathyroid hormone $(1-84)$ and biochemical markers of bone turnover: a doseresponse study. Calcified Tissue International 51, 276-281.

COMA (1991). Dietary Reference Values for Food Energy and Nutrients for the United Kingdom. Report of the Panel on Dietary Reference Values, Committee on Medical Aspects of Food and Nutrition Policy. HMSO, London.

Goldsmith, R.S., Bartos, H, Hulley, S.B., Ingbar, S.H., Moloney, W.C. (1968) Phosphate supplementation as an adjunct in the therapy of multiple myeloma. Archives of Internal Medicine 122, 128-133.

Goldsmith, R.S., Jowsey, J., Dube, W.J., Riggs, B.L., Arnaud, C.D. Kelly (1976) Effects of phosphorus supplementation on serum parathyroid hormone and bone morphology in osteoporosis. Journal of Clinical Endocrinology and Metabolism 43, 523-532.

JECFA-Summary of evaluations performed by the joint FAO/WHO Expert Committee on Food Additives (1994). ILSI Press.

Whybro, A., Jagger, H., Barker, M., Eastell, R. (1998) Phosphate supplementation in young men: lack of effect on calcium homeostasis and bone turnover. European Journal of Clinical Nutrition 52, 29-33. 


\section{Risk Assessment}

\section{Potassium}

\section{General information}

\section{Chemistry}

Potassium is an alkaline, metallic element. It is not found in the elemental form in nature and is always found combined with other substances, most commonly as the chloride salt $(\mathrm{KCl})$. Potassium is widely distributed in silicate rocks, and occurs in salt beds and seawater. Within this risk assessment, the word potassium refers to ionic potassium, except where specific potassium compounds are stated.

\section{Occurrence in food, food supplements and medicines}

Potassium is present in all animal and plant tissues. Major dietary sources include milk (1.4-1.5 mg/kg), fruit and vegetables (0.8-4.4 mg/kg), fish (1.9-3.5 mg/kg), shellfish (0.3 to $3.9 \mathrm{mg} / \mathrm{kg})$, beef $(2-3.5 \mathrm{mg} / \mathrm{kg})$, chicken and turkey ( $3 \mathrm{mg} / \mathrm{kg}$ ); liver is also a rich source of potassium ( 2.5 to $4.2 \mathrm{mg} / \mathrm{kg}$ ). Potassium chloride is used in fertilisers and plant nutrients and thus may increase the potassium content of plants. Potassium chloride is the major ingredient used in salt substitutes to satisfy the taste for salt whilst reducing sodium intake.

A range of food additives are potassium salts. Potassium iodide can be added to foods and is used in other countries to supplement iodine intake in iodine deficient regions.

UK food supplements can contain up to $200 \mathrm{mg}$ potassium per tablet as potassium chloride. Licensed medicines containing potassium chloride (300 mg/dose) are available by general sale, for replacement of potassium loss following acute diarrhoea.

\section{Other sources of exposure}

The potassium content of mineral, spring, table and spa water varies widely.

\section{Recommended amounts}

The RNI for potassium is $3500 \mathrm{mg} /$ day for adults (over 18 years) of both sexes (COMA, 1991). There is no increased requirement during pregnancy or lactation.

\section{Analysis of tissue levels and potassium status}

Potassium levels can be measured by flame emission spectroscopy, electron microprobe $\mathrm{X}$ ray analysis or measuring the dilution of radioactive isotopes of potassium. Total body potassium is estimated to be approximately $135 \mathrm{~g}$ in a $70 \mathrm{~kg}$ adult man.

\section{Brief overview of non-nutritional beneficial effects}

Potassium exerts a beneficial effect on hypertension by lowering blood pressure. 


\section{Function}

Potassium, together with sodium, is essential for the maintenance of normal osmotic pressure within cells. About $98 \%$ of the total body potassium is located intracellularly where the concentration can be 30 times that of the extracellular concentration. The extracellular potassium concentration is a critical determinant of neuromuscular excitability. Potassium is also a cofactor for numerous enzymes and is required for secretion of insulin by the pancreas, for phosphorylation of creatine and for carbohydrate metabolism and protein synthesis.

\section{Deficiency}

Hypokalaemia most commonly results from increased loss of the element, secondary to diarrhoea, diabetic acidosis, vomiting, intense and prolonged sweating, body burns or diuretic drugs. Rarely, 'crash' or very low calorie diets can result in reduced intake, sufficient to cause potassium deficiency. Hypokalaemia can cause rapid and irregular heart rhythm, muscle weakness and irritability, occasional paralysis, nausea and vomiting, diarrhoea and low muscle tone in the gut, and has been reported to predispose to hypertension.

\section{Interactions}

The balance between sodium and potassium is very important. Excess sodium intake can deplete potassium. Magnesium deficiency results in failure to retain potassium and conversely, excessive levels of potassium may interfere with magnesium absorption. Thallium interacts with potassium in the body, because the active transport mechanisms for potassium do not differentiate between thallium and potassium. In animals, the rate of loss of thallium from the body increases as dietary potassium increases.

\section{Absorption and bioavailability}

About $90 \%$ of ingested potassium is absorbed, irrespective of the amount consumed. The majority of potassium absorption occurs in the small intestine, mainly through passive mechanisms.

\section{Distribution and metabolism}

Potassium is transported in the extracellular fluid, and its distribution between cells is tightly controlled, with only $1.5-2.5 \%$ of total body potassium found in the extracellular fluid.

A large proportion of the body burden of potassium is found in muscle and the skeleton, and it is also present in high concentrations in the blood, central nervous system, intestine, liver, lung and skin.

\section{Excretion}

The major excretory route of potassium is via the kidneys. It is secreted by the renal tubules, in exchange for sodium of the glomerular filtrate (ion exchange mechanism).

Excretion in sweat and faeces is negligible, the latter changing only slightly as dietary potassium intake varies over a wide range. 


\section{Toxicity}

\section{Human data}

Potassium chloride has been associated with acute poisoning in humans. Case reports have described heart failure, cyanosis and cardiac arrest after ingestion of high doses of potassium chloride tablets. Gastrointestinal toxicity has also been described after chronic ingestion of potassium chloride in case studies and supplementation studies. This is characterised by abdominal pain, nausea and vomiting, diarrhoea, and ulceration of the oesophagus, stomach and duodenum and ileum.

Case studies of toxicity resulting from high doses of salt substitutes have described chest tightness, nausea and vomiting, diarrhoea, hyperkalaemia, shortness of breath and heart failure. For example, a fatality resulted from hyperkalaemia and resultant asystole after ingestion of 21,000 mg of salt substitute representing an oral bolus of 11,065 mg potassium (Restuccio, 1992). A 2 month old boy died after being given three doses of $1500 \mathrm{mg}$ potassium chloride with breast milk over one and a half days (Wetli and Davis, 1978).

\section{Supplementation trials}

Numerous potassium supplementation studies have examined the association between increased potassium intake and decreased risk of hypertension and heart disease. The majority of these studies have shown beneficial effects of potassium supplementation. Although adverse effects have not generally been reported, outside the gastrointestinal effect, it is often unclear whether adverse effects were investigated. Although few details were given, it was stated that no adverse effects were apparent in 18 subjects aged 66-79 given $2340 \mathrm{mg}$ potassium (as potassium chloride) for 4 weeks (Fotherby and Potter, 1992) or in subjects aged 21-61 given 1900 mg potassium (form not stated) for 15 weeks (Siani et al., 1987).

In a number of supplementation studies, potassium treatment resulted in ulceration of the gastrointestinal tract, the severity of the effect related to the formulation of the treatment and to factors such as gut transit time. Hyperkalaemia also occurs as a result of potassium treatment.

\section{Animal data}

Acute oral administration of potassium to animals causes changes in acid-base balance, hyperkalaemia, changes in respiratory rate and hypernatraemia. Acute oral administration of potassium chloride in animals has been reported to cause death by respiratory failure, with gastroenteritis and renal tubular necrosis.

The subchronic or chronic toxicity of potassium has not been investigated with inert salts such as potassium chloride. Effects produced with potassium nitrate (hypertrophy of the adrenal zona glomerulosa) and potassium iodate (haemosiderin deposition in the renal tubules) were attributed to the anions (i.e. the nitrate and iodate moieties). 


\section{Carcinogenicity and genotoxicity}

There are no data on the carcinogenicity of potassium chloride. Potassium bromate, potassium iodide and potassium hydrogen carbonate produced cancers in experimental studies, but the effects were attributed to the anions (i.e. the bromate, iodide and hydrogen carbonate moieties) and are thus not relevant to this risk assessment.

Genotoxicity studies have found that potassium bromate is mutagenic in animal and human cells and can produce DNA oxidation.

\section{Mechanisms of toxicity}

No relevant data have been identified.

\section{Dose response characterisation}

No relevant data have been identified.

\section{Vulnerable groups}

Older people may be vulnerable to potassium toxicity due to reduced physiological reserve in renal function. Individuals with pre-existing renal disease, hyperkalaemia, adrenal insufficiency, acidosis or insulin deficiency are also vulnerable, as are those using certain drugs, such as potassium-sparing diuretics, B-adrenergic blockers, angiotensin-convering enzyme (ACE) inhibitors, digitalis, non-steroidal anti-inflammatory drugs, arginine hydrochloride and succinylcholine. Infants may be vulnerable to excessive potassium due to limited renal reserve and immature function.

\section{Genetic variations}

No genetic variations that increase susceptibility to toxic effects of potassium have been identified.

\section{Studies of particular importance in the risk assessment}

(For full review see http://www.food.gov.uk/science/ouradvisors/vitandmin/evmpapers or the enclosed $C D$ ).

\section{McMahon et al., 1982}

The effect of differing formulations of potassium chloride supplementation on the gastrointestinal tracts of 48 healthy young men was investigated by endoscopy. Wax-matrix potassium chloride preparations (providing $1250 \mathrm{mg}$ potassium) three times a day for seven days (total daily dose, $3700 \mathrm{mg}$ potassium) resulted in considerable mucosal pathology, with erosions, gastric ulcers, inflammatory lesions and bleeding at endoscopy. A microencapsulated form of potassium chloride caused significantly fewer mucosal lesions. 


\section{McMahon et al., 1984}

In a further study, 225 healthy male subjects received either potassium chloride as wax-matrix tablets, in liquid form or microencapsulated, a potassium-sparer or placebo for one or two weeks. The doses used ranged from 900 - $3700 \mathrm{mg}$ potassium/day). Upper mucosal injury (erosions and ulcerations) was more frequent after wax-matrix potassium chloride treatment. It was noted that gastro-intestinal effects were mild and did not correlate with endoscopic evidence of lesions. When hypertensive subjects were treated with 1560-3120 mg potassium/day) slow-release wax-matrix potassium chloride for an average of 21 months, 6/9 subjects developed erosions compared with 1/9 matched controls. Following 7 days of in-patient treatment, one of the 6 subjects with erosions developed an ulcer and a further 2 placebo subjects developed erosions. The authors concluded that cyto-adaptation to potassium chloride treatment did not seem to occur.

Grimm et al., 1988, 1990

In a double blind study, groups of males aged 45-68 years were given $3700 \mathrm{mg}$ potassium/day (as potassium chloride) or a placebo ( $n=148$ in potassium group and 150 in placebo group). Adverse effects reported in both groups after 12 weeks and two years included abdominal pains, nausea and vomiting, diarrhoea and bright red blood in the stools. The effects were not investigated by endoscopy. The incidence was stated to be similar in the two treatment groups.

\section{Exposure assessment}

Total exposure/intake

Food

Mean: $2800 \mathrm{mg} /$ day (from NDNS, 1986/7) $97.5^{\text {th }}$ percentile: $4700 \mathrm{mg} /$ day

Water Up to $24 \mathrm{mg} /$ day (assuming $2 \mathrm{~L} /$ day at the maximum permitted level in the UK)

Supplements

Up to $200 \mathrm{mg} /$ day (Annex 4)

Estimated maximum intake: $4700+24+200=4900 \mathrm{mg} /$ day

No potential high intake groups have been identified.

\section{Risk assessment}

A number of case reports of accidental and deliberate poisoning with potassium have shown that large doses of potassium result in hyperkalaemia and hypernatraemia and lead to in changes in acid-base balance and respiratory and heart rates. However, the dose associated with the onset of hyperkalaemia and adverse effects varies depending on potassium status and clearance time. 
Supplementation studies have generally not reported side effects, although it is unclear whether side effects were specifically investigated in many of these studies. However, endoscopic investigation following periods of potassium supplementation has shown that potassium supplementation may cause local irritation in the gastrointestinal tract, leading to erosion and ulcerations.

The available animal data are not relevant to this risk assessment as the effects described are considered to be due to the anionic components, such as bromate and iodate.

Older people and infants may be more vulnerable to potassium toxicity due to lower renal function, as may patients with conditions such as pre-existing hyperkalaemia, renal disease, acidosis, insulin deficiency or digitalis intoxication.

\section{ESTABLISHMENT OF GUIDANCE LEVEL}

There are insufficient data to establish a Safe Upper Level for potassium. The amounts of potassium reported to cause adverse effects are variable and depend on factors which include formulation. In the study by Grimm et al. (1998, 1990), subjects received $3700 \mathrm{mg}$ /day potassium for 2 years, with the incidence of side effects being comparable in placebo and treatment groups. However, in studies by McMahon et al. (1982, 1984), in which subjects were treated with $3700 \mathrm{mg} /$ day potassium, endoscopic examination indicated that gastrointestinal erosions could occur with only mild symptoms being apparent.

Overall, for guidance purposes, it can be concluded that supplemental doses of up to $3700 \mathrm{mg}$ potassium/day appear to be without overt adverse effects (this is equivalent to $60 \mathrm{mg} / \mathrm{kg} \mathrm{bw} /$ day in a $60 \mathrm{~kg}$ adult), but may be associated with gastrointestinal lesions diagnosed by endoscopy. No uncertainty factor has been applied for inter-human variation as this is based on data from a number of human studies. The available animal data are not relevant to this risk assessment. Since the effect appears to relate to the ingestion of potassium supplements, rather than potassium in food, a guidance level for total potassium intake has not been calculated. Extrapolation of the guidance level to children on a bodyweight basis may be inappropriate.

Infants, older subjects and patients with conditions such as pre-existing hyperkalaemia, renal disease, acidosis, insulin deficiency or digitalis intoxication should not take potassium supplementation without medical advice. 


\section{References}

COMA (1991). Dietary Reference Values for Food Energy and Nutrients for the United Kingdom. Report of the Panel on Dietary Reference Values, Committee on Medical Aspects of Food and Nutrition Policy. HMSO, London.

Fotherby, M. D. and Potter, J. F. (1992) Potassium supplementation reduces clinic and ambulatory blood pressure in elderly hypertensive patients. Journal of Hypertension 10, 1403-1408.

Grimm, R. H., Kofron, P. M., Neaton, J. D., Svendsen, K. H., Elmer, P. J., Holland, L., Witte, L. J., Clearman, D. and Prineas, R. J. (1988) Effect of potassium supplementation combined with dietary sodium reduction on blood pressure in men taking antihypertensive medication. Journal of Hypertension 6 (suppl 4) S591-S593.

Grimm, R. H., Neaton, J. D., Elmer, P. J., Svendsen, K. H., Levin, J., Segal, M., Holland, L., Witte, L. J., Clearman, D. R., Kofron, P., LaBounty, R. K., Crow, R. and Prineas, R. J. (1990) The influence of oral potassium chloride on blood pressure in hypertensive men on a low-sodium diets. New England Journal of Medicine 322, 569-574.

McMahon, F. G., Ryan, J. R., Akdamar, K. and Ertan, A. (1982) Upper gastrointestinal lesions after potassium chloride supplements: a controlled clinical trial. Lancet 2, 1059-1061.

McMahon, F. G., Ryan, J. R., Akdamar, K. and Ertan, A. (1984) Effect of potassium chloride supplements on upper gastrointestinal mucosa. Clinical Pharmacology and Therapeutics 35, 852-855.

Restuccio, A. (1992). Fatal hyperkalaemia from a salt substitute. American Journal of Emergency Medicine 10, 171-173.

Siani, A., Strazzullo, P., Giacco, A., Pacioni, D., Celentano, E. and Mancini, M. (1991) Increasing the dietary potassium intake reduces the need for antihypertensive medication. Annals of Internal Medicine 115, 753-759.

Wetli, C. V. and Davis, J. H. (1978) Fatal hyperkalaemia from accidental overdose of potassium chloride. Journal of American Medical Association 240, 1339. 


\section{Risk Assessment}

\section{Silicon}

\section{General information}

\section{Chemistry}

Silicon ( $\mathrm{Si}$ ) is a non-metallic element with an atomic weight of 28. The term 'silica' is used to refer to naturally occurring materials composed principally of silicon dioxide $\left(\mathrm{SiO}_{2}\right)$, whereas 'silicone' (organosiloxane) refers to man-made siloxane polymers based on a structure of alternating oxygen and silicon atoms.

\section{Natural occurrence}

Silicon is not found freely in nature, but occurs chiefly as the oxide and silicates. Silica $\left(\mathrm{SiO}_{2}\right)$ occurs in nature in several different forms: crystalline (quartz, cristobalite and tridymite) and amorphous. When exposed to water, silicates liberate orthosilicic acid to a concentration of 1-15 mg/L.

\section{Occurrence in food, food supplements and medicines}

High levels of silicon are found in foods derived from plants, particularly grains such as oats (4250 $\mathrm{mg} / \mathrm{kg}$ wet weight), barley ( $2420 \mathrm{mg} / \mathrm{kg}$ wet weight) or rice. Levels are lower in foods from animal sources. Beer is also a rich source of silica containing $33-60 \mathrm{mg} / \mathrm{kg}$. Silicon is also found in drinking water as orthosilicic acid.

Amorphous silica is used as a food additive, in particular as an anti-caking agent, but also to clarify beverages, control viscosity and as an anti-foaming agent and dough modifier. It is also used as an anticaking agent and as an excipient in pharmaceuticals for various drug and vitamin preparations.

UK food supplements contain up to $500 \mathrm{mg}$ silicon.

There are no licensed medicines containing silicon as an active component.

\section{Other sources of exposure}

Consumption of $2 \mathrm{~L} /$ day drinking water could result in consumption of up to $10 \mathrm{mg}$ silicon. Exposure to high levels of airborne silica occurs in occupations such as quarry workers, miners, foundry workers and sand blasters. Silica deposited in the lungs can be slowly absorbed.

\section{Recommended amounts}

Although silicon is thought to be essential, recommendations on adequate nutritional intakes have not been established, either by COMA or other advisory bodies. 


\section{Analysis of tissue levels and silicon status}

Silicon was initially measured by a colorimetric assay, but high-performance liquid chromatographic/electrothermal atomic absorption (HPLC/ETAAS) and atomic absorption spectroscopy (AAS) methods have been developed more recently.

Methods of assessing silicon status have not been established.

\section{Brief overview of non-nutritional beneficial effects}

Silicon has been claimed to reduce the incidence and severity of atherosclerosis.

\section{Function}

Silicon is involved in the formation of bone and connective tissues. The precise mechanism is uncertain. However, it has been suggested that silicon could facilitate the formation of glycosaminoglycan and collagen components of the bone matrix through its role as a constituent of the enzyme of prolylhydrolase. Alternatively, silicon could have a structural role as a component of glycosaminoglycans and glycosamino-protein complexes, occurring as silanolate in mucopolysaccharides and linking different polysaccharides in the same polysaccharide chain, or linking acid mucopolysaccharides to protein.

\section{Deficiency}

Silicon deficiency has been produced experimentally in chicks and rats. Such deficiency produces deformities of the skull and peripheral bones, characterised by poorly formed joints, defective endochondrial bone growth and reduced contents of articular cartilage, hexosamine, collagen and water. The concentrations of elements such as calcium, magnesium, zinc, sodium, iron, potassium and manganese may also be decreased in the femur or vertebrae.

Silicon deficiency has not been observed in humans.

\section{Interactions}

Silicon has been reported to interact with a number of minerals including copper, zinc and germanium. The interaction between silicon and aluminium has been researched in more detail as a means of inhibiting aluminium toxicity. However the results are conflicting and it is possible that silicon levels are too low for any effect to occur in vivo.

\section{Absorption and bioavailability}

The bioavailability of silicon depends on the solubility of the compound or speciation concerned. It is thought that silicic acid is the form absorbed from the gastro-intestinal tract. It has been reported that the absorption of silicic acid from the gut is $20-75 \%$. 


\section{Distribution and metabolism}

Silicon is widely distributed in the tissues. High levels are present in bone, nails, tendons and the walls of the aorta, with nails containing the highest levels (up to $1500 \mathrm{mg} / \mathrm{kg}$ ). Lower levels are present in red blood cells or serum (approximately $44 \mathrm{mg} / \mathrm{kg}$ for red cells and $20 \mathrm{mg} / \mathrm{kg}$ for bound silicic acid in plasma. Silicon is also found in breast milk. Silicon has also been found in the liver, spleen and lung. Animal data suggest that high levels of silicon are found in the kidney, liver and lungs with moderate amounts being found in bone, skin, muscle, testes and spleen. Brain tissue contains negligible amounts of silicon.

No data on silicon metabolism have been identified.

\section{Excretion}

Silicon is predominantly and rapidly excreted in the urine, with smaller amounts being eliminated in the faeces.

\section{Toxicity}

\section{Human data}

Few data are available on the oral toxicity of silicon in humans and no acute or chronic toxicity data have been identified. The occurrence of silica stones has been reported in patients on long term antacid therapy with magnesium trisilicate.

If inhaled at high concentrations over prolonged periods, certain forms of silica can cause silicosis. Silica particles are inhaled into the alveoli of the lung, causing tissue damage that ultimately results in fibrosis, which reduces the efficiency of the lungs and results in shortness of breath. IARC has classified silica (by inhalation) as a Group 1 'known human carcinogen', based on human epidemiological data with support from studies in both animals and biological systems. In vitro studies have demonstrated that silicon has an inhibitory effect on superoxide dismutase and thus may potentially increase free radical damage.

It is thought that the carcinogenicity of inhaled silica particles is due to local tissue damage and inflammation with the production of reactive oxygen species, which overwhelm cellular defences and damage DNA. This process is considered not to be relevant to oral exposure to silica or silicon.

Chronic occupational exposure to silica by inhalation can also lead to a distinctive nephropathy manifested clinically by albuminuria and hypertension and characterised pathologically by changes in the glomeruli and proximal tubules. This nephrotoxic reaction (as with the respiratory toxicity described above) is thought not to occur by exposure to silicon in food.

\section{Animal data}

The acute oral toxicity of silicon (as silica) is low. No significant toxicity or mortality has been reported in animals given doses of up to $3 \mathrm{~g} / \mathrm{kg}$ bw/day. 
Growth rates were reduced, and the concentrations of certain other minerals in the plasma and tissues were affected in rats fed $500 \mathrm{ppm}$ (equivalent to approximately $50 \mathrm{mg} / \mathrm{kg}$ bw for young rats) silicon from 3 different silicate forms in the diet for 8 weeks (either tetraethylorthosilicate (TES), sodium silicate or sodium zeolite (Kayonga-male and Jia, 1999). Of the forms tested, only sodium silicate is relevant to the assessment of silicon in food. Similar results were observed in turkeys fed 270 ppm dietary silicon from the same three sources for 4 weeks.

Similarly, in rats fed up to $5 \%$ syloid (amorphous silicon dioxide) for 24 months, treatment was not associated with any significant dose-related effects on growth rate, survival, haematology or microscopic pathology. Liver weights were reduced from 12-24 months in the females fed 2.5\% and 5\% syloid respectively, although a clear dose-related trend was not apparent. No dose related pathological changes were observed. No dose related increases in tumours were apparent.

Growth was reduced transiently in mice fed up to $5 \%$ (equivalent to $7,500 \mathrm{mg} / \mathrm{kg}$ bw/day) syloid in the diet for 21 months. The treatment had no effect on haematology or gross or microscopic pathology. It was stated that an increase in tumours attributed to treatment was found in the haematopoietic organs, particularly malignant lymphoma/leukaemia which occurred in the mid-dose female mice only, but this was not considered significant because a statistical trend test was negative.

No data on reproductive toxicity have been identified.

\section{Carcinogenicity and genotoxicity}

Silicon was not carcinogenic in mice or rats at $5 \%$ in the diet. Silica was negative in the Bacillus subtilis rec assay and was not mutagenic in the Ames test. Sister chromatid exchange (SCE) was not induced in Chinese hamster V79 cells at a range of concentrations. Quartz has been reported to induce dose dependent increases in the number of morphologically transformed Syrian hamster cells. Overall, inorganic silicon compounds do not appear to have significant genotoxic potential.

\section{Mechanism of toxicity}

No data have been identified on the mechanism of oral silicon toxicity

\section{Dose response characterisation}

No data have been identified.

\section{Vulnerable groups}

No vulnerable groups have been identified.

\section{Genetic variations}

No genetic variations in the handling of silicon have been identified. 


\section{Studies of particular importance in the risk assessment}

(For full review see http://www.food.gov.uk/science/ouradvisors/vitandmin/evmpapers or the enclosed CD)

Takizawa et al., 1988

Groups of 40 B6C3F1 mice were fed 0, 12,500, 25,000 or 50,000 ppm (5\%) syloid (silicon dioxide) in the diet for up to 21 months (equivalent to $1,900-7,500 \mathrm{mg} / \mathrm{kg}$ bw silica or 900 to $3500 \mathrm{mg}$ silicon). The basal silicon level of the diet was not stated. Interim sacrifices were made at 6 and 12 months, such that up to 20 mice completed the dose period. The growth of mice in the top dose group was significantly reduced at the end of the first 10 weeks of the experiment but no other significant differences were apparent. Food consumption was significantly increased in the males fed 50,000 and 25,000 ppm. No significant dose-related effects on haematology or gross or microscopic pathology, including tumours, were observed. Since the decreased growth rate is likely to be due to nutritional imbalance the top dose (equivalent to $7,500 \mathrm{mg} / \mathrm{kg}$ bw/day silicon dioxide, or 3,495 $\mathrm{mg}$ silicon/ $\mathrm{kg}$ bw/day) was considered to be a NOAEL.

Groups of 40 Fischer rats were fed 0, 12,500, 25,000 or 50,000 ppm syloid in the diet for up to 21 months. The silicon content of the basal diet was not stated. No significant dose-related effects on food consumption, growth rate, survival, haematology or microscopic pathology were observed. Mineral levels in the tissues were not measured. Liver weights were reduced (by up to 15\%) in the 2.5 and $5 \%$ females from 12-24 months; a clear dose related trend was not apparent. No dose related pathological changes were observed. No dose related increases in tumours were apparent but a relatively small number of animals were used in the study. The few effects identified may have resulted from a nutritional imbalance and therefore may not be relevant to humans. The top dose (equivalent to 2,500 mg/kg bw/day silicon dioxide, or 1,165 mg silicon/kg bw/day) was regarded as a NOAEL.

\section{Exposure assessment}

Total exposure/intake

Food up to $50 \mathrm{mg} /$ day (Bowen and Peggs, 1984; Pennington, 1991)

Supplements up to $500 \mathrm{mg} /$ day (Annex 4; OTC, 2001)

Water $10 \mathrm{mg} /$ day (assuming 2 litres of water at maximum reported level of $5 \mathrm{mg} / \mathrm{L})$ (Pennington, 1991)

Estimated maximum intake: $50+500+10 \mathrm{mg}=560 \mathrm{mg} /$ day

No potential high intake groups were identified. 


\section{Risk assessment}

Few data are available on oral silicon toxicity in humans.

Animal data are available on certain forms of silicon, which suggest that high levels of silicon in the form of silicates can reduce growth and alter mineral levels in rats and turkeys, fed 500 ppm (approximately $50 \mathrm{mg} / \mathrm{kg}$ bw) and $270 \mathrm{ppm}$ silicon, from 3 silicon sources respectively. Some chronic studies investigating the food additive syloid ( $99 \%$ amorphous silicon dioxide) are also available. Reductions in growth rates and organ weights at the higher doses were reported but no significant effects on haematology or gross or microscopic pathology were observed. The changes reported may have been due to nutritional inadequacies in the diet. Overall it can be concluded that the chronic toxicity of silicon as amorphous silica is low.

Silica by inhalation is a known carcinogen, but this results from a mechanism specific to inhalation exposure. The available genotoxicity data are largely negative, although these are limited.

No vulnerable groups have been identified and no data on the effects of silicon on children have been identified.

\section{ESTABLISHMENT OF SAFE UPPER LEVEL}

Key study: Takizawa et al. (1988)

NOAEL: 50,000 ppm supplemental dietary silica, equivalent to $2500 \mathrm{mg} / \mathrm{kg}$ bw/day in rats, $7500 \mathrm{mg} / \mathrm{kg}$ bw/day in mice). The study in rats has been used to establish the safe upper level.

Uncertainty factors: $\quad 10$ for inter-species variation

10 for inter-individual variation

Safe Upper Level

$2500 / 100=25 \mathrm{mg} / \mathrm{kg}$ bw/day supplemental silica (equivalent to for daily $1500 \mathrm{mg} /$ day for a $60 \mathrm{~kg}$ adult)

Consumption over

a lifetime:

In terms of elemental silicon, this is equivalent to a Safe Upper Level of $12 \mathrm{mg} / \mathrm{kg}$ bw/day or $700 \mathrm{mg} /$ day for a $60 \mathrm{~kg}$ adult for supplemental silicon.

Few data are available on the oral toxicity of silicon in humans and they are inadequate for risk assessment. Therefore the animal data have been considered for this purpose. The study by KayongaMale and Jia (1999) in rats and turkeys suggested that short term intakes of 500 and 270 ppm dietary silicon respectively as silicates may result in reduced growth and other changes in mineral levels. This study used tetraethylorthosilicate (an organic silicon compound), sodium zeolite, which contains aluminium, and sodium silicate, which is the compound most representative of silicon in food. A more detailed, chronic study by Takizawa et al (1988) indicated that chronic intakes of diets containing 12,500 and 25,000 ppm amorphous silica (equivalent to 600 and $1900 \mathrm{mg}$ elemental silicon/kg bw/day) were not associated with any adverse effect in rats and mice respectively. The study did not investigate mineral levels but it is likely that any significant effects attributable to mineral deficiency would have 
become apparent during the course of the study or would have been observed in the pathological examination. The adverse effects noted at 50,000 ppm (transient reductions in growth rates in mice and reduced liver weights in female rats) were likely to be due to nutritional imbalance and are not considered relevant to human exposure. Silicon does not have significant genotoxic potential.

The Safe Upper Level of $700 \mathrm{mg} /$ day for supplemental intake of elemental silicon has been derived from a chronic dietary study in rats where no relevant adverse effects were observed at doses of up to $50,000 \mathrm{ppm}$ silica in the diet, corresponding to $2500 \mathrm{mg} / \mathrm{kg}$ bw/day. Uncertainty factors of 10 for interspecies variation and 10 for inter-individual variation have been applied. Assuming an estimated maximum intake of $60 \mathrm{mg}$ silicon from food and water, a Safe Upper Level for total silicon intake of 60 $+700=760 \mathrm{mg} /$ day can be estimated. This is equivalent to $13 \mathrm{mg} / \mathrm{kg}$ bw/day for a $60 \mathrm{~kg}$ adult

No groups particularly vulnerable to silicon toxicity have been identified.

\section{References}

Bowen, H.J.M. and Peggs, A. (1984) Determination of the silicon content of food. Journal of Science Food and Agriculture 35, 1225-1229.

Kayonga-Male, H. and Jia, X (1999). Silicon bioavailability studies in young rapidly growing rats and turkeys fed semi-purified diets. A comparative study. Biological Trace Element Research 67, 173-186.

Pennington, J.A.T. (1990) Silicon in foods and diets. Food Additives and Contaminants 8, 97-118.

Takizawa, Y, Hirasawa, F, Noritomo, E, Aida, M, Tsunoda, H, Uesugi, S. (1988). Oral ingestion of syloid to mice and rats and its chronic toxicity and carcinogenicity. Acta Medica et Biologica 36, 27-56. 


\section{Risk Assessment}

\section{Sodium chloride}

\section{General information}

No relevant data are available relating to the toxicity of the chloride ion, and therefore the EVM decided to consider sodium chloride as a salt, rather than the separate elements. SACN is currently considering salt intakes and health as part of a review and their recommendations are due to be published later this year.

\section{Chemistry}

Sodium chloride is the simple ionic salt of sodium and chlorine. It is an odourless clear or white soft crystal with a distinctive taste.

\section{Natural occurrence}

Sodium chloride occurs naturally as halite, known as rock salt, which is typically 95 to $99 \%$ sodium chloride. The principal impurity of rock salt is calcium sulphate, with smaller amounts of calcium chloride and magnesium chloride.

\section{Occurrence in food and food supplements}

The salt content of natural foods varies widely. The main sources of sodium intake (excluding table salt) in the British diet are cereals and cereal products (particularly bread) and meat and meat products. Crisps and savoury snacks tend to be high in salt and can make a significant contribution to sodium intakes. The main sources of chloride are the same as for sodium. The majority of salt intake in the UK diet comes from processed foods, with about 10\% from the natural salt content of unprocessed foods.

\section{Other sources of exposure}

No data were identified.

\section{Recommended amounts}

In adults, the RNI for sodium is $1600 \mathrm{mg} /$ day and for chloride $2500 \mathrm{mg} /$ day. COMA recommended a reduction in the average intake of sodium chloride from food by the adult population from the current level of $9000 \mathrm{mg} /$ day $(150 \mathrm{mmol} /$ day) to $6000 \mathrm{mg} /$ day (100 mmol/day) (COMA, 1994).

\section{Analysis of tissue levels, sodium and chloride status}

The steady-state concentrations of sodium and chloride in mammalian skeletal muscle are $12 \mathrm{mmol}$ per litre and $3.8 \mathrm{mmol}$ per litre in the intracellular fluid respectively and $145 \mathrm{mmol}$ per litre and $120 \mathrm{mmol}$ per litre in the extracellular fluid respectively. In nerves, depolarisation is a manifestation of $\mathrm{Na}^{+}$influx. Serum sodium levels are well maintained at approximately $140 \mathrm{mmol}$ per litre even in studies involving high or low salt diets and elderly subjects. 


\section{Brief overview of non-nutritional beneficial effects}

Sodium chloride is an essential nutrient for the normal functioning of the body. It is important for nerve conduction, muscle contraction, correct osmotic balance of extracellular fluid and the absorption of other nutrients.

\section{Function}

Sodium, together with potassium, is an essential mineral for regulating body fluid balance. Sodium is the most abundant cation in the extracellular fluid and sodium salts account for more than $90 \%$ of the osmotically active solute in the plasma and interstitial fluid. Consequently, sodium load is the major determinant of extracellular volume. Chloride is also important in maintaining the fluid balance and is an essential component of the gastric and intestinal secretions.

\section{Deficiency}

Sodium deficiency is highly unusual, but can lead to low blood pressure, dehydration and muscle cramps. A normal diet will always supply sufficient chloride.

\section{Interactions}

Patients on salt restricted diets who also take lithium carbonate, prescribed for the treatment of manic disorders, are susceptible to development of lithium toxicity. The excretion of lithium appears to be proportional to the intake of sodium and increased sodium intake can reduce both therapeutic responses to lithium and its side effects.

\section{Absorption and bioavailability}

Sodium is absorbed passively from the lumen of the entire length of the intestine. Chloride is also absorbed passively, but with decreasing efficiency along the length of the intestine and is not absorbed at all in the colon. lonic sodium can also be absorbed actively from the lumen of the small intestine and colon. Once in the intestinal epithelium it is actively transported to the interstitial fluid.

\section{Distribution and metabolism}

Sodium is the principal cation of the plasma.

\section{Excretion}

Under normal conditions, gastrointestinal and respiratory excretion of sodium is negligible and sodium is excreted primarily by the kidneys. Chloride excretion is by passive diffusion, but it also leaves the tubular lumen by active transport. 


\title{
Toxicity
}

\author{
Human data \\ Although rare, acute toxicity may be caused by ingestion of $500-1000 \mathrm{mg}$ sodium chloride $/ \mathrm{kg}$ body \\ weight. Symptoms include vomiting, ulceration of the gastrointestinal tract, muscle weakness and renal \\ damage, leading to dehydration, metabolic acidosis and severe peripheral and central neural effects.
}

Opinion is divided concerning the long-term influence of dietary sodium chloride intakes greater then $6000 \mathrm{mg}$ per day, on the development of essential hypertension. Significant increases in both systolic and diastolic blood pressures have been shown in studies in which normotensive adults have received supplementary sodium. Reduction of dietary sodium is generally recommended as a non-

pharmacological treatment for patients with essential hypertension. In normotensive adults a significant reduction in sodium intake is needed to achieve a modest reduction in blood pressure. In hypertensive patients, the most pronounced effects of dietary salt restriction have been shown to be in people aged greater than 44 years. Left ventricular hypertrophy, an important risk factor in premature cardiovascular disease, appears to be associated with high dietary sodium.

High sodium chloride intakes increase calcium excretion and may increase the risk of kidney stone formation. However, there is no substantial evidence to suggest a relationship between excess sodium chloride intake and reduced bone mineral density.

\section{Animal data}

Unilaterally nephrectomised rats treated with deoxycortisone acetate (DOCA) are used as a saltsensitive model of hypertension, whereas two kidney one clip (2K1C) rats are used as a salt-resistant hypertensive model. Using these models it has been shown that high dietary sodium chloride $(21 \%$ in chow) significantly increased systolic blood pressure in DOCA treated rats, but not in controls or 2K1C rats. The high salt diet exacerbated renal arterial and arteriolar and glomerular lesions in both the saltdependent and salt-independent hypertension models. Ad libitum feeding of rats with diets containing sodium chloride or sodium citrate, or sodium-free diet, for four weeks showed that dietary sodium restriction prevented the development of left ventricular hypertrophy.

\section{Reproductive toxicity}

In rodents, extremely high doses of sodium chloride during pregnancy caused musculoskeletal abnormalities, foetotoxicity and foetal death and post-implantation mortality and abortion. High dietary sodium chloride intakes throughout gestation and during the early life of offspring have been shown to predispose to high fluid retention in the rat. Administration of a high sodium chloride diet from conception to weaning increased adult blood pressure, whereas exposure only at weaning had no effect on blood pressure. Rats born to mothers on a normal sodium diet, but fed by females on a high sodium diet, and then weaned and raised on a high sodium diet, developed more hypertension than those only raised on a high sodium diet after weaning. Thus it appears that the transfer of sodium via maternal milk is an important factor in the development of high blood pressure in adult rodents. 


\section{Carcinogenicity and genotoxicity}

Sodium chloride has been demonstrated to be a gastric tumour promoter in experimental animals and high sodium chloride intakes have been associated with incidence of stomach cancer in human populations with traditional diets of highly concentrated, salted foods.

\section{Vulnerable groups}

It has been suggested that the blood pressure of some individuals is far more sensitive to the effects of sodium depletion or loading than others. These individuals are considered to be salt-sensitive. In a normotensive population (320 subjects) approximately $30 \%$ were shown to be salt-sensitive, irrespective of ethnic origin. Characteristics shared by 'salt-sensitive' individuals include differences in responses of the renin-angiotensin and sympathetic nervous systems, renal function, body fat distribution, insulin resistance and intracellular ion transport.

Since urinary calcium excretion increases with elevated dietary sodium chloride (by approximately 1 mmol calcium (40 mg) for each $100 \mathrm{mmol}(2300 \mathrm{mg}$ ) increase in sodium chloride), high salt intake increases susceptibility to calcium salt crystallisation in urine. In calcium stone-forming patients, high sodium chloride intake has been associated with low bone density.

Very young babies cannot excrete excess sodium chloride through their kidneys, which gives rise to the advice that salt should not be added to baby foods.

\section{Genetic variations}

It has been shown that in a hypertensive population in the USA, 73\% of black people were salt-sensitive, compared with $55 \%$ of white people. An increase in salt sensitivity with increasing age and at lower renin levels appears to be common in the three ethnic groups most commonly studied (whites, blacks and Japanese). Compared with white populations, black populations have higher blood pressure earlier in life and have a greater incidence of more severe hypertension and thus a greater risk of cardiovascular complications. Considering hypertension and associated cardiovascular complications, males are a higher risk group than females.

\section{Mechanism of toxicity}

Experiments in salt-sensitive rats indicate that increased blood pressure results initially from a combination of increased cardiac output and total peripheral resistance.

\section{Dose response characterisation}

The effect of sodium chloride on hypertension is a continuous response with no threshold. Susceptible people are affected at intakes that would not cause an effect in the rest of the population. Blood pressure is linked to salt consumption, and the magnitude of the increase in blood pressure is linked to age. 


\section{Studies of particular importance in the risk assessment:}

(For full review see http://www.food.gov.uk/science/ouradvisors/vitandmin/evmpapers or the enclosed CD)

\section{Human data}

Mascioli et al., 1991

In a randomised, double-blind cross-over trial normotensive subjects ( $n=48$, average age 52 years) were placed on a low sodium basal diet (35 mmeq sodium/day or less, approximately $800 \mathrm{mg}$ sodium/day). After six weeks acclimatisation this group received capsules containing supplementary sodium chloride (96 mmeq sodium/day, approximately $2200 \mathrm{mg}$ sodium/day) for four weeks. They then entered a twoweek washout phase, followed by a four-week placebo period. A second group had a four-week placebo period first, then a two-week washout period, which was followed by four weeks of sodium chloride capsules. Total sodium chloride intake during the treatment period was estimated to be approximately $7500 \mathrm{mg} /$ day. On average, both systolic and diastolic blood pressures increased significantly $(3.6 / 2.3 \mathrm{~mm} \mathrm{Hg})$ during the sodium chloride treatment periods, compared with the placebo control. Increases in systolic and diastolic blood pressure were recorded in $65 \%$ and $69 \%$, respectively, of study participants during the sodium chloride treatment period, compared with the placebo control.

Ganry et al., 1993

A small crossover study, of six normotensive patients (males and females), examined the effect of sodium chloride or sodium bicarbonate on blood pressure regulation. The subjects were given a basal diet containing $85 \mathrm{mmol}$ sodium chloride and each of the two treatments gave an additional $151 \mathrm{mmol}$ sodium per day for six days with similar water intake. Additional sodium chloride was given in the form of nine capsules, $9 \times 986 \mathrm{mg}=8,874 \mathrm{mg} /$ day, leading to a total sodium chloride intake of approximately $13,900 \mathrm{mg} /$ day. Sodium loading in the form of bicarbonate had no effect on blood pressure, whereas sodium chloride for the same period significantly increased the blood pressure.

\section{Exposure assessment}

Total exposure/intake:

Food

Mean: $7200 \mathrm{mg} /$ day

97.5 $5^{\text {th }}$ percentile: $13000 \mathrm{mg} /$ day (from 1986/1987 NDNS)

Note 1: this does not include salt added at the table or during cooking.

Note 2: Supplements do not contain sodium chloride, but some contain chloride at up to $72 \mathrm{mg} /$ daily dose.

Estimated maximum daily intake:13000 mg/day

No potential high intake groups have been identified. 


\section{Risk assessment}

Although rare, acute sodium chloride toxicity may be caused by ingestion of $500-1000 \mathrm{mg}$ sodium chloride/ $\mathrm{kg}$ bodyweight. Symptoms include vomiting, ulceration of the gastrointestinal tract, muscle weakness and renal damage, leading to dehydration, metabolic acidosis and severe peripheral and central neural effects. This is however, normally self-limiting.

High sodium chloride intakes increase calcium excretion and may increase the risk of kidney stone formation. There is evidence for a causal relationship between the consumption of sodium (mainly from common salt) and both blood pressure and the age-related rise in blood pressure. Data suggest that $30 \%$ of a normotensive population may be salt sensitive.

In rodents, extremely high doses of sodium chloride during pregnancy caused musculoskeletal abnormalities, foetotoxicity and foetal death and post-implantation mortality and abortion. Administration to rats of diets containing high levels of sodium chloride throughout pregnancy and during the early life of offspring have been shown to permanently alter fluid retention and to increase blood pressure in the offspring.

\section{EVM OPINION}

It is not possible to establish a Safe Upper Level for sodium chloride because there appears to be a graded response across doses that include the current estimated intake in the UK.

Increased blood pressure is seen in susceptible sectors of the population (those who are 'salt sensitive'), at intake levels that are not above average for the population as a whole. Results are available from numerous trials in hypertensive individuals, in which decreasing sodium chloride intake has been shown to have beneficial effects, by lowering blood pressure. However, the data from administration of increased levels of dietary sodium chloride are minimal. Opinion is divided concerning the long-term influence of dietary sodium chloride intakes greater then $6000 \mathrm{mg}$ per day on the development of essential hypertension. Increases in both systolic and diastolic blood pressures were observed in normotensive adults receiving $2200 \mathrm{mg} /$ day supplementary sodium, with estimate total sodium chloride intake of $7500 \mathrm{mg} /$ day. Two small trials in normotensive individuals have shown that intakes of sodium chloride of approximately $14,000 \mathrm{mg} /$ day lead to increased blood pressures. The assumption is made that similar or greater effects might follow augmentation of sodium chloride intake in hypertensive individuals.

Sodium chloride causes an increase in blood pressure at customary dietary intakes in susceptible individuals, leading to the COMA and SACN recommendations for a reduction in intake. Sodium chloride is not ordinarily suitable for use in supplements. 


\section{References}

COMA (1994). Nutritional Aspects of Cardiovascular Disease. Report of the Cardiovascular Review Group, Committee on Medical Aspects of Food and Nutrition Policy. HMSO, London.

Ganry O., Boudet J., Wargon C., Hornych A. and Meyer P. J. (1993) Effect of sodium bicarbonate and sodium chloride on arterial blood pressure, plasma renin activity and urinary prostaglandins in healthy volunteers. Journal of Hypertension 11 (suppl 5), S202-S203.

Mascioli S., Grimm Jr. R., Launer C., Svendsen K., Flack J., Gonzalez N., Elmer P. and Neaton J. (1991) Hypertension 17 (suppl. I), I-21-I-26. 


\section{EVM Statement}

\section{Sulphur}

The programme of nutrients to be considered by the EVM was drawn up to include vitamins and minerals considered to be essential and those where the evidence for essentiality was less certain but which were available as food supplements (e.g. nickel). Sulphur was included as an essential element. However, elemental sulphur is not essential per se, since the body's requirements are met via the metabolism of sulphur-containing amino acids such as cysteine and methionine obtained from dietary protein. Inorganic or elemental sulphur compounds are not available in dietary supplements.

Sulphur is present in a wide variety of compounds that occur in food. For example, as well as being naturally present in foods, sulphites, and to a lesser extent sulphates, are widely used as food additives and their safety in this context has been assessed by a variety of regulatory bodies. As noted previously, sulphur is also present in amino acids and, in other food components such as glucosolinolates. The consideration of such compounds, particularly food additives, is not within the remit of the EVM.

Elemental sulphur is used as a folk remedy. However, relatively few data in either humans or animals are available. Absorption of elemental sulphur is poor in man and other monogastrics. Absorbed sulphur is likely to be rapidly oxidised to sulphate; unabsorbed sulphur is likely to be reduced to hydrogen sulphide by gut flora.

There are a few case reports suggesting that intakes of several hundred grams of elemental sulphur are associated with symptoms such as chest pain, lethargy, confusion and, in particular, metabolic acidosis. However, in all these cases, underlying medical conditions such as renal disease and constipation may have contributed to the observed symptoms.

Few data are available on dietary sulphur intake. It has been estimated that the human diet contains approximately $1 \%$ sulphur. Assuming that adults consume $1 \mathrm{~kg}$ food/day, this would represent a sulphur intake of $10 \mathrm{~g}$ or, $143 \mathrm{mg} / \mathrm{kg}$ bw for a $70 \mathrm{~kg}$ adult. However, this intake would largely consist of sulphur containing amino acids and other food components.

In view of the scarcity of relevant data and since the majority of sulphur-containing compounds are not within its remit, sulphur has not been fully reviewed or assessed by the Group. 
Annexes 
Annexes

\section{Annex 1.}

\section{Membership of the Expert Group on Vitamins and Minerals}

\section{Chairman}

Professor M J S Langman BSC, MD, FRCP, F Medici, University of Birmingham, Birmingham

\section{Members}

Professor P Aggett OBE, MB, ChB, FRCP, MSC, DCH

University of Central Lancashire, Preston

Professor D S Davies BSc, PhD, CChem, FRSC, FRCP, FRC Path.

Imperial College School of Medicine, London

Professor A A Jackson BA, MBBCHIR, MD, FRCP

University of Southampton, Southampton

Professor B J Kirby OBE, MB, ChB, FRCP, FRCPEd

University of Exeter, Exeter

Professor A E McLean BM, BCh, PhD, FRCPath

University College, London

Professor A G Renwick OBE, BSc, PhD, DSc

University of Southampton, Southampton

Dr L Rushton BA, MSc, PhD, CStat

MRC Institute for Environment and Health, Leicester

Ms B Saunders OBE, BA

Independent Consumer Consultant, St Albans, Herts

Ms C E Shaw BSc, SRD

The Royal Marsden NHS Trust, London

Dr A J Thomas PhD, FRCP, MB, ChB

Plymouth Hospitals NHS Trust and Peninsula Medical School Universities of Exeter and Plymouth

Dr A F Williams BSC, MB, BS, D.Phil, FRCP, FRCPCH

St Georges Hospital Medical School, London 


\section{Observers}

Ms S Davies MBE BSC

Consumers Association, London

Dr M Glenville BEd, MA, PhD

Nutritional Therapist, Kent

Professor D Richardson BSc, MSc, PhD FIFST, FRSM RNutr

Specialist Consultant in Nutrition and Food Science, dprnutrition, Croydon

Dr D Shrimpton MA, PhD, F.I.Biol, FIFST

Nutrition and Food Science Consultant, Cambridge

\section{Secretariat}

Dr D Benford October 2000 to date

Ms S Church Aug 2001 to date

Dr J Greig April 2000 - September 2000

Dr D Kennedy Nov 1997 - April 1998

Ms H Lee May 1998 - May 2001

Dr L Levy May 2001 to date

Mr B Maycock May 2002 to date

Ms C Mulholland Nov 1997 - April 2000, January 2001 - August 2002

Ms S Oldreive Nov 1997 - Oct 1998

Ms J Pitt May 1999 to date

Dr A Redfern May 1998 - Jun 2001

Mr W Scriven Nov 1997 - Jun 2000

Dr N Thatcher September 1999 to date

Ms O Yeates August 2002 to date

\section{Assessors}

Dr S Amyes (Medicines Control Agency)

Dr M Donaghy (Food Standards Agency, Scotland)

Dr E Mitchell (Northern Ireland Assembly)

Dr S Reddy (Department of Health)

Dr A Wadge (Food Standards Agency)

Dr S Wall (Welsh Assembly)

Review papers not prepared by the Secretariat were provided by:

Department of Health Toxicology Unit, Imperial College

Dr C Cowles

Dr S Crosbie

Ms C Terry

Ms N Corea 


\section{Annex 2. Assessment of Information}

\section{Introduction}

The scientific data considered by the EVM in the assessment of a particular vitamin or mineral were obtained from both human and animal studies. The EVM has also considered exposure data taken from nutritional surveys conducted by UK Government Departments and from other published scientific literature.

When assessing and comparing the design and conduct of scientific investigations, a range of factors which determine the quality of the study and the reliability of the results must be considered. Formal guidelines are available for clinical trials and increasingly in other epidemiological studies, whereas in other areas assessment is more dependent on the opinion and experience of the reviewer as to the characteristics of a good study. There are many published articles available discussing the issue. This annex considers the strengths and weaknesses of different types of studies in more detail.

The authors of the EVM reviews have considered the strengths and weaknesses of study design and conduct in assessing the quality and reliability of particular studies and, where appropriate, will have included their views in both the review and risk assessment papers. EVM Members have also discussed these aspects in their assessment of the data. However, because of the diversity of the available data no formal systematic review methodology has been used during the assessment process.

\section{Human Studies}

The examination of the influence of nutrient intake upon human health may depend upon observational and interventional studies, whilst experimental studies assist characterisation of underlying metabolism, including metabolic or other aberrations. In this context, human studies therefore include nutritional and toxicological studies and may derive support from comparable studies in animals.

Some human data are available from case reports of accidental or deliberate poisonings. However, there may be difficulties in determining the precise nature of the exposure in such cases. Case reports may also be difficult to compare because of the different circumstances of exposure.

\section{Interventional trials}

These may vary from clinical trials of classic design with randomisation of individuals to receive the intervention under study and a comparator (placebo or alternative treatment), to simple field studies in which a community, or group-wide intervention is made openly and the subsequent occurrence of disease is examined. These studies also include nutritional investigations of absorption, metabolism and nutrient balance in human volunteers. 


\section{Observational studies}

Observational studies can include ecological studies, which investigate groups of individuals such as those living in a particular city or country, and studies which investigate exposure and health effects (both adverse and beneficial) in individuals in, for example, cross-sectional, cohort and case-control studies.

Ecological studies include geographical studies of spatial patterns and time trend studies. In geographical studies, the relationship between the spatial patterns of exposure (for example region, work-site or school) and disease are described. Time trend studies assess the association between changes in exposure and disease over time.

Cross-sectional studies focus on individuals at a particular point in time, i.e. they represent a 'snapshot'. They are limited to describing the frequency of the disease at that time and the variation between exposure subgroups, where exposure is also ascertained at that time. They are most useful for exposures or parameters that cannot change over time, e.g. blood group.

Cohort studies (follow-up or prospective studies) follow a group of people with a particular exposure and compare disease occurrence in the exposed group with that in a non-exposed group. Cohort studies enable rates of disease in the exposed and non-exposed populations to be estimated and any associations to be evaluated. However, they may be time consuming and costly and need large numbers of subjects where rare diseases are considered.

In case-control studies (retrospective studies), individuals with a particular condition (cases) are compared with a group of individuals without the condition (controls). Information on past exposure to possible risk factors is obtained for both cases and controls. The level of exposure in the cases is compared to that of the controls. Case-control studies are useful for studying rare diseases and can be quick and cost efficient. However, recruitment and selection of controls and exposure estimation may present problems when considered retrospectively.

\section{Bias}

Almost all epidemiological studies are subject to some degree of bias. The three most important types are:

- confounding bias

- information bias

- selection bias

Confounding occurs when another variable is both associated with the disease of interest and differently distributed across different exposure groups. Confounding factors can be adjusted for either at the design stage, e.g. matching on the factors in a case-control study, or at the analysis stage.

Information bias can occur in correctly identifying both the individuals with a disease and in allocating an exposure to an individual (e.g. recall bias). This may cause misclassification (in either disease and/or exposure classification) and can affect estimates of risk. 


\section{Annexes}

Selection bias arises in the selection of study subjects from the target study population and can be particularly problematic in case-control studies.

\section{Causality}

An association between an exposure and an effect does not necessarily mean there is a causal relationship. Case-control and cohort studies can both evaluate potential causal relationships with the magnitude of the relative risks or odds ratios giving an indication of a possible causal relationship. Evidence of a exposure-response relationship would also indicate causality. However, such results need to be considered in the context of other epidemiology studies and mechanistic or toxicological investigations.

\section{Assessing human studies}

When assessing the quality of human studies a number of points should be taken into account, though not all will apply to all studies.

- There should be a clear description of the study objectives and the hypothesis to be tested.

- The study design should be appropriate to achieve the objectives.

- There should be a clear description of the study population (including control or comparison groups if appropriate) and how the main variables were measured, in particular health effects and exposures. Validity and reliability of measurements should be addressed.

- The size of the study should be adequate to provide sufficient power to address the objectives.

- Appropriate statistical analysis methodology should be used and adequately described or referenced.

- Response or tracing rates should be reported and any potential bias through non-response discussed.

- The results should be clearly presented and include both statistical significance levels and confidence intervals if appropriate.

- Potential bias due to confounding, subject selection and data measurement should be addressed in the analyses and discussed.

- The results should be interpreted in the light of evidence from other research, both epidemiological and toxicological, including consistency, strength of association and exposure-response, and biological plausibility.

\section{Human studies considered by the EVM}

The EVM has considered a wide range of experimental, observational and interventional studies. Due to the difficulties in assessing the effects of individual micronutrients, the database is a limited one. The experimental studies have been relatively few but have included large clinical trials such as the Alpha Tocopherol-Beta Carotene (ATBC) study in male smokers. The observational studies have included investigations where populations have been exposed to trace elements such as boron or manganese in drinking water, these have generally been of poor quality, with very limited information on exposure. Other observational studies have included investigations of occupational exposure to elements such as manganese, and have generally been better conducted and reported. 
Experimental studies (such as ATBC) involved large numbers of subjects but focused on specified endpoints such as cancer or stroke and did not provide information on more minor side effects. In many studies, only a single dose level was investigated. Many of the clinical trials considered by the EVM were designed to examine the potential beneficial effects of vitamins or minerals and did not consider the issue of adverse effects in detail, if at all.

One particular difficulty has been the lack of information on total exposure to a particular nutrient, the effects of supplemental nutrients on particular parameters being investigated without quantifying the exposure from the rest of the diet.

\section{Animal and in vitro studies}

The majority of the animal data considered by the EVM are toxicity studies. However, some nutritional experiments have also been conducted in laboratory animals. These can be feeding trials to assess the effects of deficiency or of excess nutrients, studies on bioavailability or metabolic studies. When assessing such studies, the following section on assessing toxicological data covers most of the relevant issues.

\section{Types of toxicity data}

Toxicity studies in animals can be single dose (acute) studies or repeat dose studies of various lengths (generally 1 week to 2 years/lifespan) or which cover specific periods or processes during the life of the animal such as reproduction and development or cell division (for in vivo mutagenicity) studies. In vitro studies usually investigate specific tissues or cell types to investigate pre-defined end points. The most common in vitro studies investigate mutagenicity.

\section{Assessing toxicity data}

In practice, toxicity studies can be broadly divided into two categories: regulatory studies and investigative studies. Regulatory studies are conducted to assess the safety of a substance, to meet regulatory requirements prior to marketing, or in support of health and safety or environmental legislation. Regulatory studies are usually part of a standardised package and individual studies use well defined methods, and study a wide range of body tissues, without any a priori hypothesis of possible effects. Investigative studies are concerned with researching a particular issue, such as studying the mechanism of a particular effect, and have a more specialised design.

Guidelines exist for the conduct of regulatory studies. For example, the Organisation for Economic Cooperation and Development (OECD) set minimum criteria for acceptability of data under the Mutual Acceptance of Data agreement. (These apply to chemicals other than pharmaceuticals, which are covered by other harmonisation agreements). A guideline is produced for each type and/or length of study, ranging from in vitro mutagenicity studies to multi-generation animal studies. These provide specific criteria for study design and are formulated to ensure harmonisation of approach and thus mutual acceptance of data by the different regulatory agencies worldwide. Studies that comply with current OECD guidelines should be of high quality since they reflect the consensus on the most up-to-date practice. The OECD guidelines are broadly similar to the guidelines produced by the European Union which are listed in Annex V to Directive 67/548/EEC on the Classification, Labelling and 


\section{Annexes}

Packaging of Dangerous Substances. Regulatory bodies such as the US FDA also produce detailed guidelines for the conduct of regulatory studies. Guidelines for test methods used in mutagenicity studies have been produced by the United Kingdom Environmental Mutagen Society (UKEMS).

Other bodies such as the EU Scientific Committee on Food (SCF) and the UK Committees on the Toxicity, Mutagenicity and Carcinogenicity of Chemicals in Food, Consumer Products and the Environment (COT, COM and COC) have also issued guidelines. These tend to be more flexible, indicating the information or data package required rather than specifying in detail the way to obtain it. Advice has also been issued by international bodies such as the Joint WHO/FAO Expert Committee on Food Additives (JECFA).

Regulatory studies should be conducted in accordance with the principles of Good Laboratory Practice (GLP). This is a system which aims to ensure that laboratory studies are properly planned, performed, monitored and recorded. All information is recorded and tracked so that the results and conclusions can be verified. Quality assurance is an essential component of GLP. Laboratories must be registered for GLP compliance by a national authority. In the UK, GLP is regulated by the Medicines and Healthcare products Regulatory Agency, an executive agency of the Department of Health.

There are no guidelines covering investigative studies (although they may in some instances be conducted in compliance with GLP). This means that professional judgement has to be used to assess the quality of the study. A well reported study should provide enough detail of the methods used to allow the work to be reproduced by others. Data from investigative studies may be used to support or elucidate the findings of regulatory studies.

\section{Assessing animal studies}

A list of factors considered in assessing the quality of a study is given below. Other more specialised points will apply to different types of study. Not all the factors below will apply to every study and where information is missing, this does not always indicate that the study is of poor quality, though it may reduce confidence in a study.

- The methods used should be clearly stated and adequate results provided.

- The appropriate species and strain of animal should have been used.

- The numbers should be such that the study has adequate power. For example, 50 animals of each sex per dose group would be used in rodent carcinogenicity studies to ensure that an increased incidence of tumours can be identified. Fewer animals would be used for studies of shorter duration or involving non-rodent species (although in the latter case the scientific and statistical justification is not clear).

- The animals should be healthy and housed in suitable conditions. Infections may occur in laboratory animal populations and affect the results and interpretation of a study. It is important to have information on body weights and preferably on food and water consumption, since substances that affect the palatability of food or water may confuse findings by reducing intakes and causing, for example, weight loss and dehydration. Where animals have died during the study it needs to be clear whether this was scheduled or whether the animals were found dead or moribund. The cause of death should be stated as this may not always be treatment related. 
- Ideally, the doses used should cover the range of adverse effects, from no-observed effect, through subtle effects to clear effects. In long-term studies, the dose used should not exceed the maximum tolerated dose. Use of excessive doses may affect survival and thus shorten duration of treatment, as well as leaving too few survivors to assess the statistical significance of the effect. It may also produce secondary toxic effects that are of little or no relevance to lower doses. Although the dose regime should produce some indications of toxicity, feeding animals large amounts of non-toxic compounds can result in nutritional effects if the quantity of test substance in the diet results in inadequate levels of other nutrients. The limit dose for all studies is considered to be $2 \mathrm{~g} / \mathrm{kg}$ bw/day to ensure that the results are meaningful. Other limits apply to different methods of dosing such as gavage so that the animal is not harmed by the procedure. The dosing should cover the appropriate periods in the animals' life/development. This is particularly important for reproductive and development toxicity studies. It is helpful for EVM purposes when the dose is given in $\mathrm{mg} / \mathrm{kg}$ bodyweight/day.

- Appropriate statistical analyses should be conducted.

- Appropriate measures of effect, adequate pathology of all major organs (macroscopic and microscopic), biochemistry, haematology and optimised methods (especially enzyme immunoassay kits and reaction conditions for enzyme assays) should be used.

Assessing in vitro studies

Many of the points discussed above have analogies for in vitro testing. For example

- The methods used should be clearly stated and adequate results provided.

- Appropriate concentrations should be used, taking into account the possible effects of the test article on the test system (e.g. pH, osmolality, cytotoxicity etc). This also depends on the test concerned since some may require testing into the cytotoxic range. The cells or tissues should be exposed in the correct part of the cell cycle.

- Appropriate exogenous activation systems and both negative and positive controls should be used.

In addition, it is important to consider whether the tests have been validated. Test methods which are included in regulatory guidelines can be considered adequately validated. Other assays may provide supporting information, but may not be adequate in the absence of results from the accepted test battery.

\section{Interpretation}

When considering the interpretation of the study, it is important to consider whether the data support the conclusions that have been drawn. It is not possible to conclude a lack of effects from an inadequate study and the evaluation of positive effects may be confounded by poor conduct or poor reporting of a study. Where there are uncertainties due to a limited study or a poor database this may be accounted for by the use of uncertainty factors.

Other factors to consider include whether there was a dose-related response (an effect in a single group may indicate a random effect such as a statistical anomaly, a design problem or a highly specific adverse effect). 


\section{Annexes}

The findings, if any, need to be assessed for their relevance to humans. Certain effects can be either species specific or may be related to the experimental method, such as the dosing method used.

Where investigations have been published, it is worth considering whether this is as a peerreviewed article or as a letter, abstract or conference report. It is also worth noting the background and reputation of the authors in the field.

\section{Toxicity data considered by the EVM}

As noted elsewhere in this report, the available database for most of the nutrients considered by the EVM is poor, with few recent good quality toxicity studies being available for the majority. This was surprising in view of the wide availability of, and interest in, supplement products. The vast majority of studies considered by the EVM were investigative and did not comply with either GLP or regulatory guidelines. There were a few exceptions to this, such as the studies produced by the US National Toxicology Program on selenium compounds.

As the EVM considered the complete database for each vitamin and mineral, many older studies were reviewed. Though these may have been conducted in accordance with the standards prevailing at the time, they would not meet modern standards. Such data nonetheless have been considered on their merits.

\section{Exposure data}

\section{Assessing food consumption and nutrient intake in the UK}

There are currently two major survey programmes in the UK that collect data on food consumption and nutrient intake:

- National Food Survey ${ }^{23}$ which provides information about household food purchases

- National Diet and Nutrition Surveys which provides detailed information on the diets of individuals

In addition there is the Total Diet Study in which foods representative of the national diet are purchased on a continuous basis some of which are analysed for a range of food constituents including nutrients.

\section{The National Food Survey}

Household surveys use information on household food purchases to provide useful data on population trends in food expenditure, consumption and nutrient intakes. The National Food Survey (NFS) is a continuous survey of UK households over the last 50 years which continues today. The information obtained during the survey, which is published on an annual and quarterly basis, provides population average data on long term trends in food consumption and nutrient intakes per person. Results can also be broken down by various household characteristics such as region, income group and household composition. However, as the NFS is a household survey, it gives no information about age/gender sub-groups nor about the distribution of food consumption or nutrient intakes in the population.

23 The National Food Survey was replaced with the Expenditure and Food Survey from April 2001. 


\section{The National Diet and Nutrition Survey}

The National Diet and Nutrition Survey programme (NDNS) provides cross-sectional information on the dietary habits and nutritional status of nationally representative samples of specific population age groups: children aged $1 \frac{1}{2}-4 \frac{1}{2}$ years; young people aged 4-18 years; adults aged 19-64 years; adults aged 65 years and over have been published. The most recent NDNS of adults aged 19-64 years was carried out in 2000/2001. Publication of the first report of the findings is expected in Autumn 2002 with further reports to be published in 2003. Each survey collects detailed, quantitative information on food consumption and nutrient intake using a weighed dietary record, kept by the participant for four or seven days. Information on nutritional status is collected via a blood sample and physical measurements and a detailed interview provides information on socio-economic, demographic and lifestyle characteristics. Data from the NDNS provides detailed and robust food consumption data for individuals and data on diet, nutritional status and related characteristics in the same individuals to allow analysis of the links between them.

\section{The Total Diet Study}

The Total Diet Study (TDS) is a continuous study in which foods representing the average UK diet (based on the National Food Survey) are purchased, prepared and combined into 20 groups of similar foods for analysis. The TDS is widely used to estimate exposure to nonnutrients such as contaminants as well as some nutrients. It can be a useful, relatively inexpensive method for estimating average population intakes of some nutrients where food composition data are lacking. The TDS can also provide information on trends. The main drawback of the TDS is that it only provides information at population level and not for individuals.

\section{Commonly Used Methods of Dietary Assessment of Individuals}

Duplicate meal analysis and metabolic balance studies

Duplicate meal analysis is a part of metabolic balance studies and allows an accurate assessment of dietary intake with laboratory analysis of the nutrient content of the diet. An exact copy of the meal offered to the subject is prepared and analysed as are the leftovers from the subject's meal. Actual intake is thus derived. The metabolic balance study included, in addition, analysis of all output (urine, faeces and, in some cases, sweat and other body fluids). Such studies are time consuming, labour intensive, expensive and involve small numbers of subjects usually studied over periods of weeks. Metabolic balance studies allow calculation of intake, output and retention of nutrients and have been used to estimate body requirements.

\section{Weighed record}

The subject weighs and keeps a diary record of each food item immediately before consumption and all plate waste at the end of each meal, usually for 4-7 days. This methodology is time-consuming and demanding of the subjects and so is prone to underreporting. However, weighed intake records are considered one of the most accurate methods of assessing current food intake. 


\section{Annexes}

Food frequency questionnaire (FFQ)

The Food Frequency Questionnaire is a questionnaire in which the respondent is presented with a list of foods and is required to record how often each food is eaten in broad terms such as $x$ times per day/per week/per month etc. The foods listed are usually chosen for the specific purposes of the study so that the FFQ may not assess the total diet. Assessment of the quantities of each food consumed on each eating occasion/day may also be included. The advantages of this method are that it is quick and easy to complete; it may be the only suitable method for large scale epidemiology studies. The main disadvantage is that it can only provide imprecise estimates of usual food consumption or nutrient intake for both individuals and groups. Because of the imprecision of this method it is better used with groups. However, the FFQ can be used as a cross-check for other methods for individual intakes to ensure that consumption of certain foods is not overlooked.

\section{Diet histories}

Diet histories provide a retrospective estimate of 'usual' consumption. The respondent is questioned about 'typical' or 'usual' food intake by a trained interviewer with the aim of constructing a typical seven day eating pattern. The diet history may be preceded by a 24hour recall, and/or supplemented with a checklist of foods usually consumed. This method is fairly quick to carry out and involves little work for the subject. The disadvantages are that it is inaccurate, especially regarding quantities consumed, and relies on the memory of the subject.

\section{4-hour recall}

The respondent is asked, through a systematic repetition of open-ended questions, to recall and describe the types and quantity of all food and drink consumed in the 24 hours prior to the interview. For assessment of individual diets, multiple 24-hour recall may be needed depending on the level of precision required. The main advantage of this method is that it is relatively quick to carry out and involves little work for the respondent. The disadvantages relate to inaccuracy in the number of foods recalled, the quantities, and an inability to estimate the habitual diet of individuals.

\section{Estimated record}

Estimated records require a diary recording all food and drink consumed but foods are not weighed. Instead, amounts of foods and drinks consumed are estimated, usually with portions described in household measures, with or without the aid of photographs or models. This method is easier to complete than a weighed record so there is less risk of under-reporting or changing food consumption. The disadvantages are that descriptions of quantities tend to be inaccurate and that processing such descriptive data is very time-consuming. 


\section{Data interpretation}

All methods of estimating the amounts of food consumed are associated with some degree of error, some sources of which are discussed above. For example:

- the dietary recording period may not be long enough to provide information about foods that are consumed only occasionally.

- subjects may mis-report foods consumed or change their habitual diet during the recording period.

For these reasons, caution is needed in the interpretation of dietary survey data.

\section{Survey data considered by the EVM}

The EVM has considered data from a range of nutritional surveys. The quality of the nutritional surveys has been generally good, though for some of the less common minerals, the data have been sparse. However, even the data from some of the most detailed surveys (such as the NDNS adult survey) may no longer be completely up to date because of the significant changes in eating habits and the increase in supplement use which has taken place in the years since the survey was conducted. Other nutritional studies assessed by the EVM have been of variable quality. 


\section{Annex 3. Information submitted to the EVM}

The following information was submitted to the EVM by interested parties.

\begin{tabular}{|c|c|c|c|}
\hline Who Sent Paper & Date Received & Author of Paper & Title of Paper \\
\hline J. Hathcock & 25 June 1999 & Hathcock (et al.) & $\begin{array}{l}\text { Presentation on chromium picolinate } \\
\text { picolinate safety }\end{array}$ \\
\hline J. Hathcock & 25 June 1999 & Hathcock (et al.) & $\begin{array}{l}\text { Safety issues for chromium and chromium } \\
\text { picolinic acid }\end{array}$ \\
\hline J. Hathcock & 25 June 1999 & Hathcock (et al.) & Vitamins and minerals: efficacy and safety \\
\hline J. Hathcock & 25 June 1999 & Hathcock (et al.) & Safety evaluation of vitamins and minerals \\
\hline J. Hathcock & 25 June 1999 & Hathcock (et al.) & $\begin{array}{l}\text { Safety limits for nutrient intakes: concepts } \\
\text { and data requirements }\end{array}$ \\
\hline J. Hathcock & 25 June 1999 & Hathcock (et al.) & $\begin{array}{l}\text { Toxicity of unmodified and time-release } \\
\text { preparations of niacin }\end{array}$ \\
\hline J. Hathcock & 25 June 1999 & Hathcock (et al.) & $\begin{array}{l}\text { Oral cobalamin for treatment of } \\
\text { pernicious anemia? }\end{array}$ \\
\hline J. Hathcock & 25 June 1999 & Hathcock (et al.) & Evaluation of vitamin A toxicity \\
\hline J. Hathcock & 25 June 1999 & Hathcock (et al.) & $\begin{array}{l}\text { Micronutrient safety micronutrients and } \\
\text { immune functions }\end{array}$ \\
\hline J. Lunec & 08 July 1999 & Lunec & $\begin{array}{l}\text { Novel antibody-based technology for } \\
\text { studying the potential ameliorating effects of } \\
\text { vitamin E and vitamin C on in vivo oxidative } \\
\text { stress }\end{array}$ \\
\hline M.T. Deans & 17 August 1999 & Deans & Selenium \\
\hline $\begin{array}{l}\text { J. Rainer, } \\
\text { Borax Europe Ltd }\end{array}$ & 22 September 1999 & & $\begin{array}{l}\text { Boron and its compounds; advances in boron } \\
\text { essentiality research; reproductive and general } \\
\text { toxicology of some inorganic borates and risks } \\
\text { assessment for human beings }\end{array}$ \\
\hline $\begin{array}{l}\text { D. Godfrey, } \\
\text { Roche Vitamins }\end{array}$ & 27 September 1999 & & Homocysteine metabolism \\
\hline J. Lunec & 01 November 1999 & Lunec & as 001 (above) \\
\hline $\begin{array}{l}\text { S. Bennett, } \\
\text { Nature's Own }\end{array}$ & 16 November 1999 & Nature's Own & $\begin{array}{l}\text { List of Published Biological Studies Performed } \\
\text { on Re-natured Vitamins, Minerals and Trace } \\
\text { Elements (Main Part, Part } 1 \text { and Part 2) }\end{array}$ \\
\hline M.Glenville & 29 February 2000 & $\begin{array}{l}\text { Berg G, Kohlmeier L } \\
\text { and Brenner } \mathrm{H}\end{array}$ & $\begin{array}{l}\text { Effect of oral contraceptive progestins on } \\
\text { serum copper concentration }\end{array}$ \\
\hline M. Glenville & 29 February 2000 & Webb J L & $\begin{array}{l}\text { Nutrition effects of oral contraceptive use: a } \\
\text { review [Review] [92 refs] }\end{array}$ \\
\hline M. Glenville & 29 February 2000 & $\begin{array}{l}\text { Newhouse I J, } \\
\text { Clement D B, Lai C }\end{array}$ & $\begin{array}{l}\text { Effects of iron supplementation and } \\
\text { discontinuation on serum copper, zinc, } \\
\text { calcium and magnesium }\end{array}$ \\
\hline C.A. John & 24 July 2000 & C A John & Vitamin B6 and pyridoxine supplementation \\
\hline P. Aggett & 05 January 2000 & Rainey CJ et al., 1999 & Daily boron intake from the American diet \\
\hline H. Fullerton & 30 October 2000 & H Fullerton & Vitamin B6 \\
\hline
\end{tabular}




\section{Annex 4. Current Usage of Vitamin and Mineral Supplements in the UK ${ }^{24}$}

\section{Introduction}

The data that follow have been provided by the manufacturers of vitamin and mineral supplements (VMS) sold as food supplements under food law in the UK. It does not include vitamin and mineral supplements sold as medicines, either on prescription or 'over the counter' (OTC). The associations which have provided the data are:

- Council for Responsible Nutrition (CRN)

- Health Food Manufacturers' Association (HFMA)

- Proprietary Association of Great Britain (PAGB)

All the products and sales reported here have been manufactured in the UK under the industry's self-regulatory code of 'Good Manufacturing Practice' (GMP).

The data provided are the best that the associations can provide and have been assembled from information provided by individual member companies. The data do not include products that are imported into the country by non-members. Inevitably there could be some products not represented by the summary tables that follow. Nevertheless, it is reasonable to conclude that the data which follow provide a reliable guide to the content and nature of the food supplement industry in the UK.

The information is organised into sections as follows:

- Size and structure of the UK market;

- Individual products - declared levels and sales in 1998 and 1999;

- Products formulated for children;

- Manufacturing practice;

- Chemical forms of nutrients used in product manufacture;

- Appendix: Dietary Reference Values and Recommended Daily Allowances.

\section{Size and structure of the UK market}

A Mintel Study of 1999 (courtesy of a VMS manufacturer) reported that the retail sales values of VMS in 1998 and 1999 were $£ 347$ million and $£ 326$ million respectively.

A 'Taylor Nelson Superpanel' report, prepared for the Council for Responsible Nutrition (CRN), summarised the sources of VMS, their use in the animal feed and food industries, and the routes of sale of supplements in the UK. The market values quoted are from ex-factory stock and will, in some cases exclude the costs of packing and labelling. 
In 1998 comparable amounts of VMS were sold from pharmacies/drug stores and from grocery/health stores.

\section{Individual products - declared levels and sales: 1998 \& 1999}

These are summarised in Tables 1, 2 and 3 from an amalgamation of the information provided by the associations. It is reasonable to conclude that data quoted are representative of at least $70 \%$ of the UK market for vitamin and mineral supplements (VMS) sold under food law. The values quoted have been calculated from these data to provide an average annual estimate of sales of VMS.

The sales volumes for micronutrients sold individually can be totalled. The corresponding data for micronutrients sold in combination cannot be totalled because of the many permutations of blends of the same micronutrients that comprise combination products, which could result in duplication of data within the final figures.

The lowest and highest declared levels quoted cannot be used to predict the limits from sales from other sources and it is possible that, from time to time, products will be marketed with higher declared levels than those quoted here by the minority of companies that are not members of the associations and hence are not subject to their self regulatory standards of Good Manufacturing Practice.

The data does not include products sold by e-commerce but may include some sold by mail order. Whilst both sectors are expected to grow, currently both are small relative to conventional sales through retail outlets.

Median data are not available and for this reason the products have been listed with their lowest and highest unit declared levels together with total sales for all declared levels in millions units (1 unit is one tablet or capsule).

For information, tables 1-3 include the Reference Nutrient Intake (RNI) for adult males aged 1950 years and the Recommended Daily Allowance (RDA) ${ }^{25}$. However, for some nutrients COMA could not set an RNI as there were insufficient reliable data on human requirements to do so. In some instances they set a Safe Intake ${ }^{26}$. Additionally, RDAs are only set for some vitamins and minerals. Where no RNI or RDA appears in the table, no such figure exists.

\footnotetext{
25 Dietary Reference Values are not a single figure and they change with age and gender and between EU Member States. This level of detail is inappropriate for food labelling, as it is impractical to provide more than a single reference number for intake. An earlier convention was to use the highest number from the range as the 'labelling RDA'. More recently the adult male value has been favoured. Within the EU the numbers to be used for 'nutritional labelling' are defined in a Council Directive of 1990 (10). These are the numbers that appear under the heading 'RDA' on the labels of nutritional supplements, as well as on all other pre-packaged foods, and are used in Tables 1 \& 3. An EU labelling RDA is not available for all nutrients. For infants and young children Labelling Reference Values (LRV) have been set for foods intended for infants and young children in Schedule 5 of the The Processed Cereal-based Foods and Baby Foods for Infants and Young Children 1997. The Labelling Reference Values are based on Population Reference Intakes for infants and children aged 6 months to 3 years.

26 A level or range of intake at which there is no risk of deficiency, and below a level where there is a risk of undesirable effects.
} 
Table 1. Fat soluble vitamins: declared levels and annual sales (millions of units)

\begin{tabular}{|c|c|c|c|c|c|c|c|c|c|c|c|}
\hline \multirow[b]{2}{*}{ Vitamin } & \multirow[b]{2}{*}{ Unit } & \multirow[b]{2}{*}{ RNI } & \multirow[b]{2}{*}{ RDA } & \multicolumn{4}{|c|}{ Single nutrient products } & \multicolumn{4}{|c|}{ Multiple Nutrient Products } \\
\hline & & & & Lowest & Highest & $\begin{array}{l}\text { Most } \\
\text { common }\end{array}$ & Sales & Lowest & Highest & $\begin{array}{l}\text { Most } \\
\text { common }\end{array}$ & Sales \\
\hline Vitamin A & $\mu g$ & 700 & 800 & 2250 & 2400 & $2250-2400$ & 2.1 & 200 & 2300 & $750-800$ & 1053 \\
\hline ß-carotene & mg & & & 1.3 & 15 & $1.35-15$ & 4.4 & 0.4 & 20 & $3-15$ & 270 \\
\hline Vitamin D & $\mu g$ & & 5 & 10 & 10 & 10 & 1.1 & 1.25 & 12.5 & 5 & 1216 \\
\hline Vitamin E & mg & $4^{*}$ & 10 & 50 & 670 & $200-670$ & 79 & 2 & 268 & $10-40$ & 564 \\
\hline Vitamin K & $\mu g$ & $70^{*}$ & & \multicolumn{4}{|c|}{ not available as single nutrient supplement } & 10 & 200 & $30-95$ & 137 \\
\hline
\end{tabular}

Key: $\quad$ RNI Reference Nutrient Intake for adult males aged 19-50 years

RDA is that used in the EU Directive of 1990

* $\quad$ Safe Intake - see text (vitamin K based on $70 \mathrm{~kg}$ male)

Notes: Most common declared level is that used on a daily basis unless otherwise stated

Annual sales are in millions of units (1 unit is 1 tablet or 1 capsule) are the average of those for 1998 and 1999

Table 2. Water soluble vitamins: declared levels and annual sales (millions of units)

\begin{tabular}{|c|c|c|c|c|c|c|c|c|c|c|c|}
\hline \multirow[b]{2}{*}{ Vitamin } & \multirow[b]{2}{*}{ Unit } & \multirow[b]{2}{*}{ RNI } & \multirow[b]{2}{*}{ RDA } & \multicolumn{4}{|c|}{ Single Nutrient Products } & \multicolumn{4}{|c|}{ Multiple Nutrient Products } \\
\hline & & & & Lowest & Highest & $\begin{array}{l}\text { Most } \\
\text { common }\end{array}$ & Sales & Lowest & Highest & $\begin{array}{l}\text { Most } \\
\text { common }\end{array}$ & Sales \\
\hline Thiamin & mg & 0.9 & 1.4 & 50 & 100 & - & 8 & 0.3 & 100 & $1.4-39$ & 514 \\
\hline Riboflavin & $\mathrm{mg}$ & 1.3 & 1.6 & 100 & 100 & 100 & 0.8 & 0.2 & 100 & $1.6-50$ & 548 \\
\hline Nicotinamide & mg & 16 & 18 & 100 & 250 & - & $\mathrm{n} / \mathrm{a}$ & 0.25 & 150 & $18-50$ & 175 \\
\hline Nicotinic acid & mg & 16 & 18 & \multicolumn{4}{|c|}{ not available as single supplement } & 2 & 100 & 10 & 375 \\
\hline Pyridoxine & $\mathrm{mg}$ & 1.4 & 2 & 10 & 100 & $10-80$ & 18.5 & 0.35 & 100 & $2-82$ & 391 \\
\hline Folic acid & $\mu g$ & 200 & 200 & 400 & 800 & 400 & 13.7 & 0.7 & 500 & $200-500$ & 414 \\
\hline $\begin{array}{l}\text { Cyano- } \\
\text { cobalamine }\end{array}$ & $\mu g$ & 1.5 & 1 & 5 & 1000 & 100 & 4.7 & 0.5 & 3000 & $1-50$ & 433 \\
\hline Biotin* & $\mu g$ & $10-200$ & 150 & 300 & 1000 & 300 & 1.04 & 0.5 & 2000 & $50-150$ & 12 \\
\hline $\begin{array}{l}\text { Pantothenic } \\
\text { acid* }\end{array}$ & $\mathrm{mg}$ & $3-7$ & 6 & 50 & 550 & 200 & 1.8 & 0.7 & 140 & $6-50$ & 250 \\
\hline Vitamin C & $\mathrm{mg}$ & 40 & 60 & 60 & 3000 & 1000 & 252 & 10 & 1000 & $50-150$ & 522 \\
\hline
\end{tabular}

Key: $\quad$ RNI Reference Nutrient Intake for adult males aged 19 to 50 years

RDA is that used in the EU Directive of 1990

* $\quad$ Safe Intake - see text (vitamin $\mathrm{K}$ based on $70 \mathrm{~kg}$ male)

$\mathrm{n} / \mathrm{a}$ data on sales not available

Notes: Most common level is that used on a daily basis unless otherwise stated

Annual sales are in millions of units (1 unit is 1 tablet or 1 capsule) are the average of those for 1998 and 1999

Nutritional Supplements (VMS) containing cobalt, fluoride or germanium are not sold by members of the CRN, HFMA or PAGB as food supplements.

Zinc at $50 \mathrm{mg}$ is sold for short term use, usually from outlets with trained staff. 


\section{Annexes}

\section{Products formulated for children}

The declared levels of the formulations for children are given in Tables 4, 5 and 6 . Their source is dominantly from two large companies but, although there may be other products on the market, especially from imports, it is the most representative summary that is currently available.

There are few, if any, single product brands and no data are available on the sales volumes of the multi-brands. Their volume, in relation to the total market, is small and is of the order of $12 \%$ of the sales of multi-brands for adults.

The RNIs used in Tables 4-6 are those for infants and children aged 7 months to 10 years. There are no labelling RDAs for children. Labelling Reference Values for infants and young children have been set and are laid down in Schedule 5 of The Processed Cereal-based Foods and Baby Foods for Infants and Young Children Regulations 1997. The Labelling Reference Values are based on Population Reference Intakes for children aged between 6 months and 3 years. Where no RNI or LRV appears in the table no such figure exists.

Table 3. Minerals and trace minerals: lowest and highest declared levels and annual sales (millions of units)

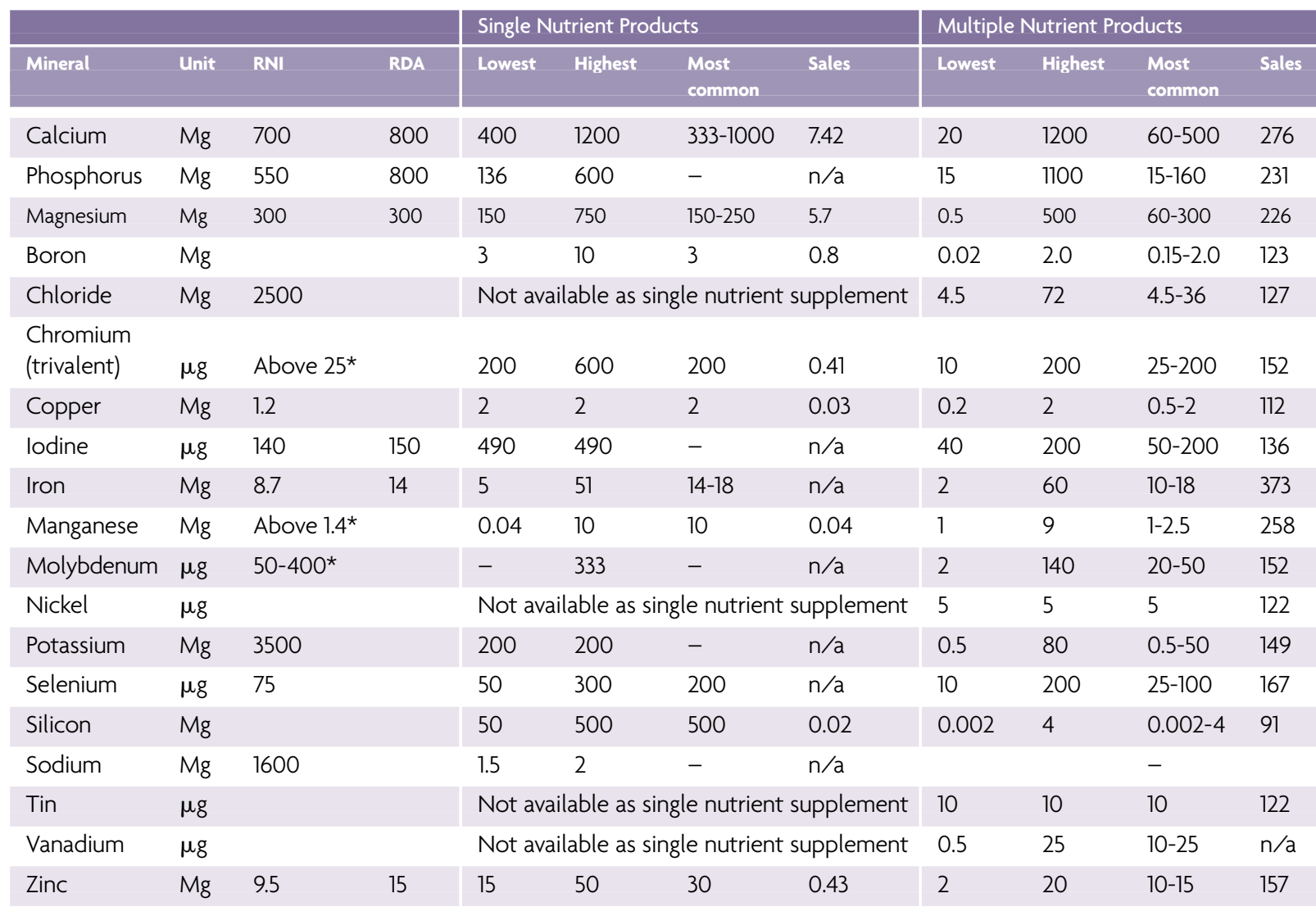

$\mathrm{n} / \mathrm{a}$ - data on sales not available

* Safe Intake 
Table 4. Fat soluble vitamins: declared levels in products formulated for children

\begin{tabular}{|llll|lll|}
\hline & & & & \multicolumn{3}{|l|}{ Multiple Nutrient Products } \\
\hline Vitamin & Unit & RN|** & LRV** & Lowest & Highest & Most common \\
\hline Vitamin A & $\mu g$ & $350-500$ & 400 & $100 \#$ & 600 & 600 \\
\hline B-carotene & $\mathrm{Mg}$ & & & 0.75 & 2.5 & $0.75-2.5$ \\
\hline Vitamin D & $\mu g$ & $7-8.5$ & 10 & $1.75 \#$ & 10 & $5-10$ \\
\hline Vitamin E & $\mathrm{Mg}$ & $0.4 \mathrm{mg} / \mathrm{g}$ PUFAs* & & $1 \#$ & 12 & 10 \\
\hline Vitamin K & $\mu g$ & $10^{*}$ & & 1 & 10 & $1-10$ \\
\hline
\end{tabular}

Key: $\quad$ RNI Reference Nutrient Intake for adult males aged 19 to 50 years LRV is Labelling Reference Value

* Safe intake

** There are no labelling RDAs for children; instead, the Labelling Reference Values from The Processed Cereal-based Foods and Baby Foods for Infants and Young Children 1997 have been used. These are based on the PRIs for infants and children aged 6 months to 3 years (4). The RNIs used in this table are for infants and children aged 7 months to 10 years. The \# indicates a syrup formulated for infants aged 4-6 months.

Notes: Declared levels are the total content of micronutrient in one unit (tablet or capsule) Most common declared level is that used on a daily basis unless otherwise stated

Table 5. Water soluble vitamins: declared levels in products formulated for children

\begin{tabular}{|c|c|c|c|c|c|c|}
\hline \multirow{2}{*}{ Vitamin } & \multirow[b]{2}{*}{ Unit } & \multirow[b]{2}{*}{ RNI** } & \multirow[b]{2}{*}{ LRV** } & \multicolumn{3}{|c|}{ Multiple Nutrient Products } \\
\hline & & & & Lowest & Highest & Most common \\
\hline Thiamin & $\mathrm{mg}$ & $0.2-0.7$ & 0.5 & $0.15 \#$ & 2.5 & 0.9 \\
\hline Riboflavin & $\mathrm{mg}$ & $0.4-1.0$ & 0.8 & $0.2 \#$ & 2.5 & 1.2 \\
\hline Nicotinamide & $\mathrm{mg}$ & $4-12$ & 9 & $2.5 \#$ & 15 & $13.5-15$ \\
\hline Pyridoxine & $\mathrm{mg}$ & $0.3-1.0$ & 0.7 & $0.175 \#$ & 2.5 & $0.86-1.2$ \\
\hline Folic acid & $\mu g$ & $50-150$ & 100 & 25 & 300 & $200-300$ \\
\hline $\begin{array}{l}\text { Cyano- } \\
\text { cobalamine }\end{array}$ & $\mu g$ & $0.4-1.0$ & 0.7 & 0.5 & 4.5 & $1.2-4.5$ \\
\hline Biotin & $\mu g$ & $10-200^{*}$ & & 75 & 150 & 150 \\
\hline $\begin{array}{l}\text { Pantothenic } \\
\text { acid }\end{array}$ & $\mathrm{mg}$ & $1.7^{*}$ & & $0.665 \#$ & 10 & $2-4.6$ \\
\hline Vitamin C & $\mathrm{mg}$ & $25-30$ & 25 & $12.5 \#$ & 60 & $35-60$ \\
\hline Inositol & $\mathrm{mg}$ & & & 5 & 5 & 5 \\
\hline
\end{tabular}

Key: $\quad$ RNI Reference Nutrient Intake for adult males aged 19 to 50 years LRV is Labelling Reference Value

* Safe intake

** There are no labelling RDAs for children instead the Labelling Reference Values from The Processed Cereal-based Foods and Baby Foods for Infants and Young Children 1997 have been used. These are based on the PRIs for infants and children aged 6 months to 3 years (4). The RNIs used in this table are for infants and children aged 7 months to 10 years. The \# indicates a syrup formulated for infants aged 4-6 months.

Notes: Declared levels are the total content of micronutrient in one unit (tablet or capsule) Most common declared level is that used on a daily basis unless otherwise stated 
Annexes

Table 6. Minerals and trace minerals: declared levels in products formulated for children

\begin{tabular}{|c|c|c|c|c|c|c|}
\hline & & & & Multip & trient Prc & \\
\hline Mineral & Unit & $\mathrm{RNI}^{*}$ & LRV* & Lowest & Highest & Most common \\
\hline Calcium & mg & $525-550$ & 400 & 100 & 134 & 120 \\
\hline Phosphorus & $\mathrm{mg}$ & $400-450$ & & 60 & 60 & 60 \\
\hline Magnesium & $\mathrm{mg}$ & $75-200$ & & 45 & 50 & 50 \\
\hline Boron & mg & & & 5 & 5 & 5 \\
\hline Chloride & mg & $500-1800$ & & - & - & - \\
\hline $\begin{array}{l}\text { Chromium } \\
\text { (trivalent) }\end{array}$ & $\mu g$ & & & 5 & 15 & $7.5-15$ \\
\hline Copper & $\mathrm{mg}$ & $0.3-0.7$ & 0.4 & 0.5 & 0.8 & $0.5-0.8$ \\
\hline lodine & $\mu g$ & $60-110$ & 70 & 25 & 130 & $37.5-130$ \\
\hline Iron & $\mathrm{mg}$ & $7.8-8.7$ & 6 & 2.8 & 11.3 & $7.5-11.3$ \\
\hline Manganese & $\mathrm{mg}$ & & & 0.32 & 0.5 & $0.32-0.5$ \\
\hline Molybdenum & $\mu g$ & & & 5 & 25 & $5-25$ \\
\hline Nickel & $\mu g$ & & & 1 & 1 & 1 \\
\hline Potassium & $\mathrm{mg}$ & $700-2000$ & & - & - & - \\
\hline Selenium & $\mu g$ & $10-30$ & 10 & 7.5 & 45 & $7.5-45$ \\
\hline Silicon & $\mathrm{mg}$ & & & - & - & - \\
\hline Sodium & $\mathrm{mg}$ & $320-1200$ & & - & - & - \\
\hline Tin & $\mu g$ & & & 1 & 1 & 1 \\
\hline Vanadium & $\mu g$ & & & 2.5 & 2.5 & 2.5 \\
\hline Zinc & $\mathrm{Mg}$ & $5.0-7.0$ & 4 & 3.75 & 9 & $3.75-9$ \\
\hline
\end{tabular}

Key: $\quad$ RNI Reference Nutrient Intake for adult males aged 19 to 50 years

LRV is Labelling Reference Value

* There are no labelling RDAs for children instead the Labelling Reference Values from The Processed Cereal-based Foods and Baby Foods for Infants and Young Children 1997 have been used. These are based on the PRIs for infants and children aged 6 months to 3 years (4). The RNIs used in this table are for infants and children aged 7 months to 10 years.

Notes: Declared levels are the total content of micronutrient in one unit (tablet or capsule) Most common declared level is that used on a daily basis unless otherwise stated

\section{Manufacturing Practice}

The majority of supplement manufacturers in the United Kingdom follow the 'Guidelines for Good Manufacturing Practice for Manufacturers of Food Supplements'.

The manufacturing processes for food supplements of tableting or encapsulating can, in some instances, result in the destruction of some of the active components of the product. Where this is likely it is customary to over-formulate so that the product at the point of sale fulfils the claim for content that is displayed on the label.

There is no consolidation of formulation practice for the whole industry, but the example which follows is a reasonable indication of current practice in the UK. 
Table 7. Over formulation

\begin{tabular}{ll}
$\begin{array}{ll}\text { Over-formulation (expressed as a } \\
\text { percentage of label declaration }\end{array}$ & Nutrient \\
\hline $30-100$ & Vitamin A \\
\hline 50 & Vitamin B12 \\
$30-50$ & Vitamin D2 \\
\hline 30 & Vitamin D3, Folic acid \\
& Vitamin B1, Biotin, B-Carotene, Vitamin K \\
\hline 5 & Vitamin B2, Vitamin B3, Vitamin B6, Vitamin C \\
\hline 0 & Vitamin E \\
\hline
\end{tabular}

\section{Chemical forms of nutrients used in product manufacture}

Nutrient

\section{Chemical form}

Fat soluble vitamins:

$\begin{array}{ll}\text { A } & \text { Esters of organic acids. } \\ D & \text { Pure vitamin. } \\ \text { E } & \text { Pure vitamin, mixture of isomers, ester. } \\ K & \text { Pure vitamin. }\end{array}$

Nutrient Chemical form

Water soluble vitamins:

B group Pure vitamin, acid salts, phosphate (vitamin $B_{2}$ ).

C Pure vitamin, sodium ascorbate, ester.

Trace minerals Inorganic salts, organic salts, amino acid chelates, biologically within yeasts, oxides.

Calcium \& phosphorus Dicalcium phosphate, tricalcium phosphate, calcium carbonate.

The choice of chemical form is influenced by many factors including: the tradition within a particular factory, demonstrable advantage in terms of bioavailability, taste or colour, space in the formulation and cost. 
Annexes

\section{References}

1. Guidelines for Good Manufacturing Practice for Manufacturers of Food Supplements. Council for Responsible Nutrition, Thames Ditton, 1997.

2. Report on Health and Social Subjects No.41, 1991. Dietary Reference values for Food Energy and Nutrients for the United Kingdom. HMSO, London.

3. Council Directive of 24 September 1990 on Nutrition labelling for Foodstuffs (90/496/EEC). Office for Official Publications of the European Communities, Luxembourg.

4. Reports of the Scientific Committee for Food (Thirty-first series). Commission of the European Communities, 1993. 


\section{Annex 5. Members' Interests.}

\section{Expert Group on Vitamins and Minerals Members}

\section{Declaration of Interests}

\begin{tabular}{|c|c|c|c|c|}
\hline & \multicolumn{2}{|c|}{ Personal Interest } & \multicolumn{2}{|c|}{ Non-personal Interest } \\
\hline Member & Company & Nature of Interest & Company & Nature of Interest \\
\hline $\begin{array}{l}\text { Prof Michael J Langman } \\
\text { (CHAIRMAN) } \\
\text { Professor of Medicine, } \\
\text { University of } \\
\text { Birmingham }\end{array}$ & None & & $\begin{array}{l}\text { Novartis } \\
\text { Merck Sharp \& Dohne } \\
\text { Astra Zeneca }\end{array}$ & $\begin{array}{l}\text { All these refer to } \\
\text { research support and } \\
\text { collaborations. } \\
\text { None in the field of } \\
\text { vitamins and minerals }\end{array}$ \\
\hline $\begin{array}{l}\text { Prof Peter Aggett } \\
\text { Head Postgraduate } \\
\text { School of Medicine and } \\
\text { Health, University of } \\
\text { Central Lancashire }\end{array}$ & $\begin{array}{l}\text { Nestlé } \\
\text { Unilever } \\
\text { Borax }\end{array}$ & $\begin{array}{l}\text { Lecture Fees } \\
\text { Lecture Fees } \\
\text { Lecture fees }\end{array}$ & $\begin{array}{l}\text { DSM-GB } \\
\text { Meat and Livestock } \\
\text { Commission } \\
\text { Abbot } \\
\text { Roche } \\
\text { Numicon }\end{array}$ & $\begin{array}{l}\text { All these refer to } \\
\text { support for } \\
\text { departmental research } \\
\text { or consultancy }\end{array}$ \\
\hline $\begin{array}{l}\text { Prof Donald S Davies } \\
\text { Head of Clinical } \\
\text { Pharmacology, } \\
\text { Director of DH } \\
\text { Toxicology Unit, } \\
\text { Faculty of Medicine, } \\
\text { Imperial College, } \\
\text { London }\end{array}$ & $\begin{array}{l}\text { ICI Plc } \\
\text { ML Laboratories } \\
\text { Astrazeneca PLC } \\
\text { SKB } \\
\text { Servier Labs }\end{array}$ & $\begin{array}{l}\text { Shareholding } \\
\text { Executive Director } \\
\text { Shareholding } \\
\text { Consultancy } \\
\text { Consultancy }\end{array}$ & $\begin{array}{l}\text { Astrazeneca } \\
\text { Bayer Plc } \\
\text { Bristol Myers Squibb } \\
\text { Curis (USA) } \\
\text { Glaxo Wellcome } \\
\text { Lilly Research } \\
\text { Merck Sharp \& Dohme } \\
\text { ML Laboratories } \\
\text { Orion-Farmos } \\
\text { Pfizer } \\
\text { Rhone-Poulenc Rorer } \\
\text { Roche } \\
\text { Sanofi Winthrop } \\
\text { Schwarz Pharma } \\
\text { Servier } \\
\text { Smithkline Beecham } \\
\text { Solvay }\end{array}$ & $\begin{array}{l}\text { All of these interests } \\
\text { relate to either } \\
\text { commissioned research, } \\
\text { meeting support or } \\
\text { advisory work }\end{array}$ \\
\hline $\begin{array}{l}\text { Prof Alan A Jackson } \\
\text { Professor of Human } \\
\text { Nutrition, University } \\
\text { of Southampton }\end{array}$ & None & & $\begin{array}{l}\text { Vitaflo } \\
\text { Hoffman la Roche } \\
\text { Unilever } \\
\text { Nutricia Research } \\
\text { Foundation }\end{array}$ & $\begin{array}{l}\text { Research support } \\
\text { Research support } \\
\text { Research support } \\
\text { Research Support }\end{array}$ \\
\hline $\begin{array}{l}\text { Prof Brian J Kirby } \\
\text { Professor of Medicine } \\
\text { University of Exeter } \\
\text { Consultant Physician } \\
\text { Royal Devon and } \\
\text { Exeter Hospitals }\end{array}$ & None & & None & \\
\hline
\end{tabular}




\section{Members (continued)}

\begin{tabular}{|c|c|c|c|c|}
\hline \multirow[b]{2}{*}{ Member } & \multicolumn{2}{|c|}{ Personal Interest } & \multicolumn{2}{|c|}{ Non-personal Interest } \\
\hline & Company & Nature of Interest & Company & Nature of Interest \\
\hline $\begin{array}{l}\text { Prof André McLean } \\
\text { Professor of Toxicology, } \\
\text { University College, } \\
\text { London }\end{array}$ & $\begin{array}{l}\text { Smith \& Nephew Ltd } \\
\text { 3M Pharmaceuticals } \\
\text { Glaxo Wellcome } \\
\text { Brittania } \\
\text { Pharmaceuticals } \\
\text { Charterhouse Clincal } \\
\text { Research Ltd } \\
\text { Galen Pharmaceuticals } \\
\text { Ltd } \\
\text { Esteve Pharmaceticals, } \\
\text { Spain } \\
\text { Omrix Pharmaceuticals } \\
\text { Ajinomoto } \\
\text { Pharmaceuticals }\end{array}$ & $\begin{array}{l}\text { Consultancy } \\
\text { Consultancy } \\
\text { Shareholding } \\
\text { Commissioned work } \\
\text { Commissioned work } \\
\text { Commissioned work } \\
\text { Commissioned work } \\
\text { Commissioned work } \\
\text { Commissioned work }\end{array}$ & & \\
\hline $\begin{array}{l}\text { Prof Andrew G Renwick } \\
\text { Professor of Biochemical } \\
\text { Pharmacology, } \\
\text { University of } \\
\text { Southampton }\end{array}$ & $\begin{array}{l}\text { International } \\
\text { Sweeteners Association } \\
\text { Neste }\end{array}$ & $\begin{array}{l}\text { Consultant } \\
\text { Fee for writing an } \\
\text { Expert Opinion }\end{array}$ & $\begin{array}{l}\text { Hoffman-La Roche } \\
\text { SmithKline Beecham } \\
\text { Pfizer } \\
\text { Flavour and Extract } \\
\text { Manufacturers } \\
\text { Association (FEMA) } \\
\text { American Chemistry } \\
\text { Council }\end{array}$ & $\begin{array}{l}\text { Research support } \\
\text { “ } \\
\text { “ } \\
\text { “ } \\
\text { “ }\end{array}$ \\
\hline $\begin{array}{l}\text { Dr Lesley Rushton } \\
\text { Head of Epidemiology, } \\
\text { Institute for } \\
\text { Environmental Health, } \\
\text { University of Leicester }\end{array}$ & None & & None & \\
\hline $\begin{array}{l}\text { Ms Barbara Saunders } \\
\text { Independent Consumer } \\
\text { Consultant }\end{array}$ & None & & None & \\
\hline $\begin{array}{l}\text { Ms Clare E Shaw } \\
\text { Chief Dietitian, Royal } \\
\text { Marsden NHS Trust }\end{array}$ & $\begin{array}{l}\text { Novartis Consumer } \\
\text { Health }\end{array}$ & $\begin{array}{l}\text { Fee for writing/editing } \\
\text { publication }\end{array}$ & None & \\
\hline $\begin{array}{l}\text { Dr Anita Thomas } \\
\text { Consultant Physician } \\
\text { and Associate Medical } \\
\text { Director (Education), } \\
\text { Plymouth Hospital NHS } \\
\text { Trust, Clinical Subdean } \\
\text { Peninsula Medical } \\
\text { School }\end{array}$ & None & & None & \\
\hline $\begin{array}{l}\text { Dr Anthony F Williams } \\
\text { Senior Lecturer and } \\
\text { Consultant in Neonatal } \\
\text { Paediatrics, St George's } \\
\text { Hospital, London }\end{array}$ & None & & None & \\
\hline
\end{tabular}




\section{Expert Group on Vitamins and Minerals Observers}

\section{Declaration of Interests}

\begin{tabular}{|c|c|c|c|c|}
\hline & \multicolumn{2}{|c|}{ Personal Interest } & \multicolumn{2}{|c|}{ Non-personal Interest } \\
\hline Observer & Company & Nature of Interest & Company & Nature of Interest \\
\hline $\begin{array}{l}\text { Ms Sue Davies } \\
\text { Principal Policy } \\
\text { Researcher, Consumers' } \\
\text { Association }\end{array}$ & $\begin{array}{l}\text { Albert Fisher } \\
\text { Marks and Spencer }\end{array}$ & $\begin{array}{l}\text { Shareholder } \\
\text { Shareholder }\end{array}$ & None & \\
\hline $\begin{array}{l}\text { Dr Marilyn Glenville } \\
\text { Nutritional Therapist/ } \\
\text { Psychologist }\end{array}$ & $\begin{array}{l}\text { The Natural Health } \\
\text { Practice Ltd. }\end{array}$ & Scientific Advisor & None & \\
\hline $\begin{array}{l}\text { Prof David Richardson } \\
\text { Specialist consultant in } \\
\text { nutrition and food } \\
\text { science, dprnutrition, } \\
\text { croydon }\end{array}$ & $\begin{array}{l}\text { Dprnutrition } \\
\text { Nestlé UK Ltd } \\
\text { Health Food } \\
\text { Manufacturers' } \\
\text { Association }\end{array}$ & $\begin{array}{l}\text { Salary } \\
\text { Fee } \\
\text { Fee }\end{array}$ & None & \\
\hline $\begin{array}{l}\text { Dr Derek Shrimpton } \\
\text { Consultant in Nutrition } \\
\text { and Food Science }\end{array}$ & $\begin{array}{l}\text { Unilever Plc } \\
\text { Henkel Ireland Ltd } \\
\text { Council for Responsible } \\
\text { Nutrition } \\
\text { Health Food } \\
\text { Manufacturers' } \\
\text { Association } \\
\text { Association of European } \\
\text { Health Food } \\
\text { Manufacturers } \\
\text { The Proprietary } \\
\text { Association of Great } \\
\text { Britain (PAGB) } \\
\text { Naturally Yours } \\
\text { Healthcare Ltd } \\
\text { Ingredients, Health and } \\
\text { Nutrition (Turret RAI) } \\
\text { International Food } \\
\text { Ingredients } \\
\text { US Council for } \\
\text { Responsible Nutrition }\end{array}$ & 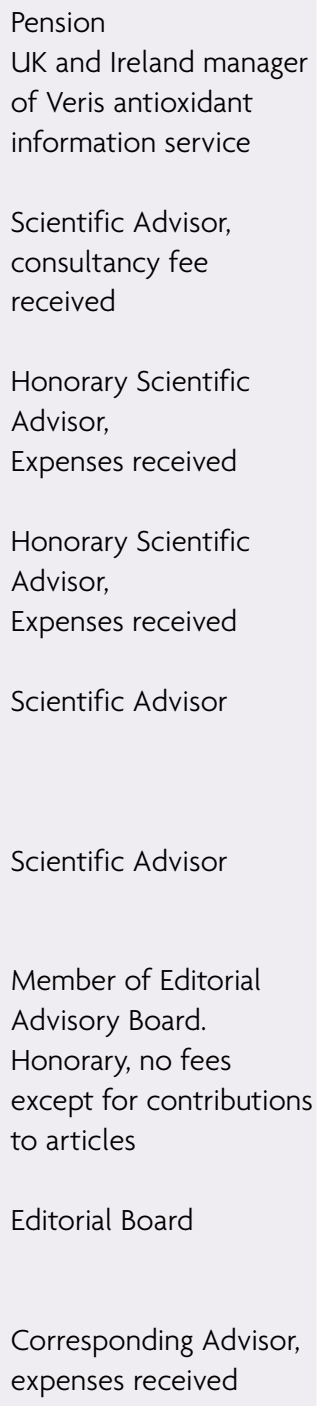 & None & \\
\hline
\end{tabular}


Annexes

\title{
Annex 6.
}

\section{List of respondents who commented on consultation}

\author{
A Revell \\ Alliance for Natural Health \\ Dr Anthony Dunstan Fox \\ Armyn Hennessy \\ Arthur Lightfoot \\ Boots Herbal Stores \\ Borax Europe Ltd \\ British Dietetic Association \\ Burckhard Viell \\ University of Capetown \\ The Caring Clinic \\ Dr C.J. Parsons \\ C McFarlane
}

Consensus Action on Salt and Health

Christina Panayiotou

Consumers for Health Choice

Council for Responsible Nutrition

Council for Responsible Nutrition (USA)

D Hartley

Dave Orr

David Grant

David Maddock

Dr David R Hawkins

Douglas Cooke

The Dove Clinic for Integrated Medicine

E.J. Bywater

Earl Kitchener

Dr Eberhard Schmidt

Dr Elizabeth Dickson

European Commission

European Scientific Advisory Group on Nutrients and Related Substances

F Kinch

Galvanizers Association

Gill Ward

Girton College

GlaxosmithKline Consumer Health Care 


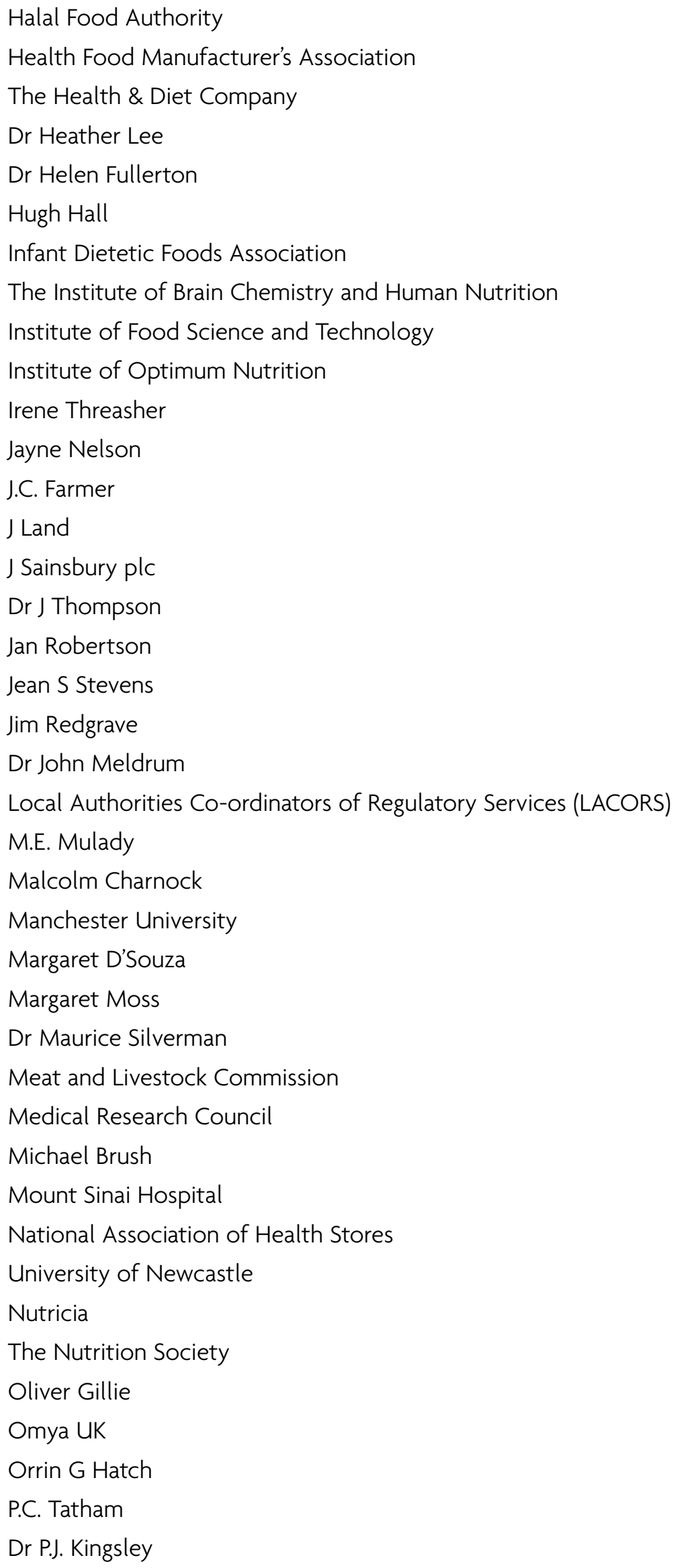


Annexes

Pat Heron

Pattern Computing plc

Peter Hinde

Proprietary Association of Great Britain

Quest Vitamins

Rachel Austin

Richard J Stanes

Robert Woodward

Dr Rodney Adeniyi-Jones

Rowett Research Institute

Royal College of General Practitioners

Royal College of Nursing

Dr Sarah Myhill

Scientific Advisory Committee on Nutrition

Sheila Gibson

Slimming Systems Ltd

University of Surrey

Dr Susan New

\section{T Dacres}

Mrs Tattersal

Tom Harkin

University of Toronto

Uni-Vite Healthcare

Valerie Elsworth

Vegetarian Economy \& Green Agriculture

Vitamin B6 Scientific Task Group

Wolfson Institute of Preventive Medicine 


\section{Annex 7}

\section{Abbreviations List}

ADI: $\quad$ Acceptable Daily Intake

AIDS: $\quad$ Acquired Immune Deficiency Syndrome

BMD: $\quad$ Bone mineral density

BMC: $\quad$ Bone mineral content

CNS: $\quad$ Central nervous systems

COMA: Committee on Medical Aspects of Food and Nutrition Policy

CoA: coenzyme A

COT: $\quad$ Committee on Toxicity of Chemicals in Food, Consumer Products and the Environment

CSM: $\quad$ Committee on Safety of Medicines

DNA: Deoxyribonucleic acid

DRV: $\quad$ Dietary Reference Value

EAR: $\quad$ Estimated Average Requirement

ESADDI: Estimated Safe and Adequate Daily Dietary Intake

EVM: The Expert Group on Vitamins and Minerals

FAC: $\quad$ The Food Advisory Committee

FAD: $\quad$ Flavin adenine dinucleotide

FAO: $\quad$ Food and Agriculture Organisation

FFQ: $\quad$ Food Frequency Questionnaire

FMN: $\quad$ Flavin mononucleotide

FSA: $\quad$ Food Standards Agency

GLP: $\quad$ Good Laboratory Practice

HDL: $\quad$ High density lipoprotein

IF: Intrinsic factor

IU: International Units

LDL: $\quad$ Low density lipoprotein

LOAEL: Lowest Observed Adverse Effect Level

LRNI: Lower Reference Nutrient Intake

MHRA: Medicines and Healthcare Products Regulatory Agency

NAD: Nicotinamide adenine dinucleotide

NADP: Nicotinamide adenine dinucleotide phosphate

NDNS: The National Diet and Nutrition Survey

NFS: $\quad$ The National Food Survey (From April 2001- Expenditure and Food Survey)

NOAEL: No Observed Adverse Effect Level

NTD: Neural tube defect

OECD: The Organisation for Economic Cooperation and Development 
Annexes

PUFA: $\quad$ Polyunsaturated fatty acid

RDA: Recommended Dietary Allowance

RDI: Recommended Dietary Intake

RE: $\quad$ Retinol equivalents

RNA: Ribonucleic acid

RNI: $\quad$ Reference Nutrient Intake

SACN: The Scientific Advisory Committee on Nutrition

SCE: $\quad$ Sister chromatid exchange

SCF: The EU Scientific Committee on Food

SUL: $\quad$ Safe Upper Level

TDS: $\quad$ Total Diet Study

THF: $\quad$ Tetrahydrofolates

TSH: $\quad$ Thyroid stimulating hormone

UF: $\quad$ Uncertainty factor

UV: $\quad$ Ultraviolet

WHO: World Health Organisation 


\section{Annex 8}

\section{Glossary}

Acceptable Daily Intake (ADI): Estimate of the amount of a substance in food or drink, expressed on a body weight basis (e.g. $\mathrm{mg} / \mathrm{kg}$ bodyweight), that can be ingested daily over a lifetime by humans without appreciable health risk.

Acute: Short term, in relation to exposure or effect.

Acute toxicity: Effects that occur over a short period of time (up to 14 days) immediately following exposure.

Adenoma: A benign growth (neoplasm) arising from a gland-forming surface tissue ( internal or external) tissue such as colon, stomach or respiratory tract.

ADI: see Acceptable Daily Intake

Adverse effect: Change in structure, function, biochemistry, growth, development or lifespan of an organism which results in impairment of capacity to function, or impairment of capacity to compensate for additional stress, or increase in susceptibility to the harmful effects of other environmental influences.

Allergy: The adverse health effects that may result from the stimulation of a specific immune response.

Allergic reaction: an adverse reaction elicited by exposure to a previously sensitised individual to the relevant substance or item causing the reaction (antigen).

Ames test: In vitro (qv) assay for bacterial gene mutations (qv) using strains of the bacterium Salmonella typhimurium developed by Ames and his colleagues.

Bias: In the context of epidemiological studies, bias is a systematic tendency to underestimate or overestimate the parameter of interest because of a deficiency in the design or execution of a study. It includes: selection bias in which subjects studied are not representative of the target population about which conclusions are to be drawn; information bias such as errors in measuring disease or exposure.

Bioavailability: A term referring to the proportion of a substance which reaches the systemic circulation unchanged after a particular route of administration.

Body burden: Total amount of a chemical present in an organism at a given time.

Cancer: Synonym for a malignant neoplasm - that is, a tumour (qv) that grows progressively, invades local tissues and spreads to distant sites (see also tumour and metastasis). 


\section{Annexes}

Carcinogenesis: The origin, causation and development of tumours (qv). The term applies to benign as well as malignant growths (neoplasms) and not just to carcinomas (qv).

Carcinogenicity study: Tests carried out in laboratory animals, usually rats and mice, to determine whether a substance causes cancer. The test material is given throughout life to groups of animals at different dose levels.

Carcinogens: The causal agents which induce tumours. They include external factors (chemicals, physical agents, viruses) and internal factors such as hormones. Chemical carcinogens are structurally diverse and include naturally-occurring substances as well as synthetic compounds. An important distinction can be drawn between genotoxic (qv) carcinogens which have been shown to react with and mutate DNA, and non-genotoxic carcinogens which act through other mechanisms. The activity of genotoxic carcinogens can often be predicted from their chemical structure - either of the parent compound or of active metabolites (qv). Most chemical carcinogens exert their effects after prolonged exposure, show a dose-response relationship and tend to act on a limited range of susceptible target tissues. Carcinogens are sometimes species- or sex-specific and the term should be qualified by the appropriate descriptive adjectives to aid clarity. Several different chemical and other carcinogens may interact, and constitutional factors (genetic susceptibility, hormonal status) may also contribute, emphasising the multifactorial nature of the carcinogenic process.

Carcinoma: Malignant tumour arising from epithelial cells lining, for example, the alimentary, respiratory and urogenital tracts and from epidermis, also from solid viscera such as the liver, pancreas, kidneys and some endocrine glands. (See also 'tumour').

Case-control study: (Synonyms - case comparison study, case referent study, retrospective study) A study which starts with the identification of a group of people with the disease of interest (the cases) and a suitable group of persons without the disease (the controls). A comparison is made of the proportion of cases that have been exposed to a particular hazard (e.g. a carcinogen) with the proportion of controls that have been exposed to the hazard.

Cell transformation: The process by which a normal cell acquires the capacity for neoplastic growth. Complete transformation occurs in several stages both in vitro and in vivo. One step which has been identified in vitro is 'immortalisation' by which a cell acquires the ability to divide indefinitely in culture. Such cells do not have the capacity to form tumours in animals, but can be induced to do so by extended passage in vitro, by treatment with chemicals, or by transfection with oncogene DNA. The transformed phenotype so generated is usually, but not always, associated with the ability of the cells to grow in soft agar and to form tumours when transplanted into animals. It should be noted that each of these stages of transformation can involve multiple events which may or may not be genetic. The order in which these events take place, if they occur at all, in vivo is not known.

Chromosome aberrations: Collective term of particular types of chromosome damage induced after exposure to exogenous chemical or physical agents which damage the DNA. 
Chromosome: In simple prokaryotic organisms, such as bacteria and most viruses, the chromosome consists of a single circular molecule of DNA containing the entire genetic material of the cell. In eukaryotic cells, the chromosomes are thread-like structures, composed mainly of DNA and protein, which are present within the nuclei of every cell. They occur in pairs, the numbers varying from one to more than 100 per nucleus in different species. Normal somatic cells in humans have 23 pairs of chromosomes, each consisting of linear sequences of DNA which are known as genes (qv).

Chronic effect: Consequence which develops slowly and has a long-lasting course (often but not always irreversible).

Chronic exposure: Continued exposures occurring over an extended period of time, or a significant fraction of the life-time of a human or animal.

Clearance: Volume of blood or plasma, or mass of an organ, effectively cleared of a substance by elimination (metabolism and excretion) in a given time interval. Total clearance is the sum or the clearances for each eliminating organ or tissue.

Cohort: A defined population that continues to exist through time.

Cohort study: (Synonyms - follow-up, longitudinal study) The study of a group of people defined at a particular point in time (the cohort), who have particular characteristics in common, such as a particular exposure. They are then observed over a period of time for the occurrence of disease. The rate at which the disease develops in the cohort is compared with the rate in a comparison population, in which the characteristics (e.g. exposure) are absent.

Complementary DNA (CDNA): CDNA is DNA that is synthesised in the laboratory from mRNA by reverse transcription. A CDNA is so-called because its sequence is the complement of the original mRNA sequence.

Confounding factor: (synonyms - confounding variable, confounder) An extraneous variable that satisfies BOTH of 2 conditions: (1) it is a risk factor for the disease under study (2) it is associated with the study exposure but is not a consequence of exposure. For example cigarette smoking is a confounding variable with respect to an association between alcohol consumption and heart disease. Failure to adjust for a confounding variable results in distortion of the apparent magnitude of the effect of the exposure under study. (In the example, smoking is a risk factor for heart disease and is associated with alcohol consumption but is not a consequence of alcohol consumption.)

Congeners: Related compounds varying in chemical structure but with similar biological properties.

Covalent binding: Chemical bonding formed by the sharing of an electron pair between two atoms. Molecules are combinations of atoms bound together by covalent bonds. 
Cytochrome P450 (CYP): An extensive family of haem-containing proteins involved in enzymic oxidation of a wide range of endogenous and xenobiotic (qv) substances and their conversion to forms that may be more easily excreted. In some cases the metabolites produced may be reactive and may have increased toxicity. In other cases the substances may be natural precursors of hormones (e.g. steroids).

DNA (Deoxyribonucleic Acid): The carrier of genetic information for all living organisms except the group of RNA viruses. Each of the 46 chromosomes in normal human cells consists of 2 strands of DNA containing up to 100,000 nucleotides, specific sequences of which make up genes (qv). DNA itself is composed of two interwound chains of linked nucleotides (qv).

Dose: Total amount of a substance administered to, taken or absorbed by an organism.

Endocrine modulator: Pertaining to hormones or to the glands that secrete hormones directly into the blood stream.

Epidemiology: Study of the distribution and the aetiology of disease in humans.

Epithelium: The tissue covering the outer surface of the body, the mucous membranes and cavities of the body.

Erythema: Reddening of the skin due to congestion of blood or increased blood flow in the skin.

Estrogen: (See oestrogen)

Exogenous: Arising outside the body.

Foetotoxic: Causing toxic, potentially lethal effects to the developing fetus.

Forestomach: The stomach in rodents consists of two separate regions - the forestomach and the glandular stomach. Only the glandular stomach is directly comparable to the human stomach.

Gavage: Administration of a liquid via a stomach tube, commonly used as a dosing method in toxicity studies.

Gene: The functional unit of inheritance: a specific sequence of nucleotides along the DNA molecule, forming part of a chromosome (qv).

Gene expression: The process by which the information in a gene is used to create proteins or polypeptides.

Genetic predisposition: Susceptibility to a disease which is related to a polymorphism, which may or may not result in actual development of the disease.

Genotoxic: The ability of a substance to cause DNA damage, either directly or after metabolic activation (see also carcinogens). 
Genotype: The particular genetic pattern seen in the DNA of an individual. 'Genotype' is usually used to refer to the particular pair of alleles that an individual possesses at a certain location in the genome. Compare this with phenotype.

Half-life: Time, in which the concentration of a substance will be reduced by half, assuming a first order elimination process.

Hazard: Set of inherent properties of a substance, mixture of substances or a process involving substances that make it capable of causing adverse effects to organisms or the environment.

Hepatic: Pertaining to the liver.

Hepatocyte: The principal cell type in the liver, possessing many metabolising enzymes (see 'metabolic activation').

Hepatotoxic: Causing toxicity to the liver.

Hyperplasia: An increase in the size of an organ or tissue due to an increase in the number of cells.

Hypertrophy: An increase in the size of an organ or tissue due to an increase in the volume of individual cells within it.

In vitro: A Latin term used to describe effects in biological material outside the living animal (literally 'in glass").

In vivo: A Latin term used to describe effects in living animals (literally "in life").

Incidence: Number of new cases of illness occurring during a given period in a specific population.

Intraperitoneal: Within the abdominal cavity.

Isomer: Isomers are two or more chemical compounds with the same molecular formula but having different properties owing to a different arrangement of atoms within the molecule. The $B$-isomer of alitame is formed when the compound degrades and the atoms within the molecule are rearranged.

LD50: The dose of a toxic compound that causes death in 50\% of a group of experimental animals to which it is administered. It can be used to assess the acute toxicity of a compound, but is being superseded by more refined methods.

Leukaemia: A group of neoplastic disorders (see tumour) affecting blood-forming elements in the bone marrow, characterised by uncontrolled proliferation and disordered differentiation or maturation. Examples include the lymphocytic leukaemias which develop from lymphoid cells and the myeloid leukaemias which are derived from myeloid cells (producing red blood cells, mainly in bone marrow). 
Ligand: A molecule which binds to a receptor.

Lipids: Fats, substances containing a fatty acid and soluble in alcohols or ether, but insoluble in water.

Lowest observed adverse effect level (LOAEL): The lowest administered dose at which an adverse (qv) effect has been observed.

Lymphocyte: A type of white blood cell that plays central roles in adaptive immune responses.

Lymphoma: Malignant tumours arising from lymphoid tissues. They are usually multifocal, involving lymph nodes, spleen, thymus and sometimes bone marrow, and other sites outside the anatomically defined lymphoid system. (See also 'tumour').

Malignancy: See 'tumour'.

Metabolism: Chemical modification of a compound by enzymes within the body, for example by reactions such as hydroxylation (see cytochrome P450), epoxidation or conjugation.

Metabolism may result in activation, inactivation, accumulation or excretion of the compound.

Metabolite: Product formed by metabolism of a compound.

Metaphase: Stage of cell division (mitosis and meiosis) during which the chromosomes are arranged on the equator of the nuclear spindle (the collection of microtubule filaments which are responsible for the movement of chromosomes during cell division). As the chromosomes are most easily examined in metaphase, cells are arrested at this stage for microscopic examination for chromosomal aberrations (qv) - known as metaphase analysis.

Metastasis: The process whereby malignant cells become detached from the primary tumour mass, disseminate (mainly in the blood stream or in lymph vessels) and 'seed out' in distant sites where they form secondary or metastatic tumours. Such tumours tend to develop at specific sites and their anatomical distribution is often characteristic; it is non-random.

Mouse lymphoma assay: An in vitro assay for gene mutation in mammalian cells using a mouse lymphoma cell line L5178Y, which is heterozygous for the gene (carries only one functional gene rather than a pair) for the enzyme thymidine kinase $\left(\mathrm{TK}^{+/-}\right)$. Mutation of that single gene is measured by resistance to toxic trifluorothymidine. Mutant cells produce two forms of colony - large, which represent mutations within the gene and small, which represent large genetic changes in the chromosome such as chromosome aberrations. Thus this assay can provide additional information about the type of mutation which has occurred if colony size is scored.

Mucosal: Regarding the mucosa or mucous membranes, consisting of epithelium (qv) containing glands secreting mucus, with underlying layers of connective tissue and muscle. 
Mutation: A permanent change in the amount or structure of the genetic material in an organism or cell, which can result in a change in phenotypic characteristics. The alteration may involve a single gene, a block of genes, or a whole chromosome. Mutations involving single genes may be a consequence of effects on single DNA bases (point mutations) or of large changes, including deletions, within the gene. Changes involving whole chromosomes may be numerical or structural. A mutation in the germ cells of sexually reproducing organisms may be transmitted to the offspring, whereas a mutation that occurs in somatic cells may be transferred only to descendent daughter cells.

Neoplasm: See 'tumour'.

Nephrotoxicity: Toxicity to the kidney.

Neurotoxicity: Toxicity to the nervous system.

No observed adverse effect level (NOAEL): The highest administered dose at which no adverse (qv) effect has been observed.

Non-genotoxic: See 'carcinogens'.

Nucleic acid: One of the family of molecules which includes the DNA and RNA molecules. Nucleic acids were so named because they were originally discovered within the nucleus of cells, but they have since been found to exist outside the nucleus as well.

Nucleotide: the 'building block' of nucleic acids, such as the DNA molecule. A nucleotide consists of one of four bases - adenine, guanine, cytosine, or thymine - attached to a phosphate-sugar group. In DNA the sugar group is deoxyribose, while in RNA (a DNA-related molecule which helps to translate genetic information into proteins), the sugar group is ribose, and the base uracil substitutes for thymine. Each group of three nucleotides in a gene is known as a codon. A nucleic acid is a long chain of nucleotides joined together, and therefore is sometimes referred to as a "polynucleotide."

Odds ratio (OR): The odds of disease in an exposed group divided by the odds of disease in an unexposed group.

Oedema: Excessive accumulation of fluid in body tissues.

Oestrogen: Sex hormone or other substance capable of developing and maintaining female characteristics of the body.

Parenteral nutrition: The administration of nutrients to patients who cannot maintain adequate nutrition themselves by a route other than the alimentary canal, e.g. intravenously.

Polymer: A very large molecule comprising a chain of many similar or identical molecular sub units (monomers) joined together (polymerised). An example is the polymer glycogen, formed from linked molecules of the monomer glucose. 


\section{Annexes}

Protoporphyrin: The most common of a rare group of disorders due to inborn errors of metabolism in which there are deficiencies in the enzymes involved in the biosynthesis of haem.

Receptor: A small, discrete protein in the cell membrane or within the cell with which specific molecules interact to initiate a change in the working of a cell.

Reference nutrient intake (RNI): An amount of the nutrient that is enough, or more than enough, for most (usually at least $97 \%$ ) of people in a group. If the average intake of a group is at the RNI, then the risk of deficiency in the group is very small.

Relative risk: A measure of the association between exposure and outcome. The rate of disease in the exposed population divided by the rate of disease among the unexposed population in a cohort study or a population-based case control study. A relative risk of 2 means that the exposed group has twice the disease risk compared to the unexposed group.

Renal: Relating to the kidney.

Risk: Possibility that a harmful event (death, injury or loss) arising from exposure to a chemical or physical agent may occur under specific conditions.

RNA (ribonucleic acid): a molecule similar to DNA (qv), which helps in the process of decoding the genetic information carried by DNA.

Safety: Practical certainty that injury will not result from a hazard under defined conditions.

SCF: The European Commission's Scientific Committee on Food (formerly the Scientific Committee for Food).

Sister chromatid exchange (SCE): Exchange of genetic material between two sub-units of a replicated chromosome.

Systematic review: A review that has been prepared using a documented systematic approach to minimising biases and random errors.

TDI: See ‘Tolerable Daily Intake’.

Teratogen: A substance which, when administered to a pregnant woman or animal, can cause congenital malformations (structural defects) in the baby or offspring.

Teratogenicity: Potential to cause structural malformations or defects in the offspring.

Threshold: Dose or exposure concentration below which an effect is not expected.

Tolerable Daily Intake (TDI): An estimate of the amount of contaminant, expressed on a body weight basis (e.g. $\mathrm{mg} / \mathrm{kg}$ bodyweight), that can be ingested daily over a lifetime without appreciable health risk. 
Toxicodynamics: The process of interaction of chemical substances with target sites and the subsequent reactions leading to adverse effects.

Toxicokinetics: The description of the fate of chemicals in the body, including a mathematical account of their absorption, distribution, metabolism and excretion. (see pharmacokinetics)

Tumour (Synonym - neoplasm): A mass of abnormal, disorganised cells, arising from preexisting tissue, which are characterised by excessive and uncoordinated proliferation and by abnormal differentiation. Benign tumours show a close morphological resemblance to their tissue of origin; grow in a slow expansile fashion; and form circumscribed and (usually) encapsulated masses. They may stop growing and they may regress. Benign tumours do not infiltrate through local tissues and they do not metastasise (qv). They are rarely fatal.

Malignant tumours (synonym - cancer) resemble their parent tissues less closely and are composed of increasingly abnormal cells in terms of their form and function. Well differentiated examples still retain recognisable features of their tissue of origin but these characteristics are progressively lost in moderately and poorly differentiated malignancies: undifferentiated or anaplastic tumours are composed of cells which resemble no known normal tissue. Most malignant tumours grow rapidly, spread progressively through adjacent tissues and metastasise to distant sites. Tumours are conventionally classified according to the anatomical site of the primary tumour and its microscopical appearance, rather than by cause. Some common examples of nomenclature are as follows:

- Tumours arising from epithelia (qv): benign - adenomas, papillomas; malignant adenocarcinomas, papillary carcinomas.

- Tumours arising from connective tissues such as fat, cartilage or bone: benign - lipomas, chondromas, osteomas; malignant - fibrosarcomas, liposarcomas, chondrosarcomas, osteosarcomas.

- Tumours arising from lymphoid tissues are malignant and are called lymphomas (qv); they are often multifocal. Malignant proliferations of bone marrow cells are called leukaemias.

Benign tumours may evolve to the corresponding malignant tumours; examples involve the adenoma $\rightarrow$ carcinoma sequence in the large bowel in humans, and the papilloma $\rightarrow$ carcinoma sequence in mouse skin.

Tumour promotion: An increasingly confusing term, originally used, like 'tumour initiation' to describe events in multistage carcinogenesis in experimental animals. In that context, promotion is regarded as the protracted process whereby initiated cells undergo clonal expansion to form overt tumours. The mechanisms of clonal expansion are diverse, but include direct stimulation of cell proliferation, repeated cycles of cell damage and cell regeneration and release of cells from normal growth-controlling mechanisms. Initiating and promoting agents were originally regarded as separate categories, but the distinction between them is becoming increasingly hard to sustain. The various modes of promotion are nongenotoxic, but it is incorrect to conclude that 'non-genotoxic carcinogen' (qv) and 'promoter' are synonymous. 
Annexes

Uncertainty factor: Value used in extrapolation from experimental animals to man (assuming that man may be more sensitive) or from selected individuals to the general population: for example, a value applied to the NOAEL to derive an ADI or TDI. The value depends on the size and type of population to be protected and the quality of the toxicological information available.

Xenobiotic: A chemical foreign to the biological system. 
(c) Crown copyright

Published by Food Standards Agency

May 2003

ISBN 1-904026-11-7

f19.95 